Anders, Yvonne; Barenthien, Julia; Hardy, Ilonca; ...

\title{
Wirkungen naturwissenschaftlicher Bildungsangebote auf pädagogische Fachkräfte und Kinder
}

\section{[1. Auflage]}

Opladen; Berlin : Verlag Barbara Budrich 2018, 314 S. - (Wissenschaftliche Untersuchungen zur Arbeit der Stiftung "Haus der Kleinen Forscher"; 10)

\section{Quellenangabe/ Reference:}

Anders, Yvonne; Barenthien, Julia; Hardy, Ilonca; Hartinger, Andreas; Kästner, Rahel; Leuchter, Miriam; Oppermann, Elisa; Pauen, Sabina; Rank, Astrid; Stetfensky, Mirjam; Taskinen, Päivi; Tietze, Sabrina; Wildemann, Anja; Ziegle, Tobias: Wirkungen naturwissenschaftlicher Bildungsangebote auf pädagogische Fachkräfte und Kinder. Opladen; Berlin : Verlag Barbara Budrich 2018, 314 S. (Wissenschaftliche Untersuchungen zur Arbeit der Stiftung "Haus der Kleinen Forscher"; 10) - URN: urn:nbn:de:0111-pedocs-178710 - DOI: 10.25656/01:17871

https://nbn-resolving.org/urn:nbn:de:0111-pedocs-178710

https://doi.org/10.25656/01:17871

in Kooperation mit / in cooperation with:

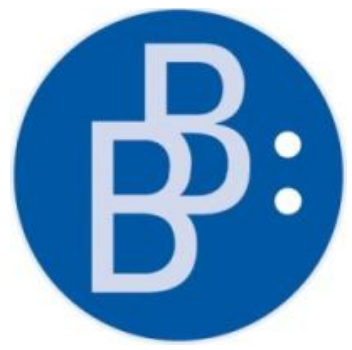

https://www.budrich.de

\section{Nutzungsbedingungen}

Dieses Dokument steht unter folgender Creative Commons-Lizenz: http://creativecommons.org/licenses/by-nc-nd/3.0/de/deed - Sie dürfen das Werk bzw. den Inhalt unter folgenden Bedingungen vervielfältigen, verbreiten und öffentlich zugänglich machen: Sie müssen den Namen des Autors/Rechteinhabers in der von ihm festgelegten Weise nennen. Dieses Werk bzw. dieser Inhalt darf nicht für kommerzielle Zwecke verwendet werden und es darf nicht bearbeitet, abgewandelt oder in anderer Weise verändert werden.

Mit der Verwendung dieses Dokuments erkennen Sie die

\section{Terms of use}

This document is published under following Creative Commons-License: http://creativecommons.org/licenses/by-nc-nd/3.0/de/deed.en - You may copy, distribute and transmit, adapt or exhibit the work in the public as long as you attribute the work in the manner specified by the author or licensor. You are not allowed to make commercial use of the work or its contents. You are not allowed to alter, transform, or change this work in any other way.

By using this particular document, you accept the above-stated conditions of use.

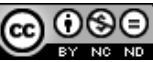

\section{Kontakt / Contact:}

\section{peDOcs}

DIPF | Leibniz-Institut für Bildungsforschung und Bildungsinformation

Informationszentrum (IZ) Bildung

E-Mail: pedocs@dipf.de

Internet: www.pedocs.de

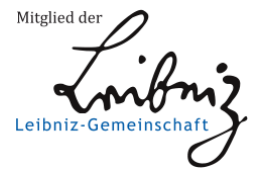


Yvonne Anders, Julia Barenthien, Iloncy Hardy, Andreas Hartinger, Rahel Kästner, Miriam Leuchter, Elisa Oppermann, Sabina Pauen,

Astrid Rank, Hans-Günther Roßbach, Mirjam Steffensky, Päivi Taskinen, Sabrina Tietze, Anja Wildemann, Tobias Ziegler

\section{Wirkungen naturwissenschaftlicher Bildungsangebote auf pädagogische Fachkräfte und Kinder}

Wissenschaftliche Untersuchungen zur Arbeit der Stiftung „Haus der kleinen Forscher“

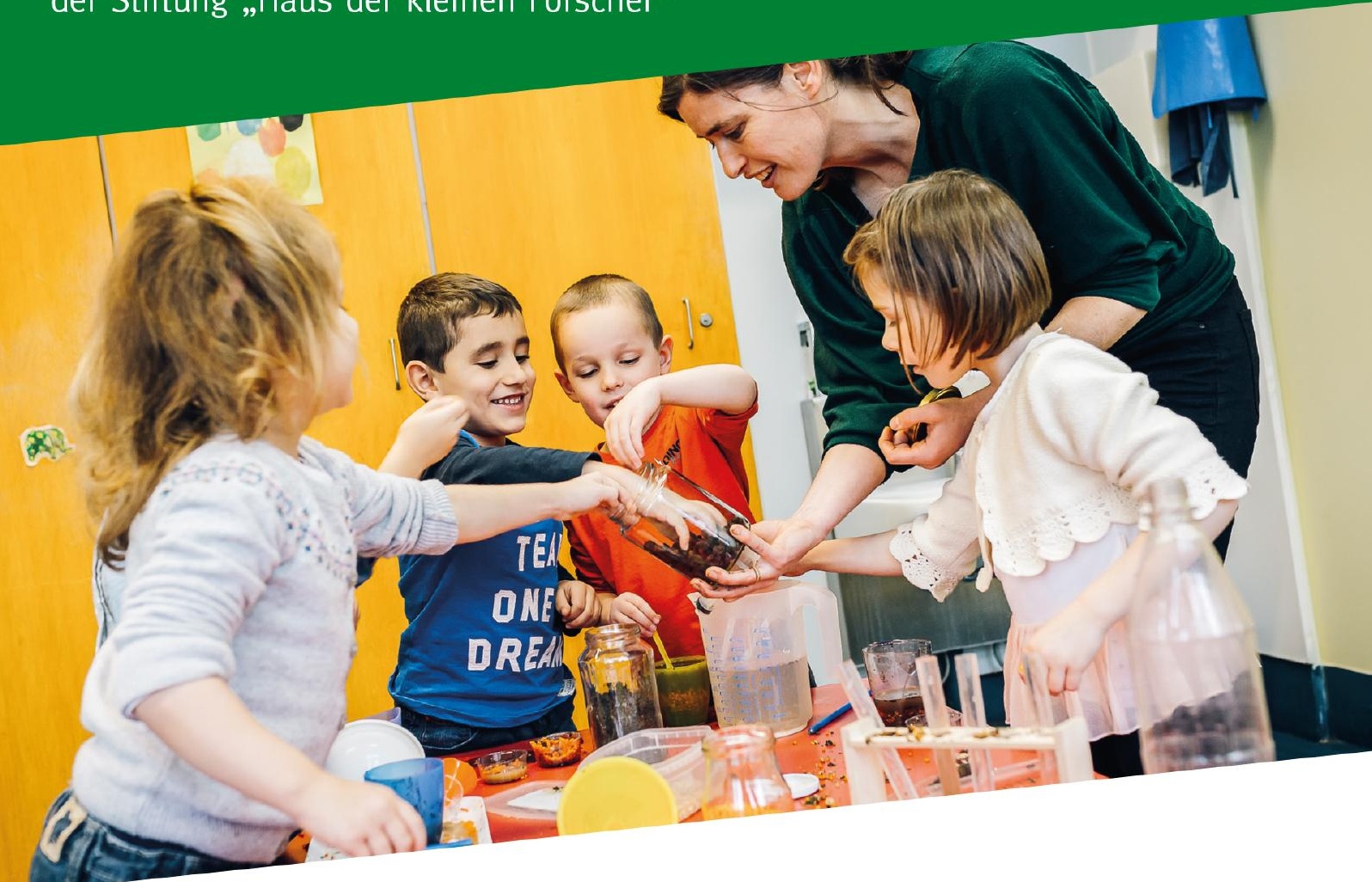

Verlag Barbara Budrich

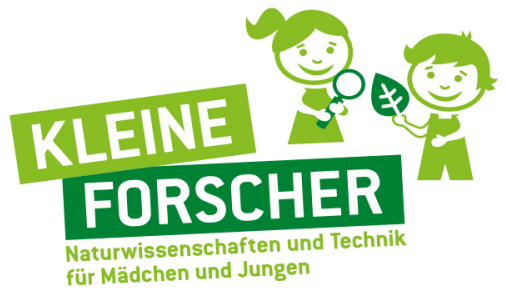


Wirkungen naturwissenschaftlicher Bildungsangebote auf pädagogische Fachkräfte und Kinder

Stiftung Haus der kleinen Forscher:

GEFÖRDERT VOM

PARTNER

Bundesministerium

Helmholtz-Gemeinschaft

für Bildung

Siemens Stiftung

Dietmar Hopp Stiftung

und Forschung

Deutsche Telekom Stiftung 
Wissenschaftliche Untersuchungen zur Arbeit der Stiftung „Haus der kleinen Forscher“

Band 10 
Stiftung Haus der kleinen Forscher (Hrsg.)

\section{Wirkungen naturwissenschaftlicher Bildungsangebote auf pädagogische Fachkräfte und Kinder}

Yvonne Anders, Julia Barenthien, Ilonca Hardy, Andreas Hartinger, Rahel Kästner, Miriam Leuchter, Elisa Oppermann, Sabina Pauen, Astrid Rank, Mirjam Steffensky, Päivi Taskinen, Sabrina Tietze, Anja Wildemann, Tobias Ziegler

Mit einem Geleitwort von Hans-Günther Roßbach

Verlag Barbara Budrich

Opladen • Berlin • Toronto 2018 
Herausgeber: Stiftung Haus der kleinen Forscher

Verantwortlich: Dr. Janna Pahnke

Projektleitung: Dr. Karen Bartling, Dr. Elena Harwardt-Heinecke

Konzeption und Redaktion: Dr. Elena Harwardt-Heinecke

Redaktionelle Mitarbeit: Irina Bitter, Karen Brünger, Dr. Paula Döge,

Victoria Escobar Heredia, Dr. Claudia Schiefer, Dr. Melanie Staats

Weitere Informationen finden Sie unter: www.haus-der-kleinen-forscher.de

Haben Sie Fragen, Anmerkungen oder Anregungen zu diesem Band oder

der wissenschaftlichen Begleitung der Stiftungsarbeit?

Wenden Sie sich an: forschung@haus-der-kleinen-forscher.de.

Weitere Informationen und Studienergebnisse finden Sie auch unter

www.haus-der-kleinen-forscher.de, Rubrik Wissenschaftliche Begleitung.

Bibliografische Information der Deutschen Nationalbibliothek

Die Deutsche Nationalbibliothek verzeichnet diese Publikation in der

Deutschen Nationalbibliografie; detaillierte bibliografische Daten sind im Internet über http://dnb.d-nb.de abrufbar.

(C) 2018 Dieses Werk ist im Verlag Barbara Budrich erschienen und steht unter folgender Creative Commons Lizenz: http://creativecommons.org/licenses/ by-nc-nd/3.0/de/

Verbreitung, Speicherung und Vervielfältigung erlaubt, kommerzielle Nutzung und Veränderung nur mit Genehmigung des Verlags Barbara Budrich

Dieses Buch steht im Open-Access-Bereich der Verlagsseite zum kostenlosen Download bereit (http://dx.doi.org/10.3224/84742274).

Eine kostenpflichtige Druckversion kann über den Verlag bezogen werden. Die Seitenzahlen in der Druck- und Onlineversion sind identisch.

$\begin{array}{ll}\text { ISBN } & 978-3-8474-2274-7 \\ \text { eISBN } & 978-3-8474-1349-3 \\ \text { DOI } & 10.3224 / 84742274\end{array}$

Umschlaggestaltung: Bettina Lehfeldt, Kleinmachnow - www.lehfeldtgraphic.de Titelbildnachweis: Christoph Wehrer/Stiftung „Haus der kleinen Forscher“ Lektorat und Satz: Ulrike Weingärtner, Gründau; info@textakzente.de Druck: SDK Systemdruck, Köln

Printed in Europe, gedruckt auf FSC-Papier 


\section{Inhaltsverzeichnis}

Informationen über die Autorinnen und Autoren ............. 9

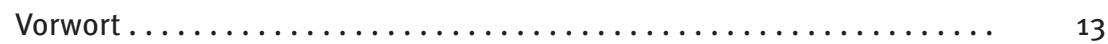

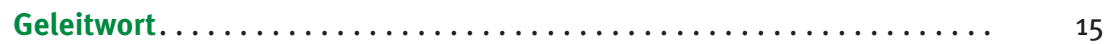

Hans-Günther Roßbach

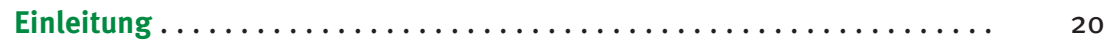

1 Überblick zur Stiftung „Haus der kleinen Forscher“ .......... 21

2 Das „N“ in MINT - Relevanz und Ziele früher naturwissenschaftlicher Bildung. ................ 30

3 Hintergründe der Studien zu Wirkungen naturwissenschaftlicher Bildungsangebote $\ldots \ldots \ldots \ldots \ldots \ldots .33$

Zusammenfassung zentraler Ergebnisse $\ldots \ldots \ldots \ldots \ldots \ldots \ldots \ldots .40$

Early Steps into Science - EASI Science

Wirkungen früher naturwissenschaftlicher Bildungsangebote auf die naturwissenschaftlichen Kompetenzen von Fachkräften und Kindern. . . . Mirjam Steffensky, Yvonne Anders, Julia Barenthien, Ilonca Hardy, Miriam Leuchter, Elisa Oppermann, Päivi Taskinen, Tobias Ziegler

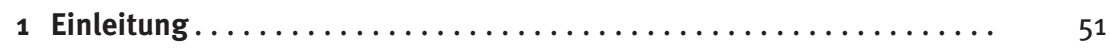

2 Theoretischer Hintergrund der Studie ................ 53

2.1 Wirkungen naturwissenschaftlicher Bildungsangebote in der Kita... 53

2.2 Naturwissenschaftliche Kompetenzen jüngerer Kinder ......... 56

2.3 Quantität und Qualität früher naturwissenschaftlicher Lerngelegenheiten ........................ 59

2.4 Naturwissenschaftliche Kompetenzen frühpädagogischer

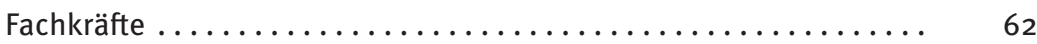

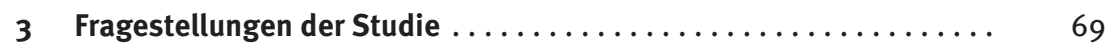

3.1 Erfassung und Struktur der Kompetenzen................. 69

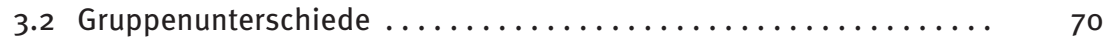

3.3 Zusammenhänge zwischen den Wirkungsebenen .......... 70

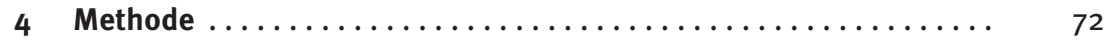

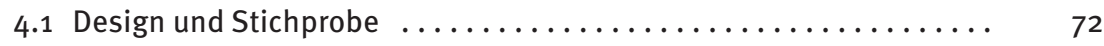

4.2 Variablen und Instrumente $\ldots \ldots \ldots \ldots \ldots \ldots \ldots \ldots \ldots \ldots \ldots \ldots \ldots \ldots$ 


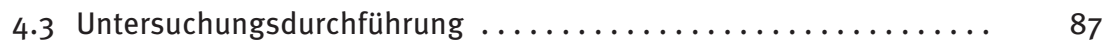

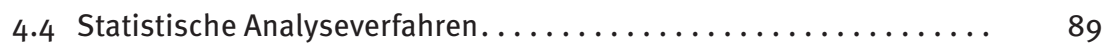

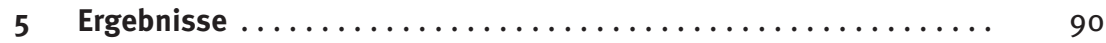

5.1 Erfassung und Struktur der Kompetenzen ............... 90

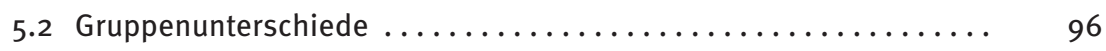

5.3 Zusammenhänge zwischen den Wirkungsebenen ........... 108

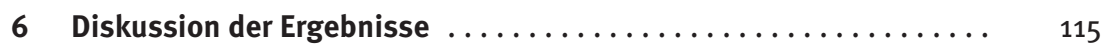

6.1 Struktur der Kompetenzen..................... 115

6.2 Gruppenunterschiede ........................ 120

6.3 Zusammenhänge zwischen den Wirkungsebenen ........... 124

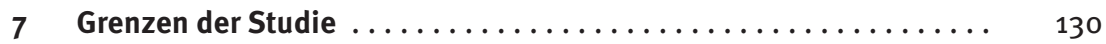

8 Zentrale Ergebnisse........................ 131

9 Folgerungen für die Stiftungsarbeit $\ldots \ldots \ldots \ldots \ldots \ldots \ldots \ldots \ldots$

Early Steps into Science and Literacy - EASI Science-L

Naturwissenschaftliche Bildung in der Kita: Gestaltung von

Lehr-Lern-Situationen, sprachliche Anregungsqualität und

sprachliche sowie naturwissenschaftliche Fähigkeiten der Kinder . . . . . . 138 Astrid Rank, Anja Wildemann, Sabina Pauen, Andreas Hartinger,

Sabrina Tietze, Rahel Kästner

Early Steps into Science and Literacy - EASI Science-L Teil 1

Wirkungen sprachlicher Anregungsqualität in naturwissenschaftlichen Bildungsangeboten auf die sprachlichen Fähigkeiten von Vorschulkindern . . . 140 Astrid Rank, Anja Wildemann, Andreas Hartinger, Sabrina Tietze

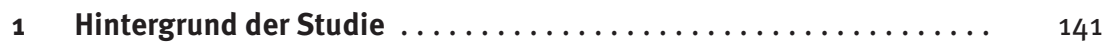

1.1 Bildungssprache und durchgängige Sprachbildung .......... 141

1.2 Bildungssprache im Kontext der kindlichen Sprachentwicklung. . . . . 145

1.3 Sprachliche Bildung durch naturwissenschaftliches Lernen....... 146

1.4 Naturwissenschaftliches Lernen und Sprachbildung im

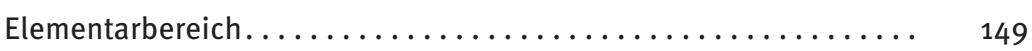

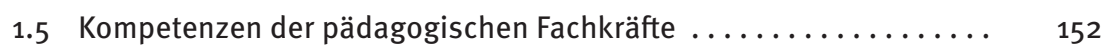

1.6 Zusammenfassung und Desiderata .................. 155 
2 Fragestellungen der Studie $\ldots \ldots \ldots \ldots \ldots \ldots \ldots \ldots \ldots \ldots \ldots \ldots$

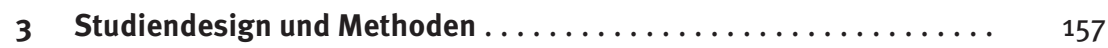

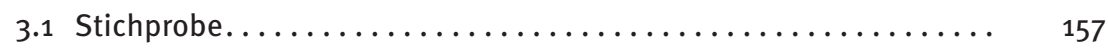

3.2 Durchführung der Erhebung.................. 166

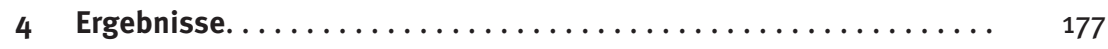

4.1 Deskriptive Ergebnisse $\ldots \ldots \ldots \ldots \ldots \ldots \ldots \ldots \ldots \ldots \ldots . \ldots \ldots$

4.2 Ergebnisse zu den Fragestellungen................. 179

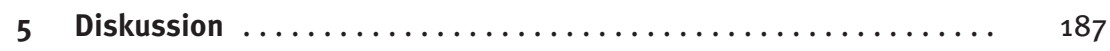

6 Zusammenfassung der zentralen Ergebnisse............. 190

$7 \quad$ Fazit und Empfehlungen $\ldots \ldots \ldots \ldots \ldots \ldots \ldots \ldots \ldots \ldots \ldots \ldots \ldots \ldots \ldots \ldots$

Early Steps into Science and Literacy - EASI Science-L Teil 2

Wirkungen naturwissenschaftlicher Bildungsangebote auf die

Gestaltung von Forschungssituationen durch pädagogische

Fachkräfte und auf die naturwissenschaftlichen Fähigkeiten

von Vorschulkindern ............................. 194

Sabina Pauen, Rahel Kästner

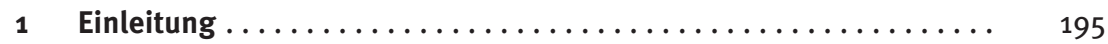

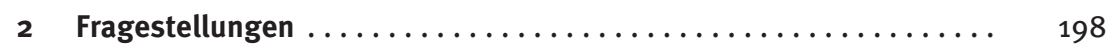

2.1 Fragestellung 1: Prozessqualität in Interaktionen und

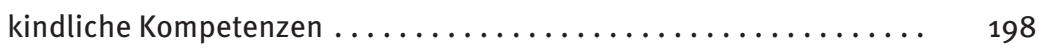

2.2 Fragestellung 2: Prozessqualität in Interaktionen und sprachliche Anregungsqualität. . . . . . . . . . . . . . 198

2.3 Fragestellung 3: Prozessqualität in Interaktionen und naturwissenschaftliche Vorbildung der Fachkräfte .......... 199

3 Datenerhebung und Methodik..................... 200

4 Entwicklung von QUOTS (Quality of Teaching Science) ......... 201

4.1 Beschreibung der fachkraftbezogenen QUOTS-Skalen ......... 201

4.2 Kindbezogene QUOTS-Skalen . . . . . . . . . . . . . . . 218

5 Zusammenhänge zwischen fachkraftspezifischen Kompetenzen und kindlichem Erkenntnisprozess. .............. 226

6 Prozessqualität in Interaktionen und sprachliche Anregungsqualität................. 230 
7 Prozessqualität in Interaktionen und naturwissenschaftliche Vorbildung der Fachkräfte

7.1 Das Fortbildungskonzept der Stiftung „Haus der kleinen Forscher“ und bisherige Wirkungsergebnisse ....

7.2 Stichproben von Fachkräften mit und ohne

Fortbildungserfahrung im naturwissenschaftlichen Bereich .......

7.3 Vergleich der QUOTS-Werte für Fachkräfte mit und ohne

Fortbildungserfahrung im naturwissenschaftlichen Bereich .......

7.4 Einfluss der Fortbildungen der Initiative

„Haus der kleinen Forscher“ auf das Scaffolding-Verhalten

8 Diskussion

8.1 Prozessqualität in Interaktionen und kindliche Kompetenzen ......

8.2 Prozessqualität in Interaktionen und sprachliche

Anregungsqualität

8.3 Prozessqualität in Interaktionen und naturwissenschaftliche

Vorbildung der Fachkräfte

9 Fazit und Empfehlungen

Fazit und Ausblick

Literatur

Anhang

Anhang I: Kodierung der Forschungskreisphasen

(EASI Science-L Teil 1)

Anhang II: Beschreibung der Indikatoren sprachlicher

Anregungsqualität (EASI Science-L Teil 1)

Bildquellenverzeichnis

Über die Stiftung „Haus der kleinen Forscher“

Bisher erschienen in der Wissenschaftlichen Schriftenreihe der Stiftung „Haus der kleinen Forscher“ 


\section{Informationen über die Autorinnen und Autoren}

\section{Prof. Dr. Yvonne Anders}

Freie Universität Berlin, Fachbereich für Erziehungswissenschaften und Psychologie, Arbeitsbereich Frühkindliche Bildung und Erziehung

Arbeitsschwerpunkte: Längsschnittanalysen der Auswirkungen frühkindlicher und schulischer Bildungsqualität, Professionelle Kompetenzen von (früh-)pädagogischen Fachkräften, Umgang mit Diversität im Kindergarten und der Schule, Internationale Vergleichsanalysen, Evaluationsforschung, Quantitative Methoden der empirischen Sozialforschung

Kontakt: Habelschwerdter Allee 45, 14195 Berlin, yvonne.anders@fu-berlin.de

\section{A. Julia Barenthien}

Leibniz-Institut für die Pädagogik der Naturwissenschaften und Mathematik an der Universität Kiel (IPN), Didaktik der Chemie

Arbeitsschwerpunkte: Professionswissen von pädagogischen Fachkräften im Elementarbereich, Lehren und Lernen von Naturwissenschaften im Kindergarten, Effekte professioneller Kompetenz pädagogischer Fachkräfte auf die Kompetenz von Kindergartenkindern

Kontakt: Olshausenstraße 62, 24118 Kiel, barenthien@ipn.uni-kiel.de

\section{Prof. Dr. Ilonca Hardy}

Goethe-Universität Frankfurt am Main, Fachbereich Erziehungswissenschaften, Institut für Pädagogik der Elementar- und Primarstufe, Arbeitsbereich Grundschulpädagogik mit Schwerpunkt Empirische Bildungsforschung

Arbeitsschwerpunkte: Kognitive Strukturierung von Lernprozessen, Fremdsprachliches Lernen und Zweisprachigkeit in vorschulischer und schulischer Bildung, Entwicklung und Förderung naturwissenschaftlicher Kompetenzen

Kontakt: Theodor-W.-Adorno-Platz 6, 60323 Frankfurt am Main, hardy@em. uni-frankfurt.de

\section{Prof. Dr. Andreas Hartinger}

Universität Augsburg, Philosophisch-Sozialwissenschaftliche Fakultät, Lehrstuhl für Grundschulpädagogik und Grundschuldidaktik

Arbeitsschwerpunkte: Frühes naturwissenschaftliches Lernen, Interessenförderung, Adaptives Unterrichten, Lehrerprofessionalität, Lehren und Lernen in jahrgangsgemischten Klassen

Kontakt: Universitätsstraße 10, 86135 Augsburg, andreas.hartinger@phil. uni-augsburg.de 


\section{M.Sc. Rahel Kästner}

Universität Heidelberg, Psychologisches Institut, Lehrstuhl für Entwicklungspsychologie und Biologische Psychologie

Arbeitsschwerpunkte: Scaffolding und Prozessqualität im naturwissenschaftlichen Lehr- und Lernprozess, Quantitative und Qualitative Interaktionsanalyse Kontakt: Hauptstr. 47-51, 69117 Heidelberg, rahel.kaestner@psychologie. uni-heidelberg.de

\section{Prof. Dr. Miriam Leuchter}

Universität Koblenz-Landau, Fachbereich 5: Erziehungswissenschaften, Institut für Bildung im Kindes- und Jugendalter, Arbeitsbereich Grundschulpädagogik ${ }^{1}$ Arbeitsschwerpunkte: Naturwissenschaftliches Lernen und Lehren in Vor- und Grundschule, Vorschulische Bildung, Conceptual Change, Professionelles Wissen und Überzeugungen von Lehrpersonen, Videobasierte Unterrichtsforschung Kontakt: August-Croissant-Straße 5, 76829 Landau, leuchter@uni-landau.de

\section{Dr. Elisa Oppermann}

Freie Universität Berlin, Fachbereich für Erziehungswissenschaften und Psychologie, Arbeitsbereich Frühkindliche Bildung und Erziehung

Arbeitsschwerpunkte: Bedingungsfaktoren und Wirkung früher naturwissenschaftlicher Förderung, Leistungsmotivation im Vorschulalter, Motivationale und pädagogische Überzeugungen von pädagogischen Fachkräften, Geschlechterunterschiede im Kontext früher mathematischer und naturwissenschaftlicher Bildung Kontakt: Habelschwerdter Allee 45, 14195 Berlin, elisa.oppermann@fu-berlin.de

\section{Prof. Dr. Sabina Pauen}

Universität Heidelberg, Lehrstuhl für Entwicklungspsychologie und Biologische Psychologie

Arbeitsschwerpunkte: Konzepterwerb in früher Kindheit, Die Rolle von Kausalverständnis für den frühen Wissenserwerb, Einfluss von Funktionswissen auf die Bildung von Artefaktkategorien, Theory of Mind - Entwicklung, Neurologische Korrelate der „Animate-Inanimate-Distinction“, Sozial-kognitive Entwicklung bei dysfunktionalen und gesunden Mutter-Kind-Interaktionen, Kategorienerwerb und -verlust über die Lebensspanne, Exekutive Funktionen und Problemlösen, Entwicklung der Selbstregulation, Beobachtung und Dokumentation frühkindlicher Entwicklung in der Praxis

1 Ehemals Westfälische Wilhelms-Universität Münster, Didaktik des Sachunterrichts, Leonardo-Campus 11, 48149 Münster. Dort wurde die Studie EASI Science von 2013 bis 2016 durchgeführt. 
Kontakt: Hauptstraße 47-51, 69117 Heidelberg, sabina.pauen@psychologie. uni-heidelberg.de

\section{Prof. Dr. Astrid Rank}

Universität Regensburg, Fakultät für Psychologie, Pädagogik und Sportwissenschaften, Institut für Pädagogik, Lehrstuhl für Pädagogik (Grundschulpädagogik) Arbeitsschwerpunkte: Sprachbildung in Sachsituationen/Bildungssprache, Kompetenzentwicklung (vor allem im Kontext von Heterogenität und Inklusion), Bildung für nachhaltige Entwicklung (vor allem Ökologie und Ökonomie), Wirksamkeit von Aus- und Fortbildung bei Lehrkräften und Erzieherinnen, Situiertes Lernen Kontakt: Universitätsstraße 31, 93053 Regensburg, astrid.rank@ur.de

\section{Prof. Dr. Hans-Günther Roßbach}

Universität Bamberg, Fakultät für Humanwissenschaften, Institut für Erziehungswissenschaften, ehemaliger Inhaber des Lehrstuhls für Elementar- und Familienpädagogik

Arbeitsschwerpunkte: Qualitätsfeststellung in Institutionen der Früherziehung, Curricularentwicklung im Kindergarten/Bildungsfragen im Kindergarten, Übergang vom Elementar- in den Primarbereich, Längsschnittanalysen der Auswirkungen frühkindlicher Betreuungen, Internationale Vergleichsuntersuchungen, Unterrichtsqualität in der Grundschule

Kontakt: An der Weberei 5, 96047 Bamberg, hans-guenther.rossbach@uni-bamberg.de

\section{Prof. Dr. Mirjam Steffensky}

Leibniz-Institut für die Pädagogik der Naturwissenschaften und Mathematik an der Universität Kiel (IPN), Didaktik der Chemie (Schwerpunkt Frühe naturwissenschaftliche Bildung)

Arbeitsschwerpunkte: Frühe naturwissenschaftliche Bildung, Fachbezogene professionelle Kompetenz von pädagogischen Fachkräften und Lehrpersonen Kontakt: Olshausenstraße 62, 24118 Kiel, steffensky@ipn.uni-kiel.de

\section{Dr. Päivi Taskinen}

Friedrich-Schiller-Universität Jena, Lehrstuhl für Pädagogische Psychologie Arbeitsschwerpunkte: Berufspräferenzen und Motivation von Jugendlichen, besonders im naturwissenschaftlichen Bereich, Einfluss des Elternhauses in Bildungsprozessen, auffälliges Sozialverhalten von Schülerinnen und Schülern Kontakt: Am Planetarium 4, 07743 Jena, p.taskinen@uni-jena.de 


\section{M.A. Sabrina Tietze}

Pädagogische Hochschule Freiburg, Institut für Psychologie, Abteilung Entwicklungspsychologie/Diagnostik

Arbeitsschwerpunkte: Bildungssprachliches Interaktionsverhalten, Professionelle Wahrnehmung, Professionsentwicklung

Kontakt: Kunzenweg 21, 79117 Freiburg, sabrina.tietze@ph-freiburg.de

\section{Prof. Dr. Anja Wildemann}

Universität Koblenz-Landau, Fachbereich 5: Erziehungswissenschaften, Institut für Bildung im Kindes- und Jugendalter, Arbeitsbereich Grundschulpädagogik Arbeitsschwerpunkte: Mehrsprachigkeit, Sprachbewusstheit, Sprachlicher Anfangsunterricht, Sprachliches Lernen in Sachsituationen

Kontakt: August-Croissant-Straße 5, 76829 Landau, wildemann@uni-landau.de

\section{Dipl.-Päd. Dipl.-Pol. Tobias Ziegler}

Goethe-Universität Frankfurt am Main, Fachbereich Erziehungswissenschaften, Institut für Pädagogik der Elementar- und Primarstufe, Arbeitsbereich Grundschulpädagogik mit Schwerpunkt Empirische Bildungsforschung. Er war bis 2017 wissenschaftlicher Mitarbeiter und ist derzeit noch Promovend.

Arbeitsschwerpunkte: Naturwissenschaftliche Lerngelegenheiten im Elementarund Primarbereich, Konzept- und prozessbezogene Aspekte naturwissenschaftlicher Kompetenz im Kindesalter, Fachbezogene Komponenten professioneller Kompetenz von pädagogischen Fachkräften

Kontakt: Theodor-W.-Adorno-Platz 6, 60323 Frankfurt am Main, tobiasziegler7@ gmail.com 


\section{Vorwort}

\section{Liebe Leserinnen und Leser,}

das „Haus der kleinen Forscher“ hat sich immer schon als lernende Organisation verstanden. Vor der Erstellung jedes einzelnen unserer Angebote fragen wir uns:

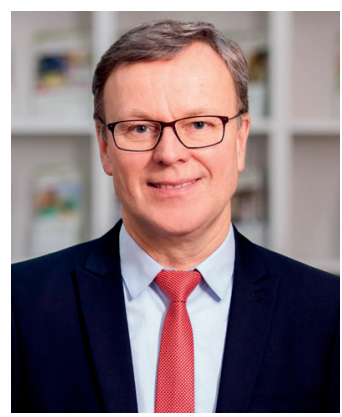
Welche Wirkung wollen wir erzielen? Wie erreichen wir das? Und wir schauen ganz genau hin, wenn es darum geht, ob unsere Arbeit etwas bewirkt.

Daher arbeiten wir seit je her fachlich fundiert: Die Stiftung lässt sich einerseits durch externe Partner wissenschaftlich begleiten und beraten. Wir reflektieren gemeinsam mit ihnen unsere Angebotsentwicklung. Andererseits nutzen wir das stiftungsinterne Monitoring zur Auswertung und fundierten Weiterentwicklung unserer Arbeit. Mit diesem Band der wissenschaftlichen Schriftenreihe stellt das „Haus der kleinen Forscher“ nun zwei umfangreiche externe Studien zu den Wirkungen naturwissenschaftlicher Bildungsangebote vor: Early Steps into Science (EASI Science) und Early Steps into Science and Literacy (EASI Science-L). Ein großer Dank gilt dem Bundesministerium für Bildung und Forschung, der Baden-Württemberg Stiftung und der Siemens Stiftung. Dank ihrer Förderung konnten die Studien ausgeschrieben und durchgeführt werden.

Die beiden Studien gingen der Frage nach, wie naturwissenschaftliche Bildungsangebote mit naturwissenschaftsbezogenen Kompetenzen von Fachkräften und Kindern zusammenhängen. Und ja: Sie zeigen, dass Fortbildungen wirken.

Oder um es konkreter auszudrücken: Fachkräfte, die Fortbildungen besucht haben, verfügen über ein höheres Fachwissen als nicht fortgebildete Fachkräfte. Kontinuierliche Fortbildungsbesuche haben auch zur Folge, dass die Erzieherinnen und Erzieher häufiger mit den Kindern forschen und den Mädchen und Jungen bei diesem Prozess mehr Freiräume lassen.

Eine weitere wichtige Erkenntnis haben wir dank der Studie EASI Science-L gewonnen: Pädagogisches Personal, das zuvor an naturwissenschaftlichen Fortbildungen teilgenommen hat, gestaltet sprachlich anregendere Lerngelegenheiten für Kinder. Am größten waren die sprachlichen Fähigkeiten der Kinder, wenn die sie betreuenden Fachkräfte zuvor eine kombinierte Fortbildung zu Naturwissenschaften und Sprache besucht hatten - wie es in der „Haus der kleinen Forscher“-Fortbildung zum Thema „Sprudelgas“ der Fall ist. 
Das sind wichtige und wegweisende Erkenntnisse. Doch wir machen hier nicht Halt, sondern werden weiter an unserer Qualitätsentwicklung arbeiten und reflektieren - zusammen mit unseren Partnern, die uns begleiten und beraten.

Michael Fritz

Vorstandsvorsitzender der Stiftung „Haus der kleinen Forscher“ 


\title{
Geleitwort
}

\author{
Von Hans-Günther Roßbach
}

Eine Besonderheit der Stiftung „Haus der kleinen Forscher“ ist, dass neben kontinuierlichen stiftungsinternen Monitoring- und Evaluationsmaßnahmen zu Zwecken der Qualitätssicherung und der Qualitätsentwicklung die Stiftungsarbeit durch externe Begleitforschung mit renommierten Partnern fachlich fundiert und in Forschungsprojekten untersucht wird. Mit der wissenschaftlichen Begleitforschung verfolgt die Stiftung das Ziel, durch einen externen Blick die Qualität ihrer Angebote und deren Wirkung zu beurteilen und Empfehlungen für die Weiterentwicklung zu erhalten. In den Studien Early Steps into Science (EASI Science) und Early Steps into Science and Literacy (EASI Science-L), deren Abschlussberichte im vorliegenden zehnten Band der Wissenschaftlichen Schriftenreihe der Stiftung „Haus der kleinen Forscher“ veröffentlicht sind, untersuchen zwei unabhängige Forschergruppen die Wirkungen naturwissenschaftlicher Bildungsangebote auf naturwissenschaftliche Kompetenzen von Fachkräften und Kindern sowie die Wirkungen sprachlicher Anregungsqualität in diesen Angeboten auf die sprachlichen Fähigkeiten von Vorschulkindern. Im Geleitwort können die vielfältigen Ergebnisse nicht angemessen zusammengefasst werden. Nur so viel: Die naturwissenschaftlichen Fortbildungsangebote der Stiftung stehen zum einen in Zusammenhang mit naturwissenschaftlichen Kompetenzen der Fachkräfte und der Kinder, zum anderen aber auch mit der sprachlichen Anregungsqualität durch Fachkräfte beim Entdecken und Forschen (teilweise auch mit den bildungssprachlichen Kompetenzen der Kinder). Zudem konnte mit dem Forschungsinstrument „Quality of Teaching Science - QUOTS“ ein Erhebungsverfahren entwickelt und getestet werden, das es erlaubt, die Prozessqualität in Forschungssituationen objektiv, reliabel und valide zu erfassen.

Die Befunde in den Studien können als Ermutigung für die Fortsetzung der Stiftungsarbeit interpretiert werden, aber auch für eine Fortsetzung der externen wissenschaftlichen Begleitforschung. Durchaus lassen die durchgeführten Studien - das ist nicht als Kritik an ihnen gemeint - einige zukünftige Herausforderungen für die Begleitforschung erkennen. Ohne Anspruch auf Vollständigkeit möchte ich vier Aspekte ansprechen: 
1. Was ist mit Wirkung gemeint? Letztlich sind damit Veränderungen in den Kindertageseinrichtungen, bei den Fachkräften und bei den Kindern gemeint, die durch die Teilnahme an einer „Haus der kleinen Forscher“-Fortbildung hervorgerufen werden. Allerdings sind in den vorliegenden Studien - wie auch bei anderen Untersuchungen von Auswirkungen der Qualität einer Kindertageseinrichtung auf die Entwicklung der Kinder - die Effekte eher niedrig. Technisch würden wir von kleinen Effekten sprechen. Aber was ist realistisch? Die kindliche Entwicklung wird von vielen Bedingungen - u. a. in der Familie - beeinflusst, und die Erfahrungen in Kindertageseinrichtungen sind nur eine Bedingung unter verschiedenen. Wir müssen hier realistisch sein und nicht übertriebenen Erwartungen hinterherträumen. Aber sind die Effekte wirklich so niedrig? Wir finden in verschiedenen Untersuchungen, dass die Unterschiede zwischen Familien etwa dreimal so bedeutsam für die Vorhersage der kindlichen Entwicklung sind wie die Unterschiede zwischen den Kindertageseinrichtungen. Und in der Familie sind genetische Aspekte und die häusliche Anregungsqualität konfundiert. In einer Untersuchung in den USA fand man heraus, dass während der vorschulischen Zeit der Effekt eines längeren Besuchs einer Kindertageseinrichtung auf die kindliche Entwicklung etwa halb so groß war wie der Effekt von Armut (NICHD, 2002). So unbedeutend sind die Effekte dann doch nicht.

2. Das Angebot der Stiftung „Haus der kleinen Forscher“ arbeitet mit einer langen Wirkungskette, vereinfacht: Fortbildung von Multiplikatoren - Fortbildung der Fachkräfte vor Ort - Auswirkungen des Besuchs der Fortbildung auf die teilnehmenden Fachkräfte - Hineintragen der Fortbildungsinhalte in die Einrichtungen durch die fortgebildeten Fachkräfte - Auswirkungen auf Quantität und Qualität naturwissenschaftlicher Lerngelegenheiten - Auswirkungen auf die Kompetenzen und Entwicklung der Kinder. Auf jeder Ebene kann es „Reibungsverluste“ geben. So ist aus der Studie EASI Science nur wenig über die Schwerpunkte und Qualität der Fortbildungen der Fachkräfte bekannt. Unterschiede zeigten sich bei der Implementation naturwissenschaftlicher Bildung in den Kindertageseinrichtungen. Fachkräfte, die Fortbildungen beim „Haus der kleinen Forscher" besucht haben, geben die Inhalte genauso häufig ins Team weiter wie Fachkräfte, die andere Fortbildungen besucht haben. In „Haus der kleinen Forscher“-Einrichtungen ist dagegen die Öffnung nach außen stärker ausgeprägt, auch finden häufiger Teamsitzungen zu naturwissenschaftlichen Themen statt als in Kindertageseinrichtungen, deren Fachkräfte andere oder bisher keine naturwissenschaftlichen Fortbildungen besucht haben. Zukünftige Begleitforschungen (in internem Monitoring und Evaluation sowie in externen Studien) sollten die gesamte Wirkungskette ins Auge fassen, um evidenzbasierte Hinweise auf Verbesserungsmöglichkeiten zu finden. Zudem sollte in der Begleit- 
forschung der Blick nicht nur auf den bisherigen personenbezogenen Ansatz der Stiftung gerichtet werden; vielmehr sollte stärker ein systemischer Ansatz verfolgt werden, der die institutionelle Verankerung von Fortbildungen in „Haus der kleinen Forscher“-Einrichtungen und dabei auch die Leitungsebene der Kindertageseinrichtungen betrachtet.

3. Die Studien zeigen, dass die Anzahl der besuchten Fortbildungsveranstaltungen bedeutsam ist. Insgesamt besuchen Fachkräfte der „Haus der kleinen Forscher"-Gruppe mehr Fortbildungen als die naturwissenschaftliche Vergleichsgruppe. Allerdings haben die in der Studie untersuchten pädagogischen Fachkräfte in „Haus der kleinen Forscher“-Einrichtungen im Durchschnitt nur an 2,3 Fortbildungen in den letzten drei Jahren teilgenommen (bei 5 bis 7 Stunden pro Thema). Dies scheint doch wenig (Egert, 2015) ${ }^{2}$. Damit stellt sich zum einen die Frage, wie die Fortbildungsquantität in Initiativen wie dem „Haus der kleinen Forscher“ erhöht werden kann, um ihre Wirkungen auf Fachkräfte und Kinder zu verstärken. Zum anderen ergibt sich die Herausforderung an die Begleitforschung, die Auswirkungen der Teilnahmedauer an Weiterbildungsprogrammen genauer zu untersuchen. Monitoring-Daten der Stiftung liefern erste Hinweise auf positive Effekte bei Fortbildungsteilnehmenden mit längerer Teilnahmedauer (Stiftung Haus der kleinen Forscher, 2017).

4. Wie bereits unter 1. gesagt, bezieht sich der Begriff „Wirkungen“ auf die Erfassung von Veränderungen, die durch Teilnahme an Bildungsangeboten hervorgerufen werden. Die vorliegenden Untersuchungen sind allerdings nur Querschnittsuntersuchungen und können somit im strengen Sinne keine Veränderungen erfassen. Obwohl plausibel, sind die Wirkrichtungen nicht abgesichert. So könnte auch der naturwissenschaftliche Entwicklungsstand der Kinder bei den Fachkräften eine anspruchsvollere naturwissenschaftliche Anregungsqualität hervorrufen (und nicht umgekehrt) oder Fachkräfte mit mehr Motivation zudem mehr Fortbildungen besuchen (und nicht umgekehrt). Um Wirkungen und die Wirkrichtung zu erfassen, sind Längsschnittuntersuchungen erforderlich, in denen z. B. Kompetenzen der Fachkräfte vor und einige Zeit nach dem Besuch einer Fortbildung erhoben werden. Dies wird eine zukünftige Aufgabe für die weitere wissenschaftliche Begleitung sein.

2 Nach der Meta-Analyse von Egert (2015) haben sich Fortbildungen mit 40 bis 60 Stunden Dauer als am effektivsten erwiesen. 
Die Initiative „Haus der kleinen Forscher“ bietet ein umfangreiches Fortbildungsangebot an, das ich nur begrüßen kann. Unter den Angeboten für Fachkräfte in Kindertageseinrichtungen und Grundschule nehmen die Stiftung und die Angebote der Bildungsinitiative „Haus der kleinen Forscher“ eine herausragende Stellung ein. Sowohl stiftungsinternes Monitoring und stiftungsinterne Evaluation als auch externe Begleitforschung ergänzen sich und sind notwendige Bestandteile einer kontinuierlichen Strategie zur Selbstvergewisserung, Selbstkritik und Weiterentwicklung. Und die in diesem Band berichteten Forschungsergebnisse unterstützen die bisherige Bildungsarbeit. Vor diesem Hintergrund sind die genannten vier zukünftigen Herausforderungen zu verstehen. Ich wünsche Ihnen viele Einsichten in die Bildungsarbeit der Stiftung und viel Vergnügen beim Lesen dieses Bandes.

Prof. Dr. Hans-Günther Roßbach

Universität Bamberg; Vorsitzender des Wissenschaftlichen Beirats der Stiftung „Haus der kleinen Forscher“ 



\section{Einleitung}

Stiftung Haus der kleinen Forscher

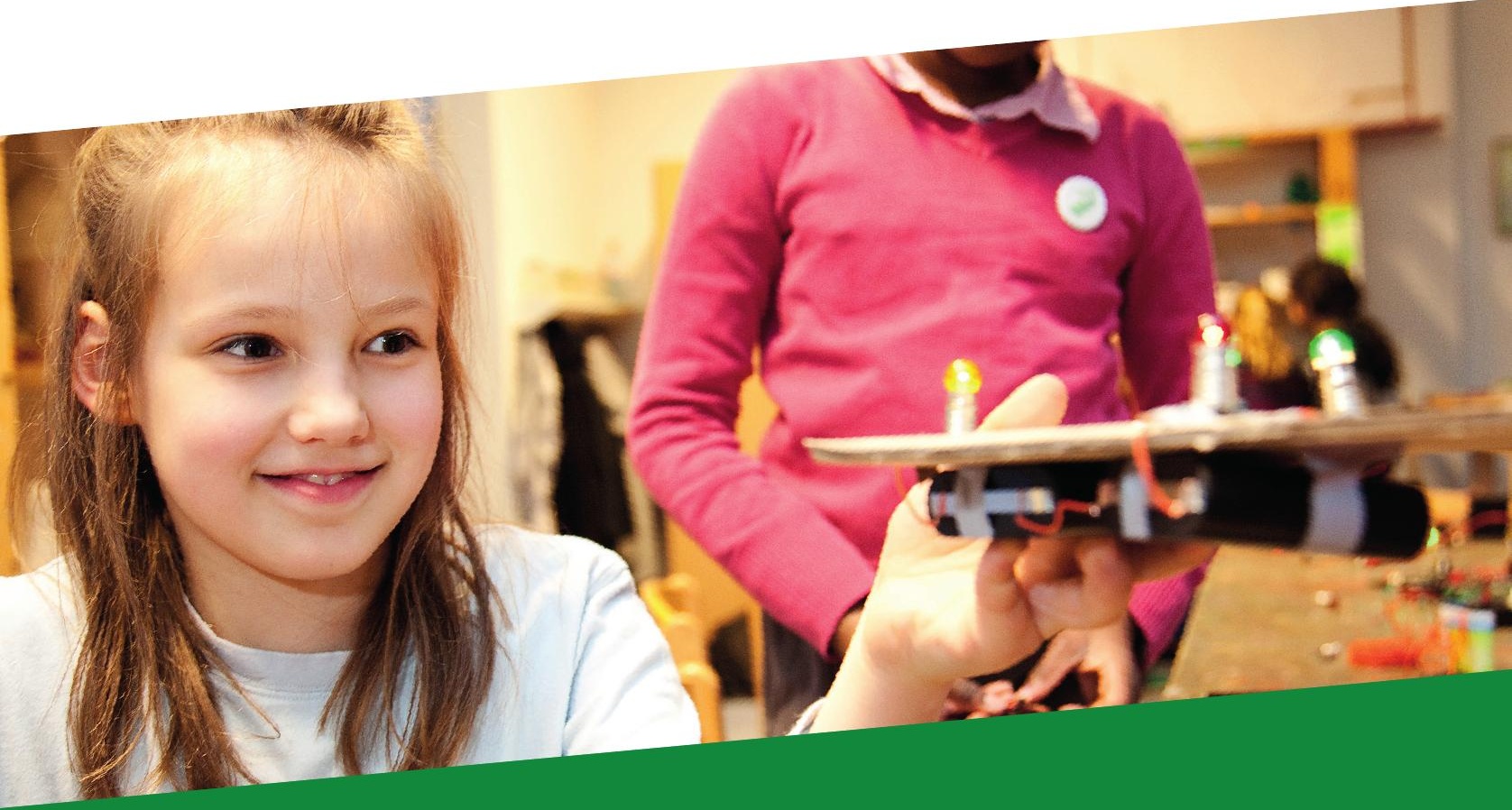

1 Überblick zur Stiftung „Haus der kleinen Forscher“

2 Das „N“ in MINT - Relevanz der frühen naturwissenschaftlichen Bildung

3 Hintergründe der Studien zu den Wirkungen naturwissenschaftlicher Bildungsangebote 


\section{1 Überblick zur Stiftung „Haus der kleinen Forscher“}

Die gemeinnützige Stiftung „Haus der kleinen Forscher“ engagiert sich seit 2006 für gute frühe Bildung in den Bereichen Mathematik, Informatik, Naturwissenschaften und Technik (MINT) - mit dem Ziel, Mädchen und Jungen stark für die Zukunft zu machen und zu nachhaltigem Handeln zu befähigen. Gemeinsam mit ihren Netzwerkpartnern vor Ort bietet die Stiftung bundesweit ein Bildungsprogramm an, das pädagogische Fach- und Lehrkräfte dabei unterstützt, Kinder in Kita- und Grundschulalter qualifiziert beim Entdecken, Forschen und Lernen zu begleiten. Das „Haus der kleinen Forscher“ verbessert Bildungschancen, fördert Interesse am MINT-Bereich und professionalisiert dafür pädagogisches Personal. Die Bildungsinitiative leistet damit einen wichtigen Beitrag in folgenden Bereichen (mehr zum Bildungsverständnis und den Bildungszielen ab S. 30):

- zur Qualifizierung des frühpädagogischen Personals

- zur Qualitätsentwicklung von Einrichtungen

- zur Persönlichkeits-, Kompetenz- und Interessenentwicklung der Kinder

- zur Nachwuchsförderung in den MINT-Bildungsbereichen

Die Hauptaktivitäten der Stiftung sind:

- der Auf- und Ausbau tragfähiger lokaler Netzwerke unter Beteiligung von Akteuren vor Ort sowie Beratung und Service für die inzwischen rund 200 Netzwerkpartner,

- die Ausbildung von Multiplikatorinnen und Multiplikatoren (Trainerinnen und Trainern), die vor Ort pädagogische Fach- und Lehrkräfte kontinuierlich fortbilden,

- die Entwicklung und Bereitstellung von Fortbildungskonzepten und Materialien für pädagogische Fach- und Lehrkräfte sowie

- die Unterstützung der Qualitätsentwicklung von Bildungseinrichtungen durch die Zertifizierung als „Haus der kleinen Forscher“. 


\section{Qualifizierungsinitiative für Pädagoginnen und Pädagogen}

Das „Haus der kleinen Forscher“ ist bundesweit die größte Qualifizierungsinitiative für Pädagoginnen und Pädagogen im Bereich der frühen Bildung. Sie unterstützt Kitas, Horte und Grundschulen dabei, mathematische, informatische, naturwissenschaftliche und/oder technische Schwerpunkte zu setzen und förderliche Entwicklungs- und Lernumgebungen für Kinder in diesen Bereichen zu bieten. Der pädagogische Ansatz der Stiftung knüpft an den Ressourcen der Kinder an und betont das gemeinsame entdeckende und forschende Lernen im dialogischen Austausch (Stiftung Haus der kleinen Forscher, in Vorb.). Die Stiftung fördert mit ihren Aktivitäten auch die Umsetzung vorhandener Bildungs- und Rahmenlehrpläne der jeweiligen Bundesländer in den Bereichen Mathematik, Informatik, Naturwissenschaften und Technik.

Die inhaltlichen Angebote der Stiftung umfassen neben den Fortbildungen für pädagogische Fach- und Lehrkräfte auch pädagogische Materialien, einen jährlichen Aktionstag sowie Anregungen für Kooperationen:

- Pädagogische Materialien: Für die praktische Umsetzung in den pädagogischen Einrichtungen stellt die Stiftung kostenlos Materialen zur Verfügung, z. B. Themenbroschüren, Forschungs- und Entdeckungskarten, didaktische Materialien und Filmbeispiele.

- Internetpräsenz: Die Website www.haus-der-kleinen-forscher.de bietet Informationen für alle Interessierten.

- Magazin „Forscht mit!“: Pädagogische Fach- und Lehrkräfte erhalten quartalsweise praktische Tipps zum Entdecken und Forschen in der Einrichtung, Informationen zur Arbeit der Stiftung sowie Best-Practice-Berichte aus anderen Einrichtungen und Netzwerken.

—Tag der kleinen Forscher“: An diesem bundesweiten Mitmachtag können Mädchen und Jungen in ganz Deutschland ein aktuelles Forschungsthema erkunden. Dazu stellt die Stiftung den pädagogischen Einrichtungen Material bereit und ruft Unterstützer und Unterstützerinnen aus Politik, Wirtschaft, Wissenschaft und Gesellschaft zum Mitmachen auf.

- Anregungen zur Kooperation: Interessierte Eltern, Patinnen und Paten sowie andere Bildungspartner unterstützen das gemeinsame Entdecken und Forschen in den Einrichtungen. 
Zertifizierung: Engagierte Einrichtungen werden anhand festgelegter Bewertungskriterien als „Haus der kleinen Forscher“ zertifiziert. Alle sich bewerbenden Einrichtungen erhalten eine detaillierte Rückmeldung mit Anregungen für die weitere Entwicklung des gemeinsamen Entdeckens und Forschens mit den Kindern.

- Kinder-Website: Unter www.meine-forscherwelt.de gelangen Kinder im Grundschulalter in einen interaktiven Forschergarten, der sie zu eigenständigen Entdeckungsreisen animiert. Für pädagogische Fach- und Lehrkräfte stehen Tipps für die Lernbegleitung zur Verfügung.

- Service-Portal Integration: Unter integration.haus-der-kleinen-forscher.de erhalten Fach- und Lehrkräfte durch vielfältige Materialien, praxisnahe Impulse sowie inspirierenden Erfahrungsaustausch Unterstützung bei der Integration geflüchteter Kinder in Kita, Hort und Grundschule.

\section{Bundesweite Vernetzung}

Das „Haus der kleinen Forscher“ lebt als bundesweite Bildungsinitiative vom Engagement vielfältiger Akteure vor Ort - den lokalen Netzwerken, die als dauerhafte Partner und Fortbildungsanbieter in den Regionen agieren. Zu den derzeit 215 Netzwerkpartnern zählen Kommunen und Kita-Träger, Wirtschaftsverbände, Science-Center, Museen, Unternehmen, Stiftungen, Vereine usw. (Stand 30. September 2018). Seit 2011 steht das Fortbildungsprogramm der Initiative auch Horten und Ganztagsgrundschulen offen.

Pädagogische Fach- und Lehrkräfte aus über 30.900 Kitas, Horten und Grundschulen haben bereits am Fortbildungsprogramm der Initiative teilgenommen, davon pädagogische Fachkräfte aus rund 25.000 Kitas sowie Fach- und Lehrkräfte aus rund 1.500 Horten und rund 4.500 (Ganztags-)Grundschulen (Stand 30. September 2018).

Deutschlandweit sind mehr als 5.100 Kitas, Horte und Grundschulen als „Haus der kleinen Forscher“ zertifiziert, darunter über 4.700 Kitas. Seit Herbst 2013 können sich auch Horte und Grundschulen zertifizieren lassen. Seitdem haben rund 200 Horte und über 200 Grundschulen das Zertifikat „Haus der kleinen Forscher" erhalten (Stand 30. September 2018). 


\section{Das kontinuierliche Fortbildungsprogramm}

Die Stiftung „Haus der kleinen Forscher“ konzentriert sich vor allem auf die Weiterqualifizierung von Pädagoginnen und Pädagogen im Hinblick auf das Entdecken und Erforschen mathematischer, informatischer, naturwissenschaftlicher und/oder technischer Themen mit Kindern. Das Ziel ist eine kontinuierliche Begleitung der pädagogischen Fach- und Lehrkräfte: Die Teilnahme an Fortbildungen zu unterschiedlichen Themen erweitert sukzessive das methodische Repertoire und vertieft das Verständnis des pädagogischen Ansatzes der Stiftung. Im Wechsel von Präsenzfortbildung und Transferphasen können die Pädagoginnen und Pädagogen das Gelernte in der Praxis ausprobieren und sich dazu in der nächsten Fortbildung austauschen.

Um möglichst vielen interessierten pädagogischen Fach- und Lehrkräften die Teilnahme an Fortbildungen zu ermöglichen, findet die Weiterqualifizierung über ein Multiplikatorenmodell statt: Die Stiftung „Haus der kleinen Forscher“ bildet an mehreren Standorten in Deutschland Trainerinnen und Trainer aus, die ihrerseits Fortbildungen für Pädagoginnen und Pädagogen in ihrem lokalen Netzwerk durchführen. Die Trainerinnen und Trainer qualifizieren sich durch die Teilnahme an den Präsenz- und Online-Fortbildungen der Stiftung dafür, Fortbildungen mit Pädagoginnen und Pädagogen durchzuführen. Als Unterstützung erhalten sie ausführliche Arbeitsunterlagen für ihre Aufgabe in der Erwachsenenbildung sowie die Möglichkeit, persönliches Feedback im Hospitationsprogramm der Stiftung oder in Form von Videocoaching zu bekommen. Für die Auffrischung und Vertiefung der Fortbildungsinhalte steht auch der Online-Campus für Trainerinnen und Trainer zur Verfügung. Die digitale Lernplattform hält neben einer Vielzahl von Online-Lernangeboten auch inhaltliche Informationen und Arbeitsunterlagen zu den einzelnen Fortbildungsmodulen bereit. Zu bestimmten Themen gibt es die Möglichkeit, eigenständig offene E-Learning-Module zu bearbeiten, an tutoriell begleiteten Kursen teilzunehmen sowie die Online-Begleitkurse zu Präsenzfortbildungen zu nutzen. Darüber hinaus können die Trainerinnen und Trainer in Themenforen oder offenen Chats miteinander in Kontakt treten und sich austauschen.

Sowohl für die pädagogischen Fach- und Lehrkräfte als auch für die Trainerinnen und Trainer werden in der Bildungsinitiative jedes Jahr unterschiedliche Fortbildungsthemen angeboten. Bis Ende 2016 besuchten neue Trainerinnen und Trainer bzw. erstmals teilnehmende Pädagoginnen und Pädagogen zunächst die Fortbildungen „Forschen mit Wasser“ (Workshop 1) und „Forschen mit Luft“ (Workshop 2), in denen der pädagogische Ansatz der Stiftung für das gemeinsame Entdecken und Forschen mit Kindern ausführlich thematisiert wird. Seit 2017 ist der Einstieg in das Bildungsangebot der Stiftung flexibel gestaltet. Sieht die Lernbegleitung noch Entwicklungsbedarf in ihrer pädagogischen Kompetenz 
bzw. möchte sich einen Überblick zum pädagogischen Konzept der Stiftung verschaffen, so erhält sie das Angebot, wie bisher mit den genannten Präsenzfortbildungen einzusteigen bzw. das Seminar oder den Online-Kurs „Grundlagen zur Pädagogik“ zu besuchen. Ebenso können die pädagogischen Fach- und Lehrkräfte bzw. die Trainerinnen und Trainer als Einstieg aber auch ein anderes Modul zu den mathematischen, informatischen, naturwissenschaftlichen oder technischen Themen wählen. Die Inhalte werden in verschiedenen Formaten angeboten: Fortbildungen vor Ort, Selbstbildungsformate (wie Online-Kurse oder gedruckte pädagogische Materialien) und Bildungsveranstaltungen. Das Zertifikat „Haus der kleinen Forscher“ unterstützt darüber hinaus bei der Qualitätsentwicklung der pädagogischen Arbeit in den Einrichtungen und macht das Engagement für gute frühe MINT-Bildung nach außen sichtbar. Die Stiftung orientiert sich somit stärker an den Bedarfen ihrer Zielgruppen in Abhängigkeit von deren Vorwissen und Vorerfahrungen sowie deren Interessen und ihrer zeitlichen Flexibilität.

Inhaltlich wurde das Stiftungsangebot zum Start des Schuljahres 2017/2018 mit dem Workshop „Informatik entdecken - mit und ohne Computer“ um den Bildungsbereich der informatischen Bildung ergänzt. Die Stiftung erweitert außerdem ihr Angebot an Fortbildungen, Inhalten und Materialien um Bildung für nachhaltige Entwicklung. Das Konzept, das sich an pädagogische Fach- und Lehrkräfte wie auch erstmals an Kitaleitungen wendet, wird seit 2017 in 29 Modellnetzwerken in der Praxis erprobt. Ab 2018 werden die Fortbildungen zur Bildung für nachhaltige Entwicklung überall dort zur Verfügung stehen, wo Netzwerkpartner diesen Bildungsschwerpunkt in ihr Angebot aufnehmen. Darüber hinaus wird ab Herbst 2018 das Thema „Technik - von hier nach da“ aus dem Bereich der technischen Bildung angeboten.

\section{Wissenschaftliche Begleitung und Qualitätsentwicklung}

Alle Aktivitäten der Bildungsinitiative werden kontinuierlich wissenschaftlich begleitet und evaluiert. Die Stiftung „Haus der kleinen Forscher“ pflegt einen offenen Austausch mit Wissenschaft und Fachpraxis und versteht sich als lernende Organisation.

Ein umfangreiches Spektrum an Maßnahmen dient der Sicherung und Weiterentwicklung der Qualität im „Haus der kleinen Forscher“ (siehe Abbildung 1). Das stiftungseigene Qualitätsmanagement erfasst mit internen Evaluationsmaßnahmen und umfassendem Monitoring alle wichtigen Aktivitäten und Angebote. Dafür nutzt die Stiftung eine ganze Reihe an Datenquellen (wie z.B. anlassbezogene Befragungen der Zielgruppen ${ }^{3}$ ).

3 Netzwerkkoordinatorinnen und -koordinatoren, Trainerinnen und Trainer sowie pädagogische Fachund Lehrkräfte. 
Eine Kombination aus Daten im Quer- und Längsschnittformat ermöglicht den Blick sowohl auf die aktuelle Situation als auch auf wichtige Veränderungen in den letzten Jahren. Um auf die Erkenntnisbedarfe der Stiftung flexibler reagieren zu können, wird die bisher jährliche Befragung sämtlicher Zielgruppen durch mehrere Erhebungen zu unterschiedlichen Zeitpunkten ersetzt. Die längsschnittliche Perspektive spielt in den internen Evaluations- und Monitoring-Maßnahmen der Stiftung eine zunehmend wichtigere Rolle, um dem Anspruch einer stärkeren Wirkungsorientierung gerecht zu werden. Mit dem regelmäßig erscheinenden Monitoring-Bericht stellt die Stiftung wichtige Ergebnisse dieser Maßnahmen bereit. So beschreibt der Monitoring-Bericht 2016/2017 auf Grundlage einer Wirkungskette, wie das Fortbildungsangebot der Initiative zur Verbesserung der frühen MINT-Bildung in Deutschland beiträgt (Stiftung Haus der kleinen Forscher, 2017a).

Im Rahmen der inhaltlichen (Weiter-)Entwicklung werden neue Stiftungsangebote auch stets im Sinne eines kollaborativen Ansatzes mit der Praxis entwickelt und getestet. In Zusammenarbeit mit einer Gruppe pädagogischer Fach- und Lehrkräfte aus Kitas sowie aus Horten und Grundschulen findet für jedes neue Fortbildungsangebot eine ausführliche Erarbeitung und Pilotierung statt, bevor die Fortbildungskonzepte und Materialien in den regionalen Netzwerken verbreitet werden. Dabei prüfen die mitwirkenden pädagogischen Fach- und Lehrkräfte erste Praxisideen auf ihre Umsetzbarkeit und geben ein Feedback zu den Unterstützungsangeboten der Stiftung. Die Fortbildungskonzepte werden auf Basis dieser Rückmeldungen überarbeitet und weiterentwickelt.

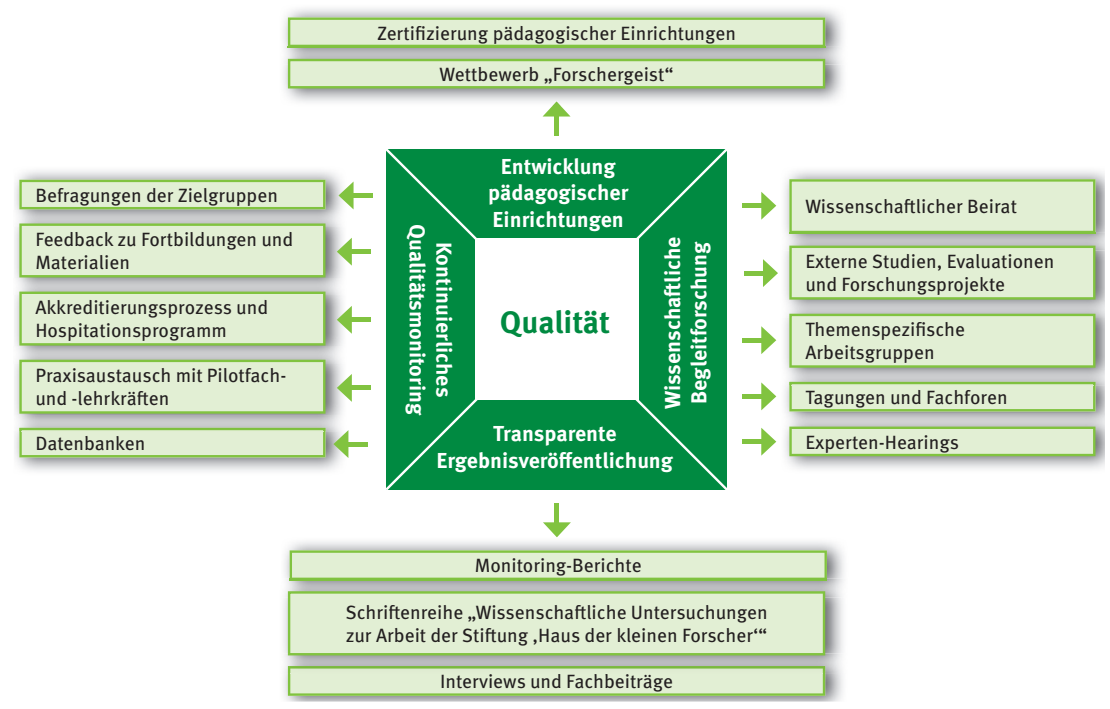

Abbildung 1. Übersicht der Maßnahmen zu Sicherung und Weiterentwicklung der Qualität der Stiftungsarbeit 
Auf Einrichtungsebene ist die Zertifizierung als „Haus der kleinen Forscher“ ein weiteres wichtiges Instrument der Qualitätsentwicklung (Stiftung Haus der kleinen Forscher, 2017b). Über die Vergabe des Zertifikats entscheidet die Stiftung in einem standardisierten Verfahren, das in Anlehnung an das Deutsche Kindergarten Gütesiegel und unter Beteiligung eines Teams aus Wissenschaftlerinnen und Wissenschaftlern ${ }^{4}$ entwickelt wurde. Die Reliabilität und Validität des Zertifizierungsverfahrens für Kitas wurde in einer externen wissenschaftlichen Studie bestätigt (Anders \& Ballaschk, 2014). Die Zertifizierung als „Haus der kleinen Forscher“ ist ein für Einrichtungen kostenfreies Verfahren zur Erfassung und Steigerung der pädagogischen Qualität bei der Umsetzung von MINT-Bildungsinhalten. Durch die Beantwortung der Fragen im Zertifizierungsfragebogen und die daraufhin erfolgende ausführliche Rückmeldung mit praktischen Anregungen und Tipps seitens der Stiftung werden Kitas, Horte und Grundschulen in ihrer Qualitätsentwicklung unterstützt. Eine Folgezertifizierung alle zwei Jahre ermöglicht die langfristige Verankerung und Weiterentwicklung der Bildungsqualität und trägt zu Qualitätsentwicklung auf Einrichtungsebene bei.

Mit dem bundesweiten Kita-Wettbewerb „Forschergeist“5 möchten die Deutsche Telekom Stiftung und die Stiftung „Haus der kleinen Forscher“ das Engagement der Kita-Fachkräfte sowie die Qualität der MINT-Bildungsarbeit in den Einrichtungen würdigen. Gesucht und prämiert werden herausragende Projekte, die Mädchen und Jungen für die Welt der Mathematik, Informatik, Naturwissenschaften oder Technik begeistert haben. Mit der Preisverleihung am 6. Juni 2018 fand der Wettbewerb bereits zum vierten Mal statt. Die prämierten Projekte werden dokumentiert und veröffentlicht, damit sie als gute Beispiele auch andere Fachkräfte für das Forschen und Entdecken in der Kita ermutigen (Stiftung Haus der kleinen Forscher, 2018c).

Neben einem kontinuierlichen Monitoring zu Zwecken der Qualitätssicherung und der Qualitätsentwicklung wird die Stiftungsarbeit im Rahmen einer langfristig angelegten externen Begleitforschung mit renommierten Partnern fachlich fundiert und in Forschungsprojekten evaluiert. Die Ergebnisse der wissenschaftlichen Begleitung veröffentlicht die Stiftung transparent in der vorliegenden wissenschaftlichen Schriftenreihe, alle Publikationen sind zudem über ihre Website frei verfügbar ${ }^{6}$. Zwei unabhängige Forschungsgruppen untersuchten die naturwis-

\footnotetext{
4 Prof. Dr. Yvonne Anders, Dr. Christa Preissing, Prof. Dr. Ursula Rabe-Kleberg, Prof. Dr. Jörg Ramseger, Prof. Dr. Wolfang Tietze.

5 www.forschergeist-wettbewerb.de

6 Alle Ergebnisse und Publikationen zur wissenschaftlichen Begleitung sind als PDF verfügbar unter: www.haus-der-kleinen-forscher.de, Rubrik „Wissenschaftliche Begleitung“. Alle Ergebnisse der externen Begleitforschung werden zudem in der vorliegenden wissenschaftlichen Schriftenreihe veröffentlicht. Eine Übersicht der bisher erschienenen Bände befindet sich auf www. haus-der-kleinen-forscher. de bzw. am Ende dieses Bandes.
} 
senschaftlichen Bildungswirkungen in der frühen Kindheit, deren Abschlussberichte in diesem Band veröffentlicht werden (vgl. Kapitel 3).

Ein Wissenschaftlicher Beirat berät die Stiftung zu Forschungsfragen sowie zur fachlichen Fundierung des Stiftungsangebots. Er setzt sich aus unabhängigen Wissenschaftlerinnen und Wissenschaftlern unterschiedlicher Fachgebiete zusammen und spricht Empfehlungen an den Vorstand und den Stiftungsrat aus. Die Mitglieder des Beirats sind hochkarätige Expertinnen und Experten relevanter Disziplinen:

- Vorsitz: Prof. Dr. Hans-Günther Roßbach, Universität Bamberg, Lehrstuhl für Elementar- und Familienpädagogik

- Prof. Dr. Fabienne Becker-Stoll, Staatsinstitut für Frühpädagogik (IFP), München

- Prof. Dr. Marcus Hasselhorn, Deutsches Institut für Internationale Pädagogische Forschung (DIPF), Frankfurt, Abteilung Bildung und Entwicklung \& Prof. Dr. Jan Lonnemann, Universität Potsdam, Lehrstuhl für Empirische Kindheitsforschung

- Prof. Dr. Christoph Igel, Deutsches Forschungszentrum für Künstliche Intelligenz (DFKI) GmbH, Abteilung Educational Technology

- Prof. Dr. Bernhard Kalicki, Deutsches Jugendinstitut e. V. (DJI), München, Abteilung Kinder und Kinderbetreuung, und Evangelische Hochschule Dresden, Lehrstuhl für Frühkindliche Bildung

- Prof. Dr. Alexander Kauertz, Universität Koblenz-Landau, Lehrstuhl für Physikdidaktik und Techniklehre

- Prof. Dr. Armin Lude, Pädagogische Hochschule Ludwigsburg, Abteilung Biologie, (Schwerpunkt Bildung für nachhaltige Entwicklung)

- Prof. Dr. Johannes Magenheim, Universität Paderborn, Lehrstuhl für Didaktik der Informatik

- Prof. Dr. Jörg Ramseger, Freie Universität Berlin, Arbeitsstelle Bildungsforschung Primarstufe 
- Prof. Pia S. Schober, Ph.D, Universität Tübingen, Lehrstuhl für Soziologie mit Schwerpunkt Mikrosoziologie und Deutsches Institut für Wirtschaftsforschung (DIW Berlin), Abteilung Bildung und Familie \&

Prof. Dr. C. Katharina Spieß, Freie Universität Berlin, Lehrstuhl für Bildungsund Familienökonomie, und Deutsches Institut für Wirtschaftsforschung (DIW Berlin)

- Prof. Dr. Mirjam Steffensky, Leibniz-Institut für die Pädagogik der Naturwissenschaften und Mathematik (IPN), Kiel, Lehrstuhl für Didaktik der Chemie (Schwerpunkt Frühe naturwissenschaftliche Bildung)

- Prof. Dr. Wolfgang Tietze, PädQUIS gGmbH, An-Institut der Alice Salomon Hochschule, Berlin \&

Prof. Dr. Catherine Walter-Laager, Universität Graz, Abteilung Elementarpädagogik

- Prof. Dr. Christian Wiesmüller, Pädagogische Hochschule Karlsruhe, Abteilung für Physik und Technische Bildung, Deutsche Gesellschaft für Technische Bildung (DGTB)

- Prof. Dr. Bernd Wollring, Universität Kassel, Lehrstuhl für Didaktik der Mathematik 


\section{Das „N“ in MINT - Relevanz und Ziele früher naturwissenschaftlicher Bildung}

Es gilt mittlerweile als unstrittig, dass frühe Bildung die Basis einer gelingenden Bildungsbiografie und Teilhabe an unserer Gesellschaft ist und dass Kinder vom Besuch einer Kita profitieren (vgl. Bundesministerium für Bildung und Forschung [BMBF], 2017; Vandell, Belsky, Burchinal, Steinberg \& Vandergrift, 2010). Dies gilt vor allem, wenn dieser Besuch in einem positiven Zusammenhang mit guter Qualität in der Kindertagesbetreuung steht (Anders, 2013; Melhuish et al., 2015; Barnett, 2011). Die gemeinnützige Stiftung „Haus der kleinen Forscher“ engagiert sich für frühe Bildung in den Bereichen Mathematik, Informatik, Naturwissenschaften und Technik (MINT). Ihr Ziel ist es, Kinder stark zu machen für die Zukunft und sie zu verantwortungsvollem Handeln im Sinne einer nachhaltigen Entwicklung zu befähigen (vgl. Vision und Mission der Stiftung „Haus der kleinen Forsch in diesem Band, S. 306f.). Kinder brauchen die Möglichkeit, ihren eigenen Fragen nachzugehen und forschend die Welt zu entdecken. Die Stiftung „Haus der kleinen Forscher“ hat das Ziel, alle Kitas, Horte und Grundschulen in Deutschland zu „Forscherorten“ zu machen, an denen Mädchen und Jungen lernen, selbstbestimmt zu denken und verantwortungsvoll zu handeln. Technologisierung und Digitalisierung sowie Folgen des Klimawandels und der sozialen Ungleichheit beeinflussen zunehmend unseren Alltag. Mit früher Bildung in den Bereichen Mathematik, Informatik, Naturwissenschaften und Technik sowie Bildung für nachhaltige Entwicklung trägt das „Haus der kleinen Forscher“ dazu bei, Kinder stark zu machen, damit sie den Herausforderungen einer komplexen Welt erfolgreich begegnen und zukunftsfähig agieren. Die Stiftung folgt dabei grundsätzlich einem kritisch-emanzipatorischen Bildungsverständnis, bei dem es darum geht, informierte Entscheidungen und informiertes Handeln im Alltag auf Grundlage einer frühen MINT-Bildung zu ermöglichen. Die Angebote der Stiftung unterstützen somit den Erwerb der Kompetenzen für das 21. Jahrhundert, wie sie etwa die OECD in ihrem Learning Framework vorgestellt hat (OECD, 2017). Die Stiftung erarbeitet ihre Angebote auf der Grundlage eines ko-konstruktivistischen Lernverständnisses entlang einer fachlichen Fundierung mit einem zugrunde liegenden Wirkungsmodell. Exploration, entdeckendes und forschendes Lernen sind für Kinder und Pädagoginnen und Pädagogen zentrale Erkenntniswege, um Wissen und Kompetenzen aufzubauen.

Im Fokus dieses Bandes stehen die Wirkungen naturwissenschaftlicher Bildungsangebote. Wirkungen werden in diesem Kontext als Veränderungen verstanden, die durch die Teilnahme am Weiterbildungsprogramm des „Hauses der kleinen Forscher“ erreicht werden. Die naturwissenschaftlichen Bildungsangebote der Stiftung wurden in ihrer MINT-Themenentwicklung als erste entwickelt und 
wissenschaftlich fundiert (vgl. Band 5 der Wissenschaftlichen Schriftenreihe der Stiftung Haus der kleinen Forscher, 2013), da zunächst ein großer Bedarf an Fortund Weiterbildungsangeboten zu naturwissenschaftlichen Themen entstand. Dieser Bedarf der Praxis entwickelte sich zunehmend, nachdem die naturwissenschaftliche Bildung nach und nach in einzelnen Bildungsplänen der Länder verankert wurde.

Mittlerweile ist die naturwissenschaftliche Bildung fester Bestandteil in allen Bildungs- und Rahmenlehrplänen (vgl. Steffensky, 2017). Der Stellenwert naturwissenschaftlicher Bildung ist enorm gewachsen und kann sich der öffentlichen Debatte um die Qualität naturwissenschaftlicher Bildungsangebote in Kitas, Horten und Grundschulen nicht mehr verschließen (Leibniz-Forschungsverbund Bildungspotenziale (kurz LERN), 2018). Die Stiftung „Haus der kleinen Forscher“ begegnet mit ihren Bildungsangeboten dem Weiterbildungsbedarf (vgl. DJI, 2018) in diesem Bildungsfeld und trägt somit zu einer Qualifizierung der pädagogischen Fach- und Lehrkräfte im Bereich der frühen MINT-Bildung bei. Mit ihren vielfältigen Angeboten verfolgt sie das Ziel, pädagogische Fach- und Lehrkräfte in ihrer Selbstwirksamkeit in der Auseinandersetzung mit MINT-Themen zu bestärken, ihre Kompetenzen zu erweitern und sie zu befähigen, sich auf die Fragen, Ideen und Vermutungen der Kinder einzulassen und diese in einem ko-konstruktiven Prozess gemeinsam zu beantworten (vgl. Stiftung Haus der kleinen Forscher, 2013, siehe Abbildung 2). Der pädagogische Ansatz der Stiftung setzt bei den Interessen und Fähigkeiten der Kinder an und betont das gemeinsame forschende Lernen im dialogischen Austausch. Kinder sollen sich in der Auseinandersetzung mit MINT-Themen ebenfalls als kompetent und selbstwirksam erleben, grundlegende Konzepte begreifen und erste Erfahrungen mit wissenschaftlichen Denkund Handlungsweisen, z. B. die prozesshafte Arbeit entlang des Forschungskreises, sammeln (Marquardt-Mau, 2004, 2011; Pädagogischer Ansatz der Stiftung Haus der kleinen Forscher, in Vorb.) ${ }^{7}$.

Diese naturwissenschaftliche Grundbildung, auch als „scientific literacy“ (Norris \& Phillips, 2003; Bybee, McCrae \& Laurie, 2009; Steffensky, 2017; Stiftung Haus der kleinen Forscher, 2013) bezeichnet, wird dabei als ein mehrdimensionales Bildungskonzept verstanden, das sowohl Wissenskomponenten als auch nicht-kognitive Komponenten enthält. Die Ziele naturwissenschaftlicher früher Bildung umfassen die Dimensionen Motivation, Verstehen naturwissenschaftlicher Grundkonzepte und prozedurales Wissen über sowie Kompetenzen zu naturwissenschaftlichen Arbeitsweisen. Zur Grundbildung in den Bereichen Mathe-

\footnotetext{
7 Das didaktische Konzept einer naturwissenschaftlichen Grundbildung zum Forschen mit Kindern und das damit verbundene Modell des Forschungskreislaufs wurde von Frau Marquardt-Mau entwickelt (2004, 2011) und im pädagogischen Ansatz der Stiftung „Haus der kleinen Forscher“ adaptiert.
} 
matik, Informatik, Naturwissenschaften und Technik gehört neben konzeptuellem Wissen vor allem die Kompetenz, Wissen durch geeignete Denk- und Handlungsweisen zu erwerben, zu erweitern, kritisch zu reflektieren und anwenden zu können. Dazu zählt auch die Fähigkeit, grundlegende Zusammenhänge selbst zu erschließen, zu beurteilen und, darauf beruhend, Entscheidungen zu treffen.

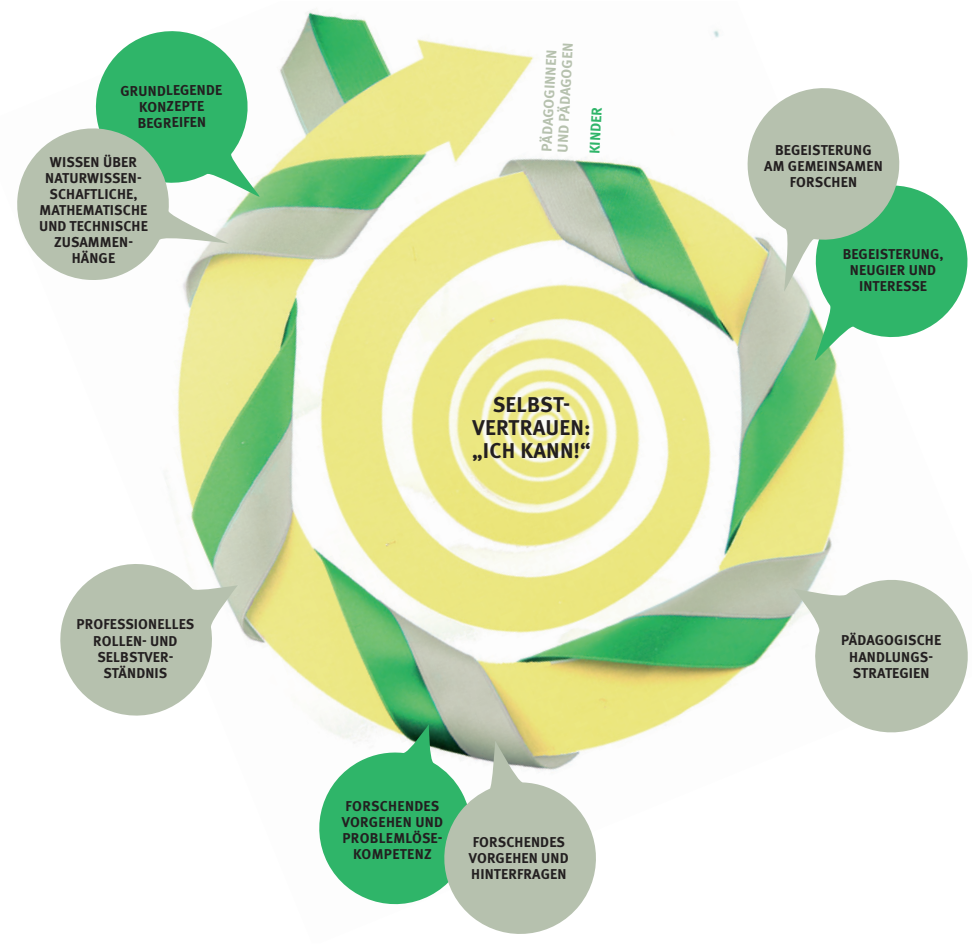

Abbildung 2: Ziele der Stiftungsarbeit auf Ebene der pädagogischen Fach- und Lehrkräfte und der Kinder (Stiftung Haus der kleinen Forscher, 2013, S. 174)

Zudem braucht es beim Entdecken und Forschen eine aktive Auseinandersetzung zwischen pädagogischer Fach- und Lehrkraft und Kind zu einem für das Kind relevanten Thema. Das Entdecken erfolgt zunächst völlig frei und explorativ durch das Kind selbst, wobei das später vertiefende, systematische Forschen oft einer Lernbegleitung durch die pädagogische Fach- und Lehrkraft bedarf. Der Prozess des Forschens gliedert sich dabei in verschiedene Phasen des Denkens und Handelns, die typischerweise in einem wiederkehrenden Zyklus auftreten. Dieser Prozess der Erkenntnisgewinnung kann als Forschungskreis (Marquardt-Mau, 2011; Stiftung Haus der kleinen Forscher, 2018b) zusammengefasst werden. Er beschreibt eine Methode naturwissenschaftlichen Vorgehens und lässt sich in sechs Phasen unterteilen (Details siehe S. 149). 


\section{Hintergründe der Studien zu Wirkungen naturwissenschaftlicher Bildungsangebote}

\section{Fragestellung und Motivation zur Ausschreibung der Wirkungsstudien}

Im Bildungsbereich Naturwissenschaften gibt es mittlerweile eine Vielzahl an Angeboten für die Praxis. Studien zur Wirkung früher naturwissenschaftlicher Bildung und ihrer Zusammenhänge zu sprachlicher Bildung stehen in Deutschland jedoch weitgehend aus. Die Stiftung „Haus der kleinen Forscher“ hat gemeinsam mit dem BMBF, der Siemens Stiftung und der Baden-Württemberg Stiftung die beiden Studien Early Steps into Science (kurz: EASI Science) und Early Steps into Science and Literacy (kurz: EASI Science-L) gefördert und somit zur Grundlagen- und Wirkungsforschung im Bereich früher Bildung beitragen. Das BMBF und die beteiligten Stiftungen teilten dabei das Erkenntnisinteresse, mehr über Wirkungen naturwissenschaftlicher Bildungsangebote zu erfahren, insbesondere mit Bezug zu möglichen sprachlichen Bildungswirkungen in Settings eines forschenden Lernens.

Ziel der ersten ausgeschriebenen Studie war es zu untersuchen, welche Wirkungen naturwissenschaftliche Bildungsangebote im Kita-Bereich, wie die der Stiftung oder anderer Initiativen im Bereich der frühen MINT-Bildung, auf die naturwissenschaftsbezogene Kompetenz von pädagogischen Fach- und Lehrkräften und Kindern erzielen. Im Blickpunkt des ersten Forschungsvorhabens standen zum einen Bildungsprozesse und -ergebnisse bei den Kindern, zum anderen bei den pädagogischen Fachkräften in den Kindertagesstätten auf den angestrebten Zieldimensionen früher naturwissenschaftlicher Bildung. Dabei sollten möglichst empirisch belastbare Aussagen über Merkmale und Faktoren (z. B. die Dauer der Teilnahme am Programm etc.) getroffen werden können, von denen die Bildungswirkungen abhängen.

Eine zweite Studie zielte in der ausgeschriebenen Fragestellung auf mögliche sprachliche Bildungswirkungen durch naturwissenschaftliche Bildungsangebote ab. Auch hier existieren große Forschungslücken zum vermuteten Zusammenhang zwischen naturwissenschaftlicher und sprachlicher Bildung. Dieser Zusammenhang wird u. a. in der „Frankfurter Erklärung“ zur frühen sprachlichen und naturwissenschaftlichen Bildung (2015) für bedeutsam gehalten. So heißt es dort „Notwendig sind empirische Studien zur Frage, wie sprachliche und naturwissenschaftliche Bildung im pädagogischen Alltag sinnvoll kombiniert, wie die Erkenntnisse aus der Spracherwerbsforschung und der Linguistik für frühpädagogische Angebote und die Praxis des Unterrichts in Kitas und Schulen genutzt werden können, und in welchem Alter welche Bildungsangebote besonders zielführend sind.“ Die zweite Studie verfolgte somit das Ziel, erste Hinweise zu Zusammenhängen von naturwissenschaftlicher und sprachlicher Bildung zu liefern. Hierbei 
sollte untersucht werden, ob bzw. in welchen Phasen naturwissenschaftlichen Vorgehens (vgl. Forschungskreis; Marquardt-Mau, 2004, 2011; Stiftung Haus der kleinen Forscher, 2013, 2018) Effekte im Hinblick auf sprachliche Kompetenzen, insbesondere auch bei Kindern mit Deutsch als Zweitsprache, erzielt werden können, und inwiefern etwaige sprachliche Bildungswirkungen von der Qualität der sprachlichen Begleitung des Forschens abhängen.

Ein wichtiges Ziel war es, erste Einblicke in Wirkungen von naturwissenschaftlichen Fortbildungen auf Ebene pädagogischer Fach- und Lehrkräfte und auf Ebene der Kinder sowie Erkenntnisse auf wirkungsbeeinflussende Faktoren zu erhalten und diese Erkenntnisse für die Weiterentwicklung von Bildungs- und Unterstützungsangeboten für pädagogische Fach-, Lehr- und Leitungskräfte zu nutzen.

In Bezug auf Erkenntnisse aus den Studien war die Stiftung des Weiteren daran interessiert, eine externe empirische Untersuchung am Beispiel ihres Ansatzes zu erhalten, um einerseits als wirksam erkannte Angebote zielgerichtet aufrechterhalten und optimieren zu können. Andererseits wollte die Stiftung erfahren, wo der Stiftungsansatz ggf. noch keine oder nur begrenzte Wirkungen zeigt, um das Angebotsportfolio forschungsbasiert in Inhalt oder Intensität wirkungsorientiert weiterentwickeln zu können.

Schließlich waren die Studien als eine der ersten ihrer Art in der frühen MINT-Bildungsforschung in Deutschland auch per se ein neues Forschungsunterfangen. Die Stiftung und ihre Mitförderer wollten damit die frühe Bildungsforschung in Deutschland stärken und waren daran interessiert zu erfahren, mit welchen Instrumenten eine Wirkungsmessung naturwissenschaftlicher Bildungsangebote in der Kita möglich ist und die Entwicklung von Messinstrumenten zu unterstützen. Ein übergeordnetes Ziel war es, Erfahrungen mit Wirkungsforschung in diesem Bereich zu sammeln, Chancen und Grenzen bestimmter Herangehensweisen kennenzulernen, die für die Interpretation von Ergebnissen zu berücksichtigen sind, und Konsequenzen für die Ausrichtung weiterer Begleitforschung ableiten zu können.

\section{Qualitätssicherung, Unabhängigkeit und Wissenschaftliche Begleitung der Studien}

Die Studien wurden in einem mehrjährigen Prozess vorbereitet und vom Wissenschaftlichen Beirat während der Studiendurchführung begleitet. Ziel war es, die Forschungsvorhaben an der Schnittstelle zwischen Entwicklungs- und Pädagogischer Psychologie, der Didaktik der Naturwissenschaften, der Früh- und Grundschulpädagogik sowie der Bildungsforschung zu verorten und Erkenntnisse aus den jeweiligen Disziplinen interdisziplinär miteinander zu verzahnen. Schon früh begann die Stiftung, unter Einbindung von Expertinnen und Experten Zielkriterien für die Wirkungsmessung zu formulieren. Dazu gehörte die Erarbeitung von Zieldimensionen früher naturwissenschaftlicher Bildung auf Ebene der pädagogischen 
Fach- und Lehrkräfte sowie auf Ebene der Kinder (vgl. Band 5 der Wissenschaftlichen Schriftenreihe der Stiftung Haus der kleinen Forscher, 2013).

Darauf aufbauend folgten die konzeptuelle Vorbereitung der Studienausschreibungen mit dem Forschungslenkungskreis der Stiftung und die Gewinnung von Förderpartnern zur Mitfinanzierung der Studien. Neben dem Bundesministerium für Bildung und Forschung konnten die Baden-Württemberg Stiftung und die Siemens Stiftung für eine finanzielle Beteiligung gewonnen werden.

Bundesministerium für Bildung und Forschung ${ }^{8}$ : Bildung und Forschung sind die Grundlagen, auf denen wir unsere Zukunft aufbauen. Die Förderung von Bildung, Wissenschaft und Forschung durch das Bundesministerium für Bildung und Forschung ist deshalb ein wichtiger Beitrag zur Sicherung des Wohlstands in unserem Land. Mit Bildung bereiten wir unsere Kinder auf die Herausforderungen einer sich verändernden und immer stärker globalisierten Welt vor. Forschung hilft uns, Neues zu entdecken und Bekanntes besser zu machen. Dank exzellenter Forschung finden wir Lösungen für globale Probleme und Strategien für nachhaltiges Wachstum.

Die Baden-Württemberg Stiftung ${ }^{9}$ engagiert sich seit vielen Jahren sehr erfolgreich in der Sprachförderung. Sprache ist gleichzeitig Schlüssel und Grundlage für den individuellen Bildungserfolg und die Lebenschancen von Kindern. Der Zugang zur deutschen Sprache ist für eine gerechte Verteilung von Bildungschancen unabdingbar. Deshalb ist für die Baden-Württemberg Stiftung die Bedeutung von Sprachkompetenz und Ausdrucksvermögen für den Bildungserfolg unbestritten. Im Jahr 2002 hat die Stiftung mit dem Programm „Sag’ mal was - Sprachförderung für Vorschulkinder“ mit dem Ziel begonnen, die sprachliche Bildung und den Spracherwerb von Kindern im vorschulischen Alter zu stärken. „Sag’ mal was“ war von Beginn an als „lernendes Programm“ angelegt. Basierend auf wissenschaftlichen Erkenntnissen und Erfahrungen aus der Praxis, wurde das Programm kontinuierlich weiterentwickelt, und es wurden neue Initiativen angestoßen. „Sag' mal was“ ist mittlerweile über seinen ursprünglichen Fokus auf Sprachförderung im Vorschulbereich hinaus aktiv. Mit der Studie EASI Science-L legt die Baden-Württemberg Stiftung gemeinsam mit anderen Förderpartnern den Fokus auf das Zusammendenken von Sprachförderung und Frühförderung im naturwissenschaftlichen Bereich, indem die Studie Zusammenhänge zwischen sprachlichen Bildungswirkungen und Interaktionsqualität sowie Kompetenzen in der naturwissenschaftlichen Bildung untersucht. Die Studie EASI Science-L und das Gesamtprogramm „Sag' mal was“ fügen sich in das Engagement der Baden-Württemberg Stiftung ein. Sie widmet sich der Spitzenforschung, der Bildung und dem Streben

8 www.bmbf.de

9 www.bwstiftung.de 
nach einem verantwortungsbewussten Umgang mit unseren Mitmenschen. Alle Aktivitäten sind darauf ausgerichtet, nachhaltige Veränderungen zu bewirken. Der frühkindliche Bereich und die sprachliche Bildung nehmen für die Gestaltung der Zukunft eine besondere Rolle ein. Hier engagiert die Baden-Württemberg Stiftung sich gemäß ihrem Motto „Wir stiften Zukunft“.

In einer technisierten Welt ist das Verständnis von naturwissenschaftlichen und technischen Zusammenhängen entscheidend für individuelle Entwicklung und gesellschaftliche Teilhabe. Die Siemens Stiftung ${ }^{10}$ engagiert sich deshalb für die Stärkung und Förderung von naturwissenschaftlich-technischer Bildung durch aktive Gremien- und operative Bildungsarbeit. Mit ihrem internationalen Bildungsprogramm Experimento bietet sie für Pädagoginnen und Pädagogen praxisorientierte Fortbildungen sowie qualitativ hochwertige Lehr- und Lernmaterialien an. Diese stellt sie digital zur freien Verfügung, um eine gleichberechtigte Förderung aller Schüler und Schülerinnen zu ermöglichen. Mit ihrem Engagement verknüpft sie forschendes Lernen mit wertebildendem Handeln und unterstützt so die Ausbildung einer sozial orientierten, starken Persönlichkeit.

Der Unterricht im Fächerkanon der Naturwissenschaften stellt für Schüler und Schülerinnen mit geringen Kenntnissen der Unterrichtssprache eine besondere Herausforderung dar. Fachvokabular, komplexe Sprachstrukturen, Nominalisierungen, Passivkonstruktionen und lange Komposita werden im Unterricht häufig verwendet und erschweren anderssprachigen Schülern und Schülerinnen, dem Unterricht zu folgen. Durch die Methode des forschenden Lernens, die dem internationalen Bildungsprogramm Experimento zugrunde liegt, wird Kindern und Schülern und Schülerinnen auch der sprachliche Zugang erleichtert. Die Experimente generieren Sprachanlässe und laden ein, sich - auch sprachlich - einzubringen. Spezielle Arbeitsblätter für den sprachsensiblen Fachunterricht liegen zu jedem Experiment vor und unterstützen additiv. Diesem folgend, können die Schüler sich durch verschiedene Lesestrategien die Fachtexte inhaltlich erschließen, durch Vorgabe von Sprachhilfen eigene Texte schreiben oder mit Lückentexten arbeiten. Der Siemens Stiftung liegt daher die Sprachvermittlung in Verbindung mit den Naturwissenschaften von Haus aus sehr am Herzen, besonders in der frühkindlichen Bildung, in der der Grundstein für das spätere Interesse gelegt wird. Die Siemens Stiftung förderte die EASI Science-L-Studie daher sehr gerne zusammen mit weiteren Partnern, da der Erkenntnisgewinn durch diese Grundlagenforschung im Bereich der frühkindlichen naturwissenschaftlichen Bildung für viele Akteure und auch für die eigene Arbeit sehr gewinnbringend ist.

Eine unabhängige wissenschaftliche Jury unter Vorsitz von Prof. Manfred Prenzel sichtete, unterstützt durch externe Fachgutachterinnen und Fachgutachter, die im Rahmen einer öffentlichen Ausschreibung im Jahr 2013 eingegangenen

10 www.siemens-stiftung.org 
Anträge. Die Jury bewertete die Anträge anhand wissenschaftlicher Exzellenzkriterien und sprach sich für die Förderung der Studien durch die beiden Forschungsgruppen aus. Die Stiftung folgte dieser Förderempfehlung.

Von 2013 bis 2017 wurden die Studien durch zwei unabhängige Forschungsgruppen extern durchgeführt.

Die erste, Early Steps into Science (kurz: EASI Science) benannte Studie wurde von Mirjam Steffensky (IPN Kiel), Yvonne Anders (FU Berlin), Julia Barenthien (IPN Kiel), Ilonca Hardy (Uni Frankfurt), Miriam Leuchter (Uni Koblenz-Landau ${ }^{11}$ ), Elisa Oppermann (FU Berlin), Päivi Taskinen (Uni Jena) und Tobias Ziegler (Uni Frankfurt) durchgeführt. Die Forschungsgruppe untersuchte Wirkungen naturwissenschaftlicher Bildungsangebote auf die kognitiven und motivationalen Kompetenzen von pädagogischen Fachkräften und Kindern in Kitas.

Die zweite, Early Steps into Science and Literacy (kurz: EASI Science-L) genannte Studie wurde von Astrid Rank (Uni Regensburg), Anja Wildemann (Uni Koblenz-Landau), Sabina Pauen (Uni Heidelberg), Andreas Hartinger (Uni Augsburg), Sabrina Tietze (PH Freiburg) und Rahel Kästner (Uni Heidelberg) umgesetzt. Diese Forschungsgruppe untersuchte schwerpunktmäßig die Interaktionsqualität und mögliche sprachliche Bildungswirkungen im Kontext naturwissenschaftlicher Bildungsangebote bei Vorschulkindern.

Bei beiden Studien wurden drei Gruppen einbezogen: pädagogische Fachkräfte aus Kitas mit regelmäßigen Fortbildungen in der Initiative HdkF (HdkF-Gruppe), solche mit naturwissenschaftlichen Fortbildungen durch andere Initiativen (NawiGruppe) und solche ohne naturwissenschaftlichen Schwerpunkt (Vergleichsgruppe). Die zentralen Fragestellungen und Ergebnisse sind in „Zusammenfassung zentraler Ergebnisse“ (Kapitel B) zu finden sowie vertiefend in den Abschlussberichten der Studien (Kapitel C und D).

Die Stiftung organisierte die administrative Betreuung der Studienprojekte, eine inhaltlich-wissenschaftliche Beratung erfolgte durch den Forschungslenkungskreis (Vorsitz: Prof. Dr. Manfred Prenzel) und ab 2015 durch das Folgegremium Wissenschaftlicher Beirat (Vorsitz: Prof. Dr. Hans-Günter Roßbach). Der Wissenschaftliche Beirat der Stiftung befasste sich in seinen Sitzungen regelmäßig mit den Studien und beriet die Forschungsgruppen während der Durchführung fünfmal im persönlichen Austausch. Abschließend berichteten die Forschungsgruppen dem Stiftungsrat über die finalen Ergebnisse. Die beiden Forschungsgruppen pflegten zudem einen intensiven Austausch untereinander, um Synergieeffekte nutzen zu können, und besuchten während der Projektlaufzeit diverse

11 Die Studie wurde an Miriam Leuchters ehemaligem Lehrstuhl für Didaktik des Sachunterrichts an der Westfälischen Wilhelms-Universität Münster durchgeführt. 
Konferenzen und Kongresse, um Input und Feedback aus der Wissenschaftscommunity zu erhalten.

Im vorliegenden Band werden die Abschlussberichte der Studien EASI Science und EASI Science-L zu den Wirkungen naturwissenschaftlicher Bildungsangebote veröffentlicht. Die Studienberichte stellen neben Fragestellungen, Studiendesigns, Erhebungsinstrumenten und wesentlichen Ergebnissen auch Empfehlungen für die naturwissenschaftliche Bildung im Elementarbereich in den Fokus und geben der Stiftung „Haus der kleinen Forscher“ Orientierung für die Qualitätsentwicklung ihrer Angebote. Die Umsetzung der wissenschaftlichen Empfehlungen in den inhaltlichen Angeboten der Stiftung „Haus der kleinen Forscher“ und ihrer fachlichen Weiterentwicklung werden im Schlusskapitel des Bandes beschrieben. 



\section{Zusammenfassung zentraler Ergebnisse}

Stiftung Haus der kleinen Forscher

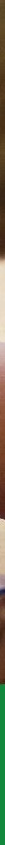




\section{Zusammenfassung zentraler Ergebnisse}

Wie wirken frühkindliche Bildungsangebote im naturwissenschaftlichen Bereich? Diese Frage konnte die Wissenschaft in Deutschland bisher kaum beantworten, denn dazu gab es bislang so gut wie keine systematische Forschung für den Elementarbereich und keine verlässlichen empirischen Messinstrumente.

Die Stiftung „Haus der kleinen Forscher“ versteht sich als lernende Organisation, die kontinuierlich an der Verbesserung der Qualität ihrer Bildungsangebote arbeitet. Die Frage, wie naturwissenschaftliche Bildungsangebote auf die Kompetenzen von pädagogischen Fachkräften und Kindern wirken, lässt sich nur mit empirischer Forschung beantworten. Deshalb hat das „Haus der kleinen Forscher" gemeinsam mit dem Bundesministerium für Bildung und Forschung, der Baden-Württemberg Stiftung und der Siemens Stiftung zwei mehrjährige Studien zu den Wirkungen naturwissenschaftlicher Frühbildung gefördert.

Folgenden zentralen Fragen sind die Bildungsforscherinnen und -forscher nachgegangen:

1. Welche Wirkungen haben naturwissenschaftliche Bildungsangebote auf naturwissenschaftsbezogene Kompetenzen von Kindern und pädagogischen Fachkräften? Dieser Frage sind Bildungsforscherinnen ${ }^{12}$ mit der Studie Early Steps into Science (EASI Science) nachgegangen.

2. Welche Wirkungen haben naturwissenschaftliche Bildungsangebote auf die sprachlichen Fähigkeiten von Kindern? Wie hängen sprachliche Anregungsqualität und naturwissenschaftsbezogene Prozessqualität von Bildungsangeboten zusammen? Diese Fragen hat eine Gruppe von Bildungswissenschaftlerinnen und -wissenschaftlern ${ }^{13}$ in der Studie Early Steps into Science and Literacy (EASI Science-L) untersucht.

3. Wie können die Wirkungen naturwissenschaftlicher Frühbildung überhaupt empirisch gemessen werden? Die beiden Forschungsgruppen haben ein metho-

12 Die Autorinnen der Studie „EASI Science“: Prof. Dr. Mirjam Steffensky (Leibniz-Institut für die Pädagogik der Naturwissenschaften und Mathematik (IPN) Kiel), Prof. Dr. Yvonne Anders (Freie Universität Berlin), Prof. Dr. Ilonca Hardy (Goethe-Universität Frankfurt am Main) und Prof. Dr. Miriam Leuchter (Universität Koblenz-Landau). Die Studie wurde vom BMBF und der Stiftung „Haus der kleinen Forscher" gefördert.

13 Die Autoren der Studie „EASI Science-L“: Prof. Dr. Astrid Rank (Universität Regensburg), Prof. Dr. Anja Wildemann (Universität Koblenz-Landau), Prof. Dr. Andreas Hartinger (Universität Augsburg) und Prof. Dr. Sabina Pauen (Universität Heidelberg). Die Studie wurde von der Baden-Württemberg Stiftung, der Siemens Stiftung und der Stiftung „Haus der kleinen Forscher“ gefördert. 
disches Instrumentarium zur Erfassung von naturwissenschaftlicher Bildungsqualität und zur Messung von Bildungswirkungen entwickelt.

Die Forscherinnen und Forscher haben Bildungswirkungen auf Ebene der Kitas und der pädagogischen Fachkräfte sowie bei den Kindern untersucht. Dabei wurden drei Gruppen einbezogen: pädagogische Fachkräfte aus Kitas mit regelmäßigen Fortbildungen in der Initiative HdkF (HdkF-Gruppe), solche mit naturwissenschaftlichen Fortbildungen durch andere Initiativen (Nawi-Gruppe) und solche ohne naturwissenschaftlichen Schwerpunkt (Vergleichsgruppe).

\section{Early Steps into Science - EASI Science}

\section{Hintergrund und Fragestellung}

Ziel des Forschungsprojekts EASI Science war es, Erkenntnisse über Wirkungen naturwissenschaftlicher Bildungsangebote in der frühen Kindheit zu gewinnen. Dabei standen zum einen die naturwissenschaftsbezogenen Kompetenzen der Kinder, zum anderen die der pädagogischen Fachkräfte in Kitas im Blickpunkt. Diese wurden im Hinblick auf angestrebte Zielbereiche früher naturwissenschaftlicher Bildung untersucht. Ein weiteres Ziel war es, reliable und valide Instrumente zur Erfassung naturwissenschaftlicher Kompetenz bei Fachkräften und Kindern zu entwickeln. Die Studie wurde von der Stiftung „Haus der kleinen Forscher“ und dem Bundesministerium für Bildung und Forschung (BMBF) gefördert. Folgende Fragen standen im Zentrum der Studie:

1. Wie lässt sich die Struktur der naturwissenschaftsbezogenen Kompetenzen von frühpädagogischen Fachkräften und Kindern beschreiben und erfassen?

2. Zeigen sich Wirkungen von Bildungsangeboten auf die naturwissenschaftsbezogenen Kompetenzen von pädagogischen Fachkräften und Kindern? Gibt es Unterschiede zwischen Einrichtungen mit bzw. ohne naturwissenschaftlichen Bildungsschwerpunkt („Haus der kleinen Forscher“-Schwerpunkt, anderer naturwissenschaftlicher Schwerpunkt, kein naturwissenschaftlicher Schwerpunkt), und hängen die Wirkungen von der Fortbildungsteilnahme der Fachkräfte ab?

3. In welchem Zusammenhang stehen die Qualität und Häufigkeit der Umsetzung naturwissenschaftlicher Bildungsangebote in der Einrichtung mit den Kompetenzen von Fachkräften und Kindern? 


\section{Durchführung}

An der Studie beteiligten sich 110 Kindertagestätten aus den Großräumen Berlin, Jena, Kiel, Münster und Frankfurt. Es nahmen 327 pädagogische Fachkräfte (Durchschnittsalter: 38 Jahre, $88 \%$ Frauen) und 283 Kinder teil (Durchschnittsalter: fünfeinhalb Jahre, $48 \%$ Mädchen).

\section{Zentrale Ergebnisse der Studie}

\section{Naturwissenschaftsbezogene Kompetenz ist mehrdimensional.}

- Naturwissenschaftliche Kompetenz besteht aus kognitiven und motivationalen Facetten. Interesse, Motivation, Wissen und Überzeugungen spielen eine Rolle.

- In der Studie gelang es, Messinstrumente von hoher wissenschaftlicher Qualität und Güte zur empirischen Erfassung naturwissenschaftsbezogener Kompetenzen von pädagogischen Fachkräften und Kindern im Elementarbereich zu entwickeln.

\section{Naturwissenschaftsbezogene Bildungsangebote zeigen Wirkungen.}

- Naturwissenschaftliche Fortbildungen stehen in einem positiven Zusammenhang mit den naturwissenschaftsbezogenen professionellen Kompetenzen der Fachkräfte.

- Pädagogische Fachkräfte, die an naturwissenschaftlichen Fortbildungen teilgenommen haben (,Haus der kleinen Forscher“ oder andere naturwissenschaftliche Fortbildungen), weisen ein höheres inhalts- und prozessbezogenes Fachwissen auf als nicht fortgebildete pädagogische Fachkräfte. Tendenzielle Unterschiede im fachdidaktischen Wissen zeigen sich, wenn man weitere naturwissenschaftliche Lerngelegenheiten der Fachkräfte (etwa in der Ausbildung) berücksichtigt.

- Naturwissenschaftlich fortgebildete pädagogische Fachkräfte haben ein stärkeres Interesse an Naturwissenschaften und höhere Fähigkeitsüberzeugungen in Bezug auf ihre pädagogische Arbeit im Vergleich zu nicht fortgebildeten Fachkräften.

- Fortgebildete pädagogische Fachkräfte vertreten eher lerntheoretische Überzeugungen, die die Eigeninitiative des Kindes bzw. die Kombination einer 
hohen Initiative auf Seiten des Kindes und auf Seiten der Fachkraft betonen; Ansätze, die dem Kind eine passive Rolle zuschreiben, lehnen sie stärker ab als nicht fortgebildete Fachkräfte.

- Fortgebildete pädagogische Fachkräfte setzen naturwissenschaftliche Bildungsgelegenheiten häufiger und in höherer (selbstberichteter) Prozessqualität um.

- Pädagogische Fachkräfte der „Haus der kleinen Forscher“-Gruppe besuchen im Mittel mehr Fortbildungen zu naturwissenschaftlichen Themen als Fachkräfte der naturwissenschaftlichen Vergleichsgruppe.

— „Haus der kleinen Forscher“-Kitas haben den naturwissenschaftlichen Bildungsbereich stärker in der Einrichtung verankert als die Vergleichsgruppen. Dies zeigt sich beispielsweise in einer häufigeren Thematisierung des Bildungsbereichs Naturwissenschaften in Teamsitzungen.

- Kinder aus Einrichtungen mit einem expliziten naturwissenschaftlichen Schwerpunkt (durch die Einrichtungsleitung bestimmt) zeigen höhere Lernfreude und höheres Selbstvertrauen in Bezug auf Naturwissenschaften als Kinder in Einrichtungen ohne naturwissenschaftlichen Schwerpunkt.

\section{Die Qualität und Regelmäßigkeit der Umsetzung naturwissenschaftlicher} Bildungsangebote sind ausschlaggebend für den Wissenszuwachs der Kinder.

- Die naturwissenschaftlichen Kompetenzen der Fachkräfte und insbesondere deren Motivation hängen positiv mit der Prozessqualität und der Häufigkeit naturwissenschaftlicher Bildungsangebote zusammen.

- Je stärker die naturwissenschaftliche Bildung in der Einrichtung verankert ist, desto höher fällt wiederum die naturwissenschaftsbezogene Motivation der Fachkräfte aus. Dies deutet darauf hin, dass Fachkräfte in ihrer Motivation über die eigenen Fortbildungseffekte hinaus von der Verankerung der Naturwissenschaften in ihrer Einrichtung profitieren.

- Es besteht ein positiver Interaktionseffekt von berichteter Qualität und Häufigkeit naturwissenschaftlicher Lerngelegenheiten auf das Wissen der Kinder in den Naturwissenschaften. Ausschlaggebend für das kindliche Wissen scheinen regelmäßige naturwissenschaftliche Lerngelegenheiten mit einem Minimum an Prozessqualität zu sein. Regelmäßige Lernangebote bei gerin- 
ger Prozessqualität bzw. qualitativ hochwertige Lernangebote, die nur selten stattfinden, allein stehen in keinem positiven Zusammenhang mit dem kindlichen Wissen in den Naturwissenschaften.

\section{Early Steps into Science and Literacy - EASI Science-L}

\section{Hintergrund und Fragestellung}

Das Forschungsprojekt EASI Science-L untersuchte die Interaktionsqualität und mögliche sprachliche Bildungswirkungen im Kontext naturwissenschaftlicher Bildungsangebote bei Vorschulkindern. Die Studie ${ }^{14}$ konzentrierte sich auf die sprachliche Anregungsqualität (Rank et al., Teilprojekt 1) und auf die Prozessqualität naturwissenschaftlicher Lehr-Lern-Prozesse (Pauen \& Kästner, Teilprojekt 2). Mithilfe neuer Instrumente zur Auswertung von Video- und Transkriptdaten wurde überprüft, ob bzw. bei welchen Phasen naturwissenschaftlichen Vorgehens Zusammenhänge mit sprachlichen Kompetenzen auftreten. Zudem interessierte, welche Bezüge zwischen der Prozessqualität einer Bildungssituation und naturwissenschaftlichen Bildungswirkungen beobachtbar sind. Die Studie wurde von der Stiftung „Haus der kleinen Forscher“ gemeinsam mit der Baden-Württemberg Stiftung und der Siemens Stiftung gefördert. Folgende Fragen standen im Zentrum der Studie:

\section{Wie hoch ist die sprachliche Anregungsqualität in einer naturwissenschaftlichen Forschungssituation mit Kindern? (Teilprojekt 1)}

- Gibt es Unterschiede in der sprachlichen Anregungsqualität zwischen Einrichtungen mit bzw. ohne naturwissenschaftlichen Bildungsschwerpunkt (,Haus der kleinen Forscher“-Schwerpunkt, anderer naturwissenschaftlicher Schwerpunkt, kein naturwissenschaftlicher Schwerpunkt)?

- Hängt die sprachliche Anregungsqualität von der Fortbildungsteilnahme der Fachkräfte ab?

- Lassen sich unterschiedliche Ausprägungen der sprachlichen Anregungsqualität während einzelner Phasen des forschenden Vorgehens (Methode „Forschungskreis“) finden?

14 Die Studie EASI Science-L wurde in einem Forschungsverbund mit zwei Schwerpunkten (Sprachliche Anregung in Teilprojekt 1 und Prozessqualität in Teilprojekt 2) durchgeführt. Die Darstellung der Ergebnisse erfolgt daher in getrennten Abschlussberichten in diesem Band. 
2. Können Zusammenhänge zwischen der sprachlichen Anregung während des Forschens und sprachlichen Kompetenzen der Kinder festgestellt werden? (Teilprojekt 1)

- Zeigen Kinder (u. a. bei Kindern mit Deutsch als Zweitsprache) aus Einrichtungen mit bzw. ohne naturwissenschaftlichen Bildungsschwerpunkt Unterschiede in der Verwendung von Bildungssprache?

- Hängt die gezeigte Bildungssprache der Kinder mit der sprachlichen Anregungsqualität der pädagogischen Fachkräfte in der Forschungssituation oder mit dem Fortbildungsbesuch zusammen?

3. Wie lässt sich naturwissenschaftsbezogene Prozessqualität mikroanalytisch beschreiben, und womit hängt diese zusammen? (Teilprojekt 2)

- Kann man die naturwissenschaftliche Prozessqualität anhand von Videodaten zuverlässig und valide erfassen?

- Hängt die naturwissenschaftsbezogene Interaktionsqualität mit der sprachlichen Anregungsqualität in der Forschungssituation zusammen?

- Gibt es Unterschiede in der naturwissenschaftsbezogenen Prozessqualität zwischen Einrichtungen mit bzw. ohne naturwissenschaftlichen Bildungsschwerpunkt, und hängt dies von der Fortbildungsteilnahme der Fachkräfte $a b ?$

- Gibt es Zusammenhänge mit den naturwissenschaftlichen Kompetenzen der Kinder?

\section{Durchführung}

An der Studie beteiligten sich 58 Kindertagestätten aus dem Großraum Karlsruhe und Heidelberg mit je einer pädagogischen Fachkraft (95\% Frauen) sowie einer Gruppe von je etwa vier Kindern. Insgesamt nahmen 222 Kinder im Durchschnittsalter von fünfeinhalb Jahren teil (46\% Mädchen). Die pädagogischen Fachkräfte gestalteten eine Forschungssituation mit den Kindern, die videografiert und im Hinblick auf die Fragestellungen ausgewertet wurde. 


\section{Zentrale Ergebnisse der Studie}

\section{Eine hohe sprachliche Anregungsqualität ist beim Forschen möglich (Teilprojekt 1).}

- Im Durchschnitt aller untersuchten Kitas zeigt sich eine sprachliche Anregungsqualität im mittleren Bereich, dabei gibt es Unterschiede je nach Einrichtungsschwerpunkt: Fachkräfte aus einer als „Haus der kleinen Forscher“ zertifizierten Einrichtung zeigen im Schnitt die höchste sprachliche Anregungsqualität.

- Fachkräfte, die Fortbildungen zu einem naturwissenschaftlichen Thema besucht hatten, zeigen eine höhere sprachliche Anregungsqualität als Fachkräfte ohne naturwissenschaftsbezogene Fortbildung - demgegenüber gibt es in der untersuchten Stichprobe keinen Effekt rein sprachlicher Fortbildungen.

- Fachkräfte, die eine kombinierte Fortbildung zu Naturwissenschaften und Sprache der Stiftung „Haus der kleinen Forscher“"15 besucht hatten, zeigen eine höhere sprachliche Anregungsqualität als Fachkräfte, die diese Fortbildung nicht besucht hatten.

- In bestimmten Phasen naturwissenschaftlichen Vorgehens („Beobachten \& Beschreiben“, „Ergebnisse dokumentieren/sammeln“, „Ergebnisse erörtern") kommt anteilig besonders viel sprachliche Anregung vor. Diese Phasen bieten ein besonderes Potenzial für die Verknüpfung von sprachlicher und naturwissenschaftlicher Bildung. Allerdings finden die besonders sprachförderlichen Episoden in den Phasen insgesamt noch eher selten statt.

\section{Die sprachlichen Kompetenzen der Kinder hängen mit der sprachlichen} Anregung während des Forschens zusammen (Teilprojekt 1).

- Die Kinder aller Untersuchungsgruppen zeigen im Durchschnitt vergleichbare bildungssprachliche Kompetenzen (unabhängig davon, ob bzw. welchen naturwissenschaftlichen Schwerpunkt eine Kita hat oder ob ein Kind Deutsch als Erst- oder Zweitsprache spricht).

15 Fortbildung „Sprudelgas und andere Stoffe - Mit Kita- und Grundschulkindern Chemie entdecken und dabei die sprachliche Entwicklung unterstützen“, vgl. gleichnamige Broschüre der Stiftung Haus der kleinen Forscher (2013). Verfügbar unter: www.haus-der-kleinen-forscher.de 
- Den stärksten Einfluss auf die Sprachfähigkeiten der Kinder haben Merkmale des Kindes wie Intelligenz, Alter und Geschlecht.

- Der Fortbildungsbesuch der Fachkräfte und die kindliche Verwendung von (Bildungs-)Sprache hängen zusammen: Kinder, deren pädagogische Fachkraft eine kombinierte Fortbildung zu Naturwissenschaften und Sprache besucht hat, zeigen in der naturwissenschaftlichen Lernsituation eine höhere bildungssprachliche Performanz als Kinder, deren Fachkraft nicht entsprechend fortgebildet ist.

3. Die naturwissenschaftsbezogene Prozessqualität hängt mit dem Fortbildungsbesuch der Fachkräfte, der sprachlichen Anregungsqualität und den kindlichen Kompetenzen zusammen (Teilprojekt 2).

- Die durch die Fachkraft gestaltete naturwissenschaftsbezogene Prozessqualität zeigt positive Zusammenhänge mit den naturwissenschaftlichen Kompetenzen der Kinder.

- Die naturwissenschaftsbezogene Prozessqualität hängt positiv mit der sprachlichen Anregungsqualität zusammen. Fachkräfte mit einer höheren Prozessqualität zeigen in der Forschungssituation auch eine höhere sprachliche Anregungsqualität.

- Die naturwissenschaftsbezogene Interaktionsqualität in der Forschungssituation ist bei Fachkräften, die an „Haus der kleinen Forscher“-Fortbildungen teilgenommen haben, höher als bei Fachkräften ohne naturwissenschaftliche Fortbildung. 



\section{Early Steps into Science - EASI Science}

Wirkungen früher naturwissenschaftlicher Bildungsangebote auf die naturwissenschaftlichen Kompetenzen von Fachkräften und Kindern

Mirjam Steffensky, Yvonne Anders, Julia Barenthien, Ilonca Hardy, Miriam Leuchter, Elisa Oppermann, Päivi Taskinen, Tobias Ziegler

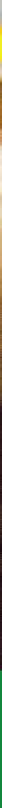

1 Enleitung

2 Theoretischer Hintergrund der Studie

3 Fragestellungen der Studie

4 Methode

5 Ergebnisse

6 Diskussion der Ergebnisse

7 Grenzen der Studie

8 Zentrale Ergebnisse

9 Folgerungen für die Stiftungsarbeit 


\section{Einleitung}

In den letzten Jahrzehnten wuchs die Aufmerksamkeit für die Potenziale früher Bildungsangebote (im Sinne von Bildungsgelegenheiten) in Kindertageseinrichtungen. Hierdurch fand eine Ausweitung der Aufgabenbereiche der Kindertageseinrichtungen (im Folgenden Kitas genannt) statt, z. B. hinsichtlich der stärkeren Akzentuierung der Förderung früher akademischer Fähigkeiten. Diese wurde in den Rahmen- und Orientierungsplänen der Bundesländer für den Elementarbereich festgeschrieben, in denen auch die Naturwissenschaften als ein Bildungsbereich benannt werden.

Verschiedene internationale und nationale Untersuchungen haben sich mit den kurz-, mittel- und langfristigen Auswirkungen früher Bildungsangebote beschäftigt und die Bedeutsamkeit der Qualität für die Art und Persistenz möglicher positiver Wirkungen herausgestellt (z. B. Anders, 2013). So konnte für die mathematische Entwicklung von Kindern gezeigt werden, dass sich der Besuch qualitativ hochwertiger Kitas positiv auf die Entwicklung auswirkt und dass sich die positiven Effekte der Qualität auch noch viele Jahre später abbilden lassen. Bisherige Studien haben kaum naturwissenschaftliche Kompetenzen von Kindern in den Blick genommen. Erste Ergebnisse zeigen, dass natur- und sozialwissenschaftliches Wissen von Kindern am Ende der Kita-Zeit und zu Beginn der Grundschule naturwissenschaftliche Leistungen in der dritten Klasse voraussagen (Morgan, Farkas, Hillemeier \& Maczuga, 2016). Auf Basis dieser Ergebnisse und der Ergebnisse aus anderen Domänen kann angenommen werden, dass frühe grundlegende Kompetenzen in den Naturwissenschaften wichtig für den weiteren Bildungserfolg sind und die Entwicklung dieser grundlegenden Kompetenzen maßgeblich von der Qualität früher Bildungsangebote abhängt.

Verschiedene Bildungsinitiativen in Deutschland streben eine stärkere Verankerung und eine Steigerung der Qualität früher naturwissenschaftlicher Bildung im Elementarbereich an. Eine dieser Bildungsinitiativen ist die Stiftung „Haus der kleinen Forscher“. Die Stiftung ist einer der größten bundesweiten Akteure, die das Ziel haben, Kindern in Kitas und Grundschulen eine intensivere Begegnung mit Mathematik, Informatik, Naturwissenschaften und Technik (kurz: MINT) zu ermöglichen. Die Stiftungsarbeit wird vom Bundesministerium für Bildung und Forschung (BMBF) und verschiedenen weiteren Partnern gefördert. Ein Schwerpunkt der Arbeit ist der Aufbau von lokalen Netzwerken, in denen pädagogische Fachkräfte mit Fortbildungsangeboten und Materialien unterstützt werden, um Lerngelegenheiten für Jungen und Mädchen in den MINT-Bildungsbereichen bereitzustellen und die Lernprozesse zu begleiten. 
Gleichzeitig unterstützt die Stiftung Studien zur Untersuchung der Stiftungsarbeit. Im Rahmen dieser Aktivitäten wurden die Wirkungsstudien mit den zwei Projekten Early Steps into Science (EASI Science) und Early Steps into Science and Literacy (EASI Science-L) von der Stiftung „Haus der kleinen Forscher“ und dem Bundesministerium für Bildung und Forschung (EASI Science) sowie der Siemens Stiftung und Baden-Württemberg Stiftung (EASI Science-L) gefördert. Im Fokus des Projektes EASI Science stehen die möglichen Wirkungen naturwissenschaftlicher Bildungsangebote in der Kita auf die professionellen Kompetenzen frühpädagogischer Fachkräfte, auf die Bildungsqualität von Kitas und die naturwissenschaftlichen Kompetenzen von Kindern. Dabei wird angenommen, dass die Bildungsinitiative „Haus der kleinen Forscher“, als ein Beispiel für ein solches Programm, über ihre Angebote die professionellen Kompetenzen der Fachkräfte und die Implementation naturwissenschaftlicher Bildungsinhalte in den Kitas positiv beeinflusst. Hierbei wird ein naturwissenschaftsspezifisches, strukturell-prozessuales Modell vorschulischer Bildungsqualität angenommen: Professionelle Kompetenzen frühpädagogischer Fachkräfte werden als strukturelle Voraussetzung für die Bereitstellung qualitativ hochwertiger Lerngelegenheiten angenommen. Diese qualitativ hochwertigen Lerngelegenheiten werden wiederum als ausschlaggebend für die (naturwissenschaftliche) Kompetenzentwicklung der Kinder angesehen (Anders, Hardy, Pauen \& Steffensky, 2013). Eine hinreichende Quantität, d. h. die regelmäßige sowie umfangreiche Bereitstellung naturwissenschaftlicher Lerngelegenheiten, ist dabei eine Voraussetzung dafür, dass Kinder überhaupt von erhöhter naturwissenschaftlicher Bildungsqualität profitieren können. Zentrale Zielebenen von naturwissenschaftlichen Bildungsangeboten stellen folglich insbesondere die Ebene der frühpädagogischen Fachkräfte und die Ebene der teilnehmenden Kinder dar.

Der vorliegende Bericht bezieht sich auf das Forschungsprojekt EASI Science. Im Rahmen dieses Projektes wurden Wirkungen naturwissenschaftlicher Bildungsangebote auf die naturwissenschaftlichen, professionellen Kompetenzen von frühpädagogischen Fachkräften und auf die naturwissenschaftlichen Kompetenzen von Kindern untersucht. Im Folgenden wird zunächst ein Gesamtmodell vorgestellt, welches mögliche Wirkmechanismen naturwissenschaftlicher Bildungsangebote in der Kita skizziert. Im Anschluss werden die Zielebenen der Kinder und Fachkräfte sowie die Bedeutung qualitativer und quantitativer Aspekte früher naturwissenschaftlicher Bildung näher beschrieben. Um naturwissenschaftliche Kompetenzen von frühpädagogischen Fachkräften und Kindern zu untersuchen, war es jedoch zunächst notwendig, geeignete empirische Messinstrumente zu entwickeln. Die Instrumente sowie das Design des Projekts EASI Science mit seinen Resultaten und Schlussfolgerungen werden im Folgenden vorgestellt. 


\section{Theoretischer Hintergrund der Studie}

\subsection{Wirkungen naturwissenschaftlicher Bildungsangebote in der Kita}

Die potenziellen Wirkungsmechanismen verschiedener naturwissenschaftlicher Bildungsangebote in der Einrichtung können über das in Abbildung 3 veranschaulichte Modell beschrieben werden. Die kindliche Kompetenzentwicklung steht als zentrale Zieldimension naturwissenschaftlicher Bildung - im Fokus des Modells. Hierbei wird im Sinne der Domänenspezifität angenommen, dass für die naturwissenschaftliche Kompetenzentwicklung insbesondere die naturwissenschaftlichen Lernangebote für die Kinder relevant sind. Ein direkter Einfluss auf den Lernerfolg der Kinder wird der Quantität sowie der Qualität früher naturwissenschaftlicher Lerngelegenheiten unterstellt. So wird davon ausgegangen, dass Kinder nur dann von hochwertigen Lerngelegenheiten profitieren können, wenn diese ausreichend häufig und ausreichend intensiv stattfinden. Eine hinreichende Regelmäßigkeit und Intensität (im Sinne von Qualität) stellen damit eine Voraussetzung für die Annahme eines positiven Fördereffekts dar. Darüber hinaus kann eine multiplikative Wirkung von Quantität und Qualität auf die kindliche Entwicklung angenommen werden. Das heißt, qualitativ hochwertige Lerngelegenheiten werden einen umso stärkeren Einfluss auf die kindliche Entwicklung haben, je häufiger sie umgesetzt werden.

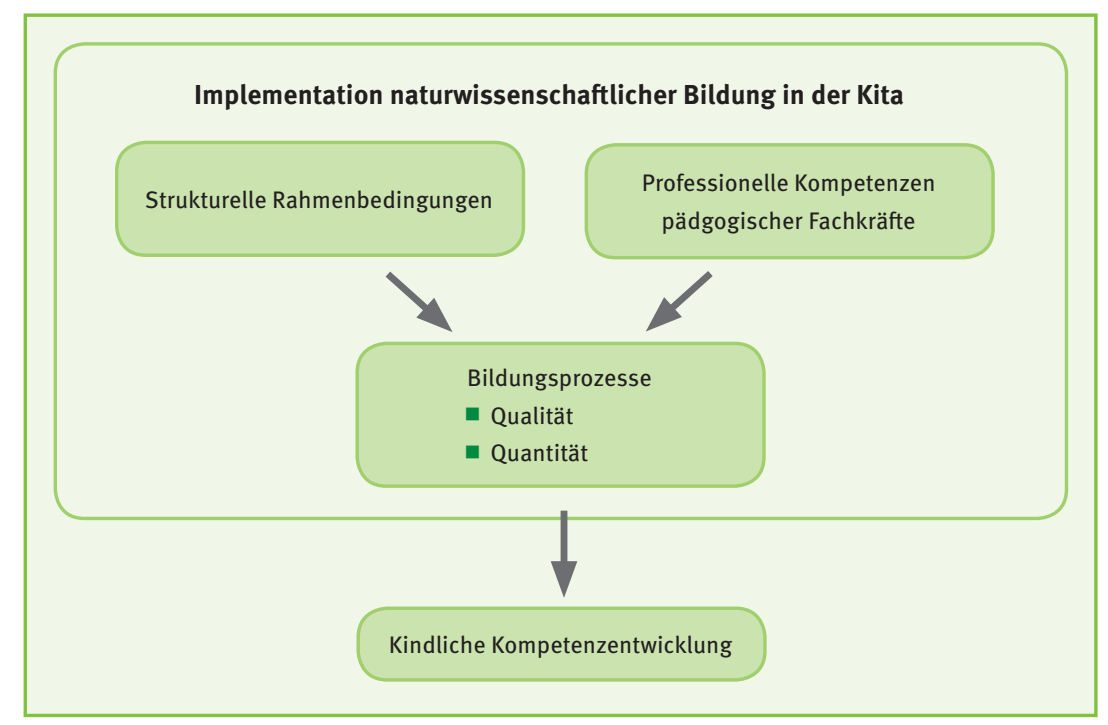

Abbildung 3. Wirkungsmodell früher naturwissenschaftlicher Bildungsangebote 
Voraussetzungen für die Gestaltung hochwertiger und regelmäßiger naturwissenschaftlicher Lerngelegenheiten sind strukturelle Qualitätsaspekte der Einrichtung sowie die notwendigen Kompetenzen aufseiten der Fachkräfte. Zu den strukturellen Qualitätsaspekten zählen z. B. die Gruppengröße, der Fachkraft-Kind-Schlüssel, die räumliche Ausstattung, die materiale Ausstattung sowie die Größe der Einrichtung. Die Bedeutsamkeit der strukturellen Rahmenbedingungen für die Qualität der pädagogischen Interaktionen konnte in zahlreichen Studien belegt werden (Blatchford, 2003; De Schipper, Riksen-Walraven \& Geurts, 2006; Kuger \& Kluczniok, 2008; Barros \& Aguiar, 2010; Rentzou \& Sakellariou, 2011), auch wenn hinsichtlich der Bedeutung einzelner Qualitätsmerkmale in den Studien zum Teil unterschiedliche Ergebnisse gefunden wurden. Für die Qualität früher naturwissenschaftlicher Bildung werden insbesondere der Anteil der in den Naturwissenschaften fortgebildeten Fachkräfte sowie die naturwissenschaftlichen Bildungsmaterialien und die Räumlichkeiten in der Einrichtung, z. B. das Vorhandensein eines Außengeländes, Möglichkeiten, mit Wasser zu spielen etc., als relevant angenommen.

Neben günstigen strukturellen Rahmenbedingungen sind die notwendigen professionellen Kompetenzen der Fachkräfte entscheidend für die Gestaltung früher Lerngelegenheiten (vgl. Anders \& Rossbach, 2015; Fröhlich-Gildhoff, Nentwig-Gesemann \& Pietsch, 2011; McCray \& Chen, 2012; Siraj-Blatchford, Sylva, Muttock, Gilden \& Bell, 2002). Die pädagogische Fachkraft plant und initiiert pädagogische Interaktionen und reagiert spontan auf die Anforderungen in bestimmten Situationen. Dementsprechend ist ihre Rolle zentral. Mit Blick auf ihre Kompetenzen wird im Allgemeinen in Anlehnung an die Schulforschung (z. B. Baumert \& Kunter, 2006; Siraj-Blatchford et al., 2002) zwischen Wissensaspekten sowie motivationalen Aspekten und Einstellungen unterschieden. So erfordert die Umsetzung naturwissenschaftlicher Lerngelegenheiten das nötige Fach- und fachdidaktische Wissen in den Naturwissenschaften. Fachwissen umfasst die fachlichen Grundlagen der relevanten Inhalte, während es beim fachdidaktischen Wissen um das Zugänglichmachen dieser Inhalte für Lerner und Lernerinnen geht. Auch eine positive Einstellung gegenüber den Naturwissenschaften sowie Selbstwirksamkeit und Freude beim Umgang mit naturwissenschaftlichen Themen können die Wahrscheinlichkeit erhöhen, dass solche Themen im Kita-Alltag überhaupt aufgegriffen werden (vgl. auch Erden \& Sönmez, 2010; Spektor-Levy, Baruch \& Mevarech, 2013).

Strukturelle Rahmenbedingungen und die professionellen Kompetenzen der Fachkräfte stehen jedoch nicht für sich, sondern sind in das System Kita eingebettet. Für eine langfristige Steigerung von Quantität und Qualität naturwissenschaftlicher Bildung ist es daher notwendig, dass Naturwissenschaften als Bildungsbereich in der gesamten Einrichtung implementiert sind (Implementationsqualität). 
Dies zeichnet sich u. a. dadurch aus, dass die naturwissenschaftliche Bildung als Einrichtungskonzept aufgegriffen wird. Zentral ist auch, dass Fortbildungsinhalte in das gesamte Team getragen werden und hier die Teamentwicklung anregen. Die Bedeutung der Implementationsqualität in der Einrichtung konnte bereits für andere Inhaltsbereiche bestätigt werden: Studien zur frühen sprachlichen Bildung und allgemeinen Anregungsqualität zeigen, dass das Ausmaß des Austausches im pädagogischen Team in einem Zusammenhang zur sprachlichen bzw. allgemeinen Anregungsqualität in der Einrichtung steht (Resa, Groeneveld, Turani \& Anders, 2017; Wertfein, Müller \& Danay, 2013). Eine Studie im Bereich der Naturwissenschaften auf Grundlage von Daten, auf denen auch der vorliegende Bericht beruht, konnte ferner zeigen, dass die Implementation des Bereichs der Naturwissenschaften als Einrichtungskonzept (Naturwissenschaften als pädagogischer Schwerpunkt der Einrichtung) in einem positiven Zusammenhang mit der naturwissenschaftlichen Lernfreude und Selbstwirksamkeitserwartung der Kinder steht (Oppermann, Brunner, Eccles \& Anders, 2017). Vor diesem Hintergrund wird die Implementation naturwissenschaftlicher Bildung als zentrale Qualitätsdomäne und Voraussetzung für die Wirksamkeit von Bildungsangeboten in der gesamten Einrichtung im Wirkungsmodell mit aufgenommen.

Die potenzielle Wirkung naturwissenschaftlicher (Fort-)Bildungsangebote ist an mehreren Punkten im Modell (Abbildung 3) zu verorten. Einerseits können Bildungsangebote durch materielle, finanzielle oder zeitliche Ressourcen die strukturellen Rahmenbedingungen verbessern. Hierzu zählen beispielweise die Bereitstellung von Materialien zum Forschen sowie die Gestaltung von Räumen, z. B. das Einrichten eines Forscherraums. Andererseits können durch Fort- und Weiterbildungen gezielt die pädagogischen Fachkräfte angesprochen werden, um diese für die Vermittlung von Naturwissenschaften weiter zu qualifizieren. Nach Weinert (2001) wird davon ausgegangen, dass professionelle Kompetenzen grundsätzlich erlernbar sind und sich dementsprechend durch Angebote der Aus-, Fort- und Weiterbildung verändern lassen. Befunde aus der Schulforschung belegen zudem positive Effekte von Fortbildungen für den Kompetenzerwerb von Fachkräften (vgl. Zaslow, Tout, Halle, Whittaker \& Lavelle, 2010; Gropen, Kook, Hoisington \& Clark-Chiarelli, 2017). Durch die Verbesserung der strukturellen Rahmenbedingungen sowie die Qualifizierung von Fachkräften können Bildungsangebote indirekt auf die Qualität und Quantität naturwissenschaftlicher Bildung wirken und so letztendlich auch die Entwicklung der Kinder positiv beeinflussen. Dies gelingt jedoch langfristig nur, wenn naturwissenschaftliche Bildungsimpulse in der Einrichtung aufgegriffen und weiterentwickelt werden. Voraussetzung für eine nachhaltige Wirkung von Bildungsangeboten ist daher auch die Implementation naturwissenschaftlicher Bildung in der gesamten Einrichtung. 


\subsection{Naturwissenschaftliche Kompetenzen jüngerer Kinder}

Kinder erwerben bildungsrelevante Fertigkeiten und Fähigkeiten nicht erst in der Schule, sondern kontinuierlich ab der Geburt. Hierzu gehört, neben dem Spracherwerb sowie der Herausbildung von sozio-emotionalen und mathematischen Kompetenzen, auch die Entwicklung von frühen Fähigkeiten im Bereich der Naturwissenschaften (Sodian \& Mayer, 2013). Naturwissenschaftliche Kompetenzen werden dabei in der internationalen und nationalen Diskussion in Anlehnung an Konzeptionen der Scientific Literacy als mehrdimensionales Konstrukt aufgefasst, das Wissenskomponenten wie auch nicht leistungsbezogene Komponenten enthält (Norris \& Phillips, 2003; Bybee, McCrae \& Laurie, 2009). Kinder kommen durch die Auseinandersetzung mit Materialien, natürlichen Phänomenen oder durch aktive Denkanstöße und Gespräche mit Erwachsenen und anderen Kindern zum Auf- und Ausbau spezifischer Vorstellungen und Erkenntnisse (Eisenberg, DeLoache \& Siegler, 2005). Diese wiederum bilden die Grundlage für spätere Lernprozesse in der Schule (Saçkes, 2013; Saçkes, Trundle, Bell \& O’Connell, 2011), so dass frühe grundlegende Kompetenzen in der nationalen und internationalen Literatur auch als anschlussfähig und zunehmend auszudifferenzierend bezeichnet werden.

\subsubsection{Wissenskomponenten naturwissenschaftlicher Kompetenzen}

Die Wissenskomponenten naturwissenschaftlicher Kompetenzen werden im Sinne von Scientific Literacy in die Bereiche naturwissenschaftliches Wissen und Wissen über die Naturwissenschaften unterteilt. Naturwissenschaftliches Wissen beinhaltet das Verständnis naturwissenschaftlicher Inhalte bzw. Erklärungen zu Phänomenen und umfasst Wissen über Konzepte, Theorien und Begriffe in verschiedenen Inhaltsbereichen. Naturwissenschaftliches Wissen bzw. Konzeptverständnis wurde bereits im Kindergartenalter in unterschiedlichen Inhaltsbereichen nachgewiesen und wird als aktiv konstruiertes Wissen verstanden, das auf der Basis von bereichsspezifischem Vorwissen entsteht (z. B. Carey, 2009). Relevante alltagsnahe Themenfelder für junge Kinder im Bereich der unbelebten Natur sind die Auseinandersetzung mit unterschiedlichen Materialien, Aggregatzuständen des Wassers, Schwimmen und Sinken von Objekten im Wasser oder Magnetismus. Studien zeigen, dass die von jungen Kindern in alltäglichen Situationen entwickelten Vorstellungen zu naturwissenschaftlichen Phänomenen häufig nur eingeschränkt tragfähig sind (Siegler \& Chen, 2008). Durch Differenzierungs-, Integrations- und Umstrukturierungsprozesse werden diese naiven Vorstellungen sukzessive in wissenschaftliche, empirisch tragfähige Vorstellungen verändert. 
Dabei spielt eine aktive Auseinandersetzung mit den kindlichen Vorstellungen durch geeignete Bildungsangebote eine bedeutsame Rolle.

Das Wissen über die Naturwissenschaften bezieht sich auf das Verständnis naturwissenschaftlicher Methoden bzw. Denk- und Arbeitsweisen und das Verständnis der Naturwissenschaften als Disziplin (Nature of Science) und wird auch als prozessbezogenes Wissen bezeichnet. Studien zeigen, dass auch junge Kinder Bezüge zwischen Theorie und Evidenz auf einfachem Niveau herstellen können (Koerber, Sodian, Thoermer \& Nett, 2005; Zimmermann, 2007) und Elemente des wissenschaftlichen Zyklus verwenden (Samarapungavan, Mantzicopoulos, Patrick \& French, 2009). Dazu zählen verschiedene Prozesse, z. B. Vermutungen aufstellen, Phänomene gezielt beobachten und vergleichen oder Begründungen ableiten (Fthenakis, 2009).

Die Erhebung naturwissenschaftlicher Kompetenzen junger Kinder erfolgt häufig mittels lernprozessbegleitender Methoden, z. B. Portfolios (Samarapungavan, Mantzicopoulos \& Patrick, 2008). Diese sind eng an die konkrete Lernsituation gebunden und ermöglichen kaum vergleichbare Aussagen. Standardisierte und validierte Tests, mit denen Wissen in unterschiedlicher Breite und Tiefe ökonomisch erfasst werden kann, sind selten. Beispiele verfügbarer Instrumente für den Kindergartenbereich sind das Science Learning Assessment (Samarapungavan et al., 2009), der SNaKE-Test (Carstensen, Lankes \& Steffensky, 2011) oder das NEPS-Instrument (Hahn et al., 2013). Obwohl sich empirische Hinweise auf konzept- und prozessbezogene Kompetenzen von Vorschulkindern finden, ist über die Struktur bzw. den Zusammenhang der verschiedenen Komponenten naturwissenschaftlicher Kompetenzen bei Kindern bislang wenig bekannt. Sodian und Mayer (2013) weisen darauf hin, dass das Wissen über die Naturwissenschaften aus theoretischer Perspektive im Sinne einer allgemeinen und bereichsübergreifenden Schlüsselkompetenz als Einflussfaktor auf das domänenspezifische Konzeptwissen angesehen werden kann. Dieser Annahme wurde jedoch in elementar- und primarpädagogischen Studien selten nachgegangen. Hinweise auf positive Zusammenhänge zwischen einzelnen Facetten naturwissenschaftlicher Wissenskomponenten im Grundschulalter finden sich bei Pollmeier und Kollegen (2017). Auch Grygier (2008) konnte im Rahmen einer Transferstudie mit Kindern im Grundschulalter positive Zusammenhänge zwischen konzeptuellem Wissen im Bereich Schwimmen und Sinken und dem Wissenschaftsverständnis ausmachen. Insbesondere für ältere Schülerinnen und Schüler sowie für Erwachsene konnte gezeigt werden, dass sich zunehmendes Wissen über naturwissenschaftliche Methoden positiv auf den konzeptuellen Wissensbereich auswirkt (u. a. Dirks \& Cunningham, 2006). Für Kinder im Vorschulalter finden sich kaum Untersuchungen zur Struktur naturwissenschaftlicher Kompetenzen. Hinweise liefern Analysen zur Validierung von Testinstrumenten. Samarapungavan et al. (2009) zeigen, dass die 
im Rahmen des Science Learning Assessments entwickelten prozess- und konzeptbezogenen Komponenten empirisch trennbar sind. Darüber hinaus liegen auch Analysen im Rahmen eher explorativer Item-Entwicklungen vor, in denen sich die prozess- und konzeptbezogenen Komponenten als nicht trennbar erwiesen (Carstensen et al., 2011; Hahn et al., 2013).

\subsubsection{Motivationale Komponenten naturwissenschaftlicher Kompetenzen}

Zu naturwissenschaftlichen Kompetenzen gehören auch motivationale Merkmale wie z. B. Lernfreude und Selbstwirksamkeitserwartung. Die Bedeutung domänenspezifischer Lernmotivation für die Kompetenzentwicklung von Kindern im Schulalter ist gut belegt (Eccles, Adler, Futterman, Goff, Kaczala, Meece \& Midgley, 1983; Wigfield, Eccles, Yoon, Harold, Arbreton, Freedman-Doan \& Blumenfeld, 1997). So lernen Kinder effektiver, wenn sie Freude an einer Lernsituation haben und sich selbst zutrauen, die Inhalte verstehen zu können (Wigfield, Eccles \& Rodriguez, 1998). Die Motivation zur Auseinandersetzung mit Naturwissenschaften wird bei jüngeren Kindern allgemein als hoch eingeschätzt (Lück, 2010), aber es fehlen dazu umfangreiche empirische Untersuchungen. Dies ist u. a. auf die Schwierigkeit der Erfassung motivationaler Aspekte im frühen Kindesalter zurückzuführen, da Kinder vor dem Schuleintritt selten in der Lage sind, differenziert über sich Auskunft zu geben (Nölke, Steffensky, Lankes \& Carstensen, 2013). Da die in der Grundschule vielfach eingesetzten Skalen nicht direkt verwendet werden können, werden zur Erfassung der Motivation jüngerer Kinder zumeist Beobachtungsverfahren und Elternbefragungen eingesetzt (Lück, 2010). Ansätze zur standardisierten Erfassung selbstbezogener bzw. motivationaler Komponenten sind z. B. das PICSES-Instrument (Puppet Interview Scales of Competence in and Enjoyment of Science; Mantzicopoulos, Patrick \& Samarapungavan, 2008) oder Fragebögen mit Ranking-Fragen zur Erfassung des Interesses (Nölke et al. 2013; Sechtig, Freund, Roßbach \& Anders, 2012). Insbesondere das US-amerikanische PICSES-Instrument leistet wichtige Vorarbeiten zur Erfassung früher naturwissenschaftlicher Lernmotivation, lässt sich aber nicht ohne Weiteres auf den deutschen Kontext übertragen, weil es die Kenntnis des Begriffs „Naturwissenschaften“ bei den Kindern voraussetzt. Diese Kenntnis ist für Kinder im Alter von 5-6 Jahren grundsätzlich fraglich. Das Verfahren ist in besonderem Maße in Deutschland infrage zu stellen, da sich die Tradition der Frühpädagogik hier als sozialpädagogisch beschreiben lässt (OECD, 2011). Frühe akademische Lerninhalte wurden erst seit der Implementation der Rahmen- und Orientierungspläne vor wenigen Jahren stärker fokussiert. Die gezielte Vermittlung von Lerninhalten spielt dennoch eine eher untergeordnete Rolle. Dementsprechend dürfte der Begriff der „Naturwissenschaften“ für viele Kinder im Vorschulalter unbekannt sein. 
Eine Adaption vorhandener Instrumente für den deutschen Kontext scheint daher notwendig.

Die Operationalisierung von kindlicher Lernmotivation erfolgte in existierenden Instrumenten auf Grundlage der theoretischen Annahmen zur Struktur der Motivation nach Wigfield und Eccles (2000). Demnach lässt sich Lernmotivation in zwei Arten von Überzeugungen unterteilen: Erfolgserwartung und Aufgabenwert. Erfolgserwartung beschreibt neben der Einschätzung der Schwierigkeit einer Aufgabe vor allem das Vertrauen in die eigenen Fähigkeiten, die Aufgabe zu bewältigen (Selbstwirksamkeitserwartung). Zum Aufgabenwert zählen der Nutzen der Aufgabe, die Freude während der Ausführung der Aufgabe (intrinsischer Wert) sowie der persönliche Wert für die eigene Identität (Eccles \& Wigfield, 2002). Bei Kindern im Vorschulalter wird in dieser Untersuchung davon ausgegangen, dass der intrinsische Wert, also die Freude an der Beschäftigung mit naturwissenschaftlichen Inhalten, für den Lernerfolg der Kinder von höherer Relevanz ist als der subjektive Nutzen oder der persönliche Wert für die Identität. Die Untersuchung fokussiert daher auf die Lernfreude der Kinder. Auf Grundlage der theoretischen Annahmen von Eccles und Wigfield (2002) sowie empirischer Befunde von Mantzicopoulos et al. (2008) für den Bereich der Naturwissenschaften wird davon ausgegangen, dass Selbstwirksamkeitserwartungen und Lernfreude trennbare Aspekte der Lernmotivation abbilden.

\subsection{Quantität und Qualität früher naturwissenschaftlicher Lerngelegenheiten}

Die kindliche Kompetenzentwicklung in den Naturwissenschaften ist maßgeblich von den Lerngelegenheiten abhängig, die Kinder vorfinden. Hierbei gelten, wie bereits im Modell zur Wirkung früher naturwissenschaftlicher Bildungsangebote beschrieben (siehe Abschnitt 2.1), sowohl die Qualität als auch die Quantität früher naturwissenschaftlicher Bildungsprozesse als entscheidend und werden deshalb im Folgenden noch einmal vertiefend dargestellt. Die Qualität früher Bildungsprozesse beschreibt die Passung der Lern- und Entwicklungsumgebung auf die Bedürfnisse des Kindes im Sinne einer ganzheitlichen Förderung (Tietze, 2008). In der Forschungsliteratur wird Qualität als mehrdimensionales Konstrukt aufgegriffen, welches die strukturellen Merkmale der Einrichtung (Strukturqualität), die Einstellungen der frühpädagogischen Fachkräfte (Orientierungsqualität) sowie die Qualität der pädagogischen Prozesse (Prozessqualität) umfasst (Pianta, Howes, Burchinal, Bryant, Clifford, Early \& Barbarin, 2005; Tietze \& Förster, 2005; Tietze, Rossbach \& Grenner, 2005). In einigen Konzeptionen wird zusätzlich die Qualität des Managements und der Abstimmungsprozesse in der Einrichtung 


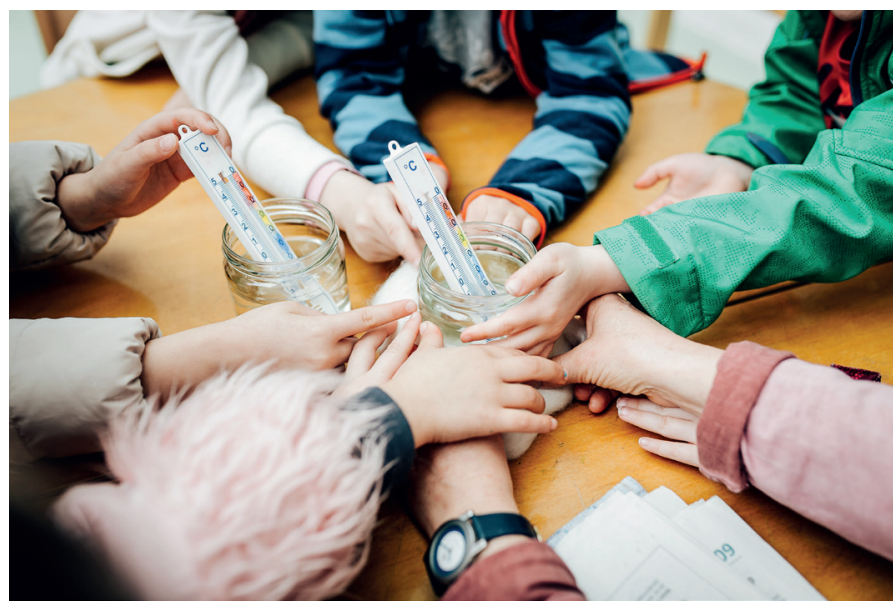

(Implementationsqualität) hinzugezählt. Hierbei liegt die Annahme zugrunde, dass sich die Qualität früher Bildungsprozesse nur dann nachhaltig erhöhen lässt, wenn sich die komplette Einrichtung in den Prozess der Qualitätsentwicklung begibt (vgl. Kapitel 2.1). Direkten Einfluss auf die kindliche Entwicklung hat nach den strukturell-prozessualen Qualitätsmodellen allein die Prozessqualität, wohingegen

die Implementationsqualität, die Strukturqualität sowie die Orientierungsqualität vermittelt über die Prozesse auf die Kinder wirken (NICHD ECCRN, 2003; Kluczniok \& Roßbach, 2014; Sylva, Melhuish, Sammons, Siraj-Blatchford \& Taggart, 2004; Tietze, Meischner, Gänsfuß, Grenner, Schuster, Völkel \& Roßbach, 1998; Tietze \& Grenner , 2005). Die Prozessqualität beschreibt die Art der Interaktionen zwischen frühpädagogischen Fachkräften und Kindern, die Interaktionen der Kinder untereinander und die Auseinandersetzung des Kindes mit Raum und Materialien. Hinsichtlich der Merkmale hoher Prozessqualität wird in der internationalen Forschungsliteratur zunehmend die Bedeutung kognitiv anregender verbaler Interaktionen zwischen Fachkraft und Kind betont (Peterson \& French, 2008; Siraj-Blatchford et al., 2002; Sylva et al., 2004). Im Sinne der Ko-Konstruktion von Wissen stehen die Kinder hierbei als aktive Lerner im Fokus: Ihre Sichtweisen, ihre Theorien und ihr Vorwissen sind Ausgangspunkt des Lernprozesses (König, 2009). Der Fachkraft kommt eine aktive Rolle in der Anregung von Lernprozessen durch gezielte Fragen und die Veranschaulichung von Problemlösungen zu (Tournier, 2016). Forschungsbefunde zur globalen Qualität der pädagogischen Interaktionen zeigen, dass diese tendenziell im geringen bis moderaten Bereich liegt (Wilcox-Herzog \& Ward, 2004). Für das Lernen in den Naturwissenschaften ist aber vor allem die naturwissenschaftliche Anregungsqualität entscheidend. Hierzu existieren bisher nur wenige empirische Befunde. Hopf (2012) untersuchte die naturwissenschaftliche Anregungsqualität in einer Videoanalyse eines Settings, bei dem die Fachkräfte die explizite Anweisung bekamen, eine naturwissenschaftliche Lernsituation zu gestalten. In diesem idealen Setting konnte Hopf (2012) zumindest 23\% aller verbalen Interaktionen als kognitiv anregend klassifizieren. Die Ergebnisse für den Bereich der Naturwissenschaften fallen damit deutlich besser aus als bisherige Befunde zur globalen Anregungsqualität (König, 2007; Roux, 2002; Siraj-Blatchford \& Manni, 2008). Kruse (2016) konn- 
te zudem in einer Mikroanalyse von Videosequenzen aus zwei Kitas zeigen, dass diejenigen Dialoge, die als besonders hochwertig identifiziert wurden, sich häufig inhaltlich auf naturwissenschaftliche Themen beziehen. Diese Befunde legen nahe, dass sich kognitiv anregende Interaktionen zwischen Fachkraft und Kindern insbesondere für den Forschungsprozess in den Naturwissenschaften anbieten, z. B. bei der Durchführung von Experimenten (Hopf, 2012). So können bereits bei der Entwicklung naturwissenschaftlicher Projekte oder Versuche die Fragen und Ideen der Kinder sowie ihr Vorwissen einbezogen werden (vgl. Bulunuz, 2013; Samuelsson \& Carlsson, 2008). Auch in der Durchführung und Auswertung sollten die Kinder zentrale Akteure sein, wobei sie von den Fachkräften unterstützt und durch gezieltes Nachfragen zum Weiterdenken und Reflektieren angeregt werden (Möller, Jonen, Hardy \& Stern, 2002; Samuelsson \& Carlsson, 2008). Inwieweit sich in dieser Weise kognitiv anregende naturwissenschaftliche Lernsituationen auf die Kompetenzen der Kinder auswirken, ist bisher für den deutschen Kontext noch nicht erforscht.

Neben der Qualität wird auch der Quantität früher naturwissenschaftlicher Bildungsprozesse eine wichtige Rolle für den Lernerfolg der Kinder zugesprochen. So können Kinder nur dann von hoher Qualität profieren, wenn Lerngelegenheiten regelmäßig und mit hinreichender Intensität erfolgen. Wie häufig Kinder mit naturwissenschaftlichen Lerngelegenheiten in der Kita in Kontakt kommen, ist im deutschen Raum bisher kaum erforscht. Erste Befunde aus Deutschland, die auf Selbstauskünften der Fachkräfte basieren, zeigen das ein Großteil der befragten Fachkräfte ein oder mehrmals in der Woche mit den Kindern forscht (Stiftung Haus der kleinen Forscher, 2015a; Stiftung Haus der kleinen Forscher, 2017). Im internationalen Raum liegen bereits in größerem Umfang empirische Befunde vor, die insgesamt verdeutlichen, dass die Erkennung und Gestaltung früher naturwissenschaftlicher Lerngelegenheiten im Kita-Alltag für Fachkräfte eine Schwierigkeit darstellt. Beispielsweise konnte Tu (2006) in einer Beobachtungsstudie im US-amerikanischen Raum feststellen, dass 13,3\% aller Aktivitäten einen Bezug zu den Naturwissenschaften hatten, diese aber selten als naturwissenschaftliche Lerngelegenheiten genutzt wurden. Auch die Ergebnisse einer Befragung frühpädagogischer Fachkräfte durch Saçkes et al. (2011) weisen darauf hin, dass Fachkräfte naturwissenschaftliche Lerngelegenheiten verhältnismäßig selten wahrnehmen: Etwa die Hälfte der Fachkräfte gab an, naturwissenschaftliche Aktivitäten ein- bis zweimal in der Woche durchzuführen und nur $15 \%$ behandelten naturwissenschaftliche Inhalte täglich. Dies deckt sich mit Befunden von Spektor-Levy et al. (2013): Der überwiegende Teil der frühpädagogischen Fachkräfte (62\%) gab an, wöchentlich naturwissenschaftliche Lerngelegenheiten anzubieten, $27 \%$ ein- bis zweimal im Monat und $11 \%$ seltener. Inwieweit die Häufigkeit der Nutzung von naturwissenschaftlichen Lerngelegenheiten in Zusammenhang 
mit den kindlichen naturwissenschaftlichen Kompetenzen steht, ist bisher nicht bekannt. Einzelne Studien konnten jedoch zeigen, dass die naturwissenschaftlichen Lerngelegenheiten die naturwissenschaftliche Lernmotivation der Kinder positiv beeinflussen (z. B. Mantzicopoulos et al., 2008).

\subsection{Naturwissenschaftliche Kompetenzen frühpädagogischer Fachkräfte}

Die Gestaltung qualitativ hochwertiger naturwissenschaftlicher Lerngelegenheiten stellt eine Herausforderung dar, für die vielfältige Kompetenzen aufseiten der Fachkraft erforderlich sind. In Anlehnung an Handlungskompetenzmodelle für Lehrkräfte der Primar- und Sekundarstufe (Baumert \& Kunter, 2006), werden für den Elementarbereich die generische Kompetenz, aber auch domänenspezifische Aspekte als bedeutsam erachtet (Fröhlich-Gildhoff, Nentwig-Gesemann, Pietsch, Köhler \& Koch, 2014). Das heißt, für die Quantität und Qualität der frühen naturwissenschaftlichen Bildung sollten auch naturwissenschaftliche professionelle Kompetenzen in besonderem Maße relevant sein (Stiftung Haus der kleinen Forscher, 2013b). Welche domänenspezifische Kompetenzen und in welcher Ausprägung pädagogische Fachkräfte diese benötigen, ist aber weitestgehend unklar.

Zentrale Kompetenzaspekte, die sich sowohl in den Handlungskompetenzmodellen für Lehrkräfte der Primar- und Sekundarstufe, aber auch in dem von Fröhlich-Gildhoff und anderen (2011) vorgeschlagenen Modell für die Kompetenzen frühpädagogischer Fachkräfte wiederfinden, sind Professionswissen, Überzeugungen/Einstellungen, Motivation und selbstregulative Fähigkeiten.

Im Rahmen des EASI-Projektes wird auf das Professionswissen, die Überzeugungen sowie die Motivation frühpädagogischer Fachkräfte fokussiert, die im Folgenden näher erläutert werden.

\subsubsection{Naturwissenschaftliches Professionswissen von Fachkräften}

Für das Professionswissen von Lehrpersonen werden Fachwissen (content knowledge, CK), fachdidaktisches Wissen (pedagogical content knowledge, PCK) und pädagogisches Wissen (pedagogical knowledge, PK) als zentral angesehen (Bromme, 1997; Baumert \& Kunter, 2006; Shulman, 1987). Auch für das fachspezifische Wissen pädagogischer Fachkräfte gelten das Fachwissen sowie das fachdidaktische Wissen als relevant (siehe Aubrey, 1997; Lee, 2010; McCray, 2008; McCray \& Chen, 2012; Siraj-Blatchford et al., 2002; Tirosh, Tsamir, Levenson \& Tabach, 2011; Oppermann, Anders \& Hachfeld, 2016, Dunekacke, Jenßen \& Blömeke, 2015). 
Entsprechend der Orientierung am Bildungskonzept Scientific Literacy, das oft als Zieldimension früher naturwissenschaftlicher Bildung genannt wird (Möller \& Steffensky, 2010), umfasst das naturwissenschaftliche Fachwissen (CK) von Fachkräften das inhaltsbezogene Fachwissen $\left(\mathrm{CK}_{\text {Inhalt }}\right)$ sowie das Fachwissen über zentrale Denk- und Arbeitsweisen im Elementarbereich (im Folgenden prozessbezogenes Fachwissen ( $\left.\mathrm{CK}_{\text {Prozess }}\right)$ genannt).

In der internationalen Diskussion wird darauf hingewiesen, dass auch frühpädagogische Fachkräfte eine Fachwissensbasis in den grundlegenden naturwissenschaftlichen Themen benötigen (Garbett, 2003), um Kindern naturwissenschaftliche Inhalte zugänglich zu machen. Bisher ist allerdings unklar, was genau unter dem naturwissenschaftlichen Fachwissen frühpädagogischer Fachkräfte zu verstehen ist und welches Niveau an naturwissenschaftlichem Wissen Fachkräfte tatsächlich benötigen. Generell wird angenommen, dass das Niveau des Wissens der frühpädagogischen Fachkräfte über das Niveau des zu „vermittelnden“ Wissens hinausgehen sollte (Krauss, Brunner, Kunter, Baumert, Blum, Neubrand \& Jordan, 2008; Garbett, 2003). Demnach würden frühpädagogische Fachkräfte mindestens das Wissen benötigen, welches dem angestrebten Wissen von Kindern im Grundschulalter und in Ansätzen dem Wissen von Kindern, die den naturwissenschaftlichen Anfangsunterricht der weiterführenden Schulen besuchen, entspricht (Anders et al., 2013). Im Rahmen dieser Studie wird das inhaltsbezogene Fachwissen $\left(\mathrm{CK}_{\text {Inhalt }}\right)$ in Naturwissenschaften nicht als reines Faktenwissen gesehen, sondern es wird vielmehr als ein anwendbares Wissen über alltägliche naturwissenschaftliche Phänomene definiert, das es ermöglicht, Prozesse zu beschreiben und naturwissenschaftliche Themen auf der Basis von beobachtbaren Zusammenhängen zu erklären.

Um Kinder bei der Verwendung und der Entwicklung eines Verständnisses von naturwissenschaftlichen Denk- und Arbeitsweisen, beispielsweise beim Beobachten, Vergleichen oder Messen, unterstützen zu können (Hardy, Kloetzer, Moller \& Sodian, 2010), benötigen frühpädagogische Fachkräfte selbst ein Wissen über diese Denk- und Arbeitsweisen (prozessbezogenes Fachwissen $\left(\mathrm{CK}_{\text {Prozess }}\right)$ ). Ziel ist es, dass Fachkräfte nicht nur Denk- und Arbeitsweisen anwenden können, sondern auch ein übergeordnetes Verständnis dieser Verfahren besitzen, z. B. den Unterschied zwischen einer wissenschaftlichen und einer alltäglichen Beobachtung kennen (Stiftung Haus der kleinen Forscher, 2013b).

Das fachdidaktische Wissen (PCK) bezieht sich auf das Wissen darüber, wie Fachinhalte Lernenden verfügbar gemacht werden können. Als besonders relevant wird hierfür das Wissen über kognitive Unterstützung (z. B. die Darstellung naturwissenschaftlicher Inhalte anhand alltäglicher Beispiele) sowie das Wissen über Vorstellungen von Lernenden (z. B. über typische inhaltsspezifische Vorstellungen, Erfahrungen oder Schwierigkeiten von Kindern) angesehen (Shulman, 1986; 
Grossman, 1990). Grundsätzlich ist davon auszugehen, dass geeignete Lernumgebungen das Lernen von Kindern unterstützen können (Siraj-Blatchford \& Manni, 2008). Dabei wird das Wissen über die kognitive Unterstützung der Lernenden als bedeutsam für die Gestaltung dieser Lernumgebungen angenommen (Baumert \& Kunter, 2011). Es umfasst neben generischen Aspekten, die dem pädagogischen Wissen zuzuordnen wären, Kenntnisse über geeignete Versuche oder Alltagssituationen, um spezifische naturwissenschaftliche Inhalte (z. B. das Schmelzen oder Gefrieren von Wasser) zu illustrieren, und kann damit eine wichtige Grundlage für anregende Lernumgebungen in Naturwissenschaften darstellen.

Da Kinder bereits in jungen Jahren schon erste Vorstellungen über naturwissenschaftliche Phänomene entwickeln (z. B. Trundle \& Saçkes, 2015), die nicht notwendigerweise den wissenschaftlichen Vorstellungen entsprechen müssen, wird auch das Wissen über die Vorstellungen der Lernenden als bedeutsam für die Lernbegleitung und die Gestaltung von Lerngelegenheiten angesehen. Diese bestehenden Vorstellungen der Kinder können beispielsweise im Kita-Alltag aufgegriffen, von den Kindern überprüft und um neue Vorstellungen erweitert oder umstrukturiert werden (conceptual change) (Vosniadou, loannides, Dimitrakopoulou \& Papademetriou, 2001; diSessa, 2006). Das Wissen über die Vorstellungen der lernenden Kinder, d. h. darüber, welche Vorstellungen Kinder zu bestimmten Konzepten haben, welche naturwissenschaftlichen Themen schwer oder einfach sind, hilft den Fachkräften, Vorstellungen in komplexen Lehr-Lern-Situationen schnell zu erkennen und das Denken der Kinder zu interpretieren und zu erklären sowie ihre Lernunterstützung adaptiv an die Vorstellungen der Kinder anzupassen (Tirosh, 2000).

Insgesamt gibt es kaum Untersuchungen zum naturwissenschaftlichen Professionswissen von frühpädagogischen Fachkräften. Erste Befunde aus dem Bereich der Naturwissenschaften deuten auf ein niedrig ausgeprägtes inhaltliches Fachwissen ( CK $_{\text {Inhalt }}$ ) der Fachkräfte hin (Garbett, 2003; Kallery \& Psillos, 2001). So wurde in einer Studie von Garbett (2003) mit einem schriftlichen Wissenstest, der zuvor schon bei angehenden Grundschullehrkräften eingesetzt wurde, das inhaltliche Fachwissen angehender frühpädagogischer Fachkräfte zu Themen der Biologie, Chemie, Physik und Astronomie erfragt. Die Ergebnisse zeigten, dass die frühpädagogischen Fachkräfte geringere Werte im Wissenstest erzielten als die angehenden Grundschullehrkräfte. Auch in der Studie von Kallery und Psillos (2001) weisen die empirischen Befunde auf eine Differenz zwischen dem von den Autoren als notwendig erachteten naturwissenschaftlichen Fachwissen und den tatsächlich vorhandenen Vorstellungen der frühpädagogischen Fachkräfte hin.

Gleichzeitig machen erste Befunde auf die Bedeutung des domänenspezifischen Professionswissens von Fachkräften für die Qualität der frühen Bildung aufmerksam (Siraj-Blatchford et al., 2002). So scheint das mathematische PCK frühpädagogischer Fachkräfte beispielsweise die instruktionale Qualität sowie 
die Lernzuwächse der Kinder im Kindergarten vorherzusagen (McCray \& Chen, 2012). Weniger eindeutig ist die Bedeutung des reinen Fachwissens frühpädagogischer Fachkräfte für die Qualität der pädagogischen Interaktionen und die Lernfortschritte der Kinder. Ergebnisse erster Studien weisen jedoch darauf hin, dass das mathematische Fachwissen bedeutsam für die Wahrnehmung von mathematischen Inhalten im Spiel der Kinder und die Analyse von mathematischen Lernsituationen ist (Oppermann et al., 2016 bzw. Dunekacke et al., 2015). Die Wahrnehmung von fachspezifischen Inhalten und potenziellen Lernsituationen wird wiederum als Voraussetzung für die Implementation früher Bildung angesehen, da frühes Lernen typischerweise auf Alltagssituationen sowie dem Interesse der Kinder aufbaut (Oppermann et al., 2016).

Auch wenn die Forschungslage zur Bedeutung von Professionswissen von frühpädagogischen Fachkräften, insbesondere im Bereich Naturwissenschaften, bisher eher spärlich ist, kann das fachspezifische professionelle Wissen insgesamt aufgrund theoretisch-konzeptioneller Überlegungen sowie erster Befunde aus dem Bereich der Mathematik als wichtige Voraussetzung für die Bereitstellung aktivierender, bereichsspezifischer Lerngelegenheiten angenommen werden (Blömeke, Kaiser \& Lehmann, 2008; Baumert et al., 2010; McCray \& Chen, 2012). Insgesamt mangelt es allerdings noch immer an empirischen Befunden zum naturwissenschaftlichen Wissen von frühpädagogischen Fachkräften, was möglicherweise auf das Fehlen von geeigneten Messinstrumenten zur Erfassung des naturwissenschaftlichen Wissens von frühpädagogischen Fachkräften zurückzuführen ist.

\subsubsection{Motivationale Aspekte professioneller Kompetenzen}

Neben dem Professionswissen werden auch motivationale Orientierungen als wichtige Komponenten professioneller Kompetenzen verstanden. Die motivationalen Aspekte professioneller Kompetenz sind mit den pädagogischen Überzeugungen eng verwandt, werden aber dennoch als eigenständige Kompetenzfacette behandelt (Anders, 2012; Fröhlich-Gildhoff et al., 2011). Motivation wird als relevante Voraussetzung für die Gestaltung qualitativ hochwertiger Lerngelegenheiten diskutiert (Anders, 2012; Baumert \& Kunter, 2006). Als besonders bedeutsame motivationale Komponenten werden Interesse, Enthusiasmus in Bezug auf die Gestaltung von Lernprozessen sowie das Selbstkonzept und die Selbstwirksamkeitserwartung in Bezug auf die Begleitung naturwissenschaftlicher Lernprozesse angenommen (vgl. Erden \& Sönmez, 2010; Faulkner-Schneider, 2005).

Im Hinblick auf die Ausprägung motivationaler Kompetenzaspekte lassen sich einerseits Studien anführen, die auf eine geringe Selbstwirksamkeitserwartung in Bezug auf die Gestaltung naturwissenschaftlicher Lerngelegenheiten hinweisen, wobei es deutliche Unterschiede zwischen der Biologie und den „harten“ Naturwis- 
senschaften zu geben scheint (Erden \& Sönmez, 2010; Koballa \& Crawley, 1985; Eshach, 2003). Hinsichtlich der Bedeutung motivationaler Kompetenzaspekte für das pädagogische Handeln weisen internationale Befunde auf eine positive Assoziation zwischen der Selbstwirksamkeitserwartung von frühpädagogischen Fachkräften und der Qualität der Gestaltung früher Lerngelegenheiten hin (Guo, Piasta, Justice \& Kaderavek, 2010; Justice, Mashburn, Hamre \& Pianta, 2008; Lohse-Bossenz, Zimmermann, Janke \& Müller, 2015). Zudem zeigen Befunde, dass eine niedrige Selbstwirksamkeitserwartung dazu führen kann, dass naturwissenschaftliche Lerngelegenheiten vermieden werden (Erden \& Sönmez, 2010; Spektor-Levy et al., 2013). Aus der Unterrichtsforschung existieren empirische Belege dafür, dass der Enthusiasmus für die Gestaltung von Lerngelegenheiten signifikant mit der realisierten Prozessqualität assoziiert ist (Kunter et al., 2007).

\subsubsection{Pädagogische Überzeugungen}

Der Begriff pädagogische Überzeugungen beschreibt ein weit gefasstes Konstrukt, welches die Einstellungen, Werte und Annahmen von frühpädagogischen Fachkräften zusammenfasst (Anders, 2012). Dies beinhaltet beispielsweise lerntheoretische Überzeugungen zum Erwerb von domänenspezifischen Kompetenzen oder Einstellungen hinsichtlich der Bedeutsamkeit einzelner Bildungsbereiche wie den Naturwissenschaften (Anders, 2012). Es wird angenommen, dass die pädagogischen Überzeugungen von frühpädagogischen Fachkräften in den Naturwissenschaften handlungsleitend sind (Anders, 2012) und dadurch auch die Lernerfahrungen der Kinder beeinflussen können.

Der Mannigfaltigkeit des Konstruktes pädagogischer Überzeugungen entsprechend gestaltet sich der Forschungsstand zu den einzelnen Facetten. Hinsichtlich der Annahmen zur Bedeutsamkeit einzelner Bildungsbereiche verdeutlichen Studien, dass die Förderung sozioemotionaler Kompetenzen als besonders wichtig erachtet wird (Tietze et al., 2005; Mischo, Wahl, Hendler \& Strohmer 2012), während die Förderung akademischer Fähigkeiten (bislang) als weniger bedeutsam eingeschätzt wird. Hinsichtlich der lerntheoretischen Überzeugungen wird in der Forschungsliteratur, insbesondere in der Schulforschung, im Allgemeinen zwischen transmissiven und (ko-)konstruktiven Überzeugungen unterschieden (Kleickmann, 2008; Voss, Kleickmann, Kunter \& Hachfeld, 2011). Untersuchungen zu domänenunspezifischen lerntheoretischen Überzeugungen zeigen, dass frühpädagogische Fachkräfte eher (ko-)konstruktive als transmissive Überzeugungen zum Lehren und Lernen haben (Mischo et al., 2012). Auch die Ergebnisse einer naturwissenschaftsspezifischen Untersuchung legen die Verbreitung (ko-)konstruktiver Überzeugungen nahe (Kuhn, Lankes \& Steffensky, 2012). Vor diesem Hintergrund mag im Kontext der frühen Bildung vielmehr die Differenzierung innerhalb des Spektrums (ko-)konstruktiver Überzeugungen gewinnbringend sein als 
die grobe Unterscheidung transmissiver und (ko-)konstruktiver Überzeugungen. Da lerntheoretische Überzeugungen letztendlich Annahmen über geeignete pädagogische Ansätze und Methoden sind, lohnt ein Blick in die Forschungsliteratur zu frühpädagogischen Ansätzen. Hier wird, analog zur Klassifizierung pädagogischer Überzeugungen, häufig zwischen fachkraftorientierten (teacher-directed)/didak-

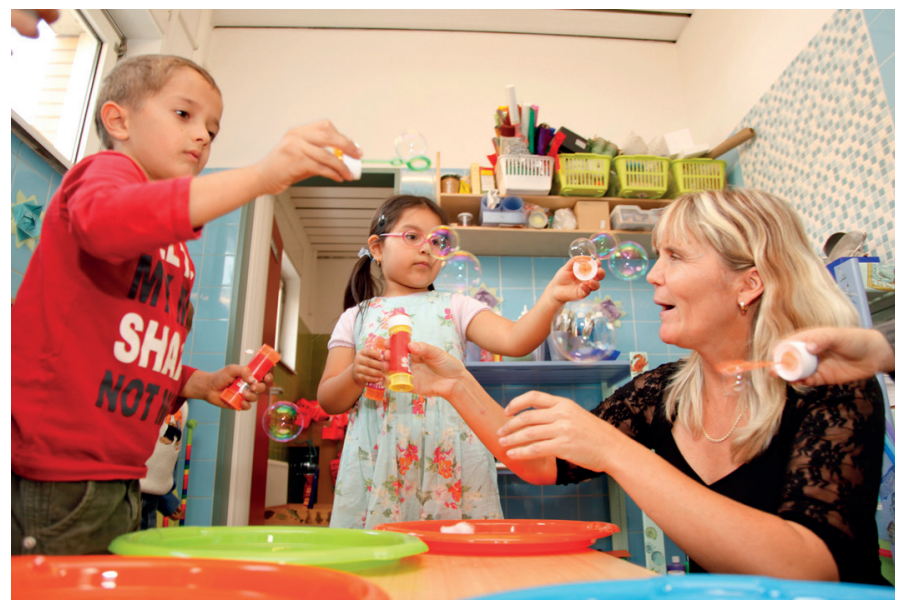
tischen Ansätzen und kindzentrierten (child-centred) Ansätzen unterschieden (vgl. Stipek, 1991). Diese Klassifizierung wurde in einer 2×2-Kategorisierung erweitert, die die Rolle der Fachkraft (hohe Initiative, geringe Initiative) und die Rolle des Kindes (hohe Initiative, geringe Initiative) als zwei Faktoren auffasst (z. B. Weikart, 2000; Siraj-Blatchford et al., 2002; Stipek, 1991). Hierdurch entsteht ein Muster, das zwischen vier Ansätzen unterscheidet: (1) dem programmatischen Ansatz, (2) dem kindzentrierten Ansatz, (3) dem Open-Framework-Ansatz und (4) dem Obhutsansatz.

Der programmatische Ansatz (1) zeichnet sich durch eine hohe Initiative aufseiten der Fachkraft und eine eher passive Rolle des Kindes aus, wohingegen beim kindzentrierten Ansatz (2) die Eigeninitiative des Kindes betont wird und sich die Fachkraft, u. a. aus Angst, das Kind in seiner Exploration zu stören, zurückhält. Der Open-Framework-Ansatz (3) verbindet eine hohe Initiative aufseiten der Fachkraft und mit einer hohen Initiative und Beteiligung des Kindes und kommt damit einer kognitiv anregenden Interaktion zwischen Fachkraft und Kind im Sinne der Ko-Konstruktion von Wissen am nächsten. Der Obhutsansatz (4) beschreibt die Betreuung ohne Förderung und ist damit streng genommen kein pädagogischer Ansatz, da hier kein pädagogisches Konzept vertreten wird.

Möchte man die drei verbliebenen pädagogischen Ansätze der zuvor eingeführten Dichotomie pädagogischer Überzeugungen zuweisen, so wäre der programmatische Ansatz mit den transmissiven Überzeugungen vergleichbar und der Open-Framework-Ansatz ließe sich tendenziell den ko-konstruktiven Überzeugungen zuordnen. Die Hinzunahme des kindzentrierten Ansatzes erscheint gerade für die frühe naturwissenschaftliche Förderung sinnvoll, da hier häufig eine Haltung vertreten wird, welche die autonome Kompetenzentwicklung des Kindes betont. Dabei wird die Einstellung vertreten, dass das Kind in seiner spielerischen Erkun- 
dung und Auseinandersetzung mit naturwissenschaftlichen Phänomenen nach Möglichkeit nicht gestört werden sollte.

Die theoretische Unterscheidung zwischen den drei Dimensionen frühpädagogischer Überzeugungen wurde bisher noch nicht empirisch überprüft. Es existieren allerdings Befunde hinsichtlich der allgemeinen Bedeutung pädagogischer Überzeugen von Fachkräften für die Gestaltung von Lerngelegenheiten (Kowalski, Pretti-Frontczak \& Johnson, 2001; McCarty, Abbott-Shim \& Lambert, 2001). Kluczniok, Anderes und Ebert (2011) konnten zeigen, dass grundlegende Fördereinstellungen von Fachkräften nicht nur die Prozessqualität der Lerngelegenheiten vorhersagen, sondern sich, darüber vermittelt, auch auf die numerischen Kompetenzen der Kinder auswirken. Ähnliche Befunde zur Bedeutung epistemologischer Überzeugungen für die Qualität existieren bereits aus der Unterrichtsforschung (vgl. u. a. Kunter et al., 2007; Staub \& Stern, 2002; Stipek, Givin, Salmon \& MacGyvers, 2001, Dubberke, Kunter, McElvany, Brunner \& Baumert, 2008; Smith, Maclin, Houghton \& Hennessey, 2000; Sodian, Jonen \& Thoermer, 2006). 


\section{Fragestellungen der Studie}

Die Fragestellungen der Studie beziehen sich (1) auf die Erfassung und Struktur der naturwissenschaftlichen Kompetenzen von Fachkräften und Kinder, (2) auf Unterschiede zwischen Gruppen von Kitas mit naturwissenschaftlich fortgebildeten und nicht fortgebildeten Fachkräften sowie (3) auf Zusammenhänge zwischen den Wirkungsebenen des Wirkungsmodells (vgl. Kapitel 2.1).

\subsection{Erfassung und Struktur der Kompetenzen}

Bisher ist wenig über die naturwissenschaftlichen Kompetenzen von frühpädagogischen Fachkräften und Kindern bekannt. Dies lässt sich auch auf einen Mangel an Messinstrumenten zur Erfassung der Kompetenzen von Fachkräften und Kindern in den Naturwissenschaften zurückführen. Ferner ist unklar, wie sich die Struktur der Kompetenzen von frühpädagogischen Fachkräften und Kindern beschreiben lässt. Vor diesem Hintergrund befasst sich die erste Zielstellung des Projektes mit der Entwicklung reliabler und valider Messinstrumente sowie der Überprüfung der Kompetenzstruktur auf Fachkraft- und Kinderebene.

Konkret wird auf Ebene der Fachkräfte und Kinder folgenden Fragestellungen nachgegangen:

- In welcher Struktur liegt das naturwissenschaftliche professionelle Wissen der pädagogischen Fachkräfte vor?

- In welcher Struktur liegen die motivationalen Aspekte professioneller Kompetenz bei pädagogischen Fachkräften vor?

- In welcher Struktur liegen die lerntheoretischen Überzeugungen der pädagogischen Fachkräfte in Bezug auf die Vermittlung naturwissenschaftlicher Bildungsinhalte vor?

- In welcher Struktur liegen die Wissenskomponenten naturwissenschaftlicher Kompetenzen von Kindern vor?

- In welcher Struktur liegt die Motivation der Kinder in den Naturwissenschaften vor? 


\subsection{Gruppenunterschiede}

Die Angebote früher naturwissenschaftlicher Bildung sind in Deutschland sehr heterogen. Vor diesem Hintergrund war die Frage nach der Wirkung naturwissenschaftlicher Bildungsangebote auf die Kompetenzen von Fachkräften und Kindern zentral für das Forschungsvorhaben. Hierfür wurden exemplarisch Kitas, die an der Initiative „Haus der kleinen Forscher“ teilnehmen (HdkF-Gruppe), und Kitas mit einem naturwissenschaftlichen Schwerpunkt außerhalb der Initiative „Haus der kleinen Forscher" (Nawi-Kitas) mit einer Gruppe an Kitas ohne naturwissenschaftlichen Schwerpunkt (Vergleichsgruppe) verglichen.

Frühe naturwissenschaftliche Bildungsangebote haben primär das Ziel, über die Kompetenzentwicklung der Fachkräfte die Qualität und Häufigkeit naturwissenschaftlicher Bildung in den Kitas zu verbessern und damit auch auf die Kinder zu wirken. Vor diesem Hintergrund ist die Frage nach Gruppenunterschieden auf diesen drei Zielebenen relevant. Bildungsangebote können jedoch nicht losgelöst von dem Kontext betrachtet werden, in dem sie wirken. Daher wird zunächst die Einrichtungsebene fokussiert. Als Voraussetzung für eine nachhaltige Wirkung naturwissenschaftlicher Bildung wird die Implementation des Bildungsbereiches Naturwissenschaften in der gesamten Kita angenommen. Folglich wird mit Blick auf die Gruppenunterschiede folgenden Fragen nachgegangen:

- Unterscheiden sich die Untersuchungsgruppen anhand des Ausmaßes der Implementation der Naturwissenschaften als Bildungsbereich in der Einrichtung?

- Unterscheiden sich die pädagogischen Fachkräfte in den drei Untersuchungsgruppen in der Ausprägung ihrer naturwissenschaftlichen Kompetenzen?

- Unterscheiden sich die Kitas in den drei Untersuchungsgruppen in der Häufigkeit und Qualität naturwissenschaftlicher Bildungsangebote?

- Unterscheiden sich die Kinder in den drei Untersuchungsgruppen in der Ausprägung ihrer naturwissenschaftlichen Kompetenzen?

\subsection{Zusammenhänge zwischen den Wirkungsebenen}

Die Wirkungen von naturwissenschaftlichen Bildungsangeboten können über das theoretische Wirkungsmodell beschrieben werden (vgl. Kapitel 2.1). Diese Annahmen sollen im Rahmen des Projektes überprüft werden. Es ergeben sich folgende Fragestellungen: 
- Wie hängt die Implementationsqualität mit den naturwissenschaftlichen Kompetenzen der pädagogischen Fachkräfte zusammen?

- Wie hängen die naturwissenschaftlichen Kompetenzen der pädagogischen Fachkräfte mit der Qualität und Häufigkeit naturwissenschaftlicher Lerngelegenheiten zusammen?

- Wie hängt die Implementationsqualität mit der Qualität und Häufigkeit naturwissenschaftlicher Lerngelegenheiten zusammen?

- Wie hängt die Qualität und Häufigkeit naturwissenschaftlicher Lerngelegenheiten mit den Kompetenzen der Kinder zusammen? 


\section{Methode}

\subsection{Design und Stichprobe}

Das Untersuchungsdesign legte das in Kapitel 2 beschriebene Wirkungsmodell früher naturwissenschaftlicher Bildungsangebote zugrunde. Dabei spielen die professionellen Kompetenzen der Fachkräfte sowie die Qualität und Häufigkeit der Interaktionen zwischen der Fachkraft und dem Kind (Qualität der Interaktionen der angebotenen Lerngelegenheiten) eine zentrale Rolle für die Kompetenzentwicklung der Kinder. Um die Fragestellungen dieser Studie (siehe Kapitel 3) adäquat untersuchen zu können, ist die Untersuchung von jeweils einer weiteren Gruppe von Kitas mit und ohne Teilnahme an naturwissenschaftlichen Bildungsinitiativen nötig, um auszuschließen, dass potenzielle Unterschiede zwischen Kitas der HdkF-Gruppe und Kitas ohne Teilnahme an Bildungsinitiativen ausschließlich durch Faktoren wie beispielsweise die Grundmotivation der pädagogischen Fachkräfte, die Haltung der pädagogischen Fachkräfte hinsichtlich der Förderung früher Bildung in Kitas, unterschiedliches Familienklientel etc. erklärbar sind. Aufgrund dessen wurde ein 3-Gruppen-Design mit drei verschiedenen Modalitäten (Gruppen von Kitas) umgesetzt:

- HdkF-Kitas: Kitas, die an der HdkF-Initiative und regelmäßig an naturwissenschaftlichen Fortbildungen teilnehmen. (Die Einrichtung ist als HdkF-Kita zertifiziert, und mindestens eine Fachkraft in der Einrichtung hat in den letzten 3 Jahren an 2 HdkF-Fortbildungen teilgenommen.)

- Kitas mit Nawi-Schwerpunkt (Nawi-Kitas): Kitas außerhalb der HdkF-Initiative, die Naturwissenschaften als Schwerpunkt ihrer Bildungsarbeit definieren und regelmäßig an naturwissenschaftlichen Fortbildungen teilnehmen. (Mindestens eine Fachkraft in der Einrichtung hat in den letzten 3 Jahren an 2 naturwissenschaftlichen Fortbildungen teilgenommen.)

- Kitas ohne Nawi-Schwerpunkt (Vergleichsgruppe): Kitas, die keinen expliziten naturwissenschaftlichen Schwerpunkt haben und in denen die Fachkräfte weniger als 2 oder gar keine naturwissenschaftlichen Fortbildungen in den letzten 3 Jahren besucht haben.

- Die Untersuchung wurde als Querschnittsuntersuchung mit einem Messzeitpunkt auf Ebene der Kitas, Fachkräfte und Kinder angelegt. An der Untersuchung nahmen insgesamt 110 Kitas teil, davon beantworteten 98 Leitungen 
den Leitungsfragebogen. In 54 Kitas wurden pädagogische Fachkräfte und Kinder befragt. In 53 Kitas wurden lediglich die pädagogischen Fachkräfte befragt. Die Rekrutierung der Gesamtstichprobe und ihre Zusammensetzung werden im Folgenden genauer beschrieben.

\section{Stichprobe auf Ebene der Kitas}

Die 110 Kitas teilten sich auf in: 37 HdkF-Kitas, 25 Kitas mit anderem Nawi-Schwerpunkt und 39 Kitas der Vergleichsgruppe. 9 Kitas ließen sich aufgrund fehlender Angaben der Leitungen nicht den Untersuchungsgruppen zuordnen und wurden daher für die Analyse der Gruppenunterschiede ausgeschlossen. Bei allen anderen Analysen wurden diese Fälle berücksichtigt.

Die Rekrutierung der Kitas für die Studie erfolgte telefonisch. Zusätzlich wurde schriftliches Informationsmaterial, beispielsweise ein Flyer zur Studie, an die Kitas verschickt. In Telefonaten mit der Kita-Leitung wurde anhand eines standardisierten Vorgehens ermittelt, ob die Kita die gewählten Kriterien für eine HdkF-Kita, Nawi-Kita oder Vergleichskita erfüllt; sie wurde dementsprechend einer der drei Gruppen zugeordnet. Nach Zusage zur Studienteilnahme durch die Kita-Leitung wurden anschließend die Kinder und frühpädagogischen Fachkräfte gewonnen. Für die Kinderstichprobe kamen nur Kitas infrage, in denen sich frühpädagogische Fachkräfte für die Teilnahme an der Untersuchung bereiterklärten und zum Befragungszeitpunkt Kinder im Zielalter betreuten. Aufgrund dieser Notwendigkeit war es nicht möglich, die Stichprobe rein zufällig zu ziehen.

Die Rekrutierung der Kitas fand gleichzeitig an fünf Standorten statt: in Berlin, Hessen (Frankfurt am Main und angrenzende Gemeinden), Nordrhein-Westfalen (Münsterland ohne Stadt Münster), Schleswig-Holstein (Großraum Kiel, Flensburg und Lübeck) und Thüringen (Großraum Jena: Gera, Erfurt, Jena und Weimar mit umliegenden Gemeinden). Ziel der Stichprobenziehung war es, sowohl die regionale als auch die strukturelle Varianz der Rahmenbedingung abzubilden. Dies sollte durch die Verteilung auf die fünf unterschiedlichen Standorte berücksichtigt werden. Dabei repräsentieren die Kitas aus Berlin $(N=19)$ und Frankfurt $(N=24)$ Kitas aus einer Großstadt; die Kitas aus dem Großraum Jena $(N=15)$ und Kiel $(N=26)$ aus mittelgroßen Städten. Die Kitas aus Münsterland (ohne Stadt Münster) repräsentieren Kitas mit eher ländlichem Charakter $(N=26)$. Trotz des strategischen Vorgehens bei der Stichprobenziehung kann die vorliegende Stichprobe nicht als repräsentativ eingeordnet werden.

In Tabelle 1 ist die Stichprobenzusammensetzung der Studie für die drei Gruppen dargestellt. 
Tabelle 1. Stichprobenzusammensetzung der Studie. ${ }^{16}$

$\begin{array}{cccc} & \text { HdkF-Kitas } & \text { NAWI-Kitas } & \begin{array}{c}\text { Vergleichs- } \\ \text { gruppe }\end{array} \\ & 37 & 25 & 39 \\ & 125 & 97 & 127 \\ & 104 & 67 & 86\end{array}$

\section{Stichprobe auf Ebene der Fachkräfte}

Der Zugang zu den Fachkräften erfolgte über die Einrichtungsleitung. Bei Telefonaten mit den Leitungen von Kitas mit naturwissenschaftlichem Schwerpunkt wurde ermittelt, ob mindestens eine Fachkraft, die naturwissenschaftliche Fortbildungen besucht hat, an der Studie teilnehmen würde und ob die Kita an der HdkF-Initiative teilnimmt. Bei den Kitas, in denen auch Kinder untersucht wurden, soll diese Fachkraft zusätzlich möglichst auch Kinder im Zielalter betreuen. In den Kitas ohne naturwissenschaftlichen Schwerpunkt wurden die Fachkräfte untersucht, die sich freiwillig für die Studienteilnahme bereiterklärt hatten. Durch eine monetäre Aufwandsentschädigung für die Fachkräfte (30€) sowie das Angebot einer kostenfreien Teilnahme an einer naturwissenschaftlichen Fortbildung wurde die Motivation zur Teilnahme an der Studie erzeugt.

Die finale Stichprobe auf Fachkraftebene besteht aus $N=349$ pädagogischen Fachkräften. Im Durchschnitt sind die teilnehmenden pädagogischen Fachkräfte 38.29 Jahre alt ( $M i n=18$ Jahre; $\operatorname{Max}=65$ Jahre). Die Mehrheit (91\%) der teilnehmenden pädagogischen Fachkräfte sind Frauen. Die Stichprobenbeschreibung der Fachkräfte in den jeweiligen Gruppen hinsichtlich Alter und Berufserfahrung sowie Geschlecht und Berufsausbildung befindet sich in Tabelle 2 und 3.

Tabelle 2. Stichprobenzusammensetzung der Studie auf Fachkraftebene.

\begin{tabular}{lllllllllllllll}
\hline & \multicolumn{3}{l}{ HdkF-Gruppe } & \multicolumn{3}{c}{ Nawi-Gruppe } & \multicolumn{5}{c}{ Vergleichsgruppe } \\
\hline & $N$ & $M$ & $S D$ & Range & $N$ & $M$ & $S D$ & Range & $N$ & $M$ & $S D$ & Range \\
\hline Alter & 125 & 38.38 & 11.32 & $19-65$ & 97 & 38.75 & 12.20 & $22-62$ & 127 & 37.94 & 11.68 & $18-61$ \\
\hline $\begin{array}{l}\text { Berufs- } \\
\text { erfahrung }\end{array}$ & 125 & 16.12 & 11.64 & $2-47$ & 97 & 15.97 & 12.20 & $2-45$ & 127 & 14.76 & 11.38 & $2-42$ \\
\hline
\end{tabular}

16 Die Stichprobenzahl für die Berechnungen zu Fragestellung 1 und 3 können von der Anzahl in Tabelle 1 abweichen, da hier auch die Fachkräfte und Kinder aus den Kitas ohne Zuordnung zu den drei Untersuchungsgruppen einbezogen wurden. 
Für die pädagogischen Fachkräfte der HdkF-Gruppe ergibt sich folgende Stichprobenbeschreibung: Die pädagogischen Fachkräfte sind im Durchschnitt 38 Jahre alt $(\operatorname{Min}=19 ;$ Max $=65)$ und durchschnittlich seit 16 Jahren im Beruf tätig (siehe Tabelle 2). 91 \% der teilnehmenden Fachkräfte sind Frauen, und $75 \%$ haben eine Erzieherausbildung absolviert (siehe Tabelle 3).

Die teilnehmenden pädagogischen Fachkräfte der Kitas mit Nawi-Schwerpunkt sind im Durchschnitt 39 Jahre alt $($ Min $=22$; Max $=62)$ und arbeiten seit 16 Jahren als pädagogische Fachkraft in der Kita. $89 \%$ der teilnehmenden pädagogischen Fachkräfte sind Frauen und $69 \%$ haben eine Erzieherausbildung.

Die pädagogischen Fachkräfte der Vergleichsgruppe sind im Durchschnitt 38 Jahre alt $($ Min $=18 ;$ Max $=61)$ und haben im Durchschnitt 15 Berufsjahre. $88 \%$ teilnehmenden Fachkräfte sind Frauen, und $68 \%$ der pädagogischen Fachkräfte haben eine Erzieherausbildung.

Tabelle 3. Stichprobenzusammensetzung in der Studie auf Fachkrafteben

\begin{tabular}{lccccccc}
\hline & \multicolumn{1}{l}{ HdkF-Gruppe } & Nawi-Gruppe & \multicolumn{2}{c}{ Vergleichsgruppe } \\
\hline & $N$ & Prozent & $N$ & Prozent & $N$ & Prozent \\
\hline Geschlecht (Prozent Frauen) & 125 & $91 \%$ & 97 & $89 \%$ & 127 & $88 \%$ \\
\hline $\begin{array}{l}\text { Pädagogische Fachkräfte } \\
\text { (Ausbildung) }\end{array}$ & 125 & $75 \%$ & 97 & $69 \%$ & 127 & $68 \%$ \\
\hline Hochschulabschluss & 125 & $15 \%$ & 97 & $8 \%$ & 127 & $19 \%$ \\
\hline
\end{tabular}

Bei den Analysen zeigten sich keine Gruppenunterschiede im Alter und dem Geschlecht der pädagogischen Fachkräfte. Signifikante Gruppenunterschiede zum Vorteil der HdkF-Fachkräfte gegenüber den Fachkräften der Nawi-Gruppe bestehen beim Berufsabschluss sowie bei der Anzahl der besuchten naturwissenschaftlichen Fortbildungen in den letzten 3 Jahren (Vorteil HdkF-Fachkräfte gegenüber Fachkräften der Nawi- und Vergleichsgruppe). Um einen fairen Gruppenvergleich zu ermöglichen, wurden bei allen Analysen zu den Gruppenunterschieden stets u. a. für den Berufsabschluss und die Anzahl der naturwissenschaftlichen Fortbildungen in den letzten 3 Jahren kontrolliert.

\section{Stichprobe auf Ebene der Kinder}

Der Kontakt zu den Familien der Kinder wurde über die Kitas hergestellt (Leitungen bzw. betreuende Fachkräfte der Gruppen). Die Verteilung des Informationsmaterials für Eltern und der Rücklauf von Einverständniserklärungen erfolgten ebenfalls durch die Kitas. 
Die Kinder für die Untersuchung stammten aus Gruppen, die kurz vor dem Übergang zur Schule standen. Das Zielalter der Kinder lag bei 5-6 Jahren. Der Fokus auf dieser Altersstufe begründete sich darin, dass viele Kitas gezielt Bildungsangebote für diese Altersstufe anbieten und die Möglichkeiten der Testung (z. B. Dauer) im Vergleich zu noch jüngeren Kindern größer sind.

Um die Zusammenhänge zwischen den professionellen Kompetenzfacetten, der Quantität der naturwissenschaftlichen Aktivitäten, der Qualität der Interaktionen und den kindlichen naturwissenschaftlichen Kompetenzfacetten analysieren zu können, war es notwendig, dass jene Kinder einbezogen wurden, die auch regelmäßig von an der Studie teilnehmenden Fachkräften betreut wurden.

Die finale Stichprobengröße betrug $N=257$ (48\% Mädchen). Das mittlere Alter lag bei 5.6 Jahren.

Die Stichprobenbeschreibung der Kinder in den jeweiligen Gruppen hinsichtlich Alter, Geschlecht und Deutsch als Erstsprache befindet sich in Tabelle 4 und 5.

Tabelle 4. Stichprobenzusammensetzung der Studie auf Kinderebene

\begin{tabular}{lcccccccccc}
\hline & \multicolumn{3}{c}{ HdkF-Gruppe } & \multicolumn{3}{c}{ Nawi-Gruppe } & \multicolumn{3}{c}{ Vergleichsgruppe } \\
\hline & $N$ & $M$ & $S D$ & $N$ & $M$ & $S D$ & $N$ & $M$ & $S D$ \\
\hline Alter & 104 & 5.56 & 0.54 & 67 & 5.76 & 0.50 & 86 & 5.47 & 0.55 \\
\hline
\end{tabular}

Für die Kinder der HdkF-Gruppe ergibt sich folgende Stichprobenbeschreibung: Die Kinder sind im Durchschnitt 5.5 Jahre alt. $46 \%$ der befragten Kinder sind Mädchen; die Mehrheit (74\%) spricht Deutsch als Erstsprache.

Die teilnehmenden Kinder der Kitas mit Nawi-Schwerpunkt sind im Durchschnitt 5.7 Jahre alt. $43 \%$ der befragten Kinder sind Mädchen; die Mehrheit (69\%) spricht Deutsch als Erstsprache.

Die teilnehmenden Kinder in der Vergleichsgruppe sind im Durchschnitt 5.5 Jahre alt. $55 \%$ der befragten Kinder sind Mädchen; die Mehrheit (80\%) spricht Deutsch als Erstsprache. 
Tabelle 5. Stichprobenzusammensetzung der Studie auf Kinderebene

\begin{tabular}{lccccccc}
\hline & \multicolumn{2}{c}{ HdkF-Gruppe } & \multicolumn{2}{c}{ Nawi-Gruppe } & \multicolumn{2}{c}{ Vergleichsgruppe } \\
& $N$ & Prozent & $N$ & Prozent & $N$ & Prozent \\
\hline Geschlecht (Mädchen) & 104 & $46 \%$ & 67 & $43 \%$ & 86 & $55 \%$ \\
\hline Deutsch als Erstsprache & 104 & $74 \%$ & 67 & $69 \%$ & 86 & $80 \%$ \\
\hline
\end{tabular}

Bei den Analysen zeigten sich keine Gruppenunterschiede hinsichtlich der deutschen Sprache als Muttersprache und dem Geschlecht der Kinder. Die Kinder der Kitas mit Nawi-Schwerpunkt sind signifikant älter als die Kinder der anderen beiden Gruppen.

Im Zuge der Studie wurden noch weitere Hintergrundvariablen der Kinder erhoben (z. B. der Wortschatz der Kinder). Es zeigten sich keine signifikanten Gruppenunterschiede in den kognitiven Fähigkeiten, im Wortschatz und in den mathematischen Fähigkeiten.

\subsection{Variablen und Instrumente}

\subsubsection{Implementation naturwissenschaftlicher Bildung in der Einrichtung}

Die Implementation naturwissenschaftlicher Bildung in der Einrichtung beschreibt ein Qualitätsmerkmal auf Einrichtungsebene (Implementationsqualität) und wurde mithilfe eines Fragebogens für pädagogische Fachkräfte erfasst.

Die Implementationsqualität wurde anhand folgender Merkmale operationalisiert (siehe Tabelle 6).

Darüber hinaus wurde ein Index über die drei Merkmale gebildet, der den Grad der Implementation der naturwissenschaftlichen Bildung in der Kita wiedergibt. Hierfür wurde der Mittelwert der zuvor z-standardisierten Variablen gebildet. Die interne Konsistenz des Indexes liegt mit $\alpha=0.45$ im geringen Bereich, was auf die geringe Itemanzahl (3 Items) sowie die Heterogenität des Konstruktes zurückzuführen ist. Für die Analysen zu den Zusammenhängen der Implementationsqualität mit den Kompetenzen der Fachkräfte und den Prozessmerkmalen wurde der Implementationsindex auf Kita-Ebene aggregiert. 
Tabelle 6. Merkmale zur Operationalisierung von Implementationsqualität

\begin{tabular}{|c|c|}
\hline Merkmal & Kodierung \\
\hline $\begin{array}{l}\text { Die Weitergabe naturwissenschaftli- } \\
\text { cher Fortbildungsinhalte im Kollegium. }\end{array}$ & $\begin{array}{l}\text { Dieses Item konnte nur beantwortet werden, wenn } \\
\text { Fachkräfte zuvor angaben, mindestens eine natur- } \\
\text { wissenschaftliche Fortbildung in den letzten } 3 \\
\text { Jahren besucht zu haben. } \\
\text { Sofern naturwissenschaftliche Fortbildungsinhalte } \\
\text { im Kollegium weitergegeben wurden, wurde eine } 1 \\
\text { zugewiesen, andernfalls eine } 0 .\end{array}$ \\
\hline $\begin{array}{l}\text { Die Häufigkeit von Teamsitzungen, in } \\
\text { denen naturwissenschaftliche Bildung } \\
\text { thematisiert wurde. }\end{array}$ & $\begin{array}{l}\text { Hierbei wurden die Angaben zur Besprechung } \\
\text { naturwissenschaftlicher Bildung in Teamsitzungen } \\
\text { ( } 0=\text { kein Mal bis } 3=\text { auf allen Teamsitzungen) ge- } \\
\text { wichtet an der Gesamtzahl aller Teamsitzungen im } \\
\text { Umfang von mindestens } 60 \text { Minuten in den letzten } \\
3 \text { Monaten. }\end{array}$ \\
\hline $\begin{array}{l}\text { Die Absprache naturwissenschaftlicher } \\
\text { Inhalte innerhalb der Einrichtung sowie } \\
\text { mit den Eltern und dem Träger (Öffnung } \\
\text { nach außen). }\end{array}$ & $\begin{array}{l}\text { Sofern naturwissenschaftliche Bildungsinhalte in } \\
\text { der Einrichtung abgestimmt wurden, wurde eine } 1 \\
\text { zugewiesen, andernfalls eine } 0 .\end{array}$ \\
\hline
\end{tabular}

\subsubsection{Naturwissenschaftliche Kompetenzen frühpädagogischer Fachkräfte}

\section{Naturwissenschaftliche Kompetenzen der Fachkräfte: \\ Wissenskomponenten}

Um das naturwissenschaftliche Wissen von frühpädagogischen Fachkräften zu erfassen und empirisch zu untersuchen, wurde im Rahmen des EASI-Science-Projektes ein Wissenstest entwickelt. Die verschiedenen Teile des Wissenstests erfassen dabei das inhalts- und prozessbezogene Fachwissen sowie das fachdidaktische Wissen der pädagogischen Fachkräfte in der Domäne Naturwissenschaften (siehe Kapitel 2.4.1). Hierbei wurde analog zu den Wissenstests der Kinder (siehe Abschnitt 4.2.3) das inhaltsbezogene Fachwissen $\left(\mathrm{CK}_{\text {Inhalt }}\right)$ und das fachdidaktische Wissen in den Inhaltsbereichen Material, Schwimmen und Sinken, Aggregatzustände und Magnetismus erhoben. Dabei erfragen die Aufgaben zum Inhaltsbereich Magnetismus beispielsweise, welche Kenntnisse die pädagogischen Fachkräfte über Gegenstände/Materialien, die von einem Magneten angezogen werden, über magnetische Pole, über Anziehung und Abstoßung sowie zur Anziehung durch einen anderen Stoff haben. Im Inhaltsbereich Schwimmen und Sinken wurden Aufgaben entwickelt, die das Wissen zu den Konzepten Dichte, Auftriebskraft und Verdrängung erfassen (Beispielitem vgl. Abbildung 4). Die Aufgaben zum Inhaltsbereich Material erfassen das Wissen der frühpädagogischen Fachkräfte zu Materialklassen und Materialeigenschaften. Für den Inhaltsbereich Aggregatzustände zielen die Fragen auf die Erfassung des Wissens über feste, flüssige und 
gasförmige Zustände und die Übergänge zwischen Aggregatzuständen ab. Das erfasste Wissen kann als phänomenbasiertes Wissen Zusammenhänge beschreiben; komplexere Erklärungsmodelle, die z. B. auf Teilchenvorstellungen basieren, wurden nicht erhoben.

Die Kinder legen Kugeln in ein mit Wasser gefülltes Becken. Die Kugeln sind gleich schwer,
aber unterschiedlich groß. Alle gehen im Wasser unter.
Bitte schauen Sie sich die vier verschiedenen Bildreihen an und entscheiden Sie sich
unten auf dieser Seite, welche Bildreihe die richtigen Wasserstände zeigt.
Kreuzen Sie genau eine Antwort an!

Abbildung 4. Beispielitem für die Erfassung des inhaltsbezogenen Fachwissens

Das prozessbezogene Fachwissen ( $\mathrm{CK}_{\text {Prozess }}$ ) wird durch Aufgaben, die das Wissen der Fachkräfte über zentrale Denk- und Arbeitsweisen im Elementarbereich (z. B. Beobachten, Vermuten und Begründen) sowie ihr Wissen über den Forschungszyklus (Vermuten, Erproben/Überprüfen, Schlussfolgern) erfassen, gemessen. Die Aufgaben zum fachdidaktischen Wissen (PCK) erfragen das Wissen der frühpädagogischen Fachkräfte über Vorstellungen der Kinder und über Strategien zur möglichen Umsetzung zu den genannten Inhaltsbereichen, z. B. geeignete Versuche oder Alltagssituationen, in denen ein Phänomen erfahrbar ist. 
In einer Pilotphase wurden die neu entwickelten Aufgaben zunächst auf Verständlichkeit und Inhaltsvalidität in einer Gruppendiskussion mit Experten und in mehreren Einzelgesprächen mit externen Wissenschaftlerinnen und Wissenschaftlern überprüft. Danach erfolgte eine Pilotierung in einer kleinen Gruppe von Fachkräften, die die Aufgaben lösten und kommentierten. Abschließend wurden die Aufgaben in Pilotstudien anhand kleinerer Stichproben mit 38 bis 46 frühpädagogischen Fachkräften überprüft.

Das finale Testinstrument besteht aus 22 Aufgaben zum inhaltsbezogenen Fachwissen, 13 Aufgaben zum prozessbezogenen Fachwissen und 24 Aufgaben zum fachdidaktischen Wissen. Die Reliabilität (die Zuverlässigkeit der Messung durch das Testinstrument) der finalen Skala zum inhaltsbezogenen Fachwissen ( $\alpha=.71$ ) und zum fachdidaktischen Wissen $(\alpha=.69$ ) ist zufriedenstellend. Die Reliabilität für die Skalen zum prozessbezogenen Fachwissen $(\alpha=.61)$ fällt niedriger aus.

\section{Naturwissenschaftliche Kompetenzen der Fachkräfte:}

\section{Motivationale Komponente}

Auf Grundlage der theoretischen Vorarbeiten wurden die folgenden zentralen Aspekte naturwissenschaftlicher Motivation erfasst: allgemeines naturwissenschaftliches Selbstkonzept, Selbstwirksamkeitserwartung in Bezug auf die Vermittlung von Naturwissenschaften, allgemeines Interesse an den Naturwissenschaften sowie Enthusiasmus in Bezug auf die Vermittlung von Naturwissenschaften. Hierbei wurden bereits vorhandene Instrumente aus der Schulforschung an den frühkindlichen Kontext sowie den Inhaltsbereich der Naturwissenschaften angepasst. Die entwickelten Instrumente wurden anschließend mit Blick auf die Verständlichkeit der Items sowie die Reliabilitäten der Skalen anhand einer kleinen Stichprobe von $\mathrm{N}=33$ Fachkräften in Berlin überprüft und erneut überarbeitet. Die finalen Skalen wiesen moderate bis sehr gute Reliabilität in der Pilotierungsstichprobe von $\mathrm{N}=33$ auf $(\alpha=.69-.91)$.

\section{Naturwissenschaftliche Kompetenzen der Fachkräfte: Lerntheoretische Überzeugungen}

Die Entwicklung der Skalen zur Erfassung der lerntheoretischen Überzeugungen frühpädagogischer Fachkräfte erfolgte auf Grundlage der theoretischen Vorarbeiten (vgl. Kapitel 2.4.3). Entsprechend der drei Dimensionen (programmatischer Ansatz, kindzentrierter Ansatz und Open-Framework-Ansatz) wurden drei Skalen zur Erfassung der lerntheoretischen Überzeugungen entwickelt und anhand einer Stichprobe von N = 33 Fachkräften in Berlin überprüft. Im Anschluss an die erste Pilotierung wurden die Skalen überarbeitet und erneut mit N $=25$ Fachkräften er- 
probt. Die Reliabilitäten der finalen Skalen auf Grundlage der Pilotierungsstichprobe von $\mathrm{N}=25$ waren zufriedenstellend $(\alpha=.79-.83)$.

\subsubsection{Qualität und Quantität früher naturwissenschaftlicher Lerngelegenheiten}

Die Quantität, also Häufigkeit, naturwissenschaftlicher Bildungsaktivitäten wurde anhand des folgenden Items im Fragebogen für pädagogische Fachkräfte erfragt: „Denken Sie an die vergangenen 3 Monate: Wie häufig haben Sie als Lernbegleitung mit den Kindern in Ihrer Einrichtung zu naturwissenschaftlichen Fragestellungen geforscht?“ (Antwortoptionen: täglich; mehrmals pro Woche; einmal pro Woche; 1-3 Mal im Monat; seltener; nie). Im Mittel forschen die Fachkräfte 1-3 Mal im Monat mit den Kindern ( $=319 ; M=2.04 ; S D=1.33$; Min = 0/„nie“; Max $=5$ /“täglich").

Die Erfassung der Qualität naturwissenschaftlicher Bildungsaktivitäten erfolgte anhand mehrerer geschlossener Items zur Art und Weise der Umsetzung eines naturwissenschaftlichen Lernangebotes. Diese wurden auf Basis der Resultate zur Überprüfung des Zertifizierungsverfahrens der Stiftung „Haus der kleinen Forscher" (Anders \& Ballaschk, 2014) weiterentwickelt. Die Fachkräfte wurden gebeten, sich an die letzte größer angelegte Forschungsaktivität zu erinnern und diejenigen Antworten auszuwählen, die am ehesten der Umsetzung des Projektes entsprachen. Hierbei wurde auf die unterschiedlichen Stadien des Forschungsprozesses Bezug genommen (z. B. Entwicklung der Fragestellung, Einbezug der Ideen der Kinder bei der Aufstellung der Hypothesen, Durchführung, Dokumentation und Auswertung des Forschungsprojektes; siehe Tabelle 7). Die unterschiedlichen Antwortoptionen entsprachen den verschiedenen pädagogischen Praktiken (Ko-Konstruktion durch kognitiv anregende Lernbegleitung; programmatisch/ transmissiv; Open-Framework-Ansatz). Beispielweise konnten Fachkräfte angeben, ob die Fragestellung von innen vorbereitet wurde (programmatischer Ansatz) oder ob Ideen und Fragen der Kinder mit einbezogen wurden (Ko-Konstruktion). Die Angaben der Fachkräfte wurden im Anschluss kodiert und zu einem Prozessqualitätsindex zusammengefasst. 
Tabelle 7. Merkmale zur Operationalisierung der Qualität früher naturwissenschaftlicher Lerngelegenheiten

\begin{tabular}{|c|c|c|c|c|}
\hline Merkmal & Kodierung & Range & $M$ & $S D$ \\
\hline $\begin{array}{l}\text { Durchführung } \\
\text { einer geplanten } \\
\text { Forschungs- } \\
\text { aktivität }\end{array}$ & $\begin{array}{l}\text { Sofern mit den Kindern eine größer angelegte } \\
\text { Forschungsaktivität in den letzten } 12 \text { Monaten } \\
\text { durchgeführt wurde, wurde eine } 1 \text { zugewiesen, } \\
\text { andernfalls eine } 0 . \\
\text { Die nachfolgenden Items konnten nur dann be- } \\
\text { antwortet werden, wenn eine Forschungsaktivität } \\
\text { stattgefunden hat. }\end{array}$ & $0-1$ & 0.55 & 0.50 \\
\hline $\begin{array}{l}\text { Entwicklung der } \\
\text { Fragestellung }\end{array}$ & $\begin{array}{l}\text { Wurde eine Fragestellung gewählt, die von mehre- } \\
\text { ren Kindern vorgeschlagen wurde, so wurde die An- } \\
\text { gabe höher gewichtet, als wenn die Fragestellung } \\
\text { von einer Fachkraft vorgegeben wurde. }\end{array}$ & $0-2$ & 1.11 & 0.60 \\
\hline $\begin{array}{l}\text { Hypothesen- } \\
\text { entwicklung }\end{array}$ & $\begin{array}{l}\text { Antwortoptionen, die die Vermutungen der Kinder } \\
\text { berücksichtigen, wurden höher kodiert. }\end{array}$ & $0-8$ & 3.66 & 2.31 \\
\hline Umsetzung & $\begin{array}{l}\text { Die Umsetzung der Forschungsaktivität in der } \\
\text { Interaktion mit den Kindern wurde am höchsten } \\
\text { kodiert; geringer kodiert wurden Vorgehen, bei } \\
\text { denen die Fachkräfte den Versuch vorstellten oder } \\
\text { die Kinder ohne Begleitung forschten. }\end{array}$ & $0-6$ & 2.66 & 1.22 \\
\hline $\begin{array}{l}\text { Interaktion/ } \\
\text { Unterstützung }\end{array}$ & $\begin{array}{l}\text { Kognitiv anregende Interaktionen durch Be- } \\
\text { sprechung der Ideen der Kinder, Rückfragen der } \\
\text { Fachkräfte sowie durch Aufzeigen alternativer Vor- } \\
\text { gehensweisen wurden höher kodiert als Vorgehen, } \\
\text { bei denen nur die Fachkraft oder nur das Kind aktiv } \\
\text { beteiligt war. }\end{array}$ & $0-14$ & 7.00 & 3.70 \\
\hline Reflexion & $\begin{array}{l}\text { Vorgehen, bei denen die Ergebnisse der For- } \\
\text { schungsaktivität im Anschluss besprochen und mit } \\
\text { den Vermutungen in Beziehung gesetzt wurden, } \\
\text { wurden höher kodiert als Vorgehen, bei denen dies } \\
\text { nicht erfolgte. }\end{array}$ & $0-4$ & 2.17 & 1.09 \\
\hline $\begin{array}{l}\text { Entstehen } \\
\text { weiterführender } \\
\text { Fragen }\end{array}$ & $\begin{array}{l}\text { Sofern sich neue Fragen aus den Forschungsergeb- } \\
\text { nissen ergeben haben, wurde dies höher kodiert, } \\
\text { als wenn dies nicht erfolgte. }\end{array}$ & $0-3$ & 1.44 & 1.06 \\
\hline
\end{tabular}

Die kodierten Items wurden anschließend z-standardisiert und durch Aufsummierung zu einem „Prozessqualitätsindex“ zusammengefasst. Somit entspricht ein höherer Wert höherer Qualität im Sinne des Indexes. Die interne Konsistenz des Indexes liegt mit $\alpha=0.64 \mathrm{im}$ akzeptablen Bereich. 


\subsubsection{Naturwissenschaftliche Kompetenzen jüngerer Kinder}

\section{Naturwissenschaftliche Kompetenzen der Kinder: Wissenskomponenten}

Im Rahmen der Studie wurden Items entwickelt, die auf das konzeptuelle Wissen von Vorschulkindern in den Inhaltsbereichen Material, Magnetismus, Schwimmen und Sinken und Aggregatzustände sowie auf das prozessbezogene Wissen im Sinne naturwissenschaftlicher Denk- und Arbeitsweisen in den Einzelprozessen Beobachten, Vergleichen, Vermuten und Begründen abzielen.

Die gewählten Inhalte stellen eine repräsentative Auswahl der für den Naturwissenschaftsbereich der Kita typischen und in Rahmen- und Orientierungsplänen geforderten Themen dar. Die konzeptbezogenen Wissenstests beziehen sich auf verschiedene Themen eines Inhaltsgebietes, wobei berücksichtigt wird, ob die Kinder relevante Begriffe kennen und anwenden sowie entsprechende Alltagsphänomene korrekt vorhersagen und beschreiben. Beispielsweise wurden im Inhaltsgebiet Magnetismus anhand von Learning Progressions zu konzeptbezogenen Kompetenzen (Steffensky \& Hardy, 2013) Items bezogen auf vier Kompetenzbereiche entwickelt: 1) werden Magnete erkannt und entsprechende Begrifflichkeiten genutzt („,ein Magnet zieht Sachen an“), 2) wissen die Kinder, dass Magnete bestimmte Gegenstände anziehen, und sie können begründen, dass das Material des Gegenstandes ursächlich ist, 3) wissen die Kinder, dass Magnete über eine gewisse Entfernung und durch Materialien hindurch wirken können, und 4) kann die Wirkung zwischen zwei Magneten vorhergesagt werden? Die Items im Inhaltsbereich Material schließen sowohl das Wissen über Objekte und Materialien als auch über dazugehörige Materialeigenschaften ein. Die Items im Bereich Schwimmen und Sinken zielen auf das Schwimmverhalten von Gegenständen ab und die Items im Bereich Aggregatzustände auf feste, flüssige und gasförmige Zustände. Das Prozesswissen wurde im Rahmen der Denk- und Arbeitsweisen Vermuten, Beobachten, Vergleichen und Begründen unter Berücksichtigung von zwei Fragestellungen erfasst: 1) Können die Kinder zwischen verschiedenen Denk- und Arbeitsweisen unterscheiden, und 2) können sie qualitativ höherwertige Aussagen („das Boot schwimmt, weil es aus Papier ist“) von Aussagen mit geringerer Qualität („das Boot schwimmt, weil ein Boot schwimmt“) trennen? Die Items beziehen sich in ihrem Kontext sowohl auf alltägliche Situationen als auch auf spezifische Situationen der vier erfassten Inhaltsgebiete.

Der Einsatz der Items ist auf Einzeltestungen ausgerichtet, in welchen den Kindern Aufgaben und Antwortalternativen vorgelesen und Antworten protokolliert werden. Die Aufgabenkontexte sind in der Regel mit Bildmaterial und/oder passenden Gegenständen visualisiert. Die Kinder wurden durch Beispielaufgaben aus Alltagskontexten in das Testformat der Mehrfachwahlaufgaben eingeführt, 
welche vor Beginn der Testung ggf. durch Unterstützung der Testleitung korrekt gelöst werden sollen, um ein Verständnis der Vorgehensweise sicherzustellen.

Alle Items wurden im Rahmen einzelner Pilotierungsstudien anhand kleiner Stichproben getestet. Die Pilotierungsergebnisse mit insgesamt 158 Kinderbefragungen im Rahmen verschiedener Erhebungswellen deuten auf eine ausreichende Reliabilität der Instrumente im Bereich des Konzeptwissens mit den Inhaltsgebieten Material ( $\mathrm{N}=36 ; \alpha=.70)$, Magnetismus ( $\mathrm{N}=34 ; \alpha=.83$ ) sowie Schwimmen und Sinken ( $\mathrm{N}=27 ; \alpha=.61$ ) hin. Die Instrumente des Prozesswissens mit den zwei Teilbereichen der inhaltsbezogenen Denk- und Arbeitsweisen $(\mathrm{N}=30 ; \alpha=.65)$ und alltagsbezogenen Denk- und Arbeitsweisen $(\mathrm{N}=27 ; \alpha=.58)$ wiesen niedrige, aber mit Blick auf die geringe Itemanzahl akzeptable Werte auf. Im Bereich der Aggregatszustände wurden einzelne Items aus der SNAKE-Studie (Carstensen et al., 2011) ausgewählt und auf Reliabilität geprüft $(\mathrm{N}=29 ; \alpha=.58)$. Darüber hinaus wurden 4 ergänzende Items zur Erfassung des Prozesswissens eingesetzt, die sich auf den Umgang mit Hypothesen und Evidenz beziehen (Stephan-Gramberg \& Hardy, 2014).

Auf Grundlage der Pilotierungen wurde ein Itempool für die jeweiligen Instrumente ausgewählt, wobei die Items verschiedenen Wissens- und Inhaltsbereichen zugeordnet werden können. Die Items bestehen aus 28 Multiple-Choice-Aufgaben, 24 offenen Aufgaben, 11 Richtig-Falsch-Aufgaben und 4 Zuordnungsaufgaben. Die Bewertungen der Aufgaben erfolgt über zwei- und dreistufige Kodierungen, wobei bei jeder Aufgabe 2 Punkte erzielt werden können. Ein Teil der Aufgaben werden mit 0,1 oder 2 Punkten, alle anderen Aufgaben mit 0 oder 2 Punkten kodiert. Die finalen Skalen für die Analysen der Haupterhebung ergeben sich aus einem Instrument im Bereich Prozesswissen (21 Items) und vier Konzeptwissensinstrumenten in den Bereichen Material (13 Items), Magnetismus (13 Items), Schwimmen und Sinken (10 Items) sowie Aggregatzustände (10 Items).

\section{Naturwissenschaftliche Kompetenzen der Kinder: Motivationale Komponente}

Zur Messung von motivationalen Komponenten der naturwissenschaftlichen Kompetenzen von Kindern wurden neue Instrumente entwickelt. Der Instrumentenentwicklung ging eine Literaturaufarbeitung existierender Instrumente zur Erfassung motivationaler Überzeugungen von Kindern im Alter von 5-6 Jahren voraus. Verfahren (Itemformulierungen, Antwortformate), die sich in existierenden Instrumenten bewährt haben, wurden übernommen und an den deutschen Kontext sowie den Inhaltsbereich der Naturwissenschaften angepasst. Da die Kenntnis des Begriffs „Naturwissenschaften“ bei Kindern im Alter von 5-6 Jahren nicht vorausgesetzt werden kann, wurde ein Vorgehen gewählt, bei dem die motivationalen Überzeugungen hinsichtlich verschiedener naturwissenschaftlicher Themen im 
Alltag erfasst wurden. Die Auswahl der naturwissenschaftlichen Themen erfolgte auf Grundlage der Rahmen- und Orientierungspläne sowie wissenschaftlicher Erkenntnisse zu alltagsnahen naturwissenschaftlichen Aktivitäten und Materialien (Tu, 2006). Hierbei wurden sowohl Themen der belebten als auch der unbelebten Natur berücksichtigt.

Analog zu den Wissenstests erfolgte die Befragung der Kinder in standardisierten Einzelinterviews. Auch für die Durchführung der Interviews wurde auf existierende Instrumente zurückgegriffen. So hat sich die Verwendung von Handpuppen in vorherigen Studien bei Kindern im Vorschulalter bewährt (vgl. Edens und Potter, 2013; Manticopoulos et al., 2008; Measelle, Ablow, Cowan \& Cowan, 1998). Die Puppen erleichtern den Kindern den Umgang mit der ungewohnten Interviewsituation, dienen zur Veranschaulichung der Items und Antwortformate (z. B. „... diese Puppe weiß bereits sehr viel über .../möchte gern mehr über ... lernen") und verdeutlichen, dass es keine richtigen oder falschen Antworten gibt.

Die Items und Antwortformate wurden in insgesamt vier subsequenten Pilotstudien mit einer kleinen Stichprobe von Kindern überprüft und sukzessive weiterentwickelt. Die Stichprobengrößen betrugen jeweils $\alpha=14, \alpha=8$ und $\alpha=12$ Kinder in den ersten drei Pilotstudien sowie $\alpha=52$ Kinder in einer finalen Pilotstudie. Die Erprobung verschiedener Antwortformate zeigte, dass die Antworten der Kinder auf einer dichotomen Antwortskala (Ja/Nein) zu $85 \%$ mit den Antworten auf einer 4-Punkt-Likert-Skala übereinstimmen. Aufgrund der besseren psychometrischen Eigenschaften wurde das 4-Punkt-Antwortformat für die finale Version der Skala verwendet. Die Pilotstudie zeigte ferner, dass weniger extreme Antworttendenzen (Deckeneffekte) auftraten und die Skala insgesamt eine höhere Reliabilität aufwies, wenn statt Emoticons ein liegendes Dreieck mit vier Ausprägungen als visuelle Unterstützung der Antwortalternativen verwendet wurde (vgl. Abbildung 5).

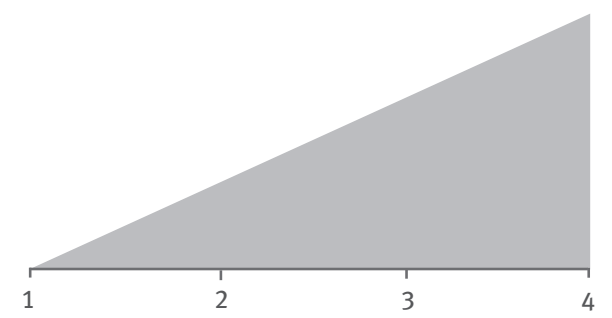

Abbildung 5. Grafisches Antwortformat

Neben den Antwortformaten wurden auch die neu entwickelten Items erprobt und überarbeitet. Das finale Instrument besteht aus 28 Items, davon 15 Items zur Erfassung der Selbstwirksamkeitserwartungen ( 8 Items im Bereich der belebten 
und 7 Items im Bereich der unbelebten Natur) sowie 13 Items zur Erfassung der Lernfreude (7 Items im Bereich der belebten und 6 Items im Bereich der unbelebten Natur).

\subsubsection{Kontroll- und Hintergrundvariablen}

Neben der Erfassung naturwissenschaftlicher Kompetenzen der Kinder und professioneller Kompetenzen von Fachkräften wurden im Rahmen der Studie verschiedene individuelle Voraussetzungen der befragten Kinder und Fachkräfte sowie Hintergrundvariablen, bezogen auf Kinder, Eltern, Fachkräfte und Kitas, erhoben.

\section{Einrichtungsebene}

Auf Ebene der Kitas wurden insbesondere Strukturmerkmale als Kontrollvariablen erhoben. Dazu zählen Fragen nach der Anzahl und Qualifikation der Fachkräfte, der Anzahl an Gruppen und betreuten Kindern, der Altersstruktur der Kinder, der Anzahl an Kindern mit Migrationshintergrund, der Anzahl an Räumen und weiteren räumlichen Rahmenbedingungen. Ferner wurden das pädagogische Konzept (z. B. offenes Konzept) und die inhaltlichen Schwerpunkte der Bildungsarbeit (z. B. Mathematik) erfragt.

\section{Fachkraftebene}

Auf Ebene der Fachkräfte wurden sowohl individuelle, kognitive Voraussetzungen wie auch verschiedene nicht leistungsbezogene Daten erhoben. Die kognitiven Fähigkeiten wurden durch den Subtest Figurenanalogien des Instruments Kognitiver Fähigkeitstest (Heller \& Perleth, 2000) erhoben. Individuelle Hintergründe wurden in Bezug auf Alter, Geschlecht, Qualifikation und Ausbildung sowie den sprachlichen, kulturellen und bildungsbezogenen Hintergrund der Fachkräfte erfasst.

\section{Kinderebene}

Auf Ebene der Kinder wurden verschiedene individuelle Vorrausetzungen als Kontrollvariablen erhoben: Die nonverbale Intelligenz der Kinder wurde durch die Unterskalen Substitution und Ähnlichkeiten des Culture Fair Intelligence Test (Weiß \& Osterland, 2012) ermittelt. Zur Erfassung mathematischer Fähigkeiten wurde der Untertest Rechnen des Kaufman-Assessment Battery for Children (Melchers \& Preuß, 2009) eingesetzt und der Wortschatz der Kinder durch den Peabody Picture Vocabulary Test (Dunn \& Dunn, 2004) erhoben. Darüber hinaus wurden Angaben der Kinder zum Alter, zum Geschlecht und zur gesprochenen Sprache in der Familie erfasst. 
Die Kinder erzielten im Durchschnitt beim nonverbalen Intelligenztest 23 von 75 Punkten beim Subtest „Substitutionen“ und 6 von 15 Punkten beim Subtest „Ähnlichkeiten“.Im Durchschnitt lösten sie 18 von 25 Aufgaben zur Arithmetik (erfasst durch den K-ABC) und 47 von 60 Aufgaben beim Wortschatztest (PPVT).

\section{Elternebene}

Auf Ebene der Eltern wurden u. a. Angaben zum sozialen, kulturellen, bildungsbezogenen und sprachlichen Hintergrund erhoben.

\subsection{Untersuchungsdurchführung}

Die EASI-Science-Studie zielt auf die Wirkung naturwissenschaftlicher Bildungsangebote hinsichtlich professioneller Kompetenzen von Fachkräften und naturwissenschaftlicher Kompetenzen der betreuten Kinder ab. Um die aus dieser allgemeinen Zielsetzung ergebenden Teilfragestellungen empirisch zu beantworten, war der Einsatz vielfältiger Messinstrumente notwendig, die sich an unterschiedliche Personengruppen richten. Im Rahmen der Studie wurden Leiterinnen und Leiter von Kitas, pädagogische Fachkräfte sowie Kinder und deren Eltern befragt. Dabei wurden sowohl Fragebögen und Instrumente zur Erfassung von motivationalen Orientierungen, Fachwissen und fachdidaktischem Wissen, naturwissenschaftlichem Prozess- und Konzeptwissen eingesetzt wie auch Kontroll- und Hintergrundvariablen auf Einrichtungsebene, Fachkraftebene und Kinderebene. Die Befragungen der Kinder erfolgten von Juni bis September 2015. Da eine Nacherhebung zur Realisierung der Gesamtstichprobe nötig war und die Altersstruktur der bereits erhobenen Kinder berücksichtigt werden sollte, fanden erst von Januar bis Mai 2016 die Nacherhebungen statt. Die Befragungen von pädagogischen Fachkräften erfolgten über den gesamten Erhebungszeitraum von Juni 2015 bis Mai 2016.

\section{Einrichtungsebene}

Die Erfassung der Einrichtungsmerkmale basierte auf (halb-)standardisierten Fragebögen, die sich an die Einrichtungsleitungen richtete. Die Befragung war als Online-Befragung konzipiert und wurde den Leitungen über die webbasierte Software UNIPARK zugänglich gemacht. Nach Absprache konnte der Fragebogen auch als Paper-Pencil-Version schriftlich ausgefüllt und postalisch an den Projektstandort gesendet werden. Sofern keine Rückmeldungen der Einrichtungsleitungen erfolgte, wurden zwei Online-Erinnerungen über UNIPARK versendet oder telefonisch Kontakt durch die jeweiligen Projektstandorte aufgenommen. Die Befragungen der Einrichtungsleitungen fanden im Anschluss an die Befragungen der 
Kinder und Fachkräfte statt und sollten spätestens drei Wochen nach Abschluss der Gesamtbefragung einer Einrichtung erfolgen.

\section{Fachkraftebene}

Die Daten der pädagogischen Fachkräfte wurden im Rahmen einer Befragung im Vorfeld und einer Befragung vor Ort (Haupterhebung) erfasst. Zunächst wurde im Vorfeld der Haupterhebung eine Online-Befragung über UNIPARK durchgeführt. Die Befragung bezog sich auf motivationale Aspekte und Hintergrundvariablen der pädagogischen Fachkräfte. Die Online-Befragung war auch als Paper-Pencil-Version erhältlich. Fachkräfte, die bis zur Haupterhebung nicht an der Online-Befragung teilgenommen hatten, konnten am Tag der Haupterhebung eine gekürzte Version des Paper-Pencil-Fragebogens ausfüllen, damit die Erhebung der motivationalen Aspekte vor der Erhebung der Wissensaspekte stattfinden konnte. Der restliche Teil der Online-Befragung wurde im Nachhinein ausgefüllt. Die Befragung zu den leistungsbezogenen Bereichen des inhalts- und prozessbezogenen Fachwissens und fachdidaktischen Wissens sowie zu kognitiven Fähigkeiten fand in den teilnehmenden Kitas statt. Dabei wurden die pädagogischen Fachkräfte im Rahmen von kontrollierten Einzel- oder Gruppentestungen befragt. Die Befragungen wurden durch Paper-Pencil-Testhefte umgesetzt, die die pädagogischen Fachkräfte eigenständig ausfüllen mussten. Um die Qualität der Daten zu gewährlisten, wurden die Befragungen von geschulten Erheberinnen und Erhebern organisiert und begleitet.

\section{Kind- und Elternebene}

Die naturwissenschaftlichen Kompetenzen und weitere individuelle Voraussetzungen (Wortschatz, mathematische Fähigkeit und nonverbale Intelligenz) der Kinder sowie einige Hintergrundmerkmale wurden in den jeweiligen Kitas durch geschulte Erheberinnen und Erheber in Einzeltestungen erhoben. Um Ermüdungseffekte bei den Kindern zu vermeiden, fanden die Befragungen an zwei Tagen statt. In einer Machbarkeitsstudie im Vorfeld $(\mathrm{N}=6)$ erwies sich die zweitägige Befragung als umsetzbar. Um eine kognitive Überforderung der Kinder zu vermeiden und das Interesse aufrechtzuerhalten, wurden den Kindern im Verlauf der je nach Testtag und Kind 60- bis 120-minütigen Befragungszeit viele Spielpausen ermöglicht. Die Erhebung weiterer Hintergrundvariablen der Kinder erfolgte über Online-Befragungen der Eltern. Diese wurde den Eltern nach Abschluss der Vor-Ort-Befragung ihrer Kinder ermöglicht und konnte auch in Form eines Paper-Pencil-Fragebogens erfolgen. 


\subsection{Statistische Analyseverfahren}

Um die Fragestellungen der vorliegenden Studie zu untersuchen, wurden deskriptive, inferenzstatistische und multivariate Analysen durchgeführt. Um zu untersuchen, in welcher Struktur die Komponenten der Kompetenzen (Wissen und Motivation) der Fachkräfte und Kindern vorliegen, wurden konfirmatorische Faktorenanalysen berechnet. Bei der Berechnung konfirmatorischer Faktorenanalysen werden auf Basis theoretischer Annahmen verschiedene Modelle der Kompetenzstruktur verglichen. Die Unterschiede zwischen den Gruppen wurden mit kovarianzanalytischen Ansätzen ermittelt, die es ermöglichen, bei der Berechnung der Gruppenunterschiede die Einflüsse von relevanten Hintergrund- und Strukturvariablen zu kontrollieren.

Zur angemessenen Berücksichtigung der Mehrebenenstruktur (Fachkräfte und Kinder geschachtelt in Kitas) wurden in MPlus (Muthén \& Muthén, 2014) mit Type Complex eine Standardfehlerkorrektur vorgenommen. Für die Analysen wurde eine Haupterzieherin bzw. ein Haupterzieher des Kindes bestimmt und der Einfluss der Kompetenzen der pädagogischen Fachkraft auf die kindlichen Kompetenzen berechnet. Als Haupterzieherin bzw. Haupterzieher wurde die pädagogische Fachkraft bestimmt, die im Vergleich den höchsten Betreuungsumfang für das jeweilige Kind hatte. Falls dies auf mehrere pädagogische Fachkräfte zutraf, wurde die pädagogische Fachkraft ausgewählt, die die meisten naturwissenschaftlichen Fortbildungen besucht hat. Fehlende Werte wurden bei den Analysen mit FIML-Prozedur geschätzt. 


\section{Ergebnisse}

\subsection{Erfassung und Struktur der Kompetenzen}

Die erste Zielstellung des Projektes war die Entwicklung neuer Instrumente zur Erfassung unterschiedlicher Aspekte naturwissenschaftlicher Kompetenzen. Da bisher kaum Forschungsbefunde zu den naturwissenschaftlichen Kompetenzen von Fachkräften und Kindern existieren, war zunächst die Frage nach der Struktur der Kompetenzen zentral. Auf Grundlage der faktoriellen Validierung wurden anschließend die Reliabilitäten der Skalen sowie die deskriptiven Statistiken überprüft.

\subsubsection{Fachkraftebene}

\section{Naturwissenschaftliche Kompetenzen der Fachkräfte:}

Wissenskomponenten

Um zu prüfen, in welcher Struktur das naturwissenschaftliche Wissen der pädagogischen Fachkräfte vorliegt, wurden drei konfirmatorische Faktorenanalysen durchgeführt und die Fit-Indices miteinander abgeglichen. Drei Modelle wurden als plausibel angenommen und gegeneinander getestet: 1) ein eindimensionales Modell, das ein naturwissenschaftliches Wissen der Fachkräfte annimmt, das nicht weiter ausdifferenziert wird, 2) ein zweidimensionales Modell, bei dem das fachdidaktische Wissen und das inhalts- und prozessbezogene Fachwissen jeweils einen Faktor darstellen, 3) ein dreidimensionales Modell, das das inhalts- und prozessbezogene Fachwissen sowie das fachdidaktische Wissen als drei trennbare Wissensdimensionen annimmt. Der Vergleich der Fit-Indices zeigte, dass das dreidimensionale Modell die Datenstruktur am besten widerspiegelt. Der Vergleich der Fit-Indices spricht somit für eine Trennbarkeit des naturwissenschaftlichen Wissens der frühpädagogischen Fachkräfte in die drei Wissensfacetten inhaltsund prozessbezogenes Fachwissen ( $\mathrm{CK}_{\text {Inhalt }}$ und $\mathrm{CK}_{\text {Prozess }}$ ) sowie fachdidaktisches Wissen (PCK). Jedoch korrelieren alle drei Wissensfacetten substanziell miteinander. Das bedeutet: Je höher Z.B. das inhaltsbezogene naturwissenschaftliche Fachwissen ( $\mathrm{CK}_{\text {Inhalt }}$ ) einer frühpädagogischen Fachkraft ist, desto höher ist auch ihr naturwissenschaftliches PCK und ihr prozessbezogenes Fachwissen ( $\mathrm{CK}_{\text {Prozess }}$ ). Auf Grundlage dieser Ergebnisse wurden drei Skalen gebildet. Die Reliabilitäten der finalen Skalen fallen für das inhaltsbezogene Fachwissen höher aus als für das fachdidaktische Wissen und das prozessbezogene Fachwissen (siehe Tabelle 8). Die deskriptiven Ergebnisse zeigen, dass die frühpädagogischen Fachkräfte im Mittel ca. 50 Prozent der Aufgaben zum inhaltsbezogenen Fachwissen und zum fachdidaktischen Wissen korrekt lösten. Im Hinblick auf das prozessbezogene 
Fachwissen zeigt sich, dass die Fachkräfte im Durchschnitt fast 60 Prozent richtig lösten. Insgesamt weisen die deskriptiven Befunde eine große Heterogenität hinsichtlich des naturwissenschaftlichen Wissens der Fachkräfte auf. So zeigen die Ergebnisse der drei Skalen beispielsweise eine große Spannbreite zwischen den minimal zu maximal erreichten Punktzahlen.

Tabelle 8. Skalenreliabilitäten und deskriptive Ergebnisse zum naturwissenschaftlichen Wissen der Fachkräfte

\begin{tabular}{lccccccc}
\hline & $N$ & $M$ & $S D$ & Min & Max & $\alpha$ & Items \\
\hline $\begin{array}{l}\text { Inhaltsbezogenes } \\
\text { Fachwissen }\end{array}$ & 318 & 11.33 & 3.85 & 2 & 20 & .71 & 22 \\
\hline $\begin{array}{l}\text { Prozessbezogenes } \\
\text { Fachwissen }\end{array}$ & 318 & 7.77 & 2.28 & 0 & 13 & .61 & 13 \\
\hline $\begin{array}{l}\text { Fachdidaktisches } \\
\text { Wissen }\end{array}$ & 318 & 11.63 & 3.89 & 0 & 23 & .69 & 24 \\
\hline
\end{tabular}

\section{Naturwissenschaftliche Kompetenzen der Fachkräfte:}

\section{Motivationale Komponente}

Auf Grundlage des theoretischen Hintergrundes wurde angenommen, dass die Skalen zur Erfassung (1) des allgemeinen naturwissenschaftlichen Selbstkonzeptes, (2) der Selbstwirksamkeitserwartung in Bezug auf die Vermittlung von Naturwissenschaften, (3) des allgemeinen Interesses an den Naturwissenschaften sowie (4) des Enthusiasmus in Bezug auf die Vermittlung von Naturwissenschaften separate Aspekte der naturwissenschaftlichen Kompetenzen abbilden. Dementsprechend wurde ein 4-Faktor-Modell mit einem einfaktoriellen Modell verglichen, bei dem alle Items auf einen gemeinsamen Faktor laden. Die Ergebnisse der Faktorenanalysen zeigen einen deutlich besseren Model-Fit beim 4-Faktor-Modell im Vergleich zum einfaktoriellen Modell und bestätigen damit die empirische Trennbarkeit der vier Konstrukte. Die vier latenten Faktoren korrelieren positiv untereinander, wobei das Selbstkonzept und die Selbstwirksamkeitserwartung am höchsten $(r=.85)$ und die Selbstwirksamkeitserwartung und das Interesse vergleichsweise am geringsten zusammenhängen $(r=.59)$.

Die Skalenbildung erfolgte durch Mittelung aller Items, die auf einem Faktor laden. Die Reliabilitäten der vier Skalen fallen gut aus (siehe Tabelle 9). Die deskriptiven Ergebnisse zeigen, dass die befragten Fachkräfte im Mittel ihre Fähigkeiten in den Naturwissenschaften allgemein (Selbstkonzept) und in Bezug auf die Vermittlung naturwissenschaftlicher Inhalte (Selbstwirksamkeitserwartung) 
sowie ihr Interesse und ihren Enthusiasmus als hoch einschätzen. Bei einem Antwortformat von 1-4 (1 = stimme gar nicht zu; 4 = stimme ganz zu) fallen die Angaben der Fachkräfte auf allen vier Skalen höher aus als der theoretische Mittelwert von 2.5 .

Tabelle 9. Skalenreliabilitäten und deskriptive Ergebnisse zu den motivationalen Kompetenzfacetten der Fachkräfte

\begin{tabular}{lllllll}
\hline & $N$ & $M$ & $S D$ & $\alpha$ & Items & Beispielitem \\
\hline Selbstkonzept & 337 & 2.85 & 0.63 & .82 & 4 & $\begin{array}{l}\text { Ich bin gut in Naturwissen- } \\
\text { schaften. }\end{array}$ \\
$\begin{array}{lllllll}\text { Selbstwirksam- } \\
\text { keitserwartung }\end{array}$ & 343 & 2.98 & 0.49 & .75 & 5 & $\begin{array}{l}\text { Es fällt mir leicht, ein naturwis- } \\
\text { senschaftliches Lernangebot } \\
\text { für Kinder vorzubereiten. }\end{array}$ \\
\hline Interesse & 338 & 3.04 & 0.59 & .87 & 5 & $\begin{array}{l}\text { Im Allgemeinen macht es } \\
\text { senschaftlichen Themen zu }\end{array}$ \\
\hline befassen.
\end{tabular}

\section{Naturwissenschaftliche Kompetenzen der Fachkräfte: Lerntheoretische Überzeugungen}

Auch für die Überprüfung der Dimensionalität lerntheoretischer Überzeugungen in Bezug auf die Vermittlung naturwissenschaftlicher Inhalte wurde ein einfaktorielles Modell, bei dem alle Items auf einen gemeinsamen Faktor laden, mit einem mehrfaktoriellen Modell verglichen. Hierbei wurde auf Grundlage der theoretischen Unterscheidung zwischen dem programmatischen, kindzentrierten und Open-Framework-Ansatz ein dreifaktorielles Modell gewählt. Der Vergleich der Fit-Indices beider Modelle zeigt eine signifikant bessere Passung des dreifaktoriellen Modells gegenüber dem einfaktoriellen Modell auf die Daten. Die theoretisch begründete Unterscheidung lerntheoretischer Überzeugungen lässt sich demnach auch empirisch bestätigen. Die latenten Korrelationen des kindzentrierten sowie Open-Framework-Ansatzes mit dem programmatischen Ansatz fallen negativ aus ( $r=-.36$ bzw. $r=-.32$ ), wohingegen der kindzentrierte und Open-Framework-Ansatz positiv korrelieren $(r=.60)$.

Auf Grundlage dieser Ergebnisse wurden drei Skalen durch Mittelung aller Items, die auf einem Faktor laden, gebildet. Die Reliabilitäten der finalen Skalen sind zufriedenstellend (siehe Tabelle 10). Hinsichtlich der Ausprägung der drei unterschiedlichen Überzeugungen bei den befragten Fachkräften zeigen die 
deskriptiven Ergebnisse im Mittel eine deutliche Zustimmung zum Open-Framework-Ansatz: Der mittlere Wert von 3.46 lässt sich auf der Antwortskala von 1-4 in etwa den Antwortoptionen „stimme eher zu“ bis „stimme ganz zu“ zuordnen. Auch dem kindzentrierten Ansatz stehen die Fachkräfte durchschnittlich positiv gegenüber. Der programmatische Ansatz wird unter den befragten Fachkräften dagegen eher abgelehnt: Der Mittelwert von 2.03 entspricht in etwa der Antwortoption „stimme eher nicht zu“.

Tabelle 10. Skalenreliabilitäten und deskriptive Ergebnisse zu den lerntheoretischen Überzeugungen der Fachkräfte

\begin{tabular}{|c|c|c|c|c|c|c|}
\hline & $N$ & $M$ & $S D$ & $\alpha$ & Items & Beispielitem \\
\hline $\begin{array}{l}\text { Programmatischer } \\
\text { Ansatz }\end{array}$ & 334 & 2.03 & 0.46 & .72 & 5 & $\begin{array}{l}\text { Am besten verstehen Kinder } \\
\text { naturwissenschaftliche Inhalte } \\
\text { aus den Erklärungen und Dar- } \\
\text { stellungen der Fachkraft. }\end{array}$ \\
\hline $\begin{array}{l}\text { Kindzentrierter } \\
\text { Ansatz }\end{array}$ & 335 & 3.07 & 0.44 & .64 & 6 & $\begin{array}{l}\text { Kinder lernen am besten, wenn } \\
\text { sie sich ganz selbstbestimmt } \\
\text { mit naturwissenschaftlichen } \\
\text { Inhalten beschäftigen. }\end{array}$ \\
\hline $\begin{array}{l}\text { Open-Framework- } \\
\text { Ansatz }\end{array}$ & 335 & 3.46 & 0.37 & .62 & 5 & $\begin{array}{l}\text { Es ist wichtig, naturwissen- } \\
\text { schaftliche Bildung in der Kita } \\
\text { individuell auf den Wissens- } \\
\text { stand der Kinder anzupassen. }\end{array}$ \\
\hline
\end{tabular}

\subsubsection{Kinderebene}

Bislang liegen kaum vertiefende Analysen zur Dimensionalität naturwissenschaftlicher Kompetenzen im jungen Kindesalter vor. Über die Kompetenzstruktur junger Kinder im Allgemeinen und über die spezifische Struktur der einzelnen Komponenten des konzept- und prozessbezogenen Wissens sowie der Motivation ist demnach wenig bekannt. In den folgenden Analysen werden die Bereiche Wissen und Motivation getrennt betrachtet. Die Analysen zur Kompetenzstruktur in den beiden Bereichen liefern Hinweise darauf, ob die mit den Items erfassten Kompetenzen der Kinder am besten durch Gesamtskalen beschrieben werden oder ob es sich um ein- bzw. mehrdimensionale Konstrukte handelt.

\section{Naturwissenschaftliche Kompetenzen der Kinder: Konzeptbezogenes und prozessbezogenes Wissen}

Zur Überprüfung der Dimensionalität von Wissenskomponenten im Bereich der naturwissenschaftlichen Kompetenzen wurden durch konfirmatorische Faktorenanalysen mit dem Statistikprogramm Mplus (Muthén \& Muthén, 2014) unter Verwendung des WLMSV-Schätzers verschiedene Modelle gegeneinander getestet: 1) ein eindimensionales Modell, bei dem alle Items auf einem Faktor laden, 2) ein 
zweidimensionales Modell, bei dem die verschiedenen Items den Faktoren des prozessbezogenen Wissens und des konzeptbezogenen Wissens zugeordnet wurden, 3) ein mehrdimensionales Modell, das den Prozesswissensbereich und die einzelnen Inhaltsbereiche des konzeptbezogenen Wissens berücksichtigt, wobei die Einzelfaktoren einem gemeinsamen Faktor 2. Ordnung untergeordnet wurden.

Auf Grundlage von Fit-Indices (CFI, RMSEA) der Modellanalysen zeigen sich nur geringfügige Unterschiede zwischen Modell 1 und Modell 2, was mit einer hohen latenten Korrelation zwischen den Faktoren des prozessbezogenen Wissens und konzeptbezogenen Wissens einhergeht $(r=.85)$. Eine empirische Trennung der beiden Faktoren kann somit nicht bestätigt werden. Die besten Fit-Indices liefert Modell 3, verbunden mit hohen Korrelationen zwischen allen Faktoren, die stark auf einen gemeinsamen Globalfaktor laden. Die Modellanalysen deuten einen Informationsgewinn an, wenn jeweils die einzelnen Inhaltsbereiche und das Prozesswissen getrennt betrachtet werden. Anlehnend an diese Ergebnisse wurde sowohl eine Globalskala aller Items gebildet sowie Unterskalen im Bereich Prozesswissen und in den Inhaltsgebieten Material, Magnetismus, Schwimmen und Sinken und Aggregatzustände.

Tabelle 11. Skalenreliabilitäten und deskriptive Ergebnisse zum konzept- und prozessbezogenen Wissen der Kinder in Naturwissenschaften

\begin{tabular}{|c|c|c|c|c|c|c|}
\hline & $N$ & $M$ & $S D$ & $\alpha$ & Items & Beispielitem \\
\hline $\begin{array}{l}\text { Gesamtskala } \\
\text { Wissen }\end{array}$ & 271 & 66,59 & 18,33 & .86 & 67 & \\
\hline Prozesswissen & 281 & 21.77 & 6.78 & .65 & 21 & $\begin{array}{l}\text { Hast du eine Idee, wie } \\
\text { man diese Legosteine der } \\
\text { Reihenfolge nach ordnen } \\
\text { oder sortieren kann? }\end{array}$ \\
\hline Material & 280 & 12.57 & 4.69 & .68 & 13 & $\begin{array}{l}\text { Sachen aus Holz können } \\
\text { brennen. Ist das richtig } \\
\text { oder ist das falsch? }\end{array}$ \\
\hline Magnetismus & 279 & 12.81 & 4.69 & .70 & 13 & $\begin{array}{l}\text { Vor dir liegen verschiedene } \\
\text { Sachen. Weißt du, welche } \\
\text { Sachen an einem Magne- } \\
\text { ten dran bleiben? }\end{array}$ \\
\hline $\begin{array}{l}\text { Schwimmen } \\
\text { und Sinken }\end{array}$ & 273 & 8.21 & 4.48 & .67 & 10 & $\begin{array}{l}\text { Was passiert, wenn ich } \\
\text { das da (Zahnstocher) ins } \\
\text { Wasser lege? }\end{array}$ \\
\hline $\begin{array}{l}\text { Aggregat- } \\
\text { zustände }\end{array}$ & 272 & 10.43 & 3.90 & .60 & 10 & $\begin{array}{l}\text { Die Kinder dürfen sich } \\
\text { ein Eis kaufen. Die Sonne } \\
\text { scheint, es ist richtig warm } \\
\text { und das Eis beginnt zu } \\
\text { tropfen. Wie nennt man } \\
\text { das, was da mit dem Eis } \\
\text { passiert? }\end{array}$ \\
\hline
\end{tabular}


Die Globalskala aller Items weist eine hohe Reliabilität auf (siehe Tabelle 11), wobei die Kinder knapp die Hälfte der erreichbaren Punkte erzielen. Die Unterskalen im Bereich des Prozesswissens und den Inhaltsbereichen Material, Magnetismus, Schwimmen und Sinken sowie Aggregatzustände zeigen eine ausreichende Reliabilität. Die Beantwortung des Tests zum Schwimmen und Sinken fiel den Kindern dabei am schwersten. Alle anderen Testinstrumente sind normal verteilt, wobei die Kinder etwa die Hälfte der erreichbaren Punkte erzielten. Vertiefende Analysen auf Itemebene deuten zudem nicht auf Boden- oder Deckeneffekte hin. Lediglich ein Item im Test zum Schwimmen und Sinken weist eine Lösungshäufigkeit von unter 15 Prozent bzw. ein Item im Aggregatzustandstest eine Lösungshäufigkeit von über 85 Prozent auf. Beide Items wurden aus inhaltlichen Gründen nicht aus den Analysen ausgeschlossen.

Zusammenhangsanalysen weisen auf eine niedrige Korrelation zwischen der nonverbalen Intelligenz ( $r=.31)$ sowie der mathematischen Kompetenz $(r=.29)$ und einem latenten Globalfaktor der naturwissenschaftlichen Kompetenzen hin. Ein ähnliches Ergebnis zeigen Analysen, bezogen auf die nonverbale Intelligenz bzw. mathematische Kompetenz der Kinder, und die einzelnen Unterskalen (Prozesswissen, Material, Magnetismus, Schwimmen und Sinken, Aggregatzustände) wieder. Deutlich höher ist der Zusammenhang zwischen dem latenten Globalfaktor der naturwissenschaftlichen Kompetenzen und dem Wortschatz der Kinder $(r=.75)$. Dieser zeigt sich auch bei einer differenzierten Betrachtung der einzelnen Unterskalen, wobei ein Vergleich der Modell-Fits auf die Trennbarkeit des prozess- und konzeptbezogenen Wissens vom Wortschatz hindeutet. Vertiefende Modellanalysen zur Varianzerklärung zeigen, dass zwar ein Teil der Varianz des prozess- und konzeptbezogenen Wissens durch den Wortschatz erklärt wird, aber dennoch wesentliche Varianzanteile auf globale und bereichsspezifische naturwissenschaftliche Kompetenzen zurückgeführt werden können.

\section{Naturwissenschaftliche Kompetenzen der Kinder:}

\section{Motivationale Komponenten naturwissenschaftlicher Kompetenzen}

Zur Überprüfung der Struktur der naturwissenschaftlichen Motivation der Kinder wurden drei Faktormodelle gegeneinander getestet: (1) ein eindimensionales Modell, in dem alle Items auf einen gemeinsamen Motivationsfaktor laden, (2) ein zweidimensionales Modell, welches zwischen der Selbstwirksamkeitserwartung und der Lernfreude unterscheidet, sowie (3) ein vierdimensionales Modell, welches innerhalb der Aspekte der Selbstwirksamkeitserwartung und Lernfreude zwischen den Inhaltsbereichen der belebten und unbelebten Natur unterscheidet. Die Ergebnisse zeigen, dass Selbstwirksamkeitserwartung und Lernfreude separate Konstrukte darstellen: Das zweidimensionale war dem eindimensionalen Modell in der Güte der Modellanpassung überlegen. Die latenten 
Faktoren der Selbstwirksamkeitserwartung und Lernfreude korrelieren mittelhoch $(r=.45)$.

Der Vergleich des zweidimensionalen (2) und des vierdimensionalen (3) Modells zeigte, dass Letzteres die beste Anpassung an die Datenstruktur aufweist. Allerdings fielen hier die latenten Korrelationen zwischen der Selbstwirksamkeitserwartung in der belebten und unbelebten Natur sowie zwischen der Lernfreude in der belebten und unbelebten Natur sehr hoch aus $(r \geq 0.91)$. Dies verdeutlicht, dass sich die motivationalen Überzeugungen der Kinder in diesem Alter noch nicht in die Bereiche der belebten und unbelebten Natur trennen lassen. Zugunsten der Sparsamkeit wurde daher das zweidimensionale Modell für die Skalenbildung und weitere Analysen verwendet.

Auf Grundlage der Ergebnisse der konfirmatorischen Faktorenanalysen wurden zwei Skalen gebildet: Selbstwirksamkeitserwartung und Lernfreude in den Naturwissenschaften. Die Reliabilitäten der Skalen fallen gut aus (siehe Tabelle 12). Die deskriptiven Analysen zeigen deutliche Deckeneffekte auf beiden Skalen: Die Kinder schätzen ihre Fähigkeiten im Mittel eher hoch ein und berichten eine hohe Freude an der Beschäftigung mit den naturwissenschaftlichen Inhalten.

Tabelle 12. Skalenreliabilitäten und deskriptive Ergebnisse zu den motivationalen Kompetenzfacetten der Kinder

\begin{tabular}{lcccccl}
\hline & $N$ & $M$ & $S D$ & $\alpha$ & Items & Beispielitem \\
\hline $\begin{array}{l}\text { Selbstwirksam- } \\
\text { keitserwartung }\end{array}$ & 282 & 2.99 & 0.68 & .87 & 15 & $\begin{array}{l}\text { Zeig mir mal, wie viel du schon } \\
\text { über Tiere weißt! }\end{array}$ \\
\hline Lernfreude & 282 & 3.51 & 0.60 & .89 & 13 & $\begin{array}{l}\text { Zeig mir mal, wie gern du noch } \\
\text { mehr über Tiere lernen möchtest! }\end{array}$ \\
\hline
\end{tabular}

\subsection{Gruppenunterschiede}

Die zweite Fragestellung des Projektes zielte auf potenzielle Unterschiede zwischen „Haus der kleinen Forscher“-Kitas (HdkF), Kitas mit naturwissenschaftlichem Schwerpunkt, die nicht an der „Haus der kleinen Forscher“-Initiative teilnehmen (Nawi-Kitas), und Kitas ohne naturwissenschaftlichen Schwerpunkt (Vergleichsgruppe). Die Einordnung der Kitas in die drei Untersuchungsgruppen erfolgte - neben der Zuordnung anhand der Teilnahme an der Bildungsinitiative „Haus der kleinen Forscher“ - auf Grundlage der Anzahl besuchter Fortbildungen: Eine Einrichtung wurde nur dann der HdkF- oder Nawi-Gruppe zugewiesen, wenn mindestens eine Fachkraft in der Einrichtung mindestens 2 naturwissenschaftliche Fortbildungen in den letzten 3 Jahren besucht hat oder die Einrichtung in den letzten 3 Jahren als „Haus der kleinen Forscher Kita“ zertifiziert wurde und damit zwangsläufig mindestens eine Fachkraft in der Einrichtung mindesten 2 Fortbil- 
dungen in diesem Zeitrahmen besucht hat. HdkF- und Nawi-Kitas sollten sich daher nicht in der Fortbildungsaktivität, sondern nur in der Teilnahme an der Initiative „Haus der kleinen Forscher“ unterscheiden. Daher erschien es zunächst sinnvoll, die Fortbildungshäufigkeit in den drei Untersuchungsgruppen zu vergleichen. Die Ergebnisse in Tabelle 13 zeigen, dass Fachkräfte aus HdkF-Kitas häufiger naturwissenschaftliche Fortbildungen in den letzten 3 Jahren besuchten als Fachkräfte der Nawi-Kitas ( $p$ <.001). Im Mittel besuchten Fachkräfte der HdkF-Gruppe 2.28 Fortbildungen in den letzten 3 Jahren, wohingegen Fachkräfte der Nawi-Kitas nur 1.31 Fortbildungen besuchten. In der Vergleichsgruppe wurden im Mittel nur etwa 0.21 Fortbildungen in den letzten 3 Jahren und nahezu keine Fortbildungen in den letzten 12 Monaten besucht. Wenn man berücksichtigt, dass die Zuordnung der Kitas zur Vergleichsgruppe nur dann erfolgte, wenn weniger als 2 Fortbildungen in den letzten 3 Jahren besucht wurden, ist dieser Befund nicht überraschend. Vielmehr verdeutlicht dies, dass die Einordnung der Vergleichsgruppe - also der Kitas ohne naturwissenschaftlichen Schwerpunkt - gelungen ist. Der signifikante Unterschied in der Fortbildungsaktivität der HdkF-Gruppe gegenüber der Nawi-Gruppe zeigt hingegen, dass hier die Vergleichbarkeit beider Gruppen eingeschränkt ist. Auf Basis dieses Befundes werden im Folgenden die Gruppenunterschiede auf Fachkraft- und Prozessebene jeweils unter Berücksichtigung der Fortbildungen in den letzten 3 Jahren berichtet, um einen fairen Vergleich der HdkF-Gruppe mit der Nawi-Gruppe zu ermöglichen. Anschließend werden die Ergebnisse von Fachkräften, die an mindestens 2 Fortbildungen innerhalb der letzten 3 Jahre teilgenommen hatten, mit den Fachkräften, die keine oder weniger naturwissenschaftliche Fortbildungsteilnahme angegeben hatten, verglichen. Dieses entspricht dem Vergleich der HdkF-Gruppe/Nawi-Gruppe mit der Vergleichsgruppe.

Tabelle 13. Fortbildungshäufigkeit nach Gruppen

\begin{tabular}{|c|c|c|c|c|c|c|c|c|c|c|c|c|c|}
\hline & \multicolumn{4}{|c|}{ HdkF-Gruppe } & \multicolumn{4}{|c|}{ Nawi-Gruppe } & \multicolumn{5}{|c|}{ Vergleichsgruppe } \\
\hline & $N$ & $M$ & $S D$ & Range & $N$ & $M$ & $S D$ & Range & $N$ & $M$ & $S D$ & Range & e $p$ \\
\hline $\begin{array}{l}\text { Anzahl Fort- } \\
\text { bildungen in } \\
\text { den letzten } \\
3 \text { Jahren }\end{array}$ & 114 & 2.28 & 2.17 & $0-10$ & 95 & 1.31 & 1.66 & $0-7$ & 103 & 0.21 & .44 & $0-2$ & .000 \\
\hline $\begin{array}{l}\text { Anzahl Fort- } \\
\text { bildungen in } \\
\text { den letzten } \\
12 \text { Monaten }\end{array}$ & 110 & 0.71 & .87 & $0-4$ & 88 & 0.52 & .79 & $0-3$ & 77 & 0.03 & .16 & $0-1$ & .000 \\
\hline
\end{tabular}

Anmerkung: Kontrolliert für Alter, Geschlecht, kognitive Fähigkeiten und Berufsabschluss. 


\subsubsection{Unterschiede in der Implementation naturwissenschaftlicher Bildung in der Kita}

Die Ergebnisse zur Implementation naturwissenschaftlicher Bildung in den drei Gruppen zeigen insgesamt, dass die Kitas der HdkF-Gruppe eine höhere Implementationsqualität laut des Gesamt-Indexes aufweisen als die Nawi-Gruppe bzw. die Vergleichsgruppe (siehe Tabelle 14). Die Differenz zwischen der HdkF-Gruppe zur Nawi-Gruppe und Vergleichsgruppe lässt sich vor allem auf die vergleichsweise stärkere Öffnung nach außen sowie die häufigere Thematisierung naturwissenschaftlicher Inhalte in Teamsitzungen zurückführen. Bei der Weitergabe naturwissenschaftlicher Fortbildungsinhalte ans Kollegium zeichnen sich hingegen keine signifikanten Unterschiede zwischen der HdkF und Nawi-Gruppe bzw. HdkF und Vergleichsgruppe ab, sondern lediglich zwischen Nawi-Gruppe und Vergleichsgruppe.

Tabelle 14. Implementation naturwissenschaftlicher Bildung nach Gruppen

\begin{tabular}{|c|c|c|c|c|c|c|c|c|c|c|}
\hline & \multicolumn{3}{|c|}{ HdkF-Gruppe } & \multicolumn{3}{|c|}{ Nawi-Gruppe } & \multicolumn{3}{|c|}{ Vergleichsgruppe } & \multirow[b]{2}{*}{$p$} \\
\hline & $N$ & M & $S D$ & $N$ & $M$ & $S D$ & $N$ & $M$ & $S D$ & \\
\hline $\begin{array}{l}\text { Weitergabe natur- } \\
\text { wissenschaftlicher } \\
\text { Fortbildungsinhalte }\end{array}$ & 52 & 0.16 & 0.93 & 36 & 0.28 & .85 & 10 & -0.80 & 1.03 & .023 \\
\hline $\begin{array}{l}\text { Teamsitzungen zu } \\
\text { naturwissenschaftli- } \\
\text { chen Themen }\end{array}$ & 102 & 0.28 & 1.10 & 84 & -0.13 & .96 & 90 & -0.22 & .88 & .012 \\
\hline Öffnung nach außen & 114 & 0.36 & 1.11 & 94 & -0.13 & .96 & 101 & -0.31 & .77 & .000 \\
\hline Gesamt-Index & 114 & 0.38 & 1.07 & 95 & -0.06 & .95 & 101 & -0.34 & .84 & .000 \\
\hline
\end{tabular}

Anmerkung: Kontrolliert für Alter, Geschlecht, kognitive Fähigkeiten, Berufsabschluss und Anzahl der Fortbildungen in den letzten 3 Jahren.

\subsubsection{Kompetenzunterschiede auf Fachkraftebene}

\section{Naturwissenschaftliche Kompetenzen der Fachkräfte:}

\section{Wissenskomponenten}

Die deskriptiven Ergebnisse der einzelnen Gruppen zu den Wissenskomponenten sind in Tabelle 15 dargestellt. Pädagogische Fachkräfte der HdkF-Gruppe lösten im Durchschnitt 52 Prozent der Aufgaben zum inhaltsbezogenen Fachwissen, 59 Prozent der Aufgaben zum prozessbezogenen Fachwissen und 48 Prozent der Aufgaben zum fachdidaktischen Wissen korrekt. Pädagogische Fachkräfte der Nawi-Gruppe lösten im Durchschnitt 52 Prozent der Aufgaben zum inhaltsbezogenen Fachwissen, 63 Prozent der Aufgaben zum prozessbezogenen Fachwissen und 50 Prozent der Aufgaben zum fachdidaktischen Wissen korrekt. Teilnehmen- 
de Fachkräfte aus der Vergleichsgruppe erreichten 51 Prozent der maximal erreichbaren Punktzahl bei den Aufgaben zum inhaltsbezogenen Fachwissen, 58 Prozent bei den Aufgaben zum prozessbezogenen Fachwissen und 48 Prozent bei den Aufgaben zum fachdidaktischen Wissen.

Die Ergebnisse zeigen keine signifikanten Unterschiede im inhalts- und prozessbezogenen

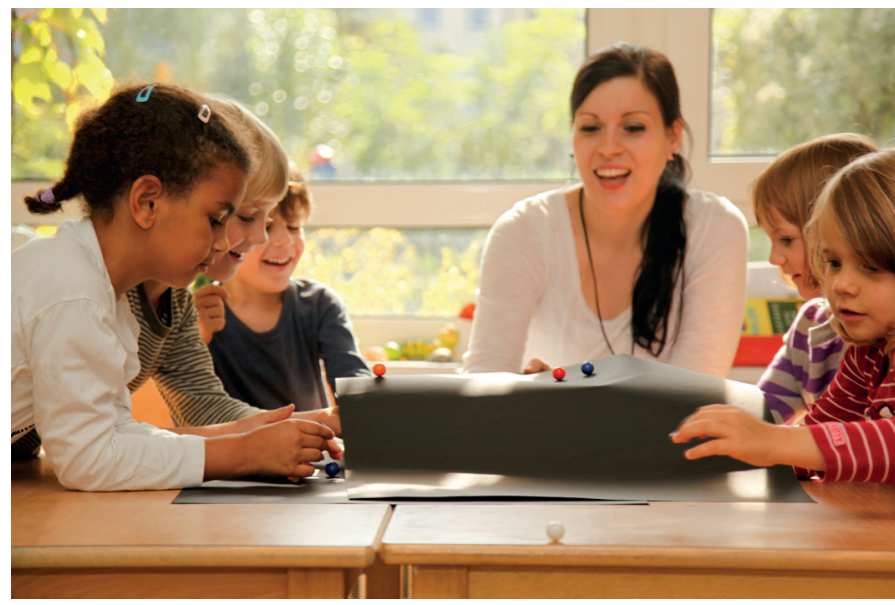
Fachwissen sowie im fachdidaktischen Wissen zwischen den frühpädagogischen Fachkräften aus den verschiedenen Gruppen. Das bedeutet, dass keine Gruppe besser in den Wissenstests abschneidet als eine andere Gruppe (siehe Tabelle 16).

Dies ist insofern erwartungsgemäß, da sich in den dargestellten Gruppen sowohl Fachkräfte mit als auch Fachkräfte ohne Fortbildung befinden und auf Wissensebene aufgrund der Ausrichtung der Fortbildungen nicht zwingend Implementationseffekte erwartet wurden. Effekte von Bildungsangeboten durch die Implementation naturwissenschaftlicher Inhalte in den Kitas und die Weitergabe von Fortbildungsinhalten im Team auf die Fachkräfte, die nicht an Fortbildungen teilgenommen haben, werden eher in Bezug auf motivationale Aspekte angenommen. 


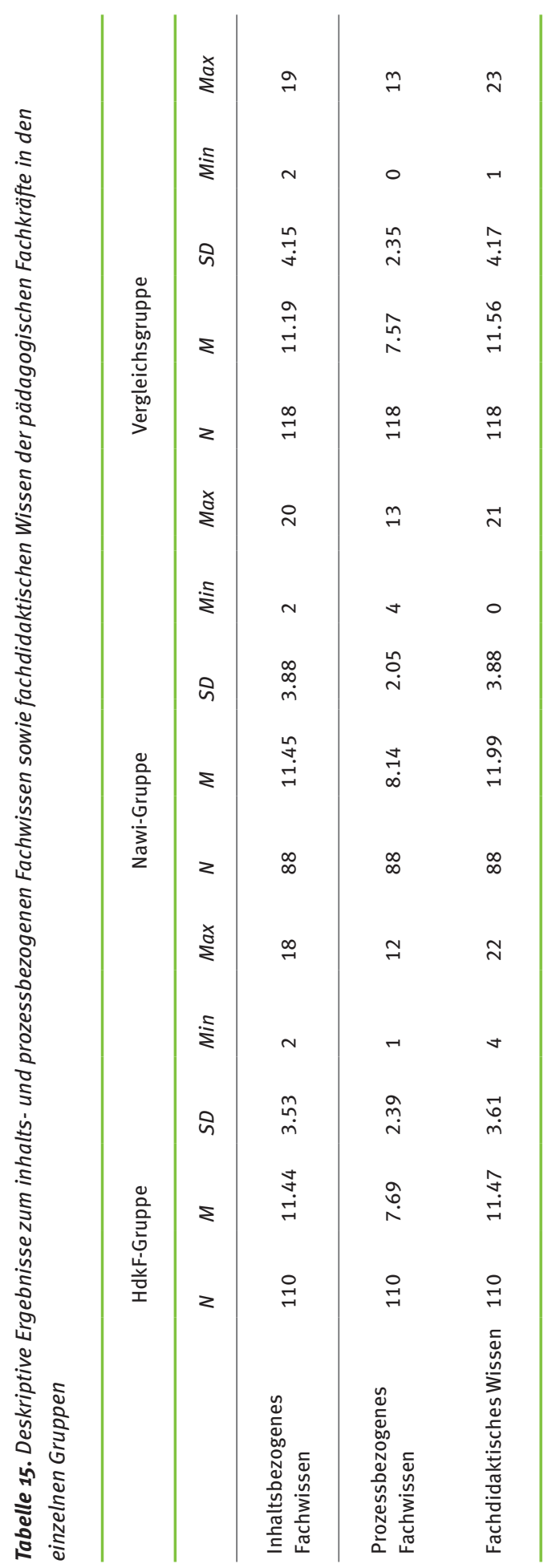


Tabelle 16. Gruppenunterschiede in den Wissenskomponenten

\begin{tabular}{lcccccccccccc}
\hline & \multicolumn{1}{c}{ HdkF-Gruppe } & \multicolumn{7}{c}{ Nawi-Gruppe } & \multicolumn{7}{c}{ Vergleichsgruppe } \\
& $N$ & $M$ & $S D$ & $N$ & $M$ & $S D$ & $N$ & $M$ & $S D$ & $p$ \\
\hline $\begin{array}{l}\text { Inhaltsbezogenes } \\
\text { Fachwissen }\end{array}$ & 105 & 11.48 & 3.58 & 87 & 11.41 & 3.89 & 103 & 11.59 & 4.03 & .120 \\
\hline $\begin{array}{l}\text { Prozessbezogenes } \\
\text { Fachwissen }\end{array}$ & 105 & 7.65 & 2.41 & 87 & 8.15 & 2.06 & 103 & 7.67 & 2.27 & .056 \\
\hline $\begin{array}{l}\text { Fachdidaktisches } \\
\text { Wissen }\end{array}$ & 105 & 11.50 & 3.63 & 87 & 12.00 & 3.90 & 103 & 11.81 & 4.20 & .116 \\
\hline
\end{tabular}

Anmerkung: Kontrolliert für Geschlecht, kognitive Fähigkeiten, Berufserfahrung, Berufsabschluss und Fortbildungsteilnahme in den letzten 3 Jahren.

Deswegen wurden in einem nächsten Schritt die Vergleichsanalysen ausschließlich für Fachkräfte vorgenommen, die an naturwissenschaftlichen Fortbildungsveranstaltungen teilgenommen haben. Vergleicht man hierbei die Fachkräfte mit mindestens 2 Fortbildungen in den letzten 3 Jahren mit den pädagogischen Fachkräften mit einer oder keiner naturwissenschaftlichen Fortbildung in den letzten 3 Jahren, so zeigen sich lediglich marginal signifikante Zusammenhänge im inhaltsbezogenen Fachwissen, aber keine signifikanten Unterschiede im prozessbezogenen Fachwissen sowie im fachdidaktischen Wissen der pädagogischen Fachkräfte (siehe Tabelle 17). Die pädagogischen Fachkräfte mit mindestens 2 Fortbildungsteilnahmen in den letzten 3 Jahren erzielten also keine signifikant höheren Leistungen in den drei Wissenstests (inhalts- und prozessbezogenes Fachwissen sowie fachdidaktisches Wissen) als die anderen pädagogischen Fachkräfte.

Tabelle 17. Unterschiede in den Wissenskomponenten nach Fortbildungsteilnahme in den letzten 3 Jahren

\begin{tabular}{llllllll}
\hline & \multicolumn{2}{l}{$\begin{array}{l}\text { Fachkräfte mit naturwissen- } \\
\text { schaftlichen Fortbildungen }\end{array}$} & \multicolumn{2}{l}{$\begin{array}{l}\text { Fachkräfte ohne naturwis- } \\
\text { senschaftliche Fortbildungen }\end{array}$} \\
& $N$ & $M$ & $S D$ & $N$ & $M$ & $S D$ & $p$ \\
\hline $\begin{array}{l}\text { Inhaltsbezogenes } \\
\text { Fachwissen }\end{array}$ & 97 & 11.97 & 3.58 & 198 & 11.27 & 3.92 & .057 \\
\hline $\begin{array}{l}\text { Prozessbezogenes } \\
\text { Fachwissen }\end{array}$ & 97 & 7.96 & 2.18 & 198 & 7.73 & 2.31 & .168 \\
\hline $\begin{array}{l}\text { Fachdidaktisches } \\
\text { Wissen }\end{array}$ & 97 & 11.89 & 3.62 & 198 & 11.70 & 4.05 & .350 \\
\hline
\end{tabular}

Anmerkung: Kontrolliert für Geschlecht, kognitive Fähigkeiten, Berufserfahrung und Berufsabschluss. 
Da basierend auf dem Forschungsstand zur Wirksamkeit von Fortbildungen bisherige empirische Befunde darauf hindeuten, dass oft keine langfristigen Effekte von kurz angelegten Fortbildungen auf das Wissen der Fachkräfte zu erwarten sind, wurden in einem nächsten Schritt Fachkräfte mit Fortbildungen in Naturwissenschaften im letzten Jahr mit Fachkräften ohne Fortbildungen verglichen (siehe Tabelle 18). Die Ergebnisse zeigen, dass fortgebildete Fachkräfte über ein signifikant höheres inhalts- und prozessbezogenes Fachwissen verfügen als nicht fortgebildete Fachkräfte. Jedoch liegen keine signifikanten Unterschiede im fachdidaktischen Wissen vor.

Tabelle 18. Unterschiede in den Wissenskomponenten nach Fortbildungsteilnahme in den letzten 12 Monaten

\begin{tabular}{llllllll}
\hline & \multicolumn{2}{l}{$\begin{array}{l}\text { Fachkräfte mit naturwissen- } \\
\text { schaftlichen Fortbildungen }\end{array}$} & \multicolumn{2}{l}{$\begin{array}{l}\text { Fachkräfte ohne naturwis- } \\
\text { senschaftliche Fortbildungen }\end{array}$} \\
& $N$ & $M$ & $S D$ & $N$ & $M$ & $S D$ & $p$ \\
\hline $\begin{array}{l}\text { Inhaltsbezogenes } \\
\text { Fachwissen }\end{array}$ & 85 & 12.36 & 3.44 & 172 & 10.99 & 3.89 & .001 \\
\hline $\begin{array}{l}\text { Prozessbezogenes } \\
\text { Fachwissen }\end{array}$ & 85 & 8.08 & 2.08 & 172 & 7.56 & 2.37 & .023 \\
\hline $\begin{array}{l}\text { Fachdidaktisches } \\
\text { Wissen }\end{array}$ & 85 & 11.91 & 3.66 & 172 & 11.42 & 3.88 & .107 \\
\hline
\end{tabular}

Anmerkung: Kontrolliert für Geschlecht, kognitive Fähigkeiten, Berufserfahrung und Berufsabschluss.

Lediglich bei der zusätzlichen Kontrolle möglicher Lerngelegenheiten im Bereich Naturwissenschaften (Seminare zu naturwissenschaftlichen Themen in der beruflichen Ausbildung bzw. Studium) zeigen sich marginal signifikante Unterschiede im fachdidaktischen Wissen zugunsten der Fachkräfte mit Fortbildungen (siehe Tabelle 19). 
Tabelle 19. Unterschiede in den Wissenskomponenten nach Fortbildungsteilnahme in den letzten 12 Monaten

\begin{tabular}{llllllll}
\hline & \multicolumn{2}{l}{$\begin{array}{l}\text { Fachkräfte mit naturwissen- } \\
\text { schaftlichen Fortbildungen }\end{array}$} & $\begin{array}{l}\text { Fachkräfte ohne naturwis- } \\
\text { senschaftliche Fortbildungen }\end{array}$ \\
& $N$ & $M$ & $S D$ & $N$ & $M$ & $S D$ & $p$ \\
\hline $\begin{array}{l}\text { Inhaltsbezogenes } \\
\text { Fachwissen }\end{array}$ & 85 & 12.36 & 3.44 & 170 & 10.98 & 3.89 & .001 \\
\hline $\begin{array}{l}\text { Prozessbezogenes } \\
\text { Fachwissen }\end{array}$ & 85 & 8.08 & 2.08 & 170 & 7.57 & 2.38 & .023 \\
\hline $\begin{array}{l}\text { Fachdidaktisches } \\
\text { Wissen }\end{array}$ & 85 & 11.91 & 3.66 & 170 & 11.39 & 3.89 & .092 \\
\hline
\end{tabular}

Anmerkung: Kontrolliert für Geschlecht, kognitive Fähigkeiten, Berufserfahrung, Berufsabschluss und Seminare zu naturwissenschaftlichen Themen in der beruflichen Ausbildung/im Studium.

\section{Naturwissenschaftliche Kompetenzen der Fachkräfte:}

\section{Motivationale Komponente}

Analog zu den Ergebnissen der Wissenskomponenten ergeben sich, unter Kontrolle der Anzahl der Fortbildungen, keine signifikanten Unterschiede in der Motivation der Fachkräfte zwischen den drei Untersuchungsgruppen (siehe Tabelle 20).

Tabelle 20. Gruppenunterschiede in den motivationalen Kompetenzfacetten

\begin{tabular}{lccccccccccc}
\hline & \multicolumn{1}{c}{ HdkF-Gruppe } & \multicolumn{7}{c}{ Nawi-Gruppe } & \multicolumn{7}{c}{ Vergleichsgruppe } \\
& $N$ & $M$ & $S D$ & $N$ & $M$ & $S D$ & $N$ & $M$ & $S D$ & $p$ \\
\hline Selbstkonzept & 105 & 2.89 & 0.62 & 85 & 2.75 & 0.59 & 98 & 2.77 & 0.55 & .460 \\
\hline Selbstwirksamkeit & 105 & 3.10 & 0.51 & 87 & 3.04 & 0.45 & 102 & 2.97 & 0.44 & .946 \\
\hline Interesse & 105 & 3.15 & 0.60 & 87 & 3.05 & 0.58 & 101 & 2.98 & 0.53 & .865 \\
\hline Enthusiasmus & 105 & 3.39 & 0.49 & 87 & 3.36 & 0.51 & 100 & 3.18 & 0.48 & .325 \\
\hline
\end{tabular}

Anmerkung: Kontrolliert für Geschlecht, kognitive Fähigkeiten, Berufserfahrung, Berufsabschluss und Anzahl der Fortbildungen in den letzten 3 Jahren.

Die Gegenüberstellung von Fachkräften mit und ohne Fortbildungen zeigt hingegen deutliche Unterschiede in allen vier Skalen: Fachkräfte, die in den letzten 3 Jahren mindestens 2 naturwissenschaftliche Fortbildungen belegt hatten, berichten signifikant höhere Fähigkeitsüberzeugungen (Selbstkonzept, Selbstwirksamkeitserwartung) sowie mehr Interesse an den Naturwissenschaften und mehr Enthusiasmus bei der Vermittlung naturwissenschaftlicher Inhalte (siehe Tabelle 21). 
Tabelle 21. Unterschiede in den motivationalen Komponenten nach Fortbildungsteilnahme in den letzten 3 Jahren

\begin{tabular}{lllllllll}
\hline & \multicolumn{7}{l}{ Fachkräfte mit Fortbildung } & \multicolumn{5}{l}{ Fachkräfte ohne Fortbildung } \\
& $N$ & $M$ & $S D$ & $N$ & $M$ & $S D$ & $p$ \\
\hline Selbstkonzept & 97 & 2.97 & 0.60 & 191 & 2.73 & 0.57 & .001 \\
\hline Selbstwirksamkeit & 97 & 3.18 & 0.47 & 197 & 2.97 & 0.46 & .001 \\
\hline Interesse & 97 & 3.23 & 0.56 & 196 & 2.98 & 0.56 & .000 \\
\hline Enthusiasmus & 97 & 3.51 & 0.42 & 195 & 3.21 & 0.51 & .000 \\
\hline
\end{tabular}

Anmerkung: Kontrolliert für Geschlecht, kognitive Fähigkeiten, Berufserfahrung und Berufsabschluss.

\section{Naturwissenschaftliche Kompetenzen der Fachkräfte: \\ Lerntheoretische Überzeugungen}

Der Vergleich der lerntheoretischen Überzeugungen der Fachkräfte nach Gruppen zeigt keine signifikanten Unterschiede (siehe Tabelle 22).

Tabelle 22. Gruppenunterschiede in den lerntheoretischen Überzeugungen

\begin{tabular}{lccccccccccc}
\hline & \multicolumn{1}{c}{ HdkF-Gruppe } & \multicolumn{5}{c}{ Nawi-Gruppe } & \multicolumn{7}{c}{ Vergleichsgruppe } \\
& $N$ & $M$ & $S D$ & $N$ & $M$ & $S D$ & $N$ & $M$ & $S D$ & $p$ \\
\hline $\begin{array}{l}\text { Programmatischer } \\
\text { Ansatz }\end{array}$ & 104 & 1.95 & 0.44 & 86 & 2.04 & 0.41 & 101 & 2.06 & 0.46 & .953 \\
\hline $\begin{array}{l}\text { Kindzentrierter } \\
\text { Ansatz }\end{array}$ & 104 & 3.06 & 0.41 & 86 & 3.04 & 0.40 & 101 & 3.05 & 0.41 & .525 \\
\hline $\begin{array}{l}\text { Open-Frame- } \\
\text { work-Ansatz }\end{array}$ & 104 & 3.47 & 0.35 & 86 & 3.46 & 0.38 & 101 & 3.37 & 0.37 & .247 \\
\hline
\end{tabular}

Anmerkung: Kontrolliert für Geschlecht, kognitive Fähigkeiten, Berufsabschluss, Berufserfahrung und Anzahl der Fortbildungen in den letzten 3 Jahren.

Vergleicht man Fachkräfte nach Fortbildungen in den letzten 3 Jahren, so zeigen sich signifikante Unterschiede in den Überzeugungen: Fachkräfte, die in den letzten 3 Jahren mindestens 2 Fortbildungen besuchten, lehnen den programmatischen Ansatz eher ab (siehe Tabelle 23) und befürworten eher kindzentrierte und Open-Framework-Ansätze als Fachkräfte mit einer oder keiner Fortbildung in den letzten 3 Jahren. 
Tabelle 23. Unterschiede in den lerntheoretischen Überzeugungen nach Fortbildungsteilnahme in den letzten 3 Jahren

\begin{tabular}{|c|c|c|c|c|c|c|c|}
\hline & \multicolumn{3}{|c|}{ Fachkräfte mit Fortbildung } & \multicolumn{4}{|c|}{ Fachkräfte ohne Fortbildung } \\
\hline & $N$ & $M$ & $S D$ & $N$ & $M$ & $S D$ & $p$ \\
\hline $\begin{array}{l}\text { Programmatischer } \\
\text { Ansatz }\end{array}$ & 96 & 1.94 & 0.48 & 195 & 2.05 & 0.42 & .019 \\
\hline $\begin{array}{l}\text { Kindzentrierter } \\
\text { Ansatz }\end{array}$ & 96 & 3.12 & 0.42 & 195 & 3.02 & 0.39 & .041 \\
\hline $\begin{array}{l}\text { Open-Framework- } \\
\text { Ansatz }\end{array}$ & 96 & 3.49 & 0.35 & 195 & 3.40 & 0.37 & .049 \\
\hline
\end{tabular}

Anmerkung: Kontrolliert für Geschlecht, kognitive Fähigkeiten, Berufserfahrung und Berufsabschluss.

\subsubsection{Unterschiede in Qualität und Quantität naturwissenschaftlicher Lerngelegenheiten}

Der Vergleich der Qualität und Quantität früher naturwissenschaftlicher Bildung nach Gruppen zeigt keine signifikanten Unterschiede: Fachkräfte in der HdkF-Gruppe bieten naturwissenschaftliche Lerngelegenheiten nicht signifikant häufiger an als Fachkräfte in der Nawi-Gruppe bzw. der Vergleichsgruppe, und die angebotenen Lerngelegenheiten sind zudem nicht von höherer Qualität (siehe Tabelle 24).

Tabelle 24. Gruppenunterschiede in der Qualität und Quantität naturwissenschaftlicher Bildung

\begin{tabular}{lccccccccccc}
\hline & \multicolumn{1}{c}{ HdkF-Gruppe } & \multicolumn{5}{c}{ Nawi-Gruppe } & \multicolumn{7}{c}{ Vergleichsgruppe } \\
& $N$ & $M$ & $S D$ & $N$ & $M$ & $S D$ & $N$ & $M$ & $S D$ & $p$ \\
\hline $\begin{array}{l}\text { Index } \\
\begin{array}{l}\text { Prozess- } \\
\text { qualität }\end{array}\end{array}$ & 69 & 0.09 & 0.66 & 47 & 0.04 & 0.56 & 52 & -0.24 & 0.67 & .451 \\
\hline Häufigkeit & 106 & 2.23 & 1.24 & 92 & 1.95 & 1.27 & 96 & 1.95 & 1.43 & .729 \\
\hline
\end{tabular}

Anmerkung: Kontrolliert für Alter, Geschlecht, kognitive Fähigkeiten, Berufsabschluss und Anzahl der Fortbildungen in den letzten 3 Jahren.

Bei der Gegenüberstellung der Qualität und Quantität naturwissenschaftlicher Bildungsprozesse nach Fortbildungsteilnahme zeigen sich Unterschiede: Fachkräfte mit mindestens 2 Fortbildungen in den letzten 3 Jahren bieten im Mittel mehr und auch qualitativ hochwertigere naturwissenschaftliche Lerngelegenheiten an als Fachkräfte mit weniger oder gar keinen Fortbildungen (siehe Tabelle 25). 
Tabelle 25. Unterschiede in der Qualität und Quantität naturwissenschaftlicher Bildung nach Fortbildungsteilnahme

\begin{tabular}{llllllll}
\hline & \multicolumn{3}{l}{ Fachkräfte mit Fortbildung } & \multicolumn{5}{l}{ Fachkräfte ohne Fortbildung } \\
& $N$ & $M$ & $S D$ & $N$ & $M$ & $S D$ & $p$ \\
\hline Index Prozessqualität & 66 & 0.20 & 0.60 & 106 & -0.14 & 0.65 & .002 \\
\hline Häufigkeit & 96 & 2.34 & 1.19 & 203 & 1.90 & 1.34 & .007 \\
\hline
\end{tabular}

Anmerkung: Kontrolliert für Alter, Geschlecht, kognitive Fähigkeiten und Berufsabschluss.

\subsubsection{Kompetenzunterschiede auf Kinderebene}

\section{Naturwissenschaftliche Kompetenzen der Kinder:}

Konzeptbezogenes und prozessbezogenes Wissen

Mögliche Gruppenunterschiede nach der Zugehörigkeit zu einer Gruppe werden anlehnend an die Strukturanalysen sowohl für die Gesamtskala der naturwissenschaftlichen Kompetenzen (alle Items des Prozess- und Konzeptwissens) und getrennt für die Unterskalen in den Bereichen Prozesswissen, Material, Magnetismus, Schwimmen und Sinken sowie Aggregatzustände abgebildet. Es zeigen sich keine signifikanten Unterschiede in den Mittelwerten der Kinder aus den drei Untersuchungsgruppen unter Kontrolle der individuellen Merkmale im Wortschatz, der kognitiven Fähigkeiten, der zuhause gesprochenen Sprache, des Alters und des Geschlechts. Die Kinder aller Gruppen schneiden somit in allen Wissenstests zu naturwissenschaftlichen Kompetenzen ähnlich gut ab (siehe Tabelle 26).

Tabelle 26. Gruppenunterschiede im konzeptbezogenen und prozessbezogenen Wissen

\begin{tabular}{lcccccccccccc}
\hline & \multicolumn{1}{c}{ HdkF-Gruppe } & \multicolumn{3}{c}{ Nawi-Gruppe } & \multicolumn{7}{c}{ Vergleichsgruppe } \\
& $N$ & $M$ & $S D$ & $N$ & $M$ & $S D$ & $N$ & $M$ & $S D$ & $p$ \\
\hline $\begin{array}{l}\text { Gesamtskala } \\
\text { Wissen }\end{array}$ & 101 & 66.09 & 17.60 & 61 & 69.10 & 18.41 & 84 & 67.95 & 17.20 & .331 \\
\hline Prozesswissen & 101 & 22.39 & 6.75 & 61 & 22.44 & 5.48 & 84 & 22.71 & 6.97 & .369 \\
\hline Material & 101 & 12.60 & 4.40 & 61 & 12.47 & 5.36 & 84 & 13.76 & 4.00 & .060 \\
\hline Magnetismus & 101 & 12.69 & 4.45 & 61 & 13.79 & 4.94 & 84 & 13.10 & 4.28 & .562 \\
\hline $\begin{array}{l}\text { Schwimmen und } \\
\text { Sinken }\end{array}$ & 101 & 11.56 & 5.76 & 61 & 12.39 & 5.47 & 84 & 10.61 & 4.82 & .294 \\
\hline Aggregatzustände & 101 & 10.00 & 3.58 & 61 & 11.21 & 4.00 & 84 & 10.60 & 3.71 & .227 \\
\hline
\end{tabular}

Anmerkung: Kontrolliert für Alter, Geschlecht, Sprache zuhause, CFT-, KABC- und PPVT-Score.

Darüber hinaus wurde untersucht, ob sich Kinder, die vorwiegend von Fachkräften mit mindestens 2 oder ohne Fortbildungen in den letzten 3 Jahren betreut werden, 
in ihrem Wissen unterscheiden. Aus Tabelle 27 wird ersichtlich, dass sich keine signifikanten Wissensunterschiede feststellen lassen.

Tabelle 27. Unterschiede im konzeptbezogenen und prozessbezogenen Wissen nach Fortbildungsteilnahme der betreuenden Fachkräfte

\begin{tabular}{llllllll}
\hline & \multicolumn{2}{l}{$\begin{array}{l}\text { Kinder betreut von Fach- } \\
\text { kräften mit Fortbildung }\end{array}$} & \multicolumn{4}{l}{$\begin{array}{l}\text { Kinder betreut von Fach- } \\
\text { kräften ohne Fortbildung }\end{array}$} \\
\hline & $N$ & $M$ & $S D$ & $N$ & $M$ & $S D$ & $p$ \\
\hline Gesamtskala Wissen & 87 & 68.56 & 18.03 & 110 & 67.78 & 16.56 & .184 \\
\hline Prozesswissen & 87 & 22.78 & 7.30 & 110 & 21.62 & 7.05 & .159 \\
\hline Material & 87 & 12.80 & 4.78 & 110 & 13.04 & 4.23 & .639 \\
\hline Magnetismus & 87 & 13.24 & 4.77 & 110 & 13.43 & 4.29 & .381 \\
\hline Schwimmen und Sinken & 87 & 9.02 & 2.87 & 110 & 9.07 & 3.10 & .799 \\
\hline Aggregatzustände & 87 & 10.54 & 3.74 & 110 & 10.63 & 3.74 & .431 \\
\hline
\end{tabular}

Anmerkung: Kontrolliert für Alter, Geschlecht, Sprache zuhause, CFT-, KABC- und PPVT-Score auf Kinderebene sowie Alter, Geschlecht, kognitive Fähigkeiten und Berufsabschluss auf Fachkraftebene.

\section{Naturwissenschaftliche Kompetenzen der Kinder:}

\section{Motivationale Komponente}

Auch bei den motivationalen Aspekten naturwissenschaftlicher Kompetenzen zeigen sich keine signifikanten Gruppenunterschiede: Kinder in allen drei Untersuchungsgruppen sind ähnlich motiviert in den Naturwissenschaften (siehe Tabelle 28).

Tabelle 28. Gruppenunterschiede in den motivationalen Kompetenzkomponenten

\begin{tabular}{lcccccccccccc}
\hline & \multicolumn{3}{c}{ HdkF-Gruppe } & \multicolumn{7}{c}{ Nawi-Gruppe } & \multicolumn{7}{c}{ Vergleichsgruppe } \\
& $N$ & $M$ & $S D$ & $N$ & $M$ & $S D$ & $N$ & $M$ & $S D$ & $p$ \\
\hline Selbstwirksamkeit & 102 & 3.03 & 0.70 & 61 & 2.96 & 0.62 & 84 & 2.99 & 0.66 & .747 \\
\hline Lernfreude & 102 & 3.55 & 0.69 & 61 & 3.44 & 0.52 & 84 & 3.52 & 0.57 & .302 \\
\hline
\end{tabular}

Anmerkung: Kontrolliert für Alter, Geschlecht, Sprache zuhause, CFT-, KABC- und PPVT-Score.

Bei der Gegenüberstellung von Kindern, die von Fachkräften mit mindestens 2 oder ohne Fortbildungen in den letzten 3 Jahren betreut werden, zeigen sich keine signifikanten Unterschiede in der Selbstwirksamkeitserwartung oder der Lernfreude der Kinder (siehe Tabelle 29). 
Tabelle 29. Unterschiede in den motivationalen Kompetenzkomponenten nach Fortbildungsteilnahme der betreuenden Fachkräfte

\begin{tabular}{lllllllll}
\hline & \multicolumn{3}{l}{$\begin{array}{l}\text { Kinder betreut von } \\
\text { Fachkräften mit Fortbildung }\end{array}$} & \multicolumn{4}{l}{$\begin{array}{l}\text { kinder betreut von Fach- } \\
\text { kräften }\end{array}$} \\
& $N$ & $M$ & $S D$ & $N$ & $M$ & $S D$ & $p$ \\
\hline Selbstwirksamkeit & 87 & 3.07 & 0.69 & 110 & 2.94 & 0.66 & .292 \\
\hline Lernfreude & 87 & 3.52 & 0.69 & 110 & 3.51 & 0.54 & .567 \\
\hline
\end{tabular}

Anmerkung: Kontrolliert für Alter, Geschlecht, Sprache zuhause, CFT-, KABC- und PPVT-Score auf Kinderebene sowie Alter, Geschlecht, kognitive Fähigkeiten und Berufsabschluss auf Fachkraftebene.

\subsection{Zusammenhänge zwischen den Wirkungsebenen}

Die dritte Fragestellung der Studie zielte auf die Zusammenhänge zwischen den Wirkungsebenen ab. Auf Grundlage des Wirkungsmodells in Kapitel 2.1 wird angenommen, dass die Implementation der Naturwissenschaften als Bildungsbereich sich positiv auf die Kompetenzen der Fachkräfte auswirkt (5.3.1), welche wiederum zentral für die Qualität und Häufigkeit der Umsetzung naturwissenschaftlicher Bildung sind (5.3.2). Darüber hinaus ist auch denkbar, dass die Implementationsqualität die Qualität und Häufigkeit naturwissenschaftlicher Bildungsprozesse direkt beeinflusst (5.3.3). Die Qualität und Häufigkeit naturwissenschaftlicher Bildungsprozesse werden wiederum als zentral für die kindliche Kompetenzentwicklung angenommen (5.3.4).

\subsubsection{Zusammenhänge zwischen der Implementationsqualität und den naturwissenschaftlichen Kompetenzen der Fachkräfte}

Die Ergebnisse der Regressionsanalysen zum Einfluss der Implementation naturwissenschaftlicher Bildung in den Kitas auf die unterschiedlichen Wissensfacetten der Fachkräfte sind in Tabelle 30 dargestellt. Die Ergebnisse zeigen, dass der Grad der Implementation naturwissenschaftlicher Bildung in den Kitas nicht prädiktiv für das inhalts- und prozessbezogene Fachwissen sowie das fachdidaktische Wissen ist.

Tabelle 30. Ergebnisse der Regressionsanalysen zum Einfluss des Implementationsindexes auf das Wissen der Fachkräfte

\begin{tabular}{lccc}
\hline Prädiktor: Implementation & Beta & $S E$ & $p$ \\
\hline Inhaltsbezogenes Fachwissen & -.02 & .05 & .662 \\
\hline Prozessbezogenes Fachwissen & -.07 & .06 & .239 \\
\hline Fachdidaktisches Wissen & -.05 & .06 & .435 \\
\hline
\end{tabular}

Anmerkung: N = 358. Kontrolliert für Alter, Geschlecht, kognitive Fähigkeiten, Berufsabschluss und Fortbildungsteilnahme in den letzten 3 Jahren. 
Die Ergebnisse der Regressionsanalysen zum Einfluss der Implementation naturwissenschaftlicher Bildung in der Einrichtung auf die unterschiedlichen Aspekte der Motivation der Fachkräfte sind in Tabelle 31 zusammengefasst. Es wird ersichtlich, dass der Grad der Implementation naturwissenschaftlicher Bildung in der Einrichtung im Zusammenhang mit dem allgemeinen Interesse und dem allgemeinen Selbstkonzept in den Naturwissenschaften steht.

Tabelle 31. Ergebnisse der Regressionsanalysen zum Einfluss des Implementationsindexes auf die Motivation der Fachkräfte

\begin{tabular}{lccc}
\hline $\begin{array}{l}\text { Prädiktor: } \\
\text { Implementation auf Kita-Ebene }\end{array}$ & Beta & SE & $p$ \\
\hline Selbstkonzept & .12 & .06 & .020 \\
\hline Selbstwirksamkeit & .09 & .06 & .141 \\
\hline Interesse & .17 & .05 & .001 \\
\hline Enthusiasmus & .09 & .06 & .149 \\
\hline
\end{tabular}

Anmerkung: $\mathrm{N}=358$. Kontrolliert für Alter, Geschlecht, kognitive Fähigkeiten, Berufsabschluss und Fortbildungsteilnahme in den letzten 3 Jahren.

Die Ergebnisse zum Einfluss der Implementation auf die lerntheoretischen Überzeugungen zeigen keine signifikanten Zusammenhänge (siehe Tabelle 32). Der Grad der Implementation naturwissenschaftlicher Bildung in der Einrichtung steht demnach nicht mit den lerntheoretischen Überzeugungen der Fachkräfte in den Naturwissenschaften im Zusammenhang.

Tabelle 32. Ergebnisse der Regressionsanalysen zum Einfluss des Implementationsindexes auf die lerntheoretischen Überzeugungen der Fachkräfte

\begin{tabular}{llll}
\hline $\begin{array}{l}\text { Prädiktor: } \\
\text { Implementation auf Kita-Ebene }\end{array}$ & Beta & $S E$ & $p$ \\
\hline Programmatischer Ansatz & .02 & .05 & .732 \\
\hline Kindzentrierter Ansatz & -.05 & .08 & .545 \\
\hline Open-Framework-Ansatz & .04 & .05 & .374 \\
\hline
\end{tabular}

Anmerkung: $\mathrm{N}=358$. Kontrolliert für Alter, Geschlecht, kognitive Fähigkeiten, Berufsabschluss und Fortbildungsteilnahme in den letzten 3 Jahren. 


\subsubsection{Zusammenhänge zwischen den naturwissenschaftlichen Kompetenzen der Fachkräfte und Prozessmerkmalen}

\section{Wissenskomponenten}

Die Ergebnisse der Regressionsanalysen zur Vorhersage der Prozessqualität der naturwissenschaftlichen Lerngelegenheiten durch das naturwissenschaftliche Wissen der pädagogischen Fachkräfte sind in Tabelle 33 dargestellt. Die Ergebnisse zeigen, dass das naturwissenschaftliche Fachwissen der Fachkräfte mit der Prozessqualität der Lerngelegenheiten zusammenhängt. Je mehr inhalts- und prozessbezogenes Fachwissen die pädagogischen Fachkräfte haben, desto höher ist auch die Prozessqualität.

Tabelle 33. Ergebnisse der Regressionsanalysen zum Einfluss des Wissens der Fachkräfte auf die Prozessqualität der Lerngelegenheiten

\begin{tabular}{lccc}
\hline & Beta & SE & $p$ \\
\hline Inhaltsbezogenes Fachwissen & .45 & .06 & .000 \\
\hline Prozessbezogenes Fachwissen & .54 & .06 & .000 \\
\hline Fachdidaktisches Wissen & -.01 & .09 & .901 \\
\hline
\end{tabular}

Anmerkung: N = 298. Kontrolliert für Alter, Geschlecht, kognitive Fähigkeiten und Berufsabschluss.

Die Ergebnisse der Regressionsanalysen zur Vorhersage der Quantität der naturwissenschaftlichen Lerngelegenheiten durch das naturwissenschaftliche Wissen der pädagogischen Fachkräfte sind in Tabelle 34 dargestellt. Die Ergebnisse zeigen weiterhin, dass weder das fachdidaktische Wissen noch das inhalts- oder prozessbezogene Fachwissen der pädagogischen Fachkräfte prädiktiv für die Häufigkeit der angebotenen Lerngelegenheiten sind. ${ }^{17}$

Tabelle 34. Ergebnisse der Regressionsanalysen zum Einfluss des Wissens der Fachkräfte auf die Häufigkeit der Lerngelegenheiten

\begin{tabular}{lccc}
\hline & Beta & SE & $p$ \\
\hline Inhaltsbezogenes Fachwissen & .03 & .06 & .599 \\
\hline Prozessbezogenes Fachwissen &. .03 & .02 & .065 \\
\hline Fachdidaktisches Wissen & .08 & .07 & .240 \\
\hline
\end{tabular}

Anmerkung: $\mathrm{N}=$ 298. Kontrolliert für Alter, Geschlecht, kognitive Fähigkeiten und Berufsabschluss.

17 In ersten Analysen zeigte sich für Fachkräfte, die mindestens 3 Fortbildungen in den letzten 3 Jahren besuchten, ein Zusammenhang zwischen fachdidaktischem Wissen und Häufigkeit der angebotenen Lerngelegenheiten. 


\section{Motivationale Komponenten}

Die Ergebnisse der Regressionsanalysen zur Vorhersage der Prozessqualität naturwissenschaftlicher Lerngelegenheiten durch die Motivation der Fachkräfte sind in Tabelle 35 zusammengefasst. Es zeigt sich, dass alle vier Aspekte der Motivation einen signifikanten positiven Einfluss auf die Prozessqualität der angebotenen Lerngelegenheiten haben. Die Fähigkeitsüberzeugungen der Fachkräfte in den Naturwissenschaften (Selbstkonzept und Selbstwirksamkeitserwartung) haben den stärksten Einfluss, gefolgt vom Enthusiasmus der Fachkräfte. Je sicherer sich die Fachkräfte in den Naturwissenschaften sowie der Vermittlung naturwissenschaftlicher Inhalte fühlen und je mehr Freude sie an der Vermittlung haben, desto höher ist die Prozessqualität der Lerngelegenheiten. Das allgemeine Interesse an den Naturwissenschaften hat einen etwas geringeren Einfluss auf die Prozessqualität.

Tabelle 35. Ergebnisse der Regressionsanalysen zum Einfluss der Motivation der Fachkräfte auf die Prozessqualität naturwissenschaftlicher Lerngelegenheiten

\begin{tabular}{lccc}
\hline & Beta & SE & $p$ \\
\hline Selbstkonzept & .27 & .07 & .000 \\
\hline Selbstwirksamkeit & .27 & .07 & .000 \\
\hline Interesse & .18 & .07 & .000 \\
\hline Enthusiasmus & .23 & .07 & .000 \\
\hline
\end{tabular}

Anmerkung: $\mathrm{N}=358$. Kontrolliert für Alter, Geschlecht, kognitive Fähigkeiten und Berufsabschluss.

Auch mit Blick auf die Häufigkeit früher naturwissenschaftlicher Lernangebote zeigt sich ein positiver Zusammenhang mit der Motivation pädagogischer Fachkräfte. Vergleicht man die Beta-Gewichte, so zeigt sich, dass die Selbstwirksamkeitserwartung sowie der Enthusiasmus in Bezug auf die Vermittlung naturwissenschaftlicher Inhalte einen etwas stärkeren Einfluss auf die Häufigkeit der Lerngelegenheiten haben als das allgemeine naturwissenschaftliche Selbstkonzept und Interesse (siehe Tabelle 36).

Tabelle 36. Ergebnisse der Regressionsanalysen zum Einfluss der Motivation der Fachkräfte auf die Häufigkeit naturwissenschaftlicher Lerngelegenheiten

\begin{tabular}{lccc}
\hline & Beta & SE & $p$ \\
\hline Selbstkonzept & .23 & .06 & .000 \\
\hline Selbstwirksamkeit & .28 & .06 & .000 \\
\hline Interesse & .24 & .06 & .000 \\
\hline Enthusiasmus & .31 & .06 & .000 \\
\hline
\end{tabular}

Anmerkung: $\mathrm{N}=358$. Kontrolliert für Alter, Geschlecht, kognitive Fähigkeiten und Berufsabschluss 


\section{Lerntheoretische Überzeugungen}

Mit Blick auf die lerntheoretischen Überzeugungen zeigt sich, dass Überzeugungen im Sinne des Kind-orientierten Ansatzes einen positiven Einfluss auf die Prozessqualität naturwissenschaftlicher Lerngelegenheiten haben, wohingegen Überzeugungen im Sinne des programmatischen Ansatzes einen negativen Einfluss haben (siehe Tabelle 37). Die Überzeugungen im Sinne des Open-Framework-Ansatzes verfehlen in ihrem Zusammenhang mit der Prozessqualität knapp die Signifikanzgrenze.

Tabelle 37. Ergebnisse der Regressionsanalysen zum Einfluss der lerntheoretischen Überzeugungen der Fachkräfte auf die Prozessqualität naturwissenschaftlicher Lerngelegenheiten

\begin{tabular}{lccc}
\hline & Beta & $S E$ & $p$ \\
\hline Programmatischer Ansatz & -.25 & .07 & .001 \\
\hline Kindzentrierter Ansatz & .15 & .08 & .048 \\
\hline Open-Framework-Ansatz & .14 & .07 & .055 \\
\hline
\end{tabular}

Anmerkung: N = 358. Kontrolliert für Alter, Geschlecht, kognitive Fähigkeiten und Berufsabschluss

In Bezug auf die Häufigkeit früher naturwissenschaftlicher Lerngelegenheiten zeichnet sich ein etwas anderes Bild ab: Hier hat der Open-Framework-Ansatz einen signifikanten positiven Einfluss, wohingegen der Zusammenhang des kindzentrierten Ansatzes mit der Häufigkeit der Lerngelegenheiten nicht mehr signifikant ist (siehe Tabelle 38). Der programmatische Ansatz steht in einem negativen Zusammenhang mit der Häufigkeit.

Tabelle 38. Ergebnisse der Regressionsanalysen zum Einfluss der lerntheoretischen Überzeugungen der Fachkräfte auf die Häufigkeit naturwissenschaftlicher Lerngelegenheiten

\begin{tabular}{lccl}
\hline & Beta & $S E$ & $p$ \\
\hline Programmatischer Ansatz & -.15 & .06 & .022 \\
\hline Kindzentrierter Ansatz & .11 & .06 & .062 \\
\hline Open-Framework-Ansatz & .14 & .05 & .007 \\
\hline
\end{tabular}

Anmerkung: $\mathrm{N}=358$. Kontrolliert für Alter, Geschlecht, kognitive Fähigkeiten und Berufsabschluss. 


\subsubsection{Zusammenhänge zwischen der Implementationsqualität und den Prozessmerkmalen}

Neben den Analysen zu den indirekten Zusammenhängen zwischen der Implementationsqualität und den Prozessmerkmalen, vermittelt über die Kompetenzen der Fachkräfte, waren auch die direkten Zusammenhänge von Interesse. So wäre denkbar, dass der Austausch im Team sowie die Öffnung nach außen auch direkt auf die Prozessqualität und die Häufigkeit naturwissenschaftlicher Bildung wirken. Hierfür wurde der Implementationsindex auf Kita-Ebene aggregiert und als unabhängige Variable ins Analysemodell eingeführt. Tabelle 39 fasst die Ergebnisse der Regressionsanalysen zur Vorhersage der Prozessqualität und Häufigkeit naturwissenschaftlicher Lerngelegenheiten durch den Implementationsindex zusammen. Es zeigen sich keine signifikanten Zusammenhänge zwischen dem Grad der Implementation auf Kita-Ebene und der Prozessqualität bzw. der Häufigkeit naturwissenschaftlicher Lerngelegenheiten.

Tabelle 39. Ergebnisse der Regressionsanalysen zum Einfluss des Implementationsindexes auf die Prozessqualität und die Häufigkeit naturwissenschaftlicher Lerngelegenheiten

\begin{tabular}{lccc}
\hline $\begin{array}{l}\text { Prädiktor: } \\
\text { Implementation auf Kita-Ebene }\end{array}$ & Beta & SE & $p$ \\
\hline Prozessqualität & .03 & .08 & .732 \\
\hline Häufigkeit & .07 & .06 & .200 \\
\hline
\end{tabular}

Anmerkung: N = 358. Kontrolliert für Alter, Geschlecht, kognitive Fähigkeiten, Berufsabschluss, Fortbildungsteilnahme in den letzten 3 Jahren sowie die Motivation, lerntheoretischen Überzeugungen und das professionelle Wissen der Fachkräfte

\subsubsection{Zusammenhang zwischen Prozessmerkmalen und kindlichen Kompetenzen}

Die Ergebnisse zur Vorhersage des Wissens der Kinder zeigen keine signifikanten Haupteffekte. Es zeigt sich jedoch ein signifikanter Interaktionseffekt zwischen der Prozessqualität und der Häufigkeit naturwissenschaftlicher Lerngelegenheiten auf das Wissen der Kinder (siehe Tabelle 40). Der Einfluss der Prozessqualität auf das Wissen der Kinder ist demnach abhängig von der Häufigkeit naturwissenschaftlicher Lerngelegenheiten und umgekehrt. 
Tabelle 40. Ergebnisse der Regressionsanalysen zum Einfluss der Prozessvariablen auf das Wissen der Kinder

\begin{tabular}{llll}
\hline \multicolumn{4}{c}{ Gesamtskala Wissen } \\
\hline Prädiktoren & Beta & $S E$ & $p$ \\
\hline Prozessqualität & .06 & .06 & .331 \\
\hline Häufigkeit & -.03 & .04 & .428 \\
\hline Prozessqualität * Häufigkeit & .29 & .13 & .025 \\
\hline
\end{tabular}

Anmerkung: $\mathrm{N}=$ 282. Kontrollvariablen der Kinder: Alter, Geschlecht, Sprache zuhause, CFT-, KABC- und PPVT-Score

Hinsichtlich der Motivation der Kinder zeigen sich keine signifikanten Haupteffekte der Prozessqualität oder Häufigkeit sowie kein signifikanter Interaktionseffekt (siehe Tabelle 41).

Tabelle 41. Ergebnisse der Regressionsanalysen zum Einfluss der Prozessvariablen auf die Motivation der Kinder

\begin{tabular}{llllllll}
\hline & \multicolumn{3}{l}{$\begin{array}{l}\text { Selbstwirksamkeits- } \\
\text { erwartung der Kinder }\end{array}$} & \multicolumn{3}{c}{ Lernfreude der Kinder } \\
\hline Prädiktoren & Beta & SE & $p$ & Beta & SE & $p$ \\
\hline Prozessqualität & .02 & .07 & .788 & -.01 & .06 & .907 \\
\hline Häufigkeit & .07 & .08 & .359 & -.07 & .07 & .323 \\
\hline Prozessqualität * Häufigkeit & -.03 & .17 & .862 & -.19 & .15 & .203 \\
\hline
\end{tabular}

Anmerkung: $N=282$. Kontrollvariablen der Kinder: Alter, Geschlecht, Sprache zuhause, CFT-, KABC- und PPVT-Score 


\section{Diskussion der Ergebnisse}

\subsection{Struktur der Kompetenzen}

Da in der Forschungsliteratur bisher kaum Instrumente zur Erfassung der naturwissenschaftlichen Kompetenzen von pädagogischen Fachkräften und Kindern existieren, war die erste Zielstellung des Projektes die Entwicklung neuer Messinstrumente sowie die Überprüfung der Kompetenzstruktur auf Fachkraft- und Kinderebene.

\subsubsection{Fachkraftebene: Professionelles Wissen}

Im Rahmen des Projektes wurde ein Testinstrument zur Erfassung des naturwissenschaftlichen professionellen Wissens von frühpädagogischen Fachkräften entwickelt. Eine Herausforderung bei der Entwicklung des Testinstruments war u. a. das mangelnde Wissen darüber, was Fachkräfte über naturwissenschaftliche Themen wissen, sowie der fehlende Konsens darüber, welches Niveau frühpädagogische Fachkräfte an naturwissenschaftlichem Wissen benötigen, um qualitativ hochwertige Lerngelegenheiten anzubieten. Folglich war auch ungeklärt, welches Niveau der Aufgaben des Wissenstests angemessen ist. In dieser Studie wurde die Entscheidung getroffen, ein sehr grundlegendes und basales naturwissenschaftliches Fachwissen zu erheben. Berechnungen zur Reliabilität zeigten, dass das inhaltsbezogene naturwissenschaftliche Fachwissen genauer und zuverlässiger (reliabler) gemessen werden konnte als das prozessbezogene Fachwissen und das fachdidaktische Wissen. Auch wenn dieses Phänomen bereits aus anderen Studien bekannt (Jenßen et al., 2015; Krauss et al., 2008) und insbesondere hinsichtlich des fachdidaktischen Wissens auch plausibel ist, da es sich um ein sehr heterogenes Konstrukt handelt, ist die geringe Reliabilität der Messung dennoch als problematisch einzustufen.

Das entwickelte Testinstrument ermöglicht es, explorativ das naturwissenschaftliche Wissen der frühpädagogischen Fachkräfte zu untersuchen und dadurch erste Erkenntnisse über die Ausprägung und Struktur des naturwissenschaftlichen Wissens von frühpädagogischen Fachkräften zu gewinnen. Die Ergebnisse der Strukturanalysen dieser Studie deuten auf die Mehrdimensionalität des naturwissenschaftlichen professionellen Wissens der Fachkräfte hin. Gemäß den Analysen lässt sich das naturwissenschaftliche professionelle Wissen der frühpädagogischen Fachkräfte empirisch in inhaltsbezogenes Fachwissen, prozessbezogenes Fachwissen und fachdidaktisches Wissen aufteilen. Alle drei Wissensdimensionen hängen allerdings substanziell miteinander zusammen. Dieser Befund steht im Einklang mit Befunden zum mathematischen Wissen 
von frühpädagogischen Fachkräften (Jenßen et al., 2015), aber auch mit Befunden aus der Lehrerprofessionsforschung (Krauss et al., 2008). Das gewonnene Wissen über die Struktur des Wissens auf Fachkraftebene bildet die Grundlage, um in einem nächsten Schritt Aussagen über die Bedeutung von inhalts- und prozessbezogenem Fachwissen und fachdidaktischem Wissen für die Quantität und Prozessqualität der naturwissenschaftlichen Lerngelegenheiten, aber auch über die Zusammenhänge des Fachkraftwissens auf die naturwissenschaftliche Kompetenzen der Kinder machen zu können. Weiterhin können erste Befunde über das naturwissenschaftliche professionelle Wissen der frühpädagogischen Fachkräfte auch bedeutsame Hinweise für die Weiterentwicklung der in den naturwissenschaftlichen Bildungsinitiativen angesiedelten Fortbildungen in Naturwissenschaften für frühpädagogische Fachkräfte geben.

\subsubsection{Fachkraftebene: Motivationale Kompetenzaspekte}

Auch zur Erfassung der motivationalen Kompetenzaspekte wurden neue, reliable Instrumente für den naturwissenschaftlichen Bereich entwickelt. Hinsichtlich der Struktur der motivationalen Kompetenzfacetten auf Fachkraftebene bestätigen die Ergebnisse die empirische Trennbarkeit der vier Skalen (Selbstkonzept, Selbstwirksamkeitserwartung, Lernfreude und Enthusiasmus). Dies ist im Einklang mit Kompetenzmodellen aus der frühkindlichen Bildungsforschung (Anders, 2012) sowie der Schulforschung (Baumert \& Kunter, 2006), die die Motivation als mehrdimensionales Konstrukt auffassen. Erwartungsgemäß korrelieren die theoretisch näher verwandten Konstrukte höher. So fällt der Zusammenhang zwischen dem Selbstkonzept und der Selbstwirksamkeitserwartung am höchsten und zwischen dem allgemeinen Interesse und der Selbstwirksamkeitserwartung am geringsten aus. Die mittleren Häufigkeiten zeigen, dass sich die Fachkräfte im Umgang mit den Naturwissenschaften sowie bei der Vermittlung naturwissenschaftlicher Inhalte sicher fühlen. Auch das allgemeine Interesse an den Naturwissenschaften sowie der Enthusiasmus in der Vermittlung naturwissenschaftlicher Inhalte werden als hoch eingeschätzt. Diese Befunde sind in Anbetracht bisheriger Ergebnisse, die eine überwiegend negative Ausprägung der Motivation der Fachkräfte in MINT-Fächern finden, überraschend (vgl. Erden \& Sönmez, 2010; Koballa \& Crawley, 1985; Maier, Greenfield \& Bulotsky-Shearer, 2013). Die Diskrepanz der Ergebnisse zu früheren Befunden mag auf die Spezifika der Stichprobe dieser Studie zurückzuführen sein, in der aufgrund des Studiendesigns überdurchschnittlich viele Kitas mit naturwissenschaftlichem Schwerpunkt vertreten sind. 


\subsubsection{Fachkraftebene: Lerntheoretische Überzeugungen}

Die im Rahmen der Studie neu entwickelten Skalen zur Erfassung der lerntheoretischen Überzeugungen pädagogischer Fachkräfte fokussieren auf Überzeugungen im Sinne von drei unterschiedlichen pädagogischen Ansätzen: den programmatischen Ansatz, den kindzentrierten Ansatz und den Open-Framework-Ansatz. Im Einklang mit der Typologie nach Siraj-Blatchford et al. (2002) sowie Weikart (2000) lassen sich die Facetten von Überzeugungen (programmatisch, kindzentriert und Open-Framework) auch empirisch trennen. Die beiden eher (ko-)konstruktiv-orientierten Überzeugungen (kindzentrierter und Open-FrameworkAnsatz) korrelieren hierbei erwartungsgemäß positiv miteinander, wohingegen die Korrelation dieser beiden Überzeugungen mit dem programmatischen Ansatz negativ ausfällt. Die Ergebnisse zur Struktur der lerntheoretischen Überzeugungen bestätigen damit, dass sich die Typologie zur Einordnung der Ansätze und Methoden in der Frühpädagogik u. a. nach Siraj-Blatchford et al. (2002) auch auf die lerntheoretischen Überzeugungen in den Naturwissenschaften übertragen lässt. Hinsichtlich der Ausprägung der lerntheoretischen Überzeugungen bestätigen die Ergebnisse frühere Befunde (vgl. Kuhn et al., 2012): Die befragten Fachkräfte vertreten überwiegend (ko-)konstruktive Überzeugungen und lehnen die transmissiven (programmatischen) Aussagen eher ab.

\subsubsection{Kinderebene: Wissen}

Es liegen nur wenige Instrumente zur standardisierten Erfassung prozess- und konzeptbezogenen Wissens im Kindergartenalter vor. Dementsprechend finden sich in der Forschungsliteratur auch nur wenige Untersuchungen zur Struktur naturwissenschaftlicher Kompetenzen im vorschulischen Alter. Standardisierte Testverfahren zur Erfassung naturwissenschaftlicher Kompetenz sind wichtig, um mit Blick auf große Stichproben Kompetenzen von Kindern ökonomisch zu erfassen und die Dimensionalität dieser Kompetenzen zu modellieren. Erkenntnisse zur Kompetenzstruktur können als Grundlage genutzt werden, um Kompetenzentwicklungsmodelle zu testen, und letztendlich, um entsprechende didaktische Konzepte für das sukzessive naturwissenschaftliche Lernen in unterschiedlichen Inhaltsgebieten zu entwickeln und zu prüfen. Um der Frage nach der Struktur naturwissenschaftlicher Kompetenzen im Bereich des prozess- und konzeptbezogenen Wissens von Vorschulkindern nachzugehen, wurden im Rahmen dieser Studie neue Testinstrumente entwickelt. Da Kinder im Kindergartenalter noch nicht lesen können, lag ein Fokus der Testentwicklung in der Umsetzung standardisierter Einzelinterviews. Um die kognitive Anforderung der Kinder durch die standardisierte Befragung gering zu halten, wurden die Items nach Möglichkeit mit Materialien oder Fotos kontextualisiert. 
Die Analysen zur Dimensionalität der naturwissenschaftlichen Kompetenzen sprechen nicht für eine Trennbarkeit von prozess- und konzeptbezogenen Items. Allerdings weist ein Globalfaktormodell mit hoch korrelierten Subskalen eine bessere Passung auf als das eindimensionale Modell. Innerhalb des Globalfaktormodells laden die Einzelfaktoren (Prozesswissen, Material, Magnetismus, Schwimmen und Sinken, Aggregatzustände) hoch auf einen gemeinsamen Faktor zweiter Ordnung. Kinder mit einem hohen Wissen in einem der Inhaltsgebiete (z. B. Inhaltsgebiet Magnetismus) verfügen demnach im Sinne von globalen naturwissenschaftlichen Kompetenzen über ein höheres Wissen in den anderen Inhaltsbereichen und dem Prozesswissen. Gleichzeitig deutet sich ein Informationsgewinn an, wenn jeweils die einzelnen Inhaltsbereiche (Material, Magnetismus, Schwimmen und Sinken und Aggregatzustände) und das Prozesswissen separat betrachtet werden. Der starke Bezug auf einen Globalfaktor naturwissenschaftlicher Kompetenzen entspricht den Ergebnissen der eher explorativen Studien von Carstensen et al. (2011) und Hahn et al. (2013), in denen sich Hinweise für eine eindimensionale Kompetenzstruktur finden. Die Ergebnisse unterscheiden sich jedoch insbesondere von den Ergebnissen von Pollmeier et al. (2017), die eine Trennbarkeit von konzept- und prozessbezogenem Wissen bei Grundschulkindern nachweisen konnten. Möglicherweise bietet der Grundschulunterricht zunehmend differenzielle Lerngelegenheiten zu naturwissenschaftlichen Inhalten bzw. Prozessen. Ein zentraler und nach wie vor offener Diskussionspunkt zeigt sich in diesem Kontext in Bezug auf die Kontextabhängigkeit des Prozesswissens. Ungefähr der Hälfte der prozessbezogenen Items liegt ein naturwissenschaftlicher Kontext in der Fragestellung zugrunde. Die andere Hälfte der Items ist durch alltägliche Situationen kontextualisiert. Analysen für getrennte Skalen mit naturwissenschaftlichen und alltäglichen Kontexten konnten aufgrund der geringen Itemanzahl nicht vorgenommen werden. Weiterführende Analysen in diesem Sinne könnten neue Erkenntnisse zur Trennbarkeit von Prozess- und Konzeptwissen im Vorschulalter liefern.

Die Analysen zur Güte der Instrumente lehnen sich an die durch die Modellanalysen ermittelte Struktur der erhobenen naturwissenschaftlichen Kompetenzen an. Dabei weist die Gesamtskala aller Items eine hohe Reliabilität auf. In weiterführenden Analysen zeigte sich zudem, dass aus dem Itempool auch eine reliable Kurzskala unter Berücksichtigung aller Konzeptbereiche (Material, Magnetismus, Schwimmen und Sinken und Aggregatzustände) und des Prozesswissens entwickelt werden konnte ( $\alpha=.76 ; 29$ Items). Zudem zeigt sich eine ausreichende Reliabilität der einzelnen Unterskalen des Prozesswissens und der verschiedenen Konzeptwissensbereiche. Auch die Abgrenzbarkeit der neu entwickelten Testinstrumente zu individuellen Vorrausetzungen wie der Intelligenz und dem Wortschatz der Kinder konnte nachgewiesen werden. Demnach wurde 
das Ziel erreicht, mit dem Einsatz von Materialien und Bildern den Einfluss von kognitiven Aspekten (Intelligenz) gering zu halten. Allerdings weisen die Analysen auf eine hohe Korrelation der naturwissenschaftlichen Kompetenzen mit dem Wortschatz der Kinder hin, der sich ebenfalls bei vertiefenden Modellierungen mit latenten Faktoren zeigt. Auch wenn sich eine empirische Trennbarkeit der naturwissenschaftlichen Kompetenzen vom Wortschatz belegen lässt, bleibt die hohe Korrelation der Kompetenzbereiche auffällig. Die Befunde decken sich grundsätzlich mit theoretischen Konzeptionen zur Bedeutung von Sprache für den domänenspezifischen Kompetenzerwerb (z. B. Honig, 2010), wobei insbesondere der Wortschatz - als sehr grundlegende Komponente der sprachlichen Entwicklung - eine hohe Bedeutung aufzuweisen scheint.

\subsubsection{Kinderebene: Motivation}

$\mathrm{Da}$ in der Forschungsliteratur keine adäquaten Instrumente zur Erfassung der naturwissenschaftlichen Lernmotivation von Kindern im Vorschulalter vorliegen, wurde im Rahmen der Studie ein neues Messinstrument entwickelt. Die besondere Herausforderung der Instrumentenentwicklung war hierbei, die Verständlichkeit der Items für Kinder im Alter von 5-6 Jahren mit und ohne naturwissenschaftliche Vorerfahrungen zu gewährleisten. Auf Grundlage der Sichtung bewährter Verfahren in der Forschungsliteratur wurde ein inhaltsspezifisches Vorgehen gewählt, bei dem die Kinder zu ihrer Selbstwirksamkeitserwartung und Lernfreude bei der Beschäftigung mit alltagsnahen naturwissenschaftlichen Inhalten befragt wurden. Die empirische Erprobung des Instrumentes in mehreren Pilotstudien bestätigte die Verständlichkeit der neu entwickelten Items und des Antwortformates. Im Einklang mit der theoretischen Differenzierung der Motivation in die Aspekte Selbstwirksamkeitserwartung und Lernfreude (vgl. Eccles \& Wigfield, 2002) passt die zweifaktorielle Struktur am besten auf die Daten. Selbstwirksamkeitserwartung und Lernfreude lassen sich also schon bei Kindern im Alter von 5-6 Jahren empirisch trennen. Die Lernmotivation der befragten Kinder fällt auf beiden Skalen hoch aus: Die Kinder schätzen sich selbst als sehr gut in unterschiedlichen naturwissenschaftlichen Lernbereichen ein und weisen eine hohe Lernfreude auf. Dieses erfreuliche Ergebnis deckt sich mit Befunden zur Lernmotivation in früheren Studien, die typischerweise von einer hohen Lernmotivation bei sehr jungen Kindern berichten (z. B. Eccles, Wigfield, Harold \& Blumenfeld, 1993; Harter \& Pike, 1984; Mantzicopoulos et al., 2008; Marsh, Ellis \& Craven, 2002). 


\subsection{Gruppenunterschiede}

Eine Fragestellung der Studie war es, die Wirkungen naturwissenschaftlicher Bildungsangebote hinsichtlich der Kompetenzen der Fachkräfte, der Prozessqualität und -quantität naturwissenschaftlicher Bildung sowie der Kompetenzen der Kinder zu untersuchen. Hierfür wurden Einrichtungen, die an der Initiative „Haus der kleinen Forscher" teilnehmen, und Kitas, die nicht an der Initiative teilnehmen, aber auch ebenso einen naturwissenschaftlichen Bildungsschwerpunkt haben, mit einer Gruppe von Kitas, die keinen naturwissenschaftlichen Bildungsschwerpunkt haben, verglichen. Die Zuteilung der Kitas zu den Untersuchungsgruppen erfolgte anhand der Anzahl der naturwissenschaftlichen Fortbildungen, wobei Kitas nur dann der HdkF- bzw. Nawi-Gruppe zugewiesen wurden, wenn mindestens eine Fachkraft in der Einrichtung mindestens 2 naturwissenschaftliche Fortbildungen in den letzten 3 Jahren besucht hat. Der Einordnung auf Grundlage der Fortbildungen liegt die Annahme zugrunde, dass die Wirkung naturwissenschaftlicher Bildungsangebote -wie die Angebote der Stiftung „Haus der kleinen Forscher“ - in den Kitas primär über Fortbildungen zu verorten ist. Vor diesem Hintergrund wurde im Anschluss an die Gruppenzuteilung die Fortbildungshäufigkeit in den drei Untersuchungsgruppen verglichen. Erwartungsgemäß besuchten Fachkräfte in den Vergleichskitas nahezu keine naturwissenschaftlichen Fortbildungen. Hinsichtlich der beiden Untersuchungsgruppen mit naturwissenschaftlichem Schwerpunkt zeigt der Gruppenvergleich, dass die Fachkräfte der Kitas in der HdkF-Gruppe im Mittel mehr Fortbildungen besucht haben als die Fachkräfte der Nawi-Kitas (HdkF: 2.28 Fortbildungen in den letzten 3 Jahren; Nawi-Gruppe: 1.31 Fortbildungen in den letzten 3 Jahren). Dies kann man auch als Outcome der Initiative „Haus der kleinen Forscher“ verstehen, die möglicherweise durch ein regelmäßiges Angebot Fachkräfte dazu motiviert, an Fortbildungen teilzunehmen. Einschränkend muss allerdings gesagt werden, dass wir keine tiefergehenden Informationen über die Fortbildungsangebote für Kitas der Vergleichsgruppe haben, so dass der direkte Vergleich schwierig ist.

Für einen fairen Vergleich wurde daher in allen folgenden Analysen die Anzahl der Fortbildungen in den letzten 3 Jahren kontrolliert. Somit ließen sich potenzielle Gruppenunterschiede zwischen der HdkF- und Nawi-Gruppe tatsächlich auf die unterschiedlichen naturwissenschaftlichen Bildungsinitiativen (HdkF vs. andere Nawi-Initiativen) und nicht lediglich auf die Fortbildungshäufigkeit - unabhängig vom Anbieter der Fortbildungen - zurückführen. 


\subsubsection{Einrichtungsebene}

Eine Voraussetzung für eine nachhaltige Wirkung naturwissenschaftlicher Bildungsangebote ist die Implementation der Naturwissenschaften als Bildungsbereich in der gesamten Kita (vgl. Kapitel 2.1). Hierzu gehören u. a. die Weitergabe von Fortbildungsinhalten im Team, regelmäßige Teamsitzungen zur naturwissenschaftlichen Bildung sowie die Absprache naturwissenschaftlicher Bildungsangebote im Kollegium, mit der Leitung, dem Träger sowie mit den Eltern („Öffnung nach außen“). Vor diesem Hintergrund wurde in einem zweiten Schritt die Implementation der Naturwissenschaften als Bildungsbereich in den Untersuchungsgruppen verglichen. In Abhängigkeit von den drei genannten Indikatoren erfolgreicher Implementation zeigen sich sehr unterschiedliche Ergebnisse: Die HdkF-Gruppe schneidet bei den Teamsitzungen sowie der Öffnung nach außen signifikant besser ab als beide anderen Gruppen. Bei der Weitergabe naturwissenschaftlicher Fortbildungsinhalte fallen hingegen die Werte der Nawi-Gruppe am besten aus, wobei zwischen HdkF-Gruppe und Nawi-Gruppe sowie HdkF-Gruppe und Vergleichsgruppe keine signifikanten Unterschiede bestehen. In der HdkF-Gruppe werden die Fortbildungsinhalte also nicht häufiger ins Team weitergegeben, obgleich die Fachkräfte in den HdkF-Kitas mehr Fortbildungen besucht haben. Die sehr geringen Werte bei der Weitergabe naturwissenschaftlicher Fortbildungsinhalte in der Vergleichsgruppe sind naheliegend, da in dieser Gruppe überwiegend Kitas ohne Fortbildungen vertreten sind.

\subsubsection{Fachkraftebene}

\section{Wissen}

Im Hinblick auf das Wissen zeigten sich keine signifikanten Unterschiede zwischen den frühpädagogischen Fachkräften in den Gruppen, die an Bildungsinitiativen teilnehmen bzw. nicht teilnehmen. Dies könnte darauf hindeuten, dass die Fortbildungsinhalte, die das Wissen betreffen, nicht in die Kitas weitergetragen werden.

Bei der Betrachtung von Fachkräften mit mindestens 2 naturwissenschaftlichen Fortbildungen in den letzten 3 Jahren zeigte sich, dass pädagogische Fachkräfte mit Fortbildungen im Wissenstest nicht signifikant besser bei den Aufgaben zum inhalts-, prozessbezogenen Fachwissen und dem fachdidaktischen Wissen abschnitten als Fachkräfte ohne Fortbildung (lediglich beim inhaltsbezogenen Fachwissen finden sich marginal signifikante Unterschiede). Da auf Basis von empirischen Befunden nicht notwendigerweise eine langfristige Wirkung der in der Regel eher kurz angelegten Fortbildungen auf das Wissen der Fachkräfte erwartet wurde, wurde in einem nächsten Schritt das Abschneiden von Fachkräften mit und ohne Fortbildungen in Naturwissenschaften im letzten Jahr untersucht. Hier 


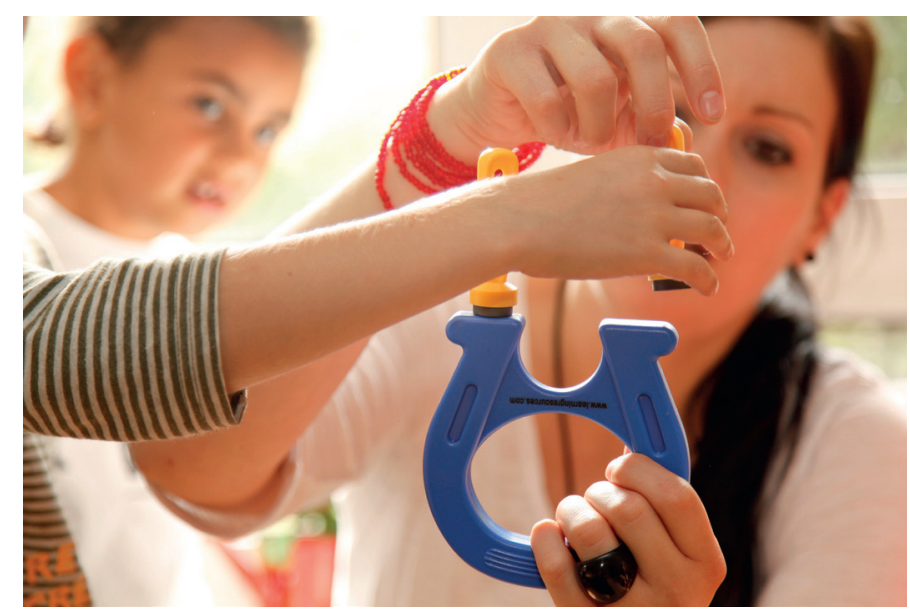

zeigen die Ergebnisse signifikante Unterschiede im inhalts- und prozessbezogenen Fachwissen. Berücksichtigt man bei weiteren Analysen mögliche Lerngelegenheiten in Naturwissenschaften in der Ausbildung zeigen sich neben den signifikanten Unterschieden im inhalts- und prozessbezogenen Wissen marginal signifikante Unterschiede im fachdidaktischen Wissen zugunsten der Fachkräfte mit Fortbildungen. Es scheint, als würden die pädagogischen Fachkräfte von den naturwissenschaftlichen Fortbildungen vor allem hinsichtlich der beiden Fachwissenskomponenten profitieren. Dies würde im Einklang mit bisherigen Befunden stehen, die Fortbildungsteilnahmen in einem positiven Zusammenhang mit dem Wissen der pädagogischen Fachkräfte darstellen (Snider \& Fu, 1990; Carlisle, Correnti, Phelps \& Zeng, 2009). Da es sich bei der vorliegenden Untersuchung um eine querschnittliche Untersuchung ohne Prä-Post-Test handelt, können auf Grundlage der vorliegenden Daten keine kausalen Schlüsse gezogen werden. Somit wäre es auch möglich, dass pädagogische Fachkräfte, die schon ein höheres Wissen haben, eher an Fortbildungen teilnehmen.

\section{Motivation}

Auch bei den motivationalen Merkmalen zeigen die Ergebnisse keine Gruppenunterschiede. Vergleicht man hingegen Fachkräfte mit und ohne Fortbildungen in den letzten 3 Jahren, zeichnen sich deutliche Unterschiede ab: Fachkräfte mit Fortbildungen sind im Durchschnitt motivierter als Fachkräfte ohne Fortbildungen. Dies deckt sich mit Ergebnissen früherer Untersuchungen, die zeigen, dass sich die Motivation der Fachkräfte, insbesondere die Selbstwirksamkeitserwartung, durch Fort- und Weiterbildung erhöhen lassen (Appleton, 1995; Guo et al., 2010; Hoy \& Woolfolk, 1990). Weniger Befunde gibt es bisher zum Einfluss von Fortbildungen auf das Interesse und den Enthusiasmus von Fachkräften. Die Ergebnisse der vorliegenden Untersuchung weisen auch hier auf einen positiven Zusammenhang hin. Da jedoch nur querschnittliche Daten vorliegen, können hierbei keine Kausalaussagen gemacht werden. Es ist demnach auch denkbar, dass Fachkräfte mit mehr Interesse und Enthusiasmus in den Naturwissenschaften eher naturwissenschaftliche Fortbildungen besuchen. 


\section{Lerntheoretische Überzeugungen}

Analog zu den Ergebnissen bezüglich des Wissens und der Motivation zeichnen sich auch in den lerntheoretischen Überzeugungen der Fachkräfte keine signifikanten Gruppenunterschiede ab. Auch hier lassen sich aber Unterschiede nach Fortbildungsteilnahmen finden: Fachkräfte mit mehr Fortbildungen vertreten im Mittel weniger programmatische Überzeugungen. Auch hinsichtlich der Überzeugungen im Sinne des kindzentrierten und Open-Framework-Ansatzes zeichnen sich signifikante Unterschiede nach Fortbildungsteilnahme ab: Fachkräfte mit mehr Fortbildungen vertreten eher Überzeugungen im Sinne des kindzentrierten oder Open-Framework-Ansatzes als Fachkräfte mit einer oder keiner Fortbildung. Dieser Befund mag darauf zurückzuführen sein, dass in den allermeisten naturwissenschaftlichen Fortbildungen eher ko-konstruktive Ansätze vermittelt werden (Stiftung Haus der kleinen Forscher, 2015b; Fthenakis, 2009). Allerdings können auch keine Kausalaussagen gemacht werden: Der positive Zusammenhang mag auch darauf zurückzuführen sein, dass Fachkräfte, die eher programmatische Überzeugungen vertreten, seltener an Fortbildungen teilnehmen, bzw. dass Fachkräfte, die eher kindzentrierte und Open-Framework-Ansätze vertreten, häufiger an Fortbildungen teilnehmen.

\subsubsection{Prozessqualität und Quantität naturwissenschaftlicher Bildungsangebote}

Für die Prozessqualität und Häufigkeit naturwissenschaftlicher Bildungsangebote konnten, unter Kontrolle der Fortbildungen, keine Gruppenunterschiede festgestellt werden. Vor dem Hintergrund fehlender Gruppenunterschiede in den Kompetenzen der Fachkräfte, welche als zentral für die Prozessqualität und Quantität naturwissenschaftlicher Bildung gelten, ist dieser Befund nicht verwunderlich. Analog zu den Ergebnissen in den Fachkraftkompetenzen zeichnen sich deutliche Unterschiede ab, wenn Fachkräfte mit mindestens 2 oder ohne Fortbildungen in den letzten 3 Jahren verglichen werden. Diese Ergebnisse könnten darauf hindeuten, dass sowohl die Häufigkeit als auch die Prozessqualität der naturwissenschaftlichen Bildungsangebote sich möglicherweise durch Fortbildungen erhöhen lassen. Es ist jedoch anzunehmen, dass dieser Zusammenhang vermutlich nicht direkt auf die Fortbildungen, sondern vielmehr indirekt auf die Kompetenzzuwächse der Fachkräfte zurückzuführen sein könnte (vgl. Kapitel 2.1). Diese Zusammenhänge werden im Abschnitt 6.3 näher beleuchtet. 


\subsubsection{Kinderebene}

Die Ergebnisse des Kita-Gruppenvergleichs auf Kinderebene zeigen keine signifikanten Unterschiede zwischen den drei Untersuchungsgruppen im Wissen oder der Motivation der Kinder. Da sich bereits auf Fachkraftebene keine Gruppenunterschiede abzeichnen, überrascht dieser Befund nicht. Darüber hinaus ließen sich keine Unterschiede zwischen Kindern, die von Fachkräften mit mindestens 2 oder ohne Fortbildungen in den letzten 3 Jahren betreut wurden, abbilden. Dies mag darauf zurückzuführen sein, dass die Fortbildungsteilnahme zwar mit unterschiedlichen Kompetenzfacetten der Fachkräfte im Zusammenhang steht, diese aber nur indirekt, vermittelt über die Prozessmerkmale, mit einzelnen Kompetenzfacetten der Kinder zusammenhängen (vgl. Kapitel 6.3). In einer weiterführenden Studie auf Grundlage von Daten, auf denen auch der vorliegende Bericht beruht, wurde untersucht, inwieweit die Implementation des Bereichs der Naturwissenschaften als Einrichtungskonzept (Naturwissenschaften als pädagogischer Schwerpunkt der Einrichtung) mit den motivationalen Überzeugungen der Kinder zusammenhängt. Die Ergebnisse konnten zeigen, dass Kinder in Einrichtungen mit einem naturwissenschaftlichen Schwerpunkt eine höhere naturwissenschaftliche Lernfreude und Selbstwirksamkeitserwartung aufweisen als Kinder aus Einrichtungen ohne naturwissenschaftlichen Schwerpunkt (vgl. Oppermann, Brunner, Eccles \& Anders, 2017). Dieser Befund kann als erster Hinweis auf die Bedeutsamkeit früher naturwissenschaftlicher Bildung für die motivationalen Überzeugungen der Kinder interpretiert werden.

\subsection{Zusammenhänge zwischen den Wirkungsebenen}

Die potenziellen Wirkungen naturwissenschaftlicher Bildungsangebote auf die naturwissenschaftlichen Kompetenzen der Fachkräfte und die Kinder wurden im Wirkungsmodell (Kapitel 2.1) beschrieben. Als besonders relevant für die kindliche Kompetenzentwicklung werden die Prozessqualität und die Quantität naturwissenschaftlicher Lerngelegenheiten angenommen, welche wiederum von den Kompetenzen der Fachkräfte abhängen. Die Kompetenzen der Fachkräfte werden beeinflusst durch Fortbildungen sowie durch die Implementationsqualität in der Einrichtung. Die Implementationsqualität kann zudem auch direkt auf die Prozessmerkmale der Prozessqualität und der Quantität naturwissenschaftlicher Bildungsprozesse wirken. Diese angenommenen Zusammenhänge zwischen den unterschiedlichen Wirkungsebenen wurden in der dritten Zielstellung des Projektes empirisch überprüft. 


\subsubsection{Zusammenhänge zwischen der Implementationsqualität und Fachkraftkompetenzen}

Dem Forschungsvorhaben lag die Annahme zugrunde, dass naturwissenschaftliche Bildungsangebote mithilfe von Fortbildungen für Fachkräfte nur dann auf allen Ebenen wirken können, wenn Fortbildungsinhalte ins Team getragen werden und sich die Einrichtung als Ganzes in den Prozess der Qualitätsentwicklung begibt. Vor diesem Hintergrund waren die Zusammenhänge zwischen dem Grad der Implementation naturwissenschaftlicher Bildung auf Einrichtungsebene und den Kompetenzen auf Fachkraftebene sowie der Prozessqualität und Häufigkeit naturwissenschaftlicher Bildung von hoher Relevanz.

Im Hinblick auf das inhalts- und prozessbezogene Fachwissen $\left(\mathrm{CK}_{\text {Inhalt }}\right.$ und $\mathrm{CK}_{\text {Prozess }}$ ) sowie fachdidaktische Wissen der pädagogischen Fachkräfte erwies sich der Implementationsindex als nicht prädiktiv. Eine stärkere Implementation scheint somit nicht mit mehr inhalts- und prozessbezogenem Fachwissen oder mehr fachdidaktischem Wissen einherzugehen. Eine mögliche Erklärung für diese Ergebnisse könnte sein, dass kaum Zeit damit verbracht wird, sich über konkrete naturwissenschaftliche Inhalte auszutauschen, sondern z. B. eher über organisatorische Aspekte bezüglich der naturwissenschaftlichen Bildungsarbeit gesprochen wird, beispielsweise: wer benötigt bestimmte naturwissenschaftliche Materialien, wer hat Materialien von einer Fortbildung mitgebracht? etc. Der negative Zusammenhang zum fachdidaktischen Wissen ist schwierig zu interpretieren. Eine Möglichkeit wäre, dass das fachdidaktische Wissen nicht korrekt weitergegeben wurde.

Hinsichtlich der Motivation der Fachkräfte ist ein positiver Zusammenhang der Implementationsqualität mit dem allgemeinen Interesse sowie dem allgemeinen Selbstkonzept der Fachkräfte in den Naturwissenschaften festzuhalten. Hierbei wurde für die Anzahl der Fortbildungen kontrolliert. Fachkräfte profitieren folglich in ihrem Interesse und Selbstkonzept von einer stärkeren Implementation der Naturwissenschaften als Bildungsbereich in der Kita. Dass Motivation veränderbar ist, konnte in Bezug auf Fort- und Weiterbildungen in der Forschungsliteratur mehrfach belegt werden (Appleton, 1995; Guo et al., 2010; Hoy \& Woolfolk, 1990). Die vorliegende Studie ist aber die erste Studie, die zeigen konnte, dass das Interesse und Selbstkonzept der Fachkräfte über die individuelle Fortbildungsaktivität hinaus auch mit der Implementationsqualität zusammenhängen. Da nur querschnittliche Daten vorliegen, können jedoch keine Aussagen über die Richtung des Zusammenhangs gemacht werden. Es ist daher auch denkbar, dass motivierte Fachkräfte eher Fortbildungsinhalte in ihrer Einrichtung weitergeben.

Im Hinblick auf die lerntheoretischen Überzeugungen existieren deutlich weniger Untersuchungen und nach unserem Wissen bisher keine zum Zusammenhang mit der Implementation und dem Austausch im Team. Die Ergebnisse der 
vorliegenden Studie zeigen, dass die Implementationsqualität für die lerntheoretischen Überzeugungen nicht prädiktiv ist. Die Überzeugungen der Fachkräfte zu angemessenen pädagogischen Ansätzen in den Naturwissenschaften lassen sich demnach über die Fortbildungseffekte hinaus nicht durch den Grad der Implementation in der Einrichtung verändern. Der Befund, dass sich die Motivation, nicht aber die lerntheoretischen Überzeugungen durch die Implementationsqualität erhöhen lassen, mag darauf zurückzuführen sein, dass in Teammeetings sowie internen Prozessen der Qualitätsentwicklung eher neu gewonnene Ideen aus Fortbildungen sowie die Begeisterung für das Thema weitergegeben werden als Spezifika der pädagogischen Ansätze bei der Vermittlung naturwissenschaftlicher Inhalte.

\subsubsection{Zusammenhänge zwischen Fachkraftkompetenzen und Prozessmerkmalen}

In Anlehnung an das Wirkungsmodell wird angenommen, dass die unterschiedlichen Kompetenzen pädagogischer Fachkräfte handlungsrelevant sind (Anders, 2012; Fröhlich-Gildhoff et al., 2011; Fröhlich-Gildhoff et al., 2014; McCray \& Chen, 2012) und somit die Prozessqualität und Quantität naturwissenschaftlicher Bildung beeinflussen. Diese Annahme wurde aber bisher für den Bereich der naturwissenschaftlichen Bildung nicht überprüft. Die vorliegende Studie kann hier erste Einblicke geben.

Im Hinblick auf das naturwissenschaftliche professionelle Wissen der pädagogischen Fachkräfte zeigte sich kein direkter signifikanter Zusammenhang zur Häufigkeit der angebotenen naturwissenschaftlichen Lerngelegenheiten. Auf der einen Seite könnte dies verwunderlich sein; so würde man auf Grundlage von bisherigen Befunden annehmen, dass Fachkräfte mit beispielsweise mehr Fachwissen auch in der Lage sind, mehr potenzielle Lerngelegenheiten wahrzunehmen (vgl. Oppermann et al., 2016; Dunekacke et al., 2015). Jedoch wäre es auch möglich, dass die pädagogischen Fachkräfte diese Lerngelegenheiten zwar wahrnehmen, aber diese Gelegenheiten letztendlich nicht nutzen. Als Gründe dafür werden in der internationalen Diskussion ein geringes Fachwissen angegeben (Kallery \& Psillos, 2001; Garbett, 2003) sowie der Druck, die Kinder sprachlich zu fördern, angeführt (Greenfield, Jirout, Dominguez, Greenberg, Maier \& Fuccilo, 2009). Weiterhin werden auch der Besuch von naturwissenschaftlichen Fortbildungen, die Verfügbarkeit von naturwissenschaftlichem Versuchsmaterialien in den Kitas und die Wahrnehmung der Lernkapazitäten der Kinder durch die pädagogischen Fachkräfte als wichtige Einflussfaktoren für die Häufigkeit der angebotenen Lerngelegenheiten gehandelt (Saçkes, 2014).

Auf der anderen Seite wäre auch denkbar, dass es einen indirekten Zusammenhang zwischen naturwissenschaftlichem Wissen und der Häufigkeit natur- 
wissenschaftlicher Aktivitäten geben könnte: So wird im Kompetenzmodell von Fröhlich-Gildhoff et al. (2011) die professionelle Haltung der pädagogischen Fachkräfte als übergeordneter Einflussfaktor für die Handlungsplanung und -bereitschaft sowie die tatsächliche Handlung angenommen. Auch motivationale Orientierungen und Überzeugungen werden oftmals neben dem Wissen als wichtige Einflussfaktoren im Zusammenhang mit dem professionellen Handeln diskutiert (Baumert \& Kunter, 2011). Einschränkend sollte hier noch angemerkt werden, dass es möglich ist, dass die pädagogischen Fachkräfte bei der Beantwortung der Frage nach der Häufigkeit der Lernbegleitung bei naturwissenschaftlichen Aktivitäten eine unterschiedliche Definition von Lernbegleitung oder naturwissenschaftlicher Aktivität zugrunde gelegt haben und deswegen möglicherweise kein Zusammenhang besteht.

Für das inhalts- und prozessbezogene Fachwissen von pädagogischen Fachkräften zeigten sich erwartungsgemäße signifikante Zusammenhänge zu den Indikatoren für die Prozessqualität der angebotenen Lerngelegenheiten. Diese Ergebnisse stehen im Einklang mit bisherigen Annahmen (Siraj-Blatchford et al., 2002; Lee, 2010). Für das fachdidaktische Wissen konnte dieser Zusammenhang nicht gezeigt werden. Dieser Befund ist erwartungswidrig, da das fachdidaktische Wissen als bedeutsam für die Prozessqualität der Interaktionen angenommen wird (McCray \& Chen, 2012). Möglicherweise spielen hier Interaktionen mit weiteren Moderator- oder Mediatorvariablen eine Rolle, auch die hier gewählte Operationalisierung des fachdidaktischen Wissens, die stark auf ein explizierbares deklaratives Wissen abzielt, könnte ursächlich für den nicht beobachtbaren Zusammenhang sein.

In Bezug auf die Motivation der Fachkräfte und die Prozessqualität sowie die Häufigkeit naturwissenschaftlicher Lerngelegenheiten zeigen sich theoriekonforme Ergebnisse: Je motivierter die Fachkräfte sind, desto häufiger initiierten sie naturwissenschaftliche Lerngelegenheiten und desto höher war die Prozessqualität dieser Lerngelegenheiten. Dies deckt sich mit Ergebnissen existierender Studien, die die Bedeutsamkeit der Motivation für die realisierte Prozessqualität (Anders \& Roßbach, 2015; Baumert \& Kunter, 2006) und die Quantität der Lerngelegenheiten in MINT-Fächern belegen (Erden \& Sönmez, 2010; Spektor-Levy et al., 2013). Bei den lerntheoretischen Überzeugungen hängen erwartungsgemäß konstruktive (kindzentrierte) Überzeugungen positiv und transmissive Überzeugungen negativ mit der Prozessqualität zusammen. Ein negativer Zusammenhang transmissiver Überzeugungen wurde auch in bisherigen Studien in Bezug auf die (Unterrichts-)Qualität berichtet (Dubberke et al., 2008; McCarty et al., 2001). Die Zusammenhänge der Prozessqualität mit den ko-konstruktiven Überzeugungen (Open-Framework-Ansatz) sind positiv, aber nicht signifikant. Hinsichtlich der Zusammenhänge zwischen den lerntheoretischen Überzeugungen von Fachkräften 
und der Häufigkeit naturwissenschaftlicher Lerngelegenheiten existieren bisher keine empirischen Untersuchungen. Hier lieferte die vorliegende Studie erste Hinweise, dass ko-konstruktive Überzeugungen für die Häufigkeit naturwissenschaftlicher Bildungsprozesse bedeutsam sind. Einschränkend muss an dieser Stelle angemerkt werden, dass die Qualitätsangaben lediglich auf Selbstberichten der Fachkräfte beruhen, welche nicht unbedingt mit dem Handeln einhergehen. Zudem sind die selbstberichteten Indikatoren für Prozessqualität konzeptionell nah mit den Überzeugungen der Fachkräfte verwandt.

\subsubsection{Zusammenhänge zwischen der Implementationsqualität und den Prozessmerkmalen}

Wie bereits in Abschnitt 6.3.1 und 6.3.2 diskutiert, beeinflusst die Implementationsqualität die Motivation der Fachkräfte und kann so indirekt auf die Prozessqualität und Häufigkeit naturwissenschaftlicher Lerngelegenheiten wirken. Darüber hinaus ist auch denkbar, dass die Implementationsqualität, also der Austausch im Team und die Öffnung nach außen, die Prozessqualität und Häufigkeit über strukturelle Rahmenbedingungen, z. B. die Raumgestaltung, oder die Elternzusammenarbeit beeinflusst. Diese Hypothese wurde empirisch geprüft. Die Ergebnisse zeigen, dass die Implementationsqualität, unter Kontrolle der Fachkraftkompetenzen, keinen signifikanten Einfluss auf die Prozessqualität oder Häufigkeit naturwissenschaftlicher Lerngelegenheiten hat. Dieser Befund steht im Kontrast zu vorherigen Untersuchungen zur Bedeutsamkeit der Implementationsqualität für die sprachliche bzw. allgemeine Prozessqualität in der Kita (Resa et al., 2017; Wertfein et al., 2013). Diese Diskrepanz mag darauf zurückzuführen sein, dass in den genannten Untersuchungen die Prozessqualität auf Kita-Ebene erhoben und nicht für die Kompetenzen der Fachkräfte kontrolliert wurde. Somit ist denkbar, dass die Zusammenhänge zwischen der Implementations- und Prozessqualität über die Kompetenzen der Fachkräfte vermittelt wurden.

\subsubsection{Zusammenhang zwischen Prozessmerkmalen und kindlichen Kompetenzen}

Die Prozessqualität und Häufigkeit naturwissenschaftlicher Lerngelegenheiten wird als zentral für die Kompetenzentwicklung der Kinder angenommen. So wird davon ausgegangen, dass regelmäßige sowie kognitiv anregende naturwissenschaftliche Lerngelegenheiten eine Voraussetzung für die kindliche Kompetenzentwicklung sind. Darüber hinaus wird im Sinne einer Schwellenwerthypothese angenommen, dass Kinder nur dann von hochwertigen Lerngelegenheiten profitieren können, wenn diese ausreichend häufig stattfinden. Gleichzeitig können regelmäßige Lerngelegenheiten nur dann einen positiven Einfluss haben, wenn 
ein Minimum an Prozessqualität gegeben ist. Die Ergebnisse zu den Haupteffekten der Prozessqualität und Häufigkeit auf die kindliche Kompetenzen fallen nicht signifikant aus: Prozessqualität bzw. die Häufigkeit allein stehen nicht im Zusammenhang mit dem Wissen oder der Motivation der Kinder. In Bezug auf das Wissen der Kinder ist jedoch der Interaktionseffekt von Prozessqualität und Quantität signifikant positiv. Dieser Befund, in Kombination mit den fehlenden Haupteffekten, bestätigt die Annahme im Sinne eines Schwelleneffektes von Quantität und Prozessqualität: Prozessqualität kann nur dann wirken, wenn Lerngelegenheiten ausreichend häufig stattfinden, und regelmäßige Lerngelegenheiten sind nur dann positiv für den Wissenszuwachs der Kinder, wenn diese Gelegenheiten qualitativ hochwertig sind.

Entgegen unserer Annahme stehen weder die Pro-

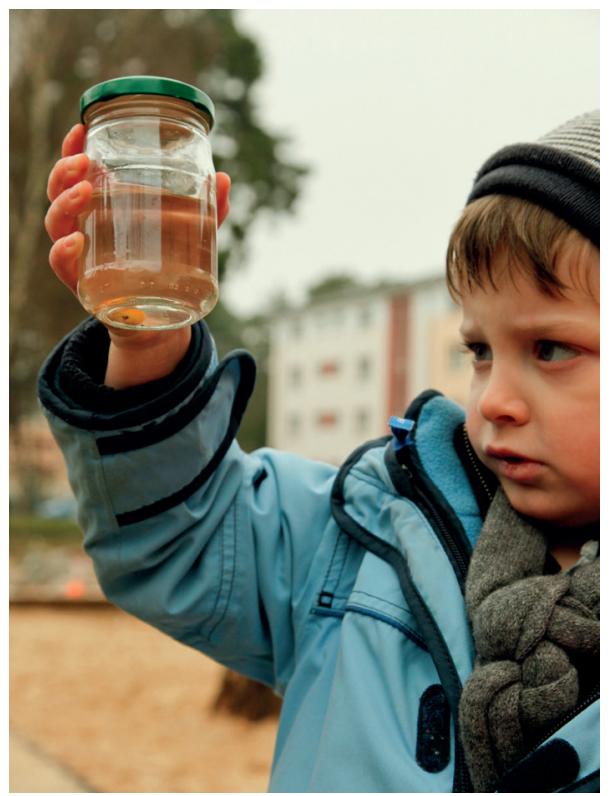
zessqualität, die Häufigkeit noch die Interaktion beider Prädiktoren in einem Zusammenhang mit der Motivation der Kinder. Dies mag u. a. damit zusammenhängen, dass die Kinder bereits hoch motiviert sind und sich die Motivation nicht durch mehr Lerngelegenheiten erhöhen lässt. Eine weitere mögliche Erklärung betrifft die Operationalisierung von Prozessqualität: Hohe Prozessqualität wurde im Sinne von kognitiv anregenden Lerngelegenheiten erfasst, d.h. Lerngelegenheiten, die die Kinder zum Nach- und Mitdenken anregen. Hier liegt der Fokus klar auf den kognitiven Outcomes der Kinder und weniger auf den motivationalen Outcomes. 


\section{Grenzen der Studie}

Im Folgenden werden die Limitationen der Studie erörtert. Zunächst ist anzumerken, dass die Stichprobe in vier Bundesländern erhoben wurde und damit nicht als repräsentativ für Gesamtdeutschland zu sehen ist. Es erfolgte bei der vorliegenden Stichprobe keine zufällige Auswahl, da einerseits die Teilnahme an der Studie auf freiwilliger Basis stattfand und andererseits die genannten Auswahlkriterien für die Teilnahme erfüllt sein mussten. Die Stichprobe ist folglich dahingehend selektiv, dass mehr Kitas mit naturwissenschaftlichem Schwerpunkt vertreten sind als im gesamtdeutschen Durchschnitt.

Ferner ist das querschnittliche Untersuchungsdesign der Studie anzumerken. Da die Studie lediglich mit einem Messzeitpunkt angelegt war und kein Prä-PostTest umgesetzt wurde, können im Rahmen dieser Studie die Ergebnisse nicht kausal interpretiert werden.

Eine weitere Begrenzung betrifft die Operationalisierung und Erhebung von Prozessqualität. Die Operationalisierung und Entwicklung des Instrumentes erfolgte theoriegeleitet und auf Basis aktueller Forschungsergebnisse zur Erfassung allgemeiner und naturwissenschaftsbezogener Prozessqualität (Anders \& Ballaschk, 2014; Hachfeld \& Anders, 2016). Für einen Teil der Fragen konnten bereits bedeutsame Assoziationen mit beobachteter Prozessqualität ermittelt werden. Auf der anderen Seite handelt es sich um ein Fragebogeninstrument, also selbstberichtete Einschätzungen der pädagogischen Fachkräfte, die entsprechend auch durch Überzeugungen, eigene und soziale Erwartungen beeinflusst sein können. Aus der Lehrerprofessionsforschung ist bekannt, dass Selbstberichte häufig nicht mit dem realisierten Handeln einhergehen (Hill, Beisiegel \& Jacob, 2013). In zukünftigen Studien sollte dieses Instrument anhand von weiteren Validierungsstudien bestätigt werden.

Gleiches betrifft auch die Selbsteinschätzung der Häufigkeit naturwissenschaftlicher Aktivitäten durch die pädagogischen Fachkräfte, auch wenn nicht von bewussten Täuschungen ausgegangen werden kann. Hier tritt allerdings das Problem auf, dass keine genauen Angaben zur Beschreibung der naturwissenschaftlichen Aktivitäten gemacht wurden. Es kann also nicht ausgeschlossen werden, dass dieses Item von unterschiedlichen Fachkräften anders verstanden wurde. So wäre denkbar, dass einige Fachkräfte lediglich geplante Versuche als Aktivität definieren und wieder andere Fachkräfte auch anregende Gespräche mit den Kindern in einer Routinesituation als naturwissenschaftliche Aktivität zählen. Auch dieses Item sollte in zukünftigen Untersuchungen anhand Beobachtungsverfahren oder einer detaillierteren Erfassung von naturwissenschaftlichen Aktivitäten validiert werden. 


\section{Zentrale Ergebnisse}

Ziel des Forschungsvorhabens war es, die Wirkungen früher naturwissenschaftlicher Bildungsangebote auf die professionellen Kompetenzen frühpädagogischer Fachkräfte sowie die Kompetenzen der Kinder zu untersuchen.

(1) Die erste Fragestellung des Projektes zielte auf die Erfassung und Struktur der Kompetenzen auf Fachkraft- und Kinderebene. Die Ergebnisse zur ersten Fragestellung lassen sich wie folgt zusammenfassen:

- Für beide Ebenen gelang es weitestgehend, reliable sowie valide Messinstrumente zu entwickeln.

- Die Ergebnisse zur Dimensionalität der unterschiedlichen Kompetenzaspekte bestätigen die theoriegeleiteten Annahmen und liefern somit auch Evidenz für die Konstruktvalidität der neu entwickelten Instrumente.

(2) Als zweite Fragestellung stand die Wirkung naturwissenschaftlicher Bildungsangebote im Fokus des Projektes. Hierfür wurden drei Kita-Gruppen (HdkF-Gruppe, Gruppe mit anderem Nawi-Schwerpunkt, Vergleichsgruppe ohne Nawi-Schwerpunkt) verglichen. Die Ergebnisse zur zweiten Fragestellung lassen sich wie folgt zusammenfassen:

- Fachkräfte der HdkF-Gruppe besuchen im Mittel mehr Fortbildungen zu naturwissenschaftlichen Themen als Fachkräfte der Nawi-Gruppe und der Vergleichsgruppe.

- HdkF-Kitas haben den naturwissenschaftlichen Bildungsbereich stärker implementiert als Kitas der Nawi-Gruppe und der Vergleichsgruppe. Dies zeigt sich beispielsweise in einer häufigeren Thematisierung des Bildungsbereichs Naturwissenschaften in Teamsitzungen.

- Es ergeben sich keine Unterschiede zwischen den drei Kita-Untersuchungsgruppen hinsichtlich der Kompetenzen der Fachkräfte. Der Vergleich von Fachkräften mit mindestens 2 oder ohne Fortbildungen in den letzten 3 Jahren lässt hingegen deutliche Unterschiede in den motivationale Kompetenzen (Interesse, Selbstkonzept, Selbstwirksamkeitserwartung und Enthusiasmus) sowie pädagogischen Überzeugungen (programmatischer Ansatz, kindzentrierter Ansatz, Open-Framework-Ansatz) erkennen. Die Ergebnisse des Ver- 
gleichs von Fachkräften mit und ohne Fortbildung im letzten Jahr zeigen signifikante Unterschiede im inhalts- und prozessbezogenen Wissen zugunsten fortgebildeter Fachkräfte. Tendenzielle Unterschiede im fachdidaktischen Wissen zeigen sich, wenn man zusätzlich für weitere naturwissenschaftliche Lerngelegenheiten (etwa in der Ausbildung) kontrolliert.

- In den selbstberichteten Angaben zur Quantität und Qualität naturwissenschaftlicher Bildungsgelegenheiten finden sich keine Unterschiede zwischen den Gruppen von Kitas. Fortgebildete pädagogische Fachkräfte geben jedoch an, naturwissenschaftliche Bildungsgelegenheiten häufiger umzusetzen. Die (selbstberichtete) Prozessqualität ist bei der Umsetzung der Lerngelegenheiten ebenfalls stärker ausgeprägt.

- In den naturwissenschaftlichen Kompetenzen der Kinder zeigen sich keine Unterschiede zwischen den drei Kita-Untersuchungsgruppen. Auch der Vergleich von Kindern, die hauptsächlich von fortgebildeten bzw. von nicht fortgebildeten Fachkräften betreut werden, zeigt keine Unterschiede. Kinder in Einrichtungen mit einem expliziten naturwissenschaftlichen Schwerpunkt weisen dagegen eine höhere naturwissenschaftliche Lernfreude und Selbstwirksamkeitserwartung auf als Kinder aus Einrichtungen ohne naturwissenschaftlichen Schwerpunkt.

(3) Im Fokus der dritten Fragestellung des Projektes standen die Zusammenhänge zwischen den unterschiedlichen Wirkungsebenen. Insgesamt lassen sich die Ergebnisse wie folgt zusammenfassen:

- Fortbildungen stehen in einem positiven Zusammenhang mit den Kompetenzen der Fachkräfte in den Naturwissenschaften.

- Die naturwissenschaftlichen Kompetenzen der Fachkräfte und insbesondere die motivationalen Kompetenzaspekte hängen wiederum positiv mit der Prozessqualität und Häufigkeit naturwissenschaftlicher Bildungsangebote zusammen.

- Motivationale Kompetenzaspekte stehen in einem positiven Zusammenhang mit der Implementation der Naturwissenschaften als Einrichtungskonzept. Je stärker die naturwissenschaftliche Bildung in der Einrichtung verankert ist, desto höher fällt die naturwissenschaftsbezogene Motivation der Fachkräfte aus. Dies könnte darauf hindeuten, dass Fachkräfte in ihrer Motivation über 
die eigenen Fortbildungseffekte hinaus von der Verankerung der Naturwissenschaften in ihrer Einrichtung profitieren.

Ausschlaggebend für das Wissen der Kinder in den Naturwissenschaften scheinen regelmäßige naturwissenschaftliche Lerngelegenheiten bei einem Minimum an Prozessqualität zu sein. Regelmäßige Lernangebote bei geringer Prozessqualität bzw. seltene, aber qualitativ hochwertige Lernangebote allein stehen in keinem positiven Zusammenhang mit dem kindlichen Wissen in den Naturwissenschaften. 


\section{Folgerungen für die Stiftungsarbeit}

Die Arbeit der Stiftung wirkt sich nach den Ergebnissen dieser Studie in erster Linie auf die Qualität der Implementation des Bildungsbereichs Naturwissenschaften im pädagogischen Konzept der Einrichtung aus. Denkbar ist, dass die höhere Fortbildungsintensität sowie die Rolle des Bildungsbereichs Naturwissenschaften für die Einrichtung sich auch auf die kontinuierliche Weiterentwicklung des Bildungsbereichs Naturwissenschaften, die professionelle Weiterentwicklung des Teams und auf das Selbstverständnis, eine „HdkF-Kita zu sein“, zurückführen lässt. Eine wichtige Rolle spielen sicherlich auch die ausgeprägteren Fähigkeitsüberzeugungen fortgebildeter Fachkräfte. Dies ist ein wichtiger Befund und stützt das Konzept der Stiftungsarbeit, da andere Forschungsarbeiten gezeigt haben, dass die Qualität der Implementation des Bildungsbereichs Naturwissenschaften in der Einrichtung grundlegend für Prozessqualität bei der Umsetzung naturwissenschaftlicher Inhalte ist. Diese wiederum ist die zentrale Qualitätskomponente mit Blick auf mögliche Effekte für die kindliche Entwicklung.

Auch zeigt sich, dass Fortbildungen ein grundsätzlich geeigneter Weg sind, um die professionelle Kompetenz zu fördern. Allerdings scheint die Teilnahme an der Initiative „Haus der kleinen Forscher“ nicht anders auf die erhobenen Wissenskomponenten, motivationalen Orientierungen oder Überzeugungen zu wirken als andere Fortbildungen. Beispielsweise schneiden die fortgebildeten pädagogischen Fachkräfte im Test zum prozessbezogenen Wissen, u. a. dem Forschungskreis, nicht besser ab als durch andere Anbieter fortgebildete Fachkräfte, obwohl dieses Wissen ein wichtiges Merkmal des Ansatzes ist, der vom „Haus der kleinen Forscher"vertreten wird. Allerdings kann für Fortbildungen außerhalb der Stiftung nicht nachgezeichnet werden, inwieweit hier inhaltlich ggf. ähnliche Schwerpunktsetzungen gemacht werden.

Das Potenzial eines kontinuierlichen und strukturierten Fortbildungsangebots ließe sich vermutlich steigern, wenn die Fortbildungsrate gesteigert würde. So haben auch die pädagogischen Fachkräfte der HdkF-Kitas im Mittel nur an 2.3 Fortbildungen in den letzten 3 Jahren teilgenommen, die, zieht man die Angaben der Fortbildungsanbieter heran, 5-7 Stunden pro Thema umfassen. Legt man internationale Fortbildungsstudien zugrunde, entspricht dies lediglich einem geringen zeitlichen Umfang. Insbesondere im Hinblick auf das fachdidaktische Wissen ist anzumerken, dass der geringe Zeitumfang der Fortbildungen möglicherweise mit den nur geringen Unterschieden im fachdidaktischen Wissen von fort- bzw. nicht fortgebildeten Fachkräften einhergeht. So nehmen die Erarbeitung fachlicher Hintergründe sowie die Erprobung von Versuchen oft so viel Zeit ein, dass die 
zusätzliche Thematisierung fachdidaktischer Inhalte in der kurzen Fortbildungsdauer nur wenig Raum findet.

Neben der Frage des Umfangs der Fortbildungen erscheint es für die Weiterentwicklung der Arbeit der Stiftung notwendig, Erkenntnisse über Schwerpunkte und die Qualität der Fortbildungen zu erhalten. Aus den vorliegenden Daten ist beispielsweise nicht zu erkennen, ob der Schwerpunkt einzelner Fortbildungsveranstaltungen eher auf fachspezifischen oder auch stärker allgemeinpädagogischen oder motivationalen Aspekten liegt. Angesichts der angenommenen Heterogenität der Fortbildnerinnen und Fortbildner (Multiplikatorinnen und Multiplikatoren) erscheinen genauere Erkenntnisse über die realisierten Fortbildungen (Schwerpunkte, Qualität) notwendig, um hier eine entsprechende Qualitätssicherung betreiben zu können. Entsprechend sind die Ansätze der Stiftung, die Kompetenz der Multiplikatorinnen und Multiplikatoren zu untersuchen, sehr zu begrüßen.

In der Studie EASI Science konnten - außer in Einrichtungen mit einem expliziten naturwissenschaftlichen Schwerpunkt - kaum Zusammenhänge auf der Ebene der Kinder gefunden werden. Dieser Befund mag auf den ersten Blick enttäuschend sein, letztlich überrascht er aber nicht. So finden sich auch in vielen anderen Studien keine Befunde bis zum Ende der Wirkungskette, da hier viele unterschiedliche und zum Teil wechselseitig interagierende Einflussfaktoren zum Tragen kommen, die in dieser Studie nicht umfassend erfasst werden konnten. Hier müssen vor allem der große Einfluss familiärer Faktoren auf individueller Ebene sowie der enorme Spielraum bei der Umsetzung naturwissenschaftlicher Bildung und damit die Variation der Umsetzungsqualität auf Einrichtungsebene berücksichtigt werden. Auch muss bedacht werden, dass die fortgebildeten pädagogischen Fachkräfte, die ihren Schwerpunkt auf den Bildungsbereich Naturwissenschaften gelegt haben, ggf. nicht immer die hauptverantwortlichen Fachkräfte für die jeweilige Gruppe sind; stattdessen bieten sie möglicherweise gezielt Angebote im Rahmen eines Vorschulprogramms an, welches nicht für alle Kinder der Einrichtung geöffnet ist. Fortbildungsstudien, in denen Effekte auf der Kindebene nachgewiesen werden konnten, sind häufig Interventionsstudien, bei denen Fortbildungsinhalte und Kindertests spezifisch aufeinander abgestimmt werden. Im Rahmen von EASI Science umfassen dagegen sowohl die Tests zum fachbezogenen Wissen der pädagogischen Fachkräfte als auch der Test zur Erfassung des kindlichen Wissens verschiedene Themenbereiche, um das Wissen über Themen der unbelebten Natur breit zu erfassen. Zudem erfassen die Tests grundlegende Konzepte, die in verschiedenen Themenbereichen und entsprechend verschiedener Fortbildungsthemen aufgegriffen werden können, z. B. Materialien. Der Bereich des prozessbezogenen Wissens umfasst Aspekte des grundlegenden naturwissenschaftlichen Denkens auf der Kind- und der Fachkraftebene. Trotz der Breite der Inhalte gibt es bewusste Überschneidungen der Tests mit den Fortbildungs- 
inhalten der Angebote der Stiftung „Haus der kleinen Forscher“, beispielsweise beim Forschungskreis (Stiftung Haus der kleinen Forscher, 2013a), welche sich weder auf Kind- noch auf Fachkraftebene im Vergleich der Untersuchungsgruppen nachweisen ließen. Ermutigend ist der beobachtete positive Interaktionseffekt von berichteter Qualität und Häufigkeit naturwissenschaftlicher Lerngelegenheiten auf das Wissen der Kinder. Eine vertiefende Untersuchung der Kopplung von Fortbildungsangebot, Umsetzungsqualität und Wissenseffekten auf Kindebene erscheint deshalb sinnvoll. 



\section{Early Steps into Science and Literacy - EASI Science-L}

Naturwissenschaftliche Bildung in der Kita: Gestaltung von Lehr-Lern-Situationen, sprachliche Anregungsqualität und sprachliche sowie naturwissenschaftliche Fähigkeiten der Kinder

Astrid Rank, Anja Wildemann, Sabina Pauen, Andreas Hartinger, Sabrina Tietze, Rahel Kästner

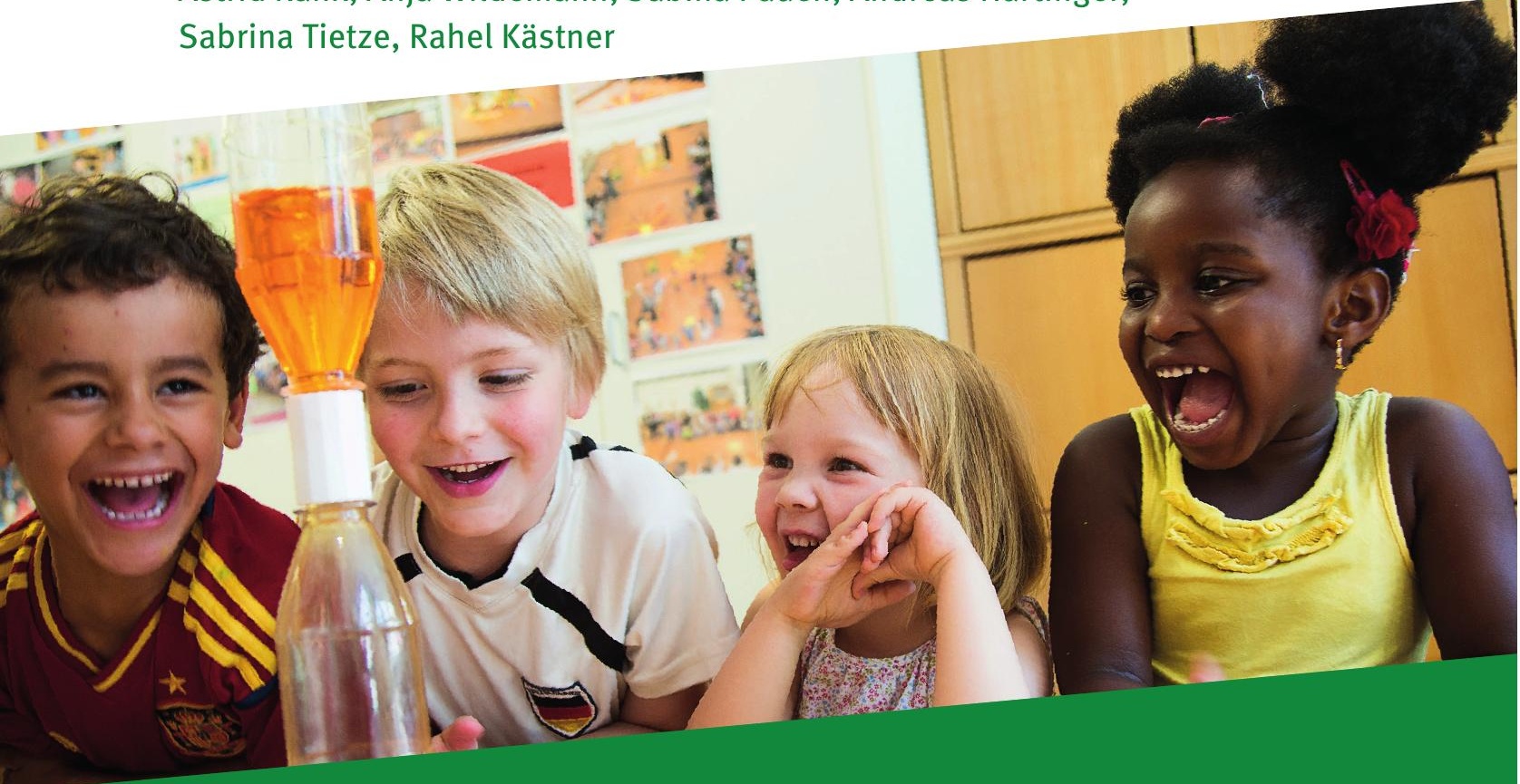





\section{Early Steps into Science and Literacy - EASI Science-L Teil 1}

Wirkungen sprachlicher Anregungsqualität in naturwissenschaftlichen Bildungsangeboten auf die sprachlichen Fähigkeiten von Vorschulkindern

Astrid Rank, Anja Wildemann, Andreas Hartinger, Sabrina Tietze

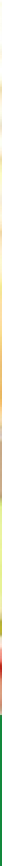

1 Hintergrund der Studie

2 Fragestellungen der Studie

3 Studiendesign und Methoden

4 Ergebnisse

5 Diskussion

6 Zusammenfassung der zentralen Ergebnisse

7 Fazit und Empfehlungen 


\section{Hintergrund der Studie}

Schon seit längerer Zeit steht die Hypothese im Raum, dass naturwissenschaftliche Situationen in der Kita auch sprachliche Bildungswirkungen haben können (vgl. die Frankfurter Erklärung zur frühen sprachlichen und naturwissenschaftlichen Bildung (Stiftung Haus der kleinen Forscher, 2015b)). Bisherige Befunde aus dem Schulkontext deuten auf eine wesentliche Rolle der Sprache bei der Entwicklung fachlicher Konzepte hin (Grießhaber, 2013). Allerdings gilt Sprachförderung in Kitas trotz hohen Aufwands als verhältnismäßig wirkungslos (Egert, 2015; Egert \& Hopf, 2016) und die sprachliche Interaktion zwischen Kind und Fachkraft als ungünstig (Weinert, Ebert, Lockl \& Kuger, 2012; Fried, 2011). Es gibt einige Ansätze zur Verbesserung der sprachlichen Interaktion im Alltag (Beller, Merkens \& Preissing, 2007; Weitzmann \& Grennberg, 2002). Untersuchungen sprachlich und kognitiv herausfordernder Interaktionen im Rahmen spezifischer Bildungsangebote hingegen beginnen gerade erst (etwa Li, 2017).

So hat auch die Stiftung „Haus der kleinen Forscher“ 2013 in der Ausschreibung für die Begleitstudien klar benannt: „Eine zusätzliche Fragestellung zielt auf mögliche sprachliche Bildungswirkungen durch naturwissenschaftliche Bildungsangebote. Hierbei soll untersucht werden, ob bzw. bei welchen Phasen naturwissenschaftlichen Vorgehens (vgl. z. B. ,Forschungskreis ${ }^{186}$, Marquardt-Mau, 2004, 2011; Stiftung Haus der kleinen Forscher, 2013) Effekte im Hinblick auf sprachliche Kompetenzen, insbesondere auch bei Kindern mit Deutsch als Zweitsprache, erzielt werden können; und inwiefern etwaige sprachliche Bildungswirkungen von der Qualität der sprachlichen Begleitung des Forschens abhängen.“ Genau dieser Fragestellung widmet sich die vorliegende Studie EASI Science-L (Early Steps into Science and Literacy). Als sprachliche Kompetenz wird hierbei vor allem die sog. „Bildungssprache“ betrachtet.

\subsection{Bildungssprache und durchgängige Sprachbildung}

Die Beherrschung von Bildungssprache gilt als zentrale Voraussetzung für schulischen Erfolg (Ahrenholz, 2010; Gogolin \& Lange, 2010; Schleppegrell, 2004). Dabei verändern und steigern sich die (bildungs-)sprachlichen Anforderungen im Laufe der Bildungsbiografie. Insbesondere Kinder mit Deutsch als Zweitsprache benötigen eine langfristige, durchgängige sprachliche Bildung, die im besten Falle vorhandene Bildungsungleichheiten kompensiert (Gogolin \& Lange, 2010).

18 Das didaktische Konzept einer naturwissenschaftlichen Grundbildung zum Forschen mit Kindern und das damit verbundene Modell des Forschungskreislaufs wurde von Frau Marquardt-Mau entwickelt (2004, 2011) und im pädagogischen Ansatz der Stiftung „Haus der kleinen Forscher“ adaptiert. 
Das Konstrukt Bildungssprache wird im aktuellen Diskurs von verschiedenen Disziplinen beleuchtet, u. a. der Soziologie, Erziehungswissenschaft, Fremdsprachendidaktik und den verschiedenen Fachdidaktiken (Ortner, 2009; zusammenfassende siehe Wildemann \& Fornol, 2016; Rank, 2016). Bildungssprache ist dabei, rekurrierend auf die Registertheorie von Halliday (1978), als sprachliches Register zu verstehen (Schleppegrell, 2001; 2004). Hallidays Registertheorie liegen drei grundlegende Konzepte zugrunde - field of discourse, tenor of discourse und mode of discourse. Das Wissen über diese Konzepte wird laut Halliday benötigt, um die Relevanz spezifischer linguistischer Mittel für den situativen Kontext bestimmen zu können. Field beschreibt den Inhalt oder Gegenstand, mit dem die entsprechenden Interaktionspartner sich in der Situation auseinandersetzen. Tenor beleuchtet die Beziehung zwischen den Interaktionspartnern und die Rolle, die diese in der Situation einnehmen. Mode betrifft die Relevanz der Sprache selbst im Rahmen der situativen Interaktion und deren Funktion.

Soziologisch steht die Ergründung von Chancenungleichheiten im Vordergrund. Bereits Bernstein (1964) stellte heraus, dass sich divergente Sprachkompetenzen auf schichtspezifische Unterschiede zurückführen lassen. Die schichtspezifisch unterschiedlichen Sprachkompetenzen beschrieb er mit dem restricted und dem elaborated code. Der restricted code zeichnet sich durch einfache Konstruktionen und eine eingeschränkte Ausdrucksfähigkeit der unteren Schichten aus. Der elaborated code hingegen wird als variantenreich und ausdifferenziert beschrieben. Er steht für die Sprachverwendung der sog. Mittel- und Oberschicht. Kinder der sog. Unterschicht, denen es nicht gelingt, ihre sprachlichen Fähigkeiten über den restricted code hinaus auszubauen, erzielen nach Bernsteins Theorie geringere Bildungserfolge (Bernstein, 1964; 1973). Die rein schichtspezifische Sichtweise auf sprachbezogenen Bildungserfolg, die Bernstein verfolgt, wurde im Nachgang jedoch scharf kritisiert. Vorgeworfen wird ihm bis heute, dass seine Defizithypothese eine „unvoreingenommene Analyse“ des Sprachmaterials verhindert hätte (Knobloch, 2016, S. 85).

Linguistische Forschungsarbeiten wenden sich der kategorialen Beschreibung von Bildungssprache als Register zu. Dabei findet ein Rückbezug auf Hallidays (1978) Registertheorie statt, wie beispielsweise bei Schleppegrell (2001), Gogolin und Lange (2011) oder Quehl und Trapp (2013). Ein Register versteht sich dabei als Beschreibung spezifischer Sprachvariationen, welche sich kontextabhängig unterscheiden. Die Wahl der sprachlichen Mittel ergibt sich aus der jeweiligen Situation (Halliday 1978).

Schleppegrell (2001) beschreibt unter Rückgriff auf die Registertheorie die in der Schule verwendete Sprache (language of schooling) in Abgrenzung zum alläglichen Sprachgebrauch. Diese schulische Sprache ist durch die fachspezifische Verwendung von Sprache sowie durch eine Distanzierung der Kommunika- 
tionspartner geprägt. Darüber hinaus dient die in der Schule verwendete Sprache dem Wissensaufbau abstrakter Inhalte.

Angeschlossen werden erziehungswissenschaftliche Perspektiven. Cummins (2000) untersucht Divergenzen zwischen Zweitsprachlernern und beschreibt das Konzept der basic interpersonal communicative skills (BICS) und cognitive academic language proficiency (CALP). Cummins' (2000) Konzeptualisierung trägt dazu bei, eine mögliche Erklärung dafür zu finden, weshalb zweisprachig aufwachsende Kinder trotz der anscheinend ausreichenden Sprachfähigkeiten (BICS) in der Schule häufig Schwierigkeiten haben, dem Unterricht zu folgen. Um fachliche Kompetenzen und Wissen aufbauen zu können, wird die CALP als zentrales Medium angenommen. Die BICS hingegen werden vor allem kontextgebunden eingesetzt.

Neben der Disziplin ist bei der Beschreibung des Konstruktes Bildungssprache auch die jeweilige Zugangsweise von Relevanz, welche in den Ausführungen von Morek und Heller (2012) Berücksichtigung findet. Diese betrachten Bildungssprache funktional und beziehen dabei die unterschiedlichen Disziplinen mit ein. Diese funktionale Sichtweise auf Sprache lässt sich ebenfalls auf Halliday zurückführen. Halliday (1978) betrachtet jede Form des Sprechens und jede Form der Sprache als funktional, wobei dabei stets der soziale Kontext entscheidend ist, der die Wahl, Form und Ausdrucksweise bestimmt. Morek und Heller (2012) stellen unter einer heuristischen Zugangsweise zunächst drei Funktionen von Bildungssprache heraus. Die erste besteht in einer kommunikativen Funktion, die „Bildungssprache als Medium von Wissenstransfer“ (S. 70) reflektiert. Daran angeschlossen und nicht strikt abzugrenzen ist die epistemische Funktion, die „Bildungssprache als Werkzeug des Denkens“(S. 74) versteht. „Bildungssprache als Eintritts- und Visitenkarte“ (S. 77) diskutiert die ungleichheitsreproduzierende und sozialsymbolische Funktion von Bildungssprache.

Bildungssprache dient im Kontext der kommunikativen Funktion von Bildungssprache dem Wissensaufbau. Schleppegrell (2001) und Ortner (2009) beschreiben diese Art von Wissen als Weltwissen, welches durch den Gebrauch von Bildungssprache auf- und ausgebaut wird. Auch Habermas (1977) schreibt Bildungssprache die Funktion zu, Wissen aufzubauen, und zwar bei jedem, der sich ein Orientierungswissen verschaffen möchte.

Ungleiche Bildungsvoraussetzungen (Cummins, 2000) und kognitive Bedingungen für den Erwerb und Gebrauch von Bildungssprache (Feilke, 2012) werden im Rahmen der epistemischen Funktion von Bildungssprache aufgegriffen (Morek und Heller, 2012). Neben Cummins (2000) Konzeptionalisierung der CALP und BICS führt auch Quasthoff (2009) aus, dass sprachliche Kompetenzen eine Schlüsselqualifikation für Lernprozesse darstellen und vorherrschende Bildungsbenachteiligungen erklären. Entscheidend sind „die externen und internen Res- 
sourcen, die Kinder in unterschiedlicher Weise zur Verfügung haben und nutzen, um sich die jeweiligen Kompetenzen anzueignen und sie auszubauen“ (Quasthoff, 2009, S. 95).

Kognitive Bedingungen für den Erwerb und Gebrauch von Bildungssprache beschreibt Feilke (2012), unabhängig von der sozialen Ausgangslage. Die Verwendung bildungssprachlicher Mittel braucht immer ein „abstrahierendes Sprachdenken“ (Feilke, 2012, S. 6), das ohne ausreichende kognitive Fähigkeiten nicht möglich wäre. Mit dem zunehmenden Gebrauch bildungssprachlicher Mittel (rezeptiv und produktiv) zur Wissensrepräsentation erweitern die Lernenden ihr fachliches Repertoire an Können und damit wiederum ihre bildungssprachlichen Fähigkeiten. „Wer Bildungssprache adäquat verwenden kann, der ist auch in der Lage zu den damit in Zusammenhang stehenden komplexen kognitiven Operationen (wie z. B. Abstraktion, Verallgemeinerung, Kausalität)“ (Morek \& Heller, 2012, S. 75).

Im Rahmen der epistemischen Funktion wird auch die sozialsymbolische Funktion aufgegriffen, die die eigene Identifikation mit sprachlichen Registern reflektiert.

„Wer Bildungssprache verwendet, gibt sich als member einer bildungsnahen akademisch orientierten community (zu erkennen), und definiert die vorliegende Kommunikationssituation als eine, in der man sich auf gebildete Weise an der Darlegung oder Diskussion eines Sachverhaltes beteiligt“ (Morek \& Heller, 2012, S. 79).

Dabei ist nicht jeder Lerner bzw. jede Lernerin dazu bereit, sich bewusst auf das geforderte sprachliche Register einzulassen (Snow \& Uccelli, 2009). Treten zwischen der eigenen Selbst- und Fremddarstellung und der Identifikation mit der Wahl sprachlicher Mittel Diskrepanzen auf, kann dies eine bewusste Distanzierung zur Folge haben und sich hinderlich sowohl auf den Gebrauch als auch den Erwerb bildungssprachlicher Kompetenzen auswirken (Preece, 2009).

Bedingungen zur Förderung von Bildungssprache kann man anhand des Konzepts der Durchgängigen Sprachbildung verdeutlichen. Dieses Konzept geht auf das von 2004 bis 2009 durchgeführte Modellprogramm FörMig zurück (Gogolin \& Lange, 2010). FörMig fokussierte in zehn Bundesländern die Entwicklung und Erprobung von Ansätzen, die die sprachliche Bildung von Kindern und Jugendlichen mit Migrationshintergrund optimal fördern.

Bei der Konzeption des Konzeptes Durchgängige Sprachbildung wird dabei auf drei zum Teil auch aus der Fremdsprachendidaktik etablierte englischsprachige Ansätze rekurriert (Gogolin \& Lange, 2010, S. 15): 
1. Language Across the Curriculum (LAC): In allen Fächern, durch das Curriculum hinweg, wird Sprachförderung durchgeführt.

2. Content and Language Integrated Learning (CLIL): Im Fachunterricht erfolgt gezielt der Fremdsprachenerwerb.

3. Language Awareness: umfasst die Förderung von Sprachgefühl und Sprachbewusstheit (Awareness).

Durchgängige Sprachbildung hat den sukzessiven Aus- und Aufbau bildungssprachlicher Kompetenzen zum Ziel (Gogolin \& Lange, 2010). Daraus ergeben sich die beiden grundlegenden Elemente durchgängiger Sprachbildung - Durchgängigkeit und Bildungssprache. Mit Durchgängigkeit ist dabei die konkrete Umsetzung gemeint und mit Bildungssprache der Gegenstand sprachlicher Bildung (Gogolin \& Lange, 2010; vgl. zusammenfassend Rank \& Wildemann, 2015). Speziell fokussiert werden Kinder, die Deutsch als Zweitsprache erwerben. „Eine planvolle und bewusste Förderung dieser sprachlichen Fähigkeiten soll für die Kinder und Jugendlichen, die Deutsch als Zweitsprache sprechen, Grundlagen für eine erfolgreiche Bildungsbiografie schaffen“ (Gogolin \& Lange, 2010, S. 14).

\subsection{Bildungssprache im Kontext der kindlichen Sprachentwicklung}

Bereits mit Schuleintritt wird Bildungssprache vorausgesetzt. Somit stellt sich die Frage, ob bildungssprachliche Kompetenzen bereits im Vorschulalter ausgeprägt werden können. Aus Sicht der kindlichen Sprachentwicklung ist die Aneignung bildungssprachlicher Muster bereits im Vorschulalter möglich, allerdings abhängig vom Input (siehe dazu Juska-Bacher \& Nodari, 2015; Leseman, Scheele, Mayo \& Messer, 2007). Kindliche Sprachentwicklung wird häufig auf drei Ebenen kategorisiert. Auf der lexikalischen Ebene finden vorrangig der Auf- und Ausbau des mentalen Lexikons und damit eine Ausdifferenzierung des Wortschatzes statt. Grammatikalische Strukturen auf der Wort- und Satzebene werden auf der morphologisch-syntaktischen Ebene erworben. Die dritte Ebene - die pragmatische - beinhaltet das Verständnis darüber, Sprache situativ angemessen als Kommunikationsmittel zu gebrauchen (Albers, 2009; Dittmann, 2002; Kany \& Schöler, 2014; Wildemann, Rank, Hartinger \& Sutter, 2016; Rank \& Wildemann, 2016).

Auf lexikalischer Ebene wird ein grundlegender Wortschatz mit steigenden Sprachkompetenzen immer weiter ausdifferenziert, bis hin zur Verwendung von komplexen, abstrakten Begriffen. Dieser Erwerb (rezeptiv und produktiv) komplexer, bildungssprachlicher Begriffe dauert bis ins späte Schulalter hinein (Szagun, 2013) und ist auch im Erwachsenalter nicht abgeschlossen (Meibauer \& Rothwei- 
ler, 1999). Auf der lexikalischen Ebene lassen sich im Laufe der konventionellen Sprachentwicklung bereits früh erste bildungssprachliche Strukturen beobachten. So werden z. B. bereits im Alter von 28 Monaten Funktionswörter wie Hilfsverben, Artikel, Konjunktionen und Präpositionen erworben und in der Regel produktiv umgesetzt (Dittmann, 2002; Weinert \& Grimm, 2012; Wildemann et al., 2016).

Mit der Produktion von Zweiwortäußerungen wird in der Regel der Beginn der morpho-syntaktischen Entwicklung beschrieben (Kany \& Schöler, 2014). Die Verwendung von Pluralformen sowie die Entwicklung von Kasus, Numerus, Genus und Verbflexion entwickeln sich zwischen dem zweiten und fünften Lebensjahr (Dittmann, 2002; Szagun, 2013; Weinert \& Grimm, 2012). Die als besonders bildungssprachlich geltenden Passivkonstruktionen sind bereits zwischen dem dritten und vierten Lebensjahr zu beobachten (Kany \& Schöler, 2014).

Die letzte Ebene betrifft die Entwicklung pragmatischer Kompetenzen. Sie beinhaltet die Fähigkeit, Sprache in unterschiedlichen Kontexten angemessen zu gebrauchen (Grimm, 1999; Rickheit, Sichelschmidt \& Strohner, 2007), sowie den korrekten Einsatz bestimmter Diskursfunktionen, z. B. das Beschreiben, Benennen, Erklären oder Vermuten. In der ersten Klasse werden einfache Diskursfunktionen wie das Beschreiben und Benennen häufiger realisiert als komplexere, wie beispielsweise Vermutungen zu äußern oder Hypothesen aufzustellen (Hövelbrinks, 2014). Grundsätzlich geht der Verwendung von Diskursfunktionen die narrative Entwicklung voraus, die sich - abhängig von der Anregung - erst im Laufe der Grundschulzeit ausdifferenziert (siehe dazu Becker, 2013).

Grundlegende sprachliche Kompetenzen, darin eingeschlossen sind auch bereits bildungssprachliche Strukturen, lassen sich somit bereits früh im konventionellen Sprachentwicklungsverlauf beobachten. Voraussetzungen für eine frühe Ausbildung komplexer bildungssprachlicher Strukturen sind damit bereits im Vorschul- und frühen Schulalter vorhanden (Gogolin, 2010; Wildemann et al., 2016).

\subsection{Sprachliche Bildung durch naturwissenschaftliches Lernen}

Fachbezogenes Lernen scheint den Aufbau sprachlicher Kompetenzen besonders zu fördern, da fachbezogene Arbeits- und Handlungsweisen oftmals mit Sprache einhergehen und Sprache fordern (Beese \& Benholz, 2013; Röhner, Blümer, Hopf, Li \& Hövelbrinks, 2009; Rank, Wildemann \& Hartinger, 2016). Verschiedene Forschungsprojekte liefern hierzu ermutigende Ergebnisse.

Im Projekt „Sprachförderung von Migrantenkindern im Kontext frühen naturwissenschaftlich-technischen Lernens“ (Röhner et al., 2009) wurden 80 Kindern mit Migrationshintergrund aus dem Elementar- und Primarbereich gefördert. Die 
sprachliche Förderung wurde dabei eng mit naturwissenschaftlichen Inhalten verknüpft. Davon ausgehend, dass in naturwissenschaftlich-technischem Lernen besonderes Potenzial für sprachliches Lernen steckt, wurden insgesamt 18 Fördereinheiten durchgeführt. Durch handlungsbegleitendes Sprechen sollten Naturphänomene wahrgenommen werden, ein sprachlicher Austausch initiiert und relevante

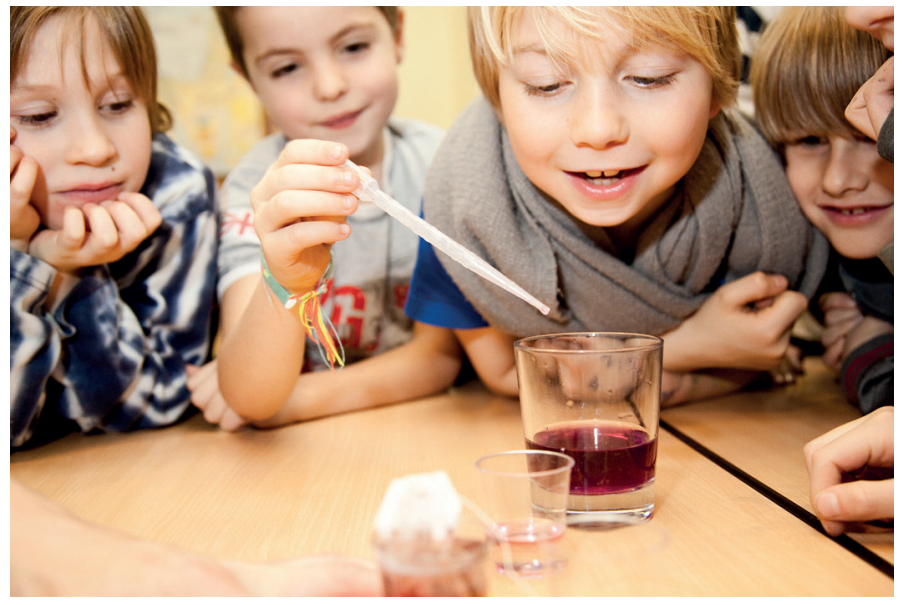

Diskurskompetenzen zum Einsatz kommen. Ziel war, die Sprache als Medium des Lernens zu verstehen. Die teilnehmenden 80 Kinder wurden in zehn Gruppen aufgeteilt und nahmen an insgesamt 18 Experimentiereinheiten teil. Diese fanden wöchentlich statt und behandelten die drei Themenfelder Elektrizität und Magnetismus; Optik, Licht und Farben sowie Materialeigenschaften. Für 48 Kinder (21 aus der Elementarstufe und 27 aus der Primarstufe) wurden videografierte Einheiten transkribiert und analysiert (Röhner et al., 2009). Es zeigte sich, „,dass sich die implizite Form der handlungsbegleitenden Sprachförderung positiv auf die Sprachleistungen der Migrantenkinder auswirkt“" (Röhner et al., 2009, S. 90). Um umfangreichere Äußerungen von den Kindern zu provozieren, sind insbesondere das Erklären und Beschreiben geeignet. Insgesamt profitieren sowohl die leistungsschwachen als auch die leistungsstarken Kinder von den Fördereinheiten. Durch eine angeschlossene Nacherhebung sechs Monate nach Abschluss der Fördereinheiten konnte darüber hinaus gezeigt werden, dass die Lerninhalte effektiv und langfristig von den Kindern aufgenommen wurden (Röhner et al., 2009).

Die Notwendigkeit sprachlicher Mittel für fachliche Inhalte stellt auch Patterson (2001) heraus. Im Rahmen einer Interventionsstudie wurden Texte von sprachlich schwachen Schülerinnen und Schülern von der zweiten bis zur sechsten Jahrgangsstufe analysiert. Dabei zeigte sich in der Analyse, dass die weniger kognitiv anspruchsvolle Diskursfunktion Beschreiben in den Texten dominiert, wohingegen kaum Erklärungen aufzufinden sind. Eine Intervention mit zwölf Schülerinnen und Schülern umfasste das Zur-Verfügung-Stellen von Konnektiven, die benötigt werden, um fachliche Inhalte zu erklären. In den anschließend produzierten Texten konnten nun weitaus mehr Erklärungen festgestellt werden. Um der kognitiv anspruchsvollen Leistung gerecht zu werden, fachliche Inhalte zu erklären, braucht es demnach nachweislich die notwendigen sprachlichen Mittel. 
Die Interventionsstudie BeFo - Bedeutung und Form (2009-2012) schließt sich - mit dem Ziel „fachbezogene und sprachsystematische Förderung in der Zweitsprache“ (Rösch, 2014, S. 195) zu untersuchen - an Vorarbeiten der Studie zum Jacobs-Sommercamp an. Im Rahmen des Jacobs-Sommercamps wurde 2004 das Ziel verfolgt, empirisch fundiert herauszuarbeiten, wie Sprachförderprogramme speziell für Kinder mit Deutsch als Zweitsprache effektiv zu gestalten sind. Die stärksten Leistungen zeigten dabei die Kinder, die sowohl die implizite als auch die explizite Förderung erhielten. In Bezug auf die Grammatik war der Leistungsvorsprung nach drei Monaten zwar nicht mehr vorhanden, im Bereich des Lesens konnte er jedoch gehalten werden (Rösch, 2006).

Die beiden Ansätze, Focus on Form (FoF) (explizite Sprachförderung) und Focus on Meaning (FoM) (implizite Sprachförderung), wurden im Rahmen des oben genannten BeFo-Projektes weiterentwickelt und auf ihre Wirksamkeit hin überprüft. Focus on Form beschreibt dabei eine explizite Form der Sprachförderung im Rahmen eines sprachsystematischen DaZ-Unterrichtes, Focus on Meaning steht für eine Form impliziter Sprachförderung durch fachbezogenen Unterricht in den Fächern Mathematik und Sachunterricht (Rösch, 2014). Die sprachsystematische Förderung (FoF) wurde mit 125 Kindern durchgeführt, die fachspezifische Förderung mit 127 - daneben gab es eine Wartekontrollgruppe, bestehend aus 110 Kindern. Zu vier Messzeitpunkten wurde der Sprachentwicklungsstand der Schülerinnen und Schüler erhoben. Zum ersten Messzeitpunkt befanden sich die Kinder in der dritten Jahrgangsstufe. Die Ergebnisse zeigen, dass bei allen Gruppen in den Bereichen Leseverstehen, Wortschatz und Grammatik ein Leistungszuwachs vorliegt. Für die Bereiche Grammatik und Wortschatz konnten keine differenziellen Effekte herausgestellt werden. Die fachbezogene Förderung in Mathematik deutet auf Fördereffekte hin. Die durch das Jacobs-Sommercamp angenommenen positiven Fördereffekte der beiden Ansätze FoF und FoM können nach den aktuellen Analysen nicht bestätigt werden. Es bleibt u. a. der Frage nachzugehen, ob Intensivphasen effektiver sind als eine Förderung, die ein Jahr anhält (Rösch, 2014).

Anhand einer Stichprobe von 80 Schülerinnen und Schülern der vierten und fünften Jahrgangsstufe untersuchte Runge (2013) die Verwendung bildungssprachlicher Verben im Kontext naturwissenschaftlicher Aufgabenstellungen. Im Zentrum der Analysen stehen verbale Äußerungen, die die Schülerinnen und Schüler bei der Beschreibung eines Videoclips zum Thema Strom produzierten. Ausgangsfrage dabei ist: „Wie kann man sich selbst eine Lampe bauen?“ (Runge, 2013, S. 155). Die Analyse der genutzten Verben einer für die Beschreibung zentralen Sequenz (Anschließen des Kabels an die Glühlampe) zeigt, dass „,bei der Verbalisierung der Sequenz vorzugsweise Präfix- und Partikelverben genutzt werden“ (Runge, 2013, S. 162). Unspezifische Verben wie machen oder tun werden deutlich häufiger eingesetzt als spezifische wie z. B. befestigen oder festmachen. 
Spezifische (Partikel-)Verben (binden, schreiben, wickeln) werden am dritthäufigsten verwendet. Verben, mit denen der Einbezug der Handlung selbst einhergeht (befestigen, anschließen), kommen im Vergleich zu unspezifischen Verben und (Partikel-)Verben weniger als halb so häufig vor. Am seltensten kommen Verben wie setzen und bauen mit Simplex (zusammen) zum Einsatz. Der Feinanalyse ging eine globale Einschätzung voraus, indem die Schülerproduktionen in stark, mittel und schwach eingestuft wurden. Die global als stark eingestuften Schülerinnen und Schüler gebrauchen weniger unspezifische Verben. Bei den global schwach eingeschätzten Schülerinnen und Schülern ist ein Auslassen des Verbes auffallend, oder es erfolgt überhaupt keine Beschreibung. Aufgrund einer unterrichtlichen Fokussierung werden von den Schülerinnen und Schülern der mittleren Stufe verstärkt die komplexen Verben wie verbinden und anschließen verwendet. Insgesamt wird bei dieser Gruppe auch auf zahlreiche unspezifische Verben zurückgegriffen (Runge, 2013). Dieses Ergebnis bestätigt die enge Verknüpfung sprachlicher und fachlicher Inhalte sowie die Bedeutung des Unterrichtes selbst. Weiterführend werden bei den Jungen bessere Leistungen als bei den Mädchen festgestellt. Dies wird darauf zurückgeführt, dass Jungen „generell ein größeres, auch außerschulisches Interesse“ (Runge, 2013, S. 171) am Themengebiet haben und die Gruppe der Mädchen höchstwahrscheinlich lediglich im Unterricht damit konfrontiert wird. Daraus lässt sich weiterführend eine gewisse Bedeutung fachlicher Kenntnis folgern.

Eine Möglichkeit, sprachliches und fachliches Lernen optimal miteinander zu verknüpfen, wird im Scaffolding-Prinzip gesehen (Quehl \& Trapp, 2013). Das Bild des Scaffolding (,ein Gerüst bauen“) verdeutlicht, „wie Lernende in der Interaktion mit einem wissenden Anderen Problemlösungen finden, zu denen sie selbstständig noch nicht in der Lage sind“ (Quehl, 2010, S. 28). Dieses Gerüst kann sowohl sprachlich als auch inhaltsbezogen sein, es wirkt behutsam unterstützend, schränkt die Eigenaktivität des Lernenden nicht ein und wird nach und nach zurückgenommen.

\subsection{Naturwissenschaftliches Lernen und Sprachbildung im Elementarbereich}

Die gezielte Förderung bildungssprachlicher Kompetenzen durch naturwissenschaftliche Bildungsangebote im Elementarbereich ist bislang wenig erforscht. Dass fachliches und sprachliches Lernen eng miteinander verbunden sind, kann durch die oben dargestellten Studien aus dem Primar- und Sekundarbereich sowie aus der Fremdsprachenforschung belegt werden. 
Neben der sprachlichen beginnt auch die naturwissenschaftliche Bildung bereits im Elementarbereich. Wie im Folgenden dargestellt, liegen Erkenntnisse vor, wie naturwissenschaftliches Lernen mit sehr jungen Kindern umgesetzt werden kann, welche Bedingungen dafür gegeben sein müssen und welche Bedeutung forschendes Lernen für den Aufbau wissenschaftlichen Denkens hat. Eine grundsätzliche Verknüpfung mit dem Aufbau sprachlicher Kompetenzen ist dabei anzunehmen. Bereits im Elementarbereich „werden pädagogische Diskurse geführt (z. B. Wissensgespräche) und der Wortschatz erweitert und damit die Kinder zu bildungssprachlichen Mustern hingeführt“ (Gogolin \& Lange, 2010, S. 16).

Eine weitere Voraussetzung besteht in der Tatsache, dass bereits Elementarkinder über die Grundlagen wissenschaftlichen Denkens verfügen (Croker \& Buchanan, 2011; Lück, 2009). Croker und Buchanan (2011) überprüften das Hypothesenwissen von 144 Kindern im Alter von 3 bis 11 Jahren. Die Kinder erhielten zunächst eine bildbasierte Aufgabe, die eine Hypothese zur Mundhygiene erforderte; dabei wurden sie aufgefordert, ihre Antwort zu begründen. Anschließend erhielten die Kinder Informationen, die entweder im Einklang oder im Widerspruch zu ihren eigenen Vorstellungen standen. Insgesamt wurden die Kinder mit vier Problemstellungen konfrontiert. Die Frage an die Kinder bestand darin, eine Möglichkeit zu erkennen, wie die Person ihre eigenen Überzeugungen prüfen kann. Dabei fiel es einem Großteil der Kinder leicht herauszufinden, dass die Person ihre Annahme nur testen kann, wenn sie die eine Gewohnheit aufgibt, während sie die anderen beibehält, um anhand der Veränderungen festzustellen, ob die Mundhygiene deshalb gut oder schlecht ist. Dabei spielen das vorhandene Vorwissen sowie die Übereinstimmung mit den eigenen Vorstellungen bei den Kindern im Elementarbereich eine wesentliche Rolle für die Fähigkeit des wissenschaftlichen Argumentierens.

„The pattern of results for children's explanations of their choices suggest that they use their knowledge of factors leading to good or bad oral health when presented with a good outcome and evidence which is inconsistent with their beliefs, or when presented with a bad outcome and evidence consistent with their beliefs“ (Croker \& Buchanan, 2011, p. 420).

Das Projekt „Versuch macht klug“ (Sterner, Skolaude, Ruberg \& Rothweiler, 2014) hat zum Ziel, Kindern im Elementarbereich physikalisch-technische Phänomene näherzubringen. Dabei steht das eigenaktive Handeln, Experimentieren und Ausprobieren im Zentrum. Durch Experimentierstationen, die die Themen Kräfte, Licht, Farben, Wasser, Luft, Magnetismus und Strom behandeln, sollen die Kinder frei wählen und ihre eigenen Fragen ergründen. Die Evaluation ergab, „dass sich die Kinder ausgiebig mit einzelnen Phänomenen beschäftigen und ausdauernd 
an den Stationen arbeiten. Teilweise sind die Kinder in der Lage, Ursache-Wirkungs-Zusammenhänge zu erkennen und Abläufe vorherzusagen“ (Sterner et al., 2014, S. 37).

Bildung wird häufig eng verknüpft mit der Fachkraft-Kind-Interaktion. Dieser wird eine grundsätzlich hohe Bedeutung für die soziale, kognitive und emotionale Entwicklung von Kindern zugesprochen (Denker, 2012; Gispert, 2004; zusammenfassend Early, Maxwell, Burchinal et al., 2007).

„Kinder, deren Erzieherinnen aktives Interesse an den kindlichen Aktivitäten zeigen und ihnen hinreichend Unterstützung zukommen lassen, zeigen ein ausgeprägtes und angstfreies Explorationsverhalten, vermehrt selbstinitiiertes Spielen, positive Interaktionen mit Gleichaltrigen, mehr Sozialkompetenz sowie eine fortgeschrittenere sprachliche und kognitive Entwicklung“(Gispert, 2004, S. 57).

Die Initiative „Haus der kleinen Forscher“ bietet im Rahmen ihres Weiterbildungsprogramms für frühpädagogische Fachkräfte eine Fortbildung an, die naturwissenschaftliche und sprachliche Aspekte kombiniert. In dieser Fortbildung zum Forschen mit Sprudelgas wird der Fokus auf die sprachliche Bildung beim naturwissenschaftlichen Forschen und Entdecken gelegt (vgl. Stiftung Haus der kleinen Forscher, 2013b, Broschüre „Sprudelgas und andere Stoffe - Mit Kita- und Grundschulkindern Chemie entdecken und dabei die sprachliche Entwicklung unterstützen“). Eine wesentliche Methode in allen Fortbildungen vom „Haus der kleinen Forscher" ist der sog. Forschungskreis, der im Einklang mit Modellen zur Förderung des frühen naturwissenschaftlichen Denkens (Anders, Hardy, Pauen \& Steffensky, 2013; Marquardt-Mau, 2004, 2011) typische Elemente naturwissenschaftlichen Vorgehens als Prozess der Erkenntnisgewinnung beschreibt (Stiftung Haus der kleinen Forscher, 2013a; Stiftung Haus der kleinen Forscher, 2015a). Der Prozess des Forschens gliedert sich in verschiedene Phasen des Denkens und Handelns, die typischerweise in einem wiederkehrenden Zyklus auftreten: (1) Frage an die Natur stellen, (2) Ideen \& Vermutungen sammeln, (3) Ausprobieren \& Versuch durchführen, (4) Beobachten \& Beschreiben, (5) Ergebnisse dokumentieren/sammeln, (6) Ergebnisse erörtern. Der Forschungskreis ist laut Stiftung „Haus der kleinen Forscher" als ein Modell oder Werkzeug zu verstehen, das pädagogischen Fachkräften aufzeigt, wie sie gemeinsam mit Kindern experimentieren und in einen Dialog über naturwissenschaftliche Phänomene treten können. Er soll Orientierung für das ausgangsoffene Forschen mit Kindern bieten und betont die Interaktion im Sprechen und Handeln der Akteure beim gemeinsamen forschenden Vorgehen. 
Das Progress-Person-Context-Time-Model („PPCT-Model“) von Bronfenbrenner und Morris (2006) beschreibt die direkten Einflüsse der Fachkraft-Kind-Interaktion auf die frühe kindliche Entwicklung. Dabei wird unterschieden zwischen distalen und proximalen Einflussfaktoren. Distale Einflussfaktoren beschreiben dabei Rahmenbedingungen, wie beispielsweise die Gruppengröße oder die Qualifikation der pädagogischen Fachkraft. Proximale Einflussfaktoren sind direkt in der Interaktion sichtbare Faktoren, anhand derer die Qualität der Interaktion beurteilt werden kann. Es wird zugrunde gelegt, dass die proximalen Faktoren eine direkte Wirkung auf die kindliche Entwicklung, insbesondere auf die Sprachentwicklung, haben. Distale Faktoren hingegen sind weniger zentral, sofern sie keine Auswirkungen auf die Verbesserung der Interaktionsqualität haben.

Während es mittlerweile Ansätze gibt, die die Verbesserung der sprachlichen Interaktion pädagogischer Fachkräfte im Alltag zum Ziel haben (Beller, Merkens \& Preissing, 2007; Weitzmann \& Grennberg, 2002), steht die Untersuchung sprachlich-kognitiv herausfordernder Interaktionen im Rahmen spezifischer Bildungsangebote noch aus (Briedigkeit, 2011).

\subsection{Kompetenzen der pädagogischen Fachkräfte}

Eine zentrale Position in diesem Gefüge nehmen die pädagogischen Fachkräfte ein. Hierzu finden wir drei Richtungen von Forschungsaktivitäten und -ergebnissen. Zum einen werden Elemente der Interaktion identifiziert, die als förderlich gelten, zum anderen wird die tatsächliche Qualität der Interaktion eingeschätzt, und zum dritten gibt es Studien zu den allgemeinen Auswirkungen von Sprachförderprogrammen in Kitas.

Zur ersten Forschungstradition lassen sich viele Hinweise finden, dass die Qualität der Sprachanregung bedeutsam ist und sich inhaltsgebundene Gespräche auf die Sprachkompetenzen der Kinder auswirken. Schon 1985 verwiesen Brophy und Hancock auf die Bedeutung der Qualität des sprachlichen Inputs. Extended teacher discourse, also auch inhaltlich reichhaltige Gespräche mit Kindern im Vorschulalter, zeigen signifikante Zusammenhänge mit dem Wortschatz der Kinder und lassen sich noch bis ins vierte Schuljahr nachweisen (Snow, Tabors \& Dickinson, 2001; vgl. auch Huttenlocher, Vasilyeva, Cymerman \& Levine, 2002 für den Bereich der Syntax). Ähnliche Befunde berichten Rydland, Grøver und Lawrence (2014) in ihrer norwegischen Preschool-Langzeit-Studie mit norwegisch-türkischen Kindern. Sie betonen die Langzeitwirkung sowohl von lehrergeleiteten als auch Peer-Gesprächen im Vorschulalter und zeigen, dass sich intensive inhaltsbezogene Wortschatzarbeit an Büchern vor allem auf den Wortschatz auswirkt. Die Kinder verwenden insbesondere die Wörter, die sie in der Intervention gelernt haben. Diese verwenden sie auch spontan. 
Instructional support, also die Qualität der Lernunterstützung, ist (im Gegensatz zur emotionalen Unterstützung und zur Gruppenführung) in deutschen Kindergärten nicht hoch ausgeprägt (vgl. für Rheinland-Pfalz Kammermeyer, Roux \& Stuck, 2011). Unter instructional support im sprachlichen Bereich wird Wortschatzentwicklung, Feedbackqualität und language modelling (Sprachanregung) subsummiert. Sprachanregung beinhaltet u. a. offene Fragen, korrektives Feedback, Wiederholung und Erweiterung sowie handlungsbegleitendes Sprechen (vgl. auch Fried \& Briedigkeit, 2008). Eine hohe Qualität in diesem Bereich ist sehr vielversprechend im Hinblick auf die Sprachkompetenzen der Kinder (Downer, Sabol \& Hamre, 2010; vgl. Curby et al., 2009; Howes et al., 2008; Mashburn et al., 2008).

Als besonders hochwertige Interaktion erwies sich das Sustained Shared Thinking (vgl. die Studien von Siraj-Blatchford, Sylva, Muttock, Gilden \& Bell, 2002), eine Interaktionsform, die sowohl Scaffolding als auch Ko-Konstruktion berücksichtigt. Beide Interaktionspartner tragen zu diesem geteilten Denkprozess bei. Das naturwissenschaftliche Setting ist ein gutes Umfeld für hochwertige kognitive Situationen in diesem Sinn. Ein gemeinsames Finden der Lösung, wie auch Wagenschein schon vorgeschlagen hat (vgl. zusammenfassend Köhnlein, 2012, S. 100ff.), gilt als extrem hochwertig und führt zu Entwicklungsfortschritten der Kinder (vgl. die Längsschnittstudie EPPE (Effective Provision of Preschool Education) von Sylva, Melhuish, Sammons, Siraj-Blatchford, Taggart \& Elliot (2004) und die Best-Practice-Studie REPEY (Research in Effective Pedagogy in the Early Years) von Siraj-Blatchford, Sylva, Muttock, Gilden \& Bell (2002)). Kognitiv-sprachliches Anregungspotenzial im Sinne von Sustained Shared Thinking untersuchte Hopf (2012) in einer qualitativen Studie. Interaktionen, die man dem Sustained Shared Thinking zuordnen kann, eignen sich zur Sprachförderung innerhalb naturwissenschaftlicher Lernangebote. Allerdings werden diese „gemeinsam geteilten Denkprozesse“ nur sehr selten nachgewiesen (Hopf, 2012, bei ihr waren 30 \% der länger anhaltenden Interaktionssituationen den „gemeinsam geteilten Denkprozessen“ zuzuordnen; vgl. auch Siraj-Blatchford et al., 2002, S. 10; König, 2009). Auch Forschungsergebnisse aus Deutschland besagen deutlich, dass im Rahmen der Interaktion zwischen Fachkraft und Kind die Qualität der sprachlichen Interaktion oftmals ungünstig ist (Weinert, Ebert, Lockl \& Kuger, 2012; Fried, 2011; König, 2009; Albers, 2009; Gasteiger-Klicpera, Knapp \& Kucharz, 2010, S. 204).

Investitionen in die professionelle Entwicklung von Fachkräften im Sprachförderbereich versprechen mitunter wenig. So zeigt eine Studie von Yoshikawa et al. (2015), dass eine zweijährige Fortbildung von Fachkräften in Chile zwar die Professionalität des Fachpersonals positiv veränderte, dass aber auf Ebene der Kinder kaum Effekte registriert werden konnten. Erklären lässt sich dieser Befund durch die Analyse der Interaktionen in der Gruppe. Inhaltliche Arbeit mit den Kindern wäre am sinnvollsten und würde sich am meisten aufs Vokabular auswir- 


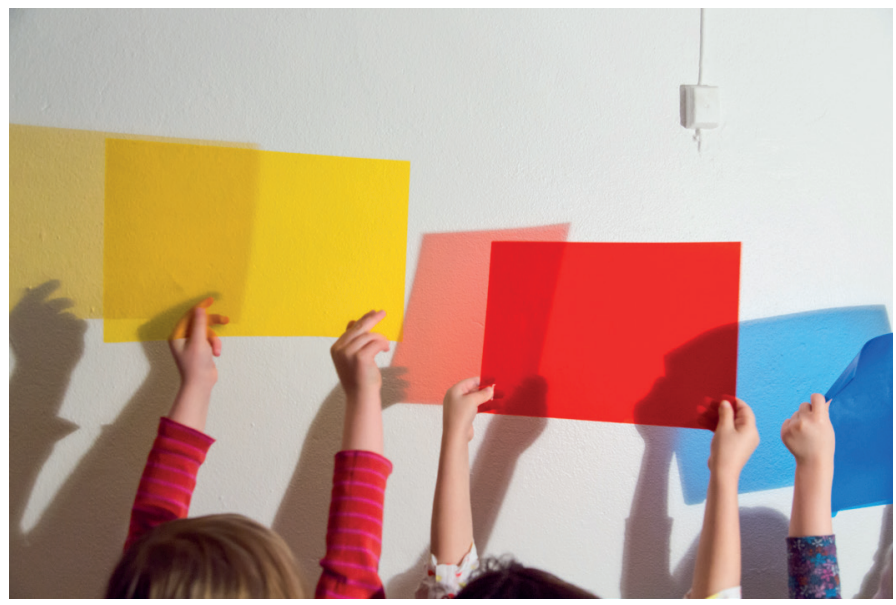

ken, findet aber kaum statt. Auch Beller und Beller (2009) konnten für ihre Intervention zur Verbesserung der Qualität des sprachlichen Anregungsniveaus der in der Kita tätigen Fachkräfte nur sehr geringe Effekte, und diese nur für die vierjährigen Kinder, nachweisen.

Der dritte Fragenkomplex befasst sich mit Studien zur Qualität von Sprachförderprogrammen. Hier sind die Ergebnisse größtenteils ernüchternd. Es gibt praktisch keine Effekte expliziter Sprachförderprogramme (Gasteiger-Klicpera, Knapp \& Kucharz, 2010; Roos, Polotzek \& Schöler, 2010; Sachse, Budde, Rinker \& Groth, 2012; Jülisch \& Häuser, 2003; Wolf, Felbrich, Stanat \& Wendt, 2011; vgl. die Metaanalyse von Egert, 2015). Die am besten evaluierten Sprachförderprogramme sind wahrscheinlich diejenigen zur phonologischen Bewusstheit, doch auch hierzu gibt es für die deutsche Sprache deutliche Einschränkungen. Die Metaanalyse von Wolf, Schroeders und Kriegbaum (2016) belegt für die deutschsprachigen Förderprogramme deutlich niedrigere Effekte als sie aus den internationalen Metaanalysen bekannt sind.

Die neuere Metaanalyse von Egert und Hopf (2016) zur Wirksamkeit von Sprachförderung in der Kita bezieht auch alltagsintegrierte Sprachförderung ein und zeigt hier inkonsistente Ergebnisse für die über Dreijährigen. Hingegen gibt es Befunde, die die Wirkung von alltagsintegrierter Förderung auf die Sprachleistungen von jüngeren Kindern aufzeigen. Fasst man all diese Ergebnisse zusammen, so erscheint nicht die Existenz von Sprachförderung per se als ausschlaggebend, sondern die Qualität dieser Förderung. Insbesondere ist es bedeutsam, dass sich innerhalb der Sprachförderung eine hohe Prozessqualität (z. B. adaptive Sprachförderstrategien) zeigt (Kammermeyer, Roux \& Stuck, 2011). 


\subsection{Zusammenfassung und Desiderata}

Es lassen sich also diese Folgerungen ziehen:

- Dem Sprachregister „Bildungssprache“ kommt eine wesentliche Bedeutung zu. Bildungssprache ist für den Wissensaufbau, die Unterrichtsbeteiligung und die Teilhabe an Bildungsprozessen schon von Schuleintritt an bedeutsam und im Rahmen der kindlichen Sprachentwicklung auch schon davor.

- Sprachliche Bildung, gerade auch der Aufbau des bildungssprachlichen Registers, kann sehr gut in naturwissenschaftlichen Lernsituationen erfolgen.

- Es gibt hierfür gut benennbare Merkmale der Gestaltung sprachförderlicher Situationen durch die Fachkräfte (im Sinne von Language Modelling, sprachanregender Interaktionsgestaltung und inhaltsanregenden Gesprächen).

- Es ist jedoch auch erwiesen, dass Fachkräfte solche Merkmale kaum in ihrer Bildungsarbeit mit Kindern umsetzen.

Interessant ist also, ob und wie sich in dezidiert auf naturwissenschaftliche Bildung ausgerichteten Situationen die Sprachanregung zeigt, ob es Unterschiede gibt und wie sich diese auf die Kinder auswirken. Im Folgenden werden die Fragestellungen und Hypothesen der vorliegenden Studie im Detail dargestellt. 


\section{Fragestellungen der Studie}

Die Forschungsfragen zur Sprachanregung in naturwissenschaftlichen Bildungssettings werden hier am Beispiel der Initiative „Haus der kleinen Forscher“ sowie an weiteren Einrichtungen mit anderem naturwissenschaftlichen Schwerpunkt untersucht. Neben diesen beiden Gruppen mit naturwissenschaftlichen Schwerpunkt wird eine Vergleichsgruppe von Kitas ohne naturwissenschaftlichen Bildungsschwerpunkt einbezogen. Im Folgenden werden zwei Zielvariablen untersucht:

„Sprachliche Anregungsqualität“ bezieht sich auf die sprachliche Gestaltung der Forschungssituation durch die Fachkräfte.

„Bildungssprachliche Performanz" bezieht sich auf die sprachlichen Äußerungen der Kinder in dieser Situation.

Fragestellung 1: Sprachliche Anregungsqualität durch die pädagogischen Fachkräfte

Wie hoch ist die sprachliche Anregungsqualität in den naturwissenschaftlichen Lehr-Lern-Situationen ...

— in Abhängigkeit vom Nawi-Bildungsschwerpunkt der Kita?

— in Abhängigkeit vom Fortbildungsbesuch der Fachkräfte?

— in Abhängigkeit von der Phase des Forschungskreises?

\section{Fragestellung 2: Bildungssprachliche Performanz der Kinder}

- Gibt es Unterschiede in den verschiedenen Untersuchungsgruppen bezüglich der Verwendung von Bildungssprache durch die Kinder?

- Gibt es Zusammenhänge zwischen der sprachlichen Anregungsqualität und der Verwendung von Bildungssprache durch die Kinder?

Im Fokus stehen dabei vor allem auch Kinder, die Deutsch als Zweitsprache erwerben. 


\section{Studiendesign und Methoden}

Die Zusammensetzung der Stichprobe beruht auf vorab festgelegten Kriterien: Sowohl die Geschlechter der Kinder als auch die Spracherwerbssituation sollten in etwa hälftig sein, weshalb jede Fachkraft gebeten wurde, immer zwei Mädchen bzw. zwei Jungen sowie zwei Kinder mit Deutsch als Erstsprache und zwei mit Deutsch als Zweitsprache im entsprechenden Vorschulalter auszuwählen. Dies führte zu einer weitestgehend ausgeglichenen Stichprobe, die gut hinsichtlich Geschlecht und Spracherwerbssituation vergleichbar ist.

Die videografierten Lehr-Lern-Einheiten fanden in einem standardisierten Setting statt. Den teilnehmenden pädagogischen Fachkräften war bekannt, dass sie eine naturwissenschaftliche Experimentiereinheit zum Thema „Schwimmen und Sinken“ gestalten sollten. Dass im Rahmen des Forschungsprojektes EASI Science-L ein besonderes Augenmerk auf die Sprachverwendung gelegt wird, war den Fachkräften ebenfalls bekannt.

\subsection{Stichprobe}

\subsubsection{Die teilnehmenden Einrichtungen}

Im Folgenden werden die Einrichtungen, die an der Studie teilgenommen haben, statistisch beschrieben (Tabelle 42). Die Gruppeneinteilung erfolgte anhand des naturwissenschaftlichen Schwerpunkts der Kita. Die „HdkF-Gruppe“ (31 Fachkräfte, 118 Kinder) besteht aus Kitas mit einer Zertifizierung als „Haus der kleinen Forscher“, die „Nawi-Gruppe“ besteht aus Kitas (9 Fachkräfte, 35 Kinder) mit einem anderen Nawi-Schwerpunkt, und die „Vergleichsgruppe“ sind Kitas (18 Fachkräfte, 69 Kinder) ohne Qualifizierungsmaßnahmen im Bereich naturwissenschaftlicher Bildung. Die statistischen Eigenschaften der Einrichtungen (sozioökonomischer Hintergrund der Eltern, Gruppenanzahl, Gruppengröße, Anteil an DaZ-Familien) unterscheiden sich nicht signifikant zwischen den Gruppen. 
Tabelle 42. Beschreibung der teilnehmenden Einrichtungen. Informationen, basierend auf den Aussagen der jeweiligen Einrichtungsleitung

\begin{tabular}{|c|c|c|c|c|c|}
\hline $\begin{array}{l}\text { Stichproben- } \\
\text { gruppe Kitas }\end{array}$ & & $\begin{array}{c}\text { HdkF-Gruppe } \\
n=31\end{array}$ & $\begin{array}{c}\text { Nawi- } \\
\text { Gruppe } \\
n=9\end{array}$ & $\begin{array}{l}\text { Vergleichs- } \\
\text { gruppe } \\
n=18\end{array}$ & $\begin{array}{l}\text { Gesamt } \\
N=58\end{array}$ \\
\hline Kriterium & & $\begin{array}{c}\text { Zertifizierung } \\
\text { als „Haus } \\
\text { der kleinen } \\
\text { Forscher“ }\end{array}$ & $\begin{array}{c}\text { anderer } \\
\text { naturwissen- } \\
\text { schaftlicher } \\
\text { Schwerpunkt }\end{array}$ & $\begin{array}{l}\text { ohne } \\
\text { naturwissen- } \\
\text { schaftlichen } \\
\text { Schwerpunkt }\end{array}$ & \\
\hline \multirow[t]{4}{*}{$\begin{array}{l}\text { Einrichtungs- } \\
\text { größe }\end{array}$} & & $\begin{array}{c}\left(n=30^{a}\right) \\
M\end{array}$ & $\begin{array}{c}(n=8) \\
M\end{array}$ & $\begin{array}{c}(n=17) \\
M\end{array}$ & $\begin{array}{c}(N=55) \\
M\end{array}$ \\
\hline & Anzahl Gruppen & 5 & 4 & 4 & 4 \\
\hline & $\begin{array}{c}\text { Anzahl } \\
\text { Kinder/Gruppe }\end{array}$ & 26 & 19 & 21 & 24 \\
\hline & $\begin{array}{l}\text { Anzahl der } \\
\text { Fachkräfte }\end{array}$ & 13 & 12 & 12 & 13 \\
\hline Anteil & & $(n=29)$ & $(n=8)$ & $(n=14)$ & $(N=53)$ \\
\hline \multirow[t]{7}{*}{ Familien DaZ ${ }^{b}$} & & $\begin{array}{c}\text { Anzahl } \\
(\text { Anteil in \%)c }\end{array}$ & $\begin{array}{c}\text { Anzahl } \\
\text { (Anteil in \%) }\end{array}$ & $\begin{array}{c}\text { Anzahl } \\
\text { (Anteil in \%) }\end{array}$ & $\begin{array}{c}\text { Anzahl } \\
\text { (Anteil in \%) }\end{array}$ \\
\hline & unter $15 \%$ & $8(28 \%)$ & $2(25 \%)$ & $2(14 \%)$ & $12(22 \%)$ \\
\hline & $15-25 \%$ & $9(32 \%)$ & $0(0 \%)$ & $2(14 \%)$ & $11(21 \%)$ \\
\hline & $25-35 \%$ & $0(0 \%)$ & $3(38 \%)$ & $4(29 \%)$ & $7(13 \%)$ \\
\hline & $35-45 \%$ & $3(10 \%)$ & $1(13 \%)$ & $0(0 \%)$ & $4(8 \%)$ \\
\hline & $50 \%$ & $1(3 \%)$ & $0(0 \%)$ & $0(0 \%)$ & $1(2 \%)$ \\
\hline & über $50 \%$ & $8(28 \%)$ & $2(25 \%)$ & $6(43 \%)$ & $16(30 \%)$ \\
\hline
\end{tabular}

${ }^{a}$ Die hier und im Folgenden auf die Gesamtzahl fehlenden Einrichtungen haben diese Angaben nicht übermittelt.

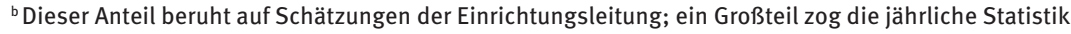
für das Bundesamt heran.

'Aufgrund der Rundung kann der Prozentanteil sich hier und im Folgenden auf etwas über/unter $100 \%$ addieren.

\subsubsection{Die teilnehmenden pädagogischen Fachkräfte}

Jede teilnehmende Kita wählte eine Fachkraft für die Teilnahme an der Studie aus. Je nach Stichprobengruppe gab es verschiedene Kriterien für die Auswahl der teilnehmenden Fachkräfte. Fachkräfte aus der HdkF-Gruppe kommen aus einer vom HdkF zertifizierten Einrichtung und sollten mindestens zwei HdkF-Fortbildungen besucht haben. Die Vorgabe für Fachkräfte aus Einrichtungen mit einem anderen Nawi-Schwerpunkt war die Teilnahme an mindestens einer Fortbildung mit naturwissenschaftlichem Schwerpunkt. Und die Fachkräfte aus der Vergleichsgruppe sollten an keiner Fortbildung zu einem naturwissenschaftlichen Thema teilgenommen haben. 
Die Fachkräfte wurden mittels eines Fragenbogens zu biografischen Themen befragt (siehe Tabelle 43). Tabelle 44 zeigt, wie viele Fachkräfte an Fortbildungen zum Thema Sprache bzw. Naturwissenschaften teilgenommen haben. Die Fachkräfte aus den „Haus der kleinen Forscher“-Einrichtungen waren hierbei sowohl tendenziell älter ( $p=.071)$ als auch signifikant berufserfahrener $(p=.024)$ als die Teilnehmerinnen aus den anderen beiden Gruppen. In der Vergleichsgruppe gab es einen verhältnismäßig hohen Anteil an Fachkräften mit abgeschlossenem Studium. Da sich aber keine dieser Hintergrundvariablen signifikant auf die Zielvariable der sprachlichen Anregungsqualität auswirkt, werden diese Variablen in den weiteren Analysen nicht mit einbezogen.

Tabelle 43. Beschreibung der teilnehmenden Fachkräfte

\begin{tabular}{|c|c|c|c|c|c|}
\hline \multicolumn{2}{|c|}{$\begin{array}{l}\text { Stichprobengruppe } \\
\text { Fachkräfte }\end{array}$} & $\begin{array}{c}\text { HdkF-Gruppe } \\
\quad n=31\end{array}$ & $\begin{array}{c}\text { Nawi-Gruppe } \\
n=9\end{array}$ & $\begin{array}{l}\text { Vergleichs- } \\
\text { gruppe } \\
n=18\end{array}$ & $\begin{array}{l}\text { Gesamt } \\
N=58\end{array}$ \\
\hline \multicolumn{2}{|l|}{ Kriterium } & $\begin{array}{l}\text { Mindestens } \\
\text { zwei Fort- } \\
\text { bildungen vom } \\
\text { „Haus der klei- } \\
\text { nen Forscher“ }\end{array}$ & $\begin{array}{l}\text { Mindestens } \\
\text { eine Fort- } \\
\text { bildung mit } \\
\text { naturwissen- } \\
\text { schaftlichen } \\
\text { Schwerpunkt }\end{array}$ & $\begin{array}{l}\text { Keine Fort- } \\
\text { bildung } \\
\text { zu einem } \\
\text { naturwissen- } \\
\text { schaftlichen } \\
\text { Thema }\end{array}$ & \\
\hline \multirow{8}{*}{ Alter } & & $\begin{array}{c}(n=29) \\
\text { Anzahl } \\
\text { (Anteil in \%) }\end{array}$ & $\begin{array}{c}(n=9) \\
\text { Anzahl } \\
\text { (Anteil in \%) }\end{array}$ & $\begin{array}{c}(n=14) \\
\text { Anzahl } \\
\text { (Anteil in \%) }\end{array}$ & $\begin{array}{c}(N=52) \\
\text { Anzahl } \\
\text { (Anteil in \%) }\end{array}$ \\
\hline & unter 20 Jahre & $0(0 \%)$ & $0(0 \%)$ & $0(0 \%)$ & $0(0 \%)$ \\
\hline & $\begin{array}{l}\text { zwischen } 20 \\
\text { und } 25 \text { Jahren }\end{array}$ & $2(7 \%)$ & $2(22 \%)$ & $2(11 \%)$ & $6(12 \%)$ \\
\hline & $\begin{array}{l}\text { zwischen } 25 \\
\text { und } 30 \text { Jahren }\end{array}$ & 4 (14\%) & $2(22 \%)$ & $4(29 \%)$ & 10 (19\%) \\
\hline & $\begin{array}{l}\text { zwischen } 30 \\
\text { und } 35 \text { Jahren }\end{array}$ & $3(10 \%)$ & $2(22 \%)$ & $2(11 \%)$ & 7 (13\%) \\
\hline & $\begin{array}{l}\text { zwischen } 35 \\
\text { und } 40 \text { Jahren }\end{array}$ & $2(7 \%)$ & $1(11 \%)$ & $1(7 \%)$ & $4(8 \%)$ \\
\hline & über 40 Jahre & $18(62 \%)$ & $2(22 \%)$ & $5(36 \%)$ & 25 (48 \%) \\
\hline & & $(n=31)$ & $(n=9)$ & $(n=18)$ & $(N=58)$ \\
\hline \multirow[t]{2}{*}{ Geschlecht } & weiblich & 31 & 6 & 18 & 55 \\
\hline & männlich & 0 & 3 & 0 & 3 \\
\hline
\end{tabular}




\begin{tabular}{|c|c|c|c|c|c|}
\hline \multicolumn{2}{|c|}{$\begin{array}{l}\text { Stichprobengruppe } \\
\text { Fachkräfte }\end{array}$} & $\begin{array}{c}\text { HdkF-Gruppe } \\
n=31\end{array}$ & $\begin{array}{c}\text { Nawi-Gruppe } \\
n=9\end{array}$ & $\begin{array}{l}\text { Vergleichs- } \\
\text { gruppe } \\
n=18\end{array}$ & $\begin{array}{l}\text { Gesamt } \\
N=58\end{array}$ \\
\hline \multirow{6}{*}{$\begin{array}{l}\text { Berufs- } \\
\text { erfahrung }\end{array}$} & & $\begin{array}{c}(n=29) \\
\text { Anzahl } \\
\text { (Anteil in \%) }\end{array}$ & $\begin{array}{c}(n=9) \\
\text { Anzahl } \\
\text { (Anteil in \%) }\end{array}$ & $\begin{array}{c}(n=14) \\
\text { Anzahl } \\
\text { (Anteil in \%) }\end{array}$ & $\begin{array}{c}(N=52) \\
\text { Anzahl } \\
\text { (Anteil in \%) }\end{array}$ \\
\hline & $\begin{array}{c}\text { weniger als } \\
5 \text { Jahre }\end{array}$ & $4(14 \%)$ & $5(55 \%)$ & $4(29 \%)$ & $13(25 \%)$ \\
\hline & $\begin{array}{c}\text { weniger als } \\
10 \text { Jahre }\end{array}$ & $4(14 \%)$ & $1(11 \%)$ & $3(21 \%)$ & $8(15 \%)$ \\
\hline & $\begin{array}{l}\text { zwischen } 10 \\
\text { und } 15 \text { Jahren }\end{array}$ & $3(10 \%)$ & $1(11 \%)$ & $0(0 \%)$ & $4(8 \%)$ \\
\hline & $\begin{array}{l}\text { zwischen } 15 \\
\text { und } 20 \text { Jahren }\end{array}$ & $2(7 \%)$ & $0(0 \%)$ & $6(43 \%)$ & $8(15 \%)$ \\
\hline & $\begin{array}{l}\text { mehr als } \\
20 \text { Jahre }\end{array}$ & $16(55 \%)$ & $2(22 \%)$ & $1(7 \%)$ & $19(37 \%)$ \\
\hline \multirow{4}{*}{ Ausbildung } & & $\begin{array}{c}(n=29) \\
\text { Anzahl } \\
\text { (Anteil in \%) }\end{array}$ & $\begin{array}{c}(n=9) \\
\text { Anzahl } \\
\text { (Anteil in \%) }\end{array}$ & $\begin{array}{c}(n=15) \\
\text { Anzahl } \\
\text { (Anteil in \%) }\end{array}$ & $\begin{array}{c}(N=53) \\
\text { Anzahl } \\
\text { (Anteil in \%) }\end{array}$ \\
\hline & $\begin{array}{c}\text { Ausbildung } \\
\text { zur } \\
\text { Erzieherin/ } \\
\text { zum Erzieher } \\
\text { an sozialpäd. } \\
\text { Fachschule }\end{array}$ & $26(90 \%)$ & $6(70 \%)$ & $10(70 \%)$ & 42 (79\%) \\
\hline & $\begin{array}{l}\text { Studium der } \\
\text { Früh-, } \\
\text { Heil-, Sozial-, } \\
\text { Sonderpäda- } \\
\text { gogik o.Ä. }\end{array}$ & $1(3 \%)$ & $0(0 \%)$ & $4(27 \%)$ & 5 (9\%) \\
\hline & Sonstiges & $2(7 \%)$ & $3(30 \%)$ & $1(7 \%)$ & $6(11 \%)$ \\
\hline
\end{tabular}

Die Fortbildungsaktivität der teilnehmenden Fachkräfte spielt in den Auswertungen eine große Rolle. In den Fortbildungen wird Wissen erworben, auf das die Fachkräfte bei ihrer Arbeit zurückgreifen. In der Studie geht es um die Verbindung von Sprache und naturwissenschaftlichem Forschen. Daher wird die Häufigkeit besuchter Fortbildungen zu den Themen Sprache und Naturwissenschaften in den letzten 5 Jahren betrachtet. Eine besondere Rolle spielt die Fortbildung „Sprudelgas und andere Stoffe - Mit Kita- und Grundschulkindern Chemie entdecken und dabei die sprachliche Entwicklung unterstützen“, die die Initiative „Haus der kleinen Forscher“ anbietet. In dieser Fortbildung, die von einem großen Teil der HdkF-Stichprobe ( $n=17)$ besucht wurde, wird der Fokus auf sprachliche Bildung beim naturwissenschaftlichen Forschen und Entdecken gelegt (vgl. Stiftung Haus der kleinen Forscher, 2013b), also genau auf den Aspekt, den die Studie betrachtet. 


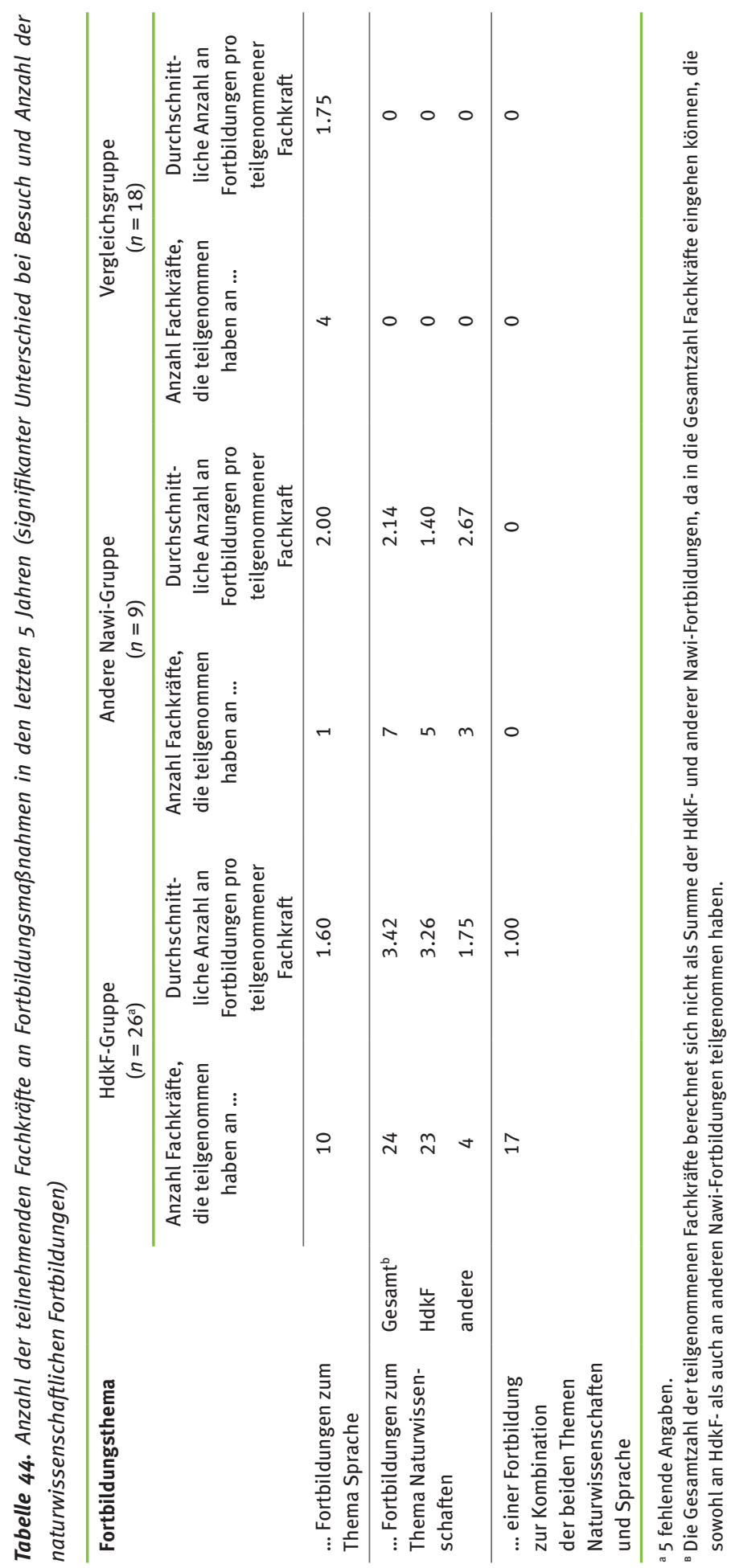




\subsubsection{Die teilnehmenden Kinder}

Jede pädagogische Fachkraft wurde gebeten, immer zwei Mädchen und zwei Jungen auszuwählen, wobei zwei der Kinder einsprachig mit Deutsch als Erstsprache und zwei mit Deutsch als Zweitsprache aufgewachsen sein sollten. Diese Zuordnung wurde weitgehend eingehalten; die Stichprobe setzt sich aus 222 Vorschulkindern zusammen, von denen 121 Deutsch als Erstsprache (DaE) und 101 Deutsch als Zweitsprache (DaZ) sprechen (Alter M 5.8 Jahre (SD=0.5), 119 Mädchen, 103 Jungen; siehe Tabelle 45).

Mit den Kindern wurde der Test LiSe-DaZ $®$ (Linguistische Sprachstandserhebung Deutsch als Zweitsprache, Schulz \& Tracy in Verbindung mit der Baden-Württemberg Stiftung, 2011) als Einzeltestverfahren durchgeführt. Die kognitiven Fähigkeiten der Kinder testeten wissenschaftliche Mitarbeiterinnen der Forschungsgruppe mithilfe des Intelligenztests CFT 1-R (Weiß \& Osterland, 2013).

Die Eltern der untersuchten Kinder füllten zudem eine verkürzte Version des Elternfragebogens aus dem Projekt BiSpra von Weinert, Stanat und Schuth (o. J.; vgl. auch Weinert, Stanat, Berendes, Dragon \& Heppt, o. J.) aus. Enthalten waren dort Fragen zum eigenen Leseverhalten, zur Unterstützung der Kinder im sprachlichen Bereich sowie zum Bildungshintergrund (Schulabschluss und berufliche Ausbildung) der Eltern.

Bezüglich dieser Hintergrundmerkmale gab es einige signifikante Unterschiede zwischen den Gruppen. Diese werden daher bei den Auswertungen berücksichtigt: Der Bildungshintergrund der Eltern ist bei der Nawi-Gruppe signifikant höher ( $p=.04$ Eta $\left.^{2}=.05\right)$ und die Kinder der HdkF-Gruppe sind signifikant älter $\left(p=.00 ;\right.$ Eta $\left.^{2}=.06\right)$. Bei den anderen Werten (Geschlecht, Spracherwerbssituation, häusliche Anregungsqualität, Intelligenz) unterscheiden sich die Gruppen dagegen nicht überzufällig.

Von den Kindern mit DaZ sprachen etwa die Hälfte $(n=53)$ bereits in den ersten drei Lebensjahren Deutsch in Kombination mit einer anderen Sprache (mit slawischen Sprachen wie Russisch, Serbisch, Bosnisch $n=16$, mit romanischen Sprachen wie Italienisch, Französisch, Spanisch $n=12$, mit Albanisch $n=5$, mit Türkisch $n=4$ ). Alle anderen DaZ-Kinder sprachen in den ersten drei Lebensjahren ausschließlich andere Erstsprachen (größtenteils slawische Sprachen $n=16$ und Türkisch $n=14$ ). Insgesamt wurden von den Eltern 44 verschiedene Sprachen genannt. Von diesen Kindern sprechen 12 mit der Mutter und 13 mit dem Vater ausschließlich und 15 (Mutter) bzw. 18 (Vater) meistens nicht Deutsch. 
Tabelle 45. Beschreibung der Kinderstichprobe

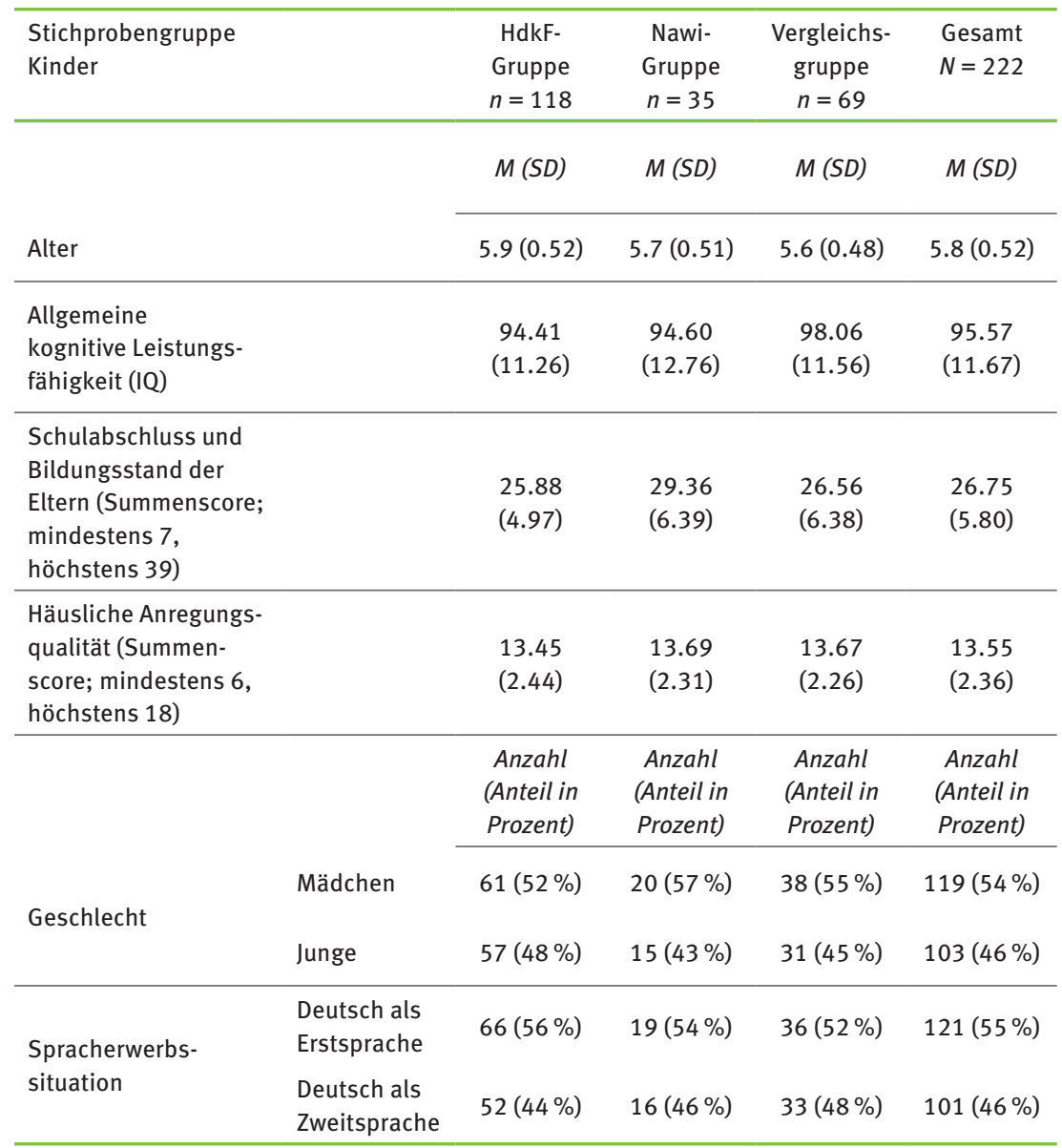

\section{Allgemeine Sprachfähigkeiten der Kinder}

Die allgemeinsprachliche Kompetenz der Kinder wurde mit LiSe-DaZ ${ }^{\circledR}$ (Schulz \& Tracy in Verbindung mit der Baden-Württemberg Stiftung, 2011) erhoben. Dieses Verfahren, in dem mit einer Bildergeschichte gearbeitet wird, unterscheidet Testaufgaben zur Sprachproduktion und zum Sprachverstehen. Die drei Stichprobengruppen unterscheiden sich nicht überzufällig für den Gesamtwert und die Dimensionen „Sprachproduktion“ und „Sprachverstehen“ (Tabelle 46). Es gibt signifikante Unterschiede in den Subtests „Verstehen von W-Fragen“ und „Subjekt-Verb-Kongruenz“, die sich aber nicht auf das Gesamtergebnis auswirken. Diese signifikanten Unterschiede sind auf die Unterschiede der HdkF-Gruppe zur Vergleichsgruppe zurückzuführen. Bei zwei Kindern kam es im Teil Sprachproduktion zu einem Abbruch der Testung. 
Tabelle 46. Allgemeine Sprachfähigkeiten der Kinder; Testverfahren LiSe-DaZ ${ }^{\circledR}$ (Schulz \& Tracy, 2011; vgl. Kapitel 3.2.2)

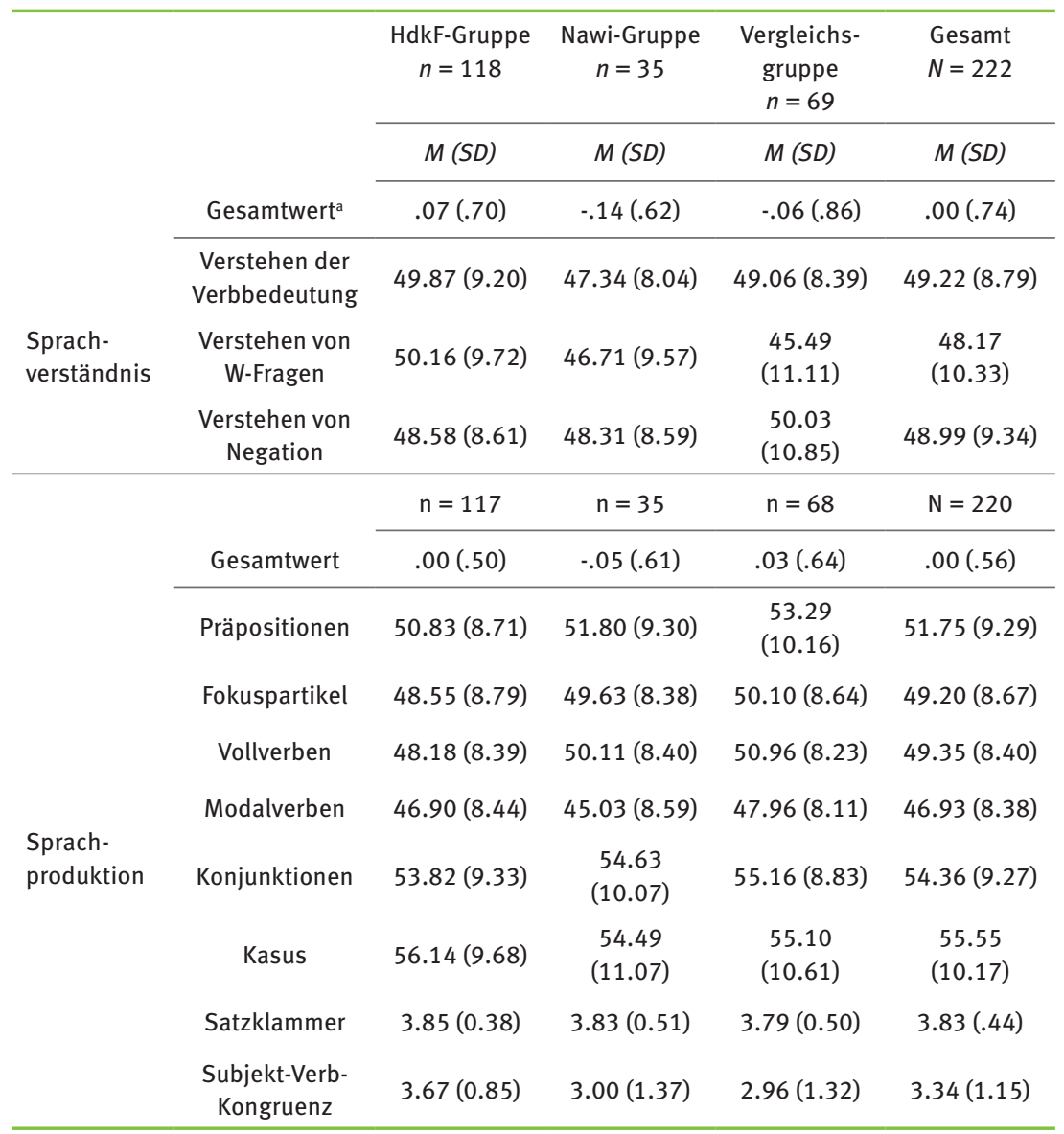

Anmerkungen: Angegeben sind die Mittelwerte der T-Werte. T-Wert unter 40 ist als unterdurchschnittlich, zwischen 40 und 60 als durchschnittlich und über 60 als überdurchschnittlich zu werten.

${ }^{a}$ Aufgrund der unterschiedlichen Skalierung in den Subtests wurden die z-standardisierten Gesamtwerte verwendet.

Bei der Sprachproduktion gibt es für die Subtests der „Satzklammer“ und der „Subjekt-Verb-Kongruenz“ keine T-Werte, sondern Entwicklungsstufen. In beiden Bereichen liegt die Gesamtstichprobe überwiegend im Normbereich. Bei „Satzklammer“ erreichen 189 Kinder (86\%) die höchste Entwicklungsstufe ${ }^{19}$ (ESS IV), 25 Kinder (11\%) sind auf der dritten Entwicklungsstufe (ESS III) und sechs Kinder (3\%) auf der zweiten (ESS II). Keines der Kinder befindet sich auf Stufe I (ESS I, siehe Tabelle 47).

19 Entwicklungsstufen siehe Schulz und Tracy (2011). 
Bei „Subjekt-Verb-Kongruenz“ sind 161 Kinder (73\%) dem oberen Viertel zuzuordnen, acht Kinder (4\%) entsprechen der Leistung im oberen Mittelbereich und 16 Kinder (7\%) dem unteren Mittelbereich. 35 Kinder (16\%) liegen im unteren Viertel.

Zur Berechnung der Unterschiede zwischen Kindern mit Deutsch als Erstsprache und Deutsch als Zweitsprache wurden die Daten Z-standardisiert. Zwischen den beiden Gruppen gibt es keine Unterschiede im Sprachverständnis, weder bezüglich der Gesamtskala noch in den Subskalen. Die Kinder mit Deutsch als Erstsprache sind in einigen Bereichen den Kindern mit Deutsch als Zweitsprache überlegen, in anderen Bereichen verhält es sich umgekehrt. In der Gesamtskala findet sich jedoch kein signifikanter Unterschied (siehe Tabelle 47).

Tabelle 47. Mittelwerte bei den Kindern mit Deutsch als Erst- und Zweitsprache über die einzelnen Bereiche der sprachlichen Kompetenz

\begin{tabular}{|c|c|c|c|c|c|c|}
\hline & $\mathrm{DaE}$ & $\begin{array}{l}\text { Entspricht } \\
\text { Prozent- } \\
\text { rang }\end{array}$ & DaZ & $\begin{array}{l}\text { Entspricht } \\
\text { Prozent- } \\
\text { rang }\end{array}$ & & \\
\hline & $M(S D)$ & & $M(S D)$ & & Sign. & $\begin{array}{c}\text { Eta- } \\
\text { Quadrat }\end{array}$ \\
\hline \multicolumn{7}{|c|}{ Sprachverständnis } \\
\hline $\begin{array}{l}\text { Verstehen der } \\
\text { Verbbedeutung }\end{array}$ & $44.81(26.96)$ & $31 \%$ & $50.74(29.68)$ & $54 \%$ & n.s. & \\
\hline $\begin{array}{l}\text { Verstehen von } \\
\text { W-Fragen }\end{array}$ & $46.01(32.17)$ & $35 \%$ & $43.49(31.29)$ & $24 \%$ & n.s. & \\
\hline $\begin{array}{l}\text { Verstehen von } \\
\text { Negation }\end{array}$ & $44.59(28.39)$ & $27 \%$ & $47.08(27.03)$ & $42 \%$ & n.s. & \\
\hline \multicolumn{7}{|c|}{ Sprachproduktion } \\
\hline Präpositionen & $55.94(30.17)$ & $73 \%$ & $50.27(27.35)$ & $50 \%$ & n.s. & \\
\hline Fokuspartikel & $46.26(28.56)$ & $35 \%$ & $48.80(24.33)$ & $46 \%$ & n.s. & \\
\hline Vollverben & $51.61(28.33)$ & $54 \%$ & $38.43(21.98)$ & $12 \%$ & $p<.001$ & .062 \\
\hline $\begin{array}{l}\text { Modal- und } \\
\text { Hilfsverben }\end{array}$ & $47.25(25.44)$ & $38 \%$ & $32.10(21.94)$ & $4 \%$ & $p<.001$ & .091 \\
\hline Konjunktionen & $56.58(29.22)$ & $76 \%$ & $65.60(25.57)$ & $95 \%$ & $p=.035$ & .026 \\
\hline Kasus & $62.00(27.79)$ & $89 \%$ & $68.41(29.41)$ & $96 \%$ & n.s. & \\
\hline Satzklammer & $3.90(.34)$ & $5 \%$ & $3.71(.54)$ & $39 \%$ & $p=.007$ & .041 \\
\hline $\begin{array}{l}\text { Subjekt-Verb- } \\
\text { Kongruenz }\end{array}$ & $3.50(1.06)$ & $>0.98$ & $3.10(1.26)$ & $\begin{array}{c}0.93- \\
0.97^{\mathrm{b}}\end{array}$ & $p=.026$ & .029 \\
\hline
\end{tabular}

Anmerkungen: Es sind jeweils die erzielten $T$-Werte angegeben. Die Prozentränge zeigen die Normwerte für die jeweilige Population im Alter von 6 Jahren.

a Bei den Subtests „Satzklammer“ und „Subjekt-Verb-Kongruenz“ gibt der Test keine $T$-Werte an, sondern Normbereiche von 1 bis 4.4 ist der höchste Wert.

${ }^{\mathrm{b}}$ Die Werte geben an, in welchem Bereich zwischen 0 und 1 die Leistung im Bereich zur Vergleichsgruppe liegt. 


\subsection{Durchführung der Erhebung}

Die Rekrutierung der Stichprobengruppen erfolgte sowohl telefonisch als auch postalisch über einen ausführlichen Informationsbrief und einen zusammenfassenden Flyer. Mit den interessierten Einrichtungen wurde ein persönlicher Gesprächstermin vereinbart. Dieser fand in der Regel mit der Einrichtungsleitung und der pädagogischen Fachkraft, die sich an der Studie beteiligen wollte, statt. In einigen Fällen wurde die Studie in der Teamsitzung vorgestellt. Beim Erstgespräch mit der Einrichtungsleitung fand per Interview die Erfassung der relevanten Hintergrundvariablen, die die Gesamteinrichtung betreffen, statt. Die teilnehmende pädagogische Fachkraft erhielt die Fragebögen, die sie ihrerseits ausfüllen sollte, sowie eine Übersicht über die anstehenden Termine und die Einverständniserklärungen für die Eltern. Der erste Termin umfasste die Testung der allgemeinen Sprachfähigkeiten der Kinder, die in der Einrichtung selbst stattfand. An einem zweiten Termin fand der Besuch an der Universität Heidelberg oder dem Labor in Karlsruhe statt, an dem die Fachkraft mit den vier Kindern an einem Vormittag eine naturwissenschaftliche Experimentiereinheit zum Thema „Sinken und Schwimmen“ gestaltete. Der Termin zur Sprachstandtestung fand immer als erster statt; in einem Abstand von ungefähr zwei bis drei Wochen folgte der Termin an den Universitäten.

Die Gestaltung des Vormittags an den Universitäten ging für jede beteiligte Einrichtung gleichermaßen vonstatten. Bei dem Experiment anwesend waren immer mindestens eine der wissenschaftlichen Mitarbeiterinnen und zwei studentische Hilfskräfte. Wenn es terminlich machbar war, waren beide wissenschaftlichen Mitarbeiterinnen anwesend. Drei bis vier Tage vor dem Termin an der Universität erhielt die pädagogische Fachkraft postalisch eine Anfahrtsskizze, einen zeitlichen Überblick sowie Bilder den Räumlichkeiten und der zur Verfügung stehenden Materialien. Außerdem erhielten die Fachkräfte folgende Instruktion zur Experimentiereinheit (bei Fachkräften, die vorher an keinem Fortbildungsprogramm im Bereich der Naturwissenschaften teilgenommen hatten, wurde der Teil „auf ihr Förderangebot“ des letzten Satzes weggelassen):

„Bitte gestalten Sie eine kleine Lehr-Lerneinheit zum Thema Sinken und Schwimmen von Objekten mit den Kindern und verwenden Sie dafür ausschließlich das im Raum befindliche Material. Sie haben insgesamt 30 Minuten mit den Kindern Zeit. Die Situation wird dabei aufgezeichnet. Weil wir uns besonders für die Reaktionen der Kinder auf Ihr Förderangebot interessieren, bitten wir Sie, allen Kindern ausreichend Möglichkeit zu geben, sich sprachlich zu äußern.“ 
Die pädagogische Fachkraft und die Kindergruppe wurden auf Wunsch von der Bahnhaltestelle abgeholt und an die Universitäten begleitet. Der Raum war bereits vorbereitet. Begonnen wurde anschließend direkt mit der Experimentiereinheit, die von der Fachkraft mit der Kindergruppe gestaltet wurde und in etwa $30 \mathrm{Mi}$ nuten dauerte. Jede Fachkraft hatte dabei dieselben Materialien zur Verfügung. Jeweils in vierfacher Ausführung standen ein Plastikteller, eine Plastikschüssel, eine dicke und kurze Kerze ohne Docht, ein halbierter Schwamm, verschieden große Murmeln, zwei Büroklammern, ein Holz-, Plastik- und Metallknopf sowie verschieden große Klötze aus Holz, Styropor und Metall zur Verfügung (Abbildung 6). Der Einsatz der Materialien wurde von jeder pädagogischen Fachkraft frei gestaltet.

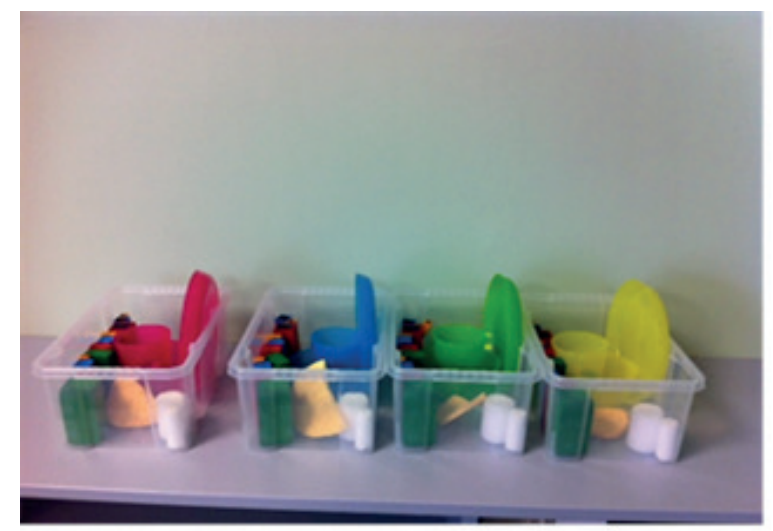

Abbildung 6. Verwendete Materialien während der Experimentiereinheit

Die Aufnahme der Experimentiereinheit erfolgte mit zwei Kameras aus unterschiedlichen Perspektiven und einem Deckenmikrofon. Im Anschluss an die 30-minütige Forschungssituation konnte die Kindergruppe eine Pause machen, die je nach Gruppe zwischen 10 und 20 Minuten variierte. Nach der Pause gingen die Kinder mit der wissenschaftlichen Mitarbeiterin und einer studentischen Hilfskraft zurück in den Experimentierraum, um dort die Gruppentestung zur allgemeinen kognitiven Leistungsfähigkeit durchzuführen. Die Fachkraft blieb mit einer weiteren studentischen Hilfskraft direkt vor dem Raum, um einen Online-Fragebogen auszufüllen. Beides dauerte ungefähr zwischen 30 und 40 Minuten, so dass die Fachkraft und die Kindergruppe in etwa zeitgleich fertig waren. Abgeschlossen wurde der Besuch an den Universitäten mit einer kleinen Schatzsuche für die Kinder. 


\subsubsection{Pädagogische Fachkräfte: Erfassung der sprachlichen Anregungsqualität}

Die sprachliche Anregungsqualität der pädagogischen Fachkräfte wurde explizit für das von der Fachkraft gestaltete naturwissenschaftliche Lehr-Lern-Setting eingeschätzt. Dafür wurde die „Dortmunder Ratingskala zur Erfassung sprachförderrelevanter Interaktionen “ (DO-RESI) von Fried und Briedigkeit (2008) herangezogen. Die Einschätzung erfolgte anhand der Videoaufnahmen. Zur Prüfung der Interrater-Reliabilität wurden 15 der insgesamt 58 Videos von zwei Mitarbeiterinnen statt einer Mitarbeiterin eingeschätzt und ihre Übereinstimmung bestimmt ( $\alpha=$.782). Insgesamt besteht die von uns genutzte Skala aus 12 Items: Kongruenz, Empathisches Zuhören, Anregung, Handlungen verbalisieren, Informationen/Berichte einholen, Lernmöglichkeiten aufzeigen, Vielfalt des Wortschatzes, Grammatisch komplexer Input (keine Fragmentsätze), Offene Fragen, Themen benennen/entdecken, Themen verbinden, Zusammenhänge eines Themas erklären/ hinterfragen $(\alpha=$.954). Jedes Item wird im Instrument DO-RESI über verschiedene Indikatoren beschrieben und über die gesamte Situation eingeschätzt.

\section{Kodierung der sprachlichen Anregungsqualität innerhalb der Forschungskreisphasen}

Um die Gestaltung der Experimentiersituation genauer betrachten zu können, wurden in den videografierten Situationen sowohl die einzelnen Elemente des naturwissenschaftlichen Vorgehens als auch die darin zu lokalisierenden Indikatoren der sprachlichen Anregungsqualität anhand eines Extremfallsamplings ( $\alpha=15$ ) mit dem qualitativen Analyseprogramm MAXQDA analysiert.

Dazu wurde die Stichprobe anhand ihrer DO-RESI-Werte der sprachlichen Anregungsqualität (SKA) in drei Extremgruppen (unzureichend; mittel bis gut; sehr gut) geteilt. Aus den Gruppen wurden insgesamt 15 Fälle gezogen mit unzureichender Anregungsqualität (SKA zwischen 1.36 und 2.55), mittlerer bis guter Anregungsqualität (SKA zwischen 4.36 und 4.91) und sehr guter Anregungsqualität (SKA zwischen 5.54 und 6.82). Da die Fälle zufällig aus den entsprechenden Gruppen gezogen wurden, sind die Merkmale willkürlich verteilt (Tabelle 48). 


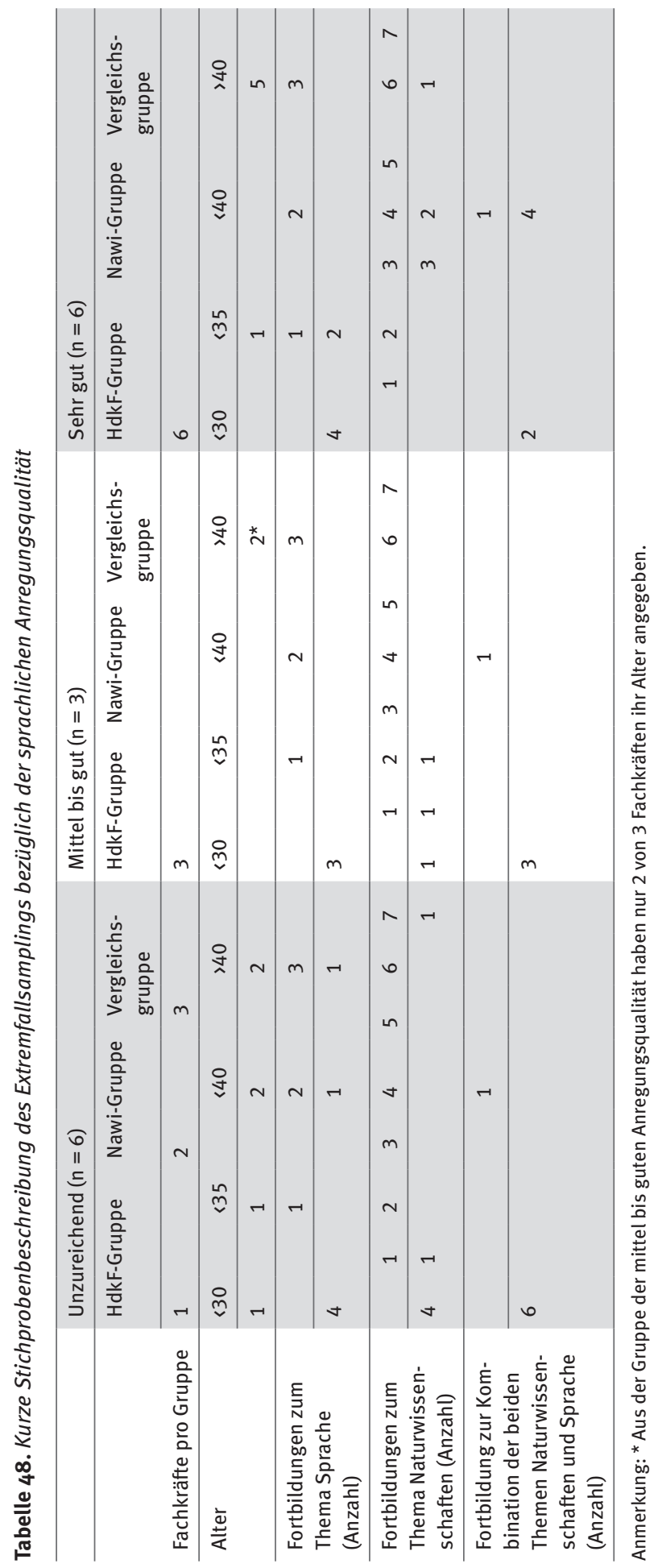


Nach der Auswahl der Fachkräfte wurden in einem ersten Kodierungsdurchlauf zunächst die einzelnen Phasen des naturwissenschaftlichen Vorgehens kodiert.

Die Kodierung erfolgte anhand der Beschreibung des Forschungskreises der Stiftung „Haus der kleinen Forscher“ (Stiftung Haus der kleinen Forscher, 2013a; Stiftung Haus der kleinen Forscher, 2015a). Insgesamt umfasst die Beschreibung sechs Phasen, die innerhalb einer Experimentierphase differenzierbar sind (1) Frage an die Natur stellen, (2) Ideen \& Vermutungen sammeln, (3) Ausprobieren und Versuch durchführen, (4) Beobachten \& Beschreiben, (5) Ergebnisse dokumentieren/sammeln, (6) Ergebnisse erörtern. Tabelle 49 zeigt exemplarisch anhand einer Phase den Kodierungsprozess. Die Beschreibung für alle Phasen sind Anhang I zu entnehmen.

Tabelle 49. Exemplarischer Auszug aus dem Handbuch zur Kodierung der Forschungskreisphase „Ideen \& Vermutungen sammeln“

\begin{tabular}{|c|c|}
\hline Beschreibung & Kodierhinweise \\
\hline $\begin{array}{l}\text { Zunächst sollten die Mädchen und Jungen } \\
\text { darüber nachdenken, welche Ideen und } \\
\text { Vermutungen sie zu dem Thema bereits } \\
\text { mitbringen. Dabei geht es nicht um ein „Ab- } \\
\text { fragen“, sondern darum, den Geist der Kinder } \\
\text { auf den Forschungsprozess einzustimmen } \\
\text { und ihnen ihr Vorwissen bewusst zu machen. } \\
\text { Neue Erkenntnisse müssen an bereits vor- } \\
\text { handenes Wissen anknüpfen, sonst können } \\
\text { sie nicht richtig verankert werden und bleiben } \\
\text { ohne Zusammenhang. Zeigen Sie den Kin- } \\
\text { dern, dass Sie ihre Ideen ernst nehmen und } \\
\text { wertschätzen. Stellen Sie Rückfragen, die die } \\
\text { Mädchen und Jungen zu weiterem Nachden- } \\
\text { ken anregen. }\end{array}$ & $\begin{array}{l}\text { Die Kodierung erfolgte gemäß der Beschrei- } \\
\text { bung. Die Fachkraft weckt durch geschicktes } \\
\text { Frageverhalten das Interesse an bestimmten } \\
\text { Phänomenen und provoziert damit, dass die } \\
\text { Kinder Ideen/Vermutungen äußern. Wurde } \\
\text { auch kodiert, wenn die Kinder von sich aus } \\
\text { Ideen/Vermutungen geäußert haben. } \\
\text { Kodierte Beispiele (K=Kind(er), F=Fachkraft): } \\
\text { 1. Fachkraft evoziert durch Fragen Vermutun- } \\
\text { gen: } \\
\text { F: aber is das wirklisch holz/ } \\
\text { K4: nei:n } \\
\text { K2: mmh } \\
\text { F: was könnte das sein/ } \\
\text { K3: meta:ll } \\
\text { K1: metall } \\
\text { F: is das metall/ } \\
\text { K1: mmh } \\
\text { F: gebma der kind2 oder dem kind4 vielleisch } \\
\text { ** was meinst du kind4/ } \\
\text { K4: des is plastik } \\
\text { K2: :a: * plastik } \\
\text { F: das könnte plastik sein) *plastik } \\
\text { K1: plastik is leichter * plastik is leichter als } \\
\text { holz } \\
\text { K4: ich glaub des is so wie beim waschlappn } \\
\text { * weil mein waschlappn das is aber komisch } \\
\text { gewesen } \\
\text { F: aha } \\
\text { 2. Kind stellt von sich aus Vermutung auf: } \\
\text { K: der schwimmt glaub ich } \\
\text { F: der sch:wimmt sagst du * oke }\end{array}$ \\
\hline
\end{tabular}


In einem daran angeschlossenen zweiten Kodierungsdurchlauf wurden die Indikatoren für die sprachliche Anregungsqualität lokalisiert. Hierfür wurden Indikatoren, die besonders repräsentativ für sprachliche Anregungen sind, aus dem Beobachtungsverfahren DO-RESI ausgewählt. Die Indikatoren dienen im Instrument DO-RESI zur genaueren Beschreibung der Items. Wir betrachten insgesamt acht Indikatoren: (1) Impuls, (2) Benennungen erarbeiten, (3) Variation des Wortschatzes, (4) Sprachliche Unterstützung - Anbieten von Lückensätzen, (5) Wiederholung unvollständiger Aussagen, (6) Längerer Satz, (7) Aufgreifen einer Kinderäußerung, (8) Zusammenfassung.

Indikator (2) Benennungen erarbeiten wird hier exemplarisch dargestellt. Die vollständige Beschreibung aller Indikatoren ist Anhang II zu entnehmen.

Indikator 2: Benennungen erarbeiten (aus Item 18: Vielfalt des Wortschatzes): Die Fachkraft erarbeitet eine Benennung mit einem Kind gemeinsam, z. B. durch Raten, Hinterfragen, Ergänzen, Sprachwitz, Nonsens. Hierfür müssen Indikatoren vorhanden sein, die eine gemeinsame Erarbeitung repräsentieren: Beispielsweise. lenkt die Fachkraft durch weitere Fragen das Kind zur richtigen Bezeichnung. Antwortet das Kind mit „weiß nicht“ und die Fachkraft beantwortet die Frage dann selbst, wird dies nicht als gemeinsame Erarbeitung gewertet.

\section{Beispiel ( $\mathrm{K}=$ Kind(er), $\mathrm{F}=$ Fachkraft):}

K3: die kugel * die schwimmt unter- * 1 *

F: die schwimmt unter/was heißt des/ *schwimmt sie jetzt oder geht sie unterl

K3: die geht unter

F: die geht unter|F: die geht unter|

Tabelle 50 stellt die kodierten Indikatoren sprachlicher Anregungsqualität dar.

Nachdem beide Kodiervorgänge abgeschlossen wurden, wurde mittels der Retrieval-Funktion in MAXQDA ermittelt, in welchen Phasen welche Indikatoren sprachlicher Anregungsqualität besonders häufig oder eben weniger häufig vorkommen. Die Ergebnisse sind dem Ergebniskapitel zu entnehmen. 


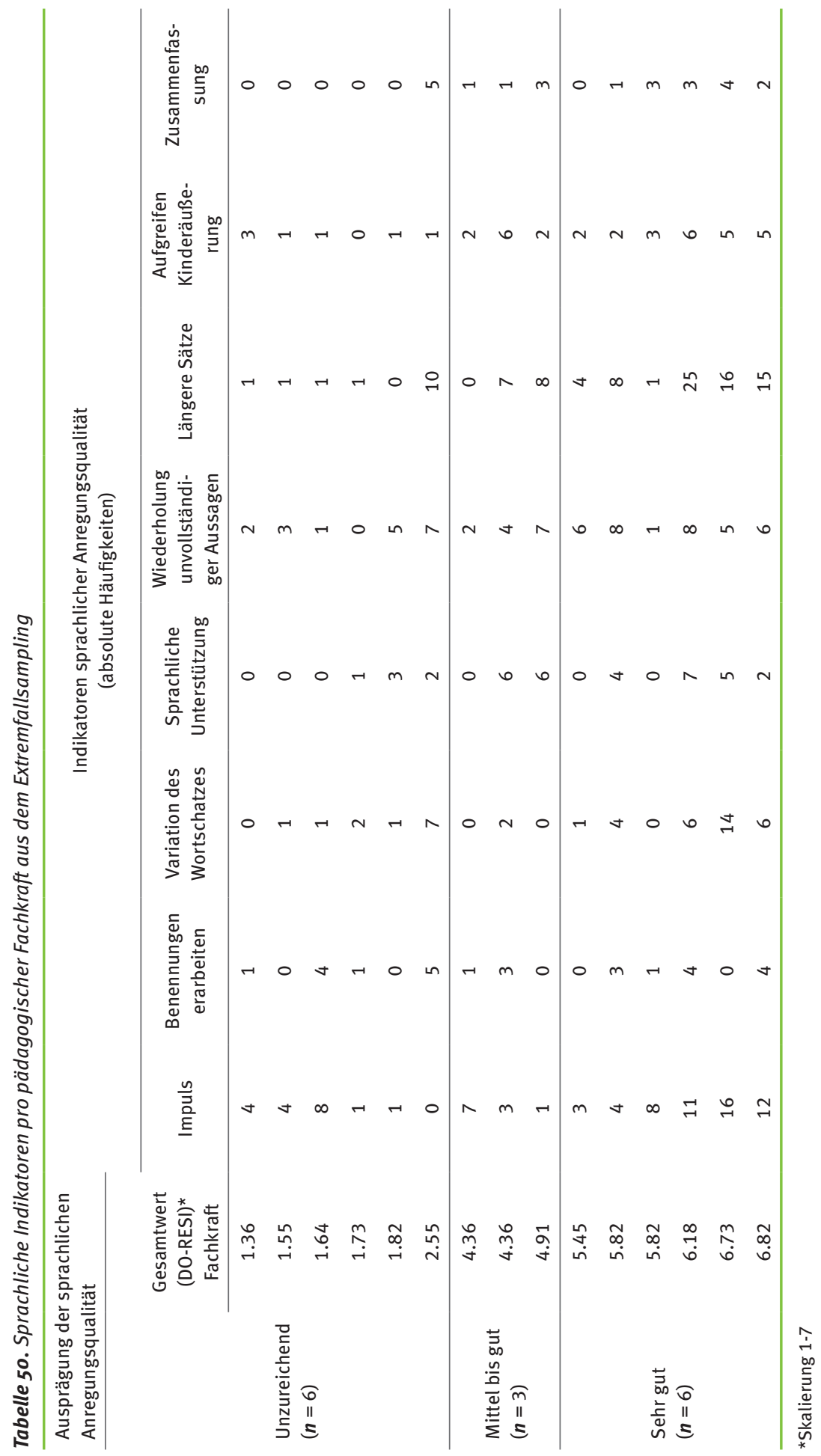




\subsubsection{Kinder: Erfassung der Sprachverwendung in naturwissenschaftlichen Interaktionssituationen}

Speziell für die Sprachverwendung in naturwissenschaftlichen Interaktionssituationen wurde zu Projektbeginn eine Ratingskala entwickelt, die die in der Situation gezeigten bildungssprachlichen Fähigkeiten der Kinder einschätzt (Tietze, Rank \& Wildemann, 2016). Diese Ratingskala zur Erfassung bildungssprachlicher Fähigkeiten (RaBi) setzt sich aus den drei Hauptdimensionen Lexikon, Morphosyntax und Sprachhandlungen zusammen. Jede Dimension beschreibt dabei Merkmale, die zum derzeitigen Erkenntnisstand als bildungssprachlich angenommen wurden und entsprechend der konventionellen Sprachentwicklung als angemessen und erwartbar herausgestellt werden konnten.

Die Dimensionen setzen sich, wie in Tabelle 51 ersichtlich, aus verschiedenen Items zusammen. Zur Kodierung wurden zunächst sämtliche Sprechhandlungen der Kinder in der naturwissenschaftlichen Lehr-Lern-Situation transkribiert. Anschließend wurde mithilfe eines Kategoriensystems zunächst eingeschätzt, inwieweit die Kinder lexikalische und morphosyntaktische Mittel verwendeten. Dies geschah in drei bzw. vier Items und jeweils vier Abstufungen ( $0=$ rudimentär, 1=alltagssprachlich, 2=alltagssprachlich mit bildungssprachlichen Elementen, 3=bildungssprachlich). Diese Analyse wird durch die Analyse der Sprachhandlungen ergänzt. Bei den Sprachhandlungen wird zwischen komplexen und nicht komplexen Sprachhandlungen unterschieden, die aufgrund der einheitlichen Skalierung innerhalb der Skala in die Werte 0 (keine komplexe Sprachhandlung) und 3 (komplexe Sprachhandlung) kodiert wurden. Die an 23 Auswertungen berechnete Interrater-Reliabilität zeigt mit Werten von .76 bis .81 (Cohens Kappa) ein gutes Maß an Übereinstimmung.

Die Reliabilität der Skalen ist mit Ausnahme der Sprachhandlungen zumindest akzeptabel (siehe Tabelle 51). Da in der Subskala „Sprachhandlungen“ nur zwei Items eingehen und insbesondere das Item Erklären/Begründen vermutlich auch durch das Wissen zum Thema „Schwimmen und Sinken“ mitbeeinflusst wird, sind die Befunde zu dieser Teilskala mit Vorsicht zu betrachten, sollen jedoch im Folgenden aufgrund der inhaltlichen Passung dennoch als vertretbarer Index berichtet werden. 
Tabelle 51. Ausprägung der bildungssprachlichen Fähigkeiten der Kinder (Werte der RaBi)

\begin{tabular}{llccc}
\hline Dimension & Items & $M$ & $S D$ & $\begin{array}{c}\text { Cronbachs } \\
\text { Alpha }\end{array}$ \\
\hline Lexikon & $\begin{array}{l}\text { Nomen } \\
\text { Adjektive } \\
\text { Verben }\end{array}$ & 0.93 & 0.70 & .675 \\
\hline Morphosyntax & Kohäsion & & & \\
& Satzgefüge & & & \\
& Unpersönliche Konstruktion \\
& Komplexes Verbgefüge & 0.99 & 0.62 & .739 \\
\hline Sprachhandlungen & Erklären/Begründen & & & \\
& Vermuten & & & \\
\hline Gesamt & & 1.33 & 1.49 & .419 \\
\hline
\end{tabular}

Die RaBi misst die in der Situation gezeigten Kompetenzen. Wir sprechen daher im Folgenden von Performanz im Sinne von Kompetenz als allgemeiner Sprachfähigkeit und Performanz als individueller Sprachverwendung (z.B. nach Chomsky). Chomsky (1981) definiert Performanz als „das beobachtbare Verhalten, in dem die Kompetenz sichtbar wird“.

Die Validität eines Verfahrens kann auf mehreren Ebenen überprüft werden. Für die RaBi-Skala wurden die konvergente Validität, die Kriterienvalidität sowie die Konstruktvalidität ermittelt.

Da zum Zeitpunkt der Entwicklung kein vergleichbares Instrument vorlag, wurden zur Ermittlung der konvergenten Validität die Ergebnisse der allgemeinen Sprachstandsmessung mit LiSe-DaZ $®$ (Schulz, Tracy, Baden-Württemberg Stiftung, 2011) herangezogen. Zwischen der bildungssprachlichen Kompetenz und den Ergebnissen der Sprachstandsmessung besteht eine signifikante, geringe Korrelation ( $r=.185, p=.006$ ) (vertiefend Tietze, Rank \& Wildemann, 2016, S. 21f.).

Die Korrelationen zwischen den Items geben Aufschluss über die Konstruktvalidität (Tabelle 52). Alle Items korrelieren auf einem mittleren Niveau untereinander. Insgesamt fallen die Korrelationen mit dem Item Adjektive etwas geringer aus, was auf die Komplexität und einen späteren Erwerbsbeginn von Adjektiven zurückzuführen ist (siehe dazu ausführlich Tietze, Rank \& Wildemann, 2016, S. 21f.). 


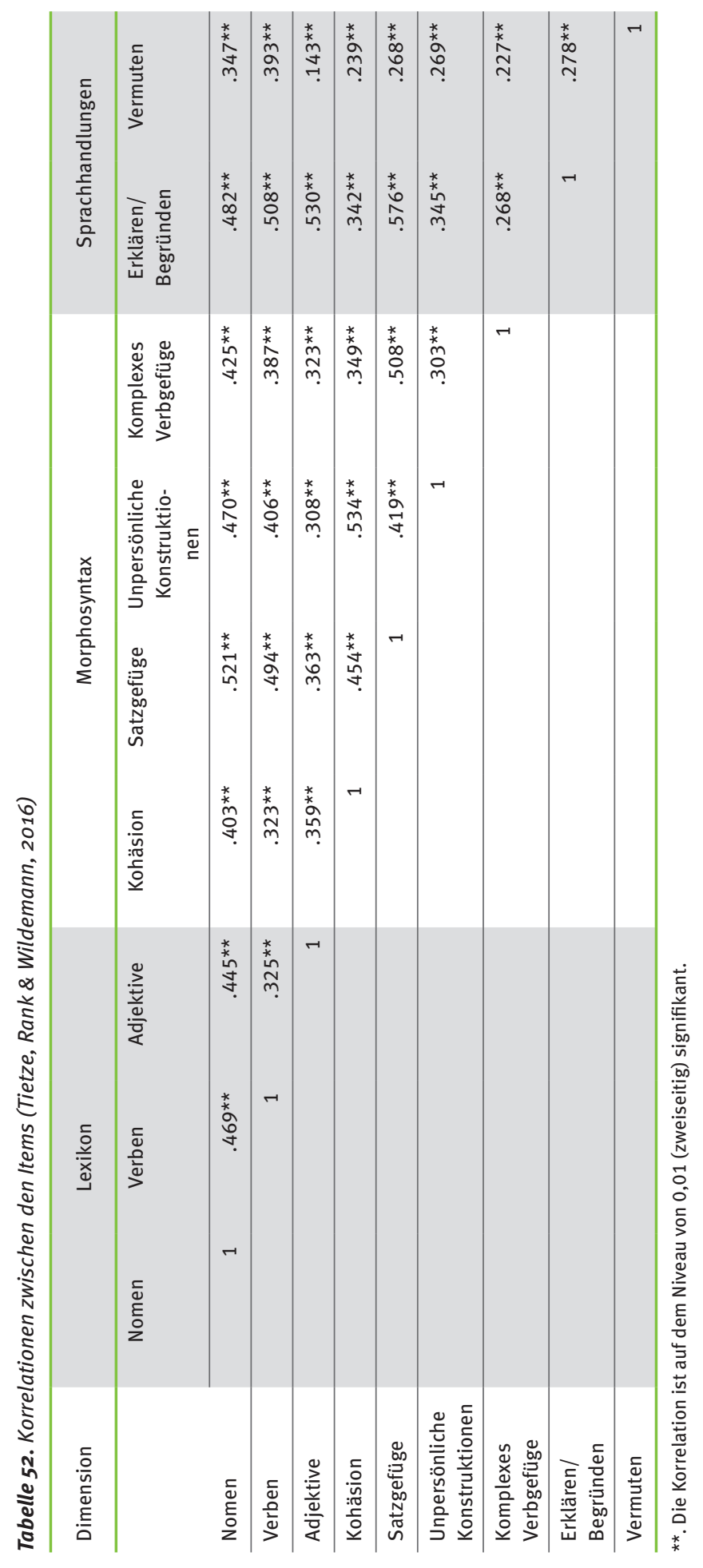




\subsubsection{Statistische Analyseverfahren}

Deskriptive statistische Analysen und inferenzstatistische Analysen auf Fachkraftebene erfolgten mit dem Statistikprogramm SPSS (IBM SPSS Statistics 22). Sowohl auf Fachkraft- als auch auf Kinderebene erfolgte die Ermittlung von signifikanten Mittelwertunterschieden zwischen den Stichprobengruppen mit dem t-Test. Für den Vergleich aller drei Stichprobengruppen wurde die Berechnung einer einfaktoriellen Varianzanalyse (ANOVA) mit Bonferroni-Korrektur durchgeführt, um dem erhöhten Alphafehler durch den Vergleich der drei Gruppen angemessen Rechnung zu tragen.

Die Zusammenhänge und Effekte zwischen den Fachkräften und Kindern wurden mehrebenenanalytisch mit den Statistikprogrammen Mplus (Muthén \& Muthén, 2010) sowie R ermittelt. Dies ist erforderlich, da ansonsten Fehler aufgrund der „Klumpung“ der Stichprobe (immer vier Kinder bei einer pädagogischen Fachkraft) entstehen können. Berechnet wurden hierarchische Regressionsanalysen. 


\section{Ergebnisse}

\subsection{Deskriptive Ergebnisse}

\subsubsection{Sprachliche Anregungsqualität durch die pädagogischen Fachkräfte}

Für eine erste Einsicht in die Daten erfolgt nachstehend die Darstellung deskriptiver Ergebnisse, deren inferenzstatistische Auswertung in Kapitel 4.2 angeschlossen wird.

Die sprachliche Anregungsqualität wurde mittels einer aus dem Beobachtungsinstrument DO-RESi (Fried \& Briedigkeit, 2008) gebildeten Subskala ( $\alpha=$ .954) eingeschätzt (siehe Kapitel 3.2.1). Die Einschätzung erfolgte auf einer Skala von eins bis sieben. Die Wertebereiche zwischen 1 und 2.5 wurden als unzureichend, zwischen 2.5 und 4.5 als wenig, zwischen 4.5 und 6.5 als gut und zwischen 6.5 und 7 als exzellent bezeichnet.

Die pädagogischen Fachkräfte aus Einrichtungen mit einer Zertifizierung als „Haus der kleinen Forscher“ zeigen im Schnitt die höchste sprachliche Anregungsqualität (Tabelle 53). In der Gesamtstichprobe lag die Qualität der sprachlichen Anregung im mittleren Bereich.

Tabelle 53. Deskriptivstatistiken der sprachlichen Anregungsqualität pro Stichprobengruppe

\begin{tabular}{lccccc}
\hline Stichprobengruppe & $N$ & $M$ & $S D$ & Min. & Max. \\
\hline HdkF-Gruppe & 31 & 4.43 & 1.37 & 2.00 & 6.82 \\
Nawi-Gruppe & 9 & 3.48 & 1.31 & 1.64 & 5.27 \\
Vergleichsgruppe & 18 & 3.33 & 1.46 & 1.36 & 5.73 \\
\hline Gesamt & 58 & 3.94 & 1.47 & 1.36 & 6.82 \\
\hline
\end{tabular}

\subsubsection{Verwendung von Bildungssprache durch die Kinder in der naturwissenschaftlichen Situation}

Die in der Situation verwendete Bildungssprache (Tabelle 54) lag sowohl bei der Gesamtskala als auch auf den einzelnen Dimensionen vorwiegend auf der ersten Niveaustufe (siehe ausführlich Tietze, Rank \& Wildemann, 2016). Die an der Studie teilnehmenden Kinder zeigen damit im Mittel ein alltagssprachliches Niveau, was der medial mündlichen Situation (Forschungssituation) und dem Stand der Sprachentwicklung angemessen ist. Allerdings ist die Streuung nach oben deutlich, und man findet Kinder vor, die sehr kompetent bildungssprachliche Muster realisieren. Das hier abgebildete Boxplot (Abbildung 7) zeigt die Streuung der Gesamtskala über die Gesamtstichprobe. 


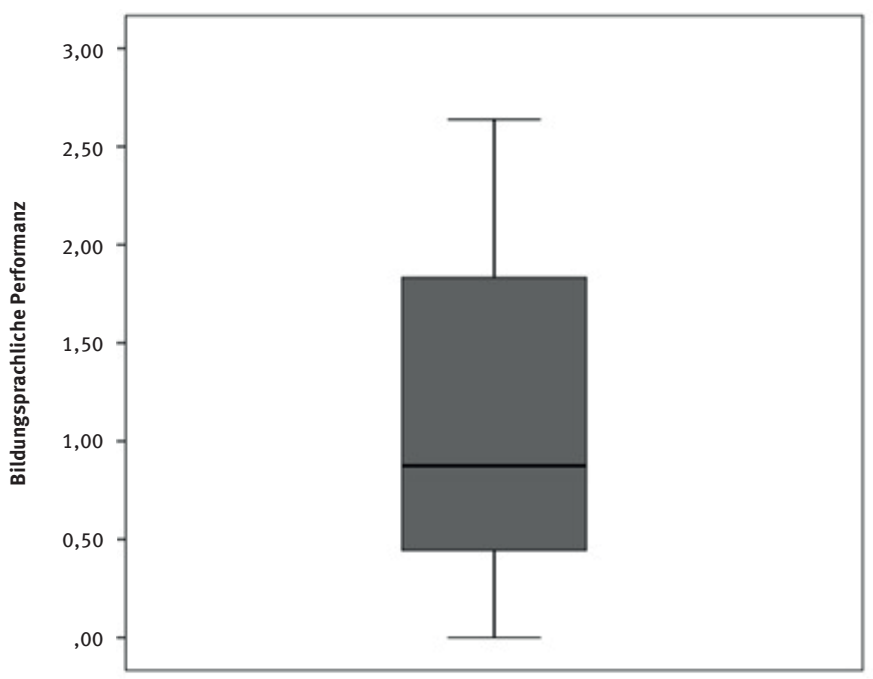

Abbildung 7. Boxplot der bildungssprachlichen Performanz in der Gesamtstichprobe (Min = $0.00, M a x=2.64, M W=1.09, S D=0.79, N=222$ )

In Tabelle 54 werden die deskriptiven Ergebnisse der gezeigten bildungssprachlichen Performanz nach Stichprobengruppe und Spracherwerbssituation dargestellt.

Tabelle 54. Deskriptive Übersicht bildungssprachlicher Performanz nach Stichprobengruppe und Spracherwerbssituation

\begin{tabular}{|c|c|c|c|c|c|c|c|}
\hline \multirow{3}{*}{$\begin{array}{l}\text { Bildungssprachliche } \\
\text { Performanz } \\
\text { Dimensionen }\end{array}$} & \multicolumn{2}{|c|}{$\begin{array}{l}\text { HdkF-Gruppe } \\
\qquad(n=118)\end{array}$} & \multicolumn{2}{|c|}{$\begin{array}{c}\text { Nawi-Gruppe } \\
\quad(n=35)\end{array}$} & \multicolumn{2}{|c|}{$\begin{array}{l}\text { Vergleichsgruppe } \\
\quad(n=69)\end{array}$} & \multirow{3}{*}{$\begin{array}{c}\text { Gesamt- } \\
\text { stich- } \\
\text { probe } \\
(N=222) \\
M(S D)\end{array}$} \\
\hline & \multicolumn{2}{|c|}{$M(S D)$} & \multicolumn{2}{|c|}{$M(S D)$} & \multicolumn{2}{|c|}{$M(S D)$} & \\
\hline & $\begin{array}{c}\mathrm{DaE} \\
(n=66)\end{array}$ & $\begin{array}{c}\mathrm{DaZ} \\
(n=52)\end{array}$ & $\begin{array}{c}\mathrm{DaE} \\
(n=19)\end{array}$ & $\begin{array}{c}\mathrm{DaZ} \\
(n=16)\end{array}$ & $\begin{array}{c}\mathrm{DaE} \\
(n=36)\end{array}$ & $\begin{array}{c}\mathrm{DaZ} \\
(n=33)\end{array}$ & \\
\hline Sprachhandlungen & $\begin{array}{c}1.55 \\
(1.51)\end{array}$ & $\begin{array}{c}1.56 \\
(1.51)\end{array}$ & $\begin{array}{c}0.79 \\
(1.36)\end{array}$ & $\begin{array}{c}1.50 \\
(1.55)\end{array}$ & $\begin{array}{c}1.08 \\
(1.46)\end{array}$ & $\begin{array}{c}1.09 \\
(1.47)\end{array}$ & $\begin{array}{c}1.33 \\
(1.49)\end{array}$ \\
\hline Lexikon & $\begin{array}{c}1.01 \\
(0.76)\end{array}$ & $\begin{array}{c}0.91 \\
(0.72)\end{array}$ & $\begin{array}{l}1.00 \\
(0.67)\end{array}$ & $\begin{array}{c}1.13 \\
(0.70)\end{array}$ & $\begin{array}{c}0.87 \\
(0.72)\end{array}$ & $\begin{array}{c}0.76 \\
(0.58)\end{array}$ & $\begin{array}{c}0.93 \\
(0.71)\end{array}$ \\
\hline Morphosyntax & $\begin{array}{c}0.91 \\
(0.63)\end{array}$ & $\begin{array}{c}0.88 \\
(0.54)\end{array}$ & $\begin{array}{l}0.96 \\
(0.65)\end{array}$ & $\begin{array}{c}1.22 \\
(0.58)\end{array}$ & $\begin{array}{c}1.11 \\
(0.65)\end{array}$ & $\begin{array}{c}1.12 \\
(0.68)\end{array}$ & $\begin{array}{c}0.99 \\
(0.62)\end{array}$ \\
\hline $\begin{array}{l}\text { Bildungssprachliche } \\
\text { Performanz gesamt }\end{array}$ & $\begin{array}{c}1.15 \\
(0.84)\end{array}$ & $\begin{array}{c}1.11 \\
(0.75)\end{array}$ & $\begin{array}{c}0.92 \\
(0.79)\end{array}$ & $\begin{array}{c}1.28 \\
(0.82)\end{array}$ & $\begin{array}{c}1.02 \\
(0.80)\end{array}$ & $\begin{array}{c}0.99 \\
(0.80)\end{array}$ & 0.79) \\
\hline
\end{tabular}




\subsection{Ergebnisse zu den Fragestellungen}

\subsubsection{Fragestellung 1: Sprachliche Anregungsqualität durch die pädagogischen Fachkräfte}

Zunächst wird untersucht, wie hoch die sprachliche Anregungsqualität in den naturwissenschaftlichen Lehr-Lern-Situationen ist.

Wie hoch ist die sprachliche Anregungsqualität in den naturwissenschaftlichen Lehr-Lern-Situationen in Abhängigkeit vom Nawi-Programm der Kita?

Insgesamt wird bei 12 pädagogischen Fachkräften (21\%) die sprachliche Anregungsqualität als unzureichend eingeschätzt. Siebenundzwanzig pädagogische Fachkräfte (47\%) zeigen eine gute sprachliche Anregungsqualität. Von diesen gehört der größte Anteil mit 17 pädagogischen Fachkräften (63\%) der Stichprobengruppe mit einer Zertifizierung als „Haus der kleinen Forscher“ an. Zwei pädagogische Fachkräfte der Gesamtstichprobe werden als exzellent eingeschätzt und sind ebenfalls der HdkF-Gruppe zuzuordnen (Abbildung 8).

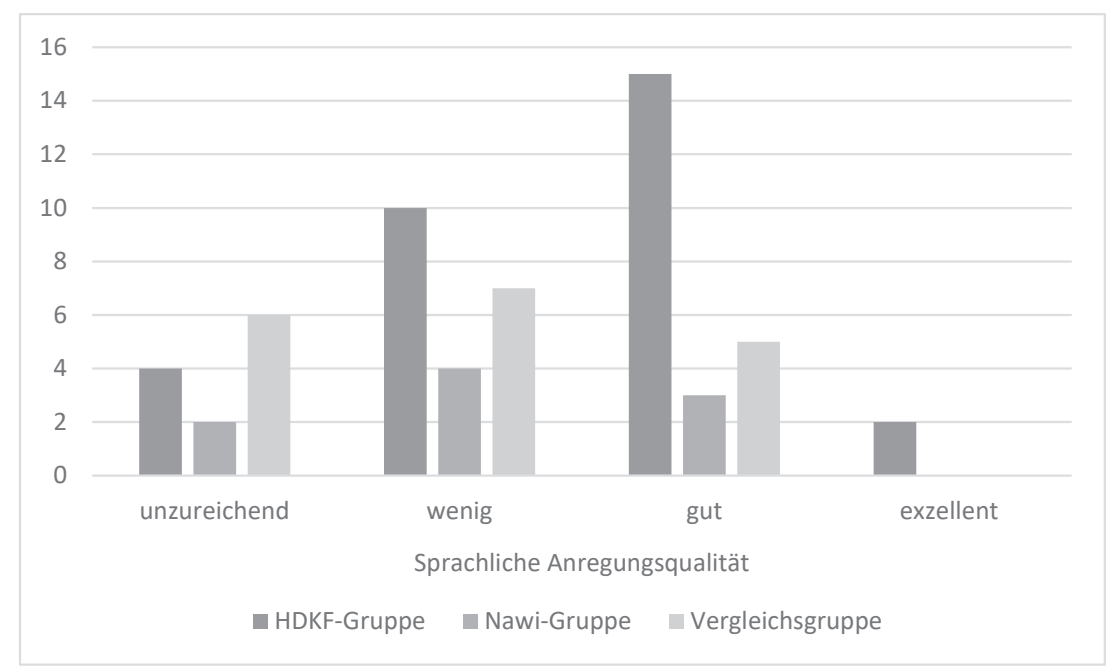

Abbildung 8. Verteilung der sprachlichen Anregungsqualität, aufgeschlüsselt nach Gruppen $(N=58)$

Bezüglich der sprachlichen Anregungsqualität wird mit einer einfaktoriellen Varianzanalyse ein mittlerer signifikanter Effekt zwischen den drei Stichprobengruppen herausgestellt $\left(F(2,55)=4.100, p=.022 ; \eta^{2}=0,13\right.$; Abbildung 9). 


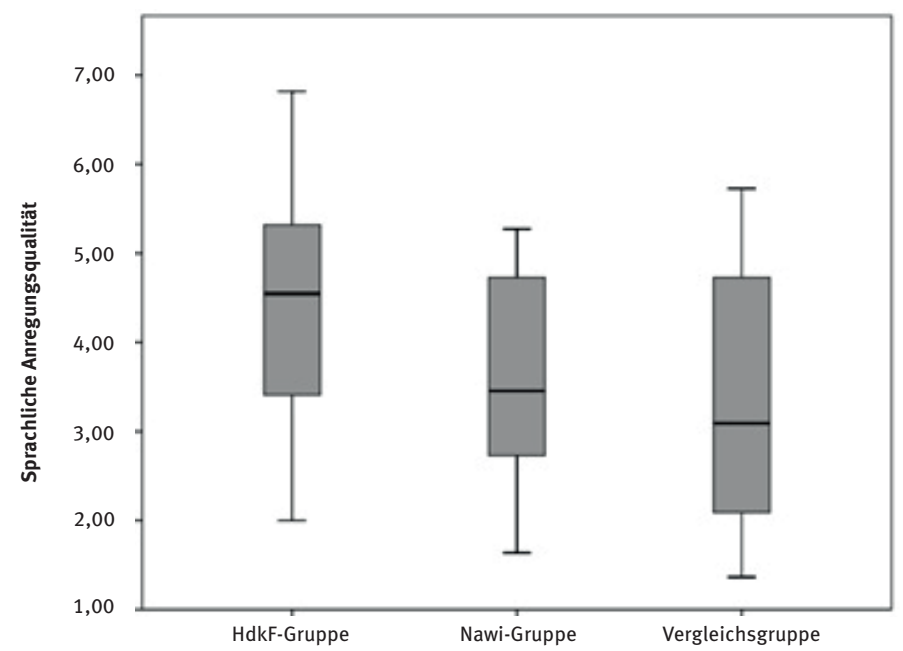

Abbildung 9. Einschätzung der sprachlichen Anregungsqualität der pädagogischen Fachkräfte, aufgeschlüsselt nach Gruppen ( $N=58)$

Die Paarvergleiche zwischen den Gruppen werden mit einem Post-hoc-Test (Bonferroni) durchgeführt. Dieser gibt an, dass die Unterschiede auf die Stichprobengruppe mit einer Zertifizierung als „Haus der kleinen Forscher“ - im Vergleich zu Einrichtungen, die an keinerlei naturwissenschaftlichen Fort- und Weiterbildungsmaßnahmen teilgenommen haben, zurückzuführen sind. Im Vergleich zur Gruppe mit einem anderweitigen naturwissenschaftlichen Schwerpunkt gibt es keine überzufälligen Effekte (Tabelle 55).

Tabelle 55. Gruppenvergleich sprachliche Anregungsqualität. Post-hoc-Test mit BonferroniKorrektur

\begin{tabular}{lccc}
\hline Stichprobengruppe & & Mittlere Differenz & Standardfehler \\
\hline \multirow{2}{*}{ HdkF-Gruppe } & Nawi-Gruppe & .94 & .53 \\
\cline { 2 - 4 } & Vergleichsgruppe & $1.09^{*}$ & .41 \\
\hline \multirow{2}{*}{ Nawi-Gruppe } & HdkF-Gruppe & -.94 & .53 \\
\cline { 2 - 4 } & Vergleichsgruppe & .15 & .57 \\
\hline \multirow{2}{*}{ Vergleichsgruppe } & HdkF-Gruppe & $-1.09^{*}$ & .41 \\
\cline { 2 - 4 } & Nawi-Gruppe & -.15 & .57 \\
\hline
\end{tabular}

* Die Differenz der Mittelwerte ist auf dem Niveau 0.05 signifikant. 
Wie hoch ist die sprachliche Anregungsqualität in den naturwissenschaftlichen Lehr-Lern-Situationen in Abhängigkeit vom Fortbildungsbesuch der Fachkräfte?

Die heterogene Gestaltung der Interaktionssituation lässt sich durch verschiedene Variablen erklären. Es kann vermutet werden, dass die Kenntnisse der Fachkraft entscheidend sind. Diese werden über die Fortbildungsaktivität der Fachkräfte modelliert. Im Fragebogen wurde nach den Fortbildungen der letzten 5 Jahre gefragt (siehe Tabelle 44).

Die Daten zeigen, dass es signifikante Zusammenhänge der sprachlichen Anregungsqualität mit den naturwissenschaftlichen Fortbildungsaktivitäten der pädagogischen Fachkräfte in den letzten 5 Jahren gibt (Tabelle 56). Es zeigt sich dabei auch, dass die sprachliche Anregung in der Situation nicht mit den sprachbezogenen Fortbildungen zusammenhängt, obwohl diese thematisch sehr nahe an dem liegen, was in der Situation bewertet wurde, nämlich der hochwertigen alltagsintegrierten Sprachbildung. Bemerkenswert ist in diesem Zusammenhang auch eine Fortbildung, in der explizit Hinweise zur Verbindung sprachlicher und naturwissenschaftlicher Inhalte gegeben werden, die Fortbildung „Forschen mit Sprudelgas“ der Stiftung „Haus der kleinen Forscher“. Nach einem Besuch dieser Fortbildung ist die sprachliche Anregungsqualität in der Experimentiersituation signifikant höher (siehe Tabelle 56).

Dieser Zusammenhang zeigt sich auch im deskriptiven Vergleich: Die fünf Fachkräfte, deren sprachliche Anregungsqualität insgesamt am höchsten eingeschätzt wurde, hatten alle an dieser Fortbildung teilgenommen, während die Fachkräfte mit der geringsten Anregungsqualität allesamt diese Fortbildung nicht besucht hatten. Es ist also davon auszugehen, dass das Wissen der Fachkraft über Naturwissenschaften, insbesondere das Wissen über den Zusammenhang von Naturwissenschaften und Sprache, die sprachliche Anregungsqualität in der Forschungssituation beeinflusst.

Tabelle 56. Zusammenhang der sprachlichen Anregungsqualitätmit dem Fortbildungsbesuch

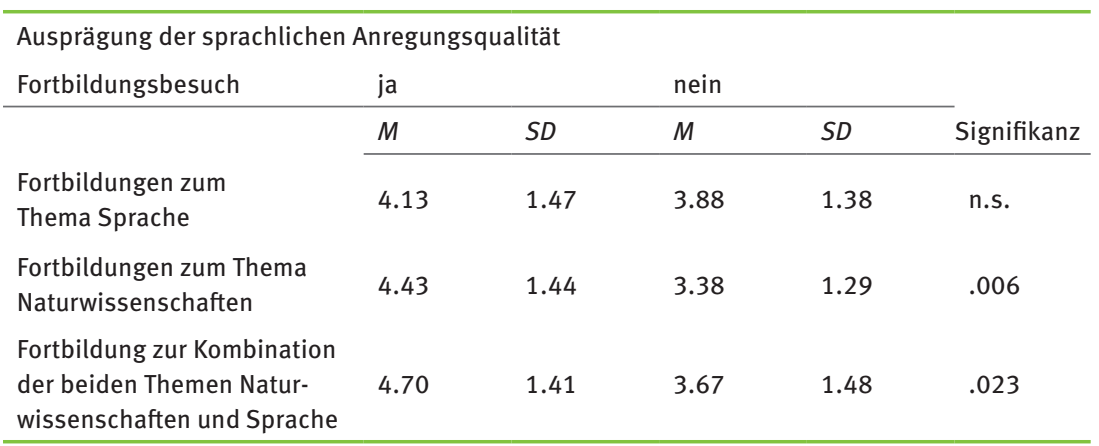


Wie hoch ist die sprachliche Anregungsqualität in den naturwissenschaftlichen Lehr-Lern-Situationen in Abhängigkeit von der Phase des Forschungskreises?

Sowohl die einzelnen Phasen des Forschungskreises als auch die darin zu lokalisierenden Indikatoren der sprachlichen Anregungsqualität der pädagogischen Fachkräfte wurden anhand des Extremfallsamplings $(n=15)$ qualitativ analysiert (siehe Kapitel 3.2.1).

Abbildung 10 zeigt alle Kodierungen. Die Kodierung einer Phase beginnt mit der erkennbaren Einleitung der Interaktion und endet sobald dieser Interaktion nicht mehr nachgegangen wird oder eine neue Phase beginnt. Somit wurden zum Teil sehr kurze Abschnitte kodiert (ein bis zwei Sätze), aber auch längere Abschnitte von 30 Sätzen. Insgesamt wurde jeweils das komplette Transkript in Phasen eingeteilt. Die Einleitung der Phase konnte sowohl von der Fachkraft als auch vom Kind ausgehen. Innerhalb einer Versuchseinheit wurden also immer wieder diese Phasen mehrfach kodiert. Die Interkoderreliabilität dieser Phasen wurde anhand zweier Videos überprüft und lag bei $\mathrm{k}=.696$.

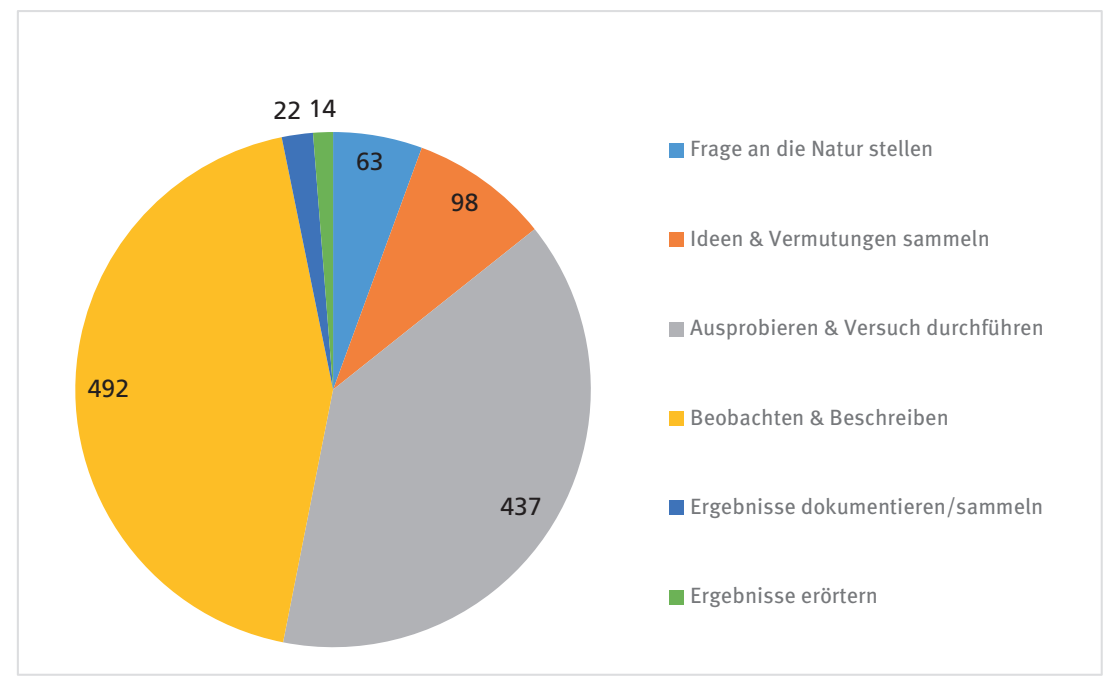

Abbildung 10. Realisierte Forschungskreisphasen - absolute Anzahl der Kodierungen ( $n=15$ videografierte Lernsituationen)

Innerhalb der 30-minütigen Settings werden die beiden Forschungskreisphasen Ausprobieren \& Versuch durchführen sowie Beobachten \& Beschreiben am häufigsten realisiert. Am seltensten lassen sich die Phasen Frage an die Natur stellen und Ergebnisse erörtern finden.

Die Phasen Ausprobieren \& Versuch durchführen sowie Beobachten \& Beschreiben kommen annähernd gleich häufig vor (437 und 492). Dabei sind in der 
Phase Beobachten \& Beschreiben doppelt so viele Merkmale der sprachlichen Anregung festzustellen wie in der Phase Ausprobieren \& Versuch durchführen (siehe Tabelle 57).

Tabelle 57. Sprachliche Anregung innerhalb der Forschungskreisphasen

\begin{tabular}{lccc}
\hline Forschungskreisphase & $\begin{array}{c}\text { Häufigkeit } \\
\text { Forschungs- } \\
\text { kreisphase }\end{array}$ & $\begin{array}{c}\text { Anzahl Merkmale } \\
\text { sprachlicher } \\
\text { Anregung }\end{array}$ & $\begin{array}{c}\text { Merkmale } \\
\text { sprachlicher } \\
\text { Anregung } \\
\text { pro Einheit }\end{array}$ \\
\hline Frage an die Natur stellen & 63 & 37 & 0.59 \\
Ideen \& Vermutungen sammeln & 98 & 50 & 0.51 \\
Ausprobieren \& Versuch & 437 & 105 & 0.24 \\
durchführen & 492 & 252 & 0.51 \\
Beobachten \& Beschreiben & 22 & 24 & 1.09 \\
Ergebnisse dokumentieren/sammeln & 14 & 17 & 1.21 \\
\hline Ergebnisse erörtern & & & \\
\hline
\end{tabular}

Betrachtet man die sprachliche Anregungsqualität differenzierter, so zeigt sich, dass in der Forschungskreisphase Beobachten \& Beschreiben im Vergleich zu den anderen Phasen der Indikator „Wiederholung unvollständiger Aussagen“ besonders häufig realisiert wird (siehe Tabelle 58), ebenso innerhalb der Phase Beobachten \& Beschreiben die Indikatoren „Impuls“. „Wiederholung unvollständiger Aussagen“ und „Längere Sätze“.

Tabelle 58. Häufigkeit der Indikatoren sprachlicher Anregung innerhalb der einzelnen Forschungskreisphasen

\begin{tabular}{|c|c|c|c|c|c|c|c|c|c|}
\hline & $\begin{array}{l}\frac{n}{3} \\
\text { 트 }\end{array}$ & 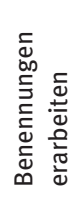 & 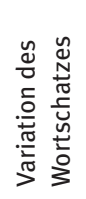 & 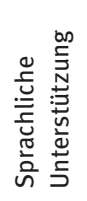 & 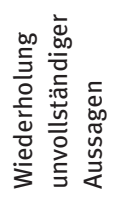 & 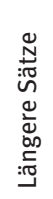 & 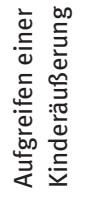 & 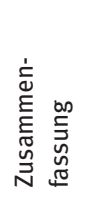 & 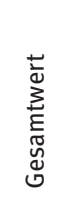 \\
\hline $\begin{array}{l}\text { Frage an die Natur } \\
\text { stellen }\end{array}$ & 7 & 4 & 2 & 3 & 6 & 13 & 2 & 0 & 37 \\
\hline $\begin{array}{l}\text { Ideen \& Vermutun- } \\
\text { gen sammeln }\end{array}$ & 13 & 3 & 4 & 6 & 5 & 7 & 10 & 2 & 50 \\
\hline $\begin{array}{l}\text { Ausprobieren \& } \\
\text { Versuch durchführen }\end{array}$ & 18 & 5 & 12 & 4 & 11 & 31 & 17 & 7 & 105 \\
\hline $\begin{array}{l}\text { Beobachten \& } \\
\text { Beschreiben }\end{array}$ & 50 & 20 & 31 & 18 & 40 & 40 & 36 & 17 & 252 \\
\hline $\begin{array}{l}\text { Ergebnisse } \\
\text { dokumentieren/ } \\
\text { sammeln }\end{array}$ & 5 & 0 & 2 & 3 & 2 & 5 & 2 & 5 & 24 \\
\hline Ergebnisse erörtern & 5 & 1 & 1 & 0 & 1 & 2 & 3 & 4 & 17 \\
\hline
\end{tabular}


Tabelle 57 zeigt, dass die beiden Phasen Ergebnisse dokumentieren/sammeln und Ergebnisse erörtern im Vergleich zu den Phasen Ausprobieren \& Versuch durchführen sowie Beobachten \& Beschreiben vergleichsweise selten realisiert werden. Werden sie jedoch realisiert, so lassen sich in nahezu jeder Einheit Merkmale der sprachlichen Anregung herausstellen.

\subsubsection{Fragestellung 2: Bildungssprachliche Performanz der Kinder}

Die zweite Fragestellung bezieht sich auf die Ebene der Kinder.

Gibt es Unterschiede in den verschiedenen Untersuchungsgruppen bezüglich der Verwendung von Bildungssprache durch die Kinder?

Die Beurteilung der Sprachverwendung in naturwissenschaftlichen Interaktionssituationen erfolgte über die Erfassung der bildungssprachlichen Performanz der Kinder. Diese wurde mit der RaBi-Skala (siehe S. 173ff.) eingeschätzt. Es gibt keine signifikanten Unterschiede $(p=.561)$ zwischen den Gruppen bezüglich der bildungssprachlichen Performanz insgesamt (siehe Tabelle 59).

Bei Betrachtung der einzelnen Dimensionen sind signifikante Unterschiede herauszustellen, jedoch mit kleinen Effekten (Tabelle 59). Hierbei ist die Morphosyntax bei den Kindern aus Einrichtungen des „Hauses der kleinen Forscher“ auf niedrigerem Niveau als bei Kindern aus den anderen beiden Gruppen. Die Sprachhandlungen sind bei den Kindern der HdkF-Gruppe hingegen auf höherem Niveau als bei Kindern aus den anderen beiden Gruppen.

Tabelle 59. Bildungssprachliche Performanz der Kinder, getrennt nach Dimensionen

\begin{tabular}{cccccc}
\hline & $\begin{array}{c}\text { HDKF-Gruppe } \\
(n=118)\end{array}$ & $\begin{array}{c}\text { Nawi-Gruppe } \\
(n=35)\end{array}$ & $\begin{array}{c}\text { Vergleichs- } \\
\text { gruppe } \\
(n=69)\end{array}$ & Signifikanz & Eta $^{2}$ \\
\hline Morphosyntax & $0.90(0.59)$ & $1.08(0.62)$ & $1.12(0.66)$ & .05 & .03 \\
Lexikon & $0.96(0.74)$ & $1.06(0.67)$ & $1.12(0.65)$ & .20 & .01 \\
Sprachhandlungen & $1.55(1.50)$ & $1.11(1.47)$ & $1.09(1.49)$ & .08 & .02 \\
\hline Gesamt & $1.14(0.80)$ & $1.08(0.81)$ & $1.01(0.80)$ & .56 & .01 \\
\hline
\end{tabular}

Bei Betrachtung der bildungssprachlichen Performanz der Kinder, getrennt nach der Spracherwerbssituation, wird deutlich, dass für Kinder mit Deutsch als Erstsprache die Gruppenvergleiche lediglich eine tendenziell bessere Performanz bei den Sprachhandlungen für die Kinder der HdkF-Gruppe ergeben (Tabelle 60). Für die Kinder mit Deutsch als Zweitsprache zeigt sich tendenziell ein Vorteil bei der Morphosyntax für die Kinder aus Kitas der Vergleichsgruppe. In beiden Fällen wirken sich diese Unterschiede allerdings nicht auf die bildungssprachliche Performanz insgesamt aus. 
Tabelle 6o. Bildungssprachliche Performanz bei Kindern mit DaE $(n=121)$ und DaZ $(n=101)$

\begin{tabular}{ccccccc}
\hline \multirow{6}{*}{ Morphosyntax } & $\begin{array}{c}\text { Sprach- } \\
\text { erwerbs- } \\
\text { situation }\end{array}$ & $\begin{array}{c}\text { HDKF- } \\
\text { Gruppe }\end{array}$ & $\begin{array}{c}\text { Nawi- } \\
\text { Gruppe }\end{array}$ & $\begin{array}{c}\text { Vergleichs- } \\
\text { gruppe }\end{array}$ & Signifikanz & Eta $^{2}$ \\
& DaE & $0.91(0.63)$ & $0.96(0.65)$ & $1.11(0.65)$ & .33 & .02 \\
Lexikon & DaZ & $0.88(0.54)$ & $1.23(0.58)$ & $1.12(0.68)$ & .06 & .06 \\
Sprach- & DaZ & $1.00(0.76)$ & $1.00(.67)$ & $0.87(0.71)$ & .66 & .01 \\
handlungen & DaE & $1.55(1.51)$ & $0.79(1.36)$ & $1.08(1.46)$ & .09 & .04 \\
\hline \multirow{2}{*}{ Gesamt } & DaZ & $1.56(1.51)$ & $1.50(1.54)$ & $1.09(1.47)$ & .37 & .02 \\
\hline & DaE & $1.15(0.84)$ & $0.92(0.78)$ & $1.02(0.80)$ & .48 & .01 \\
& DaZ & $1.01(0.61)$ & $1.28(0.82)$ & $0.99(0.80)$ & .47 & .02 \\
\hline
\end{tabular}

Gibt es Zusammenhänge zwischen der sprachlichen Anregungsqualität und der Verwendung von Bildungssprache durch die Kinder?

Für die Sprachfähigkeiten der Kinder im naturwissenschaftlichen Kontext werden die Ergebnisse aus der RaBi-Skala herangezogen. Davon ausgehend, dass die sprachliche Anregungsqualität der pädagogischen Fachkräfte in der Experimentiersituation einen Einfluss auf die in der Situation gezeigten bildungssprachlichen Kompetenzen der Kinder hat, wird unter Berücksichtigung der hierarchischen Struktur, eine lineare Regression gerechnet.

Die lineare Regression gibt einen signifikanten Effekt der sprachlichen Anregungsqualität $\left(R^{2}=.026, p=.015\right)$ an. Dieser Effekt kann bei Kontrolle der weiteren Variablen allerdings nicht aufrechterhalten werden. Auch die Tatsache, ob die Kinder Deutsch als Erst- oder Zweitsprache erwerben, spielt hier keine Rolle (siehe Tabelle 61). Den größten Einfluss haben die Variablen der Kinder (Alter, Geschlecht, Intelligenz).

Weiterhin wurde untersucht, inwieweit die Verwendung von Bildungssprache durch die Kinder in der Forschungssituation mit dem Fortbildungsbesuch der pädagogischen Fachkräfte zusammenhängt. Kinder, deren pädagogische Fachkraft die Fortbildung „Forschen mit Sprudelgas“ besucht hat, zeigen eine höhere bildungssprachliche Performanz in der Nawi-Situation als Kinder, deren Fachkraft diese Fortbildung nicht besucht hat $(p=.026)$. Dieser Effekt ist unabhängig von den Variablen der Kinder wie Alter, IQ oder Mehrsprachigkeit. Reine Sprach- oder Nawi-Fortbildungen hingegen wirken sich in der Forschungssituation nicht auf die bildungssprachliche Performanz der Kinder aus. 
Tabelle 61. Betagewichte der verschiedenen Einflussvariablen auf die bildungssprachliche Performanz der Kinder $(n=220)$

\begin{tabular}{|c|c|c|c|c|c|c|}
\hline & Modell 1 & Modell 2 & Modell 3 & Modell 4 & Modell 5 & Modell 6 \\
\hline Spracherwerbssituation & .024 & & & & & .049 \\
\hline $\begin{array}{l}\text { Sprachliche } \\
\text { Anregungsqualität }\end{array}$ & & $.162^{\star}$ & & & & .119 \\
\hline $\begin{array}{l}\text { Kompetenz } \\
\text { Sprachproduktion }\end{array}$ & & & $.294^{\star}$ & & & .074 \\
\hline $\begin{array}{l}\text { Kompetenz } \\
\text { Sprachverständnis }\end{array}$ & & & .030 & & & $.187^{\star \star}$ \\
\hline Kind: Alter & & & & $.269^{\star \star \star}$ & & $.381^{\star \star \star}$ \\
\hline Kind: Geschlecht & & & & $-.206^{\star \star *}$ & & $-.217^{\star \star}$ \\
\hline Kind: Intelligenz & & & & $.252^{\star \star \star}$ & & $.272^{\star \star \star}$ \\
\hline $\begin{array}{l}\text { Eltern: Bildungshinter- } \\
\text { grund }\end{array}$ & & & & & .155 & .175 \\
\hline Eltern: Leseverhalten & & & & & .136 & .090 \\
\hline $\begin{array}{l}\text { Eltern: häusliche Unter- } \\
\text { stützung }\end{array}$ & & & & & .020 & .020 \\
\hline $\mathrm{R}^{2}$ & .001 & .026 & .051 & .137 & .064 & .294 \\
\hline
\end{tabular}

${ }^{*} \mathrm{p}<.05 ;{ }^{* *} \mathrm{p}<.01 ;{ }^{* * *} \mathrm{p}<.001 ;$ Geschlecht ist so kodiert, dass positive Werte auf höhere Werte der Mädchen deuten; Spracherwerbssituation ist so kodiert, dass positive Werte auf höhere Werte der Kinder mit DaE deuten; $\mathrm{R}^{2}$ = Stichprobenvarianz 


\section{Diskussion}

Den Ausgang der Studie EASI Science-L bildeten drei theoretische Annahmen, die im aktuellen Fachdiskurs um frühkindliche Bildung und der Entwicklung bildungssprachlicher Fähigkeiten im Elementarbereich virulent sind:

(1) der Zusammenhang zwischen konventioneller kindlicher Sprachentwicklung und der Aneignung von Bildungssprache,

(2) die Möglichkeit der Nutzung von naturwissenschaftlichen Lernsettings für die Sprachförderung sowie

(3) die Bedeutung der sprachlichen Anregungsqualität in der Fachkraft-Kind-Interaktion und deren Effekte auf die (bildungs-)sprachlichen Aktivitäten der jungen Lernerinnen und Lerner.

Die Hauptfragestellung der Studie war der Einfluss der Gestaltung der Lehr-Lern-Situation sowie der darin realisierten sprachlichen Anregungsqualität der pädagogischen Fachkraft auf die bildungssprachliche Performanz der Kinder. Andere Studien konnten Hinweise darauf geben, wie sprachliches und kognitives Anregungspotenzial in naturwissenschaftlichen Interaktionssituationen der Kita verwirklicht werden kann wie z. B. durch Sustained Shared Thinking (Hopf, 2012) oder durch die Elizitierung von Student Critical Turns im naturwissenschaftlichen Diskurs (Li, 2017).

Grundsätzlich konnte in der Studie EASI Science-L festgestellt werden, dass sich die Realisierung der sprachlichen Anregungsqualität durch die Fachkräfte über alle Ausprägungen verteilt. Signifikant am besten waren dabei die Fachkräfte, die in einer Kita mit Zertifizierung als „Haus der kleinen Forscher“ arbeiten. Außerdem zeigten die Fachkräfte eine bessere Anregungsqualität, die Fortbildungen zu einem naturwissenschaftlichen Thema besucht hatten. Hier lässt sich also vermuten, dass das naturwissenschaftliche Fachwissen der Fachkräfte einen Einfluss hat. Zudem zeigt sich ein signifikanter Einfluss der Fortbildung „Forschen mit Sprudelgas“, die von der Stiftung „Haus der kleinen Forscher“ angeboten wird. Die Fachkräfte mit der höchsten sprachlichen Anregungsqualität hatten diese Fortbildung besucht, in der Sache und Sprache kombiniert werden. Dieser Befund deutet unseres Erachtens darauf hin, dass eine gewisse Vertrautheit mit der inhaltlichen (naturwissenschaftlichen) Thematik bedeutsam auch für die sprachliche Anregungsqualität ist. Selbst auf Ebene der Kinder konnte der Einfluss dieser Fortbildung gefunden werden. Der Ansatz, in der Fortbildung Sache und Sprache inhaltlich zu koppeln, ist also erfolgversprechend. 
In der qualitativen Teilstichprobe (vgl. Abschnitt 3.2.1, Extremfallsampling) zeigt sich, dass gerade komplexe, aber anregungsreiche Diskursformen (Ergebnisse dokumentieren/sammeln und Ergebnisse erörtern) in den naturwissenschaftlichen Situationen in der Kita eher selten realisiert werden. Hier lässt sich auch die Brücke zur Sachkenntnis der Fachkräfte schlagen, denn die komplexeren Diskursformen verlangen auch eine grundlegendere Kenntnis des Gegenstandes. Als Folgerung kann man aus diesen Ergebnissen ziehen, dass die pädagogischen Fachkräfte fachliches und fachdidaktisches Wissen sowohl über den Gegenstand als auch über die Sprache benötigen.

Die Studie EASI Science-L hat weiterhin gezeigt, dass auch im Vorschulalter und in einer medial mündlichen Situation (hier: Forschungssituation) bildungssprachliche Kompetenzen beobachtet und eingeschätzt werden können. Die an der Studie teilnehmenden Kinder zeigen im Mittel ein alltagssprachliches Niveau, was der medial mündlichen Situation und dem Stand der Sprachentwicklung angemessen ist. Allerdings ist die Streuung nach oben deutlich, und man findet Kinder vor, die sehr kompetent bildungssprachliche Muster realisieren. Diese Kompetenz in der Forschungssituation ist wider Erwarten nicht von der Spracherwerbssituation im Elternhaus abhängig, es ist also für die Verwendung von Bildungssprache zunächst irrelevant, ob die Kinder Deutsch als Erst- oder Zweitsprache erwerben - Bildungssprache ist für alle Kinder gleichermaßen komplex. Allerdings sieht man signifikante Einflüsse anderer Variablen auf die Verwendung von Bildungssprache in der untersuchten Situation: Da Sprache entwicklungsabhängig ist, ist der Einfluss des Alters gut zu erklären. Der Vorteil von Jungen zeigt sich auch in anderen Studien (z. B. bei Runge, 2013) und kann eventuell mit einem höheren Interesse an naturwissenschaftlichen Themen erklärt werden. Der Einfluss der Intelligenz weist darauf hin, dass die Verwendung von Bildungssprache kognitive Kompetenzen voraussetzt, wie auch andere Autoren (z. B. Feilke, 2012; Morek \& Heller, 2012) vermuten. Zum einen ist Intelligenz für den abstrahierenden Umgang mit Sprache nötig, zum anderen aber auch zur Durchdringung des Fachgegenstandes und der Möglichkeit, diesen zu strukturieren und in Spracheinheiten zu übermitteln. Bildungssprachlich hochwertige Sprachhandlungen wie begründetes Erklären oder Vermuten können wohl nur dann stattfinden, wenn das Kind die Erklärung und Vermutung gemessen an der Sache auch kognitiv vollziehen kann. Dass hierzu auch Input und Übungsmöglichkeiten nötig sind, erklärt den hohen Einfluss des Bildungsstandes der Eltern auf die bildungssprachliche Kompetenz in der Situation.

Betrachtet man lediglich die Korrelationen der sprachlichen Anregungsqualität der pädagogischen Fachkräfte mit der bildungssprachlichen Kompetenz der Kinder in der Sachsituation, dann findet man einen Zusammenhang, der zu der Vermutung Anlass gibt, dass eine sprachlich anregende Gestaltung der Situation 
auch zu bildungssprachlichen Kompetenzen bei den Kindern führt. Mit Einbezug der Kindvariablen (Alter, Geschlecht, Intelligenz) verschwindet der Effekt. Die Zusammenhänge sind aber nicht einfach monokausal: Kinder aus bildungsnahen Familien besuchen bessere Kitas (vgl. Liebers, 2016). Fachkräfte richten ihr Sprachverhalten an den Kindern aus, das häusliche Umfeld ist wirkmächtiger als die Bildungsinstitution. Dennoch kann dieser Befund nicht entmutigen: Die Studie EASI Science-L zeigt, dass Sprachförderung in naturwissenschaftlichen Settings möglich und sinnvoll ist. Die gezielte Kombination von Sache und Sprache in Fortbildungen scheint bis auf die Ebene der Kinder zu wirken: Die Teilnahme der Fachkräfte an der Fortbildung „Sprudelgas und andere Stoffe - Mit Kita- und Grundschulkindern Chemie entdecken und dabei die sprachliche Entwicklung unterstützen“ zeigt Korrelationen mit der bildungssprachlichen Performanz bei den Kindern.

Die Limitationen der Studie sollen hierbei nicht verschwiegen werden. Die Situation war künstlich angeordnet und dauerte lediglich eine halbe Stunde. Die Fachkräfte nahmen freiwillig teil und suchten selbst die Kinder aus. Die bildungssprachliche Performanz wurde in einer medial mündlichen und nicht in einer spezifisch bildungssprachlichen Situation (z. B. Kinder diktieren etwas) erhoben. Die Gruppe der mehrsprachigen Kinder wurde (trotz der Vielfalt von über 40 neben dem Deutschen vorhandenen Sprachen) als homogene Gruppe interpretiert. 


\section{Zusammenfassung der zentralen Ergebnisse}

Die Ergebnisse der Studie EASI Science-L (Teilprojekt 1) lassen sich wie folgt zusammenfassen:

- Die pädagogischen Fachkräfte aus Einrichtungen mit einer Zertifizierung als „Haus der kleinen Forscher“ zeigen im Schnitt die höchste sprachliche Anregungsqualität in der Forschungssituation. Bei der Gesamtstichprobe liegt die Qualität der sprachlichen Anregung im mittleren Bereich.

- Die sprachliche Anregungsqualität ist in der Gruppe der pädagogischen Fachkräfte aus Einrichtungen mit einer Zertifizierung als „Haus der der kleinen Forscher" größtenteils gut, bei zwei pädagogischen Fachkräften sogar exzellent.

- Fachkräfte, die mindestens eine Fortbildung zu naturwissenschaftlichen Themen besucht haben, zeigen eine höhere sprachliche Anregungsqualität als Fachkräfte ohne entsprechende Fortbildung.

- Fachkräfte, die die kombinierte Fortbildung zu Naturwissenschaften und Sprache (,Forschen mit Sprudelgas“ vom Haus der kleinen Forscher) besucht haben, zeigen eine höhere sprachliche Anregungsqualität als Fachkräfte ohne diese Fortbildung.

- Hinsichtlich der Phasen des Forschungskreises der Stiftung „Haus der kleinen Forscher" kann festgehalten werden, dass die Phasen Ausprobieren \& Versuch durchführen sowie Beobachten \& Beschreiben etwa gleich häufig vorkommen. Dabei sind in der Phase Beobachten \& Beschreiben doppelt so viele Merkmale der sprachlichen Anregung festzustellen wie in der Phase Ausprobieren \& Versuch durchführen.

- Die Phasen Ergebnisse dokumentieren/sammeln und Ergebnisse erörtern kommen auch in der Gruppe des „Hauses der kleiner Forscher” eher selten vor. Sie gelten aber als (bildungs-)sprachlich besonders anregend und könnten daher in zukünftigen Fortbildungen verstärkt thematisiert und geübt werden.

- Eine Korrelation der bildungssprachlichen Fähigkeiten der Kinder mit der sprachlichen Anregungsqualität der pädagogischen Fachkraft in der For- 
schungssituation konnte bei Kontrolle aller Hintergrundvariablen (Eltern und Kinder) nicht gefunden werden. Den größten Einfluss auf die bildungssprachliche Kompetenz der Kinder haben kindbezogene Variablen wie das Alter, die Intelligenz und das Geschlecht.

- Die Teilnahme der Fachkräfte an der kombinierten Fortbildung zu Naturwissenschaften und Sprache zeigt Korrelationen mit der bildungssprachlichen Performanz bei den Kindern. Kinder, deren pädagogische Fachkraft die Fortbildung „Forschen mit Sprudelgas“ besucht hat, zeigen eine höhere bildungssprachliche Performanz beim Forschen als Kinder, deren Fachkraft diese Fortbildung nicht besucht hat. 


\section{Fazit und Empfehlungen}

Naturwissenschaftliche Lernsituationen bieten eine gute Möglichkeit für eine integrierte Sprachförderung. Die explizite Berücksichtigung dieser Sprachförderung, so wie sie in der von der Stiftung „Haus der kleinen Forscher“ angebotenen Fortbildung „Forschen mit Sprudelgas“ geschieht, ist sicherlich ein gewinnbringender Weg - dies zeigen die Daten zur entsprechenden Fortbildung. Dieser Weg könnte auch für weitere Themen ausgebaut werden. Es ist zudem zu überlegen, ob eine kombinierte, langfristige Fortbildung, in der sprachliche und naturwissenschaftliche Aspekte thematisiert werden, angeboten werden kann.

Es lässt sich festhalten, dass auch Fortbildungen zum naturwissenschaftlichen Lernen einen Betrag leisten, um die (bildungs-)sprachlichen Fähigkeiten von Lernerinnen und Lernern mit Deutsch als Erst- oder Zweitsprache zu fördern. Die Sachkenntnis der Fachkräfte ist auch bei der Gestaltung des Forschens entscheidend. Dabei ist in den naturwissenschaftlichen Fortbildungen zu beachten, dass die Phasen des Forschungskreises weiterhin thematisiert, dabei aber die bildungssprachlich förderlichen Phasen des Forschungskreises Ergebnisse dokumentieren/sammeln und Ergebnisse erörtern stärker in den Fokus gerückt werden. Die Studie konnte zeigen, dass deren Realisierung in nahezu jeder Einheit Merkmale der sprachlichen Anregung evoziert. Dies lässt die vorläufige Annahme zu, dass besonders in diesen beiden Phasen intensiv sprachliche Anregung stattfinden kann und eine häufigere Realisierung dieser beiden Phasen daher erstrebenswert ist.

Ergänzend könnte und sollte aufgrund der Häufigkeit der Phase Ausprobieren \& Versuch durchführen die sprachliche Anregung verbessert werden. In dieser Phase handeln die Kinder mehr als sie sprechen, somit hat die Fachkraft auch weniger Möglichkeiten, auf die Äußerungen der Kinder zu reagieren. Wenn die Fachkraft hier die Kinder mehr dazu anregt, ihr Handeln sprachlich zu begleiten (Impulse), so kann sie häufiger auf die Aussagen der Kinder variierend, erweiternd und korrigierend eingehen.

Die Stiftung „Haus der kleinen Forscher“ agiert vor allem im Elementarbereich und weitet ihr Engagement in den letzten Jahren in den Primarbereich aus. Unter dem Aspekt der Sprachbildung als Kontinuum wäre auch eine Ausweitung der Verknüpfung von Naturwissenschaften und Sprache auf das Alter von 0-10 Jahren empfehlenswert. Unter dem Aspekt der Inklusion wäre zudem eine Ausweitung auf Kinder mit Förderbedarf zu überlegen.

Für die weitere Forschung ist von Interesse, inwieweit kombinierte Fortbildungen (Sprache und Sache) die sprachliche Anregungsqualität und letztlich die kindlichen Sprachleistungen effektiver beeinflussen können als rein sprachliche 
oder naturwissenschaftliche Fortbildungen. Zu diesen Zusammenhängen könnte eine randomisierte Interventionsstudie im Pre-Post-Follow-up-Design noch detailliertere Hinweise geben.

Die Auswirkungen von naturwissenschaftlichen Fortbildungen auf die sprachliche Anregungsqualität der Fachkräfte und die bildungssprachlichen Fähigkeiten der Kinder geben einen Hinweis darauf, dass Sachkenntnis eine Rolle bei der Sprachförderung spielt. Den Zusammenhang zwischen Sachkompetenz und Sprachkompetenz sowohl auf Fachkraft- als auch auf Kind-Seite zu erforschen, wäre eine lohnende Studie.

Insgesamt wären vertiefende Längsschnittanalysen und die Dokumentation von Einzelfällen hilfreich, um Aussagen über die Qualität des sprachlichen Inputs und der kindlichen (Sprach-)Fähigkeiten treffen zu können. Auch die weitere Erprobung und Validierung der Ratingskala RaBi ist von empirischem und letztlich von didaktischem Interesse.

Insgesamt aber geht die Stiftung „Haus der kleinen Forscher“ einen guten Weg. Unsere Studie konnte zeigen, dass sich dieser auch sprachförderlich auszahlt. 


\section{Early Steps into Science and Literacy - EASI Science-L Teil 2}

Wirkungen naturwissenschaftlicher Bildungsangebote auf die Gestaltung von Forschungssituationen durch pädagogische Fachkräfte und auf die naturwissenschaftlichen Fähigkeiten von Vorschulkindern

Sabina Pauen, Rahel Kästner

1 Einleitung

2 Fragestellungen

3 Datenerhebung und Methodik

4 Entwicklung von QUOTS (Quality of Teaching Science)

5 Zusammenhänge zwischen fachkraftspezifischen Kompetenzen und kindlichem Erkenntnisprozess

6 Prozessqualität in Interaktionen und sprachliche Anregungsqualität

7 Prozessqualität in Interaktionen und naturwissenschaftliche Vorbildung der Fachkräfte

8 Diskussion

9 Fazit und Empfehlungen 


\section{Einleitung}

Nach Sodian und Mayer (2013) kann der Begriff wissenschaftliches Denken aus zwei Perspektiven betrachtet werden. Zum einen bezieht er sich auf das inhaltsbezogene wissenschaftliche Denken über Phänomene und Prozesse. Die zweite Perspektive betrachtet das formale wissenschaftliche Denken, welches den Erkenntnisprozess selbst beinhaltet. Dieser Erkenntnisprozess wird von Sodian und Mayer (2013) als ein zyklischer und kumulativer Prozess mit vier Hauptelementen beschrieben (vgl. auch Kuhn, 2010): Aus bestehenden Theorien werden Hypothesen über einen Sachverhalt abgeleitet. Daraufhin werden Experimente zur Überprüfung der Hypothesen geplant und durchgeführt und die daraus gewonnenen Informationen oder Daten interpretiert. Entsprechende Schlussfolgerungen werden genutzt, um die anfänglichen Theorien weiterzuentwickeln und zu revidieren.

Im Einklang mit Modellen zur Förderung des frühen naturwissenschaftlichen Denkens (Anders, Hardy, Pauen \& Steffensky, 2013; Marquardt-Mau, 2004, 2011) beschreibt die Stiftung mit der Methode „Forschungskreis“ praxisnah die Teilschritte des oben geschilderten naturwissenschaftlichen Erkenntnisprozesses, zu denen Kindergartenkinder bereits in der Lage sind (vgl. Pädagogischer Ansatz der Stiftung; Stiftung Haus der kleinen Forscher, 2015a). Bei einer ihrem jeweiligen Entwicklungsstand angemessenen Förderung können Kinder ihre Fähigkeiten und ihr Wissen erweitern, was bei der späteren Entwicklung wissenschaftlicher Konzepte hilft (Eshach \& Fried, 2005). Im Zentrum steht dabei das Bemühen um eine hohe Prozessqualität bildungsbezogener Interaktionen (Andersson \& Gullberg, 2012), welche sich in emotionaler Unterstützung, Organisation der Lernsituation und Unterstützung beim Lernen zeigt (Fthenakis, Wendell, Eitel, Daut \& Schmitt, 2009). Schafft es die Fachkraft, diese Aspekte umzusetzen, können Kinder rasch Lernfortschritte erzielen, ihr Verhalten und ihre Aufmerksamkeit besser regulieren sowie soziale Kompetenzen ausbauen (Mashburn et al., 2008; Burchinal, Vandergrift, Pianta \& Mashburn, 2010). Auch gibt es Hinweise darauf, dass eine hohe Interaktionsqualität Unterschiede im Lernen zwischen unterschiedlich stark engagierten Kindern vermindern kann (Williford, Maier, Downer, Pianta \& Howes, 2013), indem sie hilft, ein niedriges Engagement (z. B. aufgrund einer geringen Verhaltenskontrolle oder sozialer Konflikte) zu überwinden, was insbesondere bei Kindern aus sozial schwachen Familien Vorteile verspricht. Dabei wirkt sich die extrinsische sowie intrinsische Motivation der Fachkraft positiv auf das Engagement der Kinder aus (Demir, 2011). Eine positive Fachkraft-Kind-Interaktion kann zudem die selbstregulativen Fähigkeiten der Kinder verbessern, exekutive Funktionen und Aufgabenorientierung fördern sowie dysreguliertes Verhalten reduzieren (Williford, Whittaker, Vitiello \& Downer, 2013). 
Bei der gezielten Förderung von Kindern im naturwissenschaftlichen Bereich begleiten pädagogische Fachkräfte den Lernprozess und schaffen passende Lerngelegenheiten (Hamre, 2014). Hier wird die Relevanz des Zusammenspiels zwischen der Interaktionsqualität und der (naturwissenschaftlichen) Bildung deutlich: Selbst bei einem ausreichenden Fachwissen genügt die reine Vermittlung von Fakten und Konzepten nicht. So sind Erklärungen alleine wenig effektiv, wenn es um den Erkenntnisprozess geht (Webb \& Mastergeorge, 2003). Ziel pädagogischen Handelns ist vielmehr die Förderung naturwissenschaftlicher Denkfähigkeiten (Yoon \& Onchwari, 2006). Für eine positive Entwicklung auf diesem Gebiet bedarf es kognitiv stimulierender, täglicher Interaktionen zwischen Kindern und pädagogischen Fachkräften (Sabol et al., 2013). Damit Fachkräfte diese Rolle übernehmen können, müssen Unterstützungsstrategien herausgearbeitet werden, die Kinder in die Lage versetzen, selbstständig und strukturiert zu experimentieren sowie grundlegende Aspekte wissenschaftlichen Denkens (beispielsweise das Aufstellen von Hypothesen, deren Prüfung und Revision) anzuwenden.

Angelehnt an Vygotskys Konzept der Zone der nächsten Entwicklung (1978) geht man heute davon aus, dass Scaffolding-Strategien besonders nützlich sind, um Kinder an Aufgaben und Probleme heranzuführen, die sie alleine noch nicht lösen können (Cazden, 1979; Hsin \& Wu, 2011; Wood, Bruner \& Ross, 1976). Eine positive Prozess- oder Interaktionsqualität ist neben einer ko-konstruktiven Haltung (Fthenakis et al., 2009) durch ein hohes Maß an emotionaler, organisatorischer und lernunterstützender Interaktion gekennzeichnet (Burchinal et al., 2010; Hamre, 2014). Der Schlüssel zum Erfolg in der naturwissenschaftlichen Bildung von Kindergartenkindern liegt damit im Bemühen der Fachkraft, ihre naturwissenschaftlichen Kenntnisse in kindgerechter und aktivierender Weise zu vermitteln und dabei eine geeignete Balance zwischen Engagement und Zurückhaltung zu finden.

Trotz vielfältiger Forschung zur Frage, wie naturwissenschaftliche Bildungsprozesse am besten gelingen können, liegen erst wenige gesicherte Erkenntnisse vor (van de Pol, 2012). Ebenso fehlt es an Studien, die den Zusammenhang zwischen der Qualität der Fachkraft-Kind-Interaktion und der naturwissenschaftlichen Kompetenzentwicklung bei Kindern systematisch untersuchen. Ein Grund für diesen Mangel ist das Fehlen von standardisierten Instrumenten zur Beurteilung der Prozessqualität in interaktiven Lehr-Lern-Situationen.

Hier setzt die vorliegende Teilstudie an: Sie untersucht, welche Strategien im Verhalten der Fachkraft zur Verbesserung der Prozessqualität beitragen, ob es Zusammenhänge zwischen sprachlicher Anregungsqualität und prozessorientierter Kompetenz der Fachkraft gibt, wie entsprechende Strategien in Beziehung zu Kompetenzen und dem Erkenntnisprozess der Kinder stehen und ob sich nachweisen lässt, dass eine Fortbildung von Fachkräften hinsichtlich einer guten Prozess- 
orientierung Wirkung zeigt. Anders als viele existierende Arbeiten stützen wir uns dabei nicht auf Auskünfte der Fachkräfte, sondern auf die Kodierung von Videodaten durch unabhängige geschulte Beobachter und Beobachterinnen.

Exemplarisch für Bildungsmaßnahmen im naturwissenschaftlichen Bereich setzen wir uns mit den Auswirklungen von Fortbildungen der Stiftung „Haus der kleinen Forscher" auseinander. Dieser bundesweit verbreiteten Bildungsinitiative geht es darum, frühpädagogische Fachkräfte in die Lage zu versetzen, die Bildungschancen von Kindern im Bereich Naturwissenschaften nachhaltig zu verbessern.

In den folgenden Absätzen werden die drei zentralen Fragestellungen des Teilprojekts aufgelistet, der Ablauf der Datenerhebung kurz skizziert (siehe auch Teilprojekt 1), die Entwicklung und Überprüfung eines Instruments zur videobasierten Erfassung der auf naturwissenschaftliche Bildung bezogenen Fähigkeiten der Fachkraft (QUOTS - Quality of Teaching Science) dargestellt und Zusammenhänge mit der sprachlichen Anregungsqualität sowie mit unterschiedlichen Aspekten des kindlichen Verhaltens analysiert. Schließlich vergleichen wir das Verhalten der Fachkräfte zwischen Gruppen mit unterschiedlich vorgebildeten Fachkräften. Eine integrative Diskussion der Ergebnisse mit Empfehlungen für die Stiftung „Haus der kleinen Forscher“ schließt den Bericht ab. 


\section{Fragestellungen}

\subsection{Fragestellung 1: Prozessqualität in Interaktionen und kindliche Kompetenzen}

Wie lassen sich ko-konstruktive Verhaltensweisen von pädagogischen Fachkräften und die Gestaltung naturwissenschaftsbezogener Prozessqualität auf der Basis von Videodaten beschreiben? Das zur Beantwortung dieser Frage neu entwickelte Kodierschema QUOTS erlaubt eine Bewertung des Fachkraft-Verhaltens durch unabhängige Beobachter und Beobachterinnen anhand von Ratings. Wie das Verhalten der Fachkraft zu Erkenntnisprozessen der Kinder in Beziehung steht, untersucht Fragestellung 1. Wir gehen davon aus, dass zwischen den fachkraft- und kindbezogenen Variablen systematische Beziehungen bestehen. Die zugehörige Hypothese 1 lautet:

H1) Parameter der naturwissenschaftsbezogenen Prozessqualität aufseiten der Fachkraft korrelieren positiv mit Kompetenzmerkmalen der Kinder im naturwissenschaftlichen Denken (hier: kindlicher Erkenntnisprozess in einer Forschungssituation).

\subsection{Fragestellung 2: Prozessqualität in Interaktionen und sprachliche Anregungsqualität}

Hängen Maße der naturwissenschaftsbezogenen Prozessqualität in Interaktionen zwischen pädagogischer Fachkraft und Kindern mit Maßen der sprachlichen Anregungsqualität zusammen? Diese Frage zielt auf eine erste Verknüpfung der beiden Teilprojekte 1 (Rank, Wildemann, Hartinger \& Tietze, in diesem Band) und 2 (Pauen \& Kästner, vorliegendes Kapitel) dieses Projektes ab. Ausgehend von der Annahme, dass sprachliche Anregung ein wichtiger Bestandteil von Prozessqualität in Bildungssituationen ist, vermuten wir, dass die Einschätzung der Prozessqualität des Fachkraft-Verhaltens in einer Forschungssituation auch durch deren Sprachverhalten mitbeeinflusst wird. Hypothese 2 lautet folglich:

H2) Es besteht eine positive Beziehung zwischen der sprachlichen Anregungsqualität und der Prozessqualität in naturwissenschaftlichen Interaktionen zwischen pädagogischer Fachkraft und Kindern. 


\subsection{Fragestellung 3: Prozessqualität in Interaktionen und naturwissenschaftliche Vorbildung der Fachkräfte}

Unterscheidet sich die naturwissenschaftsbezogene Prozessqualität in Interaktionen mit Kindern zwischen Fachkräften, die an naturwissenschaftlichen Fortbildungen teilgenommen haben und Fachkräften, die keine entsprechende Qualifizierung erhalten haben? Hier gehen wir von der Annahme aus, dass sich naturwissenschaftliche Fortbildungen hinsichtlich naturwissenschaftlicher Bildungsprozesse positiv auf das Verhalten der Fachkräfte und damit auf die Prozessqualität auswirken.

H3) Die naturwissenschaftsbezogene Prozessqualität ist höher bei pädagogischen Fachkräften, die an naturwissenschaftlichen Fortbildungen teilgenommen haben, als bei pädagogischen Fachkräften ohne naturwissenschaftliche Fortbildung. 


\section{Datenerhebung und Methodik}

Wir beziehen uns auf Daten von $\mathrm{N}=58$ Videos, auf denen pädagogische Fachkräfte aus Karlsruhe und Heidelberg zu sehen sind, die jeweils in Kleingruppen mit Kindern für ca. 20 Minuten einen Versuch zum Thema Sinken und Schwimmen durchgeführt haben. Es nahmen Fachkräfte aus drei Gruppen mit unterschiedlicher naturwissenschaftlicher Vorbildung teil: eine Gruppe mit naturwissenschaftlichem Schwerpunkt „Haus der kleinen Forscher“ (HdkF-Gruppe), eine Gruppe mit anderem naturwissenschaftlichen Schwerpunkt (Nawi-Gruppe) und eine Gruppe ohne naturwissenschaftlichen Bildungsschwerpunkt (Vergleichsgruppe). Nähere Informationen zur Rekrutierung der Gruppen sowie zur Stichprobenzusammensetzung finden sich im Bericht von Rank et al. in diesem Band (Teilprojekt 1). Im vorliegenden Teilprojekt wird insbesondere der Vergleich zwischen Gruppe 1 (HdkF-Gruppe) und Gruppe 3 (Vergleichsgruppe) im Vordergrund stehen.

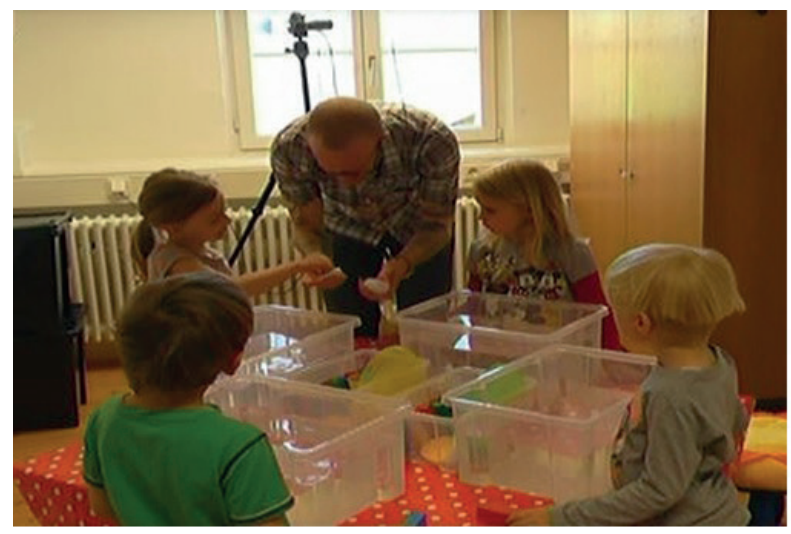

Jedes Video wurde durch zwei unabhängige Kodiererinnen (studentische Hilfskräfte der Arbeitseinheit Entwicklungs- und Biologische Psychologie an der Universität Heidelberg) anhand der QUOTS-Skalen beurteilt. Die Kodiererinnen wussten dabei nicht, welche Vorbildung die einzelnen Fachkräfte hatten. Sie waren „blind“ bezüglich der Gruppenzugehörigkeit der Fachkräfte. Die gemittelten Ratings dienten als Rohwerte für alle weiteren Analysen.

Bevor eine Überprüfung der Hypothesen erfolgen kann, wird in einem ersten Schritt, die Konzeption der QUOTS-Skalen detaillierter erläutert. 


\section{Entwicklung von QUOTS (Quality of Teaching Science)}

Quality of Teaching Science (kurz: QUOTS) ist ein Verfahren, bei dem unabhängige Rater anhand von Videodaten Globalratings über unterschiedliche Aspekte des Verhaltens der pädagogischen Fachkräfte sowie über das Verhalten der Kinder und deren naturwissenschaftlichen Erkenntnisprozess abgeben. Diese Ratings beziehen sich auf Dimensionen des Verhaltens, die in der aktuellen Fachliteratur als potenziell relevant für die Prozessqualität erachtet werden.

Der erste Teil enthält QUOTS-Skalen, die sich auf Aspekte des Verhaltens von Fachkräften im naturwissenschaftlichen Bildungsbereich beziehen. Diese Skalen wurden in Zusammenarbeit mit der Arbeitsgruppe von Prof. Dr. Silke Hertel (Institut für Bildungswissenschaften, Universität Heidelberg) konzipiert. In einem zweiten Teil geht es um das Verhalten der Kinder, d. h. den Aufgabenfokus und die Forscherhaltung in der Kindergruppe sowie die Aufgabenbearbeitung, den Unterstützungsbedarf, die Stimmung und die Erkenntnisse jedes beteiligten Kindes. Diese Items wurden speziell für das vorliegende Forschungsprojekt konzipiert.

\subsection{Beschreibung der fachkraftbezogenen QUOTS-Skalen}

In Anlehnung an die bestehende Literatur wurden unterschiedliche Konzepte und Strategien operationalisiert, die mit der naturwissenschaftsbezogenen Prozessqualität in Verbindung stehen und sich inhaltlich ergänzen bzw. überlappen. Bei der Konzeption passender Skalen war es uns wichtig, möglichst viele relevante Aspekte einzubeziehen und diese so zu beschreiben, dass ein geschulter Kodierer bzw. eine geschulte Kodiererin in der Lage ist, das Verhalten der Fachkraft auf den jeweiligen Dimensionen zuverlässig einzuschätzen. Tabelle 62 benennt sämtliche Skalen bzw. Variablen, erläutert kurz ihren Gegenstandsbereich und definiert die zugehörigen Antwortkategorien. Die Reihenfolge der Darstellung weicht dabei aus pragmatischen Gründen von der Reihenfolge im Kodierbogen ab. Eine ausführliche Begründung der Auswahl relevanter Dimensionen, eine Darstellung der Items jeder Skala und eine Zusammenfassung wichtiger Gütekriterien folgen im Anschluss. 
Tabelle 62. Fachkraftbezogene QUOTS-Skalen, Gegenstand und Antwortkategorien

\begin{tabular}{|c|c|c|}
\hline Skala & Inhalt & Antwortkategorien \\
\hline $\begin{array}{l}\text { Scaffolding- } \\
\text { Strategien } \\
\text { (SS: } 11 \text { Items) }\end{array}$ & $\begin{array}{l}\text { Nutzt die Fachkraft Strategien zur Unterstüt- } \\
\text { zung des kindlichen Erkenntnisprozesses, die } \\
\text { sich am Forschungskreis orientieren? }\end{array}$ & $\begin{array}{l}\text { gar nicht (0) } \\
\text { wenig (1) } \\
\text { etwas (2) } \\
\text { viel (3) } \\
\text { sehr viel (4) } \\
\text { nicht kodierbar (n. k.) }\end{array}$ \\
\hline $\begin{array}{l}\text { Manner of } \\
\text { Instruction } \\
\text { (MI: } 9 \text { Items) }\end{array}$ & $\begin{array}{l}\text { Strukturiert die Fachkraft die Wissensinhalte } \\
\text { und das Vorgehen der Kinder in systematischer } \\
\text { Weise? }\end{array}$ & $\begin{array}{l}\text { gar nicht (0) bis } \\
\text { sehr viel (4) }\end{array}$ \\
\hline $\begin{array}{l}\text { Transfer of } \\
\text { Responsibility - } \\
\text { Lokus Fachkraft } \\
\text { (TR-LF: } 3 \text { Items) }\end{array}$ & $\begin{array}{l}\text { Übernimmt die Fachkraft die Entscheidung über } \\
\text { das konkrete Vorgehen? }\end{array}$ & $\begin{array}{l}\text { gar nicht (0) bis } \\
\text { sehr viel (4) }\end{array}$ \\
\hline
\end{tabular}

\begin{tabular}{|c|c|c|}
\hline $\begin{array}{l}\text { Transfer of } \\
\text { Responsibility - } \\
\text { Lokus Kind } \\
\text { (TR-LK: } 3 \text { Items) }\end{array}$ & $\begin{array}{l}\text { Überlässt die Fachkraft dem Kind die Entschei- } \\
\text { dung über das konkrete Vorgehen? }\end{array}$ & $\begin{array}{l}\text { gar nicht (0) bis } \\
\text { sehr viel (4) }\end{array}$ \\
\hline $\begin{array}{l}\text { Direction } \\
\text { Maintenance } \\
\text { (DM: } 4 \text { Items) }\end{array}$ & $\begin{array}{l}\text { Lenkt die Fachkraft das Interesse/die Aufmerk- } \\
\text { samkeit der Kinder gezielt auf das Experimen- } \\
\text { tieren? }\end{array}$ & $\begin{array}{l}\text { gar nicht (0) bis } \\
\text { sehr viel (4) }\end{array}$ \\
\hline $\begin{array}{l}\text { Contingency } \\
\text { Management } \\
\text { (CM: } 3 \text { Items) }\end{array}$ & $\begin{array}{l}\text { Gelingt es der Fachkraft, sich bei Interventio- } \\
\text { nen auf die Kompetenzen der Kinder einzu- } \\
\text { stellen? }\end{array}$ & $\begin{array}{l}\text { gar nicht (0) bis } \\
\text { sehr viel (4) }\end{array}$ \\
\hline $\begin{array}{l}\text { Begrenzung von } \\
\text { Freiheitsgraden } \\
\text { (BF: } 3 \text { Items) }\end{array}$ & $\begin{array}{l}\text { Achtet die Fachkraft auf die Einhaltung von } \\
\text { Rahmenbedingungen des Experimentierens? }\end{array}$ & $\begin{array}{l}\text { gar nicht (0) bis } \\
\text { sehr viel (4) }\end{array}$ \\
\hline $\begin{array}{l}\text { Aufdringlichkeit } \\
\text { Übergriffigkeit } \\
\text { (AÜ: } 3 \text { Items) }\end{array}$ & $\begin{array}{l}\text { Wird die Fachkraft zu viel/zu oft aktiv, um den } \\
\text { Erkenntnisprozess der Kinder zu steuern? }\end{array}$ & $\begin{array}{l}\text { gar nicht (0) bis } \\
\text { sehr viel (4) }\end{array}$ \\
\hline $\begin{array}{l}\text { Allgemeine } \\
\text { Haltung } \\
\text { (AH: } 5 \text { Items) }\end{array}$ & $\begin{array}{l}\text { Inwiefern stimmt das Verhalten der Fachkraft } \\
\text { mit verschiedenen Rollenbildern (z. B. „Leh- } \\
\text { rer“, „Begleiter“, „,Möglichmacher“, „weiteres } \\
\text { Kind“) überein? }\end{array}$ & $\begin{array}{l}\text { gar nicht (0) bis } \\
\text { sehr viel (4) }\end{array}$ \\
\hline $\begin{array}{l}\text { Angemessenheit } \\
\text { von Scaffolding } \\
\text { (AC: } 1 \text { Item) }\end{array}$ & $\begin{array}{l}\text { Werden Unterstützungsstrategien in angemes- } \\
\text { senem Umfang eingesetzt? }\end{array}$ & $\begin{array}{l}\text { zu wenig }(-2) \text { bis zu } \\
\text { viel }(+2)\end{array}$ \\
\hline $\begin{array}{l}\text { Unterstützungs- } \\
\text { verhalten } \\
\text { (UV: } 1 \text { Item) }\end{array}$ & $\begin{array}{l}\text { In welcher Form und in welchem Ausmaß bietet } \\
\text { die Fachkraft den Kindern Unterstützung beim } \\
\text { Experimentieren? }\end{array}$ & niedrig (1) bis hoch (5) \\
\hline $\begin{array}{l}\text { Stimmung } \\
\text { (SI: } 5 \text { Items) }\end{array}$ & $\begin{array}{l}\text { Wie stark sind unterschiedlich gepolte Emotio- } \\
\text { nen bei der Fachkraft erkennbar? }\end{array}$ & $\begin{array}{l}\text { negativ }(-2) \text { bis } \\
\text { positiv }(+2)\end{array}$ \\
\hline $\begin{array}{l}\text { Globalurteil } \\
\text { (GU: } 1 \text { Item) }\end{array}$ & $\begin{array}{l}\text { Gelingt es der Fachkraft, die Kinder in ihrem } \\
\text { naturwissenschaftlichen Erkenntnisprozess zu } \\
\text { fördern? }\end{array}$ & $\begin{array}{l}\text { nicht erfolgreich (1) } \\
\text { bis erfolgreich (5) }\end{array}$ \\
\hline
\end{tabular}




\subsubsection{Beobachterübereinstimmung und Verteilungseigenschaften der Skalen}

Um sicherzustellen, dass die Skalen hinreichend reliabel sind, wurde zunächst die Beurteiler-übereinstimmung ermittelt. Hierbei kam die Intraklassenkorrelation zum Einsatz. Sie ist strenggenommen nur zulässig, wenn die Daten Intervallskalen-Niveau haben, kommt jedoch auch oft bei Daten zum Einsatz, die mindestens ordinal-skaliert sind, wie dies auf die hier verwendeten Likert-Skalen zutrifft (Carifio \& Perla, 2007). Wie später noch deutlich wird, liegen die Intraklassenkorrelationen alle im befriedigenden bis sehr guten Bereich, so dass von einer hinreichend objektiven Erfassung der interessierenden Variablen ausgegangen werden kann. Zur Überprüfung der Normalverteilungsannahme wurden statistische Cut-offs und Histogramme verwendet, welche bei der hier verwendeten Stichprobengröße sinnvolle Maße darstellen (Tabachnick \& Fidel, 2006). Eine visuelle Inspektion der Häufigkeitsverteilung für einzelne Skalen, sowie Wertebereiche von Kurtosis und Schiefe unterhalb der Cut-off-Werte deutete in allen Fällen auf eine Normalverteilung hin. Folglich gibt es für die Berechnung von Mittelwertvergleichen und Korrelationen keine Einschränkungen. Nachfolgend werden sämtliche Skalen detailliert vorgestellt und hinsichtlich wichtiger Kennwerte beschrieben. Dabei beziehen wir uns stets auf die gemittelten Werte beider Kodiererinnen.

\subsubsection{Scaffolding-Strategien (SCS) und Angemessenheit von Scaffolding (ASC)}

Bildlich gesprochen, beschreibt Scaffolding ein Gerüst, welches ein im Bau befindliches Gebäude von Beginn an unterstützt (Alake \& Ogunseemi, 2013). Zu Beginn trägt das Gerüst das Gebäude; sind alle Phasen des Baus abgeschlossen, kann das Gebäude ohne Unterstützung stehen. Übertragen auf Lehr-Lern-Situationen bedeutet dies, dass die pädagogische Fachkraft so lange ihre Begleitung und Unterstützung anbietet, bis das Kind das Gelernte selbstständig anwenden kann. Van de Pol, Volman und Beishuizen (2010) unterscheiden Scaffolding Intentions und Scaffolding Means.

Scaffolding Intentions beschreiben Ziele von Scaffolding, also das, was durch die Fachkraft unterstützt werden soll. Im vorliegenden Fall geht es dabei vor allem um den naturwissenschaftlichen Erkenntnisprozess. Als Werkzeuge dienen das Stellen von Fragen, das Geben von Hinweisen, das Anleiten, das Erklären sowie das Verbalisieren von Beobachtungen (van de Pol et al. 2010). Um die genannten Ziele zu erreichen, wendet der Lehrende bestimmte Strategien (Scaffolding Means) an. Die Fachkraft gibt dabei nach Möglichkeit keine detaillierte Instruktionen oder komplette Lösungen vor (van de Pol et al., 2010), sondern regt die Kinder dazu an, selbst aktiv zu werden und sich auf den naturwissenschaftlichen Denk- 
prozess einzulassen. Zu diesem Zweck stellt sie Fragen oder formuliert Hinweise und Anleitungen oder Erklärungen und regt Kinder dazu an, es ihr gleich zu tun.

Ausgehend von diesen Überlegungen wurde die erste QUOTS-Skala - Scaffolding-Strategien - in zwei Subskalen unterteilt: Initiative der Fachkraft (a-e) bezieht sich auf das Bemühen der Fachkraft, die Kinder durch Fragen, Hinweise oder Anleitungen in ihrem Erkenntnisprozess zu lenken oder ihnen Aspekte des Forschungskreises selbst vorzuleben, während Initiative der Kinder ( $\mathrm{f}-\mathrm{k}$ ) sich darauf bezieht, die Kinder zu entsprechenden Aktivitäten zu ermuntern. Hierbei gilt es zu beachten, dass sich beide Strategien durchaus ergänzen können und damit nicht im Widerspruch stehen. Tabelle 63 referiert sämtliche Items und die deskriptiven Werte für beide Subskalen sowie für die Gesamtskala.

Tabelle 63. Inhalte, Mittelwerte (M), Standardabweichungen (SD), Min- und Max-Werte für alle Items, die Subskalen und die Gesamtskala von Scaffolding-Strategien

\begin{tabular}{lcccc}
\hline Scaffolding-Strategien (SCS) & M & SD & Min & Max \\
\hline a) selber Fragen stellen & 2.85 & 0.71 & 1.50 & 4.00 \\
b) selber Hinweise geben & 2.19 & 0.59 & 1.00 & 3.50 \\
c) selber Anleitungen geben & 2.37 & 0.76 & 0.50 & 4.00 \\
d) selber Beobachtungen verbalisieren & 2.00 & 0.74 & 0.50 & 3.50 \\
e) selber Erklärungen anbieten & 1.57 & 0.71 & 0.00 & 3.50 \\
f) Kinder zum Fragen ermutigen & 0.84 & 0.64 & 0.00 & 2.50 \\
g) Kinder zur Formulierung von Vermutungen & 1.96 & 0.99 & 0.00 & 4.00 \\
$\quad$ oder Hypothesen anregen & & & & 4.00 \\
h) Kinder zur Verbalisierung von Beobachtungen & 2.67 & 0.71 & 0.50 & 4.00 \\
$\quad \begin{array}{l}\text { anregen } \\
\text { i) Kinder zu Vergleichen anregen }\end{array}$ & 1.41 & 0.68 & 0.00 & 3.50 \\
j) Kinder zu Erklärungen anregen & 2.10 & 0.78 & 0.00 & 3.52 \\
k) Kinder zu Transfer anregen & 0.87 & 0.73 & 0.00 & 2.50 \\
\hline Initiative Fachkraft (a-e) & 2.20 & 0.52 & 0.90 & 3.30 \\
\hline Initiative Kind (f-k) & 1.64 & 0.57 & 0.33 & 2.83 \\
\hline Gesamtskala Scaffolding-Strategien (a-k) & 1.89 & 0.51 & 0.59 & 2.82 \\
\hline
\end{tabular}

Beide Subskalen der QUOTS-Skala Scaffolding-Strategien weisen zufriedenstellende interne Konsistenzen (Initiative Fachkraft: $\alpha=.82$, Initiative Kind: $\alpha=.87$ ) und eine gute mittlere Beurteilerübereinstimmung auf $(I C C=.82)$. Für spätere Auswertungen war aufgrund der hohen Interkorrelation beider Subskalen vor allem der Gesamtwert relevant.

Die Kodiererinnen wurden außerdem gebeten, einzuschätzen, wie angemessen sie das Verhalten der Fachkraft fanden. Diese Variable (Angemessenheit der Scaffolding Means) weist ihr theoretisches Optimum bei $M=0$ auf, wobei sich 
die Abstufungen hier von zu wenig bis zu viel Unterstützung durch Scaffolding Means erstrecken. Tabelle 64 referiert die zugehörige deskriptive Statistik. Die Ausgangsfrage für die Kodiererinnen lautete: Inwieweit verwendet die Fachkraft folgende Strategien mit dem Ziel, Kinder beim Experimentieren zu unterstützen? Wie Tabelle 64 dokumentiert, wurde das Ausmaß an Unterstützung insgesamt als passend (nahe 0) bewertet, wobei die Kodiererinnen im Mittel etwas mehr Scaffolding angemessen gefunden hätten.

Tabelle 64. Inhalte, Mittelwerte (M), Standardabweichungen (SD), Min- und Max-Werte für Angemessenheit der Häufigkeit, mit der Scaffolding-Strategien angewendet wurden

\begin{tabular}{lcccc}
\hline $\begin{array}{l}\text { Angemessenheit der Häufigkeit der } \\
\text { Scaffolding-Strategien (ASC) }\end{array}$ & M D & Min & Max \\
\hline $\begin{array}{l}\text { Das Ausmaß der Unterstützung anhand von Scaffol- } \\
\text { ding-Strategien ist ...(zu wenig bis zu viel; -2 bis +2) }\end{array}$ & -0.21 & 0.80 & -2.00 & 1.50 \\
\hline
\end{tabular}

Auch für das Item Angemessenheit der Häufigkeit von Scaffolding-Strategien (Einzelitem) fällt die Beobachterübereinstimmung zufriedenstellend aus $(I C C=.71)$. Weil das theoretische Optimum bei 0 liegt, wurde die Variable für die Berechnung von Korrelationen mit anderen Variablen rekodiert, so dass Abweichungen des Wertes sowohl im Positiv- als auch im Negativbereich gleichermaßen zu einer Reduktion des optimalen Wertes (3) führte (ASC, rekodiert: $0=3,-1 /+1=2,-2 /+2=1$ ).

\subsubsection{Manner of Instruction (MI)}

Laut van der Pol et al. (2010) ist Manner of Instruction das Bereitstellen metakognitiven Wissens. Nach Vygotsky (1987) sind Instruktionen ein Mittel, dieses Wissen zu fördern. So können Kinder einen Lösungsweg oder das Erreichen einer Antwort besser reflektieren und ihre Handlung evaluieren (Stright, Neitzel, Sears \& Hoke-Sinex, 2001). Dazu bedarf es einer altersgerechten Ansprache und Aktivierung. Zudem sollten die kognitiven Ansprüche einer Aufgabe vereinfacht und Informationen in kleinen Schritten präsentiert werden. Auch das Protokollieren von Zwischenergebnissen und Fortschritten in Bezug auf das Ziel kann helfen. QUOTS enthält Items, die sich auf die Strukturierung der Wissensinhalte $(\mathrm{a}-\mathrm{c})$ und die Gestaltung der Gruppensituation (d-f) sowie die sprachliche Vermittlung von Fachwissen ( $\mathrm{g}-\mathrm{i}$ ) beziehen und so verschiedene Aspekte von Manner of Instruction parallel abdecken (siehe Tabelle 65). Bei der sprachlichen Vermittlung gilt es zu beachten, dass die Verwendung einfacher Sprache und die Benutzung von Fachwörtern gleichermaßen positiv bewertet wurden, weil ein Kind am ehesten profitiert, wenn die Sprache einerseits leicht verständlich ist, andererseits aber auch neue Begriffe einführt und so das Vokabular erweitert. 
Tabelle 65. Inhalte, Mittelwerte (M), Standardabweichungen (SD), Min- und Max-Werte für alle Items, der Subskalen und der Gesamtskala von Manner of Instruction

\begin{tabular}{lllll}
\hline Manner of Instruction (MI) & M SD & Min & Max \\
\hline Die pädagogische Fachkraft ... & & & & \\
a) präsentiert Informationen in überschaubaren Mengen. & 2.08 & 0.73 & 0.00 & 3.00 \\
b) formuliert Zwischenergebnisse des Erkenntnisprozesses. & 1.59 & 0.66 & 0.50 & 3.50 \\
c) protokolliert Ergebnisse. & 0.42 & 0.70 & 0.00 & 3.00 \\
d) koordiniert die Aktivität einzelner Kinder. & 2.11 & 0.85 & 0.00 & 4.00 \\
e) sorgt dafür, dass alle Kinder sich aktiv beteiligen. & 2.32 & 0.69 & 1.00 & 4.00 \\
f) geht auf jedes Kind individuell ein. & 2.50 & 0.63 & 1.00 & 4.00 \\
g) bringt fachspezifisches Wissen über Sinken/Schwimmen ein. & 1.60 & 0.80 & 0.00 & 3.50 \\
h) verwendet einfache Sprache (simple Sätze, bekannte Worte). & 2.95 & 0.44 & 2.00 & 4.00 \\
$\begin{array}{l}\text { i) benutzt Fachwörter. } \\
\text { Strukturierung Wissensinhalte (a-c) }\end{array}$ & 1.25 & 0.62 & 0.00 & 2.50 \\
Gestaltung der Gruppensituation (d-f) & 1.80 & 0.71 & 0.00 & 3.00 \\
Sprachliche Vermittlung von Fachwissen (g-i) & 1.36 & 0.52 & 0.17 & 2.67 \\
\hline Gesamtskala (a-i) & 1.93 & 0.38 & 1.00 & 2.67 \\
\hline
\end{tabular}

Die interne Konsistenz der Gesamtskala liegt mit $\alpha=.81$ hoch, die Beurteilerübereinstimmung mit ICC $=.79$ ebenfalls im guten Bereich. Einzelne Items (z. B. „protokolliert Ergebnisse“) wurden nicht häufig beobachtet, was dem speziellen Versuchssetting und dem Alter der Probanden und Probandinnen zuzurechnen ist. Dafür erreichten andere Items (z. B. „geht auf die Kinder ein“, „verwendet eine einfache Sprache“) besonders hohe Werte, da diese Form der Wissensvermittlung in der untersuchten Altersgruppe eine prominente Rolle spielt.

\subsubsection{Transfer of Responsibility (TR-LF; TR-LK)}

Generell scheint es wichtig, das Unterstützungsverhalten dem aktuellen und potenziellen Entwicklungsstand des Kindes anzupassen (Bredekamp \& Copple, 1997). Demnach beinhaltet Transfer of Responsibility einerseits die Bereitschaft zur aktiven Gestaltung der Experimentiersituation durch die Fachkraft, andererseits aber auch die Bereitschaft zur Übertragung von Verantwortung an die Kinder, in Abhängigkeit von deren Entwicklungs- und Kompetenzstand. Übernimmt das Kind graduell mehr Kontrolle über eigene Aktivitäten, benötigt es bei bestimmten Handlungen oder Lernerfahrungen weniger Unterstützung (van de Pol et al., 2010). Neitzel und Stright (2003) verbinden mit Transfer of Responsibility 
vor allem das Involvieren des Kindes in die Aufgabenbearbeitung und die Anregung, sich aktiv zu beteiligen.

Tabelle 66 referiert, wie diese Aspekte in QUOTS operationalisiert wurden, und listet die zugehörigen Item- und Skalencharakteristiken auf. Die Skala wurde dabei in zwei Subskalen untergliedert: Lokus Fachkraft (Regelung des Experimentierverhaltens und Planung des Vorgehens der Kinder durch die Fachkraft; siehe Items a, c, f), und Lokus Kind (Übertragung von Verantwortung für die Gestaltung der Lernsituation auf die Kinder, siehe Items b, d, e). Auch hier gilt es zu beachten, dass beide Aspekte für eine ko-konstruktive Haltung bedeutsam sind. So sollte die Fachkraft durchaus die Kontrolle über das Geschehen behalten, dabei aber gleichzeitig auch ausreichend Raum für Aktivitäten und Entscheidungen der Kinder lassen.

Im vorliegenden Fall fielen die Mittelwerte für die Skala Lokus Kind in der Gesamtgruppe etwas höher aus als für die Skala Lokus Fachkraft, sie lagen jedoch für alle Items im moderaten Bereich.

Die Subskala Lokus Fachkraft weist mit $\alpha=.90$ eine sehr hohe interne Konsistenz auf, ebenso wie die Subskala - Lokus Kind $(\alpha=.80)$. Mit ICC $=.74$ fiel die Beobachterübereinstimmung für die Gesamtskala zufriedenstellend aus.

Tabelle 66. Inhalte, Mittelwerte (M), Standardabweichungen (SD), Min- und Max-Werte für alle Items der Subskalen und der Gesamtskala von Transfer of Responsibility

\begin{tabular}{|c|c|c|c|c|}
\hline Transfer of Responsibility (TR) & M & SD & Min & Max \\
\hline \multicolumn{5}{|l|}{ Die pädagogische Fachkraft ... } \\
\hline a) regelt, wie die Kinder beim Experimentieren vorgehen sollen. & 2.16 & 0.83 & 0.00 & 4.00 \\
\hline $\begin{array}{l}\text { b) überlässt den Kindern die Entscheidung, wie sie } \\
\text { experimentieren wollen. }\end{array}$ & 2.35 & 0.74 & 1.00 & 3.50 \\
\hline $\begin{array}{l}\text { c) scheint einen festen eigenen Plan zu haben, nach dem sie } \\
\text { vorgeht. }\end{array}$ & 1.76 & 0.86 & 0.00 & 4.00 \\
\hline d) lässt sich von Impulsen und Ideen aus der Gruppe leiten. & 2.40 & 0.67 & 0.00 & 3.50 \\
\hline $\begin{array}{l}\text { e) überlässt den Kindern, welche Schlüsse sie aus den } \\
\text { Experimenten ziehen. }\end{array}$ & 2.08 & 0.73 & 0.50 & 4.00 \\
\hline f) will, dass die Kinder etwas ganz Bestimmtes lernen. & 1.75 & 0.87 & 0.00 & 3.50 \\
\hline Lokus Fachkraft (LF) & 1.89 & 0.77 & 0.00 & 3.33 \\
\hline Lokus Kind (LK) & 2.30 & 0.61 & 1.16 & 3.50 \\
\hline Gesamtskala & 2.08 & 0.22 & 1.30 & 2.58 \\
\hline
\end{tabular}




\subsubsection{Direction Maintenance (DM)}

Direction Maintenance ist nach van de Pol et al. (2010) ein weiterer Aspekt des Unterstützungsverhaltens von Fachkräften. Während der Interaktion kommt der pädagogischen Fachkraft die Aufgabe zu, die Kinder für das jeweilige Themengebiet zu begeistern und ihre Aufmerksamkeit aufrechtzuerhalten. Diese Skala wurde mit insgesamt vier Items erfasst, die in Tabelle 67 referiert sind. Es ist zu beachten, dass die Skala Direction Maintenance im QUOTS-Schema im Gegensatz zum gleichnamigem Scaffolding-Ziel bei van de Pol et al. (2010) neben den kognitiven Aspekten auch die affektive Aufmerksamkeitslenkung beinhaltet, die van de Pol et al. (2010) separat unter die „Strategie Recruitment“ fassen. Die Skala erwies sich als intern konsistent $(\alpha=.85)$. Auch die Beurteilerübereinstimmung erreicht mit ICC $=.74$ einen guten Wert. Tabelle 67 referiert die empirisch ermittelten Mittelwerte, Standardabweichungen und Min-/Max-Werte.

Tabelle 67. Inhalt, Mittelwerte (M), Standardabweichungen (SD), Min- und Max-Werte für alle Items der Skala Direction Maintenance

\begin{tabular}{|c|c|c|c|c|}
\hline Direction Maintenance (DM) & M & SD & Min & Max \\
\hline \multicolumn{5}{|l|}{ Die pädagogische Fachkraft ... } \\
\hline $\begin{array}{l}\text { a) lenkt die Aufmerksamkeit der Kinder erfolgreich auf das } \\
\text { Thema Sinken und Schwimmen. }\end{array}$ & 2.68 & 0.67 & 1.00 & 4.00 \\
\hline $\begin{array}{l}\text { b) hält die Aufmerksamkeit der Kinder beim Experimentieren } \\
\text { oder erlangt sie ohne Probleme und Zwang wieder. }\end{array}$ & 2.44 & 0.76 & 0.50 & 4.00 \\
\hline c) begeistert die Kinder für das Thema. & 2.54 & 0.57 & 1.00 & 3.50 \\
\hline $\begin{array}{l}\text { d) benennt und/oder verweist auf wichtige Aspekte des Experi- } \\
\text { ments (z. B. Material, Größe, Gewicht). }\end{array}$ & 2.12 & 0.92 & 0.50 & 3.50 \\
\hline Gesamtskala Direction Maintenance $(a-d)$ & 2.42 & 0.60 & 0.88 & 3.50 \\
\hline
\end{tabular}

\subsubsection{Contingency Management (CM)}

Um ein Kind weder zu unter- noch zu überfordern, sollte die pädagogische Fachkraft ihr Ausmaß an Unterstützung an das Leistungsniveau des Kindes anpassen. Das Ausmaß von Contingency Management sollte entweder auf dem Leistungsniveau des Kindes liegen oder auf einem etwas höheren Leistungsniveau ansetzen (van de Pol et al., 2010). Diese Strategie dient dazu, die Anforderungen an das Kind anzupassen, so dass weder Langeweile noch Überforderung auftreten. Hier zeigt sich die Notwendigkeit, den Entwicklungsstand des Kindes zu kennen, da sonst keine passende Unterstützungsebene gewählt werden kann. Die Formulierung entsprechender Items ist keineswegs trivial und die korrekte Beurteilung des Fachkraft-Verhaltens durch unabhängige Beobachter und Beobachterinnen setzt hohe Sachkenntnis über Entwicklungspsychologie voraus. Die QUOTS-Skala erfasst, inwieweit Fachkräfte ihre Unterstützung an den Entwicklungsstand der 
Kinder anpassen, die Hilfestellung bei Bedarf verstärken und sie zurücknehmen, wenn sie nicht benötigt wird. Sie ist nicht zu verwechseln mit dem gleichnamigen Scaffolding-Ziel bei van de Pol et al. (2010), die darunter die kontingente Verstärkung der Lernenden verstehen, um deren Motivation zu fördern.

Tabelle 68 referiert die Kennwerte der Skala. Die interne Konsistenz erreicht mit $\alpha=.34$ nur einen geringen Wert, ebenso wie die Beurteilerübereinstimmung $(I C C=.44)$. Item $c$ wies eine tendenziell negative, nicht signifikante Korrelation mit Item $a$ auf, $r(39)=-.08, p=.56$, ebenso Item $b, r(39)=-.10, p=.47$. Nach Entfernen von Item $\mathrm{c}$ lag die Interkorrelation zwischen den Items a und b bei $r=$ .80. Weitere Auswertungen wurden daher nur mit zwei Items vorgenommen (CM korrigiert). Hier gilt es zu beachten, dass eine so kurze Skala nur bedingt valide Schlussfolgerungen über Zusammenhänge mit anderen Variablen liefern kann und dass die Interpretation entsprechender Befunde unter Vorbehalt erfolgt.

Tabelle 68. Inhalte, Mittelwerte (M), Standardabweichungen (SD), Min- und Max-Werte für alle Items der Skala Contingency Management

\begin{tabular}{lrrrrr}
\hline Contingency Management (CM) & $M$ & SD & Min & Max \\
\hline $\begin{array}{l}\text { Die pädagogische Fachkraft ... } \\
\text { a) passt das Unterstützungsverhalten an das kindliche } \\
\text { Verhalten an. }\end{array}$ & 2.19 & 0.64 & 1.00 & 3.00 \\
$\begin{array}{l}\text { b) gibt Hinweise und Anregungen, wenn sie merkt, } \\
\text { dass ein Kind unsicher ist oder Fragen hat. }\end{array}$ & 1.84 & 0.57 & 0.50 & 3.00 \\
$\begin{array}{l}\text { c) nimmt sich zurück/mischt sich nicht ein, wenn } \\
\text { sie merkt, dass das Kind seine eigenen Gedanken } \\
\text { zum Thema hat. }\end{array}$ & 2.06 & 0.69 & 1.00 & 4.00 \\
\hline \begin{tabular}{l} 
Gesamtskala Contingency Management (a-c) \\
\hline
\end{tabular} & 2.03 & 0.41 & 1.00 & 2.83 \\
\hline
\end{tabular}

\subsubsection{Begrenzung von Freiheitsgraden (BF), Aufdringlichkeit/ Übergriffigkeit (AÜ)}

Die Ermutigung zur Autonomie führt nach Deci und Ryan (1985) zu gesteigerten Gefühlen der Kompetenz und des Durchhaltevermögens bei der Bearbeitung von Aufgaben. Wird den Kindern nicht genügend Freiraum eingeräumt und verhält sich die Fachkraft zu aufdringlich oder übergriffig - sei es durch zu viele Instruktionen, Aufforderungen oder gar physisches Eingreifen - kann der selbstständige und aktive Experimentierprozess gestört werden. Es ist wichtig, die Autonomie zu fördern, wodurch die Kinder ein Gefühl dafür entwickeln, welche Quellen ihre Lernergebnisse beeinflussen (Pino-Pasternak \& Whitebread, 2010). Baleghizadeh, Timcheh Memar und Timcheh Memar (2011) heben hervor, dass eine gering strukturierte Unterstützung erfolgreicher ist als eine hoch strukturierte Unterstützung. Das bedeutet aber nicht, dass die Fachkraft ihre Unterstützung komplett zurück- 
ziehen soll (Ginsburg \& Bronstein, 1993). Pino-Pasternak und Whitebread (2010) führen an, dass vielmehr die richtige Anpassung der Unterstützung entscheidend ist. Diese Annahme deckt sich mit den bereits genannten Zielen von Scaffolding. Insgesamt spricht ein Großteil der Literatur für ein geringes bis moderates Niveau an Unterstützung. Dennoch heben manche Autoren hervor, dass zu wenig oder gar keine Kontrolle negative Wirkungen hervorruft (z. B. Grolnick, 2003). Mariani (1997) argumentiert, dass die Unterstützungsebene gleichzeitig hoch und dennoch herausfordernd sein sollte, damit weder ein Gefühl der Langeweile noch Frustration bei den Lernenden entsteht. Für das naturwissenschaftliche Forschen mit Kindergartenkindern scheint es einerseits wichtig, dass die Fachkraft für die Kinder einen guten Rahmen schafft, in dem sie sich „geordnet“ mit dem Material und dem Thema befassen können und in der Gruppe gut zusammenarbeiten, andererseits aber auch bedeutsam, dass die Fachkraft den Kindern noch genügend Freiheit lässt, sich zu entfalten. Um feststellen zu können, inwiefern es der Fachkraft gelingt, diese beiden Aspekte in ihrem Handeln ausreichend zu berücksichtigen, wurden zwei weitere Skalen in QUOTS aufgenommen: Begrenzung von Freiheitsgraden bezieht sich auf die Einhaltung von Rahmenbedingungen für das Forschen. Aufdringlichkeit/Übergriffigkeit erfasst, ob die Fachkraft im Kontext des Forschens zu wenig Raum für die Kinder gelassen hat, sich selbst auszuprobieren (siehe Tabelle 69,70$)$.

Tabelle 69. Inhalt, Mittelwerte (M), Standardabweichungen (SD), Min- und Max-Werte für alle Items der Skalen Begrenzung von Freiheitsgraden

\begin{tabular}{|c|c|c|c|c|}
\hline Begrenzung von Freiheitsgraden (BF) & M & SD & Min & Max \\
\hline \multicolumn{5}{|l|}{ Die pädagogische Fachkraft ... } \\
\hline a) sorgt dafür, dass alle Kinder am Tisch bleiben. & 1.80 & 0.71 & 0.00 & 3.00 \\
\hline $\begin{array}{l}\text { b) achtet darauf, dass es nicht zu laut wird und sich alle } \\
\text { gegenseitig zuhören. }\end{array}$ & 1.64 & 0.64 & 0.00 & 3.00 \\
\hline $\begin{array}{l}\text { c) passt auf, dass die Kinder gut mit dem } \\
\text { Versuchsmaterial umgehen. }\end{array}$ & 1.83 & 0.61 & 0.00 & 3.00 \\
\hline
\end{tabular}

Tabelle 70. Inhalte, Mittelwerte (M), Standardabweichungen (SD), Min- und Max-Werte für alle Items der Skala Aufdringlichkeit/Übergriffigkeit

\begin{tabular}{|c|c|c|c|c|}
\hline Aufdringlichkeit/Übergriffigkeit (AÜ) & M & SD & Min & Max \\
\hline \multicolumn{5}{|l|}{ Die pädagogische Fachkraft ... } \\
\hline $\begin{array}{l}\text { a) redet zu viel (Fragen, Hinweise, Anmerkungen, Erklärungen, } \\
\text { Feedback). }\end{array}$ & 1.12 & 0.67 & 0.00 & 3.00 \\
\hline $\begin{array}{l}\text { b) sagt den Kindern zu oft, was sie machen sollen (Instruktionen, } \\
\text { Aufforderungen). }\end{array}$ & 1.14 & 0.77 & 0.00 & 3.00 \\
\hline $\begin{array}{l}\text { c) greift physisch in das Geschehen ein (unterbricht kindliche } \\
\text { Handlungen). }\end{array}$ & 0.63 & 0.55 & 0.00 & 2.50 \\
\hline
\end{tabular}


Beide Skalen erreichten ausreichende interne Konsistenzen ( $\alpha=.62$ bzw. $\alpha=.75$ ) und mittlere Beurteilerübereinstimmungen $(I C C=.52$ bzw. ICC $=.55)$. Wie die geringen Mittelwerte in den Tabellen 69 und 70 nahelegen, mussten die Fachkräfte selten in das Geschehen eingreifen, um sicherzustellen, dass die Kinder Rahmenbedingungen einhalten und waren insgesamt auch wenig aufdringlich oder übergriffig.

\subsubsection{Allgemeine Haltung (AH)}

Bei dieser Skala ging es darum, einzuschätzen, mit welchem Rollenbild das Verhalten der Fachkraft wie gut vereinbar ist. Während das Rollenbild eines „Lehrers“ dadurch gekennzeichnet ist, dass die Fachkraft klare inhaltliche Lernziele und eine aktive Strategie zeigt, die Kinder zu diesen Lernzielen zu führen, entspricht das Rollenbild des „Möglichmachers“ eher einer Person, die versucht zu erkennen, wo die Ziele des Kindes liegen, und es dabei unterstützt, diesen Zielen näherzukommen, auch wenn sie sich nicht mit den primären inhaltlichen Lernzielen decken. Der „Begleiter“ zeigt aktive Partizipation an den Gedanken, Gefühlen und Prozessen, die im Kind ablaufen, definiert selbst aber keine Ziele, sondern überlässt hier dem Kind die Initiative. Schließlich wird noch das Rollenbild eines „weiteren Kindes" angesprochen, das eine Person beschreibt, die sich in ihrem Verhalten nicht von anderen Mitgliedern der Kindergruppe unterscheidet. Jede Fachkraft wurde auf allen vier Rollen-Dimensionen geratet. Beobachterübereinstimmungen von ICC $=.71$ sprechen dafür, dass eine hinreichend reliable Beurteilung des Rollenverhaltens möglich ist. Tabelle 71 listet die einzelnen Rollen auf und gibt zu jeder Rolle die Mittelwerte, Standardabweichungen, Min- und Max-Werte an. Es fällt auf, dass das Rollenbild eines „Begleiters“ den numerisch höchsten Wert erzielte, also am häufigsten beobachtet wurde, gefolgt von „Lehrer“ und „Möglichmacher“, wobei alle drei Rollenbilder im mittleren Bereich bewertet wurden. Folglich scheinen die Fachkräfte insgesamt eine Mischung aus diesen Kategorien in ihrem Verhalten zu zeigen. Das Rollenbild eines „weiteren Kindes“ „erreichte mit Abstand den geringsten Wert, was nahelegt, dass die Führungsrolle der Fachkräfte gut deutlich wurde.

Tabelle 71. Inhalte, Mittelwerte (M), Standardabweichungen (SD), Min- und Max-Werte für alle Items der Skala Allgemeine Haltung

\begin{tabular}{lllll}
\hline Allgemeine Haltung (AH) & M & SD & Min & Max \\
\hline Die pädagogische Fachkraft verhält sich wie ein ... & & & & \\
(a) „Lehrer“ & 2.19 & 0.82 & 1.0 & 4.0 \\
(b) „Möglichmacher“ & 2.06 & 0.49 & 1.0 & 3.0 \\
(c) „Begleiter“ & 2.53 & 0.59 & 1.0 & 3.5 \\
(d) „weiteres Kind“ & 1.07 & 0.71 & 0.0 & 3.0 \\
\hline
\end{tabular}




\subsubsection{Unterstützungsverhalten (UV)}

Abschließend sollten die Kodiererinnen den Grad der Unterstützung durch die Fachkraft auf einer Ratingskala beurteilen. Das Unterstützungsverhalten beinhaltet die Abstufungen der child-regulation (niedriges Maß an Unterstützung), co-regulation (moderates Maß an Unterstützung) und adult-regulation (hohes Maß an Unterstützung). Tabelle 72 stellt die Bewertungsstufen einzeln dar und referiert die absolute und relative Häufigkeit, mit der einzelne Antwortkategorien gewählt wurden.

Tabelle 72. Absolute (i) und relative (\%)Häufigkeit der Wahlen aller Antwortkategorien für das Unterstützungsverhalten der pädagogischen Fachkraft

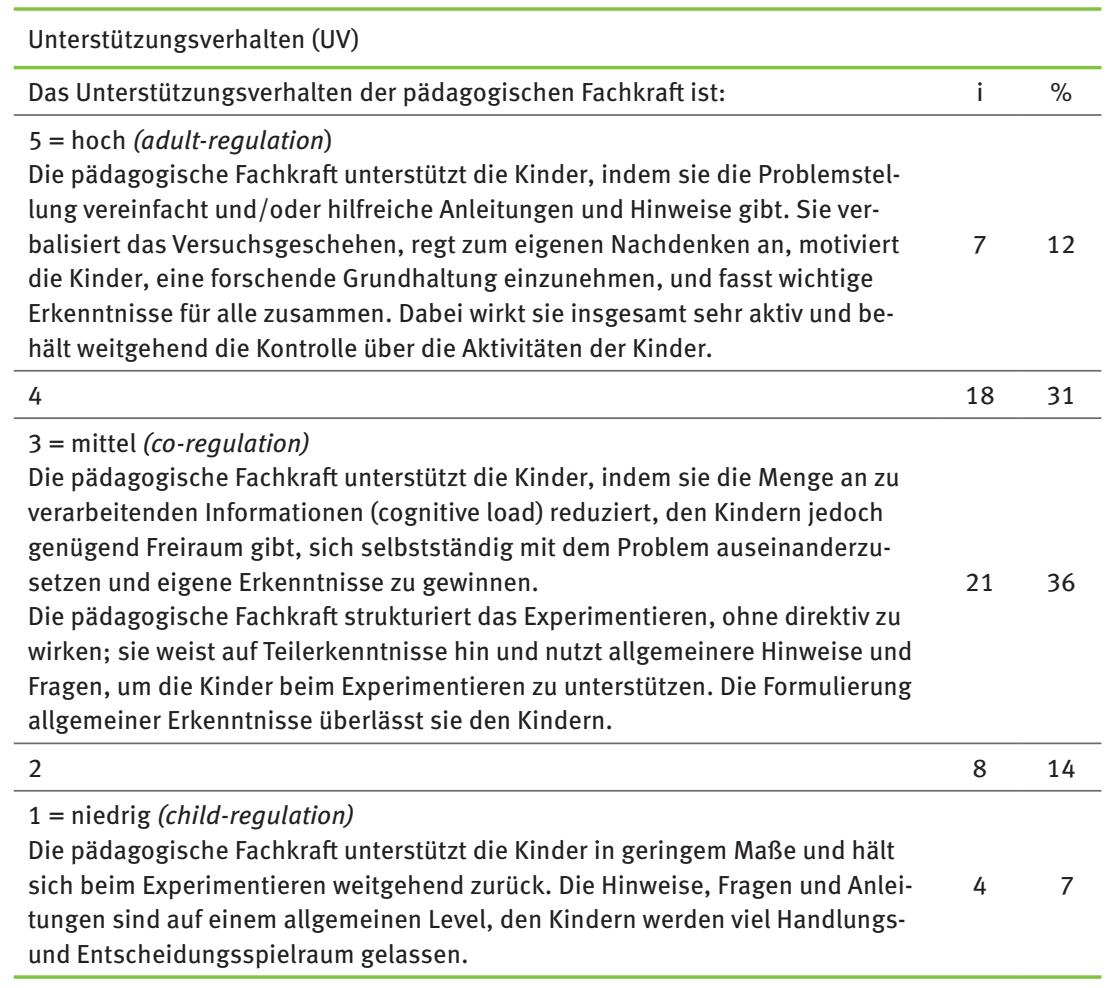

Die Beobachterübereinstimmung für diese Skala lag bei ICC $=.74$ in befriedigender Höhe. Folgt man dem Großteil der bestehenden Literatur, so scheint ein geringes bzw. moderates Maß an Unterstützung besonders günstig; von daher würde diese Variable das theoretische Optimum in der Mitte aufweisen $(M=3$, co-regulation). Gleichzeitig ist das Item so formuliert, dass höhere Werte mehr unterstützendes Verhalten der Fachkraft im Sinne von Scaffolding-Bemühungen widerspiegeln. Aus dieser Perspektive betrachtet wäre ein möglichst hoher Wert optimal. Diese Widersprüchlichkeit wurde bei der Item-Konzeption nicht ausrei- 
chend bedacht. Im vorliegenden Fall orientieren wir uns an der zweitgenannten Interpretation, d. h. höhere Werte für Unterstützung in dieser Skala weisen auf mehr Scaffolding-Verhalten hin. Es wird daher keine Rekodierung der Variablen vorgenommen, wenn später Zusammenhänge mit anderen Variablen berechnet werden. In der vorliegenden Stichprobe zeigten die meisten Fachkräfte mittleres Unterstützungsverhalten mit einer Tendenz zu höherer Unterstützung. Extremwerte traten nur bei $19 \%$ aller Fachkräfte auf.

\subsubsection{Stimmung Fachkraft (SF)}

Mit der Variable Stimmung werden neben einem Bewertungsaspekt (negative/ positive Stimmung, Ausmaß an Zufriedenheit und Entspanntheit) die Motivation sowie die Aktivität der Fachkraft erfasst. Die Skala ist so gepolt, dass der Wert 0 einen neutralen Zustand beschreibt, positive Werte eher positive und negative Werte eher negative Gefühlszustände anzeigen. Tabelle 73 berichtet die zugehörigen deskriptiven Item- und Skalenwerte. Die Beurteilerübereinstimmung für die Skala lag insgesamt bei ICC $=.52$ und damit nur im mittleren Bereich, die interne Konsistenz erreichte mit $\alpha=.70$ befriedigende Werte. Die Mittelwerte lagen sämtlich eher im positiven Bereich, wobei die höchsten Ratings für „motiviert“ und „positiv gestimmt“ vergeben wurden. Auch „Zufriedenheit“ und „Aktivität“ wurden wahrgenommen. Sogar auf der Skala angespannt - entspannt, lag der Mittelwerte über 0, was dafür spricht, dass die Rahmenbedingungen (Laborsituation, Videoaufnahme) insgesamt nicht zu großen Stressreaktionen geführt haben.

Tabelle 73. Inhalte, Mittelwerte (M), Standardabweichung (SD), Min- und Max-Werte für einzelne Stimmungsskalen

\begin{tabular}{lcccc}
\hline Stimmung der Fachkraft (SF) & M & SD & Min & Max \\
\hline Die Fachkraft ist ... & & & & \\
a) negativ gestimmt (-2) - positiv gestimmt (+2). & 0.75 & 0.52 & -0.5 & 1.5 \\
b) unzufrieden (-2) - zufrieden (+2). & 0.67 & 0.57 & -1.0 & 1.5 \\
c) angespannt (-2) - entspannt (+2). & 0.14 & 0.68 & -2.0 & 1.5 \\
d) lustlos (-2) - motiviert (+2). & 0.91 & 0.61 & -1.0 & 2.0 \\
e) passiv (-2) - aktiv (+2). & 0.56 & 0.90 & -1.5 & 2.0 \\
\hline Gesamtskala Stimmung Fachkraft (a-e) & 0.61 & 0.45 & -0.3 & 1.5 \\
\hline
\end{tabular}

\subsubsection{Globalurteil (GU) zur Prozessgestaltung beim Forschen}

Das Globalurteil spiegelt die subjektive Einschätzung des Kodierers bzw. der Kodiererin hinsichtlich der erfolgreichen Unterstützung des kindlichen Erkenntnisprozesses durch die Fachkraft wider. Es beinhaltet die Aspekte 
- Motivation der Fachkraft,

Angemessenheit der gewählten Unterstützungsstrategie und der Intensität von pädagogischen Interventionen bezogen auf die konkreten Bedürfnisse des Kindes sowie

- Aussagen zur Resonanz des Kindes.

Damit bildet dieses Urteil ganz verschiedene Aspekte der Prozessgestaltung beim Forschen gleichzeitig ab (siehe Tabelle 74).

Tabelle 74. Absolute (i) und prozentuale (\%) Häufigkeit der Wahlen aller Antwortkategorien für das Globalurteil des Verhaltens der pädagogischen Fachkraft

\begin{tabular}{|c|c|c|}
\hline \multicolumn{3}{|l|}{ Globalurteil Verhalten Fachkraft (GU) } \\
\hline $\begin{array}{l}\text { Insgesamt war das Unterstützungsverhalten der pädagogischen } \\
\text { Fachkraft in dieser Situation ... }\end{array}$ & i & $\%$ \\
\hline $\begin{array}{l}5=\text { erfolgreich umgesetzt. } \\
\text { Die Fachkraft wirkt motiviert und engagiert. Die Unterstützung ist überwiegend } \\
\text { angemessen. Die Fachkraft wählt überwiegend die passende Unterstützungsstufe } \\
\text { für jedes Kind. Die Fachkraft passt ihre Unterstützung im Prozess an die kind- } \\
\text { lichen Bedürfnisse an. }\end{array}$ & 7 & 12 \\
\hline 4 & 25 & 43 \\
\hline $\begin{array}{l}3=\text { teilweise erfolgreich umgesetzt. } \\
\text { Das Unterstützungsverhalten ist häufig angemessen, aber Verbesserungs- } \\
\text { potenzial ist klar erkennbar. Die Fachkraft wählt in einigen Fällen eine falsche } \\
\text { Unterstützungsstufe. Die Fachkraft nutzt in einigen Fällen Strategien, die Kinder } \\
\text { beim Forschen nicht voranbringen. Die Unterstützung wird von den Kindern nur } \\
\text { teilweise aufgegriffen. }\end{array}$ & 20 & 35 \\
\hline 2 & 5 & 9 \\
\hline $\begin{array}{l}1 \text { = nicht erfolgreich umgesetzt. } \\
\text { Die Fachkraft zeigt zu viel oder zu wenig Unterstützung. Die Unterstützung wird } \\
\text { von den Kindern nicht oder nur sehr selten erfolgreich aufgegriffen. Die Fachkraft } \\
\text { wählt (fast) immer eine falsche Unterstützungsstufe. }\end{array}$ & 1 & 2 \\
\hline
\end{tabular}

Trotz großer Heterogenität der im Globalurteil zusammengefassten Aspekte von Prozessqualität liegt die Beurteilerübereinstimmung mit ICC $=.74 \mathrm{im}$ mittleren bis guten Bereich. Die Kategorien „teilweise erfolgreich umgesetzt“ bzw. die nächsthöhere Kategorie wurden am häufigsten vergeben. Immerhin $12 \%$ wurden sogar in die höchste Kategorie eingestuft. 


\subsubsection{Empirische Beziehung zwischen den einzelnen Fachkraft-Dimensionen}

Zunächst soll dokumentiert werden, wie die einzelnen fachkraftbezogenen Skalen von QUOTS mit dem Globalurteil korrelieren, um besser einschätzen zu können, welche Rolle einzelne Verhaltensdimensionen für die Beurteilung des fachkraftbezogenen Verhaltens spielen. Tabelle 75 referiert die entsprechenden Koeffizienten. Anschließend referieren wir die Interkorrelationen aller übrigen fachkraftbezogenen QUOTS-Skalen mit Ausnahme der Allgemeinen Haltung (Rollenbilder) in Tabelle 76. Letztere werden dann noch einmal gesondert in ihrer Beziehung zu den einzelnen Dimensionen von QUOTS beschrieben (siehe Tabelle 77).

Tabelle 75. Interkorrelationen zwischen allen fachkraftbezogenen QUOTS-Skalen mit dem Globalurteil

\begin{tabular}{lc}
\hline QUOTS-Skalen & Korrelation $(r)$ mit Globalurteil \\
\hline Scaffolding-Strategien & $0.63^{\star \star}$ \\
\hline Angemessenheit Scaffolding & $0.65^{\star \star}$ \\
\hline Manner of Instruction & $0.74^{\star \star}$ \\
\hline Begrenzung von Freiheitsgraden & $0.45^{\star \star}$ \\
\hline Transfer of Responsibitiy - Lokus Fachkraft & $0.66^{\star \star}$ \\
\hline Transfer of Responsibility - Lokus Kind & $-0.55^{\star \star}$ \\
\hline Direction Maintenance & $0.79^{\star \star}$ \\
\hline Contingency Management & $0.75^{\star \star}$ \\
\hline Aufdringlichkeit/Übergriffigkeit & -0.20 \\
\hline Unterstützungsverhalten & $0.80^{\star \star}$ \\
\hline Stimmung Fachkraft & $0.38^{\star}$ \\
\hline Allgemeine Haltung „Lehrer“ & $0.49^{\star}$ \\
\hline Allgemeine Haltung „Möglichmacher“ & $0.41^{\star}$ \\
\hline Allgemeine Haltung „Begleiter“ & $0.70^{\star \star}$ \\
\hline Allgemeine Haltung „Weiteres Kind“ & -0.25 \\
\hline
\end{tabular}

Anmerkung: Die Helligkeit der Felder in der Tabelle markiert das Signifikanzniveau der einzelnen Koeffizienten (weiß = nicht signifikant, hellgrau $/{ }^{*}=5 \%$ Niveau, dunkelgrau $/{ }^{\star \star}=1 \%$ Niveau). Eine kritische Diskussion der Bedeutung entsprechender Signifikanzen erfolgt am Ende des Berichts.

Es wird deutlich, dass die Kodiererinnen Fachkräfte vor allem dann als besonders positiv bewerten, wenn diese sich stark engagieren und dabei motivierend, ko-konstruktiv sowie fachkompetent verhalten. Aufdringlichkeit/Übergriffigkeit sowie eine Allgemeine Haltung als „weiteres Kind“ - beides Skalen, die auf ein zu starkes oder zu schwaches pädagogisches Engagement verweisen - scheinen nicht mit dem Globalurteil assoziiert zu sein, während Transfer of Responsibility 
- Lokus Kind als einzige Skala signifikant negativ mit dem Globalurteil zusammenhängt.

\section{Interkorrelationen zwischen fachkraftbezogenen QUOTS-Skalen}

Tabelle 76 bezieht sich auf die Interkorrelationen zwischen allen fachkraftbezogenen QUOTS-Skalen (außer dem Globalurteil, siehe Tabelle 75, und der Allgemeinen Haltung, siehe Tabelle 77). Eine entsprechende Analyse gibt genaueren Aufschluss über die konkreten Bezüge zwischen unterschiedlichen Aspekten prozessorientierter Verhaltensweisen.

Tabelle 76. Interkorrelationen zwischen fachkraftbezogenen QUOTS-Skalen (außer Allgemeine Haltung und Globalurteil; siehe dazu Tabelle 77 und Tabelle 75)

\begin{tabular}{|c|c|c|c|c|c|c|c|c|c|c|c|}
\hline Pearson $r$ & SCS & ASC & MI & TR- LF & TR-LK & DM & $\mathrm{CM}$ & $\mathrm{BF}$ & AÜ & UV & SF \\
\hline SCS & 1 & $0.44^{\star \star}$ & $0.80^{\star *}$ & $0.61^{\star *}$ & $-.54^{\star \star}$ & $0.69^{* *}$ & $0.61 * \star$ & $0.46^{\star \star}$ & $0.47^{\star \star}$ & $0.69^{\star \star}$ & $0.57^{* *}$ \\
\hline ASC & & 1 & $0.34^{\star \star}$ & $0.53^{\star \star}$ & $-.48^{\star \star}$ & $0.54^{* *}$ & $0.53^{\star \star}$ & 0.40 ** & 0.05 & $0.60^{\star \star}$ & 0.04 \\
\hline MI & & & 1 & $0.77^{\star \star}$ & $-.63^{\star \star}$ & $0.72^{\star \star}$ & $0.58^{\star \star}$ & $0.41^{\star *}$ & $0.44^{\star \star}$ & $0.74^{\star \star}$ & $0.52^{\star \star}$ \\
\hline TR-LF & & & & 1 & $-.84^{\star \star}$ & $0.77^{\star \star}$ & $0.47^{\star \star}$ & $0.54^{* *}$ & $0.38^{\star \star}$ & $0.85^{\star \star}$ & 0.24 \\
\hline TR-LK & & & & & 1 & $-0.66^{\star \star}$ & $-0.27^{\star *}$ & -0.50 ** & $-0.45^{\star \star}$ & $-0.77^{\star \star}$ & -.017 \\
\hline $\mathrm{DM}$ & & & & & & 1 & $0.68^{\star \star}$ & $0.50^{\text {** }}$ & $0.35^{\star \star}$ & $0.82^{\star \star}$ & 0.39 ** \\
\hline CM & & & & & & & 1 & $0.44^{\star \star}$ & 0.13 & $0.39^{\star \star}$ & $0.46^{\star *}$ \\
\hline $\mathrm{BF}$ & & & & & & & & 1 & 0.21 & $0.57^{\star \star}$ & $0.32^{\star}$ \\
\hline AÜ & & & & & & & & & 1 & $0.36^{\star \star}$ & $0.30^{\star}$ \\
\hline UV & & & & & & & & & & 1 & $0.33^{\star}$ \\
\hline SF & & & & & & & & & & & 1 \\
\hline
\end{tabular}

Anmerkung: Die Helligkeit der Felder in der Tabelle markiert das Signifikanzniveau der einzelnen Koeffizienten (weiß = nicht signifikant, hellgrau $/ *=5 \%$ Niveau, dunkelgrau $/ * *=1 \%$ Niveau).

Es fällt auf, dass nahezu alle Skalen hoch positiv korrelieren, bis auf Transfer of Responsibility - Lokus Kind. Damit scheint das Fachkraft-Verhalten auf unterschiedlichen Dimensionen einem bestimmten Muster zu folgen. Weiterhin wird deutlich, dass Aufdringlichkeit/Übergriffigkeit keine systematischen Bezüge mit Angemessenheit von Scaffolding, Contingency Management und Begrenzung von Freiheitsgraden aufweist. Dies könnte auf eine grundsätzliche Schwierigkeit bei der Beurteilung der richtigen Balance zwischen zu viel und zu wenig Direktivität im Verhalten der Fachkraft hindeuten. Weiterhin fällt auf, dass keine statistisch signifikanten Zusammenhänge zwischen der Stimmung der Fachkraft und der Angemessenheit von Scaffolding sowie beiden Transfer of Responsibility Subskalen 
zu bestehen scheinen und dass die Korrelationen anderer Skalen mit der Stimmung tendenziell eher gering ausfallen. Damit deutet sich an, dass kognitive und emotionale Dimensionen des Verhaltens von Fachkräften getrennt variieren.

\section{Korrelationen der QUOTS-Dimensionen mit der Allgemeinen Haltung der Fachkräfte}

Tabelle 77 referiert schließlich, wie die einzelnen Dimensionen von QUOTS mit Einschätzungen der Allgemeinen Haltung der Fachkräfte zusammenhängen. Es zeigt sich, dass die meisten signifikanten Korrelationen der QUOTS-Skalen mit dem Rollenbild des Lehrers vorliegen. Dies deckt sich mit der Beobachtung, dass Transfer of Responsibility - Lokus Fachkraft hoch positiv und Transfer of Responsibility - Lokus Kind negativ mit dem Globalurteil über das Verhalten der Fachkräfte korreliert. Gleichzeitig gilt es zu beachten, dass der Großteil der Skalen, die in QUOTS beschrieben werden, aktive Strategien der Fachkraft sind. Da das Rollenbild des „Lehrers“ primär auf aktives Verhalten der Fachkraft abhebt, macht es Sinn, dass gerade dieses Rollenbild mit den unterschiedlichen Strategien positiv korreliert. Die geringste Anzahl signifikanter Zusammenhänge ergab sich mit dem Rollenbild des Begleiters als Gegenentwurf zum Lehrer hinsichtlich der Eigeninitiative in der Gestaltung der Experimentiersituation. Ganz in diesem Sinne korrelierte die Skala Transfer of Responsibility - Lokus Kind als einzige positiv mit dieser Rollenbild-Dimension (ebenso wie mit der Dimension „weiteres Kind“).

Tabelle 77. Korrelationen der QUOTS-Dimensionen mit der Beschreibung der Allgemeinen Haltung der Fachkräfte

\begin{tabular}{|c|c|c|c|c|c|c|c|c|c|c|c|}
\hline Pearson $r$ & SCS & ASC & MI & TR- LF & TR- LK & DM & $\mathrm{CM}$ & $\mathrm{BF}$ & AÜ & UV & SF \\
\hline „Lehrer“ & $0.50^{\star *}$ & $0.26^{*}$ & $0.64^{\star \star}$ & $0.63^{\star \star}$ & $-.73 * *$ & 0.50 ** & $0.27^{\star \star}$ & $0.44^{* *}$ & $0.37^{\star \star}$ & $0.63^{\star *}$ & 0.19 \\
\hline $\begin{array}{l}\text { „Möglich- } \\
\text { macher“ }\end{array}$ & $0.41^{\star *}$ & 0.31 * & 0.25 & 0.05 & -.05 & $0.30^{*}$ & $0.50^{\star \star}$ & 0.19 & 0.09 & 0.22 & $0.36^{* *}$ \\
\hline „Begleiter“ & -.03 & 0.16 & -.22 & $-.33^{\star}$ & $0.33^{*}$ & -.05 & 0.10 & -.25 & -.15 & -.20 & 0.04 \\
\hline $\begin{array}{l}\text { „weiteres } \\
\text { Kind“ }\end{array}$ & -.23 & -.24 & -.24 & -.40 ** & $0.53^{\star *}$ & $-.33^{*}$ & 0.10 & -.23 & -.22 & $-.28 *$ & 0.11 \\
\hline
\end{tabular}

Anmerkung: Die Helligkeit der Felder in der Tabelle markiert das Signifikanzniveau der einzelnen Koeffizienten (weiß $=$ nicht signifikant, hellgrau $/ *=5 \%$ Niveau, dunkelgrau $/$ ** $=1 \%$ Niveau). 


\subsubsection{Zusammenfassende Bewertung der Ergebnisse zu fachkraftbezogenen QUOTS -Skalen}

Mit QUOTS scheint es gelungen zu sein, wichtige Teilaspekte des prozessorientierten Verhaltens von Fachkräften beim naturwissenschaftlichen Forschen mit Kindern so zu beschreiben, dass eine inhaltlich sinnvolle, objektive und reliable Erfassung der Zielvariablen anhand von Videodaten möglich ist. Die Items wiesen größtenteils gute Verteilungseigenschaften auf, und die Interkorrelationen zwischen unterschiedlichen Skalen fielen überwiegend hoch aus, obwohl durchaus unterschiedliche Aspekte von Prozessqualität thematisiert wurden.

Auffällig scheint, dass Fachkräfte, die insgesamt eine besonders positive Einschätzung ihres pädagogischen Verhaltens erhielten, als hoch engagiert beschrieben wurden und in ihrem Verhalten am ehesten dem Rollenbild eines Lehrers entsprachen. Wer die Verantwortung für das Geschehen primär den Kindern überließ (Transfer of Responsibility -Lokus Kind), erhielt ein weniger gutes Globalurteil. Während positiv beschriebene Formen von aktivem Engagement der Fachkräfte eine eher positive Bewertung erhielten, solange das Verhalten nicht als aufdringlich oder übergriffig identifiziert wurde, wurde es als durchaus kritisch gesehen, wenn die Fachkraft den Gestaltungsprozess zu sehr den Kindern überließ und sich eher passiv verhielt.

Klar differenzierten die Beurteiler und Beurteilerinnen zwischen der Stimmung der Fachkraft und ihrem sachbezogenen Verhalten. Das sachbezogene Fachkraft-Verhalten wies deutlich engere Zusammenhänge mit der Globaleinschätzung des Fachkraft-Verhaltens auf als das emotionale Verhalten. Gleichzeitig fiel auf, dass es auch fachlich geschulten Beobachtern und Beobachterinnen nicht ganz leicht fällt, eine gute ko-konstruktive Haltung zu identifizieren, wenn es um die Frage geht, wie stark sich die Fachkraft beim Forschen und im Umgang mit den Kindern einbringt oder zurückhält. Hier kommen Globaleinschätzungen vermutlich auch an ihre Grenzen

\subsection{Kindbezogene QUOTS-Skalen}

Bei den kindbezogenen Skalen wurde unterschieden zwischen Skalen, die sich auf die gesamte Gruppe (Kinder plus Fachkraft) beziehen, und solchen, die sich auf einzelne Kinder beziehen. Hinsichtlich der Gruppe interessierte vor allem der Aufgabenfokus (GAF, als grobes Maß für die Involviertheit) und die Forscherhaltung (GFH, als grobes Maß für die Orientierung auf die Gewinnung naturwissenschaftlicher Einsichten). Bei den Skalen, die sich speziell auf einzelne Kinder bezogen, wurde die kindliche Aufgabenbearbeitung (KAB), der kindliche Unter- 
stützungsbedarf (KUB), die Stimmung des Kindes (SK) und sein Erkenntnisgewinn (KEG) thematisiert.

\subsubsection{Gruppe: Aufgabenfokus (GAF)}

Beim Aufgabenfokus wurde erfasst, wie hoch der Anteil der Zeit war, den die Kinder mit aktivem Experimentieren zubrachten. Dabei gingen wir von der Annahme aus, dass ein hoher Prozentsatz an aktiver Experimentierzeit mit einem großen Interesse der Kinder für die Fragestellung einhergeht. Tabelle 78 dokumentiert die Häufigkeit der unterschiedlichen Einschätzungen. Die Interrater-Übereinstimmung für diese Skala erreicht mit ICC $=.69$ befriedigende Werte.

Wie ersichtlich, waren 49 von 58 Gruppen hoch motiviert und widmeten mindestens $50 \%$ ihrer Zeit dem Experimentieren. Aber immerhin 9 Gruppen widmeten sich kaum oder gar nicht dem Experimentieren, sondern beschäftigten sich die meiste Zeit anderweitig mit dem gegebenen Material.

Tabelle 78. Angaben zum Anteil der Zeit, den die Gruppe mit Experimentieren verbracht hat; Häufigkeit (absolut: i; prozentual: \%) der unterschiedlichen Antwortkategorien

\begin{tabular}{|c|c|c|}
\hline \multicolumn{3}{|l|}{ Gruppenverhalten Aufgabenfokus (GAF) } \\
\hline $\begin{array}{l}\text { Wie hoch ist der geschätzte Zeitanteil (\%), den die Gruppe dem Experimentie- } \\
\text { ren widmet? }\end{array}$ & $\mathbf{i}$ & $\%$ \\
\hline $\begin{array}{l}4=75-100 \% \\
\text { Kinder und Fachkraft widmen sich hauptsächlich der vorgegebenen Aufgabe. }\end{array}$ & 27 & 47 \\
\hline $\begin{array}{l}\begin{array}{l}3=50-75 \% \\
\text { Kinder und Fachkraft bearbeiten die vorgegebene Aufgabe, aber nicht immer in } \\
\text { zielführender Weise (z. B. planschen mit Wasser, ohne zu experimentieren). }\end{array}\end{array}$ & 22 & 38 \\
\hline $\begin{array}{l}2=25-50 \% \\
\text { Kinder und Fachkraft beschäftigen sich mit dem vorgegebenen Material, aber } \\
\text { weitgehend ohne zu experimentieren (z. B. sind fast ausschließlich mit Plan- } \\
\text { schen oder Spielen beschäftigt). }\end{array}$ & 7 & 12 \\
\hline $\begin{array}{l}1=0-25 \% \\
\text { Kinder und Fachkraft beschäftigen sich nur in sehr geringem Ausmaß mit dem } \\
\text { Material und der Aufgabe (z. B. Kinder spielen miteinander, ohne sich für das } \\
\text { Thema Sinken und Schwimmen zu interessieren). }\end{array}$ & 2 & 3 \\
\hline
\end{tabular}

\subsubsection{Gruppe: Forscherhaltung (GFH)}

Bei der Forscherhaltung stand die Qualität des Engagements im Vordergrund. Nur wenn sich die Gruppe tatsächlich auf den Experimentierprozess einlässt und der Forschungskreis sich in den Interaktionen zwischen Fachkraft und Kind widerspiegelt, kann die Fachkraft den lernziel-orientierten Prozess in Gang setzen. Die Interrater-Übereinstimmung für diese Skala lag mit ICC $=.78 \mathrm{im}$ guten Bereich. 
Tabelle 79. Angaben zur Häufigkeit der Wahl unterschiedlicher Beurteilungskategorien bei der Frage, inwiefern die Gruppe erfolgreiches Experimentierverhalten zeigte

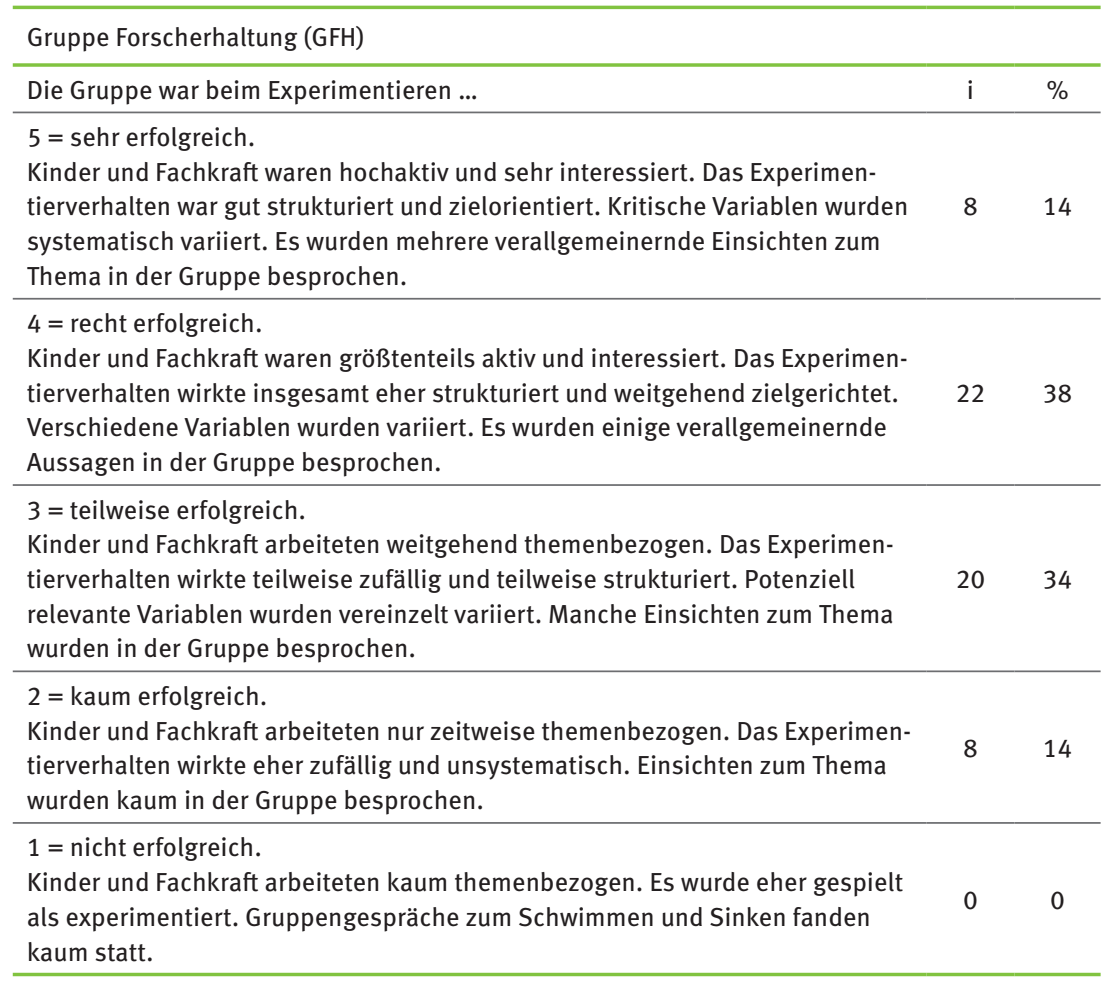

Die in Tabelle 79 dokumentierten Werte machen weiter deutlich, dass zwei Drittel der untersuchten Gruppen „teilweise“ oder sogar „recht erfolgreich“ geforscht haben. Dies scheint angesichts der heterogenen Zusammensetzung der Stichprobe, die auch Fachkräfte ohne naturwissenschaftliche Fortbildung enthält, erwartungskonform. Keine Gruppe war „nicht erfolgreich“ und nur ein kleiner Teil war entweder „kaum erfolgreich“ oder „sehr erfolgreich“.

\subsubsection{Kind: Aufgabenbearbeitung (KAB)}

Für jedes Kind wurden unterschiedliche Aspekte der Aufgabenbearbeitung von gar nicht (0) bis sehr viel (4) bewertet (siehe Tabelle 80). Die Beobachterübereinstimmung für die Skala Aufgabenbearbeitung lag bei ICC $=.68$, sie fiel damit etwas geringer als bei den Fachkraftskalen aus, befand sich aber immer noch im akzeptablen Bereich. Die interne Konsistenz lag bei $\alpha=.75$. 
Tabelle 80. Mittelwerte, Standwerte, Min- und Max-Werte der Bewertungen für die Aufgabenbearbeitung der Kinder

\begin{tabular}{lllll}
\hline Kind Aufgabenbearbeitung (KAB) & M & SD & Min & Max \\
\hline Das Kind war ... & & & & \\
a) aufmerksam und konzentriert bei der Sache. & 2.52 & 0.62 & 0.5 & 4.0 \\
b) griff Anregungen der Fachkraft erfolgreich auf. & 2.21 & 0.57 & 0.5 & 3.5 \\
c) experimentierte selbstständig. & 2.20 & 0.59 & 0.5 & 3.5 \\
d) hat wichtige Einsichten gewonnen. & 1.74 & 0.76 & 0.0 & 4.0 \\
\hline
\end{tabular}

Der Wert für die Einschätzung „hat wichtige Einsichten gewonnen“ (bezogen auf das Schwimmen und Sinken, aber ohne explizite Differenzierung zwischen Richtig und Falsch) liegt im Mittel etwas unter den anderen Werten, was angesichts der Kürze der Experimentierzeit wenig verwundert. Insgesamt scheinen die Kinder im Durchschnitt „etwas“ bis „viel“ Engagement an den Tag gelegt zu haben.

\subsubsection{Kind: Unterstützungsbedarf (KUB)}

Im nächsten Schritt interessierte der Unterstützungsbedarf der Kinder. Dieser wurde von niedrig (1) bis hoch (5) eingeschätzt (siehe Tabelle 81). Die Erfassung dieser Variable scheint wichtig, weil sich daran misst, wie aktiv die Fachkraft werden muss, um die Kinder in ihrem Bemühen um neue Einsichten zu fördern. Die Beobachterübereinstimmung für diese Skala lag bei ICC $=.70$ und bewegte sich damit im guten Bereich.

Tabelle 81. Häufigkeit (absolut, prozentual), mit der unterschiedliche Kategorien für den Unterstützungsbedarf des Kindes gewählt wurden

\begin{tabular}{|c|c|c|}
\hline \multicolumn{3}{|l|}{ Kind Unterstützungsbedarf (KUB) } \\
\hline Der Unterstützungsbedarf des Kindes ist ... & $\mathrm{i}$ & $\%$ \\
\hline $\begin{array}{l}5=\text { hoch. } \\
\text { Das Kind ist nicht in der Lage, ohne Unterstützung erfolgreich zu experimentie- } \\
\text { ren (z.B. mangelt es ihm an relevantem Vorwissen oder/und es kann Objekte } \\
\text { und Vorgänge nicht benennen); es ist daher auf Hinweise und die Anleitung der } \\
\text { Fachkraft angewiesen. (Hinweis: Auch wenn das Kind lustlos und demotiviert } \\
\text { bezüglich der Aufgabe wirkt, ist sein Unterstützungsbedarf hoch) }\end{array}$ & 8 & 4 \\
\hline 4 & 53 & 24 \\
\hline $\begin{array}{l}3=\text { mittel. } \\
\text { Das Kind ist in der Lage, in Ansätzen auch ohne Unterstützung erfolgreich zu } \\
\text { experimentieren (z. B. kann es Objekte einzeln und nacheinander ins Wasser } \\
\text { legen und beobachten, was passiert). Sein Vorwissen ist rudimentär. Es fehlen } \\
\text { ihm Ausdrucksmöglichkeiten. }\end{array}$ & 111 & 50 \\
\hline 2 & 43 & 20 \\
\hline $\begin{array}{l}1=\text { niedrig. } \\
\text { Das Kind ist in der Lage, auch ohne Unterstützung erfolgreich zu experimentie- } \\
\text { ren (z.B. kann es Objekte einzeln und nacheinander ins Wasser legen und beob- } \\
\text { achten, was passiert.) Es hat ausreichendes Vorwissen, formuliert Vermutungen } \\
\text { und kann selbst erklären, was passiert. }\end{array}$ & 5 & 2 \\
\hline
\end{tabular}


Es fällt auf, dass der Kenntnisstand der Kinder grundsätzlich als „rudimentär“ eingeschätzt wurde und bei den Kindern daher mehrheitlich ein mittlerer bis hoher Unterstützungsbedarf wahrgenommen wurde. Dies passt zu der Beobachtung, dass die pädagogischen Fachkräfte mehrheitlich aktiv werden mussten, um Erkenntnisprozesse bei den Kindern anzuregen.

\subsubsection{Kind: Stimmung (KS)}

Schließlich scheint es wichtig zu prüfen, in welcher Stimmung die Kinder beim Experimentieren waren. Es ist zu vermuten, dass die Stimmung bedeutsamen Einfluss auf Lernprozesse hat. Darüber hinaus kann spekuliert werden, dass das Fachkraft-Verhalten bedeutsamen Einfluss auf die Stimmung der Kinder nimmt. Diese wurde für unterschiedliche Aspekte (allgemeine Gestimmtheit, Zufriedenheit, Anspannung, Motivation, Aktivitätsgrad) bestimmt. Die Beobachterübereinstimmung für diese Skala lag bei ICC $=.60$ und erreichte damit akzeptable Werte; die interne Konsistenz fiel mit $\alpha=.70$ gut aus. Wie Tabelle 82 dokumentiert, war die Stimmung der Kinder insgesamt eher positiv, wies allerdings zwischen den Kindern auch erhebliche Schwankungen auf. Das Engagement der Kinder (Stimmung aktiv - passiv) wurde als moderat positiv eingeschätzt.

Tabelle 82. Inhalte, Mittelwerte (M), Standardabweichung (SD), Min- und Max-Werte für einzelne Stimmungsskalen

\begin{tabular}{lcccc}
\hline Stimmung des Kindes (KS) & M & SD & Min & Max \\
\hline Das Kind ist ... & & & & \\
a) negativ gestimmt (-2) - positiv gestimmt (+2). & 0.83 & 0.48 & -0.5 & 2.0 \\
b) unzufrieden (-2) - zufrieden (+2). & 0.68 & 0.46 & -1.0 & 1.5 \\
c) angespannt (-2) - entspannt (+2). & 0.61 & 0.49 & -1.0 & 1.5 \\
d) lustlos (-2) - motiviert (+2). & 0.62 & 0.70 & -1.5 & 2.0 \\
e) passiv (-2) - aktiv (+2). & 0.49 & 0.80 & -2.0 & 2.5 \\
\hline Gesamtskala Stimmung Kind (a-e) & 0.66 & 0.45 & -0.8 & 2.5 \\
\hline
\end{tabular}

\subsubsection{Kind: Erkenntnisgewinn (KEG)}

Neben der allgemeinen Haltung und Stimmung interessierte vor allem der Erkenntnisgewinn des Kindes als zentrale Outcome-Variable. Hier galt es zu klären, ob das Kind Schlussfolgerungen über das Schwimm-/Sinkverhalten bestimmter Eigenschaften (Material, Größe, Gewicht) ziehen konnte. Es ging also weniger darum festzustellen, ob ein physikalisch korrektes Konzept von Dichte erworben wurde, als vielmehr darum, ob die Kinder eigene Beobachtungen zum faktischen Schwimm- und Sinkverhalten von Objekten zum Ausdruck bringen können und 
bereits Hypothesen bzw. Erkenntnisse über die Bedeutung der Rolle unterschiedlicher Materialaspekte verbalisieren. Jede Form von sachbezogener Erwartung, Beschreibung oder Erklärung wurde daher unabhängig von ihrer Richtigkeit berücksichtigt und die Summe entsprechender Äußerungen pro Kind als grober Schätzwert für das Ausmaß naturwissenschaftlicher Erkenntnisse interpretiert. Tabelle 83 referiert die absoluten Häufigkeiten für bestimmte „Einsichten“. Die mittlere Übereinstimmung für den Gesamtwert lag hier bei ICC $=.80$ und damit im guten Bereich. Es kann davon ausgegangen werden, dass sich der Erkenntnisgewinn hinreichend objektiv erfassen ließ.

Wie Tabelle 83 dokumentiert, registrierte etwa die Hälfte der Kinder für jedes Material klar erkennbar, ob es sinkt oder schwimmt. Etwa ein Viertel bis die Hälfte aller Kinder scheint zu denken, dass die Frage, ob ein Gegenstand sinkt oder schwimmt, mit seinem Gewicht zusammenhängt, während die Größe als potenzielle Einflussvariabel nur selten thematisiert wurde. Diese Beobachtungen decken sich weitgehend mit den Erwartungen.

Tabelle 83. Häufigkeit, mit der Kinder bestimmte Einsichten über relevante Merkmale von Objekten, die sich auf das Schwimmen und Sinken beziehen, gewonnen haben

\begin{tabular}{|c|c|c|c|}
\hline \multicolumn{2}{|c|}{ Kindlicher Erkenntnisgewinn (KWG) } & \multirow{2}{*}{$\begin{array}{c}\text { schwimmt } \\
12 \%\end{array}$} & \multirow{2}{*}{$\begin{array}{c}\text { sinkt } \\
1 \%\end{array}$} \\
\hline Material & Styropor & & \\
\hline & Holz & $53 \%$ & $2 \%$ \\
\hline & Metall & $1 \%$ & $58 \%$ \\
\hline & Wachs & $50 \%$ & $6 \%$ \\
\hline & Plastik & $55 \%$ & $12 \%$ \\
\hline & Glas & $2 \%$ & $46 \%$ \\
\hline & Stein & $1 \%$ & $49 \%$ \\
\hline & Schwamm & $51 \%$ & $11 \%$ \\
\hline \multirow[t]{2}{*}{ Gewicht } & leicht & $22 \%$ & $5 \%$ \\
\hline & schwer & $2 \%$ & $42 \%$ \\
\hline \multirow[t]{2}{*}{ Größe } & klein & $1 \%$ & $5 \%$ \\
\hline & groß & $2 \%$ & $2 \%$ \\
\hline
\end{tabular}

Im Durchschnitt formulierte jedes Kind in der Forschungssituation fünf Erkenntnisse $(M=5.15 ; S D=2.63$; Min: 0; Max: 13). Dieses Ergebnis verdeutlicht, dass die Kinder - obwohl sie nur kurz Zeit zum Experimentieren hatten - zu einer bemerkenswert hohen Anzahl von Feststellungen/Einsichten gekommen waren, welche sie auch zum Ausdruck bringen konnten. 


\subsubsection{Empirische Beziehung zwischen den einzelnen Gruppen und kindbezogenen QUOTS-Skalen}

Wie bereits angedeutet, interessierten auch die empirischen Beziehungen zwischen gruppen- und kindbezogenen Maßen, die in Tabelle 84 nachzulesen sind. Hier zeigt sich, dass es eine ganze Reihe positiver Korrelationen zwischen verschiedenen gruppen- und kindbezogenen QUOTS-Skalen gab. So ist festzuhalten, dass auf Gruppenebene der Aufgabenfokus (GAF) und die Forscherhaltung (GFH) eng zusammenhängen und darüber hinaus substanziell mit dem kindlichen Erkenntnisgewinn (KEG) korrelieren, nicht aber mit der Stimmung der Kinder (SK). Generell korreliert die Stimmung der Kinder lediglich mit dem kindlichen Unterstützungsbedarf (KUB) in statistisch bedeutsamer Weise. Die kindliche Aufgabenbearbeitung war umso höher, je höher der allgemeine Aufgabenfokus und die Forscherhaltung der Gruppe ausfielen. Gleichzeitig waren die kindliche Aufgabenbearbeitung (KAB), der kindliche Unterstützungsbedarf (KUB) und der kindliche Erkenntnisgewinn (KEG) positiv korreliert. Mit anderen Worten: Wer einen hohen Unterstützungsbedarf signalisiert und sich bei der Aufgabenbearbeitung stark engagiert, ist in der Regel auch guter Stimmung, während eine negative Stimmung eher mit weniger Aufgabenbearbeitung und Unterstützungsbedarf gekoppelt ist.

Tabelle 84. Interkorrelation zwischen gruppen- und kindbezogenen QUOTS-Skalen

\begin{tabular}{lllllll}
\hline & GAF & GFH & KAB & KUB & SK & KEG \\
\hline GAF & 1 & & & & \\
\hline GFH & $.80^{\star *}$ & 1 & & & & \\
\hline KAB & $.62^{\star \star}$ & $.71^{\star \star}$ & 1 & & & \\
\hline KUB & $.38^{\star *}$ & $.57^{\star *}$ & $.71^{\star *}$ & 1 & 1 & \\
\hline SK & .11 & .18 & .23 & $.54^{\star \star}$ & 1 \\
\hline KEG & $.52^{\star *}$ & $.67^{\star *}$ & $.45^{\star *}$ & $.38^{\star \star}$ & .25 & 1 \\
\hline
\end{tabular}

Anmerkung: Die Helligkeit der Felder in der Tabelle markiert das Signifikanzniveau der einzelnen Koeffizienten (weiß = nicht signifikant, hellgrau $/ *=5 \%$ Niveau, dunkelgrau $/ * \star=1 \%$ Niveau).

\subsubsection{Zusammenfassende Bewertung der Ergebnisse zu gruppen- und kindbezogenen QUOTS-Skalen}

Sowohl auf Gruppen- als auch auf Individualebene war es möglich, wichtige Bewertungen bezüglich des kindlichen Verhaltens in einer Experimentiersituation mit einfachen Mitteln hinreichend objektiv und reliabel zu erfassen, wenn auch eher in groben Zügen. Eine Analyse der Zusammenhänge zwischen unterschiedlichen QUOTS-Skalen macht deutlich, dass nicht so sehr die Stimmung, wohl aber der Aufgabenfokus und die Forscherhaltung auf der Ebene einzelner Kinder und auf Gruppenebene enge Zusammenhänge mit dem Erkenntnisgewinn aufweisen. 
Interessanterweise traf Letzteres auch auf den Unterstützungsbedarf der Kinder zu. Möglicherweise sind Kinder im untersuchten Alter noch sehr davon abhängig, wie stark sie in ihrem Experimentierverhalten durch die Fachkraft unterstützt werden. Sie brauchen eine gute Anleitung, um eine angemessene Forscherhaltung zu lernen und neue Einsichten zu gewinnen. Dies deckt sich auch mit der Beobachtung, dass ein positives Globalurteil bei der Bewertung des Fachkraft-Verhaltens positiv mit den Rollenbildern „Lehrer“, „Möglichmacher“ und „Begleiter“ korreliert. Nur wenn Fachkräfte im Umgang mit den Kindern als engagiert wahrgenommen werden, werden die Erkenntnisprozesse bei den Kindern auch gefördert. Vor diesem Hintergrund scheint es besonders wichtig, im nächsten Schritt zu untersuchen, wie die Unterstützung seitens der Fachkraft am besten aussehen sollte. 


\section{Zusammenhänge zwischen fachkraftspezifischen Kompetenzen und kindlichem Erkenntnisprozess}

Eine erste zentrale Fragestellung unseres Projektes bezieht sich auf die Frage, wie das Verhalten der Fachkraft mit dem der Kinder zusammenhängt. Konkret lautet unsere Hypothese: Parameter der naturwissenschaftsbezogenen Prozessqualität aufseiten der Fachkraft korrelieren positiv mit Kompetenzmerkmalen der Kinder im naturwissenschaftlichen Denken.

Zur Überprüfung dieser Annahme werden die empirischen Zusammenhänge zwischen unterschiedlichen fachkraftbezogenen QUOTS-Skalen einerseits und den gruppen- sowie kindbezogenen Dimensionen andererseits analysiert. Zunächst erwarten wir eine signifikant positive Korrelation zwischen dem Globalurteil des Fachkraft-Verhaltens und der Forscherhaltung der Gruppe sowie dem Erkenntnisgewinn der Kinder, da Letztere zentrale Outcome-Variablen darstellen. Dieser allgemeine Zusammenhang sollte sich auch in Bezug auf einzelne QUOTS-Skalen zeigen, die mit der Qualität des Verhaltens der Fachkraft in engem Zusammenhang stehen. Das gilt konkret für folgende Dimensionen: Scaffolding-Strategien, Angemessenheit von Scaffolding, Manner of Instruction, Transfer of Responsibility - Lokus Fachkraft, Direction Maintenance, Contingency Management und Unterstützungsverhalten.

Weiterhin wird erwartet, dass die Dimensionen Transfer of Responsibility Lokus Kind und ein Rollenverständnis seitens der Fachkraft als weiteres Kind sowie eine hohe Aufdringlichkeit/Übergriffigkeit (alles Indizien für zu geringes oder zu hohes fachliches Engagement der Fachkraft) eher negativ mit der Forscherhaltung der Gruppe sowie dem Erkenntnisgewinn der Kinder zusammenhängen.

Von den Rollenbildern her sollte - ausgehend von einem ko-konstruktiven Verständnis des pädagogischen Auftrags - ein Selbstverständnis der Fachkraft als Lehrer (im zuvor definierten Sinne), „Möglichmacher“ oder „Begleiter“ besonders positiv mit dem Erkenntnisgewinn zusammenhängen.

Zudem würde es Sinn machen, dass die Begrenzung von Freiheitsgraden mit dem Aufgabenfokus der Gruppe und der Aufgabenbearbeitung des Kindes positiv korreliert.

Schließlich kann auf der Basis bisheriger Auswertungen spekuliert werden, dass die Stimmung der Fachkräfte und die der Kinder nur wenige substanzielle Zusammenhänge mit anderen Variablen aufweist.

Tabelle 85 referiert die Interkorrelationsmatrix zwischen allen fachkraft- und kindbezogenen QUOTS-Skalen in der Übersicht. 
Tabelle 85. Interkorrelationsmatrix zwischen fachkraftbezogenen Skalen und kindbezogenen QUOTS-Skalen

\begin{tabular}{|c|c|c|c|c|c|c|}
\hline $\begin{array}{l}\text { Gruppe/Kind } \\
\text { Fachkraft } \quad r\end{array}$ & $\begin{array}{l}\text { Aufgaben- } \\
\text { fokus } \\
\text { Gruppe }\end{array}$ & $\begin{array}{l}\text { Forscher- } \\
\text { haltung } \\
\text { Gruppe }\end{array}$ & $\begin{array}{l}\text { Aufgaben- } \\
\text { bearbei- } \\
\text { tung Kind }\end{array}$ & $\begin{array}{l}\text { Unterstüt- } \\
\text { zungsbe- } \\
\text { darf Kind }\end{array}$ & $\begin{array}{l}\text { Stim- } \\
\text { mung } \\
\text { Kind }\end{array}$ & $\begin{array}{l}\text { Erkenntnis- } \\
\text { gewinn } \\
\text { Kind }\end{array}$ \\
\hline $\begin{array}{l}\text { Scaffolding- } \\
\text { Strategien }\end{array}$ & $.62^{\star \star}$ & $.69^{\star \star}$ & $.58^{\star *}$ & $.33^{\star}$ & .23 & $.57^{\star \star}$ \\
\hline $\begin{array}{l}\text { Angemessenheit } \\
\text { Scaffolding }\end{array}$ & $.55^{\star \star}$ & $.55^{\star \star}$ & $.44^{\star \star}$ & .21 & .02 & $.42^{\star \star}$ \\
\hline $\begin{array}{l}\text { Manner of } \\
\text { Instruction }\end{array}$ & $.67^{\star \star}$ & $.78^{\star \star}$ & $.67^{* \star}$ & $.40^{\star \star}$ & $.37^{\star \star}$ & $.53^{\star \star}$ \\
\hline $\begin{array}{l}\text { Begrenzung von } \\
\text { Freiheitsgraden }\end{array}$ & $.42^{\star \star}$ & $.37^{\star \star}$ & $.34^{\star \star}$ & .14 & .01 & .14 \\
\hline $\begin{array}{l}\text { Transfer of } \\
\text { Responsibitiy LF }\end{array}$ & $.73^{\star \star}$ & $.80^{\star \star}$ & $.62^{\star \star}$ & $.41^{\star \star}$ & .20 & $.51^{\star \star}$ \\
\hline $\begin{array}{l}\text { Transfer of } \\
\text { Responsibility LK }\end{array}$ & $-.60^{\star \star}$ & $-.69^{\star \star}$ & $-.55^{\star \star}$ & $-.34^{\star \star}$ & -0.1 & $-.55^{\star \star}$ \\
\hline $\begin{array}{l}\text { Direction } \\
\text { Maintenance }\end{array}$ & $.82^{\star \star}$ & $.80^{\star \star}$ & $.61^{* *}$ & $.36^{\star \star}$ & .24 & $.58^{\star \star}$ \\
\hline $\begin{array}{l}\text { Contingency } \\
\text { Management }\end{array}$ & $.55^{\star \star}$ & $.55^{\star \star}$ & $.44^{\star \star}$ & .21 & .02 & $.42^{\star \star}$ \\
\hline $\begin{array}{l}\text { Aufdringlichkeit } \\
\text { Übergriffigkeit }\end{array}$ & -.20 & $-.36^{\star \star}$ & -.17 & -.18 & $-.28^{\star}$ & $-.35^{\star \star}$ \\
\hline $\begin{array}{l}\text { Unterstützungs- } \\
\text { verhalten }\end{array}$ & $.78^{\star \star}$ & $.82^{\star \star}$ & $.60^{\star \star}$ & $.31^{\star}$ & .07 & $.58^{\star \star}$ \\
\hline $\begin{array}{l}\text { Stimmung } \\
\text { Fachkraft }\end{array}$ & .24 & $.31^{*}$ & .24 & .16 & .17 & .23 \\
\hline $\begin{array}{l}\text { Globalurteil } \\
\text { Fachkraft }\end{array}$ & $.74^{\star *}$ & $.81^{* *}$ & $.69^{* *}$ & $.46^{* *}$ & .18 & $.52^{* *}$ \\
\hline $\begin{array}{l}\text { Allg. Haltung } \\
\text { „Lehrer“ }\end{array}$ & $.50^{\star \star}$ & $.57^{\star \star}$ & $.50^{\star \star}$ & .22 & .08 & .24 \\
\hline $\begin{array}{l}\text { Allg. Haltung } \\
\text { „Möglichmacher“ }\end{array}$ & .18 & .19 & .19 & -.10 & -.01 & .18 \\
\hline $\begin{array}{l}\text { Allg. Haltung } \\
\text { „Begleiter“ }\end{array}$ & -.15 & -.15 & -.19 & -.09 & -.08 & .01 \\
\hline $\begin{array}{l}\text { Allg. Haltung } \\
\text { „Weiteres Kind“ }\end{array}$ & $-.29 *$ & $-.41^{\star \star}$ & $-.46^{\star \star}$ & $-.38^{\star \star}$ & -.10 & -.24 \\
\hline
\end{tabular}

Anmerkung: Die Helligkeit der Felder in der Tabelle markiert das Signifikanzniveau der einzelnen Koeffizienten (weiß $=$ nicht signifikant, hellgrau $/ *=5 \%$ Niveau, dunkelgrau $/ * \star=1 \%$ Niveau).

Es bestehen vielfältige substanzielle Zusammenhänge zwischen den fachkraftund kindbezogenen Skalen, die weitgehend den zuvor formulierten Erwartungen entsprechen: So weist das Globalurteil für das Verhalten der Fachkraft positive Zusammenhänge mit dem Aufgabenfokus und der Forscherhaltung der Gruppe 
sowie mit der Aufgabenbearbeitung, mit dem Unterstützungsbedarf und dem Erkenntnisgewinn der Kinder auf, was gleichermaßen auch für die diesem Urteil zugrunde liegenden fachkraftbezogenen Dimensionen Scaffolding-Strategien, Angemessenheit von Scaffolding, Manner of Instruction, Transfer of Responsibility Fachkraft, Direction Maintenance und Unterstützungsverhalten gilt.

Erstaunlich scheint die Höhe der Korrelationen, die sämtlich im mittleren bis hohen Bereich lagen. Eine Ausnahme bildet Contingency Management (korrigiert). Diese Skala korreliert lediglich mit dem Aufgabenfokus und der Forscherhaltung der Gruppe, nicht aber mit Skalen, die sich auf individuelle Kinder beziehen. Dies spiegelt vermutlich den Umstand wider, dass Contingency Management für jedes individuelle Kind unterschiedlich ausfältt, in QUOTS aber kindübergreifend erfasst wird, und verweist damit auf die Grenzen einer Auswertung mit globalen Einschätzungen.

Wie vermutet, hängen Transfer of Responsibility - Lokus Kind, Aufdringlichkeit/Übergriffigkeit sowie ein Rollenverständnis als weiteres Kind negativ mit den gruppen- und kindbezogenen Variablen zusammen.

Unsere eingangs formulierten Erwartungen hinsichtlich der Bedeutung der Stimmung der Fachkräfte und der Kinder konnten ebenfalls bestätigt werden. Es ergaben sich kaum Zusammenhänge mit Dimensionen des kindlichen Erkenntnisprozesses. Auffällig war lediglich, dass ein positives emotionales Engagement der Fachkraft mit einer verstärkten Forscherhaltung der Gruppe einherging und dass Manner of Instruction positiv mit der Stimmung des Kindes zusammenhing, während Aufdringlichkeit/Übergriffigkeit negativ mit emotionalem Empfinden der Kinder korrelierte, was inhaltlich plausibel erscheint.

Das Rollenverhalten als Lehrer korreliert dagegen substanziell positiv mit dem Aufgabenfokus und der Forscherhaltung der Gruppe sowie mit dem Aufgabenfokus der Kinder. Es sagt jedoch nicht den individuellen Erkenntnisgewinn voraus. Weitere Zusammenhänge wurden nicht signifikant. Diese Beobachtung macht einerseits deutlich, dass pädagogische Fachkräfte offensichtlich durchaus aktiv und zielgerichtet vorgehen müssen, wenn sie innerhalb einer kurzen Zeit in einer Gruppe mit mehreren Kindern Erkenntnisprozesse anregen wollen. Gleichzeitig wird deutlich, dass eine globale Einschätzung des Rollenverhaltens weniger Aussagekraft zu besitzen scheint als eine stärker verhaltensbezogene Zuschreibung.

Die vorgelegten Befunde sind insgesamt plausibel und in sich stimmig. Sie sprechen dafür, dass Strategien, die in der Fachliteratur als wichtig für die frühe Vermittlung naturwissenschaftlicher Kompetenzen gelten, tatsächlich auch systematische Zusammenhänge mit dem Erkenntnisprozess der Kinder haben: Fachkräfte, die sich fach-kompetent und unterstützend verhalten, erzeugen auf Gruppen- oder/und Individualebene eine Lernumgebung, die es den Kindern erleichtert, sich auf das Experimentieren einzulassen und neue Einsichten zu ge- 
winnen, während Fachkräfte, die sich stark zurücknehmen und wenig verantwortlich für die Gestaltung der Lernsituation zeigen, oder die sich aufdringlich und übergriffig verhalten, weniger zur kindlichen Entwicklung naturwissenschaftlicher Kompetenzen beitragen. Dies ergibt sich aus dem Interkorrelationsmuster zwischen Fachkraft-Verhaltensskalen und dem kindlichen Erkenntnisgewinn (siehe Tabelle 85). Folglich besteht eine zentrale Aufgabe der Fachkraft darin, im Rahmen eines ko-konstruktiven Prozesses die richtige Balance zwischen eigener Aktivität und Aktivität der Kinder zu finden.

In einem nächsten Schritt sollen nun die Daten aus Teilprojekt 2 mit denen aus Teilprojekt 1 kombiniert werden, um festzustellen, inwiefern die sprachliche Anregungsqualität der Fachkräfte mit ko-konstruktivem pädagogischen Verhalten zusammenhängt. 


\section{Prozessqualität in Interaktionen und sprachliche Anregungsqualität}

Die zweite zentrale Fragestellung des vorliegenden Projektes bezieht sich auf Zusammenhänge zwischen Maßen des Fachkraft-Verhaltens in Interaktionen mit dem Kind und Maßen der sprachlichen Anregungsqualität. Die sprachliche Anregungsqualität der pädagogischen Fachkräfte wurde mittels der „Dortmunder Ratingskala zur Erfassung sprachförderrelevanter Interaktionen “ (DO-RESI) von Fried und Briedigkeit (2008) erfasst (siehe dazu Teilprojekt 1). Die Einschätzung erfolgte anhand der Videoaufnahmen. Die Skala besteht aus 12 Items: Kongruenz, Empathisches Zuhören, Anregung, Handlungen verbalisieren, Informationen/Berichte einholen, Lernmöglichkeiten aufzeigen, Vielfalt des Wortschatzes, Grammatisch komplexer Input (keine Fragmentsätze), Offene Fragen, Themen benennen/entdecken, Themen verbinden, Zusammenhänge eines Themas erklären/hinterfragen. Im Teilprojekt 1 wurden acht Indikatoren aus dem Beobachtungsverfahren DO-RESI verwendet, die besonders repräsentativ für sprachliche Anregung sind und zur genaueren Beschreibung der Items dienen: (1) Impuls, (2) Benennungen erarbeiten, (3) Variation des Wortschatzes, (4) Sprachliche Unterstützung -Anbieten von Lückensätzen, (5) Wiederholung unvollständiger Aussagen, (6) Längerer Satz, (7) Aufgreifen einer Kinderäußerung, (8) Zusammenfassung.

Wir gehen dabei von der Grundannahme aus, dass zwischen dem Globalurteil zum Verhalten der Fachkraft und der sprachlichen Anregungsqualität ein positiver Zusammenhang besteht. Um zusätzlich differenzierte Hinweise auf die Art der Zusammenhänge zu erhalten, werden neben dem Globalurteil auch die einzelnen fachkraftbezogenen QUOTS-Skalen in ihren empirischen Bezügen zur sprachlichen Anregungsqualität analysiert. Tabelle 86 referiert die empirischen Korrelationen zwischen dem DO-RESI Gesamtscore (siehe Teilprojekt 1) und sämtlichen fachkraftbezogenen QUOTS-Skalen. 
Tabelle 86. Korrelationen zwischen sprachlicher Anregungsqualität und der Qualität der fachkraftbezogenen Verhaltensweisen (QUOTS-Skalen)

\begin{tabular}{lc}
\hline QUOTS-Skalen (fachkraftbezogen) & $\begin{array}{l}\text { Sprachliche Anregungsqualität } \\
\text { (DO-RESI Gesamtscore) } \\
\text { (fachkraftbezogen) }\end{array}$ \\
\hline Scaffolding-Strategien & $0.38^{\star \star}$ \\
\hline Angemessenheit Scaffolding & 0.28 \\
\hline Manner of Instruction & 0.20 \\
\hline Begrenzung von Freiheitsgraden & 0.14 \\
\hline Transfer of Responsibitiy - Lokus Fachkraft & $0.27^{\star}$ \\
\hline Transfer of Responsibility - Lokus Kind & -0.19 \\
\hline Direction Maintenance & $0.28^{\star}$ \\
\hline Contingency Management & $0.31^{\star}$ \\
\hline Aufdringlichkeit/Übergriffigkeit & 0.15 \\
\hline Unterstützungsverhalten & $0.32^{\star}$ \\
\hline Stimmung Fachkraft & $0.33^{\star}$ \\
\hline Globalurteil Fachkraft & $0.33^{\star}$ \\
\hline Allgemeine Haltung „Lehrer“ & 0.05 \\
\hline Allgemeine Haltung „Möglichmacher“ & $0.30^{\star}$ \\
\hline Allgemeine Haltung „Begleiter“ & $0.35^{\star \star}$ \\
\hline Allgemeine Haltung „Weiteres Kind“ & -0.02 \\
\hline
\end{tabular}

Anmerkung: Die Helligkeit der Felder in der Tabelle markiert das Signifikanzniveau der einzelnen Koeffizienten (weiß = nicht signifikant, hellgrau $/ *=5 \%$ Niveau, dunkelgrau $/$ ** $=1 \%$ Niveau).

Tabelle 86 bestätigt unsere Vermutungen: Es besteht eine signifikante Korrelation positiver Art nicht nur mit dem Globalurteil für das Fachkraft-Verhalten, sondern auch mit einzelnen Subskalen von QUOTS wie etwa Scaffolding-Strategien, Transfer of Responsibility - Lokus Fachkraft, Direction Maintenance, Allgemeine Haltung „Möglichmacher" und Allgemeine Haltung „Begleiter“. In allen genannten Fällen handelt es sich um Qualitätsmerkmale des Fachkraft-Verhaltens, die im Sinne einer guten naturwissenschaftlichen Bildung wünschenswert scheinen. Interessanterweise ergab sich auch eine signifikante Beziehung zwischen der Stimmung der Fachkraft und der sprachlichen Anregungsqualität ihres Verhaltens.

Zusammenfassend liefern die vorliegenden Befunde damit erste Hinweise auf eine systematische Verknüpfung von Bildungssprache und Prozessqualität in naturwissenschaftlichen Bildungsprozessen. Es sei jedoch auch darauf hingewiesen, dass die gefundenen Zusammenhänge nur gering bis mittelhoch ausgefallen sind. Von daher sind die Konstrukte sprachliche Anregungsqualität und Prozessqualität in naturwissenschaftlichen Bildungsprozessen keinesfalls deckungsgleich, sondern ergänzen sich eher und sollten daher nicht unabhängig voneinander gesehen werden. 


\section{Prozessqualität in Interaktionen und naturwissenschaftliche Vorbildung der Fachkräfte}

Die dritte zentrale Fragestellung des vorliegenden Projektes bezieht sich auf einen Vergleich des bildungsbezogenen Verhaltens zwischen Fachkräften, die an naturwissenschaftlichen Fortbildungen teilgenommen haben, mit Fachkräften, die (noch) keine entsprechende Qualifizierung erhalten haben. Um diese Frage zu beantworten, fokussieren wir bei den naturwissenschaftlich fortgebildeten Fachkräften auf die Gruppe der Fachkräfte, die Fortbildungen der Stiftung „Haus der kleinen Forscher“ besucht haben (HdkF-Gruppe). Diese Fachkräfte-Gruppe ist zum einen zahlenmäßig größer und zum anderen homogener bezüglich der besuchten Fortbildungen als die kleine und sehr heterogenere Gruppe an Fachkräften, die an naturwissenschaftlichen Fortbildungen anderer Anbieter teilgenommen haben (Nawi-Gruppe). Nachfolgend wird zunächst dargelegt, worin das Fortbildungskonzept der Stiftung „Haus der kleinen Forscher“ besteht. Es folgen nähere Ausführungen zur Bildung vergleichbarer Stichproben, bevor die Ergebnisse des Gruppenvergleichs vorgestellt werden.

\subsection{Das Fortbildungskonzept der Stiftung „Haus der kleinen Forscher" und bisherige Wirkungsergebnisse}

Im Rahmen von Fortbildungen der Stiftung „Haus der kleinen Forscher“ erweitern die Fachkräfte sukzessive ihre naturwissenschaftliche, aber auch ihre pädagogische Kompetenz. Sowohl in den Einführungsworkshops als auch in weiteren Fortbildungen werden verschiedene pädagogische Schwerpunkte behandelt, z. B. das Thema sprachliche Bildung (Pahnke \& Bartling, 2013). Der Vorteil der kontinuierlichen Begleitung liegt darin, dass die Fachkräfte Gelegenheit erhalten, das Gelernte in den Altag zu transferieren, und zum anderen können sie sich nach einiger Zeit auch wieder mit anderen Fachkräften über die praktische Umsetzung im Alltag auf der nächsten Fortbildung austauschen (Stiftung Haus der kleinen Forscher, 2015b).

Um möglichst viele Fachkräfte mit dem Bildungsangebot zu erreichen, arbeitet die Stiftung mit einem Multiplikatorenmodell, mit dem sie Trainerinnen und Trainer ausbildet, die dann vor Ort kontinuierlich Fachkräfte fortbilden (Stiftung Haus der kleinen Forscher, 2015b). Neben den Präsenz-Fortbildungen stellt die Stiftung den Fachkräften unterstützende E-Learning-Angebote oder auch Arbeitsunterlagen wie Themenbroschüren, Forschungs- und Entdeckungskarten, didakti- 
sche Materialien und Filmbeispiele zur Verfügung, die den Kompetenzerwerb der Fachkräfte unterstützen sollen. Auch die viermal jährlich erscheinende Zeitschrift „Forscht mit!“ gibt den pädagogischen Fachkräften praktische Tipps zum Experimentieren und stellt weitere Informationen zur Stiftungsarbeit bereit (Pahnke \& Bartling, 2013).

Das Verständnis von Bildung in der Stiftung schließt an die aktuelle wissenschaftliche Literatur an und kann als sozial-konstruktivistischer Ansatz verstanden werden. Kind und Umwelt sind beide aktiv am Lernprozess beteiligt (Spindler \& Berwanger, 2011). Dabei sieht die Stiftung, u. a. in Anlehnung an die Kernwissenshypothese, Kinder als kompetente, individuelle Personen an, die nicht nur Fähigkeiten und Vorwissen mitbringen, sondern auch eine Neugierde und ein Interesse an der Welt, die sie umgibt (Stiftung Haus der kleinen Forscher, 2015a). Dieser sozial-konstruktivistische Ansatz bringt aber noch zwei weitere Implikationen mit sich. Auf der einen Seite sollen Lernprozesse im „Haus der kleinen Forscher" kompetenzorientiert gestaltet werden. Ziel der Stiftung ist nicht der Erwerb statischen Wissens, sondern die Entwicklung der lernmethodischen Kompetenzen. Darunter wird „das Nachdenken über das eigene Denken und ein Bewusstsein dafür, dass man lernt, was man lernt und wie man lernt" (Spindler \& Berwanger, 2011, S. 29) gefasst. Dieses nachhaltige Lernen soll die Übertragbarkeit auf andere Situationen ermöglichen. Auf der anderen Seite impliziert der Ansatz ein ko-konstruktives Bildungsverständnis. Die Arbeit der Stiftung fußt damit auf verschiedenen selbst gesetzten Zielen, die sowohl die Ebene der Kinder als auch die der Fachkräfte betrifft. Konkret soll das professionelle Wissen der Fachkräfte erweitert und die Motivation sowie das Interesse an naturwissenschaftlichen Themen gefördert werden, um so Einfluss auf das professionelle Selbstverständnis zu nehmen. Außerdem geht es darum, an den lerntheoretischen Überzeugungen zu arbeiten (Anders et al., 2013).

Dies scheint schon ab der ersten Fortbildung zu gelingen. So sinken laut der Frühjahrsbefragung der Stiftung (Stiftung Haus der kleinen Forscher, 2013b) die Vorbehalte gegenüber den Naturwissenschaften deutlich, während das Interesse an entsprechenden Themen steigt. Diese Effekte werden mit der Dauer der Teilnahme an Fortbildungen des „Hauses der kleinen Forscher“ noch größer (Stiftung Haus der kleinen Forscher, 2013b). Auch geben die Fachkräfte an, dass die wichtigsten Gründe für die Fortbildungsmotivation die „Freude am gemeinsamen Forschen mit den Kindern“ sowie die Freude „am eigenen naturwissenschaftlichen Entdecken und Forschen" sind (Stiftung Haus der kleinen Forscher, 2015b, S. 43). Die Freude sowie die Motivation zum Forschen spiegeln sich auch in der Häufigkeit des gemeinsamen Experimentierens wider. Im Durchschnitt wird pro Kita 3.2 Stunden wöchentlich geforscht (Stiftung Haus der kleinen Forscher, 2015b). In zertifizierten Kitas, also Einrichtungen, die auf die Erfüllung der Quali- 
tätsstandards der Stiftung hin geprüft wurden (Stiftung Haus der kleinen Forscher, 2013a), ist der Umfang sogar noch größer. Dort wird dem Forschen pro Woche circa eine Stunde mehr Zeit gewidmet (Stiftung Haus der kleinen Forscher, 2015b).

Ein weiteres wichtiges Ziel der Stiftung ist es, das professionelle Wissen der Fachkräfte zu erweitern. Hierunter fällt die Förderung des domänenübergreifenden prozeduralen Wissens (Stiftung Haus der kleinen Forscher, 2013a). Im Jahr 2011 hat deshalb die Methode „Forschungskreis“ Einzug in die Fortbildungen sowie in die verschiedenen Materialien erhalten, die den Fachkräften zur Verfügung gestellt werden (Stiftung Haus der kleinen Forscher, 2013a). Der Forschungskreis bietet eine Orientierung für die Fachkräfte, wie sie gemeinsam mit den Kindern forschen können (Stiftung Haus der kleinen Forscher, 2015a), und ähnelt der Konzeption des prozeduralen Wissens nach Klahr und Dunbar (1988).

Auch das domänenspezifische Fachwissen soll vergrößert werden, damit die Fachkräfte die Kinder gut bei der Auseinandersetzung mit naturwissenschaftlichen Themen unterstützen können und sich selbst sicherer fühlen (Stiftung Haus der kleinen Forscher, 2013a). Dies erfolgt natürlich durch die Fortbildungen, aber auch durch die thematisch passenden Kartensets mit Forschungs- und Entdeckungskarten, die die Fachkräfte erhalten. Denn auf den Entdeckungskarten wird auch immer fachliches Hintergrundwissen in der Rubrik „Wissenswertes für interessierte Erwachsene“ dargestellt.

Die Erweiterung der pädagogischen Handlungsstrategien ist der Stiftung ebenfalls ein wichtiges Anliegen (Stiftung Haus der kleinen Forscher, 2013a). Dabei geht es zum einen um die Aneignung pädagogisch-psychologischem Wissens als auch um die Vergrößerung des fachdidaktischen Wissens. Den Fachkräften sollen z. B. typische Kindervorstellungen hinsichtlich naturwissenschaftlicher, mathematischer und technischer Phänomene nähergebracht werden (Stiftung Haus der kleinen Forscher, 2013a). Zusätzlich sollen innen konkrete Handlungsstrategien wie das Scaffolding an die Hand gegeben werden, mittels derer sie die Kinder optimal unterstützen und mit ihnen ko-konstruktiv forschen können (Stiftung Haus der kleinen Forscher, 2013a). Wie diese Strategien dann in den Alltag transferiert werden können, wird in den Reflexionsphasen der Fortbildung diskutiert (Stiftung Haus der kleinen Forscher, 2013a).

Des Weiteren versucht die Stiftung, die epistemologischen Überzeugungen der pädagogischen Fachkräfte weg von einer statischen Perspektive, die eher zu einem transmissiven Unterrichtsstil führt, in eine konstruktivistische Richtung zu verändern (Anders et al., 2013). Zusätzlich sollen die Fachkräfte ein Gespür dafür bekommen, was eine entwicklungsangemessene Förderung naturwissenschaftlicher Kompetenzen beinhaltet, und die Bildung in dieser Wissensdomäne soll einen angemessenen Stellenwert im Elementarbereich erhalten (Anders et al., 2013). 
Noch vor einigen Jahren zeigte sich, dass die pädagogischen Leitlinien vor allem hinsichtlich der ko-konstruktiven Gestaltung von Bildungsprozessen und der Förderung der lernmethodischen Kompetenz nur mangelhaft in den pädagogischen Einrichtungen umgesetzt wurden. Zwar konnten sich in der Frühjahrsbefragung 2010 (Stiftung Haus der kleinen Forscher, 2010) 40 Prozent der Fachkräfte mit einem ko-konstruktiven Ansatz identifizieren, nichtsdestotrotz zeigte mehr als die Hälfte aller Fachkräfte zu Beginn auch Sympathien für einen instruktionsorientierten Ansatz. In der qualitativen Studie von Spindler und Berwanger (2011), bei der u.a. in vier Kitas die Durchführung von Experimenten beobachtet wurde, zeigte sich, dass weniger als die Hälfte der Experimente ko-konstruktiv angelegt war. Im Gegenteil lag der Fokus noch stark auf dem Erwerb von Faktenwissen, und nur in zwei Experimenten wurde die lernmethodische Kompetenz gefördert. Interviews ergaben, dass keine der Fachkräfte ihre pädagogische Haltung durch die Fortbildungen im „Haus der kleinen Forscher“ reflektiert hatte und für viele Fachkräfte Ko-Konstruktion überhaupt kein Begriff war. Dies ist nicht verwunderlich, wenn man die Studie von Evanschitzky (2011) betrachtet. Die Autorin fand heraus, dass die Themen Ko-Konstruktion und Metakognition zum Teil gar nicht in den Fortbildungen für die Fachkräfte behandelt oder nur am Rand erwähnt wurden. Es bestand keine Möglichkeit für die Fachkräfte, sich reflexiv mit den Leitlinien auseinanderzusetzen.

Als Reaktion auf die genannten Expertisen und Untersuchungen setzte die Stiftung „Haus der kleinen Forscher“ einige konzeptionelle Veränderungen um. Konstruktivistische Elemente sowie das kompetenzorientierte Lernen bekamen mehr Raum. Der Ansatz wurde zusätzlich um das Prinzip Scaffolding erweitert (Stiftung Haus der kleinen Forscher, 2011). Das Thema „Scaffolding“ ist seitdem fest im Fortbildungskonzept der Stiftung verankert. Aus diesem Grund ist anzunehmen, dass Fachkräfte, die eine Fortbildung der Stiftung „Haus der kleinen Forscher" besucht haben, auch häufiger Scaffolding-Strategien anwenden als Fachkräfte, denen entsprechendes Know-how nicht explizit vermittelt wurde. Durch die Vergrößerung des domänenübergreifenden prozeduralen Wissens sollten die Fachkräfte eher in der Lage sein, systematisch beim Forschen vorzugehen, selbst Fragen zu stellen, Beobachtungen zu verbalisieren und Erklärungen anzubieten (Stiftung Haus der kleinen Forscher, 2015a). Das vertiefte bereichsspezifische Wissen sollte sie zudem in die Lage versetzen, die Kinder mittels geeigneter Hinweise und Anleitungen adäquat zu unterstützen (Stiftung Haus der kleinen Forscher, 2013a). Werden Kinder als kompetente und aktiv lernende Individuen wahrgenommen, so liegt es nahe, ihr Wissen im Forschungsprozess zu berücksichtigen und sie zu einer aktiven Partizipation zu ermutigen. Gleichzeitig sollte auch die Fachkraft eigene Fragen, Vermutungen, Beobachtungen und Erklärungen mit in den Forschungsprozess einbringen (Stiftung Haus der kleinen 
Forscher, 2015a). In der von der Stiftung 2014 durchgeführten Frühjahrsbefragung (Stiftung Haus der kleinen Forscher, 2014) gaben fast alle Fachkräfte an, die Kinder beim gemeinsamen Experimentieren zu ermutigen, ihre Vermutungen und Erwartungen zu verbalisieren. In den meisten Einrichtungen orientiere sich der Forschungsprozess an den Interessen sowie dem Vorwissen der Kinder. Überdies gaben die Fachkräfte an, dass sie Scaffolding anwenden und sich dabei besonders kompetent fühlen. Speziell bei der Erhebung von Vorwissen, dem Sprechen über Beobachtungen sowie der Anregung zu eigenständigem Forschen fühlen sich die Fachkräfte offensichtlich sehr sicher.

Bislang liegt noch keine aktuelle Studie vor, die, basierend auf systematischen Beobachtungen von Forschungssituationen, die Umsetzung des pädagogischen Ansatzes der Stiftung nach dieser konzeptionellen Erweiterung überprüft hat.

Befragungsdaten deuten darauf hin, dass die Fachkräfte den Forschungsprozess mit den Kindern nun stärker ko-konstruktiv gestalten (Stiftung Haus der kleinen Forscher, 2014). Anhand einer beispielhaften Forschungsaktivität, die die Fachkräfte beschreiben sollten, wurde die Prozessqualität gemessen. Die Auswertung ergab, dass fast alle Fachkräfte ko-konstruktiv, z. B. durch den Einbezug des Vorwissens der Kinder, vorgehen (Stiftung Haus der kleinen Forscher, 2014). Der Fokus auf die Metakognition und die Entwicklung lernmethodischer Kompetenz scheint allerdings in zertifizierten Kitas noch einen deutlich höheren Stellenwert zu haben als in nicht zertifizierten Kitas (Stiftung Haus der kleinen Forscher, 2014). So dokumentierten nur ungefähr die Hälfte der Fachkräfte aus nicht zertifizierten Kitas, im Vergleich zu rund 70 \% der Fachkräfte aus zertifizierten Kitas, den Forschungsprozess und nutzten diesen zur Reflexion des Erkenntnisgewinns (Stiftung Haus der kleinen Forscher, 2014).

Zudem hat sich die Stiftung das Ziel gesetzt, die Selbstwirksamkeitserwartung der Fachkräfte im Hinblick auf die Tätigkeit als Lernbegleitung der Kinder zu verbessern (Stiftung Haus der kleinen Forscher, 2013a). Der Wissenszuwachs und das regelmäßige Forschen sollten sich auch positiv auf die Selbstwirksamkeitserwartung auswirken. Dies spiegelt sich in den Frühjahrsbefragungen wider. Die Fachkräfte fühlen sich im Hinblick auf das gemeinsame Forschen mit den Kindern kompetent (Stiftung Haus der kleinen Forscher, 2015b) und führen diese Kompetenz vor allem auf die Fortbildungen der Stiftung zurück und weniger auf andere Fortbildungen oder die eigene Ausbildung (Stiftung Haus der kleinen Forscher, 2013b). Das Kompetenzerleben steigt mit zunehmender Anzahl besuchter Fortbildungen sowie mit der Zertifizierung der eigenen Einrichtung (Stiftung Haus der kleinen Forscher, 2013b). Die Fachkräfte geben an, dass sie sich kompetent fühlen, wenn sie mit den Kindern über deren Beobachtungen sprechen, ihr Vorwissen erfragen, sie zu selbstständigem Forschen ermutigen und auf ihre Forschungsfragen und Themenwünsche eingehen (Stiftung Haus der kleinen Forscher, 2015b). 
Die Fachkräfte fühlen sich also besonders sicher in dem Bereich, der der Stiftung ein großes Anliegen ist: dem ko-konstruktiven Forschen.

Die Monitoring-Ergebnisse der Stiftung deuten also darauf hin, dass die Ziele der Stiftung erfüllt werden und die pädagogische Leitlinie in den Kindertageseinrichtungen umgesetzt wird (Stiftung Haus der kleinen Forscher, 2013b; Stiftung Haus der kleinen Forscher, 2014; Stiftung Haus der kleinen Forscher, 2015b). Die vorliegende Studie untersuchte nun, ob sich die Ziele und das pädagogische Konzept der Stiftung auch im Verhalten der Fachkräfte nachweisen lassen.

\subsection{Stichproben von Fachkräften mit und ohne Fortbildungserfahrung im naturwissenschaftlichen Bereich}

Konkret wurde analysiert, ob es systematische Unterschiede im pädagogischen Verhalten zwischen Fachkräften gibt, die in Kitas mit einer Zertifizierung als „Haus der kleinen Forscher" arbeiten und selbst bereits entsprechende Fortbildungen besucht haben (HdkF-Gruppe), und solchen, die bislang noch keinerlei Fortbildung im naturwissenschaftlichen Bereich besucht haben und deren Einrichtung auch noch keinen Kontakt zum „Haus der kleinen Forscher“ hat (Vergleichsgruppe).

Eine ausführliche Beschreibung der Ausgangsstichprobe findet sich in Teilprojekt 1. Daraus geht hervor, dass sehr viel mehr pädagogische Fachkräfte aus Kitas mit einer Zertifizierung als „Haus der kleinen Forscher“ in die Untersuchung einbezogen wurden als Fachkräfte ohne entsprechende Fortbildungen. Zudem unterscheiden sich die Gruppen deutlich hinsichtlich Alterszusammensetzung, Berufserfahrung und höchstem Bildungsabschluss. Solche Unterschiede können möglicherweise zu einer Verzerrung der Ergebnisse von Gruppenvergleichen führen. Nachfolgend stellen wir daher zunächst bedeutsame Unterschiede der Ausgangsstichprobe dar und berichten anschließend über ein Matching-Verfahren, mit dem wir von der größeren Anzahl der Fachkräfte aus HdkF-zertifizierten Kitas eine Teilgruppe auswählen, die den Fachkräften ohne Vorkenntnisse hinsichtlich potenziell bedeutsamer Variablen weitgehend vergleichbar scheint.

Ausgangspunkt für das Matching war die zahlenmäßig kleinere Vergleichsgruppe mit Fachkräften ohne Nawi-Fortbildung $\left(n=19^{20}\right)$. Es wurde das Ziel verfolgt, für jede Fachkraft der Vergleichsgruppe einen statistischen Zwilling in der HdkF-Gruppe zu suchen. Das Vorgehen bei der Selektion sah wie folgt aus: Im

20 Im Vergleich zu Teilprojekt 1 ( $n=18$ Fachkräfte ohne Nawi-Fortbildung), in dem die Auswertungen zeitlich früher erfolgten, gibt es in diesem Studienteil $(n=19)$ eine Fachkraft mehr ohne naturwissenschaftliche Fortbildung, deren Video später kodiert und in die vorliegenden Auswertungen mit einbezogen wurde. 
ersten Schritt wurden statistische Zwillinge gesucht, die in allen drei Kriterien (Alter, Berufserfahrung, Bildungsabschluss) übereinstimmten. Gab es zu viele Fachkräfte aus der HdkF-Gruppe, die passende Werte für Fachkräfte aus der Vergleichsgruppe erreichten, wurde der Erhebungsort (Karlsruhe oder Heidelberg) als weiteres Kriterium mit in das Matching einbezogen. Existierten dann immer noch zu viele potenzielle statistische Zwillinge, wurde eine Fachkraft per Zufall ausgewählt. Für den Fall, dass es zu viele Fachkräfte aus der Vergleichsgruppe gab, die passende Werte für eine Versuchsperson in der HdkF-Gruppe hatten, wurde auch hier der Erhebungsort mitberücksichtigt und, sofern dann immer noch notwendig, eine Versuchsperson per Zufall aus der Gruppe der potenziellen Zwillinge gezogen. Für neun Versuchspersonen wurde ein statistischer Zwilling gefunden, der hinsichtlich der drei Kriterien Alter, Berufserfahrung und Bildungsabschluss (zum Teil auch Erhebungsort) sehr ähnlich war (siehe Tabellen 87-90). Für die verbliebenen Fachkräfte wurden statistische Zwillinge gesucht, mit denen sie in mindestens einem dieser Kriterien übereinstimmten bzw. denen sie im Hinblick auf das Alter und die Berufserfahrung so ähnlich wie möglich waren.

Die so gebildete Stichprobe setzte sich aus $N=38$ Personen zusammen, davon $n=19$ Fachkräfte pro Gruppe. Tabelle 87 zeigt die Altersverteilung, Tabelle 88 die Verteilung der Berufserfahrung und Tabelle 89 die Verteilung der höchsten Bildungsabschlüsse der Fachkräfte im Vergleich zwischen den beiden gematchten Gruppen.

Tabelle 87. Altersverteilung der zwei Fachkräfte-Gruppen mit unterschiedlichem Fortbildungshintergrund (HdkF-Fortbildungen, ohne Nawi-Fortbildungen) nach dem Matching; Angaben in absoluten und prozentualen Häufigkeiten

\begin{tabular}{lccccccc}
\hline \multicolumn{7}{c}{ Alterskategorien [Jahre] } \\
\hline $\begin{array}{l}\text { Gruppe/ } \\
\text { Fortbildung }\end{array}$ & $20-24$ & $25-29$ & $30-34$ & $35-39$ & $40+$ & $\begin{array}{c}\text { Keine } \\
\text { Angabe }\end{array}$ & Gesamt \\
\hline HdkF & 2 & 4 & 2 & 2 & 7 & 2 & 19 \\
& $(10.5 \%)$ & $(21.1 \%)$ & $(10.5 \%)$ & $(10.5 \%)$ & $(36.8 \%)$ & $(10.5)$ & $(100 \%)$ \\
ohne Nawi & 2 & 5 & 2 & 1 & 5 & 4 & 19 \\
& $(10.5 \%)$ & $(26.3 \%)$ & $(10.5 \%)$ & $(5.3 \%)$ & $(26.3 \%)$ & $(21.1 \%)$ & $(100 \%)$ \\
Gesamt & 4 & 9 & 4 & 3 & 12 & 6 & 38 \\
& $(10.5 \%)$ & $(23.9 \%)$ & $(10.5 \%)$ & $(7.9 \%)$ & $(31.6 \%)$ & $(15.8 \%)$ & $(100 \%)$ \\
\hline
\end{tabular}


Tabelle 88. Verteilung der Berufserfahrung der zwei Fachkräfte-Gruppen mit unterschiedlichem Fortbildungshintergrund (HdkF-Fortbildungen, ohne NawiFortbildungen) nach dem Matching; Angaben in absoluten und prozentualen Häufigkeiten

\begin{tabular}{lccccccc}
\hline \multicolumn{7}{c}{ Berufserfahrung [Jahre] } \\
\hline $\begin{array}{l}\text { Gruppe } \\
\text { Fortbildung }\end{array}$ & $<5$ & $5-9$ & $10-14$ & $15-19$ & $20+$ & $\begin{array}{c}\text { Keine } \\
\text { Angabe }\end{array}$ & Gesamt \\
\hline HdkF & 4 & 4 & 2 & 2 & 5 & 2 & 19 \\
& $(21.1 \%)$ & $(21.1 \%)$ & $(10.5 \%)$ & $(10.5 \%)$ & $(26.3 \%)$ & $(10.5 \%)$ & $(100 \%)$ \\
ohne & 5 & 3 & 0 & 6 & 1 & 4 & 19 \\
& $(26.3 \%)$ & $(15.8 \%)$ & $(0.0 \%)$ & $(31.6 \%)$ & $(5.3 \%)$ & $(21.1 \%)$ & $(100 \%)$ \\
Gesamt & 9 & 7 & 2 & 8 & 6. & 6 & 38 \\
& $(23.7 \%)$ & $(18.4 \%)$ & $00(5.3 \%)$ & $(21.1 \%)$ & $(15.8 \%)$ & $(15.8 \%)$ & $(100 \%)$ \\
\hline
\end{tabular}

Tabelle 89. Vergleich der höchsten Bildungsabschlüsse von zwei Fachkräfte-Gruppen mit unterschiedlichem Fortbildungshintergrund (HdkF-Fortbildungen, ohne NawiFortbildungen) nach dem Matching; Angaben in absoluten und prozentualen Häufigkeiten

\begin{tabular}{lccccc}
\hline & \multicolumn{4}{c}{ Höchster Bildungsabschluss } \\
\hline $\begin{array}{l}\text { Gruppe/ } \\
\text { Fortbildung }\end{array}$ & Fachschule & Studium & Sonstiges & Keine Angabe & Gesamt \\
\hline HdkF & $16(84.2 \%)$ & $1(5.3 \%)$ & $0(0.0 \%)$ & $2(10.5 \%)$ & $19(100 \%)$ \\
ohne & $10(52.6 \%)$ & $4(21.1 \%)$ & $2(10.5 \%)$ & $3(15.8 \%)$ & $19(100 \%)$ \\
Gesamt & $26(68.4 \%)$ & $5(13.2 \%)$ & $2(5.3 \%)$ & $5(13.2 \%)$ & $38(100 \%)$ \\
\hline
\end{tabular}

Um den Erfolg des Matchings zu überprüfen, wurde die Verteilung der Variablen zwischen den Gruppen mittels des exakten Tests nach Fisher verglichen. Es ergaben sich keine signifikanten Gruppenunterschiede hinsichtlich des Alters ( $p=.94)$, der Berufserfahrung $(p=.21)$ und der Bildungsabschlüsse $(p=.18)$. Das Matching war demnach erfolgreich und die beiden Gruppen sind hinsichtlich Alter, Berufserfahrung, Bildungsabschluss und Geschlecht (alle weiblich) vergleichbar.

Lassen sich nun Gruppenunterschiede zwischen Fachkräften mit und ohne Fortbildung im naturwissenschaftlichen Bereich nachweisen, wäre dies ein Indiz für die Wirksamkeit des Treatments (Fortbildung durch das HdkF). Wichtig erscheint in diesem Zusammenhang der Hinweis, dass die Videokodierung im Rahmen der vorliegenden Studie „blind“ erfolgte. Die Kodiererinnen wussten also nicht, welche Vorbildung eine konkrete Fachkraft hatte, die auf einem Video beurteilt wurde und ob sie zuvor an Fortbildungen der Initiative „Haus der kleinen Forscher" teilgenommen hatte oder nicht. 


\subsection{Vergleich der QUOTS-Werte für Fachkräfte mit und ohne Fortbildungserfahrung im naturwissenschaftlichen Bereich}

Tabelle 90 bezieht sich auf die beiden gematchten Gruppen (HdkF-Gruppe, Vergleichsgruppe ohne naturwissenschaftliche Vorbildung) sowie die Gruppe mit einem anderen naturwissenschaftlichen Schwerpunkt (Nawi-Gruppe) und referiert die Mittelwerte und Standardabweichungen für sämtliche QUOTS-Skalen im deskriptiven Vergleich zwischen Fachkräften aller drei untersuchten Gruppen.

Es zeigt sich, dass Fachkräfte, die eine Fortbildung der Initiative „Haus der kleinen Forscher“ besucht hatten, auf fast allen Skalen numerisch höhere Mittelwerte aufweisen als die Vergleichsgruppe ohne naturwissenschaftliche Vorbildung. Dies gilt für die Skala Scaffolding-Strategie sowie für Angemessenheit von Scaffolding-Strategien. Weiter zeigten die Fachkräfte der HdkF-Gruppe höhere Werte bezüglich Dimensionen, die mit Scaffolding-Zielen in Verbindung stehen. Dies betrifft die Dimensionen Manner of Instruction, Begrenzung von Freiheitsgraden, Tranfer of Responsibility - Lokus Fachkraft, Direction Maintenance, Contingency Management, Unterstützungsverhalten der Fachkraft, Stimmung und Globalurteil Fachkraft sowie Allgemeine Haltung „Lehrer“, Allgemeine Haltung „Möglichmacher“ und Allgemeine Haltung „Begleiter“. Gleiches gilt zwar auch für die Skala Aufdringlichkeit/Übergriffigkeit, doch sind die entsprechenden Mittelwerte insgesamt sehr niedrig und daher nicht überzubewerten.

Umgekehrt verhielt es sich im Hinblick auf die Skalen Transfer of Responsibility - Lokus Kind sowie Allgemeine Haltung Kind. Hier waren die Werte der Fachkräfte ohne naturwissenschaftliche Fortbildung numerisch höher als die der HdkF-fortgebildeten Fachkräfte.

Insgesamt ergibt sich damit ein stimmiges Ergebnismuster, das positive Auswirkungen von Fortbildungen auf das Fachkraft-Verhalten andeutet. Schulungen scheinen dazu zu führen, dass die Fachkräfte im Kontakt mit den Kindern Verantwortung für die Gestaltung der Bildungssituation übernehmen und sich aktiver, engagierter sowie kompetenter verhalten. Die leichte Tendenz, sich eher zu stark zu engagieren (siehe Mittelwert der Skala Aufdringlichkeit/ Übergriffigkeit) ist vermutlich der Situation geschuldet, dass das Verhalten der Fachkräfte aufgezeichnet wurde und somit bei einzelnen Personen das Gefühl einer Prüfungssituation entstanden sein könnte.

Die Daten zu Fachkräften mit einer anderen naturwissenschaftlichen Vorbildung werden an dieser Stelle nicht näher interpretiert, weil die Stichprobe heterogen und ihre Größe mit $n=8$ eher gering ist. Die vorliegenden Werte für diese 
Gruppe scheinen denen der Vergleichsgruppe jedoch eher vergleichbar zu sein als gegenüber denen der HdkF-Gruppe.

Tabelle 9o. Inhalte, Mittelwerte (M) und Standardabweichungen (SD) einzelner QUOTSSkalen für Fachkräfte aus der HdkF-Gruppe, der Vergleichsgruppe von Fachkräften ohne naturwissenschaftliche Fortbildung sowie der Nawi-Gruppe von Fachkräften mit anderen naturwissenschaftlichen Fortbildungen

\begin{tabular}{lcccccc}
\hline & \multicolumn{7}{c}{ Gruppe von Fachkräften } \\
\hline & $\begin{array}{c}\text { HdkF-Gruppe } \\
(\mathrm{n}=19)\end{array}$ & $\begin{array}{c}\text { Vergleichs- } \\
\text { gruppe } \\
(\mathrm{n}=19)\end{array}$ & $\begin{array}{c}\text { Nawi-Gruppe } \\
(\mathrm{n}=9)\end{array}$ \\
& \multicolumn{7}{c}{ S } & SD & M & SD & M & SD \\
\hline Scaffolding-Strategien Initiative Fachkraft & 2.42 & 0.51 & 2.10 & 0.52 & 1.87 & 0.67 \\
Scaffolding-Strategien Initiative Kind & 1.87 & 0.55 & 1.41 & 0.46 & 1.32 & 0.64 \\
Scaffolding-Strategien & 2.12 & 0.47 & 1.72 & 0.46 & 1.57 & 0.63 \\
Angemessenheit Scaffolding-Strategien & 1.47 & 0.51 & 1.31 & 0.58 & 1.25 & 0.75 \\
Manner of Instruction & 2.00 & 0.43 & 1.72 & 0.31 & 1.90 & 0.68 \\
Begrenzung von Freiheitsgraden & 2.06 & 0.49 & 1.61 & 0.50 & 1.60 & 0.47 \\
Transfer of Responsibility LF & 2.13 & 0.86 & 1.72 & 0.63 & 1.75 & 1.05 \\
Transfer of Responsibility LK & 2.13 & 0.58 & 2.41 & 0.60 & 2.47 & 0.76 \\
Transfer of Responsibility & 2.12 & 0.20 & 2.06 & 0.23 & 2.06 & 0.34 \\
Direction Maintenance & 2.60 & 0.60 & 2.35 & 0.49 & 2.34 & 0.79 \\
Contingency Management & 2.09 & 0.43 & 2.01 & 0.32 & 2.02 & 0.55 \\
\hline Aufdringlichkeit/Übergriffigkeit & 1.10 & 0.68 & 0.80 & 0.40 & 0.96 & 0.39 \\
Unterstützungsverhalten der FK & 3.32 & 1.10 & 2.92 & 0.90 & 2.87 & 1.32 \\
Stimmung Fachkraft & 0.75 & 0.43 & 0.58 & 0.46 & 0.41 & 0.33 \\
Globalurteil Verhalten der FK & 3.44 & 0.88 & 3.15 & 0.74 & 3.12 & 1.35 \\
Allgemeine Haltung „Lehrer“ & 2.36 & 0.89 & 1.94 & 0.68 & 2.12 & 1.06 \\
Allgemeine Haltung „Möglichmacher“ & 2.18 & 0.50 & 2.02 & 0.48 & 1.87 & 0.51 \\
Allgemeine Haltung „Begleiter“ & 2.55 & 0.59 & 2.42 & 0.55 & 2.43 & 0.67 \\
Allgemeine Haltung „Weiteres Kind“ & 0.94 & 0.76 & 1.26 & 0.51 & 1.18 & 1.09 \\
\hline & & & & & & \\
\hline & & & & & &
\end{tabular}

Um die beobachteten Gruppenunterschiede hinsichtlich ihrer statistischen Bedeutsamkeit zu überprüfen, wurden alle Items der QUOTS-Skalen, die inhaltlich mit Scaffolding-Verhalten zusammenhängen, signifikant positiv untereinander korrelieren und für die zudem eine positive Assoziation mit dem Erkenntnisgewinn der Kinder besteht, zu einem QUOTS-Scaffolding-Gesamtscore zusammengefasst und zwischen den gematchten Gruppen verglichen. Auf Einzelvergleiche wurde angesichts der geringen Stichprobengröße und der hohen Anzahl von Tests, die das Alpha-Risiko falsch-positiver Ergebnisse erhöhen, verzichtet. 


\subsection{Einfluss der Fortbildungen der Initiative „Haus der kleinen Forscher" auf das Scaffolding-Verhalten}

Insgesamt sprechen unterschiedliche Indizien bisheriger Wirkungsmessungen, die auf Befragungen der Fachkräfte basieren, dafür, dass die Anwendung von Scaffolding durch das Fortbildungsprogramm der Stiftung „Haus der kleinen Forscher" systematisch gefördert wird und entsprechende Anregungen von den pädagogischen Fachkräften gut umgesetzt werden können (siehe Abschnitt 7.1). Inwiefern diese Einschätzung sich auch durch Fremdbeurteilungen bestätigen lässt, wurde im Rahmen der vorliegenden Studie geprüft. Ausgehend von entsprechenden Vorbefunden erwarten wir, dass Teilnehmende am Fortbildungsangebot der Stiftung „Haus der kleinen Forscher“ häufiger Scaffolding in seinen unterschiedlichen Aspekten zeigen als pädagogische Fachkräfte ohne entsprechende Fortbildung.

Konkret sollten sie höhere Werte in Items der Skalen Scaffolding-Strategien, Manner of Instruction, Transfer of Responsibility - Lokus Fachkraft, sowie Direction Maintenance erzielen, die zu einer neuen QUOTS-Skala Scaffolding-Gesamt zusammengefasst wurden. Die fehlende Berücksichtigung anderer Skalen erklärt sich wie folgt: Angemessenheit von Scaffolding wurde ausgeschlossen, weil die Skalierung dieser Skala das Optimum bei einem Wert von 0 vorsieht und diese Variable folglich nicht unmittelbar mit den anderen Skalen vergleichbar ist. Begrenzung von Freiheitsgraden wurde ausgeschlossen, weil diese Skala nicht mit dem Erkenntnisprozess der Kinder korrelierte; zudem ist sie nicht spezifisch für Scaffolding, sondern markiert allgemein, wie sehr die Fachkraft auf die Einhaltung bestimmter Rahmenbedingungen beim Experimentieren achtet. Transfer of Responsibility - Lokus Kind korreliert negativ mit den übrigen Scaffolding-Skalen sowie den gruppen- und kindbezogenen Skalen. Das Gleiche gilt für Aufdringlichkeit/Übergriffigkeit. Die Stimmung der Fachkraft wies nur wenige bedeutsame Zusammenhänge mit anderen Skalen auf. Schließlich wurden das Globalurteil und die unterschiedlichen Rollenbilder nicht in den Summenscore einbezogen, weil es sich in beiden genannten Fällen um bewertende integrative Einschätzungen handelt und nicht um Skalen mit verhaltensnah formulierten Items.

Der anhand der übrigen Items gebildete QUOTS-Scaffolding-Gesamtscore, in den Skalen mit mehr Items automatisch mit höherer Gewichtung eingehen als Skalen mit weniger Items, lag für die Fachkräfte der HdkF-Gruppe bei $M=2.44$ $(S D=0.64)$ und für die Fachkräfte der Vergleichsgruppe bei $M=2.09$ ( $S D=0.49)$. Dieser Mittelwertunterschied fiel signifikant aus, $t(36)=0.36, p=.04$ (einseitig getestet). Die Ausgangshypothese konnte damit bestätigt werden. Wir halten fest, dass jene Fachkräfte, die Fortbildungen vom „Haus der kleinen Forscher“ besucht 
haben, höhere Bewertungen ihres Scaffolding-Verhaltens erhielten als Fachkräfte ohne Nawi-Fortbildungen, wobei die Stichproben hinsichtlich potenziell kritischer Variablen wie Alter, Ausbildung und Berufserfahrung vergleichbar waren und die Bewertung durch zwei unabhängige fachlich geschulte Kodiererinnen erfolgte, die keine Kenntnis von der Gruppenzugehörigkeit der Fachkräfte hatten. 


\section{Diskussion}

Im Rahmen des vorliegenden Projektes kam erstmals ein neu entwickeltes Verfahren zur Anwendung, mit dem das pädagogische Verhalten von Fachkräften in naturwissenschaftlichen Experimentiersituationen anhand einer Fremdbeurteilung erfasst werden kann. Dieses Verfahren wurde in Zusammenarbeit mit den Bildungswissenschaftlern Prof. Silke Hertel und Kim Gärtner sowie der Projektmitarbeiterin Megan Bock entwickelt und auf die Zielsetzung des vorliegenden Projektes angepasst. In der hier beschriebenen Version nennt es sich QUOTS (Quality of Teaching Science) und dient der Überprüfung dreier zentraler Fragestellungen (siehe Kapitel 2). Nachfolgend werden die wichtigsten Befunde zu diesen drei Fragestellungen zusammengefasst und diskutiert.

\subsection{Prozessqualität in Interaktionen und kindliche Kompetenzen}

Wie sich zeigte, ist es möglich, mithilfe von QUOTS unterschiedliche Dimensionen von Verhalten zu erfassen, die mit einer mehr oder weniger positiven ko-konstruktiven Haltung der Fachkraft und mit einer systematischen Unterstützung des kindlichen Erkenntnisprozesses durch aktive Gestaltung von Interaktionen mit Kindern beim Experimentieren einhergehen. Bei der Betrachtung von Einzelergebnissen sind vor allem folgende Befunde bemerkenswert:

- Trotz inhaltlicher Differenzierbarkeit von Scaffolding-Strategien, Angemessenheit von Scaffolding, Manner of Instruction, Transfer of Responsibility, Direction Maintenance, Contingency Management und Unterstützungsverhalten sind diese Dimensionen empirisch positiv korreliert und scheinen damit eine generelle pädagogische Haltung widerzuspiegeln.

- Verhalten, bei dem die Fachkraft primär den Kindern die Verantwortung für das Experimentierverhalten überlässt (Transfer of Responsiblity - Lokus Kind) oder sich wie ein „weiteres Kind“ verhält (Allgemeine Haltung weiteres Kind) sind negativ mit Scaffolding-Verhalten der Fachkraft, mit der Arbeits- und Forscherhaltung der Gruppe und mit dem Erkenntnisgewinn der Kinder korreliert. Gleiches gilt für Verhalten, bei dem sich die Fachkräfte zu stark engagieren (Aufdringlichkeit/Übergriffigkeit). Damit wird deutlich, dass es für die Fachkraft vor allem darauf ankommt, die richtige Balance im Eigenengagement bei der Gestaltung des Forschungsprozesses mit den Kindern zu finden. Dies bestätigt die Relevanz einer ko-konstruktiven Haltung der Fachkraft. 
- Die Stimmung der am Forschungsprozess beteiligten Personen scheint empirisch kaum mit dem kognitiv orientierten Scaffolding-Verhalten der Fachkraft oder mit dem Erkenntnisgewinn der Kinder zusammenzuhängen. Folglich ist die kognitive Dimension einer Optimierung von Prozessqualität klar von der emotionalen Dimension zu differenzieren.

- Die meisten mit Scaffolding verbundenen Skalen sind systematisch mit der Arbeits- und Forscherhaltung der Gruppe sowie mit dem Unterstützungsbedarf und Erkenntnisgewinn der Kinder assoziiert. Dies kann als ein wichtiger Hinweis auf die hohe Bedeutung der Prozessqualität bildungsbezogener Aktivitäten der Fachkräfte bei der Unterstützung der Entwicklung naturwissenschaftlicher Kompetenzen gewertet werden.

Fragestellung 1 kann folglich positiv beantwortet werden: Parameter der naturwissenschaftsbezogenen Prozessqualität aufseiten der Fachkraft korrelieren positiv mit Kompetenzmerkmalen der Kinder im naturwissenschaftlichen Denken und ihrem Erkenntnisprozess.

\subsection{Prozessqualität in Interaktionen und sprachliche Anregungsqualität}

Unsere Analysen bezüglich der Zusammenhänge zwischen der sprachlichen Anregungsqualität der Fachkraft und der naturwissenschaftsbezogenen Prozessqualität hat ergeben, dass verschiedene Dimensionen naturwissenschaftsbezogener Interaktionsqualität (Scaffolding-Strategien, Transfer of Responsibility - Lokus Fachkraft, Direction Maintenance, Contingency Management, Unterstützungsverhalten, Stimmung der Fachkraft, das Globalurteil, die Allgemeine Haltung als „Möglichmacher" und die Allgemeine Haltung als „Begleiter") positiv mit der sprachlichen Anregungsqualität korrelieren. Dies spricht für eine enge Verzahnung des sprachlichen Verhaltens mit der Qualität des pädagogischen Vorgehens von Fachkräften in naturwissenschaftlichen Forschungssituationen. Angesichts der Tatsache, dass die Erfassung beider Aspekte (sprachlicher vs. naturwissenschaftsbezogener) durch unabhängige Personen und mit unterschiedlichen Kodierverfahren erfolgte, liefern unsere Befunde Hinweise auf robuste und substanzielle Zusammenhänge zwischen der Interaktionsqualität in beiden Bildungsbereichen, die noch genauer untersucht werden sollten.

Bemerkenswert scheint, dass vor allem jene Rollenbilder, die eine ko-konstruktive Haltung der Fachkraft repräsentieren („Möglichmacher“, „Begleiter“), positiv mit der sprachlichen Anregungsqualität korrelieren, während keine ent- 
sprechenden Zusammenhänge der genannten Rollenvorstellungen mit den anderen QUOTS-Skalen gefunden wurden. Denkbar wäre, dass dies auf generelle Unterschiede im Bildungshintergrund der Fachkräfte zurückzuführen ist. Hier könnte man spekulieren, dass die Wahrnehmung bestimmter allgemeiner Haltungen der pädagogischen Fachkräfte vor allem an ihr Sprachverhalten gekoppelt ist bzw. dass Fachkräfte, die sich entsprechende Haltungen eher zu eigen machen, auch eher Bildungssprache verwenden. In jedem Fall aber wird schon jetzt deutlich, dass die Verwendung von Fachsprache einer ko-konstruktiven Haltung in keiner Weise entgegensteht, sondern sie vielmehr zu unterstützen scheint und nicht unabhängig von ihr gesehen werden sollte.

Fragestellung 2 kann damit ebenfalls positiv beantwortet werden: Es besteht eine positive Beziehung zwischen der sprachlichen Anregungsqualität und der Prozessqualität in naturwissenschaftlichen Interaktionen zwischen pädagogischer Fachkraft und Kindern.

\subsection{Prozessqualität in Interaktionen und naturwissenschaftliche Vorbildung der Fachkräfte}

Schließlich haben wir untersucht, inwiefern sich Unterschiede in der Prozessqualität zwischen Gruppen von Fachkräften nachweisen lassen, die an naturwissenschaftlichen Fortbildungen teilgenommen haben bzw. die dies nicht getan haben. Die naturwissenschaftliche Vorbildung wurde über Fachkräfte operationalisiert, die entweder an Fortbildungen vom „Haus der kleinen Forscher“ teilgenommen haben und in einer Einrichtung arbeiten, die als „Haus der kleinen Forscher“ zertifiziert wurde, bzw. über Fachkräfte, die bislang noch nicht naturwissenschaftlich fortgebildet wurden. Bei der Blindkodierung, bei der die Kodiererinnen nicht wussten, welcher Gruppe die Fachkraft zuzuordnen war, wurden systematische Gruppenunterschiede in der erwarteten Richtung gefunden.

Fragestellung 3 kann damit ebenfalls positiv beantwortet werden: Die naturwissenschaftsbezogene Prozessqualität war bei Fachkräften, die an „Haus der kleinen Forscher"-Fortbildungen teilgenommen haben, höher als bei Fachkräften ohne naturwissenschaftliche Fortbildung. 


\section{Fazit und Empfehlungen}

Insgesamt ergibt sich damit ein konsistentes Ergebnismuster (vgl. Kapitel 8) mit vier zentralen Einsichten:

1. Die Umsetzung wichtiger Ziele naturwissenschaftlicher Fortbildungen wie der Fortbildungsarbeit der Stiftung „Haus der kleinen Forscher“ lässt sich nicht nur durch Befragung der Fachkräfte, sondern auch durch direkte Beobachtung und Bewertung ihres Verhaltens in Forschungssituationen objektiv, reliabel und valide überprüfen. Dafür steht das neu entwickelte Verfahren QUOTS zur Verfügung, dessen Skalen größtenteils befriedigende teststatistische Kennwerte aufweisen.

2. Eine gute Prozessqualität im Sinne der Anwendung von Scaffolding-Strategien und der Verwirklichung einer ko-konstruktiven Haltung seitens der Fachkraft scheint mit einer verbesserten Forscherhaltung der Gruppe, mit mehr naturwissenschaftlichem Denken und mit vermehrten Einsichten der Kinder einherzugehen.

3. Naturwissenschaftsbezogene Prozessqualität und sprachliche Anregungsqualität hängen empirisch zusammen. Eine parallele Förderung beider Aspekte scheint möglich, wünschenswert und von Nutzen bei der Optimierung des Bildungsangebotes für die Kinder.

4. Die vorliegenden Daten sprechen dafür, dass das Fortbildungsangebot der Stiftung „Haus der kleinen Forscher“ sich positiv auf das Verhalten der Fachkräfte auswirkt.

Trotz dieser ermutigenden Botschaften ist Vorsicht vor Schlussfolgerungen allgemeiner Art geboten, da die vorliegende Untersuchung einigen methodischen Einschränkungen unterliegt:

Zunächst ist festzuhalten, dass die Kodierung der fachkraft- und der gruppensowie kindbezogenen Dimensionen stets von den gleichen Ratern vorgenommen wurden. Folglich kann nicht ausgeschlossen werden, dass die Wahrnehmung des Fachkraft-Verhaltens durch die Wahrnehmung des Kind-Verhaltens mit beeinflusst wurde und umgekehrt. Vor diesem Hintergrund wäre es wünschenswert, das Kind-Verhalten nochmals durch unabhängige Beurteiler und Beurteilerinnen einschätzen zu lassen. Da der Erkenntnisgewinn der Kinder jedoch an objektivierbaren Parametern festgemacht wurde (verbale Äußerungen zum Schwimm- und 
Sinkverhalten verschiedener Objekte sowie entsprechenden Begründungen) und die Inter-Reliabilität der Einschätzungen beider Rater insgesamt hoch lag, ist zu vermuten, dass entsprechende Ergebnisse ähnlich ausfallen würden.

Es wurden zahlreiche Korrelationen anhand der gleichen Stichprobe auf Signifikanz geprüft. Dies birgt grundsätzlich die Gefahr falsch-positiver Ergebnisse. Gleichzeitig macht eine „Bonferroni-Korrektur“ im vorliegenden Fall wenig Sinn. Da die berechneten Koeffizienten primär deskriptiven Zwecken dienen, in hohem Maße konsistent Ergebnisse liefern und fast alle substanziell sind, scheinen die generellen Schlussfolgerungen, die wir übergreifend aus der Vielzahl von Einzelbefunden gezogen haben, gerechtfertigt. Selbst wenn der eine oder andere Koeffizient nur zufällig signifikant ausgefallen sein sollte, ändert das nichts am allgemeinen Ergebnismuster.

Bezüglich der Untersuchung von Fragestellung 3 ist anzumerken, dass es sich nicht um eine Interventionsstudie mit randomisierter Treatment-Zuweisung handelt, sondern um einen Post-hoc-Gruppenvergleich. Folglich sind Kausalschlüsse über die Ursachen der Gruppenunterschiede nicht möglich. Durch die Blindkodierung und das Matching der Gruppen hinsichtlich potenziell relevanter anderer Variablen, die das Ergebnis mit beeinflussen könnten, haben wir uns bemüht, einige der Fallstricke des gewählten Vorgehens, für das es aus pragmatischen Gründen keine Alternative gab, zu vermeiden. Dennoch möchten wir betonen, dass unsere Befunde lediglich als Indizien für die Wirksamkeit der Fortbildungsangebote der Stiftung „Haus der kleinen Forscher“ gewertet werden können und nicht als Nachweis im Sinne einer kausalen Interventionsstudie.

Da das primäre Ziel dieses Projektberichtes darin bestand, erste Anhaltspunkte für die Fremderfassung und Beurteilung der Effekte naturwissenschaftlicher Fortbildungsangebote wie dem der Stiftung zu liefern, haben wir uns zunächst weitgehend auf einfache statistische Verfahren zur Beantwortung der Kernfragen des Projektes beschränkt. Aufgrund der begrenzten StichprobengröBe schien die Anwendung aufwändigerer Analysen wie etwa eine faktorenanalytische Auswertung oder multiple Regressionsrechnungen, die prinzipiell wertvolle Zusatzinformationen über das konkrete Zusammenspiel einzelner Facetten des Fachkraft-Verhaltens bei der Förderung naturwissenschaftlicher Denkprozesse von Kindern liefern könnten, wenig sinnvoll.

Gerade angesichts des geringen Stichprobenumfangs scheint es jedoch erstaunlich, wie konsistent und klar die Ergebnisse ausgefallen sind. Die Ergebnisse liefern Hinweise darauf, dass die Stiftung „Haus der kleinen Forscher“ Arbeit leistet, die bei den Fachkräften und den Kindern eine positive Wirkung in Richtung der anvisierten Ziele zeigt.

Grundsätzlich können die vorgelegten Befunde daher als Ermutigung für die Fortsetzung der bisherigen Arbeit der Stiftung interpretiert werden. Gleichzeitig 
ergeben sich an verschiedenen Stellen Hinweise auf mögliche Akzentsetzungen für die Zukunft:

Zum einen fällt auf, dass die Zielvorstellung von Fachkräften, die im Rahmen ko-konstruktiven gemeinsamen Forschens mit den Kindern primär als „Begleiter“ und „Möglichmacher“ fungieren sollen, nicht ganz der Realität entspricht. Zwar scheinen viele Fachkräfte bereits verstanden zu haben, dass es wichtig ist, eine aktive Rolle im Bildungsprozess zu spielen und bedeutsame Scaffolding-Strategien ins Spiel zu bringen, ohne sich den Kindern aufzudrängen oder übergriffig zu werden, doch entspricht ihr pädagogisches Verhalten (zumindest aus der Sicht geschulter Beobachter und Beobachterinnen) oft eher dem eines „Lehrers“ als dem eines „Begleiters“ oder „Möglichmachers“. Zudem zeigen die Fachkräfte noch immer leichte Tendenzen zur Aufdringlichkeit/Übergriffigkeit. Dies mag zwar im vorliegenden Fall mit den Besonderheiten der Erhebungssituation zusammenhängen (Zeitbeschränkung, Wissen um die Videoaufnahme), sollte darüber hinaus aber trotzdem zum Nachdenken anregen.

Immerhin sprechen die vorliegenden Ergebnisse dafür, dass eine Haltung als „Lehrer“ durchaus positiv mit anderen erwünschten Verhaltensweisen korreliert ist, die wiederum positiv mit dem Erkenntnisgewinn der Kinder zusammenhängen. Gleichzeitig korrelierte das Rollenbild des „Lehrers“ nicht direkt mit dem Erkenntnisgewinn der Kinder. Insgesamt ergibt sich damit ein nicht ganz konsistentes Bild, das auf die Notwendigkeit weiterer Klärung verweist.

Spannend wäre in diesem Zusammenhang der Vergleich des Erkenntnisgewinns bei zwei Interventionsgruppen, darunter eine, die durch eine Fachkraft angeleitet wird, welche die allgemeine Haltung eines „Lehrers“ mit Vorbildfunktion verkörpert, und eine mit einer Fachkraft, die die allgemeine Haltung eines „Begleiters“ mit besonderem Fokus auf den jeweiligen Erkenntnisstand der Kinder verkörpert (möglicherweise sogar die gleiche Person, die beide Rollen einnimmt). Hier müsste allerdings sichergestellt sein, dass die Kinder der beiden Interventionsgruppen hinsichtlich ihrer Intelligenz, ihres Vorwissens und anderer potenziell relevanter Einflussfaktoren parallelisiert sind, um zu aussagekräftigen Schlussfolgerungen zu gelangen. Eine solche Studie würde es ermöglichen, die Effekte beider Haltungen und Handlungsweisen direkt zu vergleichen.

Neben dieser Forschungsfrage gibt es aber auch noch andere Möglichkeiten, mehr darüber zu erfahren, welches konkrete Verhalten Kinder im Kindergar-

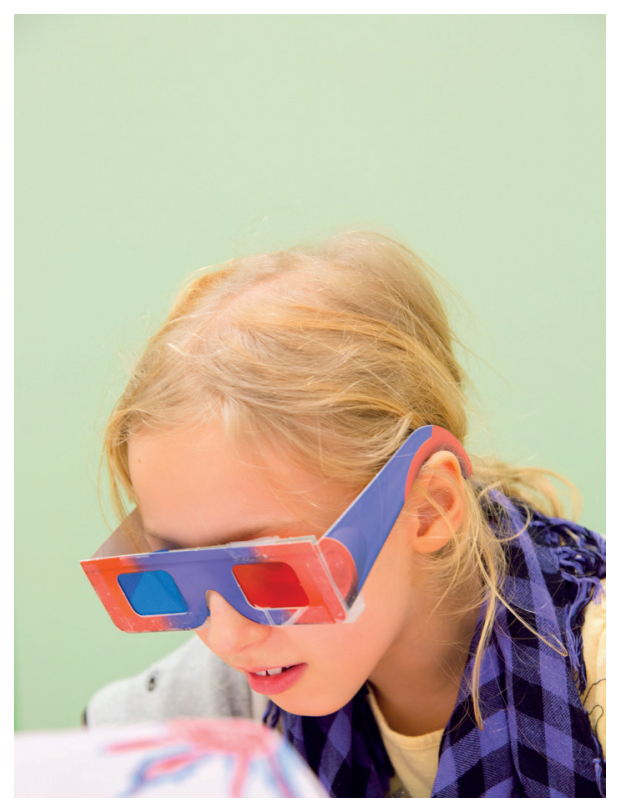


tenalter in ihrem naturwissenschaftlichen Erkenntnisprozess gezielt unterstützt: Parallel zum Globalrating über QUOTS wurde auch ein noch differenzierteres Kodiersystem (QUOTS +) entwickelt, bei dem jede Aktivität und jede sprachliche Äußerung der Fachkraft und der Kinder hochauflösend mittels Videomikroanalyse kodiert wird. Dieses Schema wurde bereits auf 20 Videos angewendet. Davon sind 10 Videos von Gruppen, bei denen Fachkräfte im QUOTS-Globalurteil besonders gut abgeschnitten haben, und 10 Videos von Fachkräften, die eine eher ungünstige Einschätzung erhalten haben. Aufgrund des enormen Kodieraufwands, der bei über 100 Stunden je Video lag, war es im Rahmen dieses Projektes noch nicht möglich, bereits eine differenzierte Analyse von möglichen Zusammenhängen zwischen konkreten Verhaltensweisen der Fachkräfte und konkreten Lernfortschritten der Kinder vorzunehmen. Eine solche Analyse ist jedoch in Vorbereitung. Spannend erscheinen uns in diesem Zusammenhang verschiedene Fragen, die hier nur beispielhaft genannt werden können:

- Was kennzeichnet das konkrete Verhalten von Fachkräften, deren pädagogische Haltung als positiv bewertet wird bzw. die als „Lehrer“, „Begleiter“, „Möglichmacher“ oder „weiteres Kind“ wahrgenommen werden. Sprechen sie mehr oder weniger? Gehen sie mehr auf Äußerungen der Kinder ein? Stellen sie mehr Fragen? Geben sie mehr Erklärungen?

- Welches Verhalten der Fachkraft geht Erkenntnisprozessen der Kinder unmittelbar voraus? Sind es eher Fragen oder Aussagen? Sind diese eher konkret oder allgemein formuliert? Ist es wichtig, dass das Kind gezielt angesprochen wird?

- Lassen sich allgemeine Parameter des Sprachverhaltens (Anteil an der Gesamtsprechzeit, Häufigkeit der Dialoge mit Kindern, Länge der Sätze etc.) identifizieren, die Auswirkungen auf die Prozessqualität haben?

Die Beantwortung entsprechender Fragen könnte wichtige Anregungen für die Formulierung von Fortbildungszielen geben und damit zur gezielten Optimierung des Fortbildungsangebotes der Stiftung „Haus der kleinen Forscher“ beitragen.

Insgesamt empfehlen wir der Stiftung, ihren bisherigen Weg der wissenschaftsbasierten Entwicklung des eigenen Bildungsprogramms konsequent weiterzuverfolgen. Er hat bereits weit geführt und kann sowohl der Forschung wichtige Impulse verleihen als auch die praktische Arbeit mit den Fachkräften und Kindern substanziell verbessern helfen.

Gleichzeitig wäre zu überlegen, ob QUOTS bzw. einzelne Skalen aus diesem neuen Instrument (z. B. Scaffolding-Strategien) für Selbstevaluationen der Fach- 
kräfte, zu Supervisionszwecken oder im Rahmen von Fortbildungen zum Einsatz gebracht werden können. Die Arbeit mit Videos und die Reflexion des eigenen Verhaltens bzw. die gezielte Beobachtung anderer Personen hinsichtlich wichtiger Ziel-Verhaltensweisen kann maßgeblich zur Verbesserung der Prozessqualität von Bildungssituationen beitragen. 


\section{Fazit und Ausblick -}

Wie die Stiftung „Haus der kleinen

Forscher" mit den Erkenntnissen umgeht

Stiftung Haus der kleinen Forscher

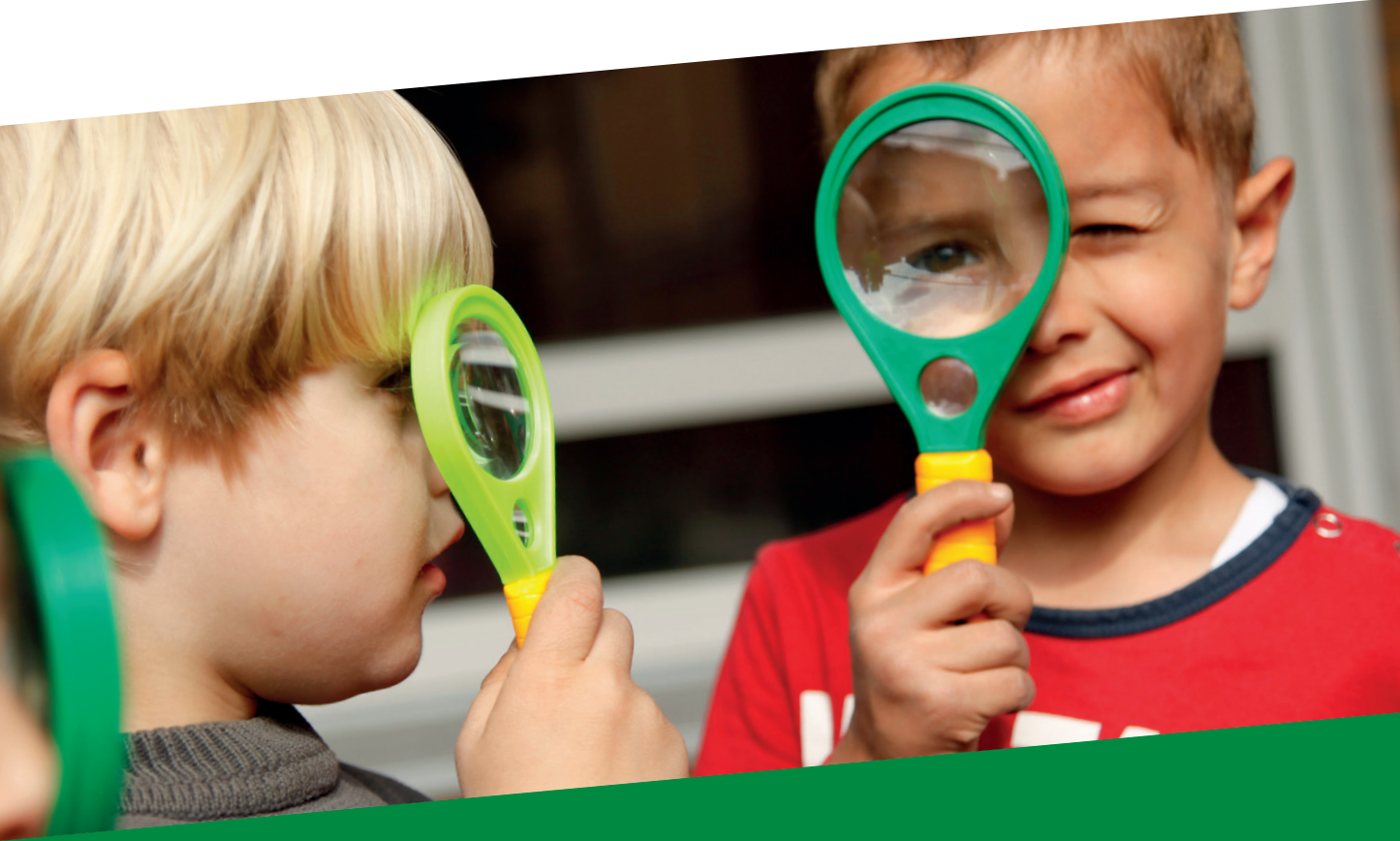




\section{Fazit und Ausblick}

\section{Ergebnisse der Studien und ihre Bedeutung für die Stiftungsarbeit}

Das erste Forschungsprojekt Early Steps into Science (EASI Science, gefördert von der Stiftung „Haus der kleinen Forscher“ und dem Bundesministerium für Bildung und Forschung) erbrachte Erkenntnisse über Wirkungen naturwissenschaftlicher Bildungsangebote auf naturwissenschaftliche Kompetenzen von pädagogischen Fachkräften und Kindern in Kitas. Die Ergebnisse zeigen, dass pädagogische Fachkräfte mit naturwissenschaftlichen Fortbildungen über ein höheres Fachwissen und mehr fachdidaktische Kenntnisse verfügen als eine Vergleichsgruppe ohne Fortbildungen. Zudem sind Motivation zu und Interesse an naturwissenschaftlicher Bildung fortgebildeter Erzieherinnen und Erzieher größer. Auch die Kinder zeigen mehr Lernfreude, Interesse an Naturwissenschaften sowie Selbstvertrauen in ihr eigenes Können, wenn ihre Kita einen naturwissenschaftlichen Schwerpunkt hat.

Das zweite Forschungsprojekt Early Steps into Science and Literacy (EASI Science-L, gefördert von der Stiftung „Haus der kleinen Forscher“, der BadenWürttemberg Stiftung und der Siemens Stiftung) untersuchte sprachliche Bildungswirkungen und die Interaktionsqualität im Kontext naturwissenschaftlicher Bildungsangebote. Im ersten Teilprojekt der Studie konnte gezeigt werden, dass sich forschendes Lernen gut für die Sprachbildung eignet. Fachkräfte mit naturwissenschaftlichen Fortbildungen gestalten sprachlich anregendere Lerngelegenheiten für Kinder als Fachkräfte ohne Fortbildungen in diesem Bereich. Die sprachlichen Fähigkeiten der Kinder waren umso größer, wenn die sie betreuenden Fachkräfte zuvor eine kombinierte Fortbildung zu Naturwissenschaften und Sprache der Stiftung „Haus der kleinen Forscher“21 besucht hatten. Zudem zeigen sich im zweiten Teilprojekt positive Zusammenhänge zwischen der durch die Fachkraft gestalteten naturwissenschaftsbezogenen Prozessqualität und den naturwissenschaftlichen Kompetenzen der Kinder.

Die Studien zeigen auf, dass die „Haus der kleinen Forscher“-Fortbildungen sowohl mit naturwissenschaftlichen Kompetenzen der Fachkräfte und der Kinder als auch mit der sprachlichen Anregungsqualität durch Fachkräfte beim Entdecken und Forschen zusammenhängen. Diese Ergebnisse weisen auf Wirkungen von Bildungsangeboten in der angezielten Richtung hin und ermutigen zur Fortführung dieser Weiterbildungsarbeit der Initiative. Gleichzeitig stimmt die Stiftung ge-

21 „Forschen mit Sprudelgas - Chemie ist überall“: Stiftung Haus der kleinen Forscher (2013). Sprudelgas und andere Stoffe - Mit Kita- und Grundschulkindern Chemie entdecken und dabei die sprachliche Entwicklung unterstützen. Berlin: Stiftung Haus der kleinen Forscher. Verfügbar unter: www. haus-der-kleinen-forscher.de 
meinsam mit dem Stiftungsrat klar mit der Empfehlung des Wissenschaftlichen Beirats überein, die Arbeit der Stiftung weiterhin regelmäßig zu hinterfragen und die Angebots(weiter-)entwicklung wie Wirkungsmessung zur Stiftungsarbeit auch künftig wissenschaftlich begleiten zu lassen. Die Stiftung nutzt die Ergebnisse der Studien für eine systematische Reflexion ihrer bestehenden Bildungsangebote und die wirkungsorientierte Entwicklung zukünftiger Fortbildungen. Hierzu fanden verschiedene Austauschtreffen sowie Diskussionen der Ergebnisse auch im Wissenschaftlichen Beirat sowie im Stiftungsrat statt, in denen neben den Kernergebnissen (was zeigen die Studien?) auch die Limitationen der Studien (was zeigen sie nicht?) berücksichtigt wurden.

\section{Grenzen der Studien}

Die Studienergebnisse sind vor dem Hintergrund der Grenzen und Limitationen der Studien zu betrachten (vgl. Fazitkapitel der jeweiligen Abschlussberichte). Die beiden Studien sind insofern limitiert, da sie im Querschnittsdesign durchgeführt worden sind. Leider war es, vor allem aufgrund begrenzter finanzieller Mittel, nicht möglich, die Studien im Längsschnitt zu fördern. Zum Zeitpunkt der Ausschreibung stand zunächst das übergeordnete Ziel im Fokus, erste empirische Belege für die Zusammenhänge von naturwissenschaftlichen Bildungsangeboten und professionellen Kompetenzen von Fachkräften sowie ihre Bezüge zur sprachlichen Bildung zu erhalten. Aufgrund der querschnittlichen Betrachtung und fehlender Längsschnittanalysen (keine Prä-Post-Testungen) sind die Ergebnisse nicht kausal und nicht eindeutig in nur eine Wirkrichtung interpretierbar.

Zudem beruhen die Ergebnisse nicht auf einer repräsentativen Stichprobe, sondern auf einer Zufallsstichprobe aus den Erhebungsregionen rund um die beteiligten Forschungsstandorte. Die pädagogischen Fachkräfte, Kinder und Eltern haben freiwillig an den Studien teilgenommen. Nicht auszuschließen ist dabei, dass in der Stichprobe vermehrt sehr motivierte und besonders forschungsinteressierte Einrichtungen vertreten sind. Gewisse Antworten sind möglicherweise im Sinne einer sozialen Erwünschtheit gemacht worden.

Bei EASI Science beruhen die Angaben zur Häufigkeit sowie die Erfassung des Verhaltens und der umgesetzten Prozessqualität auf dem Selbstbericht der Teilnehmenden. Bei EASI Science-L wurde die Prozessqualität, also das Verhalten in der Forschungssituation, durch unabhängige Beurteilerinnen anhand von Videos eingeschätzt. Die Ergebnisse beider Studien weisen in die gleiche Richtung eines positiven Zusammenhangs von Fortbildungen und Prozessqualität. Andere Validierungsstudien wie die Studie von Anders und Ballaschk (2014), die das beobachtete Verhalten von pädagogischen Fach- und Lehrkräften mit einer Selbsteinschätzung abglichen, konnten eine hohe Übereinstimmung zwischen Selbstbericht und den bei einer Begehung vorgefundenen Verhältnissen und keine 
Hinweise auf Täuschung feststellen. Weitere Studien zur Erfassung von Prozessqualität in naturwissenschaftlichen Bildungssituationen wären wünschenswert, um mehr Aufschluss über Abweichungen oder Bestätigungen bei verschiedenen Messverfahren zu erlangen.

Bei EASI Science-L muss bezüglich der Erhebungsmethodik berücksichtig werden, dass die Forschungssituation, die videografiert worden ist, nicht in einem Alltagssetting in der Kita, sondern in einer Laborsituation an einem für die Beteiligten neuen Ort stattfand. Das Verhalten der pädagogischen Fachkraft in der Interaktion mit den Kindern und das kindliche Verhalten könnten dadurch beeinflusst worden sein. Andererseits war die Standardisierung notwendig, um gleiche Rahmenbedingungen zu schaffen und eine Vergleichbarkeit der Daten gewährleisten zu können.

Diese Limitationen gilt es bei der Interpretation der Ergebnisse zu berücksichtigen und im Rahmen weiterer Forschung nach Möglichkeit zu überwinden. Analysen der Daten aus dem Stiftungsmonitoring liefern Hinweise, die zur Einordnung der Studienergebnisse beitragen können.

\section{Ergänzende Daten aus dem Stiftungsmonitoring}

Einige der empirischen Instrumente, die von den Forschungsgruppen entwickelt wurden, werden bereits im kontinuierlichen Monitoring und in der Evaluation der Arbeit der Stiftung genutzt. So wurden Items des Prozessqualitätsindex für naturwissenschaftliche Bildungsangebote (vgl. Kapitel 4.2.3) sowie weitere Items zu Selbstwirksamkeitserwartungen der Fachkräfte aus den in den Studien entwickelten Fragebögen in die Frühjahrsbefragung 2016 integriert.

Im Monitoring-Bericht 2016/2017 wurden diese Ergebnisse veröffentlicht und konkrete Annahmen und Aussagen im Sinne einer Programmtheorie dargestellt, wie die Bildungsinitiative wirkt (Stiftung Haus der kleinen Forscher, 2017a). Um die Wirkungsweise der Bildungsinitiative „Haus der kleinen Forscher“ zu verdeutlichen, präsentiert der Monitoring-Bericht 2016/2017 eine vereinfachte Wirkungskette (siehe Abbildung 11). Die Wirkungskette besteht aus Wirkungszielen, die die Initiative mit ihrer Arbeit erreichen möchte und den Prozessen, die dahinter stehen. 


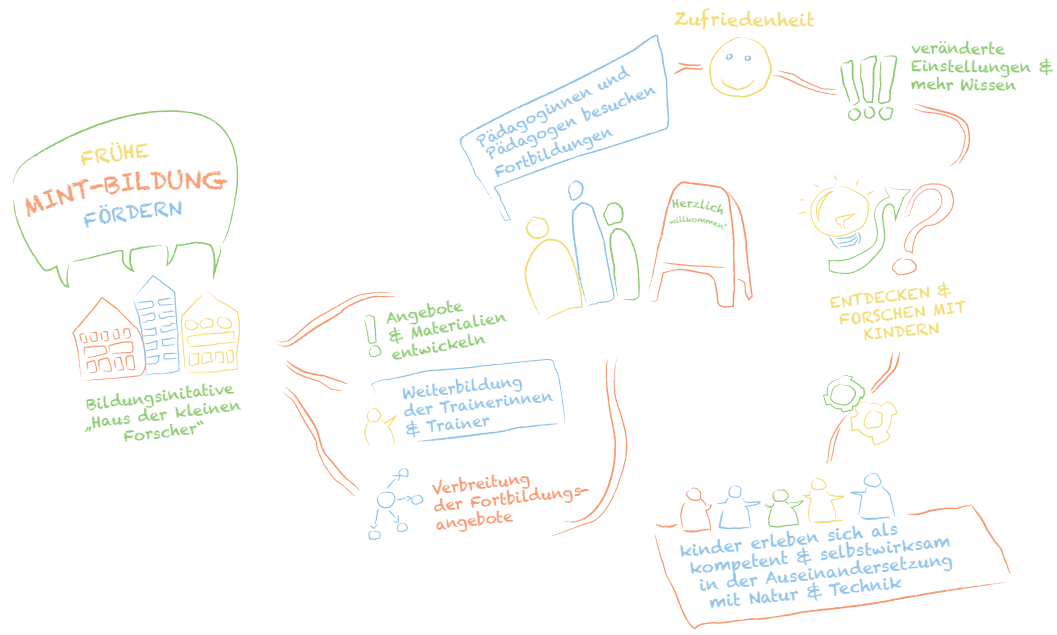

Abbildung 11. Wirkungskette der Bildungsinitiative „Haus der kleinen Forscher“ (Stiftung Haus der kleinen Forscher, 2017a)

Geleitet von der Grundfrage nach den Wirkungen von MINT-Bildungsangeboten, ist eine zentrale Fragestellung für Stiftungsmonitoring und Evaluation von Angeboten, wie die Bildungsinitiative zur Verbesserung der frühen MINT-Bildung beiträgt. Wirkungen werden in diesem Kontext als Veränderungen verstanden, die durch die Teilnahme am Weiterbildungsprogramm des „Hauses der kleinen Forscher“ in Kitas, Horten und Grundschulen erreicht werden; der Fokus liegt in diesem Bericht auf den Wirkungen bei der Zielgruppe der pädagogischen Fach- und Lehrkräfte. Die Ergebnisse des Monitoring-Berichts zeigen, wie das Fortbildungsangebot der Initiative zur Verbesserung der frühen MINT-Bildung beiträgt.

Pädagogische Fach- und Lehrkräfte nutzen das Bildungsangebot kontinuierlich und langandauernd. Der Blick auf den Verlauf der Fortbildungsteilnahmen zeigt, dass die Anzahl der durch eine Fachkraft besuchten Fortbildungen mit der Teilnahmedauer am Bildungsprogramm ansteigt (siehe Abbildung 12).

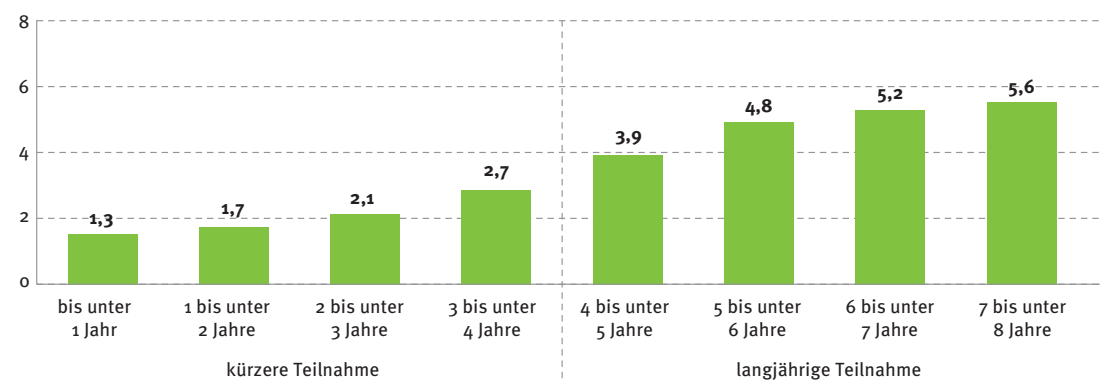

Abbildung 12. Anzahl besuchter Fortbildungen nach Teilnahmedauer der pädagogischen Fach- und Lehrkräfte 
Im Durchschnitt besuchen die teilnehmenden Pädagoginnen und Pädagogen etwa eine „Haus der kleinen Forscher“-Fortbildung pro Jahr. In den ersten zwölf Monaten sind es im Durchschnitt 1.3 Fortbildungen. Bei einer Teilnahme zwischen zwei und drei Jahren an der Initiative liegt die durchschnittliche Anzahl der Fortbildungen bei 2.1. Dies deckt sich ungefähr mit der Angabe aus der EASI Science-Studie zur durchschnittlichen Anzahl von 2.3 Fortbildungen in den letzten 3 Jahren. Diejenigen, die bereits seit sieben bis acht Jahren teilnehmen, haben insgesamt durchschnittlich 5.6 Fortbildungen besucht. Auch wenn eine noch häufigere Fortbildungsteilnahme bzw. eine Erhöhung der in Anspruch genommenen Fortbildungsstunden innerhalb eines kürzeren Zeitraums wünschenswert wären, nähert sich die Gesamtfortbildungsdauer der Langzeitteilnehmenden mit fünf bis sechs Tagesfortbildungen einer Intensität an, nach der größere Fortbildungswirkungen zu erwarten sind (nach der Metaanalyse von Egert (2015) haben sich Fortbildungen mit 40 bis 60 Stunden Dauer als am effektivsten erwiesen). Insofern ist es ermutigend, dass es der Initiative gelingt, die pädagogischen Fach- und Lehrkräfte wiederholt und stetig zur Fortbildungsteilnahme und darüber zum Forschen im Alltag mit den Kindern zu bewegen und somit vermutlich dazu beizutragen, ihr Interesse, ihr Wissen und ihre Kompetenzen und in der frühen MINT-Bildung weiterzuentwickeln.

Das Ergebnis aus EASI Science, dass Fortbildungen zu früher naturwissenschaftlicher Bildung bei pädagogischen Fachkräften, in der Kita und bei Kindern zu wirken scheinen, wird durch Wirkungsanalysen aus dem Stiftungsmonitoring bekräftigt. Die Ergebnisse weisen darauf hin, dass Pädagoginnen und Pädagogen mit dem zunehmenden Besuch von „Haus der kleinen Forscher“-Fortbildungen profitieren. Sie scheinen ihr MINT-Fachwissen, ihre pädagogisch-didaktischen Kompetenzen in der MINT-Bildung und das Vertrauen in ihre Fähigkeiten, d.h. ihre Selbstwirksamkeitserwartungen, zu steigern (Monitoring-Bericht 2016/2017, Stiftung Haus der kleinen Forscher, 2017a). Die selbstwahrgenommene Kompetenz der teilnehmenden pädagogischen Fach- und Lehrkräfte zum Forschen mit den Kindern ist insgesamt hoch; sie nimmt mit der Teilnahmedauer an der Bildungsinitiative und damit einhergehenden Fortbildungsbesuchen zu (Stiftung Haus der kleinen Forscher, 2013a, 2017a). Die Ergebnisse eines Gruppenvergleichs zwischen pädagogischen Fach- und Lehrkräften, die an ein bis zwei Fortbildungen teilgenommen haben, und solchen, die bereits sechs „Haus-der-kleinen-Forscher"-Fortbildungen und mehr besucht haben, zeigen, dass sich mit dem Besuch der Fortbildungen die Selbstwirksamkeitserwartungen der pädagogischen Fach- und Lehrkräfte erhöhen und das selbsteingeschätzte Ausmaß ihres MINT-Fachwissens sowie ihrer pädagogisch-didaktischen Kompetenzen in der MINT-Bildung erweitern (Stiftung Haus der kleinen Forscher, 2017a). Zudem beeinflusst der Besuch von „Haus der kleinen Forscher“-Fortbildungen die prakti- 
sche Umsetzung des Gelernten in der Interaktion mit den Kindern: Pädagogische Fach- und Lehrkräfte mit sechs und mehr Fortbildungen forschen häufiger mit den Mädchen und Jungen und lassen den Kindern bei diesem Prozess mehr Freiräume als diejenigen mit weniger als drei Fortbildungen.

\section{Empfehlungen aus den Abschlussberichten als Grundlage für die (Weiter-)Entwicklung der Stiftungsangebote}

Ableitend aus den Ergebnissen der beiden Studien geben die Forschungsgruppen von EASI Science und EASI Science-L der Stiftung „Haus der kleinen Forscher“ zusammenfassend folgende zentrale Empfehlungen (vgl. Fazitkapitel der jeweiligen Abschlussberichte) für ihre kontinuierliche Weiterentwicklung mit auf den Weg:

- Steigerung der Fortbildungsrate im Sinne eines kontinuierlichen und strukturierten Fortbildungsangebots mit höherer Dosis und Dauer;

- Steigerung der Bildungsqualität und Implementation früher MINT-Bildung in den Einrichtungen;

- Verknüpfung von Naturwissenschaften und Sprache, in den Bildungsangeboten;

- Qualitätssicherung der Fortbildungsarbeit im Multiplikatorenmodell;

- kontinuierliche, wissenschaftsbasierte Weiterentwicklung der Stiftungsangebote.

\section{Steigerung der Fortbildungsrate und kontinuierliches Fortbildungsangebot}

Grundansatz der Initiative ist ein bundesweites, kontinuierliches Weiterbildungsangebot zur Unterstützung der professionellen Kompetenzentwicklung („Continuous Professional Development"). Dieses wird von pädagogischen Fach- und Lehrkräften langfristig und stetig in Anspruch genommen wird (vgl. Stiftung Haus der kleinen Forscher, 2017a). Die Stiftung setzt sich mit der Empfehlung, die Dosis und Dauer der Fortbildungsangebote zu erhöhen, intensiv auseinander. In Überlegung sind Fortbildungsreihen und vertiefende Curricula, die die Stiftung in Modellprojekten erproben wird (s. u. Projekte, die zur Qualitätsentwicklung in Kitas aus systemischen Perspektive beitragen).

Ein wichtiger Ansatzpunkt der Stiftung ist die Entwicklung digitaler Lernangebote, um Fachkräften insgesamt mehr und auch unabhängig von bestimmten Orten und Zeiten zugängliche Bildungsangebote zu ermöglichen. Mit moderierten oder offenen Online-Kursen können die pädagogischen Fach- und Lehrkräften 
ergänzend zur Teilnahme an Präsenzformaten ihr Wissen und ihre Kompetenzen erweitern und sich über MINT-Bildung mit anderen austauschen. Gleiches gilt für die Qualifizierung der Trainerinnen und Trainer, die die Fortbildungen in den Netzwerken der Bildungsinitiative geben. Die digitalen Lernangebote werden verzahnt mit der Material- und Themenentwicklung der Präsenzangebote entwickelt, um den pädagogischen Fach- und Lehrkräften ein für verschiedene Bedarfe passgenaues Bildungsangebot zur Verfügung zu stellen.

Ein Beispiel dafür ist die Entwicklung des Bildungsangebots zum Thema „MINT ist überall“, das ab Herbst 2019 für alle Trainerinnen und Trainer und pädagogischen Fach- und Lehrkräfte zu Verfügung stehen wird. Dieses Bildungsangebot zielt darauf ab, verschiedene thematische Angebote aus den einzelnen MINT-Disziplinen übergreifend miteinander zu verknüpfen. Pädagogische Fachund Lehrkräfte sollen dabei unterstützt werden, die Potenziale von MINT-Lerngelegenheiten im pädagogischen Alltag zu beobachten und zu erkennen und Ideen zu entwickeln, wie diese Gelegenheiten und Situationen (z. B. Fragen der Kinder oder beobachtete Phänomene), im Sinne einer guten MINT-Bildung aufgegriffen und vertieft werden können. Dazu werden eine Präsenzfortbildung, verschiedene Online-Lernangebote sowie pädagogische Materialien (z. B. Broschüre, Karten-Sets) miteinander verzahnt entwickelt. Fachkräfte setzen sich damit auseinander, wo überall in Alltagssituationen MINT-Lerngelegenheiten enthalten sind, wie daraus Erfahrungsräume für Kinder entstehen können und diese gut begleitet werden können. Neuartig bei der Angebotsentwicklung ist, dass alle Bildungsangebote zu diesem Thema, angefangen von der Fortbildung über die Broschüre bis hin zu Online-Kursen, sowohl aufeinander aufbauend als auch unabhängig voneinander bedarfsgerecht von den pädagogischen Fachkräften genutzt werden können, je nachdem, welche Fortbildungen sie bereits besucht und welches fachspezifische Wissen sie schon erworben haben. Neben übergreifenden Grundlagen wird es immer Vertiefungsaspekte geben, die Fachkräfte für ihre weitere Qualifizierung nutzen können. Die Lerngelegenheiten in der Weiterbildung werden so aufbereitet sein, dass pädagogische Fach- und Lehrkräfte immer wieder neue Impulse erfahren und gleichzeitig von ihren eigenen Erfahrungen berichten und in einen Erfahrungsaustausch mit anderen gehen können. So können sie ihre Motivation, ihr Interesse und ihre Handlungskompetenz, sich mit MINT-Themen näher auseinanderzusetzen und diese mit den Kindern zu entdecken und zu erforschen, vertiefen und weiterentwickeln. 


\section{Steigerung der Bildungsqualität und Implementation früher MINT-Bildung in den Einrichtungen}

Der personenbezogene Ansatz der bisherigen Weiterbildungsangebote der Initiative wird künftig stärker um einen systemischen Ansatz erweitert, der die institutionelle Verankerung von Fortbildungen in „Haus der kleinen Forscher“-Einrichtungen in den Blick nimmt und sich dabei auch an die Leitungsebenen der Kitas richtet. Hiermit hat die Stiftung bereits in ihren Angeboten für Leitungskräfte im Projekt „Bildung für nachhaltige Entwicklung“ begonnen (vgl. Stiftung Haus der kleinen Forscher, 2018, in Vorb. c). Eine Chance liegt in einer noch stärkeren Verknüpfung des Fortbildungsprogramms (personenbezogen) und des Zertifizierungsprogramms der Stiftung (einrichtungsbezogen) (Stiftung Haus der kleinen Forscher, 2017b). Die Bildungsinitiative „Haus der kleinen Forscher“ bietet ihre Fortbildungen und Zertifizierungen bundesweit an und setzt auf kontinuierliche Qualifizierung und Entwicklungsunterstützung. Sie ist damit gut für die Aufgabe gerüstet, noch stärker als bislang systemisch auf Einrichtungsebene zu wirken und pädagogische Einrichtungen bei ihrer Qualitätsentwicklung zu begleiten.

Zur Förderung der pädagogischen Qualität früher MINT-Bildung plant die Stiftung „Haus der kleinen Forscher“ ab Frühjahr 2019 zwei Projekte, die die Qualitätsentwicklung in Kitas aus einer systemischen Perspektive (im Sinne eines „Whole Institution Approach“, vgl. z. B. Ferreira, Ryan \& Tilbury, 2006), auch unter Berücksichtigung der Leitungsebene, in den Blick nimmt.

Das erste Projekt „Kita-Qualitätsoffensive - Forschen im Alltag“ (kurz: KiQOff) wird vom Bundesministerium für Bildung und Forschung gefördert und soll im März 2019 starten. Die Qualitätsoffensive hat zum Ziel, einen Beitrag zur Stärkung der pädagogischen Qualität früher MINT-Bildung in Kitas zu leisten und damit ein wirksames Instrument zur Kompetenz- und Entwicklungsförderung von Kindern zu sein. Es sollen Maßnahmen entwickelt werden, die Kitas bei der Etablierung des forschenden Lernens als pädagogisches Prinzip im Kita-Alltag unterstützen. „KiQOff“ wird als Modellprojekt in vier Modellregionen und insgesamt 100 Kita-Einrichtungen erprobt und wissenschaftlich begleitet.

Zentrale Elemente des Projektes sind die Entwicklung einer Fortbildungsreihe (Dauer: ca. 18 bis 20 Monate), das aus mehreren Modulen mit pädagogischen und systemischen Schwerpunkten besteht. Diese Fortbildungsreihe schließt mit einem Zertifikat für pädagogische Fachkräfte ab. An einer Reihe von Modulen wird die Kita-Leitung beteiligt sein, so dass eine gemeinsame Arbeit im Tandem stattfindet. Des Weiteren unterstützt eine professionelle Prozessbegleitung den Prozess der Qualitäts- und Organisationsentwicklung vor Ort. Ein intensives Unterstützungsangebot für Einrichtungen zur Etablierung des forschenden Lernens als gemeinsames Selbstverständnis und integraler Bestandteil der pädagogischen 
Arbeit im Alltag (alltagsintegrierte MINT-Bildung) soll entwickelt, aufgebaut und evaluiert werden. Damit wird die Möglichkeit einer vertiefenden Professionalisierung für pädagogischer Fach- und Leitungskräfte geboten. Ausgewählte Fachkräfte und Leitungen in Elementareinrichtungen sollen im Sinne einer Mentorenfunktion befähigt werden, Veränderungsprozesse in ihrer Einrichtung anzustoßen und erfolgreich durchzuführen. Im Projekt soll eine Verschränkung von personenbezogener Qualifizierung und systembezogener Organisationsentwicklung vollzogen werden. Hierzu plant die Stiftung, das Zertifizierungsverfahren für Einrichtungen (Stiftung Haus der kleinen Forscher, 2017b) mit den Fortbildungsangeboten intensiver zu verbinden.

Das zweite Projekt, eine gemeinsame Initiative zur Kitaentwicklung, das ebenfalls zum März 2019 startet und von der Robert Bosch Stiftung gefördert wird, widmet sich dem Thema Organisationsentwicklung und Qualitätsentwicklung in Kitas. Es zeichnet sich durch eine globale, systemische Herangehensweise an das Thema aus und will übergeordnete Lösungsansätze für das System Kita erarbeiten. Das Projekt nähert sich dem Thema auf drei Ebenen. Auf wissenschaftlicher Ebene soll eine Bestandsaufnahme vom aktuellen Stand der Organisationsentwicklung in Kitas erfolgen. Darauf aufbauend erfolgt eine Theoriebildung mit fundierten, daraus abgeleiteten Handlungsempfehlungen. Zur Umsetzung der Projektziele soll ein Expertengremium beitragen, das dauerhaft etabliert werden soll. Auf der Praxisebene sollen praxisrelevante Ansätze und Maßnahmen entwickelt und exemplarisch erprobt werden. Auf bildungspolitischer Diskursebene sollen im Sinne eines Agenda-Settings Vertreter und Vertreterinnen aus Wissenschaft, Praxis, Politik und Trägern für das Thema Kitaentwicklung sensibilisiert werden. Somit soll ein Beitrag zur Anerkennung und hohen Bedeutung der Organisationsentwicklung für die Förderung von Qualitätsentwicklung im System Kita geleistet werden. Es sollen Handlungsempfehlungen publiziert und zur Etablierung des Konzepts Organisationsentwicklung in Kitas/Kitaentwicklung im Feld der frühen Bildung beigetragen werden.

\section{Verknüpfung von MINT-Bildung und sprachlicher Bildung}

Frühe Bildung in den Bereichen Mathematik, Informatik, Naturwissenschaften und Technik kann Kindern beim Sprechenlernen helfen. Diesen Ansatz verfolgt die Stiftung „Haus der kleinen Forscher“ bereits seit längerer Zeit. Mit dem Bildungsangebot „Sprudelgas und andere Stoffe - Mit Kita- und Grundschulkindern Chemie entdecken und dabei die sprachliche Entwicklung unterstützen“"(Stiftung Haus der kleinen Forscher, 2013b) werden die gemeinsamen Grundprinzipien sprachlicher und naturwissenschaftlicher Bildung thematisiert.

Mit dem Online-Angebot „Service-Portal Integration“ hat die Stiftung einen weiteren Weg erprobt, sprachliche Bildung in Kitas zu unterstützen und das Bewusst- 
sein für die Notwendigkeit guter sprachlicher Kompetenzen zu stärken. Auf dem Service-Portal Integration finden pädagogische Fach- und Lehrkräfte ein praxisnahes Angebot zur Unterstützung der pädagogischen Arbeit mit geflüchteten Kindern. Auf der Website werden Erfahrungsberichte aus der Praxis, Beispiele und Ideen für die gelungene Integration von geflüchteten Kindern in Kita, Hort und Grundschule vorgestellt, Experteninterviews, Hintergrundartikel und Reportagen veröffentlicht und praktische Infos zu Literatur, Kontakten und weiterführenden Angeboten zum Thema gegeben. Es gibt Linksammlungen und Übersichten zu hilfreichen Unterlagen anderer Anbieter, z. B. übersetzte Formulare, Elternbriefe und Dialoghilfen. Das Service-Portal berichtet über regelmäßige Austausche mit Expertinnen und Experten aus den Bereichen Integration, Pädagogik, Psychologie und Sprachforschung. Zudem können sich pädagogische Fach- und Lehrkräfte in einer eigenen, moderierten Facebook-Gruppe über ihre Erfahrungen austauschen.

Ein zentrales Thema auf dem Service-Portal Integration ist die Sprachförderung. Die Stiftung „Haus der kleinen Forscher“ gibt hier zahlreiche Hinweise, wie geflüchtete Kinder in ihrem Erwerb des Deutschen unterstützt werden können. Denn Sprache ist der Schlüssel zur Integration. Damit Sprachbarrieren möglichst schnell überwunden werden, werden in dieser Rubrik verschiedene praxisnahe Beispiele und Tipps von Expertinnen und Experten sowie Erfahrungen pädagogischer Fach- und Lehrkräfte zur Verfügung gestellt. Diese können hilfreich für die eigene pädagogische Arbeit sein und als Inspiration dienen. Die Stiftung gibt Anregungen, wie Kinder beim entdeckenden und forschenden Lernen angeregt werden, ihre Beobachtungen zu beschreiben, Vermutungen zu äußern oder aber auch Ergebnisse zu erörtern.

Zu dem Ergebnis, dass Sprache und forschendes Lernen zusammengehören, kamen auch Expertinnen und Experten aus Praxis, Ausbildung und Forschung in der im Februar 2016 veröffentlichten „Frankfurter Erklärung zur frühen sprachlichen und naturwissenschaftlichen Bildung“ (Stiftung Haus der kleinen Forscher, 2015). Dieser Zusammenhang konnte mit der Studie EASI Science-L nun weiter belegt werden: Naturwissenschaftliche Bildungsangebote haben das Potenzial auch zu sprachlichen Bildungswirkungen. Fachkräfte, die an Fortbildungen zu einem naturwissenschaftlichen Thema teilgenommen haben, zeigen eine höhere sprachliche Anregungsqualität als Fachkräfte ohne naturwissenschaftsbezogene Fortbildung; Fachkräfte aus einer als „Haus der kleinen Forscher” zertifizierten Einrichtung weisen im Schnitt die höchste sprachliche Anregungsqualität auf. Fachkräfte, die eine kombinierte Fortbildung zu Naturwissenschaften und Sprache der Stiftung „Haus der kleinen Forscher" besucht haben, zeigen eine höhere sprachliche Anregungsqualität als Fachkräfte, die diese Fortbildung nicht besucht haben (Rank et al., in diesem Band). 
Die Verbindung der Bildungsfelder von MINT und Sprache scheint somit geeignet zu sein, um Sachwie Sprachbildung zu befördern. Zum einen trägt das Entdecken und Forschen zur sprachlichen Bildung bei, indem die pädagogischen Fachkräfte mit den Kindern einen sprachlichen Dialog und einen Verstehensdialog führen. Eine qualitativ hochwertige sprachliche Interaktion stärkt somit nicht nur den Forschungspro-

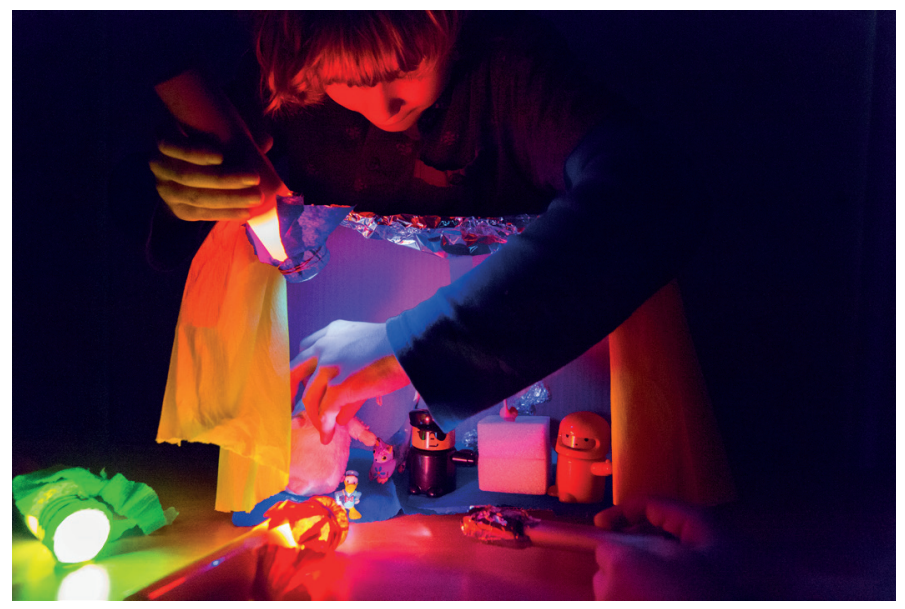
zess an sich, sondern auch die Ausbildung der bildungssprachlichen Kompetenzen der Kinder. Zum anderen gehört Sprache auch untrennbar zur MINT-Bildung, zum Aneignen der Welt und zur Kommunikation von Ideen, Vermutungen, Beobachtungen oder Erkenntnissen. Eine präzise Sprache hilft, um ein gemeinsames Verständnis der Sache zu erreichen (Ramseger, 2013).

Die Stiftung plant, die Chancen der Sprachbildung in Zusammenhang mit dem Entdecken und Forschen zu MINT-Themen künftig noch stärker zu betonen. Im Zuge der Überarbeitung des pädagogischen Ansatzes, bei der eine weitere inhaltliche Auseinandersetzung mit der pädagogischen Haltung und dem pädagogischen Verhalten im Sinne des ko-konstruktiven Ansatzes der Stiftung „Haus der kleinen Forscher" überarbeitet wird, wird auch der Fokus auf die Interaktionsqualität zwischen pädagogische Fach- bzw. Lehrkraft und den Kindern gestärkt (Stiftung Haus der kleinen Forscher, in Vorb. a). Eine hohe Interaktionsqualität geht mit einer hohen Qualität der sprachlichen Interaktion einher (Egert, in Vorb.). Auch in den Fortbildungen wird die Bedeutung der Sprachbildung während des forschenden Vorgehens als durchgängiges Thema betont werden. Zudem werden in den Fortbildungen und Online-Kursen die für die sprachliche Anregung dienlichen Phasen des Forschungskreises künftig deutlicher herausgestellt und auf deren Potenziale hingewiesen. Zukünftig soll die Angebotskapazität für die Fortbildung „Sprudelgas und andere Stoffe - Mit Kita- und Grundschulkindern Chemie entdecken und dabei die sprachliche Entwicklung unterstützen“ in der Weiterbildung der Trainerinnen und Trainer wie auch in den Netzwerken vor Ort erhöht sowie der Gewinn der Verknüpfung beider Bildungsbereiche noch stärker kommuniziert werden. Hierfür wird die Stiftung „Haus der kleinen Forscher“ weiter den Austausch mit Expertinnen und Experten aus Wissenschaft und Praxis pflegen, um fach- und forschungsbasiert wirkungsvolle Angebote (weiter-)zuentwickeln. 


\section{Qualitätssicherung der Fortbildungsarbeit im Multiplikatorenmodell}

Um naturwissenschaftsbezogene Kompetenzen auf Ebene der pädagogischen Fach- und Lehrkräfte zu steigern und qualitativ hochwertige Bildungsangebote für die Kinder anbieten und gestalten zu können, braucht es hochwertige Fortbildungen mit qualifizierten Erwachsenenbildnern. Im Projekt Trainerakademie 2.0 (2016 bis 2019, gefördert von der aqtivator $\mathrm{gGmbH}^{22}$ ) hat die Stiftung ein Qualitätssystem für Fortbildung entwickelt, das der Qualitätssicherung und Qualitätsentwicklung der Arbeit Trainerinnen und Trainern im Multiplikatorenmodell der Initiative „Haus der kleinen Forscher“ dient.

Grundlage für das Qualitätssystem für Fortbildung bildet ein Modell professioneller Kompetenz für Trainerinnen und Trainer der Stiftung „Haus der kleinen Forscher“, das sich in verschiedene für die MINT-Bildung relevante Zieldimensionen untergliedert (Stiftung Haus der kleinen Forscher, in Vorb. d). In 2016/17 trafen sich dazu wissenschaftliche Expertinnen und Experten ${ }^{23}$ aus den verschiedenen Fachdidaktiken, der Erwachsenenbildung und der Bildungsforschung sowie mehrfach Fokusgruppen aus der Trainerpraxis. Sie diskutierten mit der Stiftung, was eine optimale Lernbegleitung für pädagogische Fach- und Lehrkräfte durch Weiterbildner in der frühen MINT-Bildung ausmacht. Dieses Modell professioneller Kompetenz für Multiplikatorinnen und Multiplikatoren umfasst Wissen und Können sowie emotionale und motivationale Faktoren, die für die Fortbildungsleitung in der MINT-Bildung wichtig sind und bildet eine wichtige Basis für die Zusammenarbeit mit den Trainerinnen und Trainer in der Initiative.

Die einzelnen Maßnahmen dieses Qualitätssystems, das die Akkreditierung, regelmäßige Re-Akkreditierung und passgenaue Qualifizierung und Unterstützung der Multiplikatoren für ihre Tätigkeit umfasst (z. B. mittels Videocoaching und vertiefender Profilfortbildungen zu erwachsenenpädagogischen Kompetenzen wie „Moderieren“, „Visualisieren“ oder „Kommunikation“), stehen ab 2019 dauerhaft zur Verfügung. Die Expertise zu den Zieldimensionen für Multiplikatoren in der frühen MINT-Bildung und weitere Ergebnisse des Projekts Trainerakademie 2.0 werden Anfang 2019 in Band 11 der wissenschaftlichen Schriftenreihe der Stiftung veröffentlicht (Stiftung Haus der kleinen Forscher, in Vorb. d).

22 Der Projektpartner aqtivator $\mathrm{gGmbH}$ fördert gemeinnützige Organisationen, die sich erfolgreich für Bildung, Integration und Chancengerechtigkeit einsetzen. aqtivator unterstützt die Professionalisierung und das Wachstum der geförderten Organisationen. Ein besonderer Fokus liegt dabei auf der Entwicklung nachhaltiger Skalierungsmodelle. Gründer und Gesellschafter von aqtivator ist der Unternehmer Stefan Quandt.

23 Prof. Dr. Olaf Köller (IPN, Kiel), Prof. Dr. Johannes Magenheim (Uni Paderborn), Dr. Uwe Pfenning (DZLR, Stuttgart), Prof. Dr. Jörg Ramseger (FU Berlin), Prof. Dr. Mirjam Steffensky (IPN, Kiel), Prof. Christian Wiesmüller (PH Karlsruhe), Prof. Dr. Esther Winther (DIE, Bonn) Prof. Dr. Bernd Wollring (Uni Kassel) 


\section{Weitere wissenschaftliche Begleitung und Längsschnittansatz}

Mit EASI Science und EASI Science-L haben die Forschungsgruppen wissenschaftliches Neuland betreten und erstmals valide empirische Instrumente zur Messung früher naturwissenschaftlicher Bildungswirkungen entwickelt. Mit den entwickelten Messverfahren lassen sich die Wirkungen von naturwissenschaftlichen (Fort-)Bildungsangeboten auf naturwissenschaftliche Kompetenzen, sprachliche Anregungsqualität sowie die Prozessqualität der gestalteten naturwissenschaftlichen Bildungsangebote systematisch erfassen. Zudem sind mit den entwickelten Verfahren Aussagen zur Wirkung auf die naturwissenschaftlichen und bildungssprachlichen Kompetenzen von Kindern möglich. Die beiden von der Stiftung und ihren Partnern geförderten Studien leisten damit einen wichtigen Beitrag zur Wirkungsforschung im frühkindlichen Bereich. Durch die so mögliche Messung der Wirkungen naturwissenschaftlicher Bildungsangebote ist ein entscheidender Schritt in Richtung formative Angebotsweiterentwicklung für Fachkräfte und Qualitätsentwicklung in Kitas getan.

Zusammenfassend über alle Ergebnisse und Empfehlungen aus den Abschlussberichten hinweg lässt sich festhalten, dass weitere Forschung nötig ist, um gezielt Erkenntnisse über Wirkzusammenhänge und den Einfluss bestimmter Variablen gewinnen zu können. So werden vertiefende Studien mit Blick auf Fortbildungsangebot, Umsetzungsqualität und Wissenseffekte auf Kindebene, randomisierte Interventionsstudien, u. a. zur Überprüfung weiterer Zusammenhänge von Sach- und Sprachkompetenz oder auch zur Gegenüberstellung von pädagogischen Haltungen (z. B. „Begleiter“ vs. „Lehrer“) und Verhaltensweisen mit Bezug auf ihren Zusammenhang zum kindlichen Erkenntnisgewinn, angeregt. Zudem wird empfohlen, die in den Forschungsprojekten entwickelten Messinstrumente für weitere Forschung oder Evaluationen zu nutzen, was die Stiftung in den eigenen Monitoring- und Evaluationsmaßnahmen bereits umsetzt (s. o.).

Insgesamt bedarf es mehr und längerfristiger Forschung, um Wirkungen und Wirkungsweisen von frühen MINT-Bildungsangeboten gezielt abbilden und empirisch belegen zu können. Künftige Erhebungen sollten über längere Zeiträume und mit größeren Stichproben durchgeführt werden, um repräsentative Ergebnisse zu erzielen. Hierzu gilt es, Partner und Förderer zur Unterstützung solcher Forschungsvorhaben zu gewinnen. Die Stiftung wird die Erkenntnisse der vorliegenden Studien für die Konzeption neuer Projekte und Studien berücksichtigen. Im Rahmen der finanziellen Möglichkeiten sollen insbesondere längsschnittliche Betrachtungen umgesetzt werden.

Die längsschnittliche Perspektive spielt in den Evaluations- und Monitoring-Maßnahmen der Stiftung eine zunehmend wichtigere Rolle, um wichtige Informationen über die kurz-, mittel- und auch langfristigen Veränderungen durch 
den Besuch einer „Haus der kleinen Forscher“-Fortbildung zu erhalten. Die Stiftung „Haus der kleinen Forscher“ führt seit 2018 eine deutschlandweite Längsschnittbefragung unter pädagogischen Fach- und Lehrkräften durch. Es sollen mit dieser Befragung etwa 2.400 Pädagoginnen und Pädagogen aus Kita, Hort und Grundschule zu mehreren Zeitpunkten erreicht werden. Die pädagogischen Fachund Lehrkräften werden sowohl zu Beginn als auch unmittelbar nach dem Besuch einer „Haus der kleinen Forscher“-Fortbildung, sowie etwa zwei bis fünf Monate später und dann fortlaufend einmal jährlich befragt. Im Unterschied zur Frühjahrsbefragung der Stiftung, in der stets verschiedene Pädagoginnen und Pädagogen einmal jährlich befragt wurden (vgl. Evaluations- und Monitoring-Berichte seit $2009^{24}$ ), werden die Fach- und Lehrkräfte nun über einen langen Zeitraum bei der professionellen Entwicklung begleitet. Mit der Längsschnittbefragung werden die Wirkungen der verschiedenen „Haus der kleinen Forscher“-Fortbildungen auf die Einstellungen, das Wissen und die Umsetzung der Fortbildungsinhalte in der pädagogischen Praxis erhoben.

Im Zeitraum zwischen September 2018 und Januar 2019 werden in 17 ausgewählten Partnernetzwerken deutschlandweit in rund 220 „Haus der kleinen Forscher“-Fortbildungen zwei Fragebögen an alle Fortbildungsteilnehmenden verteilt. Einen Fragebogen erhalten die pädagogischen Fach- und Lehrkräfte zu Beginn der Fortbildung und einen weiteren Fragebogen zum Schluss. Durch den Vergleich der Angaben vor und nach der Fortbildung erhält die Stiftung Informationen darüber, ob und inwieweit der Besuch einer „Haus der kleinen Forscher“-Fortbildung unmittelbare Veränderungen bspw. hinsichtlich der Einstellungen oder des Wissens der Pädagoginnen und Pädagogen bewirkt. In der geplanten Folgebefragung werden die Einstellungen und andere Informationen der Befragten erneut erhoben, um mittelfristige Veränderungen zu messen. Außerdem wird gefragt, welche der und wie die Fortbildungsinhalte in der pädagogischen Arbeit mit Kindern in Kita, Hort und Grundschule umgesetzt wurden. In Zukunft werden dann alle teilnehmenden Fach- und Lehrkräfte einmal jährlich kontaktiert und gebeten, einige Fragen zu beantworten. Hierdurch erhält die Stiftung Informationen zu den langfristigen Veränderungen, bewirkt durch den Besuch einer oder mehrerer „Haus der kleinen Forscher“-Fortbildungen.

Die Angaben der Teilnehmer und Teilnehmerinnen zu einzelnen Fortbildungsinhalten ermöglichen zudem Rückschlüsse darauf, ob und inwieweit über alle Themenfortbildungen hinweg wichtige pädagogische und MINT-spezifische Fortbildungsinhalte thematisiert werden. So wird beispielsweise gefragt, ob die Fortbildungsteilnehmenden Informationen darüber erhalten haben, was die wichtigsten Aufgaben der Lernbegleitung sind. Zu Beginn der Längsschnittbefragung 
steht der Besuch einer Präsenzfortbildung im Fokus der Befragung, perspektivisch ist geplant, zudem Pädagoginnen und Pädagogen in Verbindung mit der Nutzung eines Online-Angebots zu befragen und den Pool der Befragungsteilnehmer und -teilnehmerinnen damit um eine sogenannte „Online-Stichprobe“ zu erweitern.

In der bisherigen Begleitforschung der Stiftung stand die Erarbeitung und Untersuchung von Zieldimensionen und Kompetenzmodellen im Fokus. Mit der bis Ende 2018 fertiggestellten externen Studie zu den „Entwicklungsverläufen von pädagogischen Fach- und Lehrkräften in der MINT-Bildung“ (EpFL MINT) wird an die bisherigen kompetenzorientierten Studien angeknüpft. Sie kann idealerweise Erkenntnisse zu individuellen Entwicklungsverläufen liefern. Die Studie wurde von der Stiftung „Haus der kleinen Forscher“ und dem Bundesministerium für Bildung und Forschung gefördert und von Prof. Dr. Diemut Kucharz (Sprecherin), Nina Skorsetz, Dr. Lisa Röder von der Goethe-Universität Frankfurt im Zeitraum von Juli 2017 bis Dezember 2018 durchgeführt.

Zielstellung der Studie war es, besser zu verstehen, welche Faktoren innerhalb der Entwicklungsverläufe von pädagogischen Fach- und Lehrkräften eine Rolle spielen und inwiefern Fortbildungen diese Aspekte beeinflussen. Erste Ergebnisse zeigen, dass sich Entwicklungsverläufe z. B. von Motivation und Einstellungen beschreiben lassen, und dass Fortbildungen diese Aspekte positiv beeinflussen können. Da die Stiftung ihre Angebote in Zukunft noch passgenauer und möglichst bedarfsgerecht (weiter-)entwickeln möchte, sollten insbesondere deren Lernbedarfe in verschiedenen Phasen ihrer Entwicklung als MINT-pädagogische Fachkraft sowie die Umsetzung von Lerninhalten aus Fortbildungsaktivitäten in den pädagogischen Alltag in der Einrichtung untersucht werden. Hierdurch möchte die Stiftung wichtige Erkenntnisse darüber gewinnen, wie sich Lernbedarfe über die Zeit bzw. individuell verändern und welche Stiftungsangebote zu welchem Zeitpunkt der Lernhistorie die Professionsentwicklung bestmöglich unterstützen. Die Ergebnisse der EpFL MINT-Studie werden in der wissenschaftlichen Schriftenreihe veröffentlicht (Stiftung Haus der kleinen Forscher, in Vorb. b).

Die Stiftung wird auch zukünftig Begleitforschung betreiben, um die Qualität ihrer Angebote wirkungsorientiert zu verbessern. Zudem versteht sich die Stiftung als eine lernende Organisation und reflektiert sich regelmäßig durch externe Evaluation und Begutachtung. 2013 fand eine institutionelle Evaluation der Stiftung in Anlehnung an das Leibniz-Verfahren statt (Köller, Euler, Hartinger, Heise, Koglin, Küpper \& Weber, 2013). In 2018 wurde die Wirkungsorientierung der Stiftung im Rahmen des MINT-Themenreports von PHINEO, einem gemeinnützigen Analyse- und Beratungshaus für wirkungsvolles gesellschaftliches Engagement, be- 
wertet $^{25}$ und erhielt das Wirkt-Siegel mit einer sehr positiven Gesamtbewertung (Phineo-Themenreport, 2018).

Auch in Zukunft wird eine regelmäßige Reflexion und wirkungsorientierte Weiterentwicklung der Stiftungsangebote unter Einbindung externer Expertinnen und Experten und unabhängiger Perspektiven erfolgen. Dabei ist es der Stiftung ein Anliegen, das Zusammenspiel zwischen Forschung und pädagogischer Praxis (z. B. Definition gemeinsamer Fragestellungen, Verständnis für Daten und ihre Nutzung, schnellere Zyklen des Diskurses über Forschungsprozesse und Ergebnisinterpretation, Bedeutung für politische Entscheidungen) weiter voranzubringen. Die Initiative „Haus der kleinen Forscher“ kann sich so gemeinsam mit allen Beteiligten weiter als wirkungsvolle Bildungsinitiative etablieren und zur Qualitätsentwicklung der Bildungsarbeit in den Einrichtungen beitragen.

25 Das unabhängige Analyse- und Beratungshaus PHINEO wurde aus der Bertelsmann Stiftung heraus gegründet und unterstützt gemeinnützige Organisationen sowie Investorinnen und Inverstoren dabei, sich wirkungsvoll gesellschaftlich zu engagieren. Diese Unterstützung erfolgt anhand von Wirkungsanalysen, Publikationen, Workshops, Beratung und der Vergabe des Wirkt-Siegels, welches nur solchen Organisationen verliehen wird, die sich nach einer intensiven Prüfung als wirkungsorientiert erweisen. 



\section{Literatur}

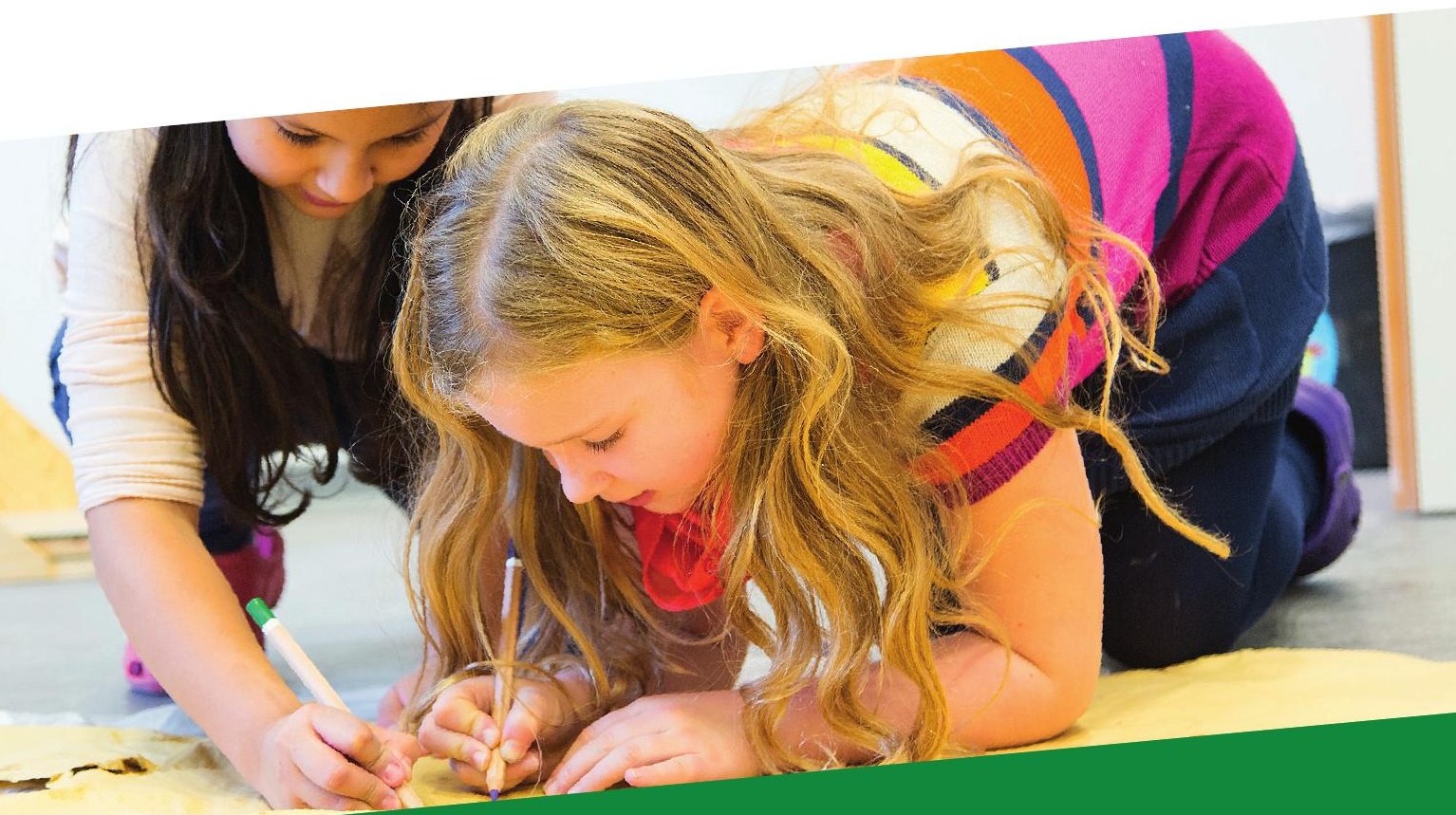




\section{Literatur}

\section{Geleitwort -}

Hans-Günther Roßbach

Egert, F. (2015). Meta-analysis on the impact of in-service professional development programs for preschool teachers on quality ratings and child outcomes. Dissertation, Bamberg: University of Bamberg Press.

NICHD Early Child Care Research Network (2002). Early Child Care and Children's Development prior to School Entry: Results from the NICHD Study of Early Child Care. American Educational Research Journal, 39, 133-164.

Stiftung Haus der kleinen Forscher (2017). Monitoring-Bericht 2016/2017 der Stiftung „Haus der kleinen Forscher“. Berlin: Stiftung Haus der kleinen Forscher. Verfügbar unter www.haus-der-kleinen-forscher.de

\section{Einleitung -}

Stiftung Haus der kleinen Forscher

Anders, Y. (2013). Stichwort: Auswirkungen frühkindlicher institutioneller Betreuung und Bildung. Zeitschrift für Erziehungswissenschaft, 16(2), 237-275.

Anders, Y. \& Ballaschk, I. (2014). Studie zur Untersuchung der Reliabilität und Validität des Zertifizierungsverfahrens der Stiftung „Haus der kleinen Forscher“. In Stiftung Haus der kleinen Forscher (Hrsg.), Wissenschaftliche Untersuchungen zur Arbeit der Stiftung „Haus der kleinen Forscher“" (Band 6, S. 35-116). Schaffhausen: Schubi Lernmedien AG.

Barnett, W. S. (2011). Effectiveness of early educational intervention. Science, 333(6045), 975-978. doi: 10.1126/science.1204534

BMBF (2017). Profis für die Kita. Ergebnisse und Impulse der Forschung zur Aus-, Fort- und Weiterbildung von pädagogischen Fachkräften. Berlin: BMBF.

Bybee, R., McCrae, B., \& Laurie, R. (2009). PISA 2006: An assessment of scientific literacy. Journal of Research in Science Teaching, 46(8), 865-883.

Deutsches Jugendinstitut (DJI)/Weiterbildungsinitiative Frühpädagogische Fachkräfte (Hrsg.). (2018). Frühe naturwissenschaftliche Bildung. Grundlagen für die kompetenzorientierte Weiterbildung. WiFF Wegweiser Weiterbildung (Band 13). München.

Köller, O. \& Spieß, C. K. (2018). „Potentiale früher Bildung: Früh übt sich, ... “Positionspapier. Berlin: Leibniz-Forschungsverbund Bildungspotenziale (LERN). Zugriff am 15.11.2018 unter http://www.leibniz-bildungspotenziale.de/pdf/Positionspapier_BPF2018.pdf

Marquardt-Mau, B. (2004). Ansätze zur Scientific Literacy. Neue Wege für den Sachunterricht. In Kaiser, A. \& Pech, D. (Hrsg.), Neuere Konzeptionen und Zielsetzungen im Sachunterricht (S. 67-83). Hohengehren: Schneider.

Marquardt-Mau, B. (2011). Der Forschungskreislauf: Was bedeutet forschen im Sachunterricht? In Deutsche Telekom Stiftung \& Deutsche Kinder- und Jugendstiftung (Hrsg.), Wie 
gute naturwissenschaftliche Bildung an Grundschulen gelingt. Ergebnisse und Erfahrungen aus Prima(r)forscher (S. 37). Berlin: DKJS.

Melhuish, E., Ereky-Stevens, K., Petrogiannis, K., Ariescu, A., Penderi, E., Rentzou, K. \& Leseman, P. (2015). A review of research on the effects of Early Childhood Education and Care (ECEC) upon child development.

Norris, S. P. \& Phillips, L. M. (2003). How literacy in its fundamental sense is central to scientific literacy. Science Education, 87(2), 224-240. doi:10.1002/sce.10066

OECD (2018). The Future of Education and Skills. Education 2030. Paris: OECD. Zugriff am 15.11.2018 unter https://www.oecd.org/education/2030/E2030\%20Position $\% 20$ Paper\%20(05.04.2018).pdf

Steffensky, Mirjam (2017). Naturwissenschaftliche Bildung in Kindertageseinrichtungen. Weiterbildungsinitiative Frühpädagogische Fachkräfte. WiFF Expertise (Band 48). München.

Stiftung Haus der kleinen Forscher (Hrsg.). (2013). Wissenschaftliche Untersuchungen zur Arbeit der Stiftung „Haus der kleinen Forscher“(Band 5). Schaffhausen: Schubi Lernmedien AG. Verfügbar unter www.haus-der-kleinen-forscher.de

Stiftung Haus der kleinen Forscher (Hrsg.) (2015). Frankfurter Erklärung zur frühen sprachlichen und naturwissenschaftlichen Bildung. Verfügbar unter www.haus-der-kleinenforscher.de

Stiftung Haus der kleinen Forscher (2017a). Monitoring-Bericht 2016/2017 der Stiftung „Haus der kleinen Forscher“. Berlin: Stiftung Haus der kleinen Forscher. Verfügbar unter www.haus-der-kleinen-forscher.de

Stiftung Haus der kleinen Forscher (2017b). Zertifizierung für Kitas, Horte und Grundschulen. So wird Ihre Einrichtung ein „Haus der kleinen Forscher“. (4. Auflage). Berlin: Stiftung Haus der kleinen Forscher. Verfügbar unter www.haus-der-kleinen-forscher.de

Stiftung Haus der kleinen Forscher (2018a). Forschergeist-Dokumentation. Verfügbar unter www.haus-der-kleinen-forscher.de

Stiftung Haus der kleinen Forscher (2018b). Forschungskreis. Berlin: Stiftung Haus der kleinen Forscher. Verfügbar unter www.haus-der-kleinen-forscher.de

Stiftung Haus der kleinen Forscher (in Vorb.). Der Pädagogische Ansatz der Stiftung „Haus der kleinen Forscher". (6. Auflage). Berlin: Stiftung Haus der kleinen Forscher.

Vandell, D. L., Belsky, J., Burchinal, M., Steinberg, L., Vandergrift, N., \& NICHD Early Child Care Research Network. (2010). Do effects of early child care extend to age 15 years? Results from the NICHD study of early child care and youth development. Child development, 81(3), 737-756.

\section{Early Steps into Science - EASI Science -}

Mirjam Steffensky, Yvonne Anders, Julia Barenthien, Ilonca Hardy, Miriam Leuchter, Elisa Oppermann, Päivi Taskinen, Tobias Ziegler

Anders, Y. (2012). Modelle professioneller Kompetenzen für frühpädagogische Fachkräfte. Aktueller Stand und ihr Bezug zur Professionalisierung. München: vbm.

Anders, Y. (2013). Stichwort: Auswirkungen frühkindlicher institutioneller Betreuung und Bildung. Zeitschrift für Erziehungswissenschaft, 16(2), 237-275. doi: 10.1007/ s11618-013-0357-5 
Anders, Y., Hardy, I., Pauen, S. \& Steffensky, M. (2013). Zieldimensionen früher naturwissenschaftlicher Bildung im Kita-Alter und ihre Messung. In Stiftung „Haus der kleinen Forscher“ (Hrsg.), Wissenschaftliche Untersuchungen zur Arbeit der Stiftung „Haus der kleinen Forscher" (Band 5, S. 19-82). Schaffhausen: Schubi Lernmedien AG.

Anders, Y. \& Ballaschk, I. (2014). Studie zur Untersuchung der Reliabilität und Validität des Zertifizierungsverfahrens der Stiftung „Haus der kleinen Forscher“. In Stiftung Haus der kleinen Forscher (Hrsg.), Wissenschaftliche Untersuchungen zur Arbeit der Stiftung „Haus der kleinen Forscher“ (Band 6, S. 35-116). Schaffhausen: Schubi Lernmedien AG.

Anders, Y. \& Rossbach, H.-G. (2015). Preschool Teachers' Sensitivity to Mathematics in Children's Play: The Influence of Math-Related School Experiences, Emotional Attitudes, and Pedagogical Beliefs. Journal of Research in Childhood Education, 29(3), 305-322. doi: $10.1080 / 02568543.2015 .1040564$

Appleton, K. (1995). Student teachers' confidence to teach science: Is more science knowledge necessary to improve self-confidence?. International Journal of Science Education, 17(3), 357-369.

Aubrey, C. (1997). Re-assessing the role of teachers' subject knowledge in early years teaching, Education Today, 25(1), 55-60.

Baumert, J. \& Kunter, M. (2006). Stichwort: Professionelle Kompetenz von Lehrkräften. Zeitschrift für Erziehungswissenschaft, 9(4), 469-520.

Baumert, J. \& Kunter, M. (2011). Das mathematikspezifische Wissen von Lehrkräften, kognitive Aktivierung im Unterricht und Lernfortschritte von Schülerinnen und Schülern. In M. Kunter, J. Baumert \& W. Blum (Hrsg.), Professionelle Kompetenz von Lehrkräften: Ergebnisse des Forschungsprogramms COACTIV (S. 163-192). Münster: Waxmann Verlag.

Baumert, J., Kunter, M., Blum, W., Brunner, M., Voss, T., Jordan, A., Klusmann, U., Krauss, S., Neubrand, M., Tsai, Y.-M. (2010). Teachers' Mathematical Knowledge, Cognitive Activation in the Classroom, and Student Progress. American Educational Research Journal, 47(1), 133-180. doi:10.3102/0002831209345157

Barros, S. \& Aguiar, C. (2010). Assessing the quality of Portuguese child care programs for toddlers. Early Childhood Research Quarterly, 25(4), 527-535.

Blatchford, P. (2003). A systematic observational study of teachers' and pupils' behaviour in large and small classes. Learning an Instruction, 13, 569-595.

Blömeke, S., Kaiser, G. \& Lehmann, R. (2008). Professionelle Kompetenz angehender Lehrerinnen und Lehrer. Wissen, Überzeugungen und Lerngelegenheiten deutscher Mathematikstudierender und -referendare - Erste Ergebnisse zur Wirksamkeit der Lehrerausbildung. Münster: Waxmann Verlag.

Blömeke, S., Gustafsson, J.-E. \& Shavelson, R. (2015). Beyond dichotomies: Competence viewed as a continuum. Zeitschrift für Psychologie, 223, 3-13.

Bromme, R. (1997). Kompetenzen, Funktionen und unterrichtliches Handeln des Lehrers. In F. E. Weinert (Hrsg.), Enzyklopadie der Psychologie: Psychologie des Unterrichts und der Schule (S. 177-212). Göttingen: Hogrefe.

Bulunuz, M. (2013). Teaching science through play in kindergarten: does integrated play and science instruction build understanding? European Early Childhood Education Research Journal, 21(2), 226-249. doi:10.1080/1350293X.2013.789195 
Bybee, R., McCrae, B. \& Laurie, R. (2009). PISA 2006: An assessment of scientific literacy. Journal of Research in Science Teaching, 46(8). 865-883. doi:10.1002/tea.20333

Carey, S. (2009). The origin of concepts. New York: Oxford University Press.

Carlisle, J. F., Correnti, R., Phelps, G. \& Zeng, J. (2009). Exploration of the contribution of teachers' knowledge about reading to their students' improvement in reading. Reading and Writing, 22(4), 457-486.

Carstensen, C. H., Lankes, E. M. \& Steffensky, M. (2011). Ein Modell zur Erfassung naturwissenschaftlicher Kompetenz im Kindergarten. Zeitschrift für Erziehungswissenschaft, 14, 651-669.

de Schipper, E. J., Riksen-Walraven, J. M. \& Geurts, S. A. (2006). Effects of child-caregiver ratio on the interactions between caregivers and children in child-care centers: an experimental study. Child Development, 77(4), 861-874.

Dirks, C. \& Cunningham, M. (2006). Enhancing diversity in science: Is teaching science process skills the answer? CBE Life Science Education, 5, 218-226.

diSessa, A. A. (2006). A history of conceptual change research: Threads and fault lines. In R. K. Sawyer, (Hrsg.), The Cambridge handbook of the learning sciences (S. 265-281). Cambridge: Cambridge University Press.

Dubberke, T., Kunter, M., McElvany, N., Brunner, M. \& Baumert, J. (2008). Lerntheoretische Überzeugungen von Mathematiklehrkräften. Zeitschrift für Pädagogische Psychologie, 22(34), 193-206. doi: 10.1024/1010-0652.22.34.193

Dunekacke, S., Jenßen, L. \& Blömeke, S. (2015). Effects of mathematics content knowledge on pre-school teachers' performance: A video-based assessment of perception and planning abilities in informal learning situations. International Journal of Science and Mathematics Education, 13(2), 267-286. doi: 10.1007/s10763-014-9596-z

Dunn, L. M. \& Dunn, L. M. (2004). Peabody Picture Vocabulary Test (PPVT) (deutsche Version). Göttingen: Hogrefe.

Eccles, J. S., Adler, T. F., Futterman, R., Goff, S. B., Kaczala, C. M., Meece, J. L. \& Midgley, C. (1983). Expectancies, values, and academic behaviors. In J. T. Spence (Hrsg.), Achievement and achievement motivation (S. 75-146). San Francisco, CA: W. H. Freeman.

Eccles, J., Wigfield, A., Harold, R. D. \& Blumenfeld, P. (1993). Age and Gender Differences in Children's Self- and Task Perceptions during Elementary School. Child Development, 64(3), 830-847.

Eccles, J. S. \& Wigfield, A. (2002). Motivational Beliefs, Values, and Goals. Annual Review of Psychology, 53(1), 109-132. doi: 10.1146/annurev.psych.53.100901.135153

Edens, K. M. \& Potter, E. F. (2013). An Exploratory Look at the Relationships Among Math Skills, Motivational Factors and Activity Choice. Early Childhood Education Journal, 41(3), 235-243. doi: 10.1007/s10643-012-0540-y

Eisenberg, N., DeLoache, J. \& Siegler, R. (2005) Entwicklungspsychologie im Kindes- und Jugendalter (deutsche Auflage herausgegeben von Sabina Pauen). München: Spektrum.

Erden, F. T. \& Sönmez, S. (2010). Study of Turkish Preschool Teachers' Attitudes toward Science Teaching. International Journal of Science Education, 33(8), 1149-1168. doi: 10.1080/09500693.2010.511295

Eshach, H. (2003). Inquiry-Events as a Tool for Changing Science Teaching Efficacy Belief of Kindergarten and Elementary School Teachers. Journal of Science Education and Technology, 12(4), 495-501. doi:10.1023/B:J0ST.0000006309.16842.c8 
Faulkner-Schneider, L. A. (2005). Child care teachers' attitudes, beliefs, and knowledge regarding science and the impact on early childhood learning opportunities. (Master of Science), University of Oklahoma, Norman, Oklahoma.

Fröhlich-Gildhoff, K., Nentwig-Gesemann, I. \& Pietsch, S. (2011). Kompetenzorientierung in der Qualifizierung frühpädagogischer Fachkräfte. WiFF Expertise (Band 19). München.

Fröhlich-Gildhoff, K., Nentwig-Gesemann, I., Pietsch, S., Köhler, L. \& Koch, M. (2014). Kompetenzentwicklung und Kompetenzerfassung in der Frühpädagogik: Konzepte und Methoden. Materialien zur Frühpädagogik (Vol. 13). Freiburg: FEL-Verl.

Fthenakis, W. E. (2009). Frühe naturwissenschaftliche Bildung. Troisdorf: Bildungsverlag Eins.

Garbett, D. (2003). Science Education in Early Childhood Teacher Education: Putting Forward a Case to Enhance Student Teachers' Confidence and Competence. Research in Science Education, 33(4), 467-481. doi:10.1023/b:rise.0000005251.20085.62

Greenfield, D.B., Jirout, J., Dominguez, X., Greenberg, A., Maier, M. \& Fuccilo, J. (2009). Science in the Preschool Classroom: A Programmatic Research Agenda to Improve Science Readiness. Early Education and Development, 20(2), 238-264. doi:10.1080/10409280802595441

Gropen, J., Kook, J. F., Hoisington, C. \& Clark-Chiarelli, N. (2017). Foundations of Science Literacy: Efficacy of a Preschool Professional Development Program in Science on Classroom Instruction, Teachers' Pedagogical Content Knowledge, and Children's Observations and Predictions. Early Education and Development, 1(4), 1-25. doi:10.1080/10 409289.2017.1279527

Grossman, P. L. (1990). The making of a teacher: Teacher knowledge and teacher education. New York: Teachers College Press.

Grygier, P. (2008). Wissenschaftsverständnis von Grundschülern im Sachunterricht. Bad Heilbrunn: Verlag Julius Klinkhardt.

Guo, Y., Piasta, S. B., Justice, L. M. \& Kaderavek, J. N. (2010). Relations among preschool teachers' self-efficacy, classroom quality, and children's language and literacy gains. Teaching and Teacher Education, 26(4), 1094-1103. doi: http://dx.doi.org/10.1016/j. tate.2009.11.005

Hachfeld, A. \& Anders, Y. (2016). Dokumentation der Validierungsstudie im Rahmen des Projekts K2ID: Untersuchung der Validität der Fragebögen zur Messung von Kita-Qualität anhand von Beobachtungsverfahren. Unveröffentlichter Abschlussbericht. Berlin: Freie Universität Berlin, Arbeitsbereich Frühkindliche Bildung und Erziehung.

Hahn, I., Schöps, K., Rönnebeck, S., Martensen, M., Hansen, S., Saß, S., Dalehefte, I. M. \& Prenzel, M. (2013). Assessing scientific literacy over the lifespan - A description of the NEPS science framework and the test development/Die Erfassung naturwissenschaftlicher Kompetenz über die Lebensspanne - eine Beschreibung der Rahmenkonzeption und der Entwicklung des NEPS-Naturwissenschaftstests. Journal for educational research online, 5(2), 110.

Hardy, I., Kloetzer, B., Moller, K. \& Sodian, B. (2010). The analysis of classroom discourse: Elementary school science curricula advancing reasoning with evidence. Educational Assessment, 15, 197-221.

Harter, S. \& Pike, R. (1984). The pictorial scale of perceived competence and social acceptance for young children. Child Development, 55(6), 1969-1982. 
Heller, K.A. \& Perleth, C. (2000): Kognitiver Fähigkeitstest für 4. bis 12. Klassen. Revision (KFT 4-12+R). Göttingen: Hogrefe.

Hill, H. C., Beisiegel, M. \& Jacob, R. (2013). Professional Development Research: Consensus, Crossroads, and Challenges. Educational Researcher, 42(9), 476-487. doi:10.3102/0013189X13512674

Honig, S. L. (2010). A framework for supporting scientific language in primary grades. The Reading Teacher, 64(1), 23-32.

Hopf, M. (2012). Sustained shared thinking im frühen naturwissenschaftlich-technischen Lernen. Münster: Waxmann.

Hoy, W. K. \& Woolfolk, A. E. (1990). Socialization of student teachers. American Educational Research Journal, 27, 279-300.

Jenßen, L., Dunekacke, S., Baack, W., Tengler, M., Koinzer, T., Schmude, C., Grassmann, M., Wedekind, H. \& Blömeke, S. (2015). KomMa: Kompetenzmodellierung und Kompetenzmessung bei frühpädagogischen Fachkräften im Bereich Mathematik. In B. Koch-Priewe, A. Köker, J. Seifried \& E. Wuttke (Hrsg.), Kompetenzerwerb an Hochschulen: Modellierung und Messung. Zur Professionalisierung angehender Lehrerinnen und Lehrer sowie frühpädagogischer Fachkräfte (S. 59-79). Bad Heilbrunn: Verlag Julius Klinkhardt.

Justice, L. M., Mashburn, A., Hamre, B. \& Pianta, R. (2008). Quality of Language and Literacy Instruction in Preschool Classrooms Serving At-Risk Pupils. Early childhood research quarterly, 23(1), 51-68. doi:10.1016/j.ecresq.2007.09.004

Kallery, M. \& Psillos, D. (2001). Pre-school Teachers' Content Knowledge in Science: Their understanding of elementary science concepts and of issues raised by children's questions. International Journal of Early Years Education, 9(3), 165-179. doi:10.1080/09669760120086929

Kleickmann, T. (2008). Zusammenhänge fachspezifischer Vorstellungen von Grundschullehrkräften zum Lehren und Lernen mit Fortschritten von Schülerinnen und Schülern im konzeptuellen naturwissenschaftlichen Verständnis. Münster: Westfälischen Wilhelms-Universität Münster.

Kluczniok, K., Anders, Y. \& Ebert, S. (2011). Fördereinstellungen von Erzieherinnen. Einflüsse auf die Gestaltung von Lerngelegenheiten im Kindergarten und die kindliche Entwicklung früher numerischer Kompetenzen. Frühe Bildung, 0, 13-21. doi: 10.1026/21919186/a000002

Kluczniok, K. \& Roßbach, H.-G. (2014). Konzeption von Bildungsqualität in Kindergärten Qualität im Bildungs- und Wissenschaftssystem (S. 145-158). Wiesbaden: Springer VS.

Koballa, T. R. \& Crawley, F. E. (1985). The Influence of Attitude on Science Teaching and Learning.SchoolScience and Mathematics, 85(3), 222-232. doi:10.1111/j.1949-8594.1985. tb09615.x

Koerber, S., Sodian, B., Thoermer, C. \& Nett, U. (2005). Scientific reasoning in young children: Preschoolers' ability to evaluate covariation evidence. Schweizerische Zeitschrift für Psychologie, 64(3), 141-152. doi:10.1024/1421-0185.64.3.141

König, A. (2007). Dialogisch-entwickelnde Interaktionsprozesse als Ausgangspunkt für die Bildungsarbeit im Kindergarten. Bildungsforschung, 4(1), 21. doi:URN: urn:nbn:de:0111-opus-46225 
König, A. (2009). Interaktionsprozesse zwischen Erzieherinnen und Kindern: Eine Videostudie aus dem Kindergartenalltag (1. Aufl.). Wiesbaden: VS Verlag für Sozialwissenschaften/GWV Fachverlage.

Kowalski, K., Pretti-Frontczak, K. \& Johnson, L. (2001). Preschool Teachers' Beliefs Concerning the Importance of Various Developmental Skills and Abilities. Journal of Research in Childhood Education, 14(1), 5-14.

Krauss, S., Brunner, M., Kunter, M., Baumert, J., Blum, W., Neubrand, M. \& Jordan, A. (2008). Pedagogical content knowledge and content knowledge of secondary mathematics teachers. Journal of Educational Psychology, 100(3), 716-725. doi:10.1037/00220663.100.3.716

Kruse, L. (2016). Pädagogische Dialoge im Alltag von Kindertageseinrichtungen: Eine Untersuchung von „Sustained Shared Thinking“ zwischen frühpädagogischen Fachkräften und Kindern. (Masterarbeit). Freie Universität Berlin, Berlin.

Kuger, S. \& Kluczniok, K. (2008). Prozessqualität im Kindergarten - Konzept, Umsetzung und Befunde. In H.-G. Roßbach \& H.-P. Blossfeld (Hrsg.), Frühpädagogische Förderung in Institutionen (S. 15-178). Wiesbaden: VS Verl. für Sozialwissenschaften.

Kuhn, N., Lankes, E.-M. \& Steffensky, M. (2012). Vorstellungen pädagogischer Fachkräfte zum Lernen von Naturwissenschaften. In H. Giest, E. Heran-Dörr \& C. Archie (Hrsg.), Lernen und Lehren im Sachunterricht. Zum Verhältnis von Konstruktion und Instruktion (Band 22, S. 183-191). Bad Heilbrunn: Verlag Julius Klinkhardt.

Kunter, M., Klusmann, U., Dubberke, T., Baumert, J., Blum, W., Brunner, M., Jordan, A., Krauss, S., Löwen, K., Neubrand, M. \& Tsai, Y.-M. (2007). Linking aspects of teacher competence to their instruction: Results from the COACTIV project. In M. Prenzel (Hrsg.), Studies on the educational quality of schools: The final report on the DFG Priority Programme (S. 39-59). Münster: Waxmann.

Kunter, M., Baumert, J. \& Blum, W. (Hrsg.) (2011). Professionelle Kompetenz von Lehrkräften: Ergebnisse des Forschungsprogramms COACTIV. Münster: Waxmann Verlag.

Lee, J. (2010). Exploring Kindergarten Teachers' Pedagogical Content Knowledge of Mathematics. International Journal of Early Childhood, 42(1), 27-41.

Lohse-Bossenz, H., Zimmermann, M., Janke, M. \& Müller, S. (2015). Selbstwirksamkeit von frühpädagogischen Fachkräften im Bereich früher naturwissenschaftlicher Bildung. Paper presented at the GEBF, Bochum, Germany.

Lück, G. (2010). Naturwissenschaften im frühen Kindesalter. In W. E. Fthenakis \& P. Oberhuemer (Hrsg.), Frühpädagogik international: Bildungsqualität im Blickpunkt (S. 331343). Wiesbaden: Verlag für Sozialwissenschaften.

Maier, M. F., Greenfield, D. B. \& Bulotsky-Shearer, R. J. (2013). Development and validation of a preschool teachers' attitudes and beliefs toward science teaching questionnaire. Early Childhood Research Quarterly, 28, 366-378.

Mantzicopoulos, P., Patrick, H. \& Samarapungavan, A. (2008). Young children's motivational beliefs about learning science. Early Childhood Research Quarterly, 23, 378-394.

Marsh, H. W., Ellis, L. A. \& Craven, R. G. (2002). How do preschool children feel about themselves? Unraveling measurement and multidimensional self-concept structure. Developmental Psychology, 38(3), 376-393. doi: 10.1037/0012-1649.38.3.376

McCarty, F., Abbott-Shim, M. \& Lambert, R. (2001). The relationship between teacher beliefs and practices, and head start classroom quality. Early Education and Development, 12(2), 225-238. 
McCray, J. (2008). Pedagogical content knowledge for preschool mathematics: Relationships to teaching practices and child outcomes. Dissertation, Erikson Institute. Chicago, Illinois.

McCray, J. S. \& Chen, J.-Q. (2012). Pedagogical Content Knowledge for Preschool Mathematics: Construct Validity of a New Teacher Interview. Journal of Research in Childhood Education, 26(3), 291-307. doi:10.1080/02568543.2012.685123

Measelle, J. R., Ablow, J. C., Cowan, P. A. \& Cowan, C. P. (1998). Assessing Young Children's Views of Their Academic, Social, and Emotional Lives: An Evaluation of the Self-Perception Scales of the Berkeley Puppet Interview. Child Development, 69(6), 1556-1576. doi: 10.1111/j.1467-8624.1998.tb06177.x

Melchers, P. \& Preuß, U. (2009). Kaufman-Assessment Battery for Children - Deutschsprachige Fassung $(K-A B C)$ (8. unveränd. Aufl.). Frankfurt: Pearson Assessment.

Mischo, C., Wahl, S., Hendler, J. \& Strohmer, J. (2012). Pädagogische Orientierungen angehender frühpädagogischer Fachkräfte an Fachschulen und Hochschulen. Frühe Bildung, 1, 34-44.

Möller, K., Jonen, A., Hardy, I. \& Stern, E. (2002). Die Förderung von naturwissenschaftlichem Verständnis bei Grundschulkindern durch Strukturierung der Lernumgebung Bildungsqualität von Schule: Schulische und außerschulische Bedingungen mathematischer, naturwissenschaftlicher und überfachlicher Kompetenzen (S. 176-191). Weinheim: Beltz Verlag.

Möller, K. \& Steffensky, M. (2010). Naturwissenschaftliches Lernen im Unterricht mit 4- bis 8-jahrigen Kindern. Kompetenzbereiche frühen naturwissenschaftlichen Lernens. In M. Leuchter (Hrsg.), Didaktik für die ersten Bildungsjahre. Unterricht mit 4- bis 8-jahrigen Kindern (S. 163-178). Seelze: Friedrich Verlag.

Morgan, P. L., Farkas, G., Hillemeier, M. M. \& Maczuga, S. (2016). Science Achievement Gaps Begin Very Early, Persist, and Are Largely Explained by Modifiable Factors. Educational Researcher, 45(1), 18-35.

Muthén, L. \& Muthén, B. (2014). Mplus Version 7.2.

NICHD Early Child Care Research Network (2003). Does quality of child care affect child outcomes at age 41 /2? Journal of Developmental \& Behavioral Pediatrics, 24(5), 386. doi:10.1037/0012-1649.39.3.451

Nölke, C., Steffensky, M., Lankes, E.-M. \& Carstensen, C. (2013). Naturwissenschaftliches Interesse bei Vorschulkindern - Welche Möglichkeiten und Schwierigkeiten ergeben sich bei der Erfassung? In E. Wannack, S. Bosshart, A. Eichenberger, M. Fuchs, E. Hardegger \& S. Marti (Hrsg.), 4- bis 12-Jährige. Ihre schulischen und außerschulischen Lern- und Lebenswelten (S. 248-259). Münster: Waxmann.

Norris, S. P. \& Phillips, L. M. (2003). How literacy in its fundamental sense is central to scientific literacy. Science Education, 87(2), 224-240. doi:10.1002/sce.10066

OECD. (2011). Starting Strong III: A Quality Toolbox for Early Childhood Education and Care. Designing and implementing curriculum and standards. Paris: OECD Publishing.

Oppermann, E., Anders, Y. \& Hachfeld, A. (2016). The influence of preschool teachers' content knowledge and mathematical ability beliefs on their sensitivity to mathematics in children's play. Teaching and Teacher Education, 58, 174-184. doi: 10.1016/j. tate.2016.05.004 
Oppermann, E., Brunner, M., Eccles, J. S. \& Anders, Y. (2018). Uncovering young children's motivational beliefs about learning science. Journal of Research in Science Teaching, 55, 399-421. doi: 10.1002/tea.21424

Peterson, S. M. \& French, L. (2008). Supporting young children's explanations through inquiry science in preschool. Early Childhood Research Quarterly, 23(3), 395-408. doi:http://dx.doi.org/10.1016/j.ecresq.2008.01.003

Pianta, R. C., Howes, C., Burchinal, M., Bryant, D., Clifford, D., Early, D. \& Barbarin, O. (2005). Features of pre-kindergarten programs, classrooms, and teachers: Do they predict observed classroom quality and child-teacher interactions? Applied Developmental Science, 9, 144-159. doi:10.1207/s1532480xads0903_2

Pollmeier, J., Tröbst, S., Hardy, I., Möller, K., Kleickmann, T., Jurecka, A. \& Schwippert, K. (2017). Science-P I. Modeling Conceptual Understanding in Primary School. In D. Leutner, J. Fleischer, J.Grünkorn \& E. Klieme (Eds.), Competence Assessment in Education. Research, Models and Instruments (pp. 9-17). Berlin: Springer.

Resa, E., Groeneveld, I., Turani, D. \& Anders, Y. (2017). The role of professional exchange in improving language-related process quality in daycare centres. Research Papers in Education, 1-20.

Rentzou, K. \& Sakellariou, M. (2011). The quality of early childhood educators: Children's interaction in Greek child care centers. Early Childhood Education Journal, 38(5), 367376.

Roux, S. (2002). Wie sehen Kinder ihren Kindergarten? Theoretische und empirische Befunde zur Qualität von Kindertagesstätten. München und Weinheim: Juventa-Verlag.

Saçkes, M. (2013). Children's Competencies in Process Skills in Kindergarten and Their Impact on Academic Achievement in Third Grade. Early Education and Development, 24(5), 704-720. doi:10.1080/10409289.2012.715571

Saçkes, M. (2014). How often do early childhood teachers teach science concepts? Determinants of the frequency of science teaching in kindergarten. European Early Childhood Education Research Journal, 22(2), 169-184.

Saçkes, M., Trundle, K. C., Bell, R. L. \& O’Connell, A. A. (2011). The influence of early science experience in kindergarten on children's immediate and later science achievement: Evidence from the early childhood longitudinal study. Journal of Research in Science Teaching, 48(2), 217-235. doi:10.1002/tea.20395

Samarapungavan, A., Mantzicopoulos, P. \& Patrick, H. (2008). Learning science through inquiry in kindergarten. Science Education, 92, 868-908.

Samarapungavan, A., Mantzicopoulos, P., Patrick, H. \& French, B. (2009). The development and validation of the Science Learning Assessment (SLA): A measure of kindergarten science learning. Journal of Advanced Academics, 20, 502-535.

Samuelsson, I. P. \& Carlsson, M. A. (2008). The Playing Learning Child: Towards a pedagogy of early childhood. Scandinavian Journal of Educational Research, 52(6), 623-641.

Sechtig, J., Freund, U., Roßbach, H.-G. \& Anders, Y. (2012). Das Modellprojekt „KiDZ - Kindergarten der Zukunft in Bayern“ - Kernelemente, zentrale Ergebnisse der Evaluation und Impulse für die Gestaltung des Übergangs vom Kindergarten in die Grundschule. In S. Pohlmann-Rother \& U. Franz (Hrsg.), Kooperation von KiTa und Grundschule. Eine Herausforderung für das pädagogische Personal (S. 174-188). Köln: Carl Link.

Shulman, L. (1986). Those Who Understand: Knowledge Growth in Teaching. Educational Researcher, 15(2), 4-14. doi:10.3102/0013189X015002004 
Shulman, L. (1987). Knowledge and Teaching: Foundations of the New Reform. Harvard Educational Review, 57(1), 1-23. doi:10.17763/haer.57.1.j463w79r56455411

Siegler, R. S. \& Chen, Z. (2008). Differentiation and integration: Guiding principles for analyzing cognitive change. Developmental Science, 11(4), 433-448.

Siraj-Blatchford, I. \& Manni, L. (2008). “Would you like to tidy up now?”: An analysis of adult questioning in the English Foundation Stage. Early Years: An International Journal of Research and Development, 28(1), 5-22. doi:10.1080/09575140701842213

Siraj-Blatchford, I., Sylva, K., Muttock, S., Gilden, R. \& Bell, D. (2002). Researching effective pedagogy in the early years. Research Report No. 356. London, Oxford: University of London, University of Oxford.

Smith, C. L., Maclin, D., Houghton, C. \& Hennessey, M. G. (2000). Sixth-Grade Students' Epistemologies of Science: The Impact of School Science Experiences on Epistemological Development. Cognition and Instruction, 18(3), 349-422.

Snider, M. H. \& Fu, V. R. (1990). The effects of specialized education and job experience on early childhood teachers' knowledge of developmentally appropriate practice. Early Childhood Research Quarterly, 5(1), 69-78.

Sodian, B., Jonen, A. \& Thoermer, C. (2006). Die Natur der Naturwissenschaften verstehen. Implementierung wissenschaftstheoretischen Unterrichts in der Grundschule. In M. Prenzel \& L. Allolio-Näcke (Hrsg.), Untersuchungen zur Bildungsqualität von Schule. Abschlussbericht des DFG-Schwerpunktprogramms (S. 147-160). Münster u. a.: Waxmann.

Sodian, B. \& Mayer, D. (2013). Entwicklung des wissenschaftlichen Denkens im Vor- und Grundschulalter. In M. Stamm. \&. D. Edelmann (Hrsg.), Handbuch Frühkindliche Bildungsforschung (S. 617-631). Wiesbaden: VS Verlag für Sozialwissenschaften.

Spektor-Levy, O., Baruch, Y. K. \& Mevarech, Z. (2013). Science and Scientific Curiosity in Pre-school - The teacher's point of view. International Journal of Science Education, 35(13), 2226-2253. doi:10.1080/09500693.2011.631608

Staub, F. C. \& Stern, E. (2002). The nature of teachers' pedagogical content beliefs matters for students' achievement gains: Quasi-experimental evidence from elementary mathematics. Journal of Educational Psychology, 94(2), 344-355. doi:10.1037/00220663.94.2.344

Steffensky, M., \& Hardy, I. (2013). Spiralcurriculum Magnetismus: Bd. 1. Elementarbereich. (Spiralcurriculum Magnetismus: Naturwissenschaftlich arbeiten und denken lernen. Ein Curriculum vom Kindergarten bis zur 7. Klasse; Band 1). Seelze: Friedrich Verlag.

Stephan-Gramberg, S. \& Hardy, I. (2014). Die Koordination von Theorie und Evidenz bei Vorschulkindern: Ergebnisse einer Vorstudie zu Scaffolding-Maßnahmen. In B. Kopp, S. Martschinke, M. Munser-Kiefer, M. Haider, E.-M. Kirschhock, G. Ranger \& G. Renner (Hrsg.), Individuelle Förderung und Lernen in der Gemeinschaft (S. 158-161). Wiesbaden: Springer Fachmedien.

Stiftung Haus der kleinen Forscher (2013a). Forschungskreis. Berlin: Stiftung Haus der kleinen Forscher. Verfügbar unter www.haus-der-kleinen-forscher.de

Stiftung Haus der kleinen Forscher (Hrsg.) (2013b). Wissenschaftliche Untersuchungen zur Arbeit der Stiftung „Haus der kleinen Forscher“ (Band 5). Schaffhausen: Schubi Lernmedien AG. Verfügbar unter www.haus-der-kleinen-forscher.de

Stiftung Haus der kleinen Forscher (2015a). Monitoring-Bericht 2015. Berlin: Stiftung Haus der kleinen Forscher. Verfügbar unter www.haus-der-kleinen-forscher.de 
Stiftung Haus der kleinen Forscher (2015b). Pädagogischer Ansatz der Stiftung „Haus der kleinen Forscher". Anregungen für die Lernbegleitung in Naturwissenschaften, Mathematik und Technik. (5. Aufl.). Berlin: Stiftung Haus der kleinen Forscher. Verfügbar unter www.haus-der-kleinen-forscher.de

Stiftung Haus der kleinen Forscher (2017). Monitoring-Bericht 2016/17. Berlin: Stiftung Haus der kleinen Forscher. Verfügbar unter www.haus-der-kleinen-forscher.de

Stipek, D. (1991). Characterizing Early Childhood Education Programs. New Direction For Child Development, 53(3), 47-55.

Stipek, D. J., Givvin, K. B., Salmon, J. M. \& MacGyvers, V. L. (2001). Teachers' beliefs and practices related to mathematics instruction. Teaching and Teacher Education, 17(2), 213-226. doi:http://dx.doi.org/10.1016/S0742-051X(00)00052-4

Sylva, K., Melhuish, E., Sammons, P., Siraj-Blatchford, I. \& Taggart, B. (2004). The effective provision of pre-school education (EPPE) project: Final report: A longitudinal study funded by the DFES 1997-2004. London: University of London.

Tietze, W. (2008). Qualitätssicherung im Elementarbereich. In E. Klieme \& R. Tippelt (Hrsg.), Qualitätssicherung im Bildungswesen. (Vol. 53, S. 16-35). Weinheim u. a.: Beltz.

Tietze, W. \& Förster, C. (2005). Allgemeines pädagogisches Gütesiegel für Kindertageseinrichtungen. In A. Diller, H. R. Leu \& T. Rauschenbach (Hrsg.), Der Streit ums Gütesiegel. Qualitätskonzepte für Kindertageseinrichtungen (3. Band, S. 31-64). München: DJIVerlag.

Tietze, W., Meischner, T., Gänsfuß, R., Grenner, K., Schuster, K.-M., Völkel, P. \& Roßbach, H.-G. (Hrsg.). (1998). Wie gut sind unsere Kindergärten? Eine empirische Untersuchung zur pädagogischen Qualität in deutschen Kindergärten. Neuwied: Leuterhand.

Tietze, W., Rossbach, H.-G. \& Grenner, K. (2005). Kinder von 4 bis 8 Jahren. Zur Qualität der Erziehungs- und Bildungsinstitution Kindergarten, Grundschule und Familie. Weinheim: Beltz.

Tirosh, D. (2000). Enhancing prospective teachers' knowledge of children's conceptions: The case of division of fractions. Journal for research in Mathematics Education, 31(1), $5-25$.

Tirosh, D., Tsamir, P., Levenson, E. \& Tabach, M. (2011). From preschool teachers' professional development to children's knowledge: comparing sets. Journal of Mathematics Teacher Education, 14(2), 113-131. doi: 10.1007/s10857-011-9172-1

Tournier, M. (2016). Kognitiv anregende Fachkraft-Kind-Interaktionen im Elementarbereich: Eine qualitativ-quantitative Videostudie. Münster: Waxmann.

Trundle, K. C. \& Saçkes, M. (Hrsg.) (2015). Research in early childhood science education. NY: Springer.

Tu, T. (2006). Preschool Science Environment: What Is Available in a Preschool Classroom? Early Childhood Education Journal, 33(4), 245-251. doi: 10.1007/s10643-005-0049-8

Vosniadou, S., loannides, C., Dimitrakopoulou, A. \& Papademetriou, E. (2001). Designing learning environments to promote conceptual change in science. Learning and instruction, 11(4), 381-419.

Voss, T., Kleickmann, T., Kunter, M. \& Hachfeld, A. (2011). Überzeugungen von Mathematiklehrkräften. In M. Kunter, J. Baumert, W. Blum, U. Klusmann, S. Krauss \& M. Neubrand (Hrsg.), Forschung zur professionellen Kompetenz von Lehrkräften - Ergebnisse des Projekts COACTIV (S. 235-258). Münster: Waxmann.

Weikart, D. P. (2000). Early Childhood Education: Needs and Opportunity. Paris: UNESCO. 
Weinert, F. E. (2001). Leistungsmessungen in Schulen. Weinheim und Basel: Beltz.

Weiß, R. H. \& Osterland, J. (2012). Grundintelligenztest Skala 1 - Revision: CFT 1-R. Göttingen: Hogrefe.

Wertfein, M., Müller, K. \& Danay, E. (2013). Die Bedeutung des Teams für die Inter. aktionsqualität in Kinderkrippen. Frühe Bildung, 2(1), 20-27.

Wigfield, A. \& Eccles, J. S. (2000). Expectancy-Value Theory of Achievement Motivation. Contemporary Educational Psychology, 25, 68-81. doi:10.1006/ceps.1999.1015

Wigfield, A., Eccles, J. S. \& Rodriguez, D. (1998). The Development of Children's Motivation in School Contexts. Review of Research in Education, 23, 73-118. doi:10.2307/1167288

Wigfield, A., Eccles, J. S., Yoon, K. S., Harold, R. D., Arbreton, A. J. A., Freedman-Doan, C. \& Blumenfeld, P. C. (1997). Change in children's competence beliefs and subjective task values across the elementary school years: A 3-year study. Journal of Educational Psychology, 89(3), 451-469. doi:10.1037/0022-0663.89.3.451

Wilcox-Herzog, A. \& Ward, S. L. (2004). Measuring teachers' perceived interactions with children: A tool for assessing beliefs and intentions. Early Childhood Research \& Practice, 6(2).

Zaslow, M., Tout, K., Halle, T., Whittaker, J. V. \& Lavelle, B. (2010). Toward the identification of features of effective professional development for early childhood educators. Literature Review. Zugriff am 26.03.2017 unter https://www2.ed.gov/rschstat/eval/professional-development/literature-review.pdf.

Zimmermann, C. (2007). The development of scientific thinking skills in elementary and middle school. Developmental Review, 27(2), 172-223.

\section{Early Steps into Science and Literacy - EASI Science-L Teil 1 -}

\section{Astrid Rank, Anja Wildemann, Andreas Hartinger, Sabrina Tietze}

Ahrenholz, B. (2010). Bildungssprache im Sachunterricht in der Grundschule. In B. Ahrenholz (Hrsg.), Fachunterricht und Deutsch als Zweitsprache (S. 15-35). Tübingen: Narr Verlag.

Albers, T. (2009). Sprache und Interaktion im Kindergarten. Eine quantitativ-qualitative Analyse der sprachlichen und kommunikativen Kompetenzen von drei- bis sechsjährigen Kindern. Bad Heilbrunn: Klinkhardt.

Anders, Y., Hardy, I., Pauen, S.\& Steffensky, M.(2013). Zieldimensionen naturwissenschaftlicher Bildung im Kita-Alter und ihre Messung. In Stiftung „Haus der kleinen Forscher“ (Hrsg.), Wissenschaftliche Untersuchungen zur Arbeit der Stiftung „Haus der kleinen Forscher"(Band 5, S. 19-82).Schaffhausen: SCHUBI Lernmedien AG.

Becker, T. (2013). Narrative Muster und literale Konzeptualisierungen in mündlichen und schriftlichen Erzählungen. In T. Becker \& P. Wieler (Hrsg.), Erzählforschung und Erzähldidaktik heute. Entwicklungslinien. Konzepte. Perspektiven (Reihe Stauffenburg Deutschdidaktik, 1. Band, S. 193-212). Tübingen: Stauffenburg-Verlag

Beese, M. \& Benholz, C. (2013). Sprachförderung im Fachunterricht. Voraussetzungen. Konzepte und empirische Befunde. In C. Röhner \& B. Hövelbrinks (Hrsg.), Fachbezogene Sprachförderung in Deutsch als Zweitsprache. Theoretische Konzepte und empirische 
Befunde zum Erwerb bildungssprachlicher Kompetenzen (S. 37-56). Weinheim/Basel: Juventa.

Beller, S. \& Beller, E. K. (2009). Abschlussbericht des Projekts Systematische sprachliche Anregung im Kindergartenalltag zur Erhöhung der Bildungschancen 4- und 5-jähriger Kinder aus sozial schwachen und Migrantenfamilien - ein Modell der pädagogischen Intervention. Zugriff am 20.12.2016 unter http://www.beller-kkp.de/downloads/ ESIA2Abschlussbericht.pdf

Beller, K., Merkens, H. \& Preissing, C. (2007). Abschlussbericht des Projektes Erzieherqualifizierung zur Erhöhung des sprachlichen Anregungsniveaus in Tageseinrichtungen für Kinder - eine Interventionsstudie. Zugriff am 10.08.2016 unter http://www.beller-kkp. de/forschung-esia-erzieherfortbildung-sprachlich-interaktive-anregung.html

Bernstein, B. (1964). Elaborated and Restricted Codes: Their Social Origins and Some Consequences. American Anthropologist, 66, 55-69.

Bernstein, B. (1973). Ein sozio-linguistischer Ansatz zur Sozialisation: Mit einigen Bezügen auf Erziehbarkeit. In C. F. Graumann \& H. Heckhausen (Hrsg.), Pädagogische Psychologie. Entwicklung und Soziologie, 1, 257-287.

Briedigkeit, E. (2011). Institutionelle Überformung sprachlicher Handlungsmuster - Realisation von Fragetypen im Erzieherin-Kind(er)-Diskurs. Empirische Pädagogik, 25(4), 499-517.

Bronfenbrenner, U. \& Morris, P. A. (2006). The bioecological model of human development. In W. Damon \& R. M. Lerner (Hrsg.), Handbook of child psychology. Theoretical models of human development (S. 793-828). New York: John Wiley.

Brophy, K. \& Hancock, S. (1985). Adult-child interaction in an integrated preschool programme: Implications for teacher training. Early Child Development and Care, 22(4), 275-294.

Chomsky, N. (1981). Regeln und Repräsentationen. Frankfurt: Suhrkamp.

Croker, S. \& Buchanan, H. (2011). Scientific reasoning in a real-world context: The effect of prior belief and outcome on children's hypothesis-testing strategies. British Journal of Development Psychology, 29, 409-424. Zugriff am 05.08.2016 unter http://onlinelibrary.wiley.com/ doi/10.1348/026151010X496906/pdf

Cummins, J. (2000). Language, power and pedagogy. Bilingual children in the crossfire (Bilingual education and bilingualism). Clevedon: Multilingual Matters.

Curby, T. W., LoCasale-Crouch, J., Konold, T. R., Pianta, R. C., Howes, C., Burchinal, M., Bryant, D., Clifford, R., Early, D. \& Barbarin, O. (2009). The relations of observed pre-k classrooms quality profiles to children's academic achievement and social competence. Early Education and Development, 20(2), 346-372.

Denker, H. (2012). Bindung und Theory of Mind. Bildungsbezogene Gestaltung von Erzieherinnen-Kind-Interaktionen. Wiesbaden: Springer VS.

Dittmann, J. (2002). Der Spracherwerb des Kindes. Verlauf und Störungen. München: Beck.

Downer, J., Sabol, T. J. \& Hamre, B. (2010). Teacher-Child Interactions in the Classroom: Toward a Theory of Within- and Cross-Domain Links to Children's Developmental Outcomes. Early Education \& Development, 21(5), 699-723.

Early, D. M., Maxwell, K. L., Burchinal, M., Alva, S., Bender. R. H., Bryant, D., Cai, K., Clifford, R. M., Ebanks, C., Griffin, J. A., Henry, G. T., Howes, C., Iriondo-Perez, J., Jeon, H. J., Mashburn, A. J., Peisner-Feinberg, E., Pianta, R. C., Vandergrift, N. \& Zill, N. (2007). Teachers' education, classroom quality, and young children's academic skills: Re- 
sults from seven studies of preschool programs. Child Development, 78(2), 558-580. Zugriff am 07.08.2016 unter http://onlinelibrary.wiley.com/ doi/10.1111/j.14678624.2007.01014.x/full

Egert, F. (2015). Meta-analysis on the impact of in-service professional development programs for preschool teachers on quality ratings and child outcomes. Dissertation, Universität Bamberg. Zugriff am 20.12.2016 unter https://opus4.kobv.de/opus4-bamberg/frontdoor/index/index/docld/45682

Egert, F. \& Hopf, M (2016). Zur Wirksamkeit von Sprachförderung in Kindertageseinrichtungen in Deutschland. Ein narratives Review. Kindheit und Entwicklung, 25, 153-163.

Feilke, H. (2012). Bildungssprachliche Kompetenzen fördern und entwickeln. Praxis Deutsch, 233, 4-13.

Fried, L. (2011). Sprachförderstrategien in Kindergartengruppen - Einschätzungen und Ergebnisse mit DO-RESI. Empirische Pädagogik, 25(4), 543-562.

Fried, L. \& Briedigkeit, E. (2008). Sprachförderkompetenz - Selbst- und Teamqualifizierung für Erzieherinnen. Fachberatungen und Ausbilder. Berlin: Cornelsen Scriptor.

Gasteiger-Klicpera, B., Knapp, W. \& Kucharz, D. (2010). Abschlussbericht der Wissenschaftlichen Begleitung des Programms „Sag' mal was - Sprachförderung für Vorschulkinder“. Zugriff am 20.12.2016 unter http://www.ph-weingarten.de/zep/Projekte/Abschlussbericht_Sprachfoerderung_Landesstiftung_PH_Weingarten.pdf

Gispert, K. (2004). Lernen lernen. Lernmethodische Kompetenzen von Kindern in Tageseinrichtungen fördern. Weinheim/Basel: Beltz.

Gogolin, I. \& Lange, I. (2010). Durchgängige Sprachbildung. Eine Handreichung. FörMig Edition 2. Münster: Waxmann.

Gogolin, I. \& Lange, I. (2011). Bildungssprache und durchgängige Sprachbildung. In S. Fürstenau \& M. Gomolla (Hrsg.), Migration und schulischer Wandel: Mehrsprachigkeit (S. 107-127). Wiesbaden: Springer VS.

Gogolin, I. (2010). Was ist Bildungssprache? Grundschulunterricht, 57(4), 4-5.

Grießhaber, W. (2013). Die Rolle der Sprache bei der Vermittlung fachlicher Inhalte. In Röhner, Ch. \& Hövelbrinks, B. (Hrsg.), Fachbezogene Sprachförderung in Deutsch als Zweitsprache. Theoretische Konzepte und empirische Befunde zum Erwerb bildungssprachlicher Kompetenzen (S. 58-74). Weinheim/Basel: Beltz Juventa.

Grimm, H. (1999). Störungen der Sprachentwicklung. Grundlagen - Ursachen - Diagnose Intervention - Prävention. Göttingen: Hogrefe Verlag für Psychologie.

Habermas, J. (1977). Umgangssprache, Wissenschaftssprache, Bildungssprache. Jahrbuch Max-Planck-Gesellschaft (S. 340-363). Göttingen: Vandenhoeck \& Ruprecht.

Halliday, M. (1978). Language as social semiotic. The social interpretation of language and meaning. London: Edward Arnold.

Hopf, M. (2012). Sustained Shared Thinking im frühen naturwissenschaftlich-technischen Lernen. Münster: Waxmann.

Hövelbrinks, B. (2014). Bildungssprachliche Kompetenz von einsprachig und mehrsprachig aufwachsenden Kindern. Eine vergleichende Studie in naturwissenschaftlicher Lernumgebung des ersten Schuljahres. Weinheim/Basel: Beltz Juventa.

Howes, C., Burchinal, M., Pianta, R., Bryant, D., Early, D. M., Clifford, R. \& Barbarin, 0. (2008). Ready to learn? Children's pre-academic achievement in pre-Kindergarten programs. Early Childhood Research Quarterly, 23(1), 27-50. 
Huttenlocher, J., Vasilyeva, M., Cymerman, E. \& Levine, S. (2002). Language input and child syntax. Cognitive Psychology, 45, 337-374.

Jülisch, B. \& Häuser., D. (2003). Handlung und Sprache - ein Programm kitaintegrierter Sprachförderung. Berlin: NIF. Zugriff am 20.12.2016 unter http://www.integrative-foerderung.de/ download/ download_05.pdf

Juska-Bacher, B. \& Nodari, C. (2015): Sprachliche Voraussetzungen für den Schulerfolg. Babylonia, 3(13), 23-31.

Kammermeyer, G., Roux, S. \& Stuck, A. (2011). Was wirkt wie? - Evaluation von Sprachfördermaßnahmen in Rheinland-Pfalz. 2. Zwischenbericht. Zugriff am 20.12.2016 unter http://kita.bildung-rp.de/fileadmin/dateiablage/Themen/Downloads/Zweiter_Zwischenbericht_Endfassung.pdf

Kany, W. \& Schöler, H. (2014). Theorien zum Spracherwerb. In L. Ahnert (Hrsg.), Theorien in der Entwicklungspsychologie (S. 468-483). Berlin/Heidelberg: Springer.

Knobloch, C. (2016). Nähe und Distanz - betrachtet aus fachlicher Nähe und historiographischer Distanz. In H. Feilke \& M. Hennig (Hrsg.), Zur Karriere von „Nähe und Distanz“. Rezeption und Diskussion des Koch-Oesterreicher-Modells (Reihe Germanistische Linguistik, S. 73-87). Berlin/Boston: De Gruyter.

Köhnlein, W. (2012). Sachunterricht und Bildung. Bad Heilbrunn: Klinkhardt.

König, A. (2009). Interaktionsprozesse zwischen ErzieherInnen und Kindern. Eine Videostudie aus dem Kindergartenalltag. Wiesbaden: Springer VS.

Li, M. (2017). Zweitsprachförderung im frühen naturwissenschaftlichen Lernen. Linguistische hochwertige Formate und interaktive Elemente in der Unterrichtskommunikation. Weinheim/Basel: Beltz Juventa.

Liebers, K. (2016). Erwerb von Early Literacy unter dem Fokus Bildungsbenachteiligung. In E. Inckemann \& R. Sigel. R. (Hrsg.), Diagnose und Förderung von bildungsbenachteiligten Kindern im Schriftspracherwerb (S. 21-34). Bad Heilbrunn: Klinkhardt.

Leseman, P. P.M., Scheele, A.F., Mayo, A.Y \& Messer, M.H. (2007). Home literacy as special language environment to prepare children for school. Zeitschrift für Erziehungswissenschaft, 10(3), 334-355.

Lück, G. (2009). Handbuch der naturwissenschaftlichen Bildung. Theorie und Praxis für die Arbeit in Kindertageseinrichtungen. Freiburg: Herder.

Marquardt-Mau, B. (2004). Ansätze zur Scientific Literacy. Neue Wege für den Sachunterricht. In Kaiser, A. \& Pech, D. (Hrsg.), Neuere Konzeptionen und Zielsetzungen im Sachunterricht (S. 67-83). Hohengehren: Schneider.

Marquardt-Mau, B. (2011). Der Forschungskreislauf: Was bedeutet forschen im Sachunterricht? In Deutsche Telekom Stiftung \& Deutsche Kinder- und Jugendstiftung (Hrsg.), Wie gute naturwissenschaftliche Bildung an Grundschulen gelingt. Ergebnisse und Erfahrungen aus Prima(r)forscher (S. 37). Berlin: DKJS.

Mashburn, A. J., Pianta, R. C., Hamre, B. K., Downer, J. T., Barbarin, O., Bryant, D., Burchinal, M., Early, D. \& Howes, C. (2008). Measures of classroom quality in pre-kindergarten and children's development of academic, language and social skills. Child Development, 79(3), 732-749.

Meibauer, J. \& Rothweiler, M. (1999). Das Lexikon im Spracherwerb. Tübingen: Francke.

Morek, M. \& Heller, V. (2012). Bildungssprache - Kommunikative, epistemische, soziale und interaktive Aspekte ihres Gebrauchs. Zeitschrift für angewandte Linguistik, 57(1), 67-101. 
Muthén, L. K. \& Muthén, B. O. (2010): Mplus User‘s Guide. Statistical Analysis with Latent Variables. Los Angeles: Muthén \& Muthén. Zugriff am 27.01.2016 unter https://www. statmodel.com/ download/usersguide/Mplus\%20Users\%20Guide\%20v6.pdf

Ortner, H. (2009). Rhetorisch-stilistische Eigenschaften der Bildungssprache. In U. Fix., A. Gardt. \& J. Knape (Hrsg.), Rhetorik und Stilistik (S. 2227-2240).

Patterson, E. W. (2001). Structuring the composition process in scientific writing. International Journal of Science Education, 23, 1-16. Zugriff am 08.08.2016 unter http://www. tandfonline.com/ doi/pdf/10.1080/09500690117425

Preece, S. (2009). Posh talk. Language and identity in higher education. Basingstoke: Palgrave Macmillan.

Quasthoff, U. (2009). Entwicklung der mündlichen Kommunikationskompetenz. In M. Becker-Mrotzek (Hrsg.), Mündliche Kommunikation und Gesprächsdidaktik (Deutschunterricht in Theorie und Praxis, 3. Band, S. 84-100). Baltmannsweiler: Schneider-Verlag Hohengehren.

Quehl, T. (2010). Die Möglichkeiten des Scaffolding. Grundschulunterricht Deutsch, 4, 2832.

Quehl, T. \& Trapp, U. (2013). Sprachbildung im Sachunterricht der Grundschule (FörMig Material. Bd. 4). Münster: Waxmann.

Rank, A. (2016). Förderung der Bildungssprache im Fachkontext bei Vor- und Grundschulkindern. In E. Inckemann \& R. Sigel (Hrsg.), Diagnose und Förderung von bildungsbenachteiligten Kindern im Schriftspracherwerb (S. 35-46). Bad Heilbrunn: Klinkhardt.

Rank, A. \& Wildemann, A. (2015). Die Sachen versprachlichen. In J. Kahlert, M. Fölling-Albers, M. Götz, A. Hartinger, S. Miller \& S. Wittkowske (Hrsg.), Handbuch Didaktik des Sachunterrichts (2. Aufl., S. 474-479). Bad Heilbrunn: Klinkhardt.

Rank, A. \& Wildemann, A. (2016). Bildungssprache im Verhältnis zur kindlichen Sprachentwicklung - eine Bedingung für Individualisierung im Anfangsunterricht. In F. Heinzel \& K. Koch (Hrsg.), Individualisierung im Grundschulunterricht. Anspruch, Realisierung und Risiken (S. 67-71). Wiesbaden: Springer VS.

Rank, A., Wildemann, A. \& Hartinger, A. (2016). Sachunterricht - der geeignete Ort zur Förderung von Bildungssprache? www.widerstreit-sachunterricht.de, 22. Zugriff am 13.12.2016 unter http://www.widerstreit-sachunterricht.de/ebenel/superworte/foerder/rank_ua.pdf

Rickheit, G., Sichelschmidt, L. \& Strohner, H. (2007). Psycholinguistik. (Die Wissenschaft vom sprachlichen Verhalten und Erleben). Tübingen: Stauffenburg.

Röhner, Ch., Blümer, H., Hopf, M., Li, M. \& Hövelbrinks, B. (2009). Sprachförderung von Migrantenkindern im Kontext frühen naturwissenschaftlich-technischen Lernens. Abschlussbericht, Universität Wuppertal. Zugriff am 08.08.2016 unter http://www.erziehungswissenschaft.uni-wuppertal.de/fileadmin/erziehungswissenschaft/fach_paedagogik-der-fruehen-kindheit/Abschlussbericht-Nawiprojekt.pdf

Rösch, H. (2006). Das Jacobs-Sommercamp - neue Ansätze zur Förderung von Deutsch als Zweitsprache. In B. Ahrenholz (Hrsg.), Kinder mit Migrationshintergrund. Spracherwerb und Fördermöglichkeiten (S. 287-302). Freiburg: Fillibach.

Rösch, H. (2014). BeFo und die Folgen für die DaZ-Didaktik. In B. Lütke \& I. Petersen (Hrsg.), Deutsch als Zweitsprache: erwerben, lernen und lehren. Beiträge aus dem 9. Workshop „Kinder mit Migrationshintergrund“(S. 195-208). Stuttgart: Fillibach. 
Roos, J., Polotzek, S. \& Schöler, H. (2010). EVAS. Evaluationsstudie zur Sprachförderung von Vorschulkindern. Wissenschaftliche Begleitung der Sprachfördermaßnahmen im Programm „Sag’ mal was - Sprachförderung für Vorschulkinder“. Abschlussbericht: Unmittelbare und längerfristige Wirkungen von Sprachförderungen in Mannheim und Heidelberg. Heidelberg: Pädagogische Hochschule.

Runge, A. (2013). Die Nutzung von (bildungssprachlichen) Verben in naturwissenschaftlichen Aufgabenstellungen bei SchülerInnen der Jahrgangsstufen 4 und 5. In A. Redder \& S. Weinert (Hrsg.), Sprachförderung und Sprachdiagnostik: interdisziplinäre Perspektiven (S. 152-173). Münster: Waxmann.

Rydland, V., Grøver, V. \& Lawrence, J. F. (2014). The second-language vocabulary trajectories of Turkish immigrant children in Norway from ages five to ten: the role of preschool talk exposure, maternal education, and co-ethnic concentration in the neighborhood. Journal of Child Language, 41(2), 352-381.

Sachse, S., Budde, N., Rinker, T. \& Groth, K. (2012). Evaluation einer Sprachfördermaßnahme für Vorschulkinder. Frühe Bildung, 1(4), 194-201.

Schleppegrell, M. J. (2001). Linguistic Features of the Language of Schooling. Linguistics and Education, 12(4), 431-459.

Schleppegrell, M. J. (2004). The language of schooling. A functional linguistics perspective. New York: Routledge.

Schulz, P. \& Tracy, R. in Verbindung mit der Baden-Württemberg Stiftung (2011). Linguistische Sprachstandserhebung - Deutsch als Zweitsprache. Göttingen: Hogrefe.

Siraj-Blatchford, I., Sylva, K., Muttock. S., Gilden, R. \& Bell, D. (2002). Researching Effective Pedagogy in the Early Years. Research Report No. 356. Norwich: Queen's Printer.

Snow, C. E. \& Uccelli, P. (2009). The Challenge of Academic Language. In D. R. Olson \& N. Torrance (Hrsg.), The Cambridge handbook of literacy (S. 112-133). Cambridge. N.Y.: Cambridge University Press.

Snow, C. E., Tabors, P. O. \& Dickinson, D. K. (2001). Language development in the preschool years. In D. K. Dickinson \& P. O. Tabors (Hrsg.), Beginning Literacy with Language (S. 1-25). Baltimore: Paul H. Brookes Publishing Co.

Sterner, F., Skolaude, D., Ruberg, T. \& Rothweiler, M. (2014). Versuch macht klug und gesprächig. Materialien zur Anregung und Unterstützung von Sprachbildungsprozessen im Kontext naturwissenschaftlichen Experimentierens. Zugriff am 10.08.2016 unter http://www.elbkinder-kitas.de/ files/versuch_macht_klug/vmkug_heft_01_web.pdf

Stiftung Haus der kleinen Forscher (2013a). Forschungskreis. Berlin: Stiftung Haus der kleinen Forscher. Verfügbar unter www.haus-der-kleinen-forscher.de

Stiftung Haus der kleinen Forscher (2013b). Sprudelgas und andere Stoffe - Mit Kita- und Grundschulkindern Chemie entdecken und dabei die sprachliche Entwicklung unterstützen. Berlin: Stiftung Haus der kleinen Forscher. Zugriff am 08.11.2017 unter https:// www.haus-der-kleinen-forscher.de/fileadmin/Redaktion/1_Forschen/Themen-Broschueren/Broschuere-Sprudelgas_2013.pdf

Stiftung Haus der kleinen Forscher (2015a). Pädagogischer Ansatz der Stiftung „Haus der kleinen Forscher". Anregungen für die Lernbegleitung in Naturwissenschaften. Mathematik und Technik (5. Aufl.). Berlin: Stiftung Haus der kleinen Forscher. Zugriff am 08.11.2017 unter https://www.haus-der-kleinen-forscher.de/fileadmin/Redaktion/1_ Forschen/Paedagogik/Paedagogikbroschuere.pdf 
Stiftung Haus der kleinen Forscher (Hrsg.) (2015b). Frankfurter Erklärung zur frühen sprachlichen und naturwissenschaftlichen Bildung. Zugriff am 22.2.2016 unter http://www. haus-der-kleinen-forscher.de/fileadmin/Redaktion/4_Ueber_Uns/Evaluation/Frankfurter-Erklaerung_2015.pdf

Sylva, K., Melhuish, E., Sammons, P., Siraj-Blatchford, I., Taggart, B. \& Elliot, K. (2004). The Effective Provision of Pre-School Education Project - Zu den Auswirkungen vorschulischer Einrichtungen in England. In G. Faust, M. Götz, H. Hacker \& H.-G. Roßbach (Hrsg.), Anschlussfähige Bildungsprozesse im Elementar- und Primarbereich (S. 154-167). Bad Heilbrunn: Klinkhardt.

Szagun, G. (2013). Sprachentwicklung beim Kind. Ein Lehrbuch (5. Aufl.). Weinheim: Beltz.

Tietze, S., Rank, A. \& Wildemann, A. (2016). Erfassung bildungssprachlicher Kompetenzen von Kindern im Vorschulalter. Grundlagen und Entwicklung einer Ratingskala (RaBi). Zugriff am 20.07.2016 unter http://www.pedocs.de/frontdoor.php?source_ opus $=12076 \&$ la $=$ de

Weinert, S., Ebert, S.. Lockl, K. \& Kuger, S. (2012). Disparitäten im Wortschatzerwerb: Zum Einfluss des Arbeitsgedächtnisses und der Anregungsqualität in Kindergarten und Familie auf den Erwerb lexikalischen Wissens. Unterrichtswissenschaft, 40(1), 4-25.

Weinert, S. \& Grimm, H. (2012). Sprachentwicklung. In W. Schneider, U. Lindenberger, Entwicklungspsychologie (S. 433-456). Weinheim: Beltz.

Weinert, S., Stanat, P. \& Schuth, E. (о. J.). Verbundprojekt Bildungssprachliche Kompetenzen. Elternfragebogen. Bamberg \& Berlin. Unveröffentlichter Test.

Weinert, S., Stanat, P., Berendes, K., Dragon, N. \& Heppt, B. (o. J.). Bildungssprachliche Kompetenzen (BiSpra): Anforderungen. Sprachverarbeitung und Diagnostik. Skalenhandbuch zur Dokumentation der Erhebungsinstrumente. Zugriff am 02.02.2017 unter https://www.iqb.hu-berlin.de/fdz/studies/BiSpra_1/02_BiSpral_Skale.pdf

Weiß, R. H. \& Osterland, J. (2013). Grundintelligenztest Skala 1. Revision. Göttingen: Hogrefe.

Weitzmann, E. \& Greenberg, J. (2002). Learning language and loving it: A guide to promoting children's social, language and literacy development. Toronto: Hanen Centre.

Wildemann, A. \& Fornol, A. (2016). Sprachsensibel unterrichten in der Grundschule. Anregungen für den Deutsch-, Mathematik- und Sachunterricht. Seelze: Klett, Kallmeyer.

Wildemann, A., Rank, A., Hartinger, A. \& Sutter, S. (2016). Bildungssprache im Kontext kindlicher Entwicklung. Die deutsche Schule, 13. Beiheft, 64-81.

Wolf, K. M., Felbrich, A., Stanat, P. \& Wendt, W. (2011). Evaluation der kompensatorischen Sprachförderung in Brandenburger Kindertagesstätten. Empirische Pädagogik, 25(4), 423-438.

Wolf, K. M., Schroeders, U. \& Kriegbaum, K. (2016). Metaanalyse zur Wirksamkeit deutschsprachiger Programme zur Förderung der phonologischen Bewusstheit. Zeitschrift für Pädagogische Psychologie, 30, 9-33.

Yoshikawa, H., Leyva, D., Snow, C., Treviño, E., Arbour, M., Barata, C., Weiland, C., Gomez, C., Moreno, L., Rolla, A. \& D'Sa, N. (2015). Experimental impacts of a teacher professional development program in Chile on preschool classroom quality and child outcomes. Developmental Psychology, 51, 309-322. 


\title{
Early Steps into Science and Literacy - EASI Science-L Teil 2 -
}

\author{
Sabina Pauen, Rahel Kästner
}

Alake, E. M. \& Ogunseemi, O. E. (2013). Effects of scaffolding strategy on learners' academic achievement in integrated science at the junior secondary school level. European Scientific Journal, 9 (19), 149-155.

Anders, Y., Hardy, I., Pauen, S. \& Steffensky, M. (2013). Zieldimensionen naturwissenschaftlicher Bildung im Kita-Alter und ihre Messung. In Stiftung „Haus der kleinen Forscher“ (Hrsg.), Wissenschaftliche Untersuchungen zur Arbeit der Stiftung „Haus der kleinen Forscher" (Band 5, S. 19-82). Schaffhausen: SCHUBI Lernmedien AG.

Andersson, K. \& Gullberg, A. (2014). What is science in preschool and what do teachers have to know to empower children? Cultural studies of science education, 9(2), 275-296. doi: 10.1007/s11422-012-9439-6

Baleghizadeh, S., Timcheh Memar, A. \& Timcheh Memar, H. (2011). A sociocultural perspective on second language acquisition: The effect of high-structured scaffolding versus low-structured scaffolding on the writing ability of EFL learners. Reflections on English Language Teaching, 10(1), 43-54.

Bredekamp, S. \& Copple, C. (1997). Developmentally appropriate practice in early childhood programs (Revised edition). NAEYC: \#234. Washington, DC: National Association for the Education of Young Children.

Burchinal, M., Vandergrift, N., Pianta, R. \& Mashburn, A. (2010). Threshold analysis of association between child care quality and child outcomes for low-income children in pre-kindergarten programs. Early Childhood Research Quarterly, 25(2), 166-176. doi:10.1016/j.ecresq.2009.10.004

Carifio, J. \& Perla, R. J. (2007). Psychological, philosophical, and educational criticisms of Harry Frankfurt's concept of and views about "bullshit" in human discourse, discussions, and exchanges. Interchange, 38(2), 119-136.

Cazden, C.B. (1979). Peekaboo as an instructional model: Discourse development at home and at school. In Bain, B. (Eds.), The Sociogenesis of Language and Human Conduct (pp. 33-58). Boston, MA: Springer US

Deci, E. L. \& Ryan, R. M. (1985). Intrinsic motivation and self-determination in human behavior. New York: Plenum Press.

Demir, K. (2011). Teachers' intrinsic and extrinsic motivation as predictors of student engagement: an application of self-determination theory. nwsa: education sciences, 6(2), 1397-1409.

Eshach, H. \& Fried, M. N. (2005). Should Science be Taught in Early Childhood? Journal of Science Education and Technology, 14(3), 315-336. doi:10.1007/s10956-005-7198-9

Evanschitzky, P. (2011). Naturwissenschaften und Technik in Kindertageseinrichtungen: Eine Expertise zur Fortbildungsstruktur der Stiftung „Haus der kleinen Forscher“. In Stiftung „Haus der kleinen Forscher“ (Hrsg.), Wissenschaftliche Untersuchungen zur Arbeit der Stiftung „Haus der kleinen Forscher“ (Band 1, S. 63-94). Köln: Bildungsverlag EINS.

Fthenakis, W. E., Wendell, A., Eitel, A., Daut, M. \& Schmitt, A. (2009). Natur-Wissen schaffen: Band 3. Frühe naturwissenschaftliche Bildung. Troisdorf: Bildungsverlag Eins. 
Ginsburg, G. \& Bronstein, P. (1993). Family factors related to children's intrinsic/extrinsic motivational orientation and academic performance. Child Development, 64, 14611474.

Grolnick, W. S. (2003). The psychology of parental control: How well-meant parenting backfires. Mahwah, N.J.: L. Erlbaum Associates.

Hamre, B. K. (2014). Teachers' Daily Interactions With Children: An Essential Ingredient in Effective Early Childhood Programs. Child Development Perspectives, 8(4), 223-230. doi:10.1111/cdep.12090

Hsin, C.-T. \& Wu, H.-K. (2011). Using Scaffolding Strategies to Promote Young Children's Scientific Understandings of Floating and Sinking. Journal of Science Education and Technology, 20, 656-666. doi:10.1007/s10956-011-9310-7

Klahr, D. \& Dunbar K. (1988). Dual Space Search During Scientific Reasoning. Cognitive Science, 12, 1-48. doi:10.1016/0364-0213(88)90007-9

Kuhn, D. (2010). What is scientific thinking and how does it develop? In U. Goswami (Hrsg.), The Wiley-Blackwell Handbook of Childhood Cognitive Development (S. 497-523). 0xford: Wiley-Blackwwell.

Mariani, L. (1997). Teacher support and teacher challenge in promoting learner autonomy. Zugriff am 03.11.2015 unter http://www.learningpaths.org/papers/papersupport.htm

Marquardt-Mau, B. (2004). Ansätze zur Scientific Literacy. Neue Wege für den Sachunterricht. In Kaiser, A. \& Pech, D. (Hrsg.), Neuere Konzeptionen und Zielsetzungen im Sachunterricht (S. 67-83). Hohengehren: Schneider.

Marquardt-Mau, B. (2011). Der Forschungskreislauf: Was bedeutet forschen im Sachunterricht? In Deutsche Telekom Stiftung \& Deutsche Kinder- und Jugendstiftung (Hrsg.), Wie gute naturwissenschaftliche Bildung an Grundschulen gelingt. Ergebnisse und Erfahrungen aus Prima(r)forscher (S. 37). Berlin: DKJS.

Mashburn, A. J., Pianta, R. C., Hamre, B. K., Downer, J. T., Barbarin, O. A., Bryant, D. et al. (2008). Measures of classroom quality in prekindergarten and children's development of academic, language, and social skills. Child development, 79(3), 732-749. doi:10.1111/j.1467-8624.2008.01154.x

Neitzel, C. \& Stright, A. D. (2003). Mothers' scaffolding of children's problem solving: Establishing a foundation of academic self-regulatory competence. Journal of Family Psychology, 17(1), 147-159. doi:10.1037/0893-3200.17.1.147

Pahnke, J. \& Bartling, K. (2013). Einleitung. In Stiftung „Haus der kleinen Forscher“ (Hrsg.), Wissenschaftliche Untersuchungen zur Arbeit der Stiftung „Haus der kleinen Forscher“ (Band 5, S. 9-16). Schaffhausen: SCHUBI Lernmedien AG.

Pino-Pasternak, D. \& Whitebread, D. (2010). The role of parenting in children's self-regulated learning. Educational Research Review, 5(3), 220-242. doi:10.1016/j.edurev. 2010. 07. 001

Sabol, T. J., Hong, S. S., Pianta, R. C. \& Burchinal, M. R. (2013). Can rating pre-K programs predict children's learning? Science, 341(6148), 845-846.

Sodian, B. \& Mayer, D. (2013). Entwicklung des wissenschaftlichen Denkens im Vor- und Grundschulalter. In M. Stamm \& D. Edelmann (Hrsg.), Handbuch frühkindliche Bildungsforschung (S. 617-631). Wiesbaden: VS Verlag für Sozialwissenschaften.

Spindler, A. \& Berwanger, D. (2011). Expertise: Pädagogischer Ansatz und Multiplikatorenmodell der Stiftung „Haus der kleinen Forscher“. In Stiftung „Haus der kleinen For- 
scher“ (Hrsg.), Wissenschaftliche Untersuchungen zur Arbeit der Stiftung „Haus der kleinen Forscher" (Band 1, S. 18-62). Köln: Bildungsverlag EINS.

Stiftung Haus der kleinen Forscher (2010). Evaluationsbericht 2010. Verfügbar unter www. haus-der-kleinen-forscher.de

Stiftung Haus der kleinen Forscher (2011). Fazit und Ausblick - Wie das „Haus der kleinen Forscher" mit diesen Erkenntnissen umgeht. In Stiftung „Haus der kleinen Forscher" (Hrsg.), Wissenschaftliche Untersuchungen zur Arbeit der Stiftung „Haus der kleinen Forscher“ (Band 1, S. 164-170). Köln: Bildungsverlag EINS. Verfügbar unter: www. haus-der-kleinen-forscher.de

Stiftung Haus der kleinen Forscher (2013a). Fazit und Ausblick - Wie die Stiftung „Haus der kleinen Forscher“ mit diesen Erkenntnissen umgeht. In Stiftung „Haus der kleinen Forscher“ (Hrsg.), Wissenschaftliche Untersuchungen zur Arbeit der Stiftung „Haus der kleinen Forscher" (Band 5). Schaffhausen: SCHUBI Lernmedien AG. Verfügbar unter: www.haus-der-kleinen-forscher.de

Stiftung Haus der kleinen Forscher (2013b). Monitoring-Bericht 2013. Verfügbar unter www.haus-der-kleinen-forscher.de

Stiftung Haus der kleinen Forscher (2014). Monitoring-Bericht 2014. Verfügbar unter www. haus-der-kleinen-forscher.de

Stiftung Haus der kleinen Forscher (2015a). Pädagogischer Ansatz der Stiftung „Haus der kleinen Forscher": Anregungen für die Lernbegleitung in Naturwissenschaften, Mathematik und Technik (5. Auflage). Verfügbar unter www.haus-der-kleinen-forscher.de

Stiftung Haus der kleinen Forscher (2015b). Monitoring-Bericht 2015. Verfügbar unter www. haus-der-kleinen-forscher.de

Stright, A. D., Neitzel, C., Sears, K. G. \& Hoke-Sinex, L. (2001). Instruction begins in the home: Relations between parental instruction and children's self-regulation in the classroom. Journal of Educational Psychology, 93(3), 456-466. doi:10.1037/00220663.93.3.456

Tabachnick, B. G. \& Fidell, L. S. (2006). Using multivariate statistics. Needham Heights, Massachusetts: Allyn and Bacon.

van de Pol, J. (2012). Scaffolding in teacher-student interaction: exploring, measuring, promoting and evaluating scaffolding. Dissertation, retrieved August 31, 2016, from http://dare.uva.nl/record/1/392689

van de Pol, J., Volman, M. \& Beishuizen, J. (2010). Scaffolding in Teacher-Student Interaction: A Decade of Research. Educational Psychology Review, 22, 271-296. doi:10.1007/s

Vygotsky, L. S. (1978). Mind in society: The development of higher psychological processes. Cambridge, Mass.: Harvard University Press.

Webb, N. M. \& Mastergeorge, A. (2003). Promoting effective helping behavior in peer-directed groups. International Journal of Educational Research, 39(1-2), 73-97.

Williford, A. P., Maier, M. F., Downer, J. T., Pianta, R. C. \& Howes, C. (2013). Understanding how children's engagement and teachers' interactions combine to predict school readiness. Journal of Applied Developmental Psychology, 34(6), 299-309. doi:10.1016/j. appdev.2013.05.002

Williford, A. P., Vick Whittaker, J. E., Vitiello, V. E. \& Downer, J. T. (2013). Children's Engagement within the Preschool Classroom and Their Development of Self-Regulation. Early education and development, 24(2), 162-187. doi:10.1080/10409289.2011.628270 
Wood, D., Bruner, J. S. \& Ross, G. (1976). The role of tutoring in problem solving. Journal of child psychology and psychiatry, and allied disciplines, 17, 89-100. doi:10.1111/j.1469-7610.1976.tb00381.x

Yoon, J. \& Onchwari, J. A. (2006). Teaching Young Children Science: Three Key Points. Early Childhood Education Journal, 33(6), 419-423. doi:10.1007/s10643-006-0064-4

\section{Fazit und Ausblick -}

\section{Stiftung Haus der kleinen Forscher}

Anders, Y. \& Ballaschk, I. (2014). Studie zur Untersuchung der Reliabilität und Validität des Zertifizierungsverfahrens der Stiftung „Haus der kleinen Forscher“. In Stiftung Haus der kleinen Forscher (Hrsg.), Wissenschaftliche Untersuchungen zur Arbeit der Stiftung „Haus der kleinen Forscher“ (Band 6, S. 35-116). Schaffhausen: Schubi Lernmedien AG.

Egert, F. (2015). Meta-analysis on the impact of in-service professional development programs for preschool teachers on quality ratings and child outcomes. Dissertation, Bamberg: University of Bamberg Press.

Egert F. (in Vorb.). Metaanalyse zur Wirkung von additiver und alltagsintegrierter Sprachförderung in Kindertageseinrichtungen. Zeitschrift für pädagogische Psychologie.

Ferreira, J., Ryan, L. \& Tilbury, D. (2006). Whole-School Approaches to Sustainability: A review of models for professional development in pre-service teacher education. Canberra: ARIES.

Köller, O., Euler, M., Hartinger, A., Heise, N., Koglin, U., Küpper, W. \& Weber, M. (2013). Evaluation der Stiftung Haus der kleinen Forscher. Evaluationsbericht. Berlin: Stiftung Haus der kleinen Forscher. Verfügbar unter www.haus-der-kleinen-forscher.de

Phineo (2018). Themenreport: MINT-Bildung neu entdecken. Zugriff am 15.11.2018 unter https://www.phineo.org/beratung/themenreports

Ramseger, J. (2013). Prozessbezogene Qualitätskriterien für den naturwissenschaftlichen Unterricht - Zehn Kriterien für wirksames didaktisches Handeln im Elementar- und Primarbereich. In Stiftung Haus der kleinen Forscher (Hrsg.), Wissenschaftliche Untersuchungen zur Arbeit der Stiftung „Haus der kleinen Forscher“ (Band 5, S. 147-171). Schaffhausen: Schubi Lernmedien AG.

Stiftung Haus der kleinen Forscher (2013a). Monitoring-Bericht 2013 der Stiftung „Haus der kleinen Forscher". Verfügbar unter www.haus-der-kleinen-forscher.de

Stiftung Haus der kleinen Forscher (2013b). Sprudelgas und andere Stoffe - Mit Kita- und Grundschulkindern Chemie entdecken und dabei die sprachliche Entwicklung unterstützen. Berlin: Stiftung Haus der kleinen Forscher. Verfügbar unter https://www.hausder-kleinen-forscher.de

Stiftung Haus der kleinen Forscher (Hrsg.) (2015). Frankfurter Erklärung zur frühen sprachlichen und naturwissenschaftlichen Bildung. Verfügbar unter www.haus-der-kleinenforscher.de

Stiftung Haus der kleinen Forscher (2017a). Monitoring-Bericht 2016/2017 der Stiftung „Haus der kleinen Forscher“. Berlin: Stiftung Haus der kleinen Forscher. Verfügbar unter www.haus-der-kleinen-forscher.de 
Stiftung Haus der kleinen Forscher (2017b). Zertifizierung für Kitas, Horte und Grundschulen. So wird Ihre Einrichtung ein „Haus der kleinen Forscher“. (4. Auflage). Berlin: Stiftung Haus der kleinen Forscher. Verfügbar unter: www.haus-der-kleinen-forscher.de

Stiftung Haus der kleinen Forscher (2018). Tür auf! Mein Einstieg in Bildung für nachhaltige Entwicklung. Berlin: Stiftung Haus der kleinen Forscher. Verfügbar unter www.hausder-kleinen-forscher.de

Stiftung Haus der kleinen Forscher (in Vorb. a). Der Pädagogische Ansatz der Stiftung „Haus der kleinen Forscher". (6. Auflage). Berlin: Stiftung Haus der kleinen Forscher.

Stiftung Haus der kleinen Forscher (Hrsg.) (in Vorb. b). Entwicklungsverläufe pädagogischer Fach- und Lehrkräfte in der MINT-Bildung. Wissenschaftliche Untersuchungen zur Arbeit der Stiftung „Haus der kleinen Forscher“(Band 13).

Stiftung Haus der kleinen Forscher (Hrsg.). (in Vorb. c). Frühe Bildung für nachhaltige Entwicklung - Ziele und Gelingensbedingungen. Wissenschaftliche Untersuchungen zur Arbeit der Stiftung „Haus der kleinen Forscher"(Band 12).

Stiftung Haus der kleinen Forscher (Hrsg.) (in Vorb. d). Zieldimensionen für Multiplikatorinnen und Multiplikatoren früher MINT-Bildung. Wissenschaftliche Untersuchungen zur Arbeit der Stiftung „Haus der kleinen Forscher“"(Band 11). 


\section{Anhang}


Anhang I: Kodierung der

Forschungskreisphasen (EASI Science-L Teil 1) 


\begin{tabular}{|c|c|c|}
\hline & 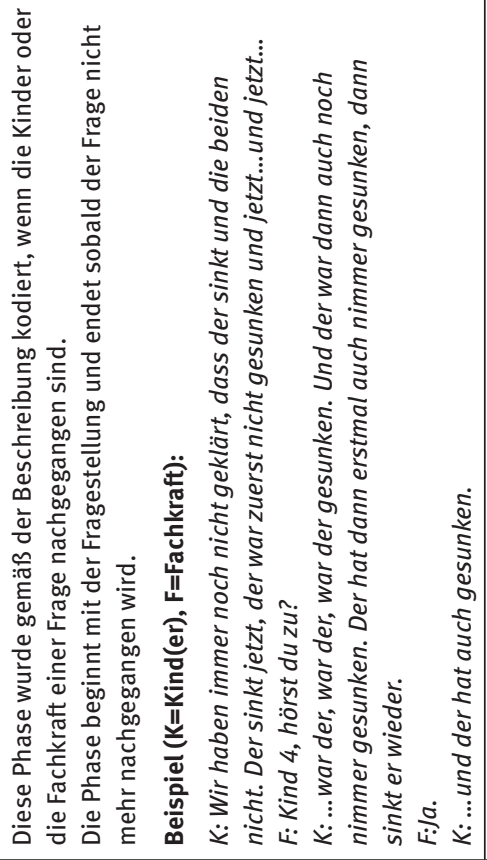 & 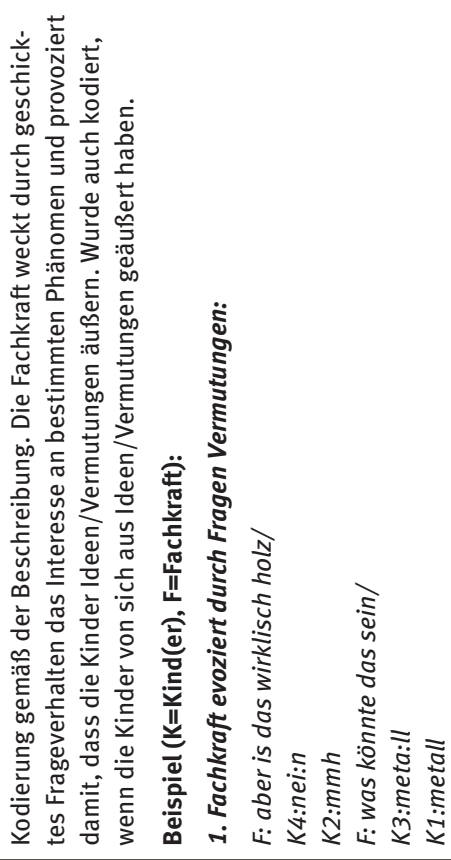 \\
\hline & 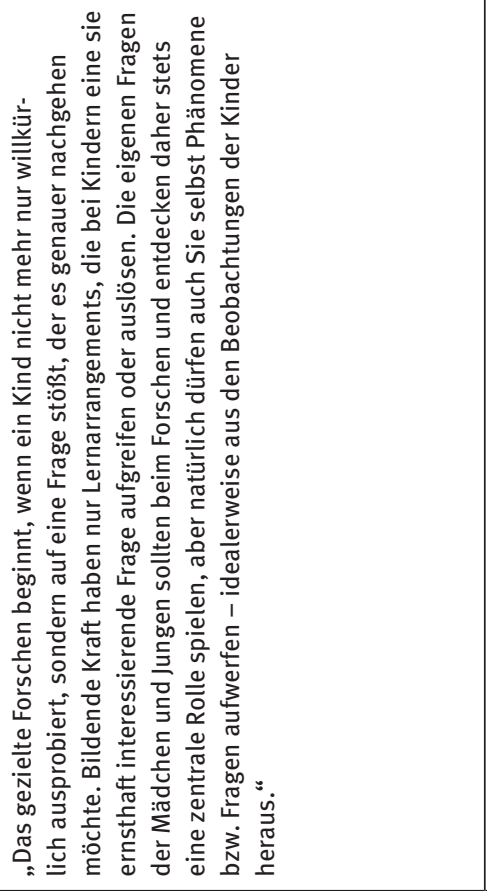 & 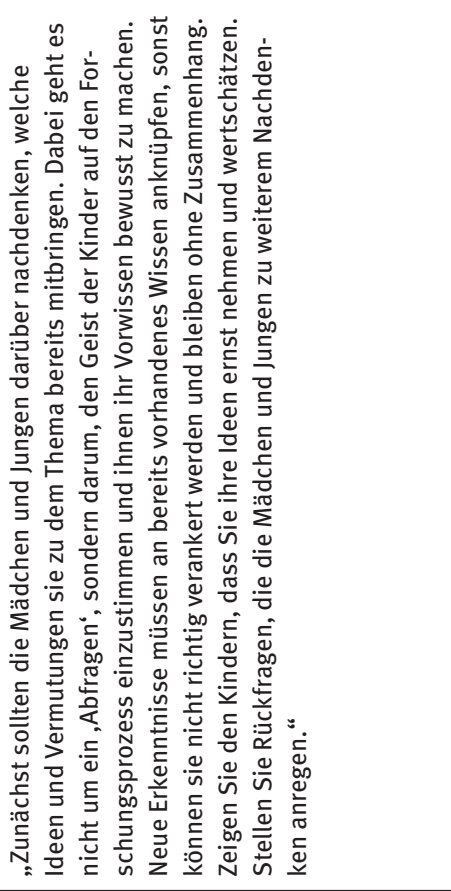 \\
\hline 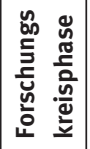 & 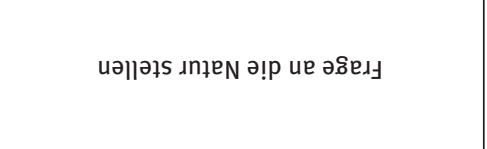 & 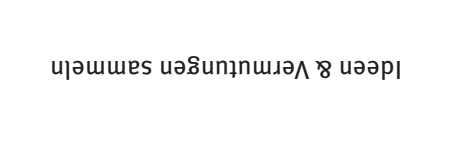 \\
\hline
\end{tabular}




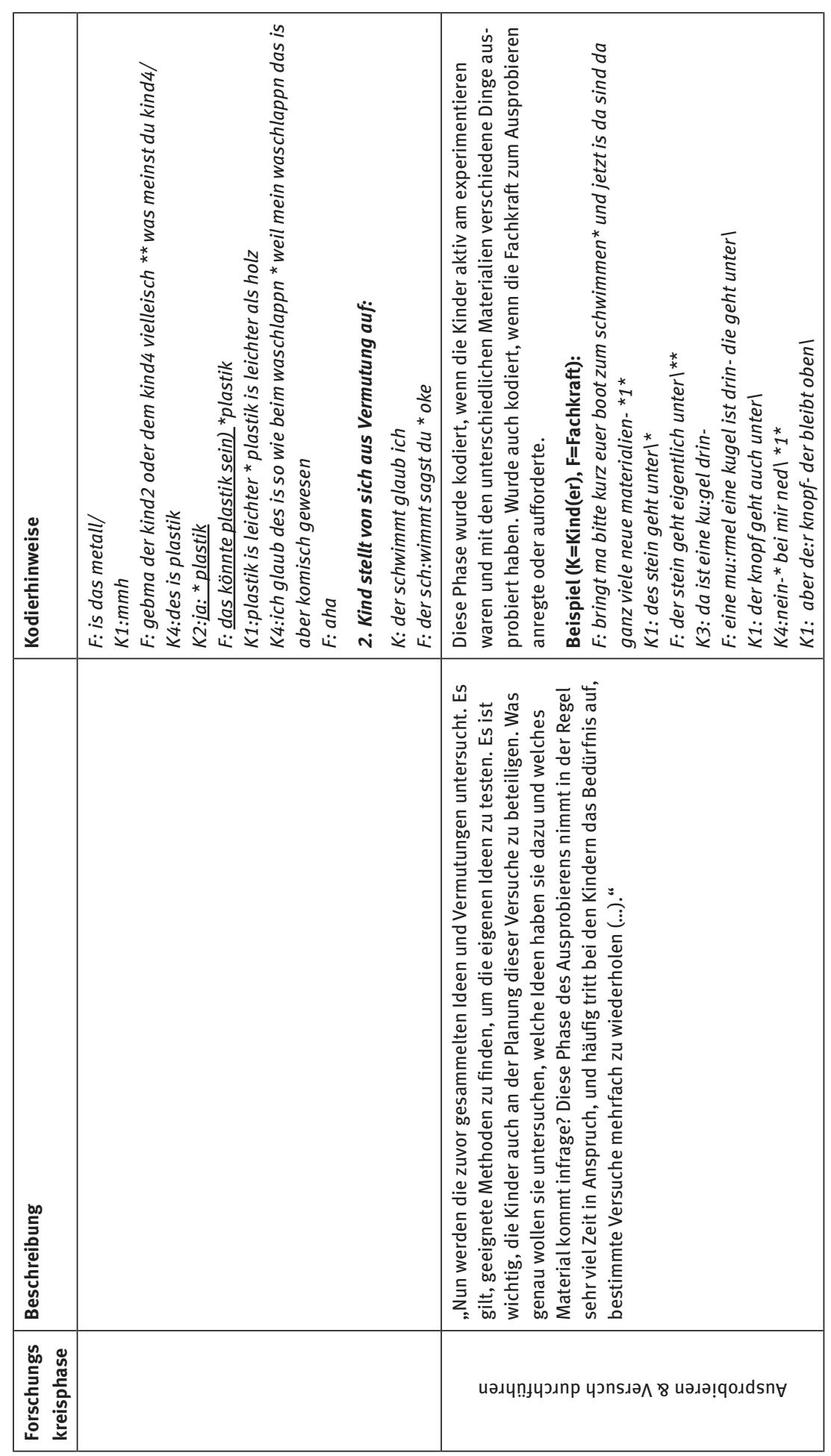




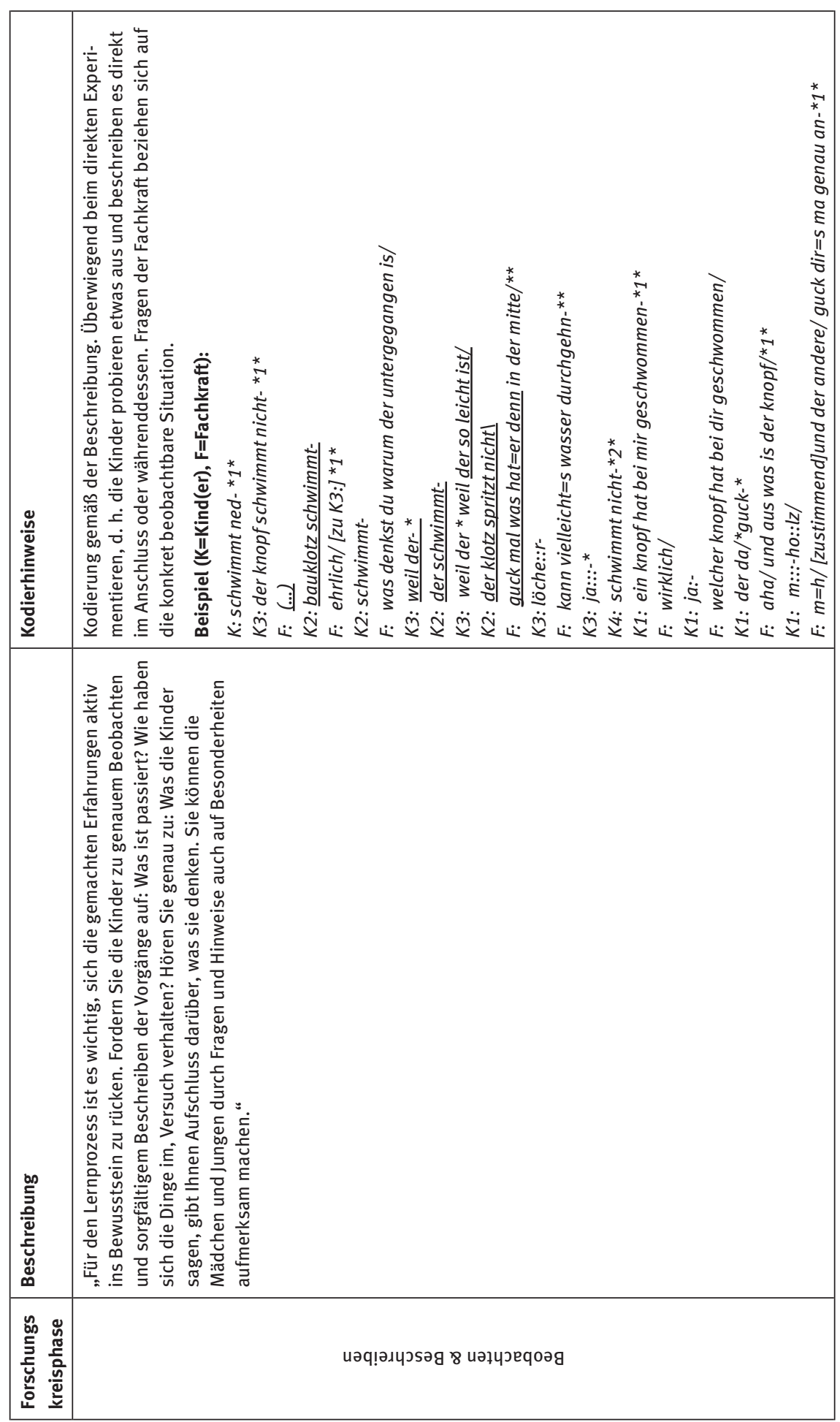




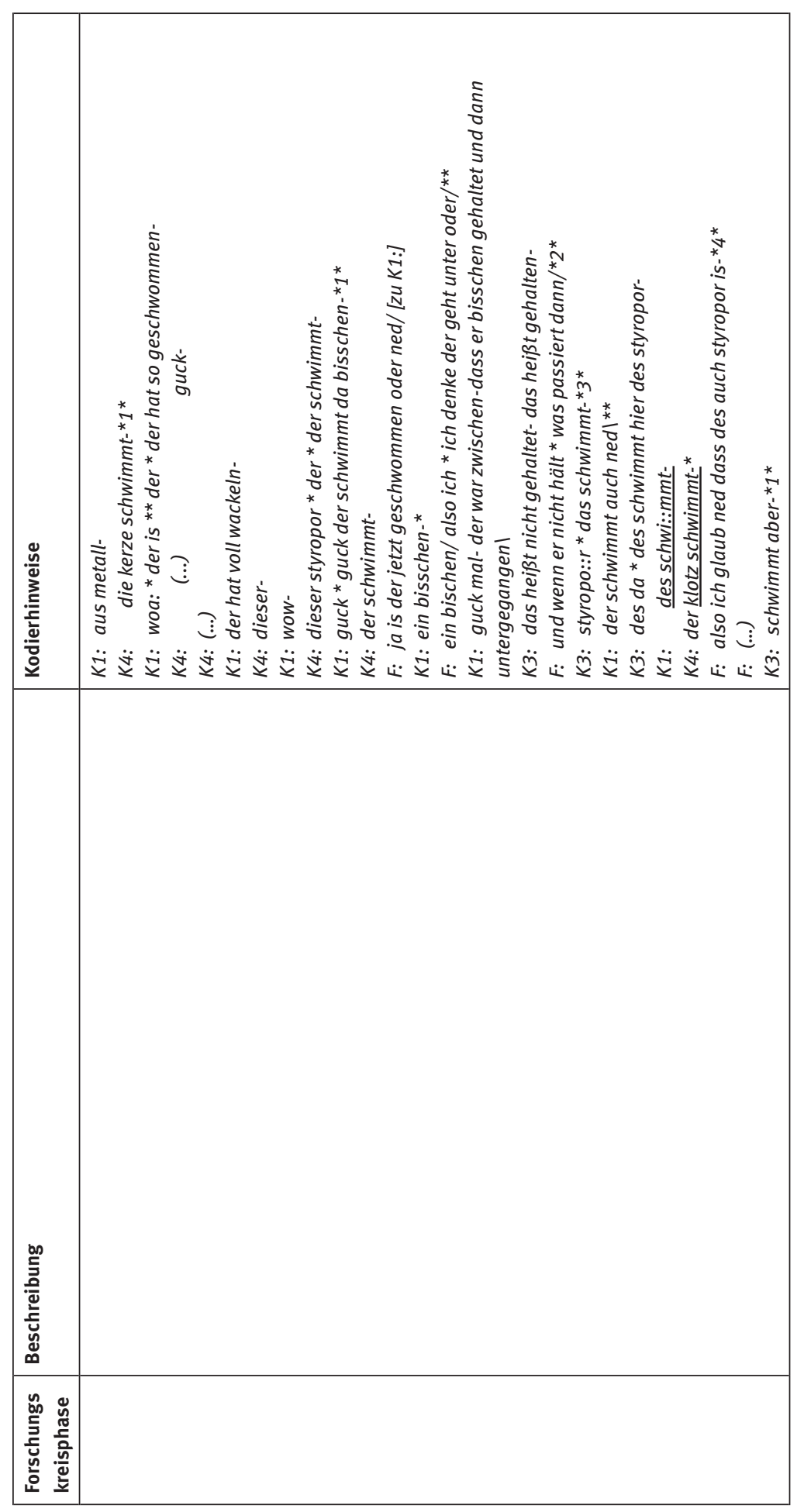




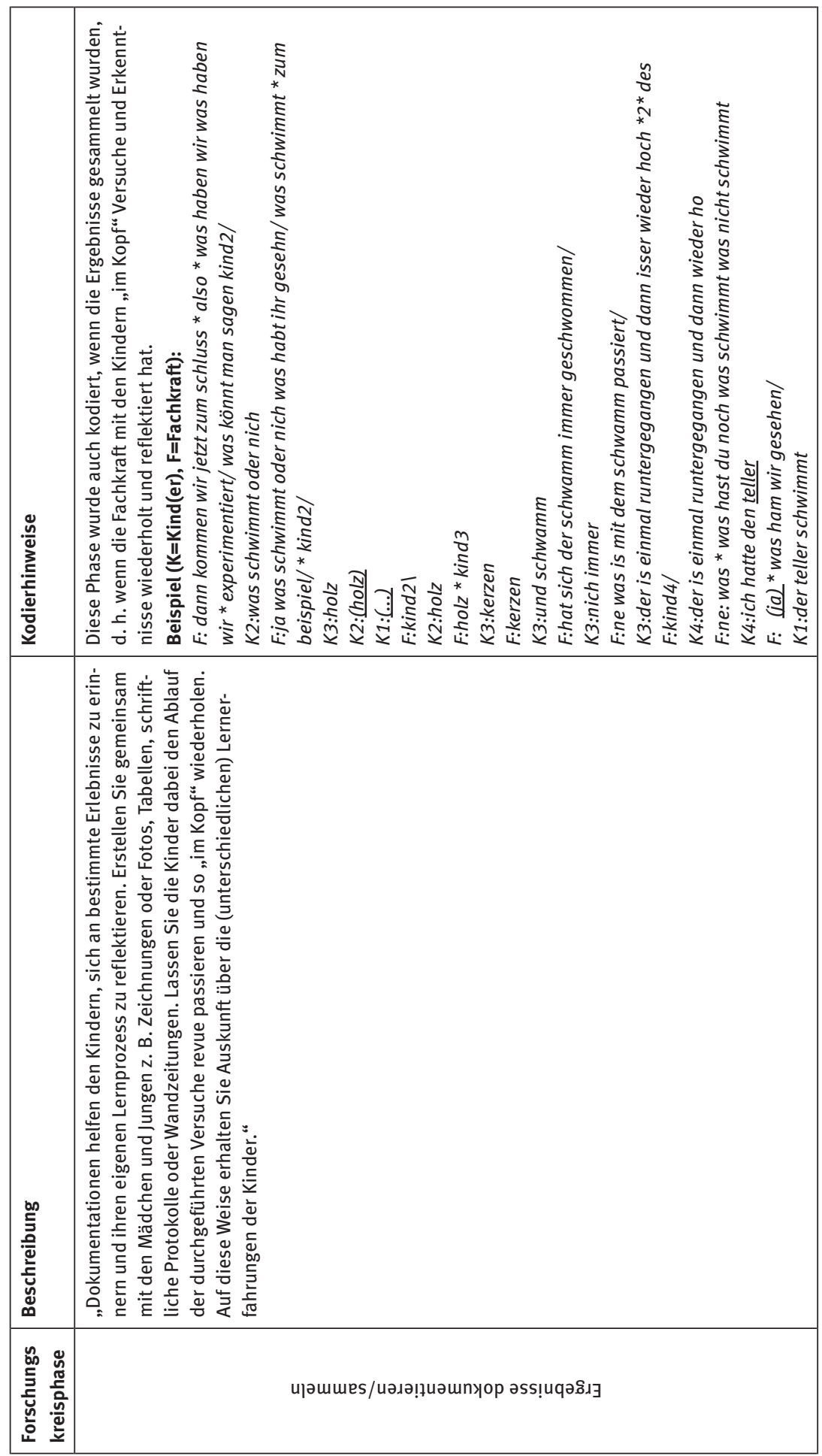




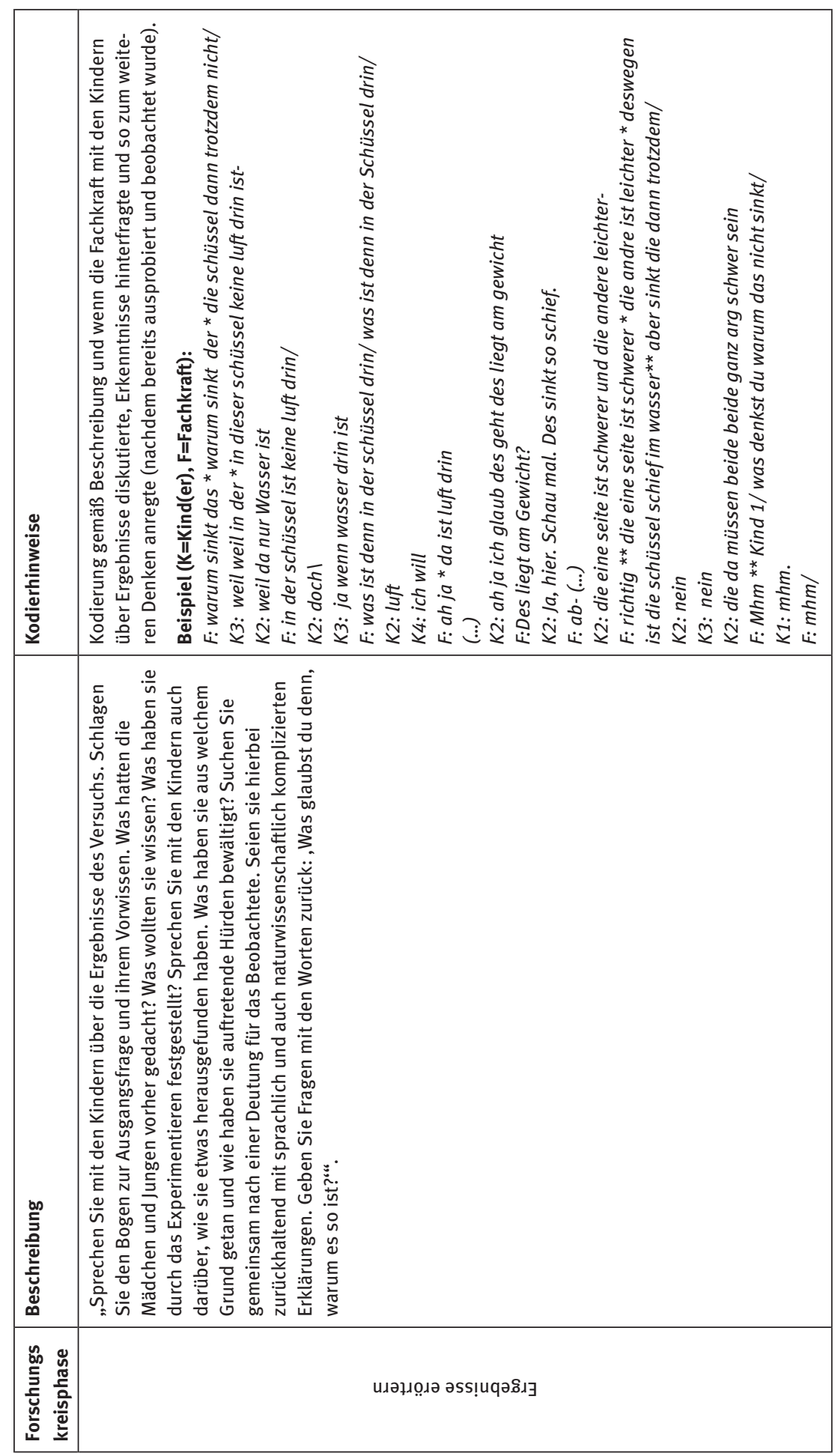




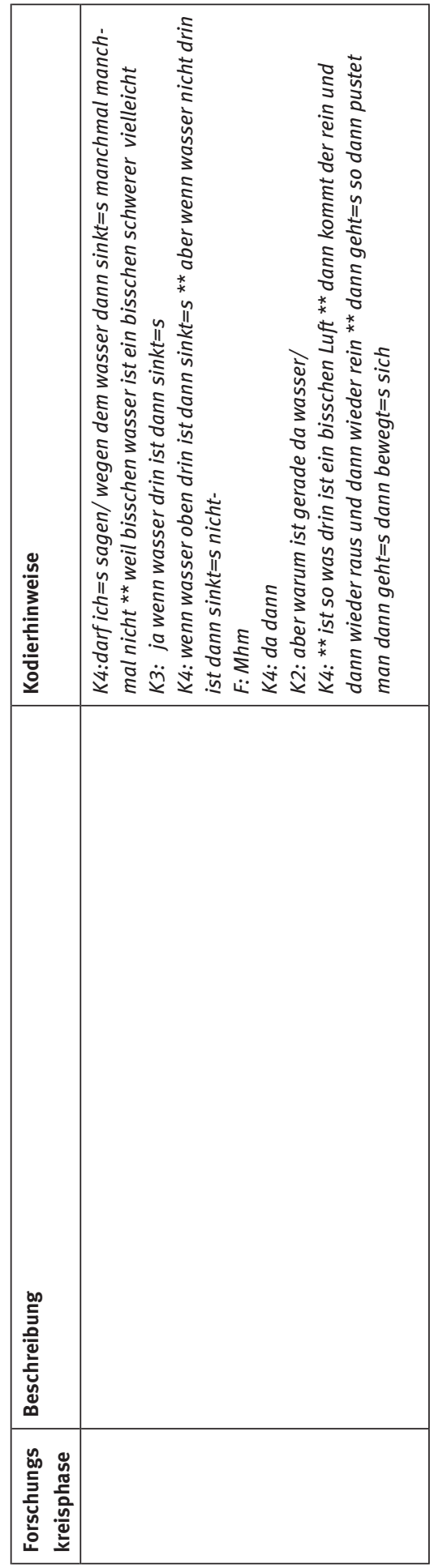


Anhang II: Beschreibung der Indikatoren sprachlicher Anregungsqualität

(EASI Science-L Teil 1) 


\section{Indikator 1: Impuls}

(aus Item 15: Informationen, Berichte einholen und Item 20: offene Fragen)

Fachkraft gibt einen Impuls, der zu einer umfangreicheren Beschreibung, einer Vermutung oder einer Erklärung führt. Äußerungen der Fachkraft, die rein sprachlich als Impuls gewertet werden könnten, die Kinder dadurch aber nicht angeregt wurden eine umfangreichere Beschreibung, Vermutung oder Erklärung zu äußern, werden nicht kodiert. Es ist davon auszugehen, dass in diesen Fällen andere Indikatoren (außer den rein sprachlichen) einer hochwertigen Interaktion nicht angemessen waren, beispielsweise wenn der Impuls in einer unangemessenen Situation erfolgte oder durch diesen eine Tätigkeit des Kindes unterbrochen wurde.

\section{Beispiel (K=Kind(er), F=Fachkraft):}

F: wie hört sich das an?

K3: wie wenn man mit dem fuss auf dem wasser ganz schnell darauf schlägt dann kann man auf dem wasser laufen

\section{Indikator 2: Benennungen erarbeiten}

\section{(aus Item 18: Vielfalt des Wortschatzes)}

Die Fachkraft erarbeitet eine Benennung mit einem Kind gemeinsam, z. B. durch raten, hinterfragen, ergänzen, Sprachwitz, Nonsens. Hierfür müssen Indikatoren vorhanden sein, die eine gemeinsame Erarbeitung repräsentieren bspw. lenkt die Fachkraft durch weitere Fragen das Kind zur richtigen Bezeichnung. Antwortet das Kind mit „weiß nicht“ und die Fachkraft beantwortet die Frage dann selbst, wird das nicht als gemeinsame Erarbeitung gewertet.

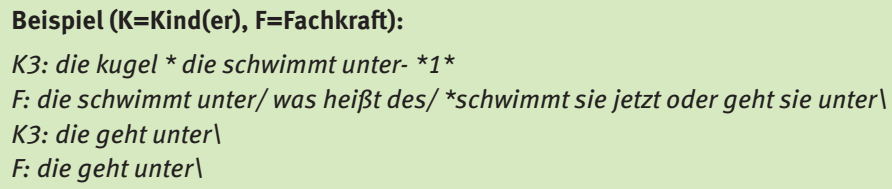

\section{Indikator 3: Variation des Wortschatzes}

(aus Item 18: Vielfalt des Wortschatzes)

Fachkraft verwendet in geeigneten Situationen auch Synonyme oder umschreibt komplexe Begriffe und Sachverhalte. 
Indikator 4: Sprachliche Unterstützung - Anbieten von Lückensätzen

(aus Item 19: Grammatisch komplexer Input)

Fachkraft bietet den Kindern in angemessenen Situationen stützende Lückensätze an.

Beispiel ( $\mathrm{K}=$ Kind(er), $\mathrm{F}=$ Fachkraft):

F: was isch=n passiert/ * nachdem die murmel gekippt isch/

dann hat se nämlich den/

K4: stein mit runter gedrückt hat sie alle mit runter gedrückt

Indikator 5: Wiederholung unvollständiger Aussagen

(aus Item 19: Grammatisch komplexer Input)

Fachkraft wiederholt und ergänzt bruchstückhafte oder falsche Äußerungen der Kinder.

Beispiel ( $\mathrm{K}=$ Kind(er), $\mathrm{F}=$ Fachkraft):

K1:ob des * runter taucht oder nich 1

F: genau * ob die gegenstände auf dem wasser oben bleiben

und schwimmen * oder * ob sie unter wasser sinken I

\section{Indikator 6: Längerer Satz}

(aus Item 19: Grammatisch komplexer Input)

Fachkraft verwendet längere Sätze. Diese müssen vollständig, nachvollziehbar und korrekt gebildet worden sein (unter Berücksichtigung der mündlichen Situation).

Beispiel (K=Kind(er), $\mathrm{F}=$ Fachkraft):

F: probiert mal die gleiche ladung-die ihr jetzt im becher habt- in die anderen * gefäße zu füllen $\backslash$ ihr habt noch ein schälchen und einen teller- ob des genauso funktioniert 


\section{Indikator 7: Aufgreifen einer Kinderäußerung}

(aus: Item 20: Offene Fragen)

Fachkraft greift eine Aussage auf und problematisiert diese und führt damit zu einem weiteren Austausch. Auch hier gelten dieselben Bedingungen wie bei Indikator 1 , Impuls geben.

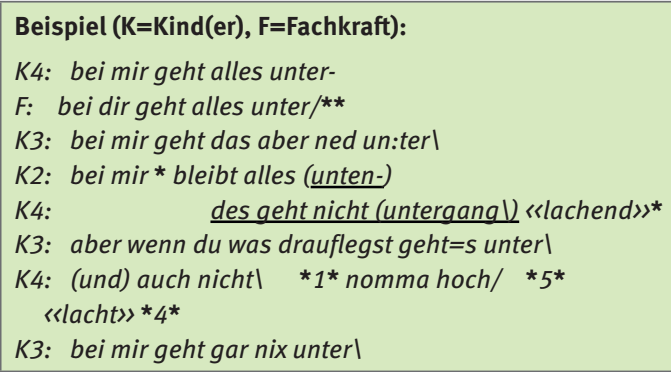

\section{Indikator 8: Zusammenfassung}

(aus Item 23: Zusammenhänge eines Themas erklären, hinterfragen)

Fachkraft fasst die Erkenntnisse der Kinder verständlich zusammen.

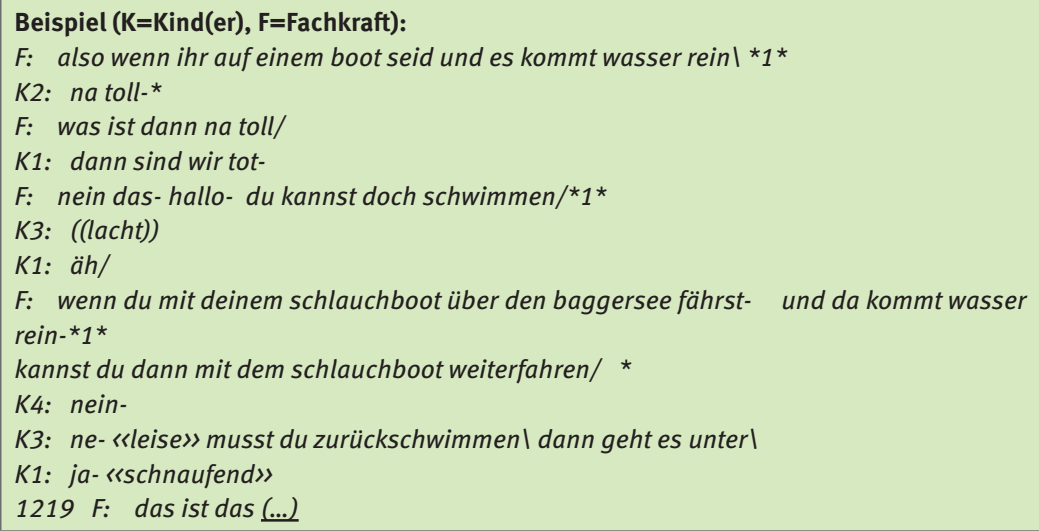




\section{Bildquellenverzeichnis}

Titelfoto, Seite 20, 40, 50, 60, 67, 99, 122, 129, 138, 140, 147, 154, 249, 252, 263, 270, 294 :

(c) Christoph Wehrer/Stiftung Haus der kleinen Forscher

Seite 13: ( Bettina Volke/Stiftung Haus der kleinen Forscher

Seite 194: ๑ Peter Lutz/Stiftung Haus der kleinen Forscher 


\section{Über die Stiftung „Haus der kleinen Forscher“}

Die gemeinnützige Stiftung „Haus der kleinen Forscher“ engagiert sich für gute frühe Bildung in den Bereichen Mathematik, Informatik, Naturwissenschaften und Technik (MINT) - mit dem Ziel, Mädchen und Jungen stark für die Zukunft zu machen und zu nachhaltigem Handeln zu befähigen. Gemeinsam mit ihren Netzwerkpartnern vor Ort bietet die Stiftung bundesweit ein Bildungsprogramm an, das pädagogische Fach- und Lehrkräfte dabei unterstützt, Kinder im Kita- und Grundschulalter qualifiziert beim Entdecken, Forschen und Lernen zu begleiten. Das „Haus der kleinen Forscher“ verbessert Bildungschancen, fördert Interesse am MINT-Bereich und professionalisiert dafür pädagogisches Personal. Partner der Stiftung sind die Helmholtz-Gemeinschaft, die Siemens Stiftung, die Dietmar Hopp Stiftung und die Deutsche Telekom Stiftung. Gefördert wird sie vom Bundesministerium für Bildung und Forschung.

\section{Vision und Mission der Stiftung „Haus der kleinen Forscher"}

Vision der Stiftung „Haus der kleinen Forscher“:

Fragen - Forschen - Zukunft gestalten

Alle Kinder in Deutschland erleben Bildungsorte, in denen sie ihren eigenen Fragen nachgehen und forschend die Welt entdecken können. Solche „Häuser der kleinen Forscher“ machen Mädchen und Jungen stark für die Zukunft. Sie befähigen Kinder, selbstbestimmt zu denken und verantwortungsvoll zu handeln.

Technologisierung und Digitalisierung sowie Folgen des Klimawandels und der sozialen Ungleichheit beeinflussen zunehmend unseren Altag. Wir tragen dazu bei, dass sich Menschen in unserer schnell verändernden Welt orientieren können und offen für Neues bleiben.

Die alltägliche Auseinandersetzung mit Natur und Technik fördert Neugier, Lern- und Denkfreude der Mädchen und Jungen. Wir sehen frühe Bildung als Schlüssel, um den Herausforderungen einer komplexen Welt erfolgreich begegnen zu können. 


\section{Mission der Stiftung „Haus der kleinen Forscher“:}

Die Stiftung „Haus der kleinen Forscher“...

befördert eine fragend-forschende Haltung bei Kindern,

- gibt Mädchen und Jungen schon in jungen Jahren die Chance, eigene Talente und Potenziale in den Bereichen Naturwissenschaften, Technik, Mathematik und Informatik zu entdecken

u und legt den Grundstein für einen reflektierten Umgang mit technologischen und gesellschaftlichen Veränderungen im Sinne einer nachhaltigen Entwicklung.

Gemeinsam mit ihren Bezugspersonen erleben die Kinder Spaß und Freude am Entdecken und Verstehen dieser Welt. Kinder gestalten Bildungsprozesse aktiv mit und erleben sich dadurch als kompetent und selbstwirksam in ihrem Alltag. Beim forschenden Lernen können Kinder Problemlösekompetenzen entwickeln, eigene Antworten finden und Selbstvertrauen spüren („Ich kann!“) - Erfahrungen und Fähigkeiten, die weit über die Kindheit hinaus für die Persönlichkeitsentwicklung und die spätere Berufsbiografie von Bedeutung sind.

In einem praxisnahen und qualitativ hochwertigen Professionalisierungsansatz unterstützt die Stiftung pädagogische Fach- und Lehrkräfte dabei, Kinder im Alter bis 10 Jahren beim Entdecken, Forschen und Lernen zu begleiten. Über vielfältige Fortbildungsangebote erleben Fach- und Lehrkräfte die Faszination eigenen Forschens für sich selbst. Sie erweitern ihre Kenntnisse und pädagogischen Kompetenzen und setzen sie in ihrer alltäglichen Arbeit mit Kindern um.

Die Initiative unterstützt Bildungseinrichtungen darin, sich als „Ort des forschenden Lernens" nachhaltig weiterzuentwickeln und in diesem Sinn als „Haus der kleinen Forscher“ förderliche Lernumgebungen für Kinder zu schaffen. 


\section{Bisher erschienen in der Wissenschaftlichen Schriftenreihe der Stiftung „Haus der kleinen Forscher“}
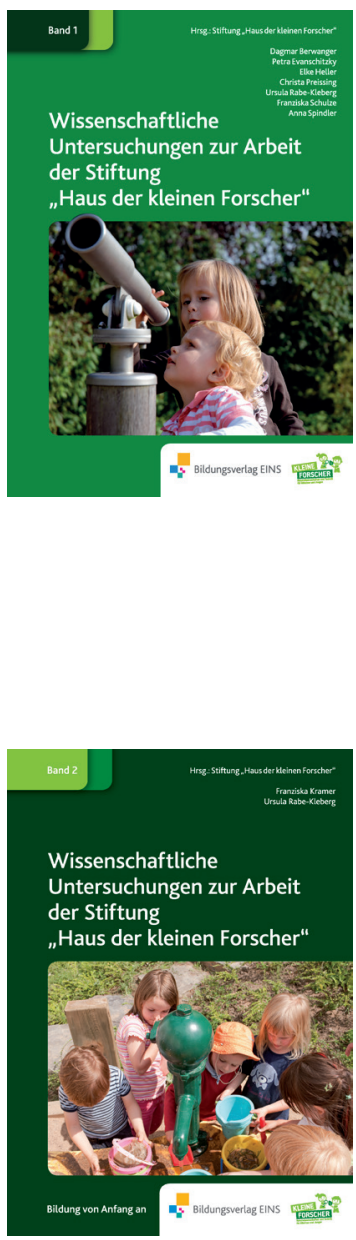

Band 1 (2011)

Dagmar Berwanger, Petra Evanschitzky, Elke Heller, Christa Preissing, Ursula Rabe-Kleberg, Franziska Schulze, Anna Spindler

Der erste Band der Schriftenreihe „Wissenschaftliche Untersuchungen zur Arbeit der Stiftung ,Haus der kleinen Forscher“" stellt vier wissenschaftliche Expertisen aus den Jahren 2009 und 2010 vor, die von renommierten Wissenschaftlerinnen und Wissenschaftlern aus dem Bereich der frühkindlichen Pädagogik verfasst wurden. Die vorliegenden wissenschaftlichen Beiträge reflektieren den pädagogischen Ansatz und das Multiplikatorenmodell der Stiftung vor dem Hintergrund der eigenen Zielvorstellungen, analysieren die Umsetzungspraxis in den Netzwerken und Kitas und zeigen weitere Entwicklungsmöglichkeiten auf.

\section{Band 2 (2011)}

Franziska Kramer, Ursula Rabe-Kleberg

Die Studie von Ursula Rabe-Kleberg und Franziska Kramer bildet eine logische Konsequenz und Ergänzung zur ersten Untersuchung der Autorinnen „Erzieherinnen und ihre Haltung zu Naturwissenschaft und Technik für Jungen und Mädchen“, die in Band 1 dieser Schriftenreihe veröffentlicht wurde (siehe oben). Die Autorinnen untersuchen die Gestaltung der Lernprozesse durch Erzieherinnen im Detail. Mit hoher Präzision und Sensibilität im Umgang mit den Möglichkeiten qualitativer Sozialforschung gelingt es den Autorinnen dabei, ko-konstruktive Augenblicke des gemeinsamen Lernens in Kitas einzufangen und intensiv im Hinblick auf wichtige Einflussvariablen zu reflektieren. 


\section{Band 3 (2012)}

Michael Fritz, Gabriele Grieshop, Katrin Hille, Maren Lau, Martin Winter

Im dritten Band werden zwei Studien vorgestellt, die sich mit der Rolle der Bedeutung der Trainerinnen und Trainer in der Initiative „Haus der kleinen Forscher“ aus jeweils unterschiedlichen Perspektiven beschäftigen: In der Studie von Maren Lau, Michael Fritz und Katrin Hille (ZNL) stehen das Rollen- und Selbstverständnis der Trainerinnen und Trainer sowie ihr subjektives Kompetenzempfinden im Mittelpunkt. In der Untersuchung von Gabriele Grieshop und Martin Winter (Institut für Didaktik der Mathematik und des Sachunterrichts (IFD), Universität Vechta) wird - im Rahmen einer vorwiegend formativen Implementierungsevaluation am Beispiel Mathematik - die Beteiligung der Trainerinnen und Trainer an der Konzept- und Materialentwicklung von Angeboten der Stiftung „Haus der kleinen Forscher" betrachtet.

\section{Band 4 (2012)}

\section{Salman Ansari, Susanna Jeschonek, Janna Pahnke, Sabina Pauen}

Band 4 enthält vier Expertisen, die basierend auf aktuellen entwicklungspsychologischen Erkenntnissen Empfehlungen für die Entwicklung weiterer naturwissenschaftlicher, technischer und mathematischer Themenschwerpunkte der Stiftung „Haus der kleinen Forscher“ aussprechen, auf mögliche Stolpersteine hinweisen und Vorschläge für die Praxis aufzeigen. Die Expertise von Janna Pahnke und Sabina Pauen gibt einen Überblick über die Entwicklung des mathematischen und naturwissenschaftlichen Denkens und Wissens in der frühen Kindheit und zieht Schlussfolgerungen für eine darauf aufbauende frühe Bildung in diesen Bereichen. Susanna Jeschoneks Expertisen behandeln die Entwicklung des kindlichen Verständnisses der Bereiche ,Magnetismus‘ und ,Akustik“ und geben Empfehlungen für die Aufbereitung von Bildungsangeboten zu diesen Themenschwerpunkten in der Praxis. In der Expertise von Salman Ansari stehen Prozesse des Lehrens und Lernens aus der Sicht der kognitiven Wissenschaften im Mittelpunkt. Ansari geht auf verschiedene Konzepte und Angebote der Stiftung „Haus der kleinen Forscher“ ein, spricht Empfehlungen für die Weiterentwicklung dieser Themen aus und verdeutlicht dies anhand von konkreten Beispielen für die praktische Umsetzung.
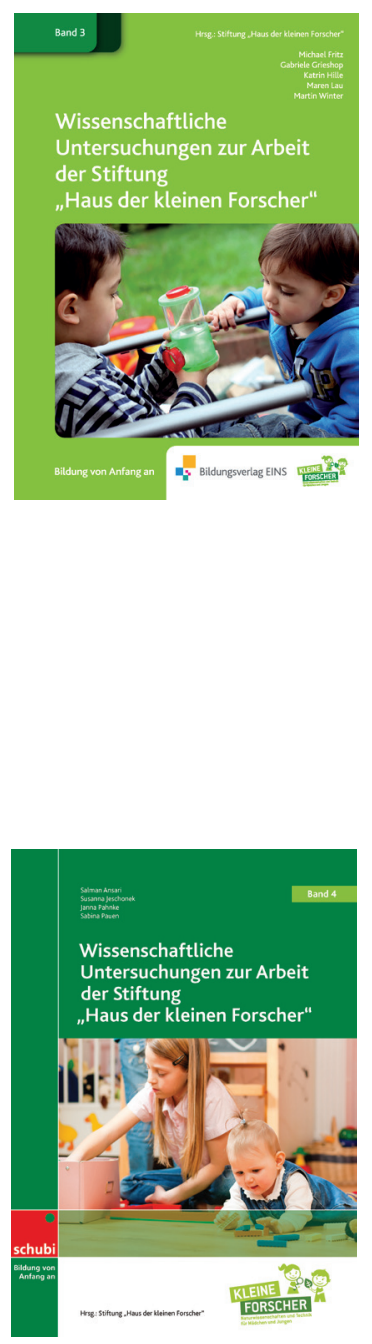


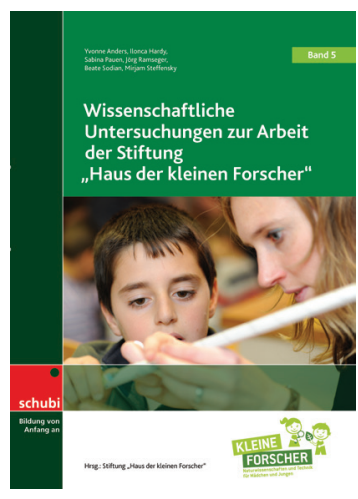

Band 5 (2013)

Yvonne Anders, Ilonca Hardy, Sabina Pauen, Jörg Ramseger, Beate Sodian, Mirjam Steffensky

Der fünfte Band stellt Ziele naturwissenschaftlicher Bildung für Kinder und pädagogische Fach-und Lehrkräfte sowie prozessbezogene Qualitätskriterien für den naturwissenschaftlichen Unterricht im Elementar- und Primarbereich in den Fokus. Yvonne Anders, Ilonca Hardy, Sabina Pauen, Beate Sodian und Mirjam Steffensky spezifizieren in ihren Expertisen pädagogisch-inhaltliche Zieldimensionen naturwissenschaftlicher Bildung im Kita- und Grundschulalter. Neben einer theoretischen Fundierung verschiedener Zielbereiche werden Instrumente für deren Messung aufgeführt. Jörg Ramseger formuliert in seiner Expertise zehn Qualitätskriterien für den naturwissenschaftlichen Unterricht. Diese prozessbezogenen Kriterien können pädagogische Fach- und Lehrkräfte bei der Unterrichtsplanung sowie bei der Selbstevaluation naturwissenschaftlicher Angebote im Elementar- und Primarbereich unterstützen.

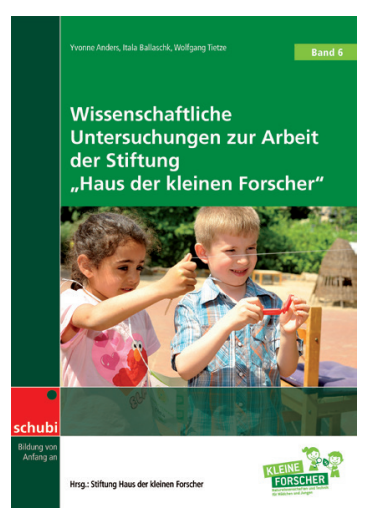

\section{Band 6 (2014)}

Yvonne Anders, Itala Ballaschk, Wolfgang Tietze

Im sechsten Band der Schriftenreihe mit einem Geleitwort von Wolfgang Tietze berichten Yvonne Anders und Itala Ballaschk die Ergebnisse ihrer Studie zur Untersuchung der Reliabilität und Validität des Zertifizierungsverfahrens der Stiftung „Haus der kleinen Forscher“, mit dem sich Bildungseinrichtungen nach bestimmten Qualitätskriterien als „Haus der kleinen Forscher“ zertifizieren lassen können. Insgesamt konnte in der Studie das Potenzial des Verfahrens für die Messung der naturwissenschaftsbezogenen Bildungsqualität in pädagogischen Einrichtungen belegt und Ansatzpunkte für weitere Optimierungen aufgezeigt werden. 


\section{Band 7 (2015)}

Gabriele Graube, Maja Jeretin-Kopf, Walter Kosack, Ingelore Mammes, Ortwin Renn, Christian Wiesmüller

Der siebte Band der Reihe mit Geleitwort von Ortwin Renn fokussiert Ziele und Konzepte technischer Bildung im Elementar- und Primarbereich. Walter Kosack, Maja Jeretin-Kopf und Christian Wiesmüller spezifizieren in ihrer Expertise pädagogisch-inhaltliche Zieldimensionen technischer Bildung im Kita- und Grundschulalter. Neben einer theoretischen Fundierung verschiedener Zielbereiche werden Instrumente für deren Messung aufgeführt. Die Autoren stellen in zwei Berichten die Ergebnisse empirischer Studien dar. Zum einen wurde der Einfluss verschiedener technikdidaktischer Materialsysteme auf die kindliche Motivation, problemlösendes Denken und technische Kreativität, und zum anderen der Einfluss verschiedener technikdidaktischer Methoden auf die kindliche Motivation sowie technikspezifische Denk- und Handlungsweisen untersucht. Gabriele Graube und Ingelore Mammes beschreiben in ihrem Beitrag ein didaktisches Konzept zur Unterstützung des professionellen Handelns pädagogischer Fach- und Lehrkräfte bei der Begleitung kindlicher Bildungsprozesse in ihrer Auseinandersetzung mit Natur und Technik.

\section{Band 8 (2017)}

Christiane Benz, Meike Grüßing, Jens Holger Lorenz, Kristina Reiss, Christoph Selter, Bernd Wollring

Der achte Band der Reihe mit einem Geleitwort von Kristina Reiss stellt die Ziele und Gelingensbedingungen mathematischer Bildung im Elementar- und Primarbereich in den Fokus. Christiane Benz, Meike Grüßing, Jens Holger Lorenz, Christoph Selter und Bernd Wollring spezifizieren in ihrer Expertise pädagogisch-inhaltliche Zieldimensionen mathematischer Bildung im Kita- und Grundschulalter. Neben einer theoretischen Fundierung verschiedener Zielbereiche werden Instrumente für deren Messung aufgeführt. Des Weiteren erörtern die Autorinnen und Autoren Gelingensbedingungen für eine effektive und wirkungsvolle frühe mathematische Bildung in der Praxis. Sie geben zudem Empfehlungen für die Weiterentwicklung der Stiftungsangebote und die wissenschaftliche Begleitung der Stiftungsarbeit im Bereich Mathematik. Das Schlusskapitel des Bandes beschreibt die Umsetzung dieser fachlichen Empfehlungen in den inhaltlichen Angeboten der Stiftung „Haus der kleinen Forscher“.
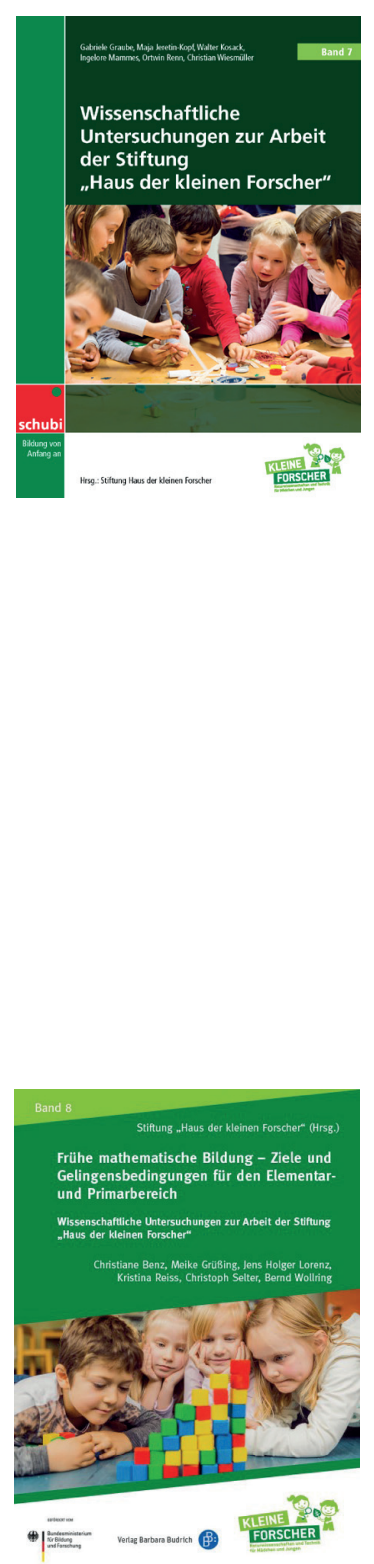


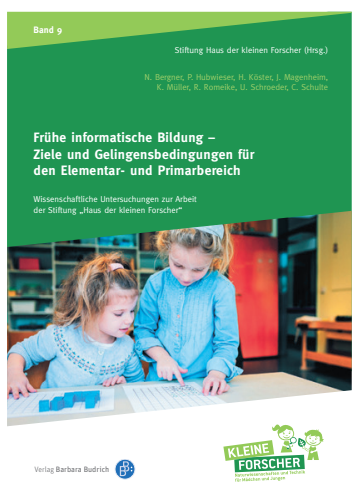

Band 9 (2018)

Nadine Bergner, Peter Hubwieser, Hilde Köster, Johannes Magenheim, Kathrin Müller, Ralf Romeike, Ulrik Schroeder, Carsten Schulte

Der neunte Band mit einem Geleitwort/Streitgespräch von Peter Hubwieser und Johannes Magenheim stellt die Ziele und Gelingensbedingungen informatischer Bildung im Elementar- und Primarbereich in den Fokus.

Nadine Bergner, Hilde Köster, Johannes Magenheim, Kathrin Müller, Ralf Romeike, Ulrik Schroeder und Carsten Schulte spezifizieren in ihrer Expertise pädagogisch-inhaltliche Zieldimensionen informatischer Bildung im Kita- und Grundschulalter. Neben einer theoretischen Fundierung verschiedener Zielbereiche erörtern die Autorinnen und Autoren Gelingensbedingungen für eine effektive und wirkungsvolle frühe informatische Bildung in der Praxis. Sie geben zudem Empfehlungen für die Entwicklung der Stiftungsangebote und die wissenschaftliche Begleitung der Stiftungsarbeit im Bereich Informatik. Nadine Bergner und Kathrin Müller beschreiben in ihrer Fachempfehlung eine Auswahl an Informatiksystemen für Kinder im Kita- und Grundschulalter und geben anhand fachlicher Kriterien Empfehlungen für besonders geeignete Informatiksysteme und deren Verwendung im Elementar- und Primarbereich.

Das Schlusskapitel des Bandes beschreibt die Umsetzung dieser fachlichen Empfehlungen in den inhaltlichen Angeboten der Stiftung „Haus der kleinen Forscher" - mit und ohne Computer. 